\title{
Sinusiaetone A, an Anti-inflammatory Norditerpenoid with \\ Bicyclo[11.3.0]hexadecane Nucleus from the Hainan Soft Coral
}

\section{Sinularia siaesensis}

Zi-Hui Chen, ${ }^{\dagger}$, Wang-Sheng Li, ${ }^{\dagger}, \S$ Zai-Yong Zhang, ${ }^{\dagger}$ Hui Luo,,${ }^{\S}$ Jian-Rong Wang, ${ }^{\dagger}$ Hai-Yan Zhang, ${ }^{*, \# ~ Z i-R o n g ~ Z e n g, ~}{ }^{\dagger}$ Bao Chen, ${ }^{\dagger} \mathrm{Xu}-\mathrm{Wen} \mathrm{Li,},+,+$ and Yue-Wei Guo ${ }^{*, \dagger, *}$

\footnotetext{
$\dagger$ State Key Laboratory of Drug Research, Shanghai Institute of Materia Medica, Chinese Academy of Sciences, 555 Zu Chong Zhi Road, Zhangjiang Hi-Tech Park, Shanghai 201203, China

$¥$ University of Chinese Academy of Sciences, No. 19A Yuquan Road, Beijing 100049, China

$\S$ The Key Lab of Zhanjiang for R\&D Marine Microbial Resources in the Beibu GulfRim, the Marine Biomedical Research Institute, Guangdong Medical University, Zhanjiang 524023, China

\# CAS Key Laboratory of Receptor Research, Shanghai Institute of Materia Medica, Chinese Academy of Sciences, 555 Zu Chong Zhi Road, Zhangjiang Hi-Tech Park, Shanghai 201203, China
} 


\section{Contents}

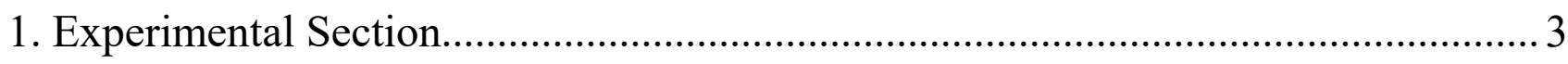

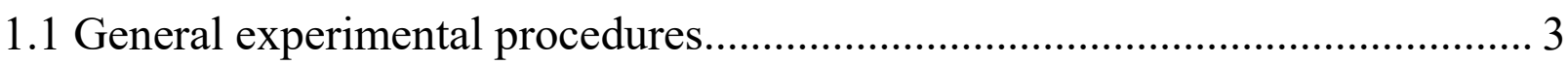

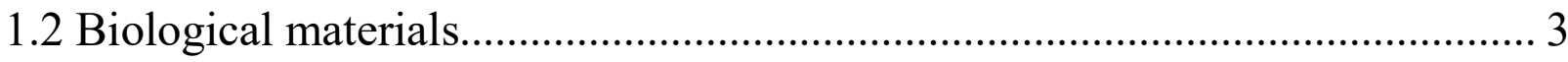

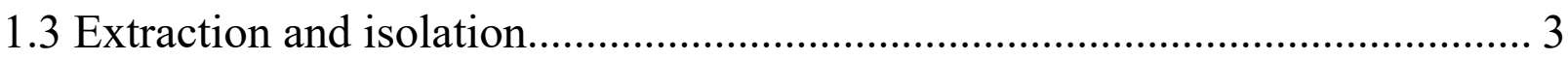

1.4 Lipopolysaccharide-induced BV-2 Inflammation..........................................4

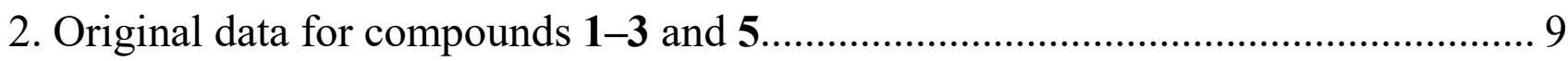

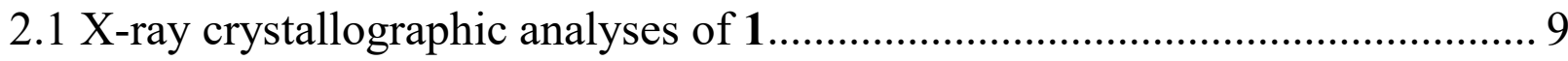

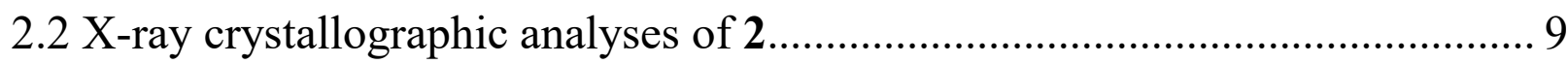

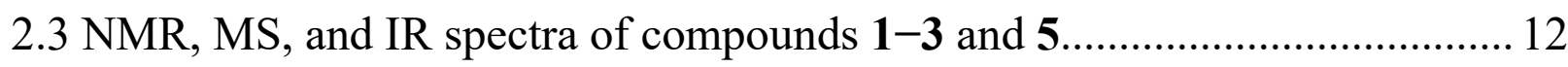

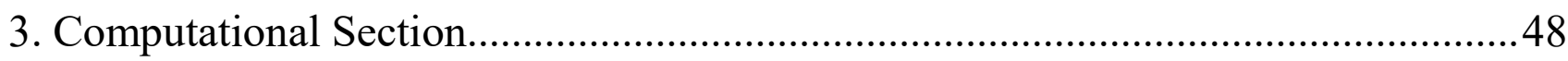

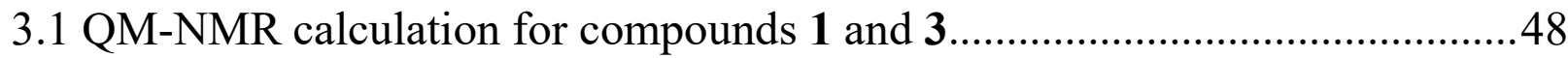

3.1.1 QM-NMR calculation for compound 1............................................. 48

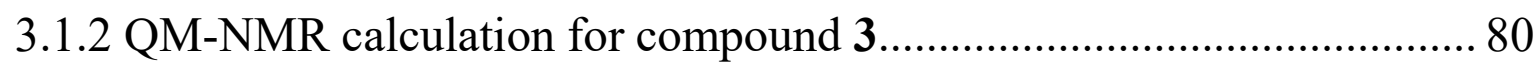




\section{Experimental Section}

\subsection{General experimental procedures}

Melting points were measured on an X-4 digital micro-melting point apparatus. Optical rotations were measured on a Perkin-Elmer $241 \mathrm{MC}$ polarimeter (PerkinElmer, Fremont, CA, USA). IR spectra was recorded on a Nicolet 6700 spectrometer (Thermo Scientific, Waltham, MA, USA); peaks are reported in $\mathrm{cm}^{-1}$. The NMR spectra were measured at $300 \mathrm{~K}$ on Bruker DRX 400, DRX 500 and Avance $600 \mathrm{MHz}$ NMR spectrometers (Bruker Biospin AG, Fallanden, Germany); Chemical shifts are reported in parts per million $(\delta)$ in $\mathrm{CDCl}_{3}\left(\delta_{\mathrm{H}}\right.$ reported referred to $\mathrm{CHCl}_{3}$ at 7.26 ppm; $\delta_{\mathrm{C}}$ reported referred to $\mathrm{CDCl}_{3}$ at $\left.77.16 \mathrm{ppm}\right)$ and coupling constants $(J)$ in $\mathrm{Hz}$; assignments were supported by ${ }^{1} \mathrm{H}-{ }^{1} \mathrm{H}$ COSY, HSQC, HMBC, and NOESY experiments. LR-ESIMS and HR-ESIMS were carried out on a Bruker Daltonics Esquire 3000 plus instrument (Bruker Daltonics K. K., Kanagawa, Japan) and a Waters Q-TOF Ultima mass spectrometer (Waters, MA, USA), respectively. Semi-preparative HPLC was performed on an Agilent-1260 system equipped with a DAD G1315D detector using ODS-HG-5 $(250 \mathrm{~mm} \times 9.4 \mathrm{~mm}, 5 \mu \mathrm{m})$ by eluting with $\mathrm{CH}_{3} \mathrm{OH}-\mathrm{H}_{2} \mathrm{O}$ or $\mathrm{CH}_{3} \mathrm{CN}-\mathrm{H}_{2} \mathrm{O}$ system at $2.5 \mathrm{~mL} / \mathrm{min}$. Commercial silica gel (100-200, 200-300 and 300-400 mesh; Qingdao, China) was used for column chromatography (CC). Precoated $\mathrm{SiO}_{2}$ plates (HSGF-254; Yantai, China) were used for analytical TLC. Spots were detected on TLC under UV light or by heating after spraying with anisaldehyde $\mathrm{H}_{2} \mathrm{SO}_{4}$ reagent. Sephadex LH-20 (Amersham Biosciences) was also used for CC. All solvents used for column chromatography and HPLC were of analytical grade (Shanghai Chemical Reagents Co., Ltd.) and chromatographic grade (Dikma Technologies Inc.), respectively.

\subsection{Biological materials}

Specimens of Sinularia siansensis, identified by Prof. Xiu-Bao Li from Hainan university, were collected along the coast of Ximao Island, Hainan province, China, in May 2019, at a depth of -20m, and were frozen immediately after collection. A voucher specimen is available for inspection at Shanghai Institute of Materia Medica, SIBS-CAS (No. 19XD-15).

\subsection{Extraction and isolation}

The frozen animals (780 g, dry weight) were cut into pieces and extracted exhaustively with acetone at room temperature $(4 \times 4.0 \mathrm{~L})$. The organic extract was evaporated to give a brown residue, which was then partitioned between $\mathrm{Et}_{2} \mathrm{O}$ and $\mathrm{H}_{2} \mathrm{O}$. The upper layer was concentrated under reduced pressure to give a brown residue $25.0 \mathrm{~g}$. The resulted residue was separated into nine fractions (A-I) by gradient silica gel column chromatography (200-300 mesh, 0-100\% $\mathrm{Et}_{2} \mathrm{O}$ in light petroleum ether). Fraction $\mathrm{B}(1.3 \mathrm{~g})$, eluted with $\mathrm{PE} / \mathrm{DCM} / \mathrm{CH}_{3} \mathrm{OH}(2: 1: 1)$, was initially chromatographed over Sephadex LH-20 column, affording subfractions (BA and BB). Subfraction BA was further subjected to silica gel $\mathrm{CC}$ (column: $20 \mathrm{~cm} \times 2 \mathrm{~cm}$ ) eluting with $\mathrm{PE}-\mathrm{Et}_{2} \mathrm{O}$ (98:2) to give four subfractions (BAA-BAD). Purification of subfraction BAC by semi-preparative RP-HPLC $\left(\mathrm{CH}_{3} \mathrm{CN}-\mathrm{H}_{2} \mathrm{O}, 80: 20\right)$ yielded compound $1\left(0.8 \mathrm{mg}, \mathrm{t}_{\mathrm{R}}=8.9 \mathrm{~min}\right)$. Compound 1 was recrystallized in $\mathrm{CH}_{3} \mathrm{OH}$ to obtain its suitable quality crystals. Fraction E $(862.8 \mathrm{mg})$, eluted with $\mathrm{PE} / \mathrm{DCM} / \mathrm{CH}_{3} \mathrm{OH}$ (2:1:1), was initially chromatographed over Sephadex LH-20 column, affording subfractions (EA-EE). Subfraction EB was further separated by silica gel CC (300-400 mesh), eluting with $\mathrm{PE}-\mathrm{Et}_{2} \mathrm{O}(9: 1)$ to give five subfractions (EBA-EBE) and subfraction EBE was further purified by semi-preparative RP-HPLC $\left(\mathrm{CH}_{3} \mathrm{CN}-\mathrm{H}_{2} \mathrm{O}, 50: 50\right)$ to afford compound 5 (10.8 $\left.\mathrm{mg}, \mathrm{t}_{\mathrm{R}}=17.8 \mathrm{~min}\right)$. Purification of subfraction EC by semi-preparative RP-HPLC $\left(\mathrm{CH}_{3} \mathrm{CN}-\mathrm{H}_{2} \mathrm{O}, 60: 40\right)$ yielded 
compound $3\left(1.0 \mathrm{mg}, \mathrm{t}_{\mathrm{R}}=14.6 \mathrm{~min}\right)$. Fraction $\mathrm{G}(962.3 \mathrm{mg})$ was first split by Sephadex LH-20 column chromatography (PE/DCM/MeOH, 2:1:1) to give two sub-fractions (GA and GB). Subfraction GA was purified by Si-gel column chromatography (300-400 mesh, PE: $\mathrm{Et}_{2} \mathrm{O}$ 5:1), yielding sub-fraction GAB. Purification of subfraction GAB by semi-preparative RP-HPLC $\left(\mathrm{CH}_{3} \mathrm{OH}-\mathrm{H}_{2} \mathrm{O}, 40: 60\right)$ yielded compound 4 (8.6 mg, $\left.\mathrm{t}_{\mathrm{R}}=15.8 \mathrm{~min}\right)$. Fraction $\mathrm{H}(813.6 \mathrm{mg})$ was first split by Sephadex LH-20 column chromatography (PE/DCM/MeOH, 2:1:1) to give three sub-fractions (HA-HC). Subfraction HC was purified by Si-gel column chromatography (300-400 mesh, PE: $\mathrm{Et}_{2} \mathrm{O}$ 1:1), yielding sub-fraction HCC. Purification of subfraction HCC by semi-preparative RP-HPLC $\left(\mathrm{CH}_{3} \mathrm{OH}-\mathrm{H}_{2} \mathrm{O}, 30: 70\right)$ yielded compound 2 (1.8 $\left.\mathrm{mg}, \mathrm{t}_{\mathrm{R}}=7.2 \mathrm{~min}\right)$. Compound 2 was recrystallized in $\mathrm{CH}_{3} \mathrm{OH} / \mathrm{DCM}(2: 1)$ to obtain its suitable quality crystals.

Sinusiaetone A (1) $(0.8 \mathrm{mg})$ : Colorless crystals; $[\alpha]_{\mathrm{D}}{ }^{16}+20.0(c 0.01 \mathrm{MeOH})$; IR $(\mathrm{KBr}) v_{\max }=2954$, 2924, 2854, 1715, 1457, 1384, 1260, 1032, $803 \mathrm{~cm}^{-1}$; $\mathrm{CD}\left(\mathrm{CH}_{3} \mathrm{CN}\right) \lambda(\Delta \varepsilon) 238.8(-0.95), 288.2$ $(+1.04) ;{ }^{1} \mathrm{H}$ and ${ }^{13} \mathrm{C}$ NMR data see Table 1; HRMS (ESI) $m / z$ : $[\mathrm{M}+\mathrm{Na}]^{+}$Calcd. for $\mathrm{C}_{19} \mathrm{H}_{28} \mathrm{NaO}_{2}$, 311.1982; Found 311.1981.

Sinusiaeolide A (2) (1.8 mg): Colorless crystals; $[\alpha]_{\mathrm{D}}{ }^{18}+83.5$ (c $\left.0.18 \mathrm{MeOH}\right)$; IR $(\mathrm{KBr}) v_{\max }=3440$, 2928, 2866, 1714, 1455, 1384, 1242, 1135, 1100, 1084, $1052 \mathrm{~cm}^{-1} ;{ }^{1} \mathrm{H}$ and ${ }^{13} \mathrm{C}$ NMR data see Table S1; HRMS (ESI) $m / z$ : $[\mathrm{M}+\mathrm{Na}]^{+}$Calcd. for $\mathrm{C}_{20} \mathrm{H}_{30} \mathrm{NaO}_{5}$, 373.1985; Found 373.1978.

Sinusiaesin A (3) (1.0 mg): Colorless oil; $[\alpha]_{\mathrm{D}}{ }^{18}+10.0(c 0.05 \mathrm{MeOH})$; IR $(\mathrm{KBr}) v_{\max }=3440,2922$, 2854, 1719, 1438, 1384, 1282, 1142, $1095 \mathrm{~cm}^{-1}$; ${ }^{1} \mathrm{H}$ and ${ }^{13} \mathrm{C}$ NMR data see Table S1; HRMS (ESI) $m / z:[\mathrm{M}+\mathrm{Na}]^{+}$Calcd. for $\mathrm{C}_{22} \mathrm{H}_{36} \mathrm{NaO}_{5}, 403.2455$; Found 403.2454.

Acetylation of flexibilisin C (5): Isolated $5(1.0 \mathrm{mg})$ were dissolved in dry pyridine $(1.0 \mathrm{~mL})$ and treated with $\mathrm{Ac}_{2} \mathrm{O}(0.5 \mathrm{~mL})$. After standing at room temperature overnight, the reaction was quenched with water. The obtained material was partitioned between water and $\mathrm{CH}_{2} \mathrm{Cl}_{2}$. The organic layer afforded colorless oil $6\left(0.9 \mathrm{mg}\right.$, yield 90\%). ${ }^{1} \mathrm{H}$ and ${ }^{13} \mathrm{C}$ NMR data of 6 were identical. ${ }^{1} \mathrm{H}$ NMR $\left(\mathrm{CDCl}_{3}, 500 \mathrm{MHz}\right) \delta_{\mathrm{H}} 6.26(1 \mathrm{H}, \mathrm{s}, \mathrm{H}-16), 5.70(1 \mathrm{H}, \mathrm{t}, J=7.0 \mathrm{~Hz}, \mathrm{H}-5), 5.60(1 \mathrm{H}, \mathrm{s}, \mathrm{H}-16)$, $5.37(1 \mathrm{H}, \mathrm{dd}, J=10.5,5.5 \mathrm{~Hz}, \mathrm{H}-3), 5.20(1 \mathrm{H}, \mathrm{t}, J=6.5 \mathrm{~Hz}, \mathrm{H}-9), 3.74\left(3 \mathrm{H}, \mathrm{s}, \mathrm{H}_{3}-21\right), 3.01$ (1H, dd, $J=8.5,4.0 \mathrm{~Hz}, \mathrm{H}-13), 3.01$ (1H, m, H-2), 2.51 (1H, m, H-1), 2.23 (2H, m, H-10), 2.16 (1H, m, H-7), 2.09 (1H, ddd, $J=13.5,5.5,3.0 \mathrm{~Hz}, \mathrm{H}-11), 2.04$ (3H, s, OAc), 2.00 (1H, m, H-2), 1.94 (1H, m, H-7), $1.92(1 \mathrm{H}, \mathrm{m}, \mathrm{H}-6), 1.78$ (1H, ddd, $J=14.0,10.5,4.0 \mathrm{~Hz}, \mathrm{H}-14), 1.69$ (1H, m, H-6), 1.67 (3H, s, $\left.\mathrm{H}_{3}-18\right), 1.65$ (3H, s, $\left.\mathrm{H}_{3}-19\right), 1.43$ (1H, ddd, $\left.J=14.0,8.5,2.0 \mathrm{~Hz}, \mathrm{H}-14\right), 1.28\left(3 \mathrm{H}, \mathrm{s}, \mathrm{H}_{3}-20\right), 1.22$ $(1 \mathrm{H}, \mathrm{m}, \mathrm{H}-11) ;{ }^{13} \mathrm{C} \mathrm{NMR}\left(\mathrm{CDCl}_{3}, 125 \mathrm{MHz}\right) \delta_{\mathrm{C}} 170.5$ (OAc), $167.6(\mathrm{C}-17), 143.4(\mathrm{C}-15), 134.5$ (C-8), 134.1 (C-4), 128.6 (C-3), 124.4 (C-16), 123.7 (C-9), 70.0 (C-5), 62.1 (C-13), 60.3 (C-12), 52.0 (C-21), 38.9 (C-1), 38.4 (C-11), 34.4 (C-7), 33.6 (C-14), 30.1 (C-2), 28.4 (C-6), 24.4 (C-10), 21.4 (OAc), 18.0 (C-18), 17.0 (C-19), 16.6 (C-20). HRESIMS m/z 413.2303 [M+Na] $]^{+}$(calcd for $\left.\mathrm{C}_{23} \mathrm{H}_{34} \mathrm{NaO}_{5}, 413.2298\right)$.

\subsection{Lipopolysaccharide-induced BV-2 Inflammation}

BV-2 microglia cells $\left(2.0 \times 10^{5}\right.$ cells $)$ were cultured in 96-well plates with DMEM high-glucose medium containing $10 \%$ fetal bovine serum in a humidified, $37{ }^{\circ} \mathrm{C}, 5 \% \mathrm{CO}_{2}$-containing incubator for $24 \mathrm{~h}$. To assess the in vitro anti-inflammatory effect of test compounds, BV-2 cells were then pretreated with 5,10 and $20 \mu \mathrm{M}$ of test compounds for $2 \mathrm{~h}$, followed by $100 \mathrm{ng} / \mathrm{mL}$ of LPS exposure for $24 \mathrm{~h}$. The culture medium of each well $(50 \mu \mathrm{L})$ was collected, and then incubated with $50 \mu \mathrm{L}$ of 
Greiss buffer for $15 \mathrm{~min}$ at room temperature. The absorbance of mixture was measured at $540 \mathrm{~nm}$ using a microplate reader, and the level of nitrite was calculated from a standard curve of sodium nitrite.

For measurement of the influence of isolates on cell viabilities, BV-2 cells were seeded at a density of $1.25 \times 10^{4}$ cells $/ \mathrm{mL}$ into 96-well plates with $100 \mu \mathrm{L} /$ well and maintained in a humidified atmosphere of $5 \% \mathrm{CO}_{2}$ and $95 \%$ air at $37{ }^{\circ} \mathrm{C}$. After $24 \mathrm{~h}$ seeding, the cells were treated with 5,10 and $20 \mu \mathrm{M}$ of test compounds for another $24 \mathrm{~h}$, and then treated with $0.5 \mathrm{mg} / \mathrm{mL}$ MTT for $3 \mathrm{~h}$. The absorbance value at $490 \mathrm{~nm}$ was measured by a microplate reader and the cell viability was calculated.

Table S1. ${ }^{1} \mathrm{H}$ NMR $(\delta \mathrm{H})$ and ${ }^{13} \mathrm{C}$ NMR $(\delta \mathrm{C})$ Data for $\mathbf{2}$ and $\mathbf{3}$ in $\mathrm{CDCl}_{3}$.

\begin{tabular}{|c|c|c|c|c|}
\hline \multirow[b]{2}{*}{ no. } & \multicolumn{2}{|l|}{2} & \multicolumn{2}{|l|}{3} \\
\hline & $\delta_{\mathrm{H}}$, mult $(J \mathrm{~Hz})^{a}$ & $\delta_{\mathrm{C}}^{b}$ & $\delta_{\mathrm{H}}$, mult $(J \mathrm{~Hz})^{a}$ & $\delta_{\mathrm{C}}^{b}$ \\
\hline 1 & $2.43 \mathrm{~m}$ & $35.6 \mathrm{CH}$ & $2.73 \mathrm{~m}$ & $38.6 \mathrm{CH}$ \\
\hline $2 \mathrm{a}$ & $2.42 \mathrm{~m}$ & $31.5 \mathrm{CH}_{2}$ & $1.64 \mathrm{~m}$ & $26.1 \mathrm{CH}_{2}$ \\
\hline $2 b$ & $1.28 \mathrm{~m}$ & & $1.46 \mathrm{~m}$ & \\
\hline $3 a$ & $2.20 \mathrm{~m}$ & $31.5 \mathrm{CH}_{2}$ & $1.34 \mathrm{~m}$ & $31.8 \mathrm{CH}_{2}$ \\
\hline $3 b$ & $1.76 \mathrm{~m}$ & & & \\
\hline 4 & & $86.9 \mathrm{qC}$ & & $78.6 \mathrm{qC}$ \\
\hline 5 & $4.35 \mathrm{~d}(9.6)$ & $67.2 \mathrm{CH}$ & $3.47 \mathrm{~d}(10.2)$ & $74.8 \mathrm{CH}$ \\
\hline $6 a$ & $2.12 \mathrm{dd}(14.4,1.2)$ & $31.0 \mathrm{CH}_{2}$ & $1.56 \mathrm{~m}$ & $29.4 \mathrm{CH}_{2}$ \\
\hline $6 b$ & 1.88 ddd $(15.0,9.6,5.4)$ & & $1.26 \mathrm{~m}$ & \\
\hline $7 \mathrm{a}$ & 4.53 brs & $75.8 \mathrm{CH}$ & $2.16 \mathrm{~m}$ & $34.2 \mathrm{CH}_{2}$ \\
\hline \multicolumn{5}{|l|}{$7 b$} \\
\hline 8 & & $137.7 \mathrm{qC}$ & & $136.6 \mathrm{qC}$ \\
\hline 9 & $5.53 \mathrm{dt}(11.4,1.8)$ & 123.9 & $5.10 \mathrm{t}(7.2)$ & $122.9 \mathrm{CH}$ \\
\hline $10 \mathrm{a}$ & 2.53 dddd $(15.6,13.8,10.8,1.8)$ & $24.8 \mathrm{CH}_{2}$ & $2.12 \mathrm{~m}$ & $23.3 \mathrm{CH}_{2}$ \\
\hline $10 \mathrm{~b}$ & $2.20 \mathrm{~m}$ & & & \\
\hline $11 \mathrm{a}$ & $2.25 \mathrm{~m}$ & $38.2 \mathrm{CH}_{2}$ & $1.99 \mathrm{~m}$ & $37.8 \mathrm{CH}_{2}$ \\
\hline $11 b$ & $1.33 \mathrm{~m}$ & & $1.40 \mathrm{~m}$ & \\
\hline 12 & & $60.3 \mathrm{qC}$ & & $60.1 \mathrm{qC}$ \\
\hline 13 & $2.97 \mathrm{dd}(10.8,3.0)$ & $64.1 \mathrm{CH}$ & $2.84 \mathrm{dd}(7.2,4.8)$ & $61.2 \mathrm{CH}$ \\
\hline $14 \mathrm{a}$ & $1.96 \mathrm{ddd}(13.2,10.2,3.0)$ & $33.3 \mathrm{CH}_{2}$ & $1.75 \mathrm{ddd}(15.0,10.2,5.4)$ & $34.5 \mathrm{CH}_{2}$ \\
\hline $14 b$ & $1.48 \mathrm{dd}(13.2,10.8)$ & & $1.61 \mathrm{ddd}(14.4,7.8,3.0)$ & \\
\hline 15 & & $144.7 \mathrm{qC}$ & & $143.3 \mathrm{qC}$ \\
\hline $16 \mathrm{a}$ & $6.26 \mathrm{~s}$ & 124.1 & $6.28 \mathrm{~s}$ & $124.5 \mathrm{CH}_{2}$ \\
\hline $16 b$ & $5.40 \mathrm{~s}$ & & $5.58 \mathrm{~s}$ & \\
\hline $17 \mathrm{a}$ & & $169.3 \mathrm{qC}$ & & $167.5 \mathrm{qC}$ \\
\hline \multicolumn{5}{|l|}{$17 \mathrm{~b}$} \\
\hline 18 & $1.35 \mathrm{~s}$ & $23.4 \mathrm{CH}_{3}$ & $1.21 \mathrm{~s}$ & $19.1 \mathrm{CH}_{3}$ \\
\hline 19 & $1.57 \mathrm{~s}$ & $15.2 \mathrm{CH}_{3}$ & $1.66 \mathrm{~s}$ & $18.1 \mathrm{CH}_{3}$ \\
\hline 20 & $1.21 \mathrm{~s}$ & $16.2 \mathrm{CH}_{3}$ & $1.29 \mathrm{~s}$ & $17.2 \mathrm{CH}_{3}$ \\
\hline 21 & & & $3.76 \mathrm{~s}$ & $52.1-\mathrm{OCH}_{3}$ \\
\hline 22 & & & $3.15 \mathrm{~s}$ & $49.3-\mathrm{OCH}_{3}$ \\
\hline
\end{tabular}

${ }^{a}$ Recorded at $600 \mathrm{MHz} .{ }^{b}$ Recorded at $125 \mathrm{MHz}$. Assignments were deduced by analysis of 1D and 2D NMR spectra. 
Table S2. The inhibitory effects of compounds 1-3 on LPS-induced inflammatory responses in BV-2 Cells.

\begin{tabular}{cccc}
\hline Compounds & & $\begin{array}{c}\text { NO production } \pm \text { SEM } \\
\text { (\% of LPS group) }\end{array}$ & $\begin{array}{c}\text { Cell viability } \pm \text { SEM } \\
\text { (\% of control group) }^{b}\end{array}$ \\
\hline \multirow{1}{*}{$5 \mu \mathrm{M}$} & $74.24 \pm 3.72^{* *}$ & $108.40 \pm 1.94$ \\
& $10 \mu \mathrm{M}$ & $54.14 \pm 7.17^{* *}$ & $113.00 \pm 1.67$ \\
$\mathbf{2}$ & $20 \mu \mathrm{M}$ & $28.62 \pm 3.96^{* * *}$ & $103.40 \pm 2.41$ \\
& $5 \mu \mathrm{M}$ & $67.64 \pm 6.35^{* * *}$ & $110.90 \pm 4.14$ \\
$\mathbf{3}$ & $10 \mu \mathrm{M}$ & $42.13 \pm 3.33^{* * *}$ & $115.30 \pm 4.16$ \\
& $20 \mu \mathrm{M}$ & $22.14 \pm 3.89^{* * *}$ & $111.70 \pm 5.63$ \\
Positive control- & $5 \mu \mathrm{M}$ & $91.16 \pm 5.53$ & $112.70 \pm 4.94$ \\
Resveratrol & $10 \mu \mathrm{M}$ & $68.82 \pm 3.99^{* *}$ & $110.90 \pm 3.17$ \\
$(20 \mu \mathrm{M})$ & $20 \mu \mathrm{M}$ & $38.08 \pm 4.66^{* * *}$ & $104.30 \pm 1.15$ \\
& $20 \mu \mathrm{M}$ & & $55.97 \pm 1.24^{* * *}$ \\
\hline
\end{tabular}

${ }^{a} \mathrm{NO}$ data were normalized by the mean value of the LPS group, which was set to $100 \% ;{ }^{b}$ Cell viability data were normalized by the mean value of the control group, which was set to $100 \%$. $* * P<0.01 * * * \mathrm{P}<0.001$ vs the LPS group.

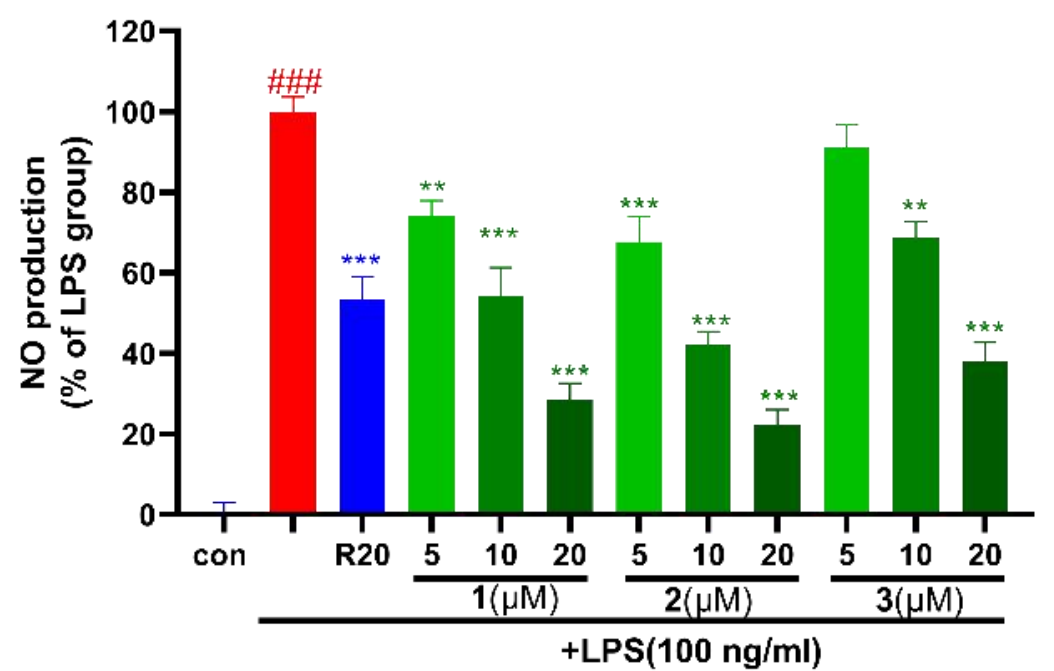

Figure S1. The inhibitory effects of compounds 1-3 on LPS-induced NO over-production in BV-2 microglial cells. Data were normalized by the mean value of the LPS group (which was set to 100\%), and expressed as Means \pm SEM $(\mathrm{n}=4)$. ${ }^{\# \#} \mathrm{P}<0.001$ vs the control group (con), $* * \mathrm{P}<0.01, * * * \mathrm{P}<$ 0.001 vs the LPS group. R20: Resveratrol $(20 \mu \mathrm{M})$. 


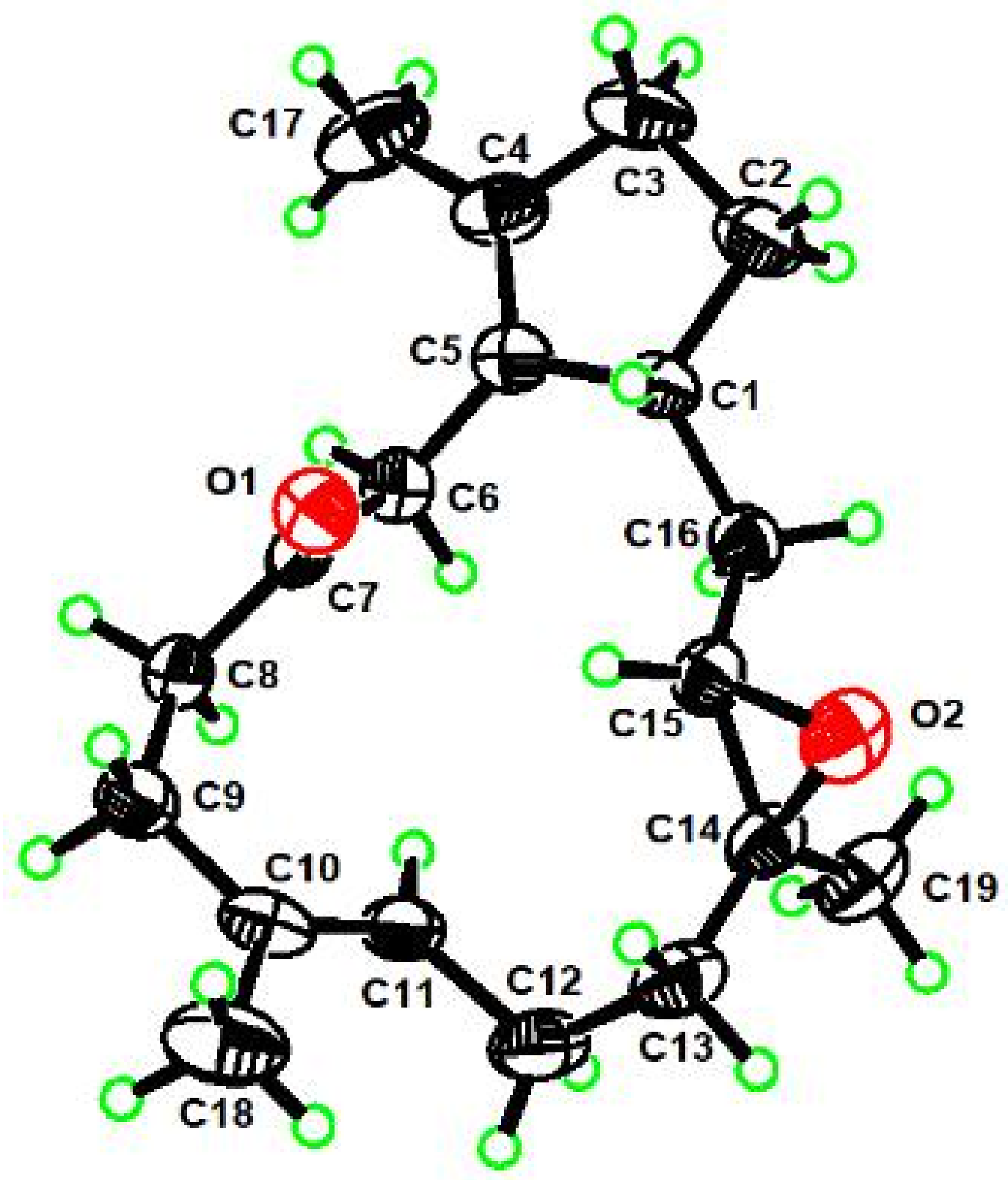

Figure S2. Perspective ORTEP drawing of X-ray structure of $\mathbf{1}$ (displacement ellipsoids are drawn at the $50 \%$ probability level). 


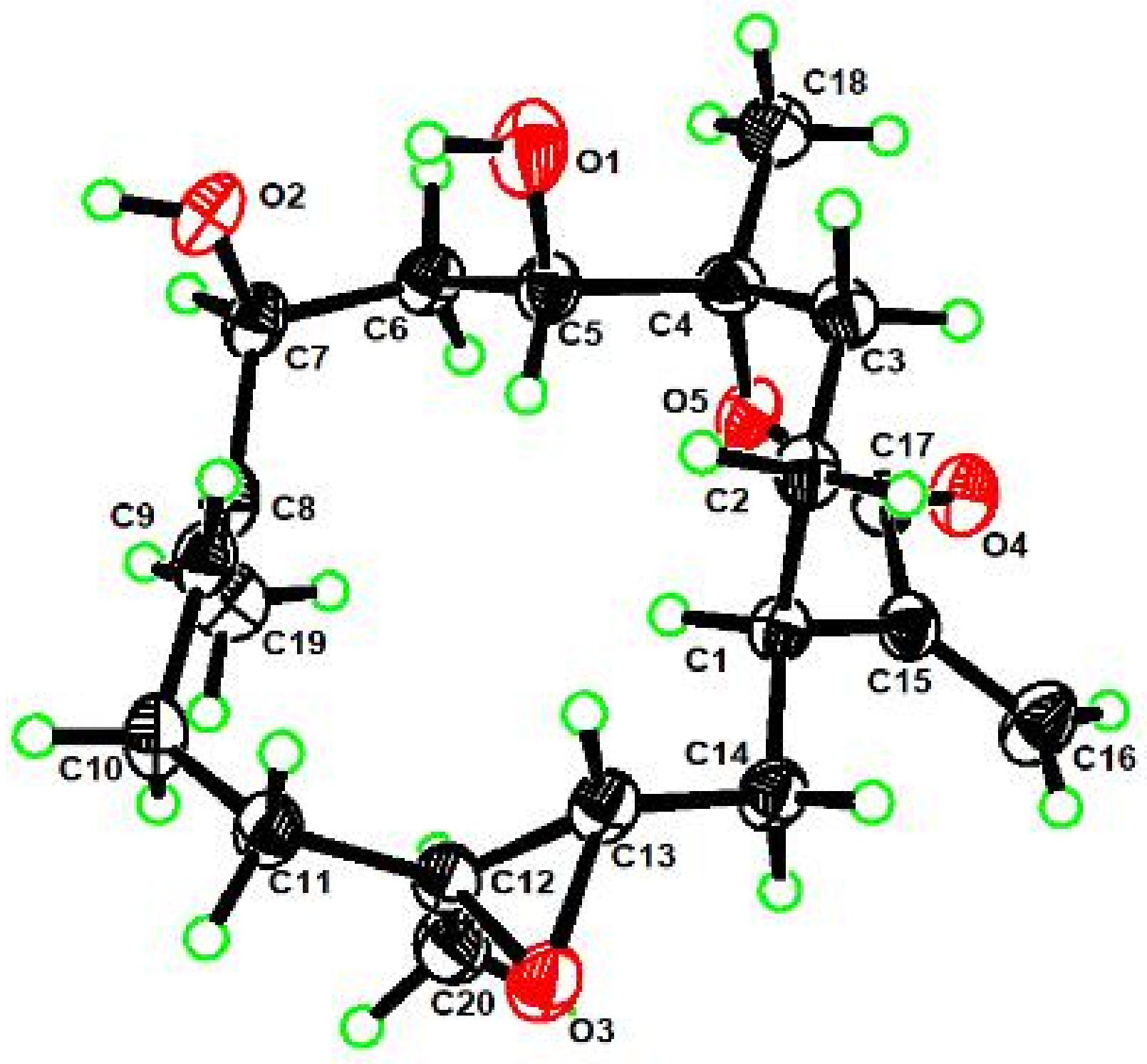

Figure S3. Perspective ORTEP drawing of X-ray structure of 2 (displacement ellipsoids are drawn at the $50 \%$ probability level). 


\section{Original data for compounds $1-3$ and 5}

\subsection{X-ray crystallographic analyses of 1}

$\mathrm{C}_{19} \mathrm{H}_{28} \mathrm{O}_{2}, \mathrm{M}_{\mathrm{r}}=288.41$, trigonal, crystal size $0.11 \times 0.03 \times 0.02 \mathrm{~mm}^{3}$, space group $\mathrm{P} 2_{1}, \mathrm{a}=11.0946$ (8) $\AA, \mathrm{b}=5.2267$ (4) $\AA, \mathrm{c}=15.4532$ (13) $\AA, \mathrm{V}=841.99$ (11) $\AA^{3}, \mathrm{Z}=2, \mathrm{D}_{\text {calcd }}=1.138 \mathrm{~g} / \mathrm{cm} 3, \mathrm{~F}(000)$ $=316.0,8178$ collected reflections, 2963 unique reflections $\left(\mathrm{R}_{\text {int }}=0.0353\right)$, final $\mathrm{R}_{1}=0.0445\left(\mathrm{wR}_{2}=\right.$ 0.0889 ) reflections with $\mathrm{I} \geq 2 \sigma(\mathrm{I}), \mathrm{R}_{1}=0.0665, \mathrm{wR}_{2}=0.1031$ for all unique data. The $\mathrm{X}$-ray measurements were made on a Bruker D8 Venture X-ray diffractometer with with Mo K $\alpha$ radiation $(\lambda=0.71073 \AA)$ at $170.0 \mathrm{~K}$. The structure was solved with the ShelXT ${ }^{1}$ structure solution program using Intrinsic Phasing and refined with the ShelXL ${ }^{2}$ refinement package using Least Squares minimisation. Crystallographic data for $\mathbf{1}$ has been deposited at the Cambridge Crystallographic Data Centre (Deposition nos. CCDC 2074984). Copies of these data can be obtained free of charge via www.ccdc.cam.ac.uk/conts/retrieving.html or from the Cambridge Crystallographic Data Centre, 12 Union Road, Cambridge CB21EZ, UK. [Fax: (+44) 1223-336-033. E-mail: deposit@ccdc.cam.ac.uk.]

\subsection{X-ray crystallographic analyses of 2}

$\mathrm{C}_{20} \mathrm{H}_{30} \mathrm{O}_{5}, \mathrm{M}_{\mathrm{r}}=350.44$, trigonal, crystal size $0.19 \times 0.12 \times 0.08 \mathrm{~mm}^{3}$, space group $\mathrm{P} 2_{1}, \mathrm{a}=9.4201$ (3) $\AA, \mathrm{b}=22.5556$ (7) $\AA, \mathrm{c}=9.4292$ (3) $\AA, \mathrm{V}=1895.64$ (11) $\AA^{3}, \mathrm{Z}=4, \mathrm{D}_{\text {calcd }}=1.228 \mathrm{~g} / \mathrm{cm} 3, \mathrm{~F}(000)=$ $760.0,34272$ collected reflections, 7686 unique reflections $\left(\mathrm{R}_{\mathrm{int}}=0.0556\right)$, final $\mathrm{R}_{1}=0.0456\left(\mathrm{w} \mathrm{R}_{2}=\right.$ $0.1136)$ reflections with $\mathrm{I} \geq 2 \sigma(\mathrm{I}), \mathrm{R}_{1}=0.0509, \mathrm{wR}_{2}=0.1190$ for all unique data. The X-ray measurements were made on a Bruker D8 Venture X-ray diffractometer with with Mo Ka radiation $(\lambda=1.54178 \AA)$ at $170.0 \mathrm{~K}$. The structure was solved with the ShelXT ${ }^{1}$ structure solution program using Intrinsic Phasing and refined with the ShelXL ${ }^{2}$ refinement package using Least Squares minimisation. Crystallographic data for $\mathbf{1}$ has been deposited at the Cambridge Crystallographic Data Centre (Deposition nos. CCDC 2074989). Copies of these data can be obtained free of charge via www.ccdc.cam.ac.uk/conts/retrieving.html or from the Cambridge Crystallographic Data Centre, 12 Union Road, Cambridge CB21EZ, UK. [Fax: (+44) 1223-336-033. E-mail: deposit@ccdc.cam.ac.uk.]

\section{References}

(1) Sheldrick, G.M. Acta Cryst. 2015, A71, 3-8.

(2) Sheldrick, G.M. Acta Cryst. 2015, C71, 3-8. 
Table S3. X-ray crystallographic data for $\mathbf{1}$

\begin{tabular}{|c|c|}
\hline Empirical formula & $\mathrm{C}_{19} \mathrm{H}_{28} \mathrm{O}_{2}$ \\
\hline Formula weight & 288.41 \\
\hline Temperature/K & 170.0 \\
\hline Crystal system & monoclinic \\
\hline Space group & $\mathrm{P} 2_{1}$ \\
\hline $\mathrm{a} / \AA$ & $11.0946(8)$ \\
\hline $\mathrm{b} / \AA$ & $5.2267(4)$ \\
\hline $\mathrm{c} / \AA$ & $15.4532(13)$ \\
\hline$\alpha /^{\circ}$ & 90 \\
\hline$\beta /^{\circ}$ & $110.013(2)$ \\
\hline$\gamma /{ }^{\circ}$ & 90 \\
\hline Volume $/ \AA^{3}$ & $841.99(11)$ \\
\hline Z & 2 \\
\hline$\rho_{\text {calc }} \mathrm{g} / \mathrm{cm}^{3}$ & 1.138 \\
\hline$\mu / \mathrm{mm}^{-1}$ & 0.071 \\
\hline $\mathrm{F}(000)$ & 316.0 \\
\hline Crystal size $/ \mathrm{mm}^{3}$ & $0.11 \times 0.03 \times 0.02$ \\
\hline Radiation & $\operatorname{MoK} \alpha(\lambda=0.71073)$ \\
\hline $2 \Theta$ range for data collection $/{ }^{\circ}$ & 3.954 to 52.13 \\
\hline Index ranges & $-13 \leq \mathrm{h} \leq 13,-6 \leq \mathrm{k} \leq 6,-19 \leq 1 \leq 19$ \\
\hline Reflections collected & 8178 \\
\hline Independent reflections & $2963\left[\mathrm{R}_{\text {int }}=0.0353, \mathrm{R}_{\text {sigma }}=0.0431\right]$ \\
\hline Data/restraints/parameters & $2963 / 1 / 193$ \\
\hline Goodness-of-fit on $\mathrm{F}^{2}$ & 1.088 \\
\hline Final $\mathrm{R}$ indexes $[\mathrm{I}>=2 \sigma(\mathrm{I})]$ & $\mathrm{R}_{1}=0.0445, \mathrm{wR}_{2}=0.0889$ \\
\hline Final $\mathrm{R}$ indexes [all data] & $\mathrm{R}_{1}=0.0665, \mathrm{wR}_{2}=0.1031$ \\
\hline Largest diff. peak/hole / e $\AA^{-3}$ & $0.24 /-0.15$ \\
\hline Flack parameter & $-0.2(8)$ \\
\hline
\end{tabular}


Table S4. X-ray crystallographic data for 2.

\begin{tabular}{|c|c|}
\hline Empirical formula & $\mathrm{C}_{20} \mathrm{H}_{30} \mathrm{O}_{5}$ \\
\hline Formula weight & 350.44 \\
\hline Temperature/K & 170.0 \\
\hline Crystal system & monoclinic \\
\hline Space group & $\mathrm{P} 2{ }_{1}$ \\
\hline $\mathrm{a} / \AA$ & $9.4201(3)$ \\
\hline $\mathrm{b} / \AA$ & $22.5556(7)$ \\
\hline $\mathrm{c} / \AA$ & $9.4292(3)$ \\
\hline$\alpha /^{\circ}$ & 90 \\
\hline$\beta /{ }^{\circ}$ & $108.884(2)$ \\
\hline$\gamma /{ }^{\circ}$ & 90 \\
\hline Volume $/ \AA^{3}$ & $1895.64(11)$ \\
\hline $\mathrm{Z}$ & 4 \\
\hline$\rho_{\text {calc }} \mathrm{g} / \mathrm{cm}^{3}$ & 1.228 \\
\hline$\mu / \mathrm{mm}^{-1}$ & 0.704 \\
\hline $\mathrm{F}(000)$ & 760.0 \\
\hline Crystal size $/ \mathrm{mm}^{3}$ & $0.19 \times 0.12 \times 0.08$ \\
\hline Radiation & $\mathrm{CuK} \alpha(\lambda=1.54178)$ \\
\hline $2 \Theta$ range for data collection ${ }^{\circ}$ & 7.838 to 149.432 \\
\hline Index ranges & $-11 \leq \mathrm{h} \leq 11,-28 \leq \mathrm{k} \leq 27,-11 \leq 1 \leq 11$ \\
\hline Reflections collected & 34272 \\
\hline Independent reflections & $7686\left[\mathrm{R}_{\text {int }}=0.0556, \mathrm{R}_{\text {sigma }}=0.0410\right]$ \\
\hline Data/restraints/parameters & $7686 / 1 / 461$ \\
\hline Goodness-of-fit on $\mathrm{F}^{2}$ & 1.025 \\
\hline Final $R$ indexes $[\mathrm{I}>=2 \sigma(\mathrm{I})]$ & $\mathrm{R}_{1}=0.0456, \mathrm{wR}_{2}=0.1136$ \\
\hline Final $\mathrm{R}$ indexes [all data] & $\mathrm{R}_{1}=0.0509, \mathrm{wR}_{2}=0.1190$ \\
\hline Largest diff. peak/hole / e $\AA^{-3}$ & $0.36 /-0.19$ \\
\hline Flack parameter & $0.02(8)$ \\
\hline
\end{tabular}


2.3 NMR, MS, and IR spectra of compounds $1-3$ and 5
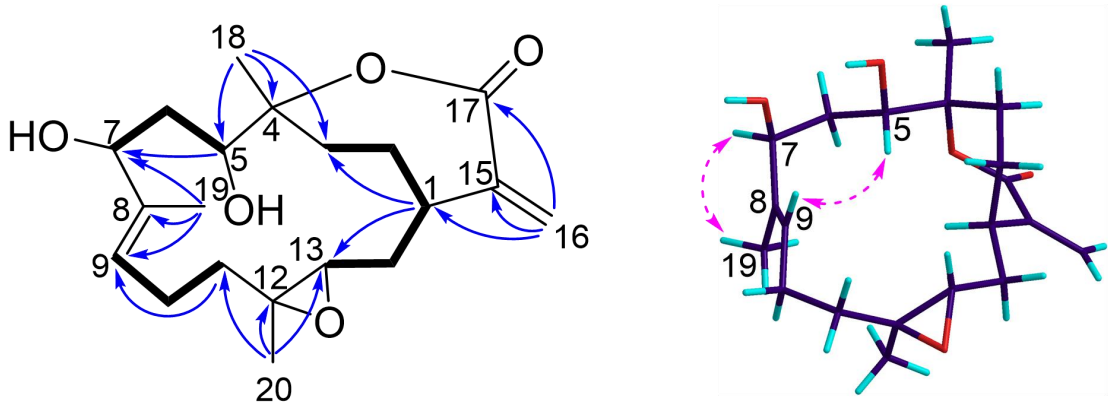

$$
2 \quad \underset{\mathrm{HMBC}}{\mathrm{COSY}}
$$
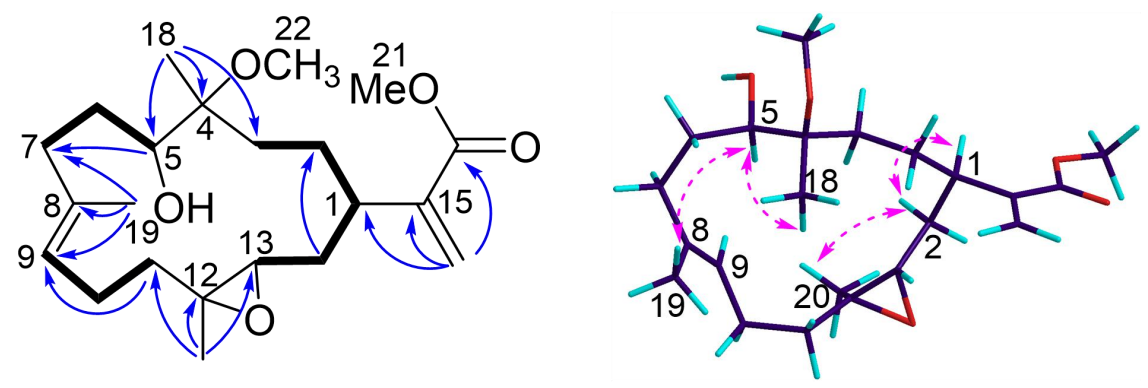

3

Figure S4 ${ }^{1} \mathrm{H}-{ }^{1} \mathrm{H}$ COSY, key HMBC and NOESY correlations of compounds 2 and $\mathbf{3}$. 


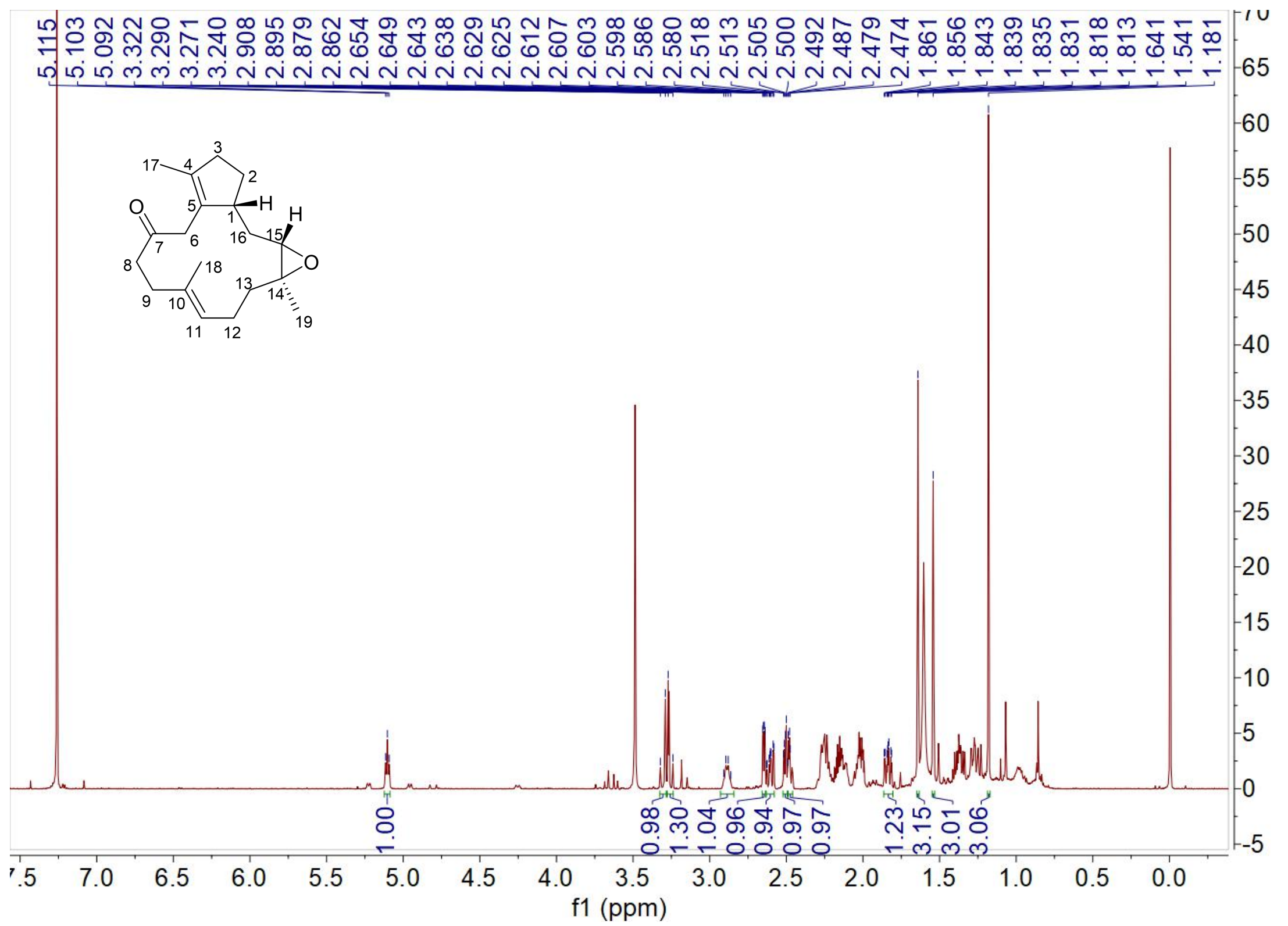

Figure S5. ${ }^{1} \mathrm{H}$ NMR spectrum $(600 \mathrm{MHz})$ of sinusiaetone $\mathrm{A}(\mathbf{1})$ in $\mathrm{CDCl}_{3}$. 

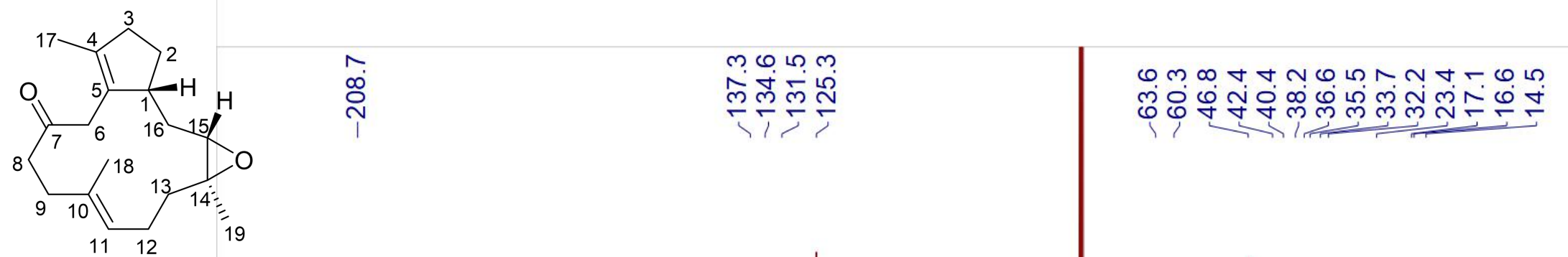

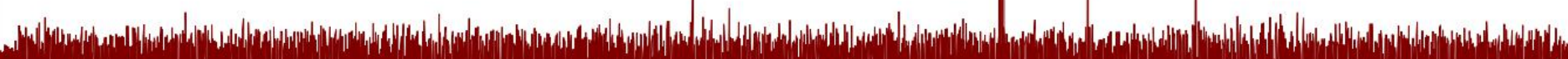

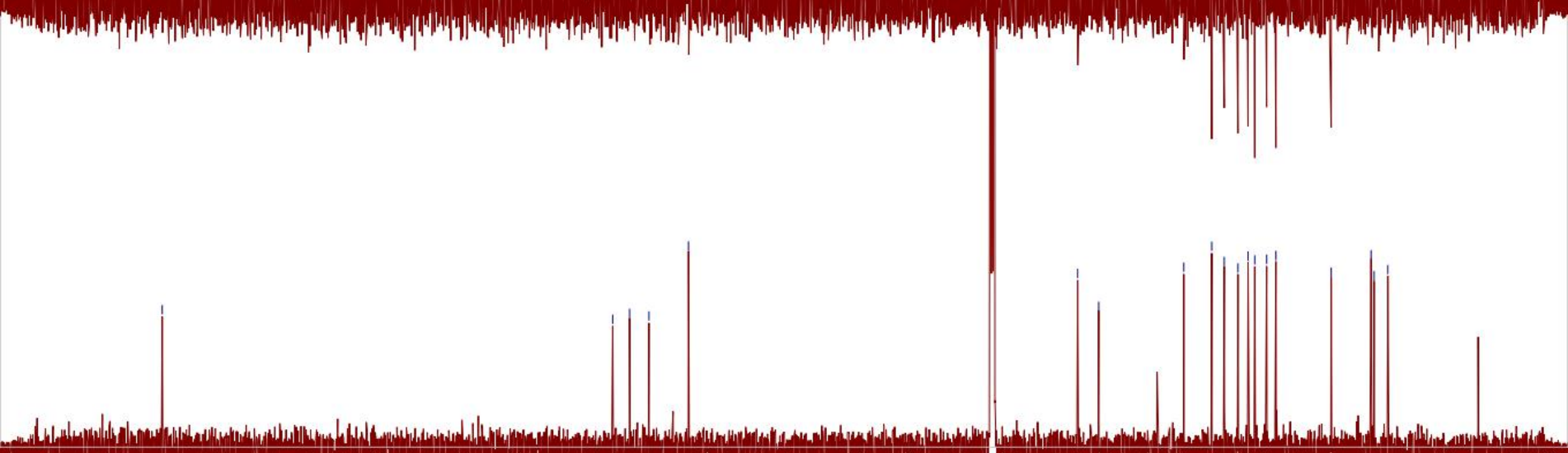

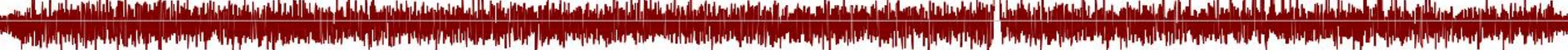

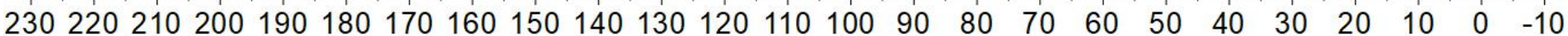
f1 (ppm)

Figure S6. ${ }^{13} \mathrm{C}$ NMR spectrum $(125 \mathrm{MHz})$ of sinusiaetone $\mathrm{A}(\mathbf{1})$ in $\mathrm{CDCl}_{3}$. 


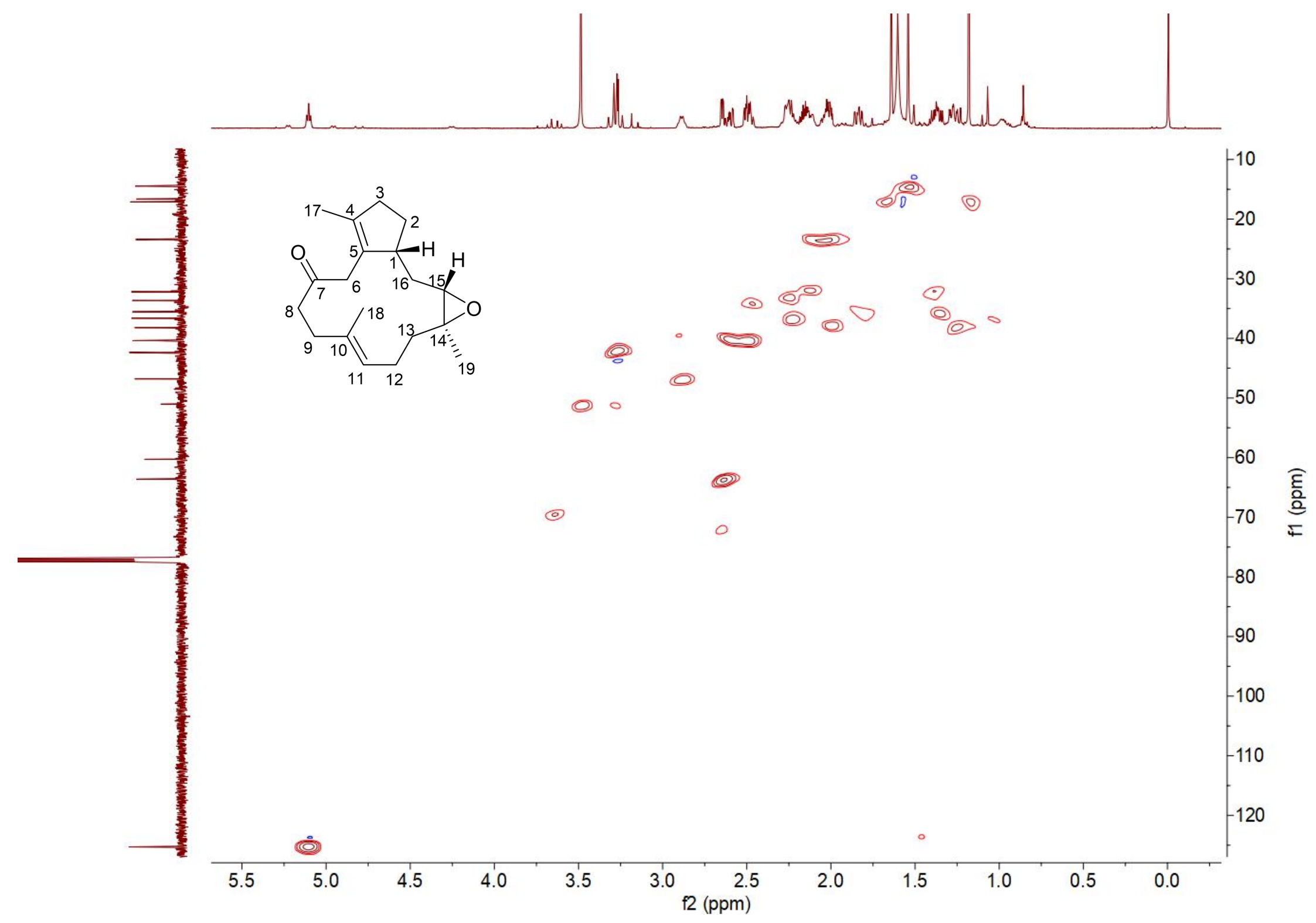

Figure S7. HSQC spectrum (500 MHz) of sinusiaetone A (1) in $\mathrm{CDCl}_{3}$. 


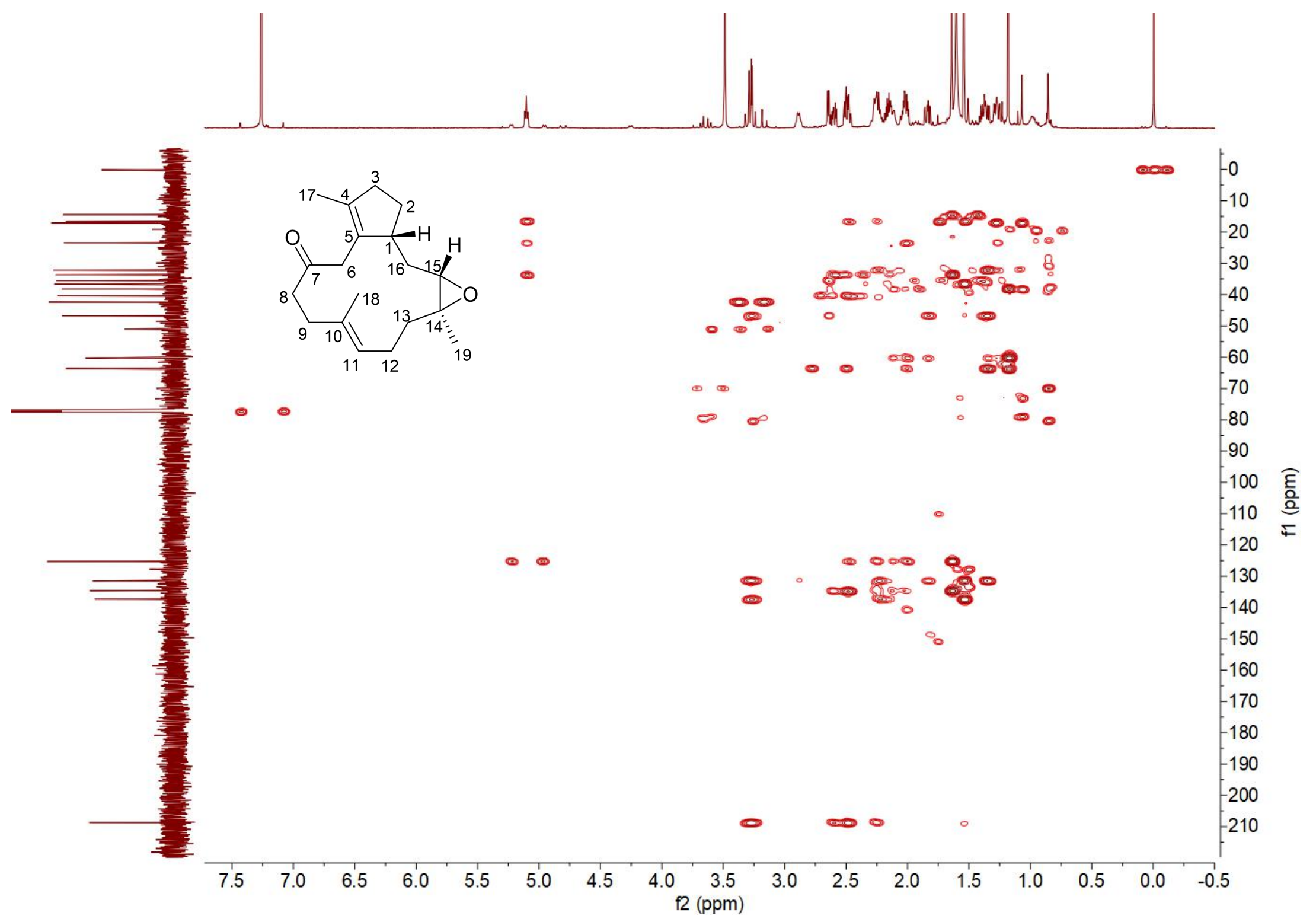

Figure S8. $\mathrm{HMBC}$ spectrum $(500 \mathrm{MHz})$ of sinusiaetone A (1) in $\mathrm{CDCl}_{3}$. 


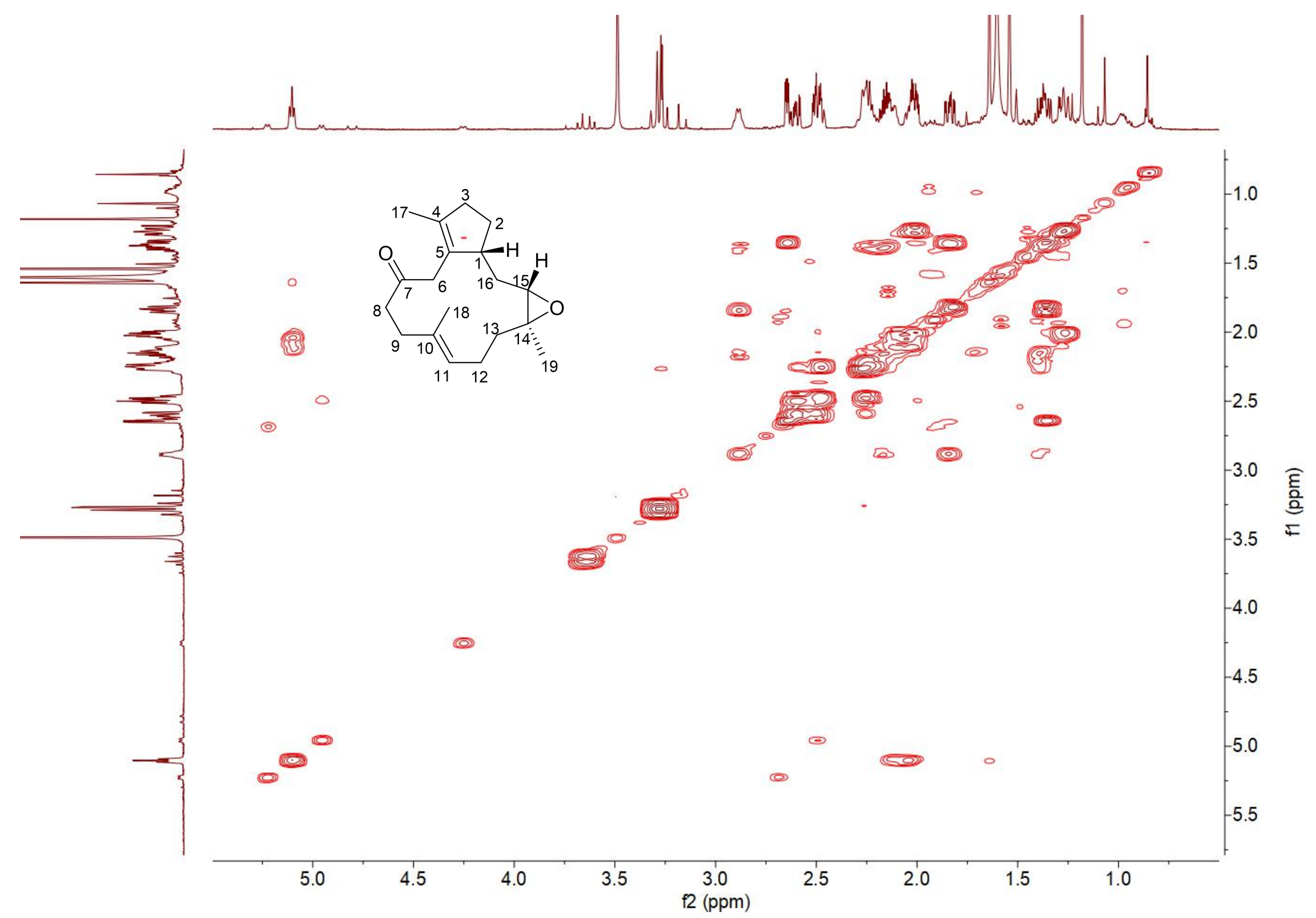

Figure S9. COSY spectrum (500 MHz) of sinusiaetone A (1) in $\mathrm{CDCl}_{3}$. 


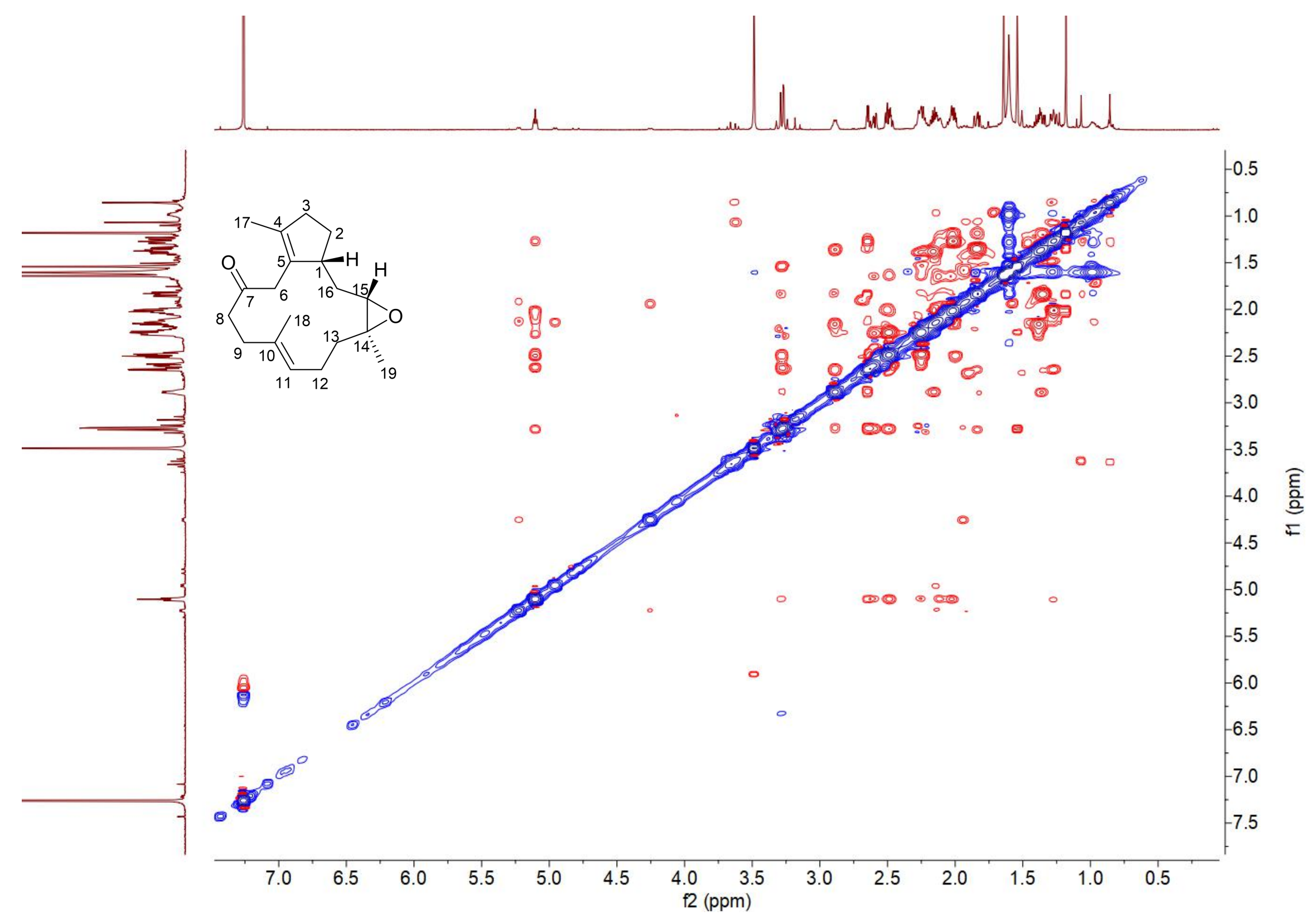

Figure S10. NOESY spectrum (500 MHz) of sinusiaetone A (1) in $\mathrm{CDCl}_{3}$. 


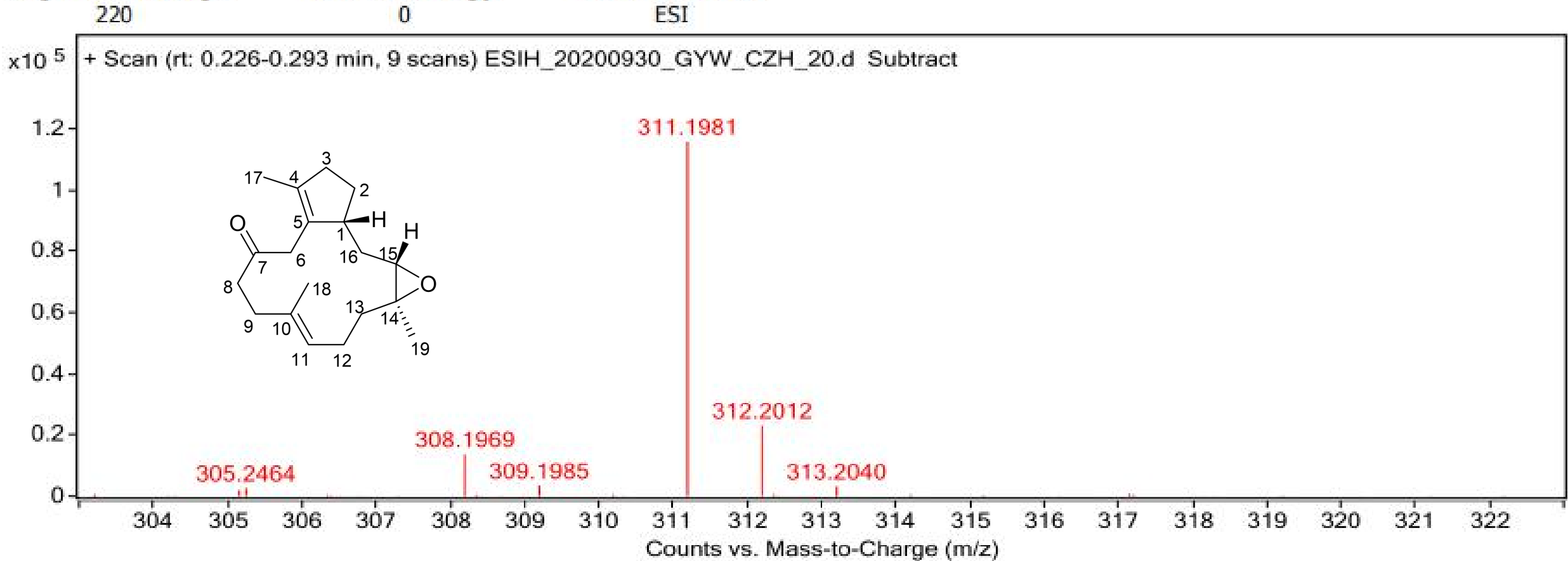

\section{Formula Calculator Results}

\begin{tabular}{|l|l|l|l|l|l|}
\hline $\mathbf{m} / \mathbf{z}$ & Calc $\mathbf{~} / \mathbf{z}$ & Diff $(\mathrm{mDa})$ & Diff $(\mathrm{ppm})$ & Ion Formula & Ion \\
\hline 311.1981 & 311.1982 & 0.06 & 0.19 & $\mathrm{C} 19 \mathrm{H} 28 \mathrm{Na} \mathrm{O} 2$ & $(\mathrm{M}+\mathrm{Na})+$ \\
\hline
\end{tabular}

Figure S11. HRESIMS spectrum of sinusiaetone A (1) in $\mathrm{MeOH}$. 


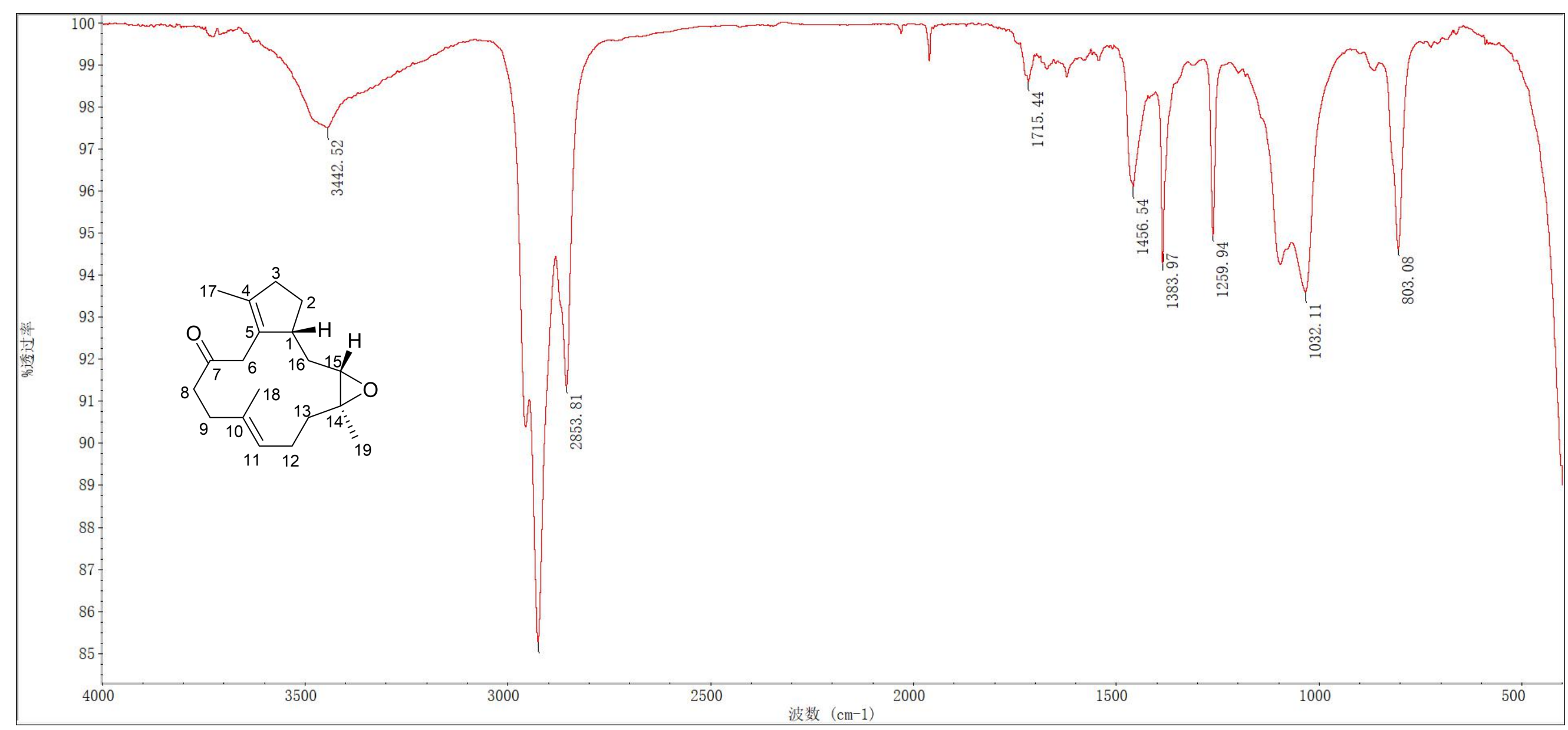

Figure S12. IR spectrum of sinusiaetone A (1). 


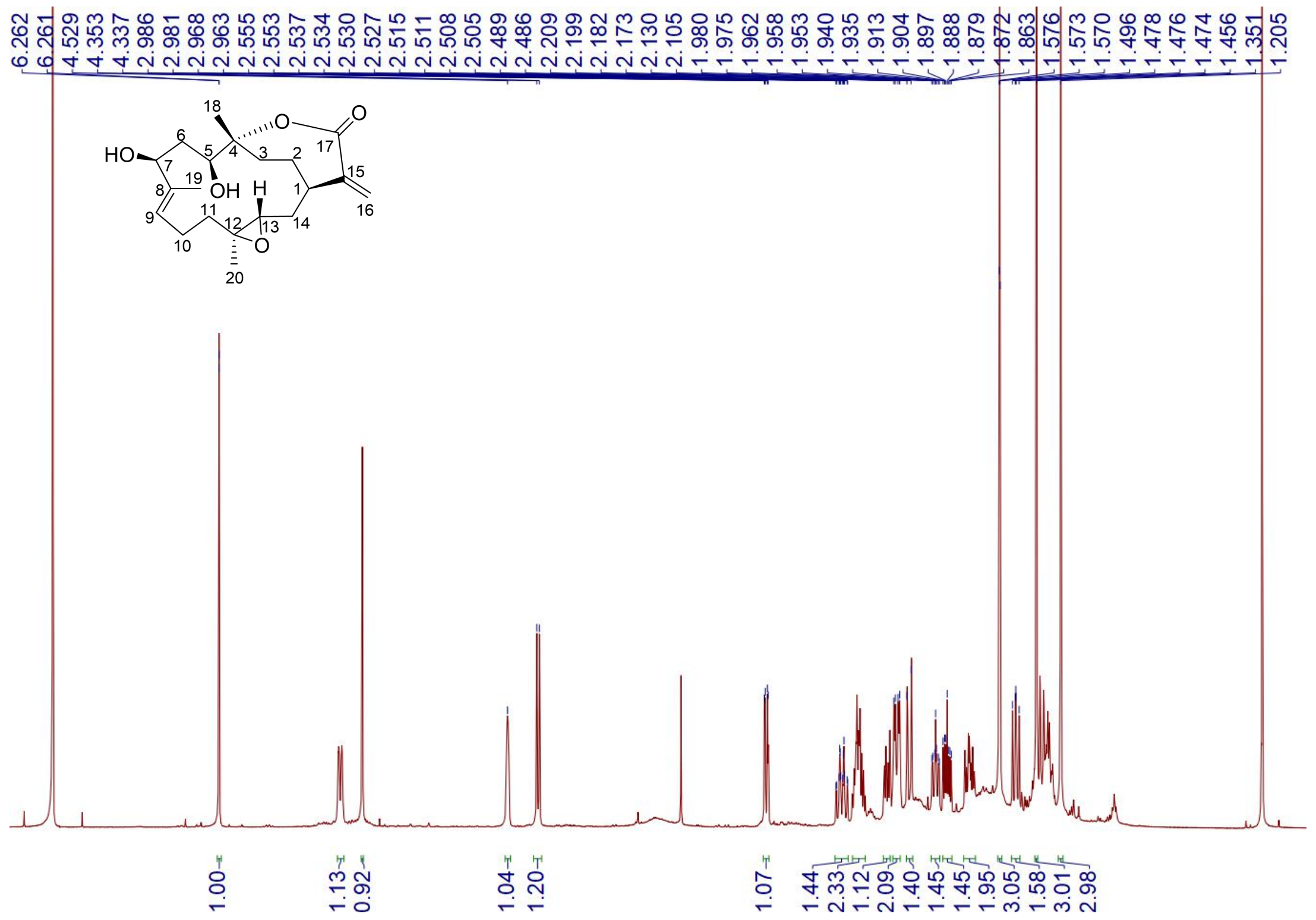

Figure S13. ${ }^{1} \mathrm{H}$ NMR spectrum $(600 \mathrm{MHz})$ of sinusiaeolide $\mathrm{A}(2)$ in $\mathrm{CDCl}_{3}$. 


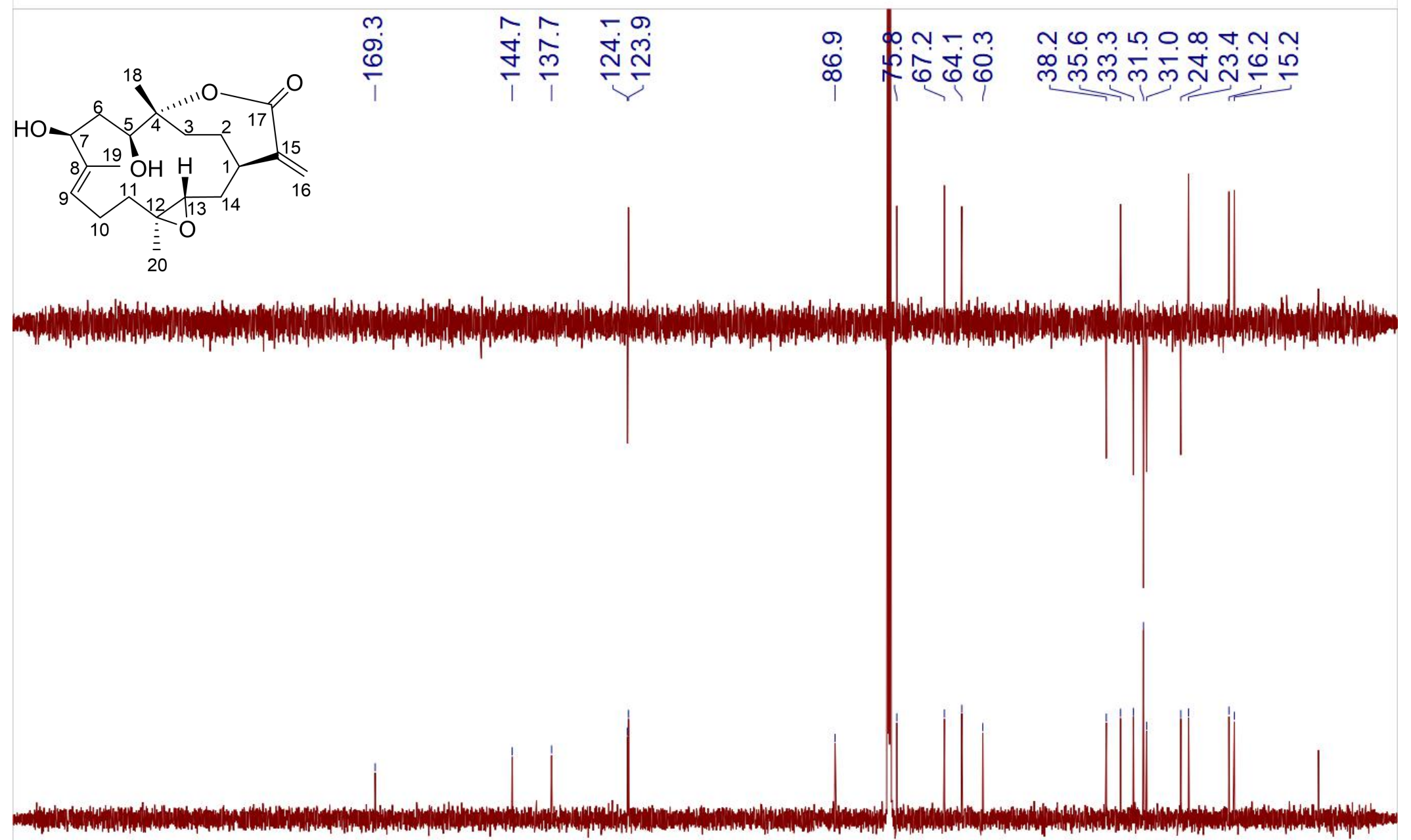

$230220210200190180170160150140130120110100 \quad 90 \quad 80 \quad 70 \quad 60 \begin{array}{llllllllll}10 & 40 & 30 & 20 & 10 & 0 & -10\end{array}$ f1 (ppm)

Figure S14. ${ }^{13} \mathrm{C}$ NMR spectrum $(125 \mathrm{MHz})$ of sinusiaeolide $\mathrm{A}(2)$ in $\mathrm{CDCl}_{3}$. 


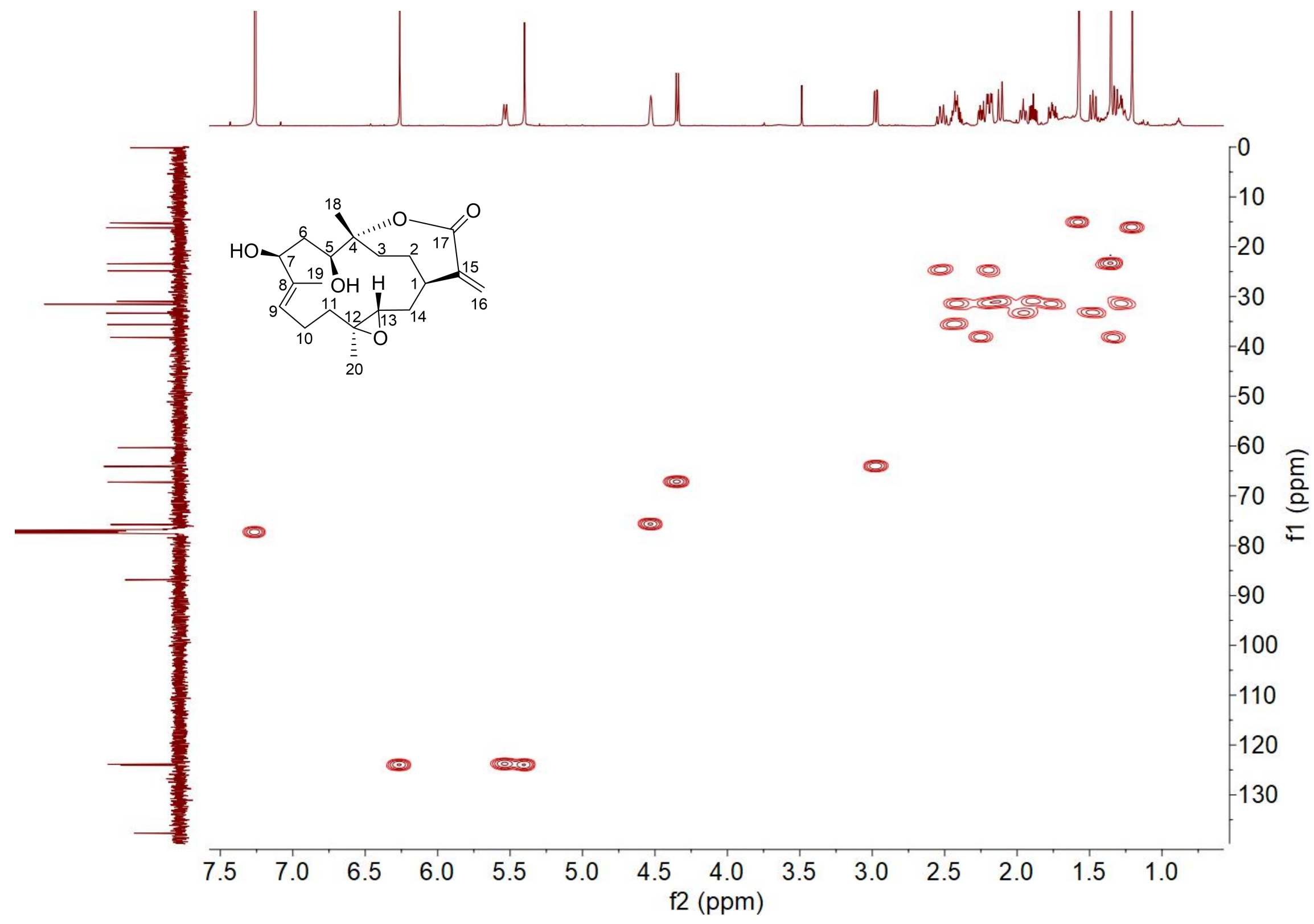

Figure S15. HSQC spectrum (500 MHz) of sinusiaeolide A (2) in $\mathrm{CDCl}_{3}$. 


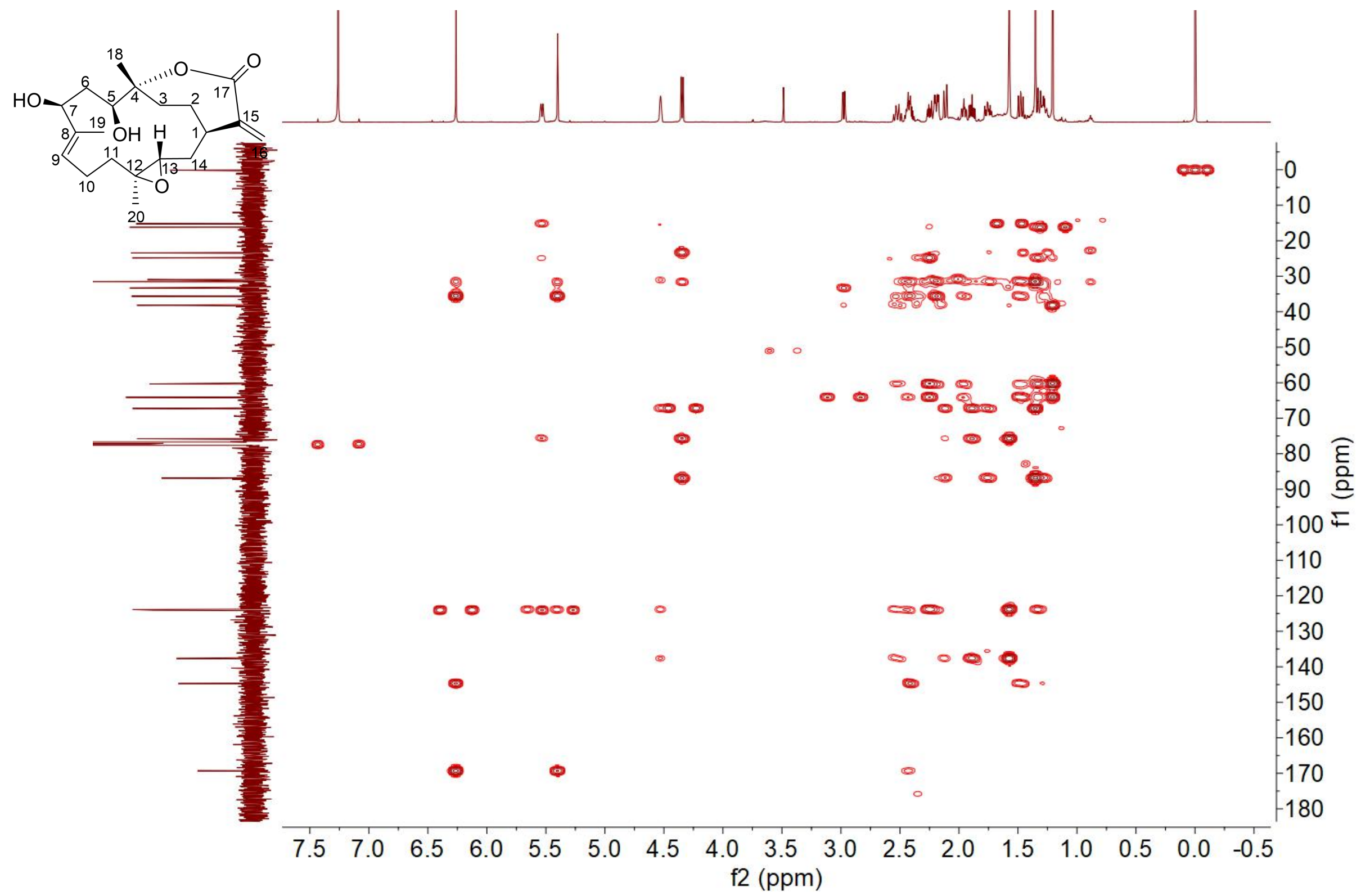

Figure S16. HMBC spectrum (500 MHz) of sinusiaeolide A (2) in $\mathrm{CDCl}_{3}$. 


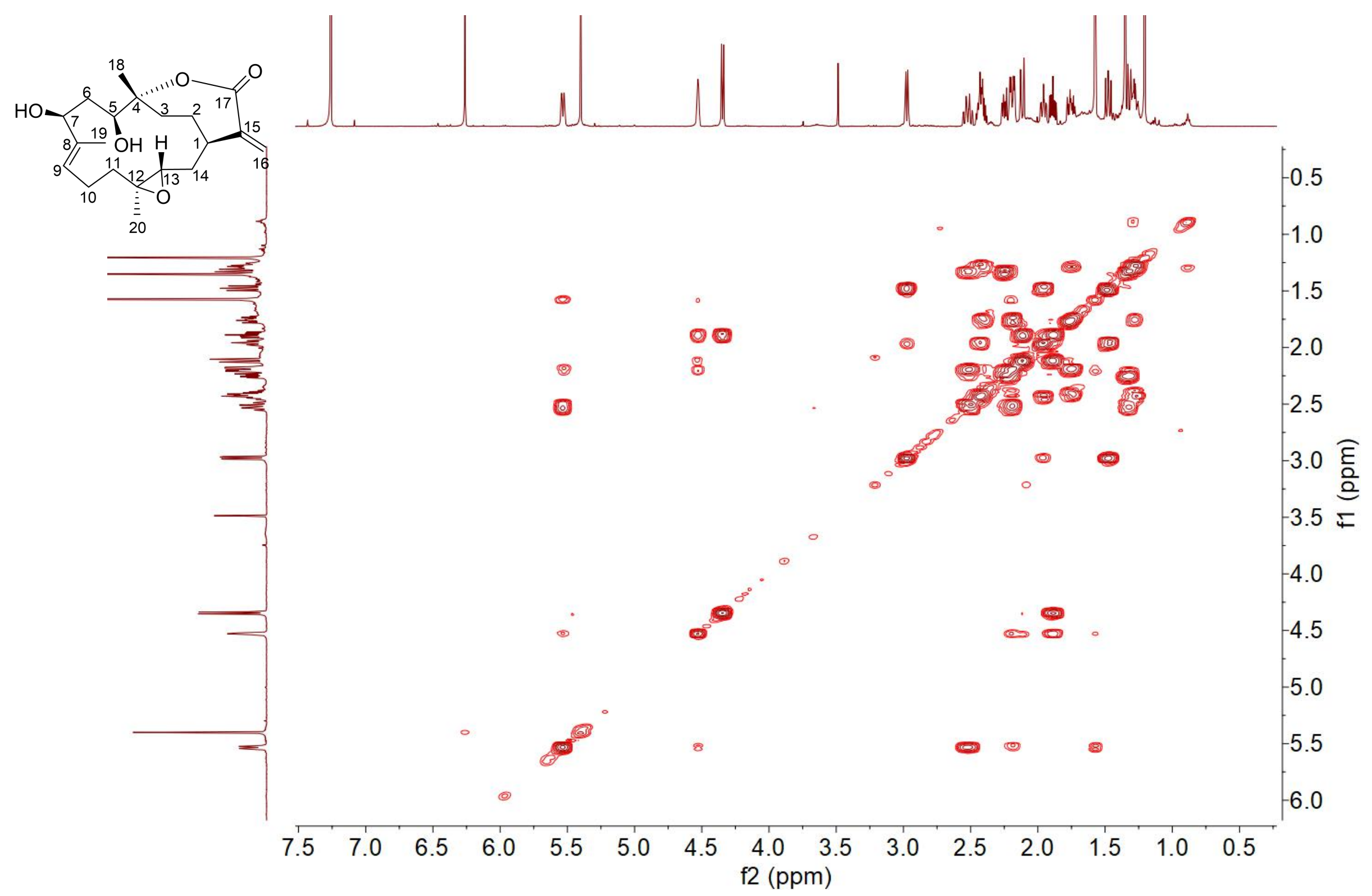

Figure S17. COSY spectrum $(500 \mathrm{MHz})$ of sinusiaeolide $\mathrm{A}(2)$ in $\mathrm{CDCl}_{3}$. 


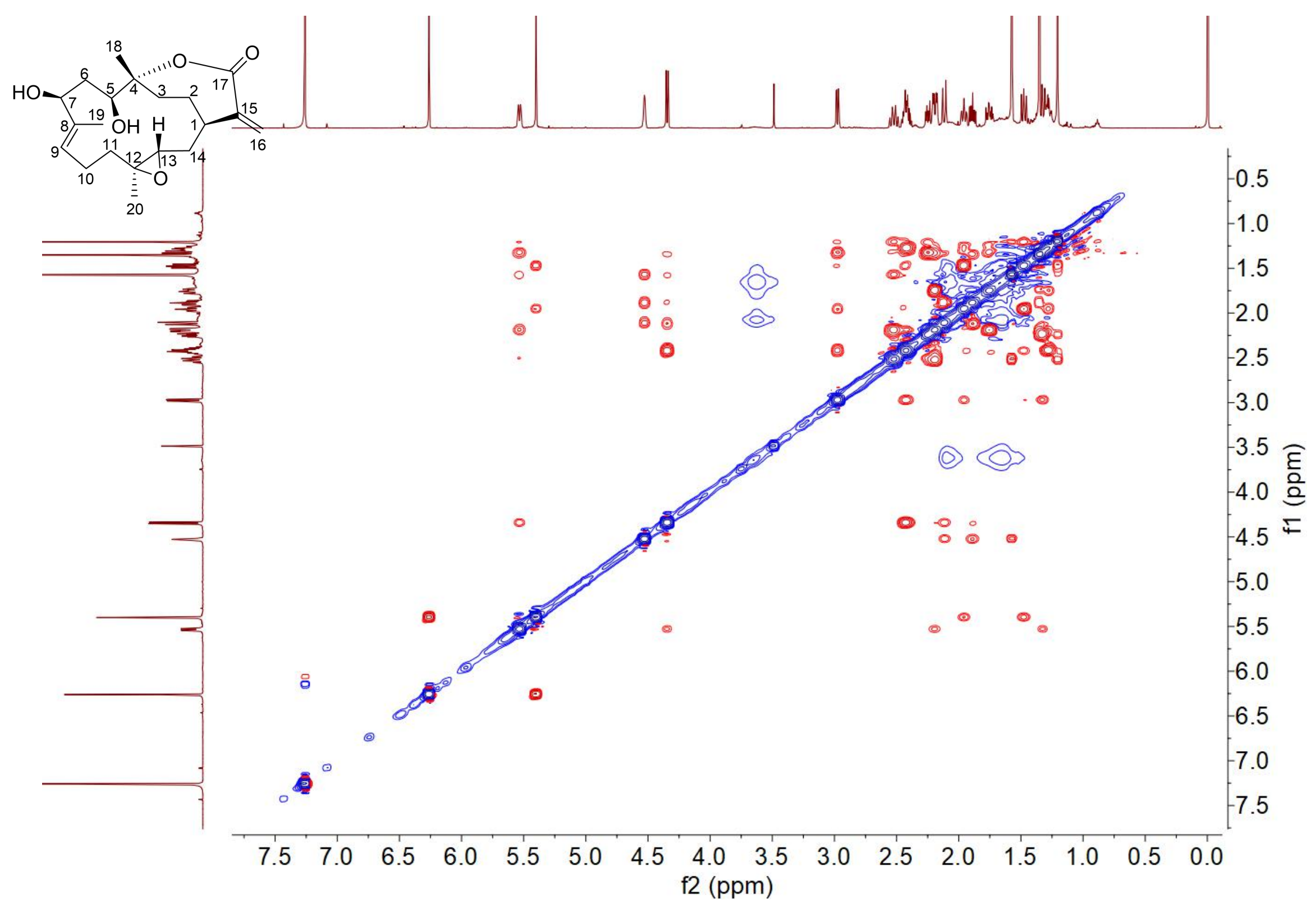

Figure S18. NOESY spectrum (500 MHz) of sinusiaeolide A (2) in $\mathrm{CDCl}_{3}$. 


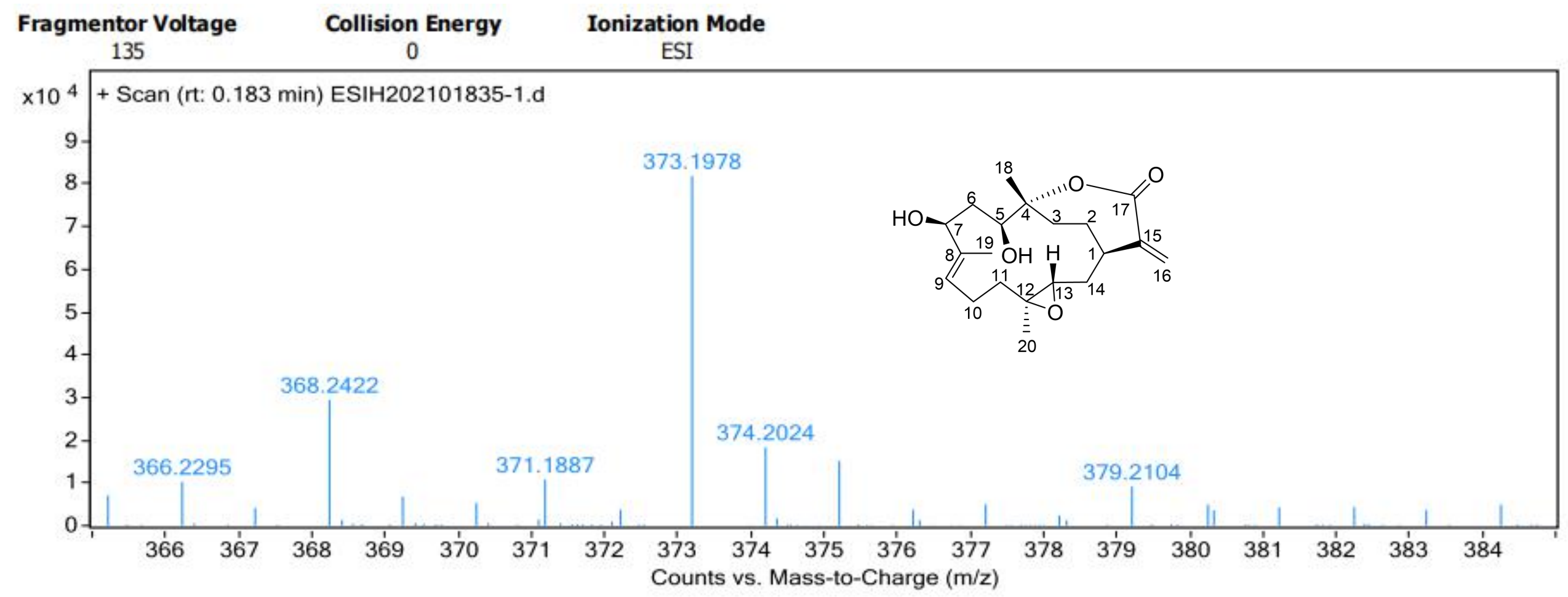

Formula Calculator Results

\begin{tabular}{|l|r|r|r|l|l|}
\hline $\mathbf{m} / \mathbf{z}$ & Calc m/z & Diff (mDa) & Diff (ppm) & Ion Formula & Ion \\
\hline 373.1978 & 373.1985 & 0.79 & 2.11 & $\mathrm{C} 20 \mathrm{H} 30 \mathrm{Na} \mathrm{O5}$ & $(\mathrm{M}+\mathrm{Na})+$ \\
\hline
\end{tabular}

Figure S19. HRESIMS spectrum of sinusiaeolide A (2) in MeOH. 


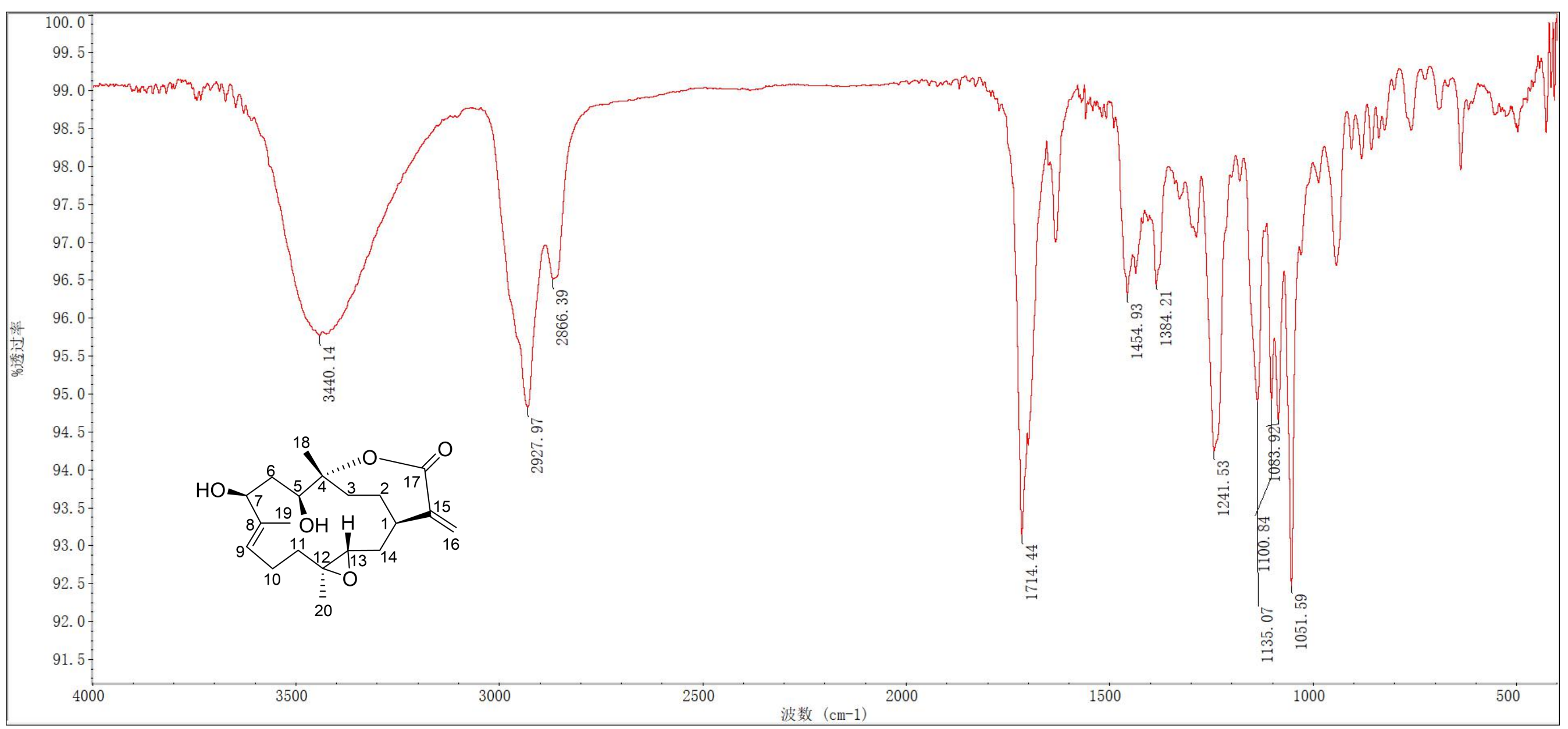

Figure S20. IR spectrum of sinusiaeolide A (2). 


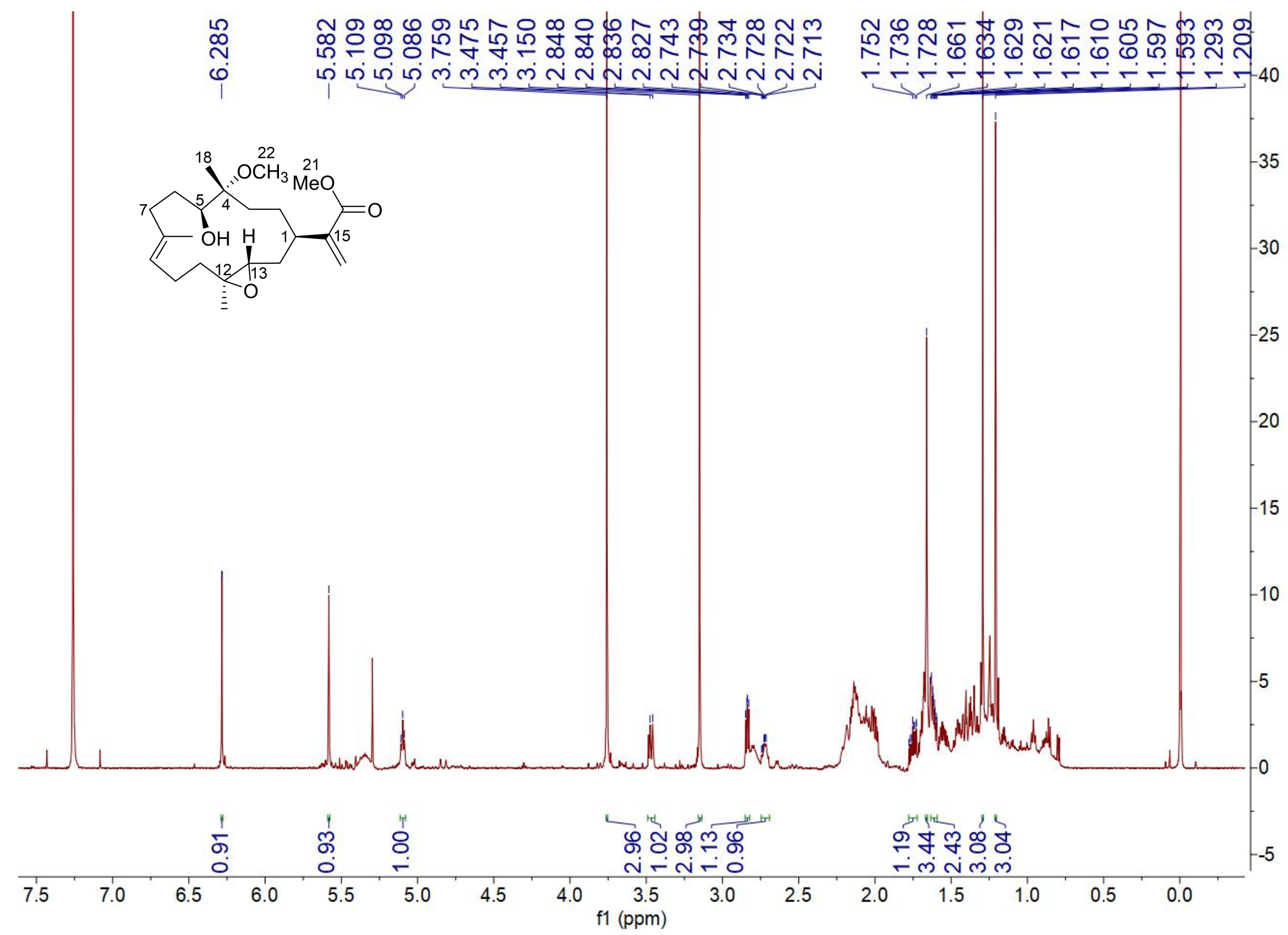

Figure S21. ${ }^{1} \mathrm{H}$ NMR spectrum $(600 \mathrm{MHz})$ of sinusiaesin $\mathrm{A}(3)$ in $\mathrm{CDCl}_{3}$. 


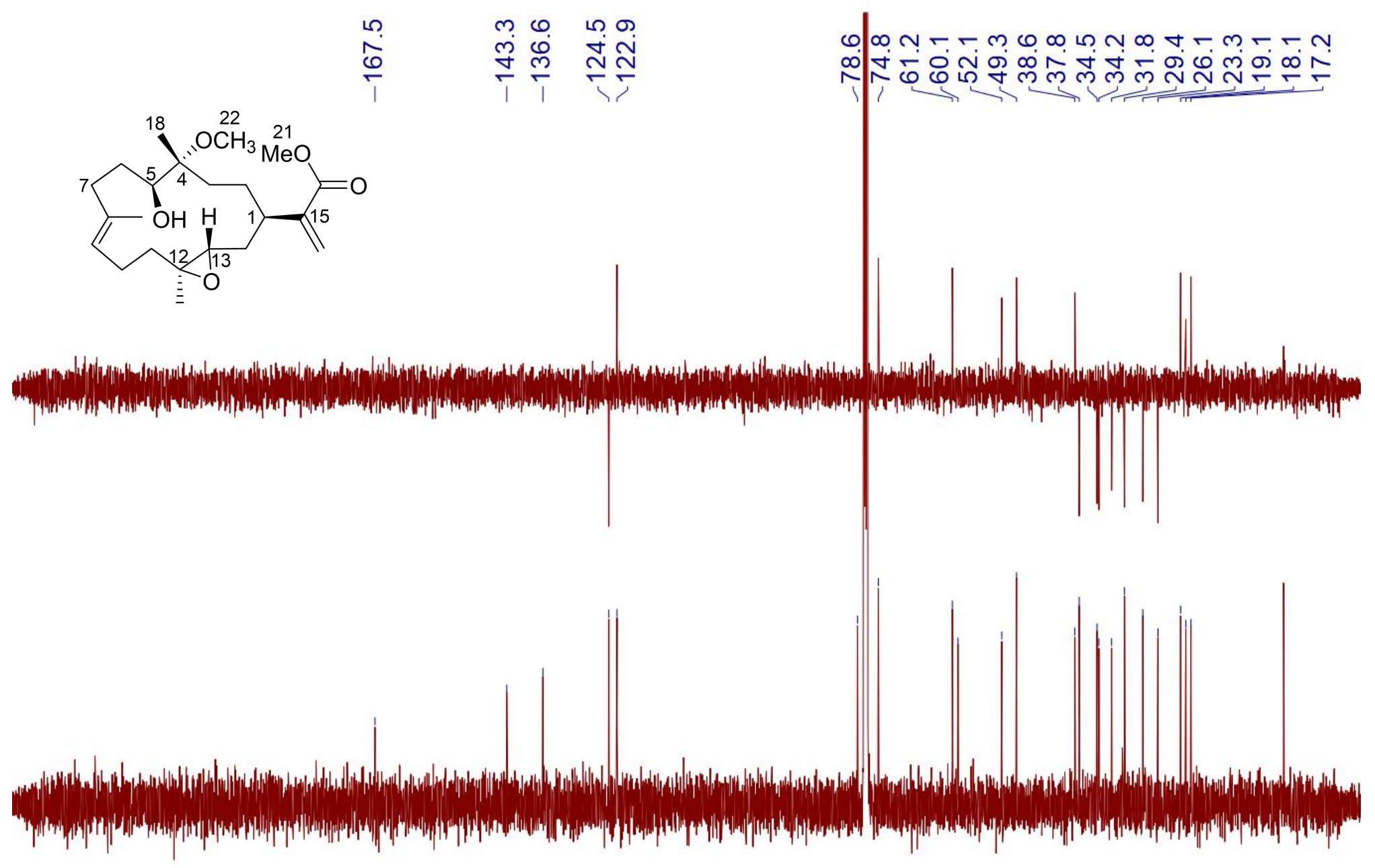

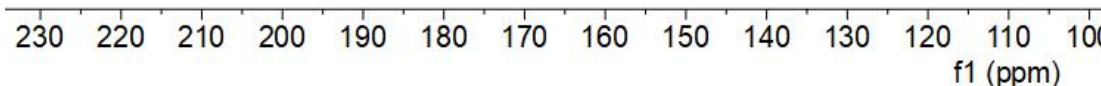

Figure S22. ${ }^{13} \mathrm{C}$ NMR spectrum $(125 \mathrm{MHz})$ of sinusiaesin $\mathrm{A}(3)$ in $\mathrm{CDCl}_{3}$. 


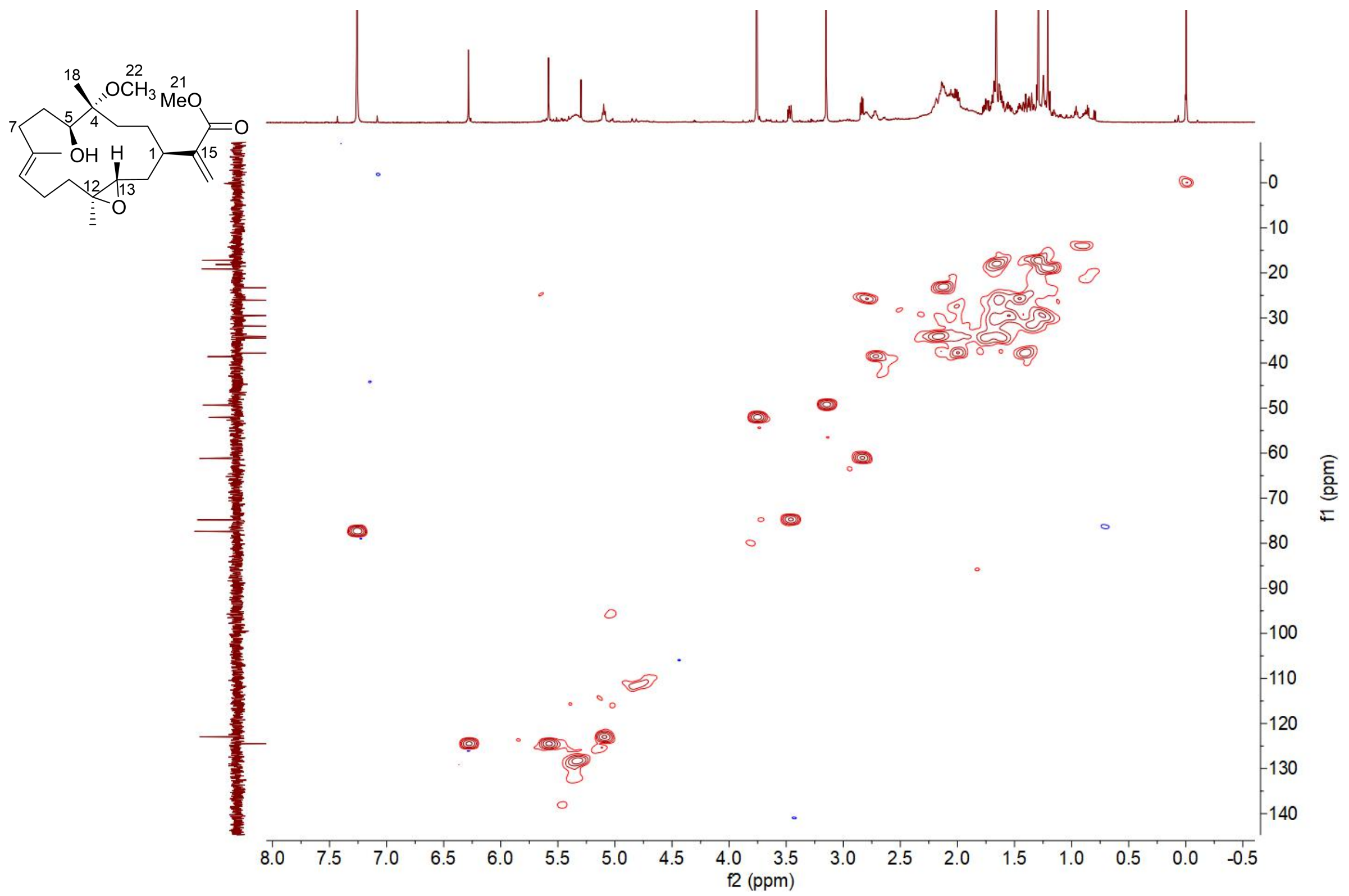

Figure S23. HSQC spectrum (500 MHz) of sinusiaesin A (3) in $\mathrm{CDCl}_{3}$. 


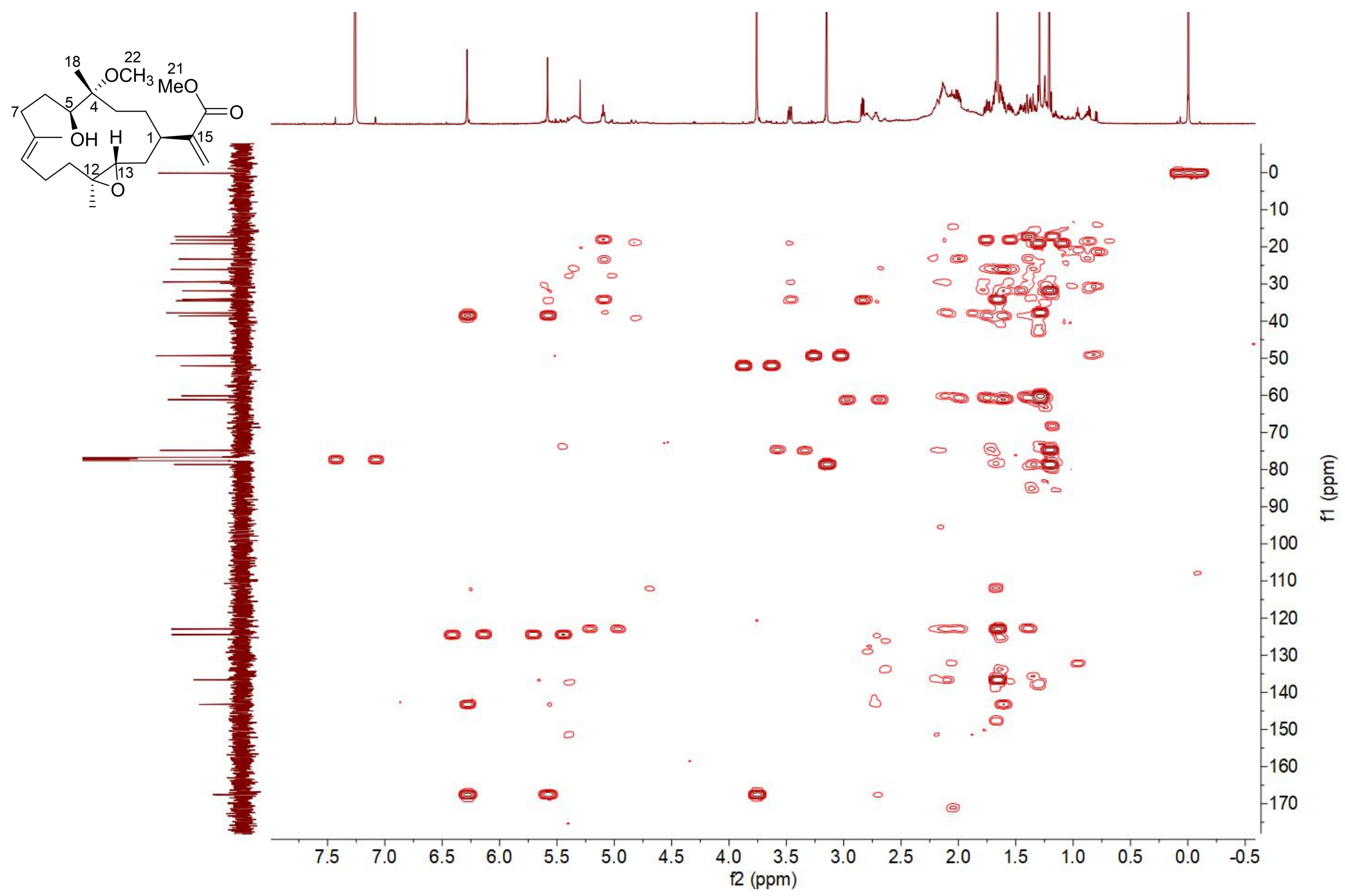

Figure S24. HMBC spectrum $(500 \mathrm{MHz})$ of sinusiaesin $\mathrm{A}(3)$ in $\mathrm{CDCl}_{3}$. 


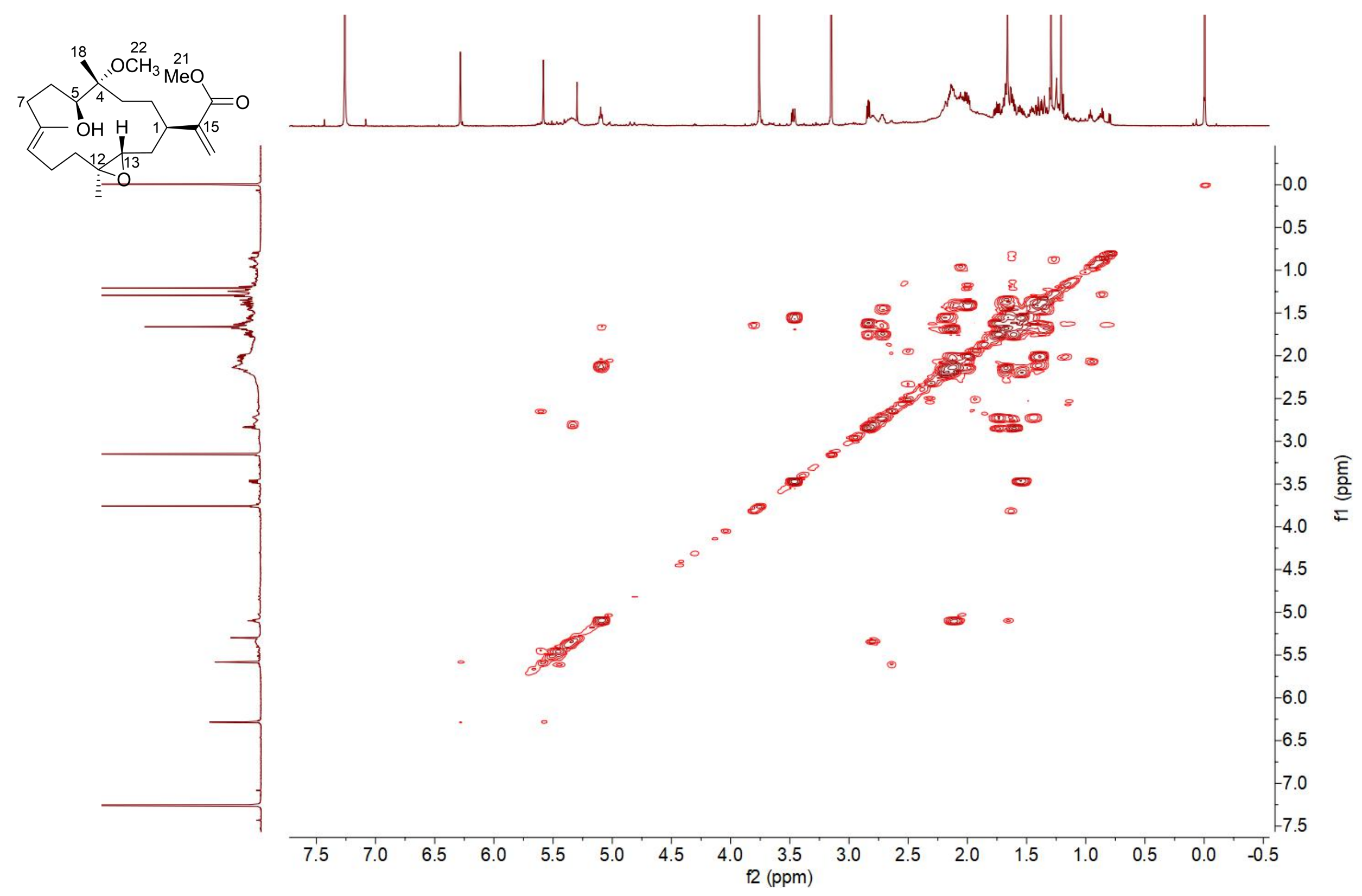

Figure S25. COSY spectrum (500 MHz) of sinusiaesin A (3) in $\mathrm{CDCl}_{3}$. 


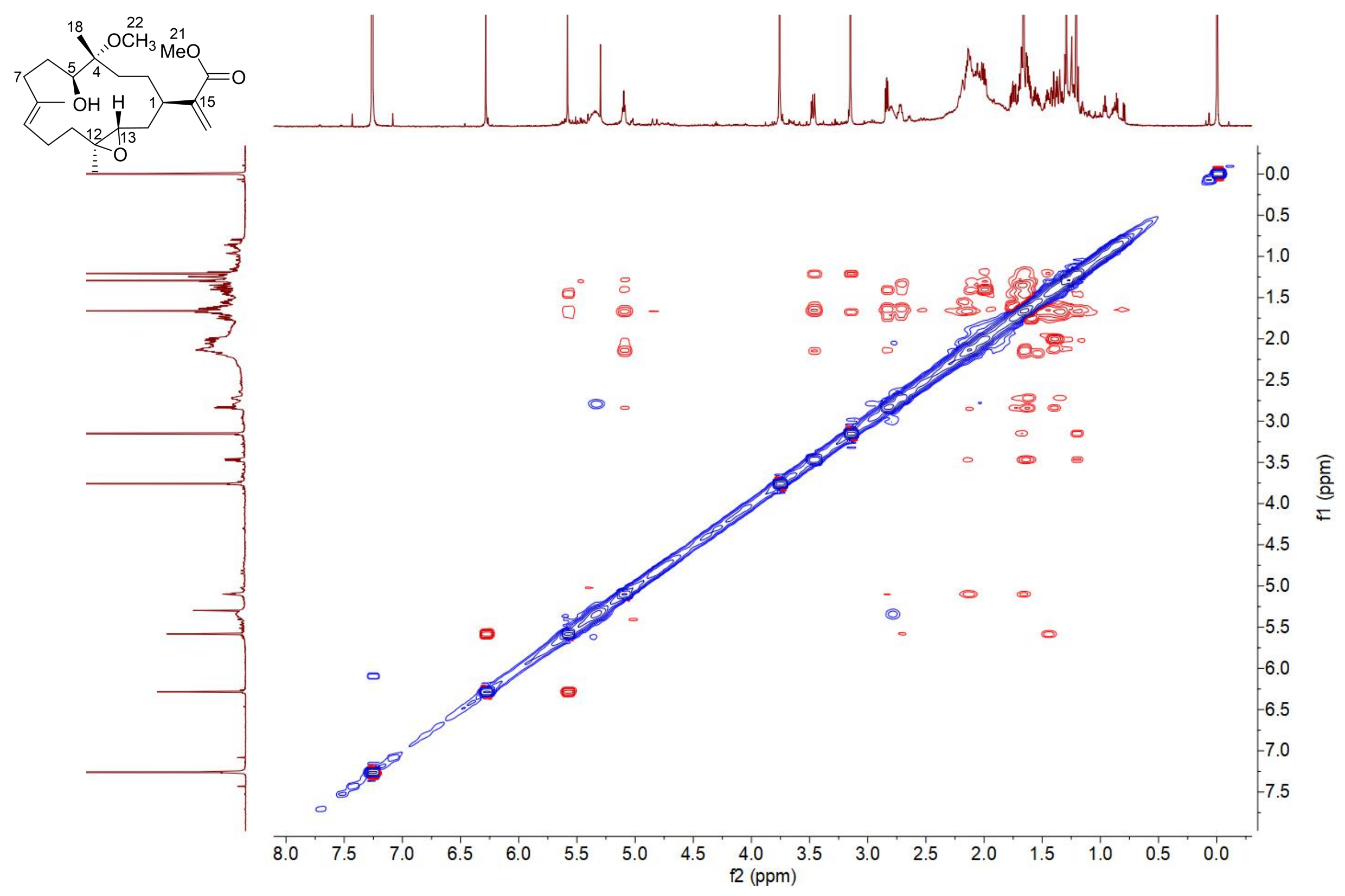

Figure S26. NOESY spectrum (500 MHz) of sinusiaesin A (3) in $\mathrm{CDCl}_{3}$. 


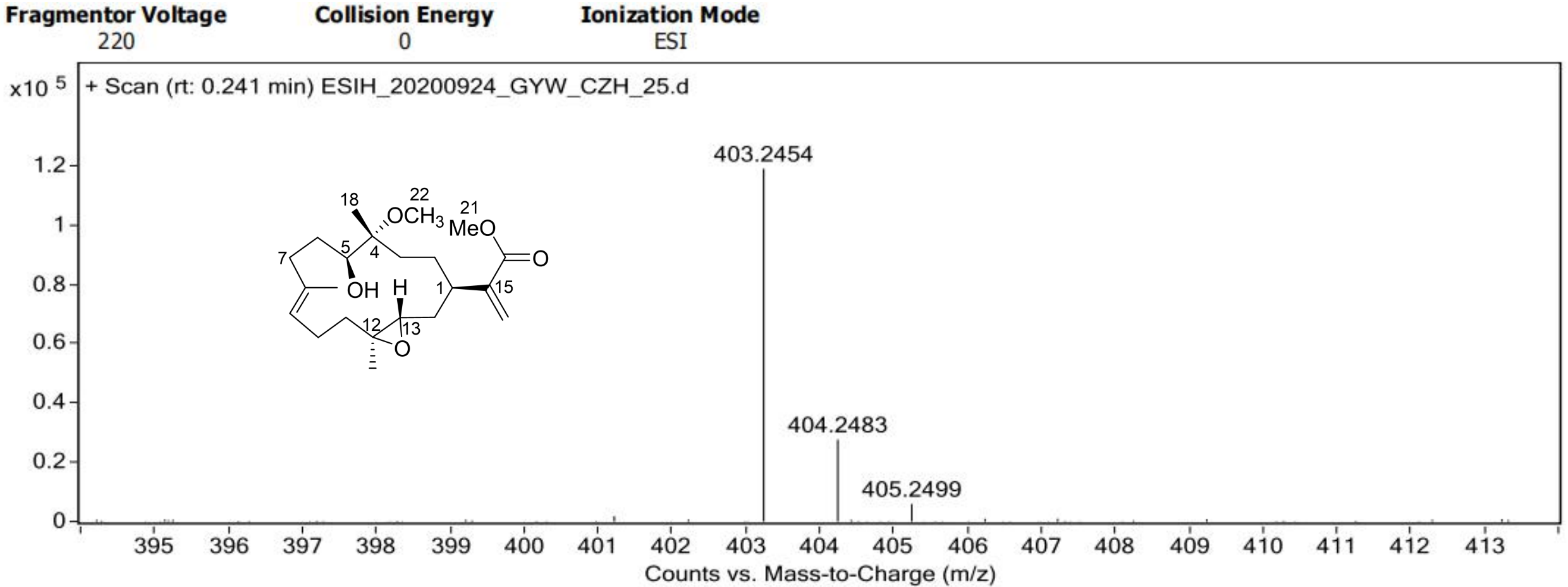

Formula Calculator Results

\begin{tabular}{|l|l|l|l|l|l|}
\hline $\mathbf{m} / \mathbf{z}$ & Calc $\mathbf{~ m / z}$ & Diff $(\mathbf{m D a})$ & Diff $(\mathbf{p p m})$ & Ion Formula & Ion \\
\hline 403.2454 & 403.2455 & 0.14 & 0.35 & $\mathrm{C} 22 \mathrm{H} 36 \mathrm{Na} \mathrm{O} 5$ & $(\mathrm{M}+\mathrm{Na})+$ \\
\hline
\end{tabular}

Figure S27. HRESIMS spectrum of sinusiaesin A (3) in $\mathrm{MeOH}$. 


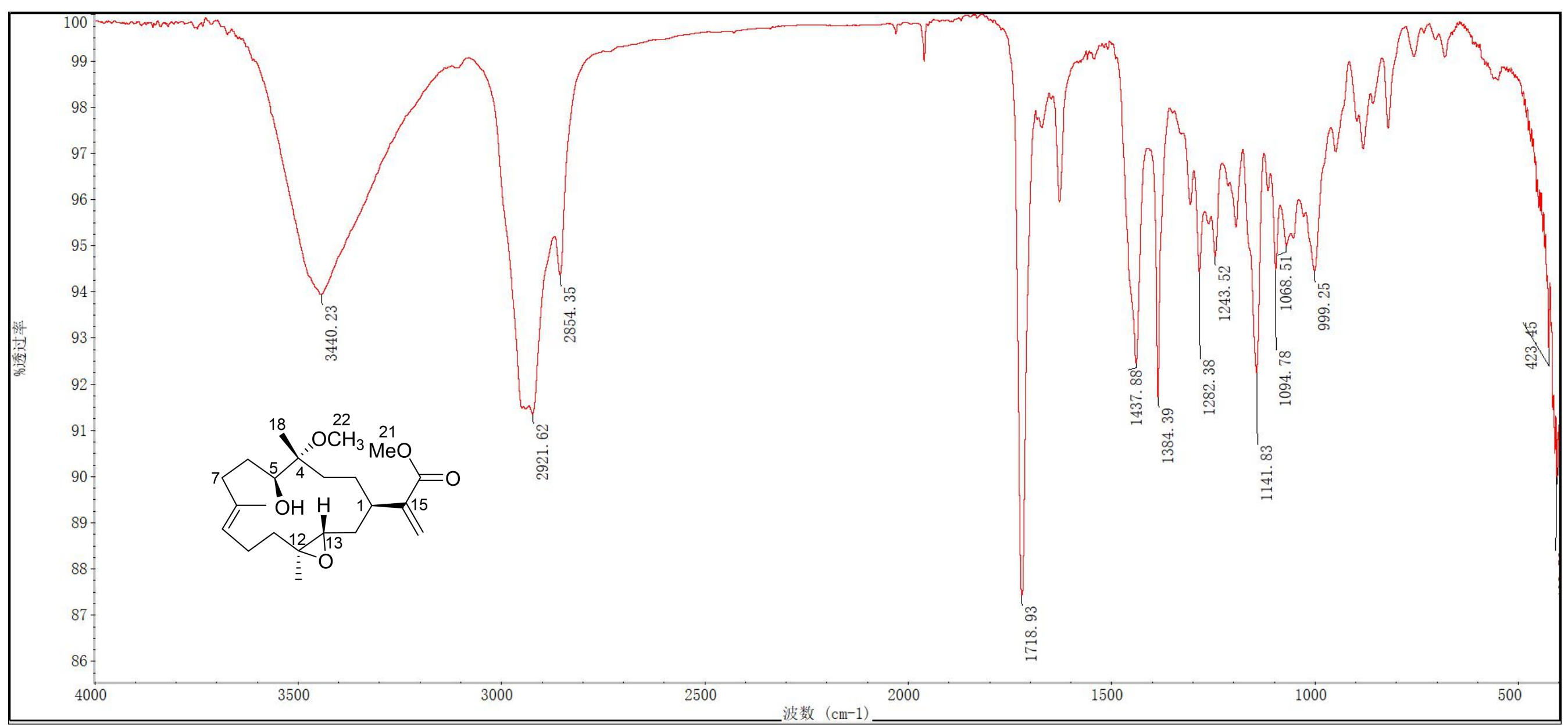

Figure S28. IR spectrum of sinusiaesin A (3). 


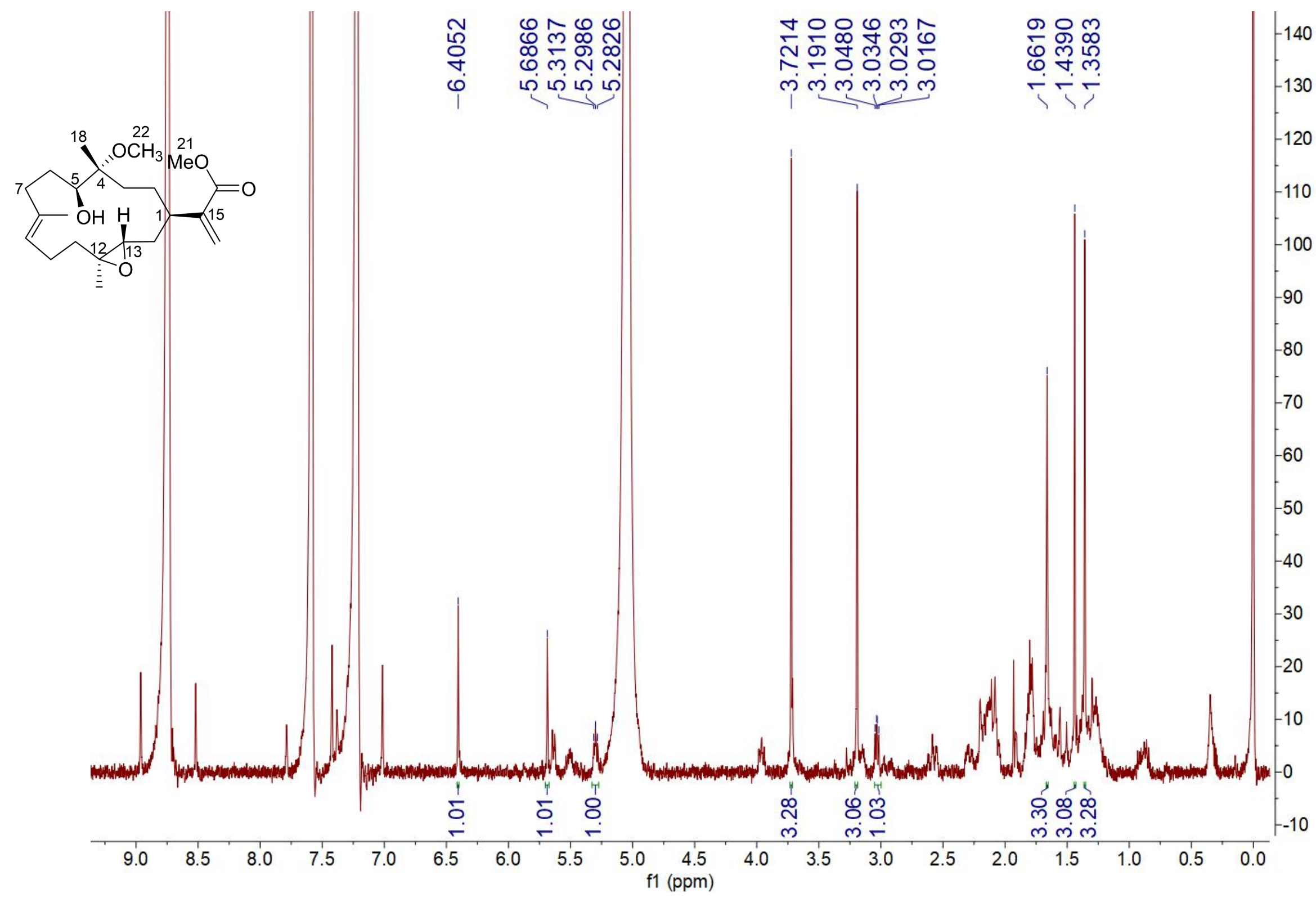

Figure S29. ${ }^{1} \mathrm{H}$ NMR spectrum $(400 \mathrm{MHz})$ of 3 in $\mathrm{C}_{5} \mathrm{D}_{5} \mathrm{~N}$. 


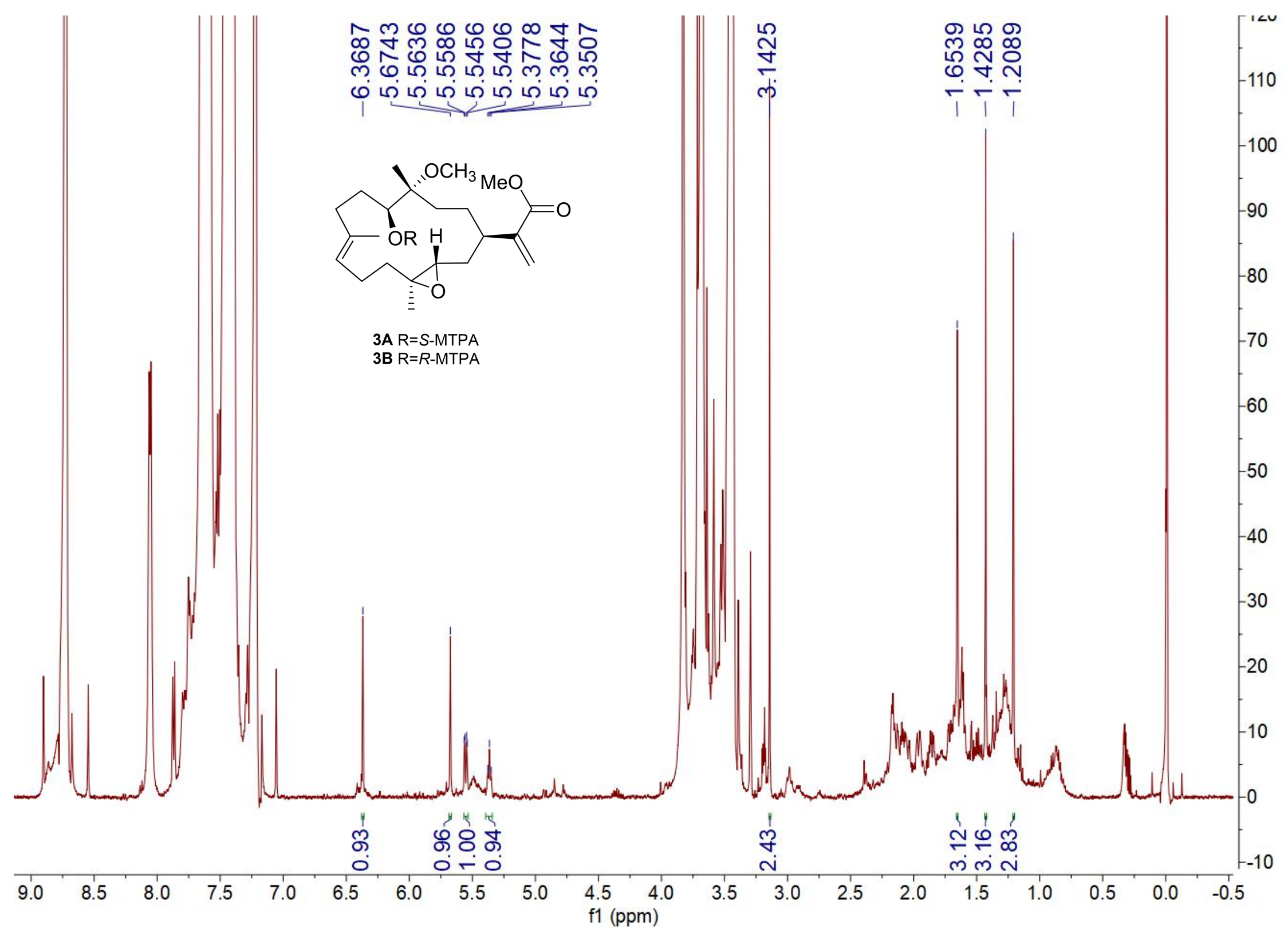

Figure S30. ${ }^{1} \mathrm{H}$ NMR spectrum $(400 \mathrm{MHz})$ of $\mathbf{3 A}$ in $\mathrm{C}_{5} \mathrm{D}_{5} \mathrm{~N}$. 


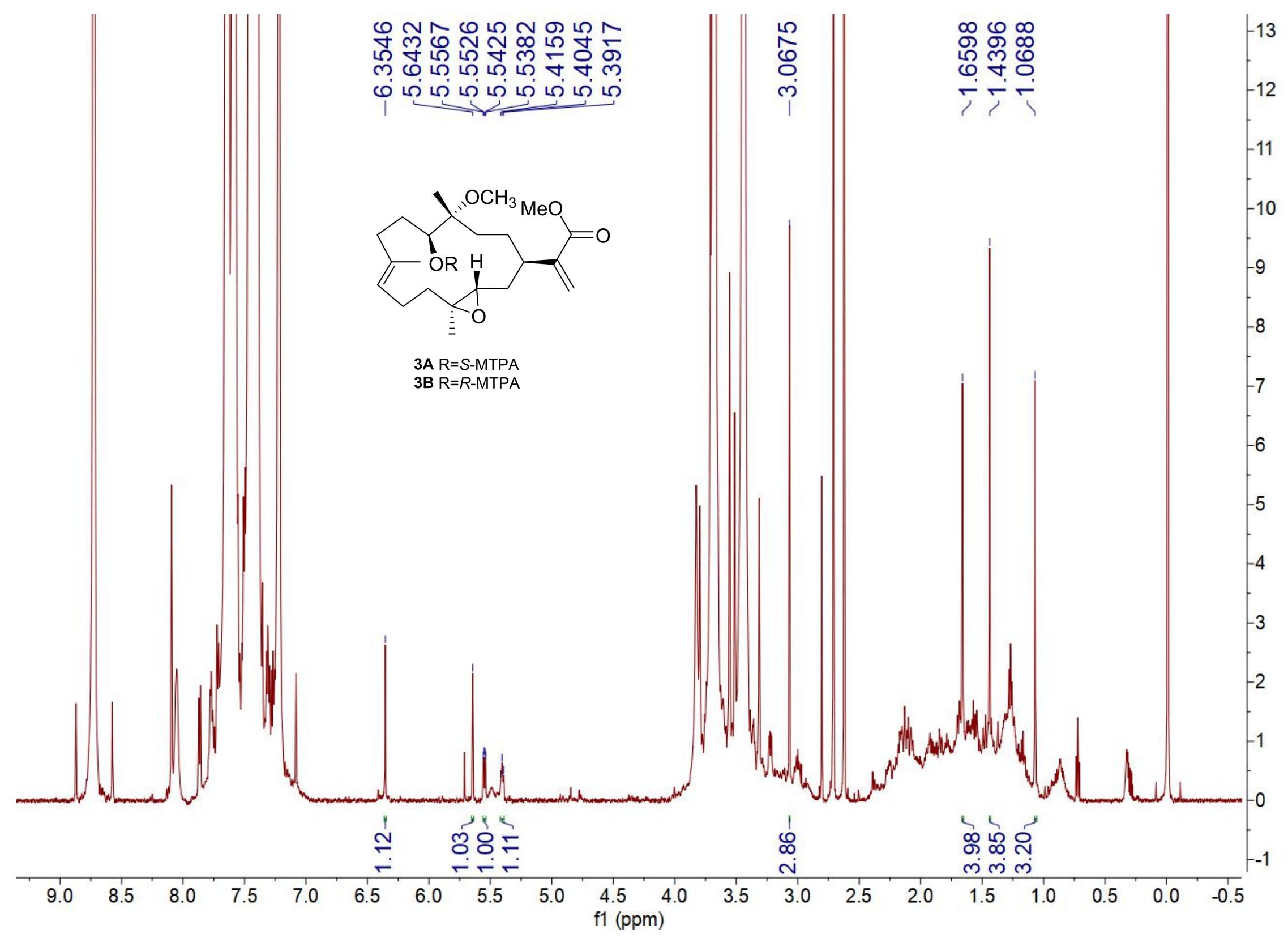

Figure S31. ${ }^{1} \mathrm{H}$ NMR spectrum $(400 \mathrm{MHz})$ of $3 B$ in $\mathrm{C}_{5} \mathrm{D}_{5} \mathrm{~N}$. 


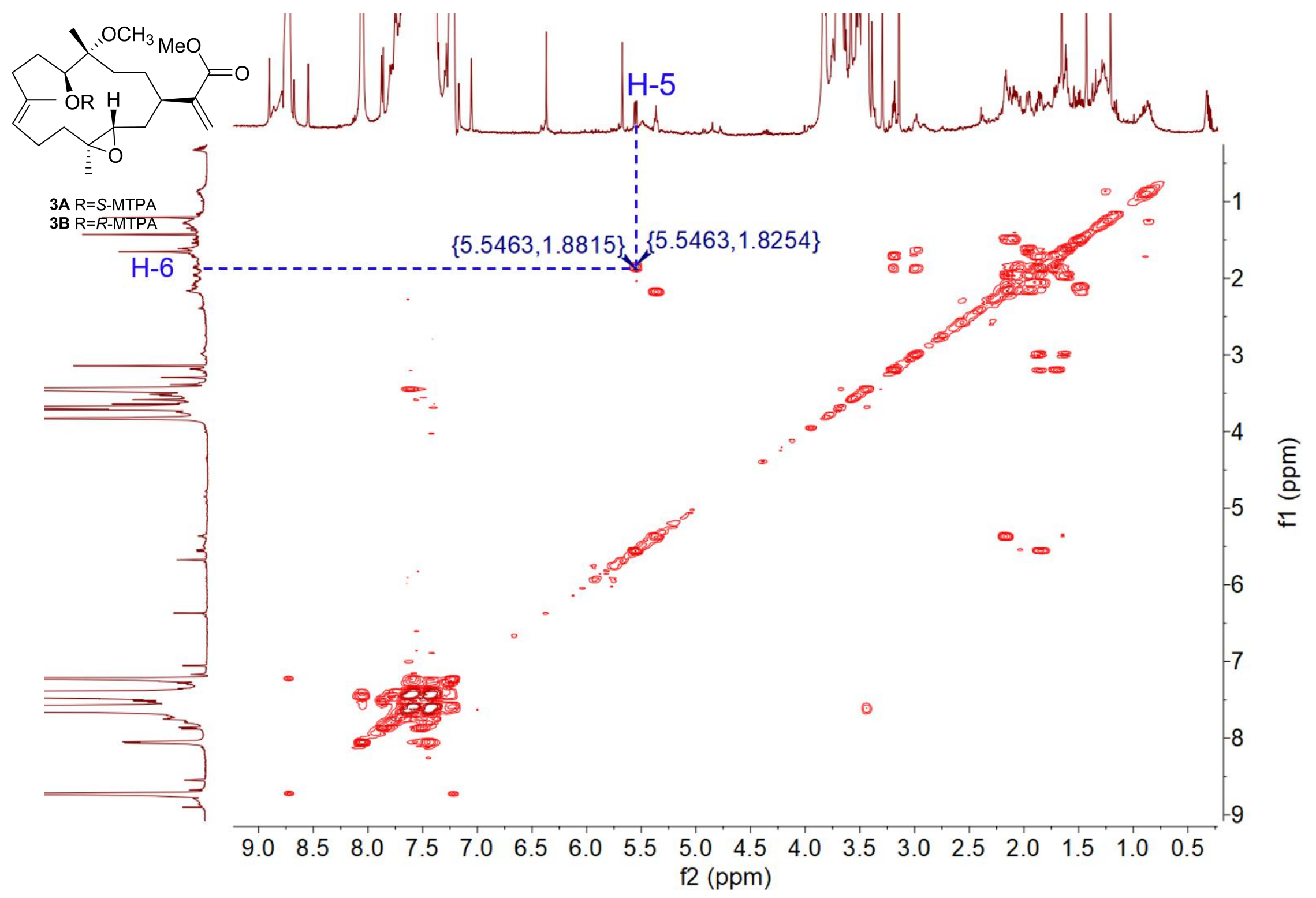

Figure S32. COSY spectrum (500 MHz) of $\mathbf{3 A}$ in $\mathrm{C}_{5} \mathrm{D}_{5} \mathrm{~N}$. 


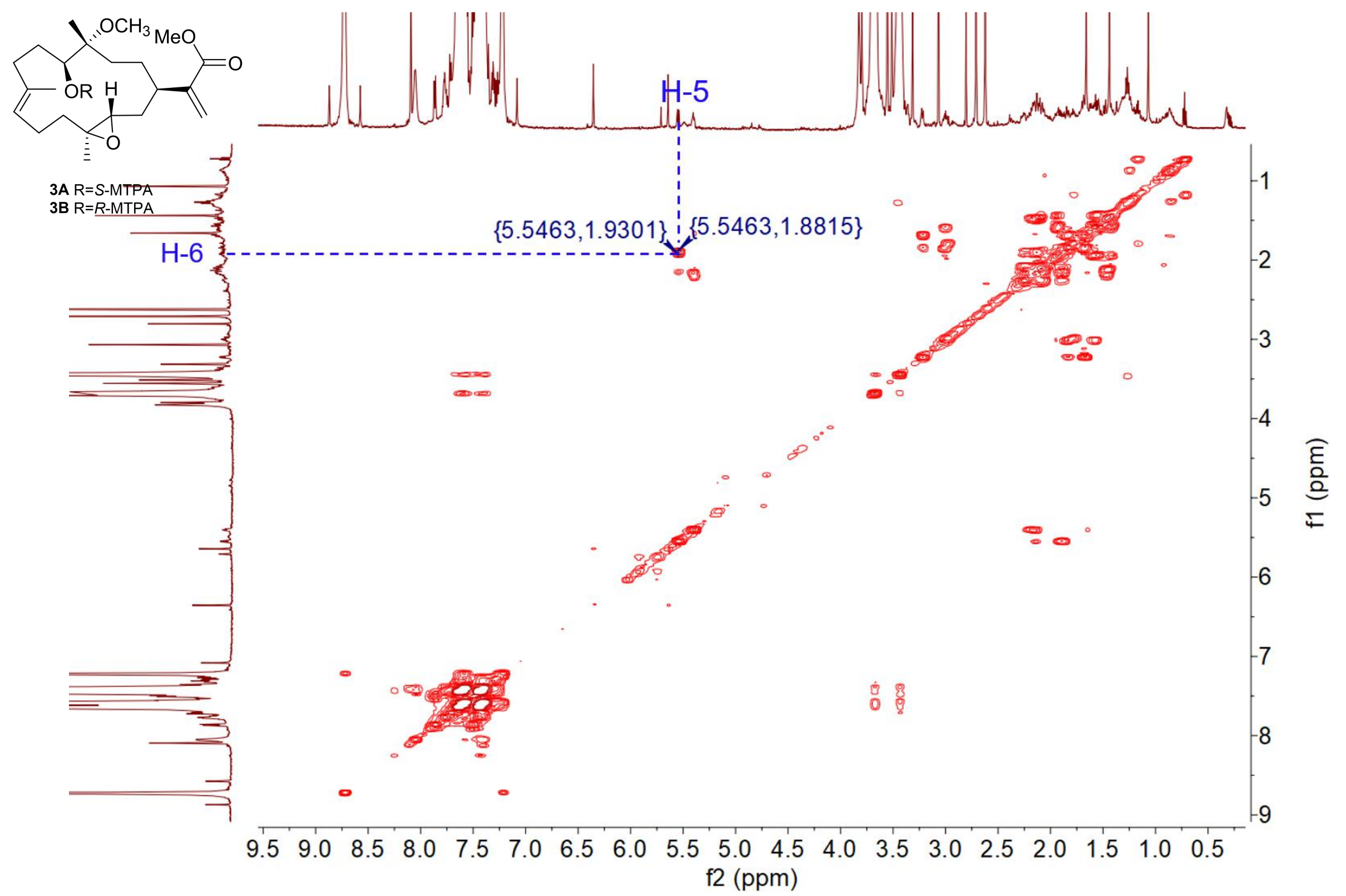

Figure S33. COSY spectrum $(500 \mathrm{MHz})$ of $\mathbf{3 B}$ in $\mathrm{C}_{5} \mathrm{D}_{5} \mathrm{~N}$. 

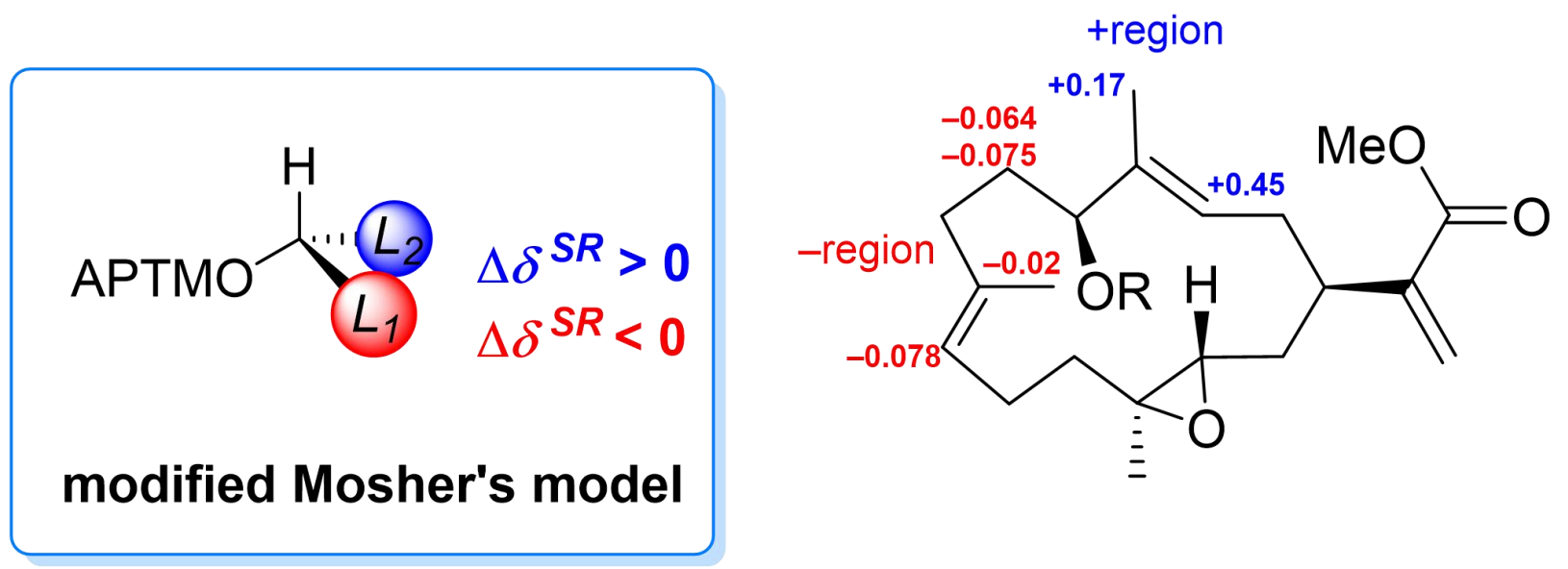

$5 A$ R $=S-M T P A$

5B R=R-MTPA

Figure S34. Application of the modified Mosher's method for AC determination of the secondary alcohol on $\mathbf{5}$. Values of $\Delta \delta_{S R}\left[\Delta\left(\delta_{S}-\delta_{R}\right)\right]$ were given in ppm. Regions (+ and - ) were marked in blue and red, respectively. 


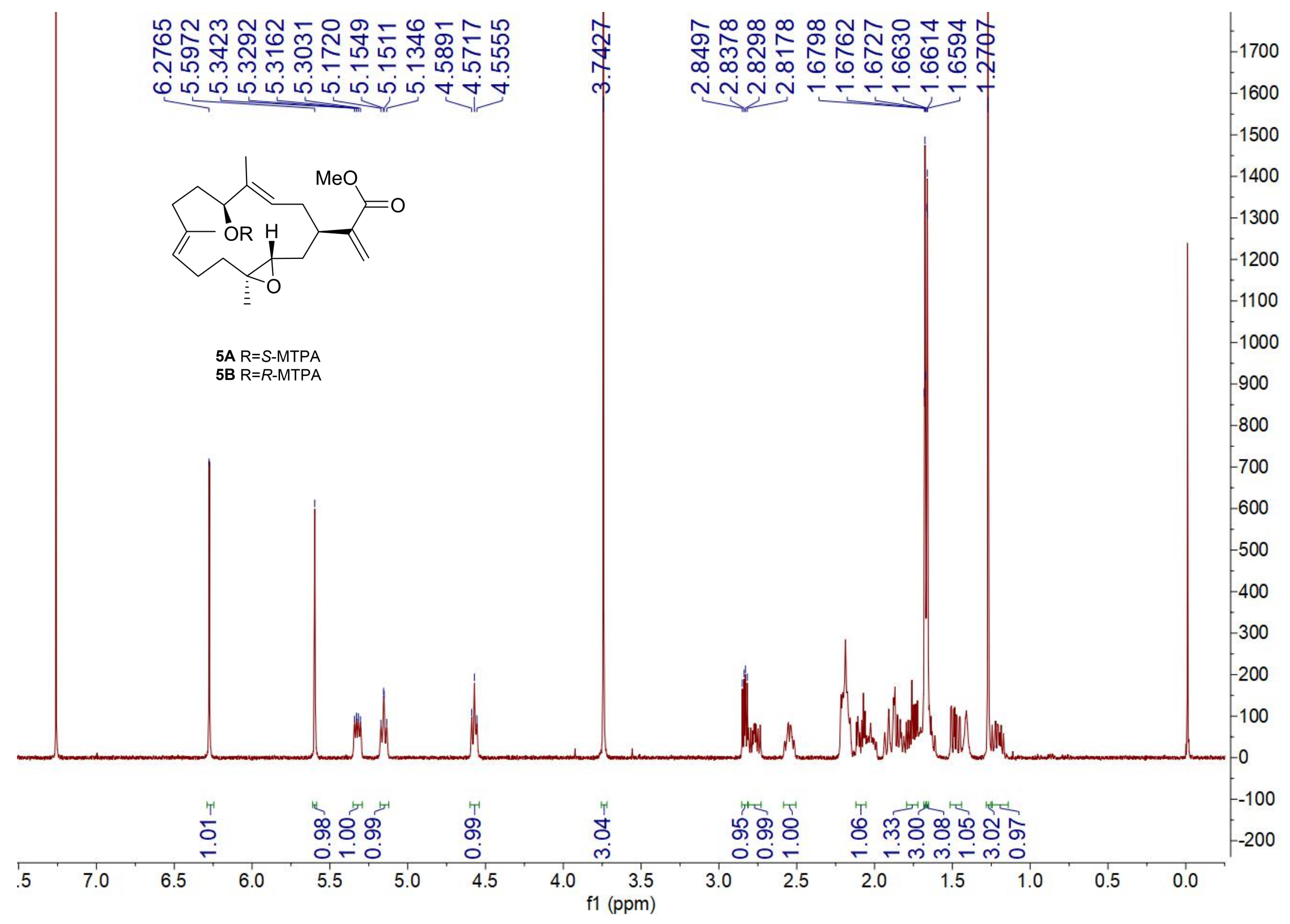

Figure S35. ${ }^{1} \mathrm{H}$ NMR spectrum $(400 \mathrm{MHz})$ of 5 in $\mathrm{CDCl}_{3}$. 


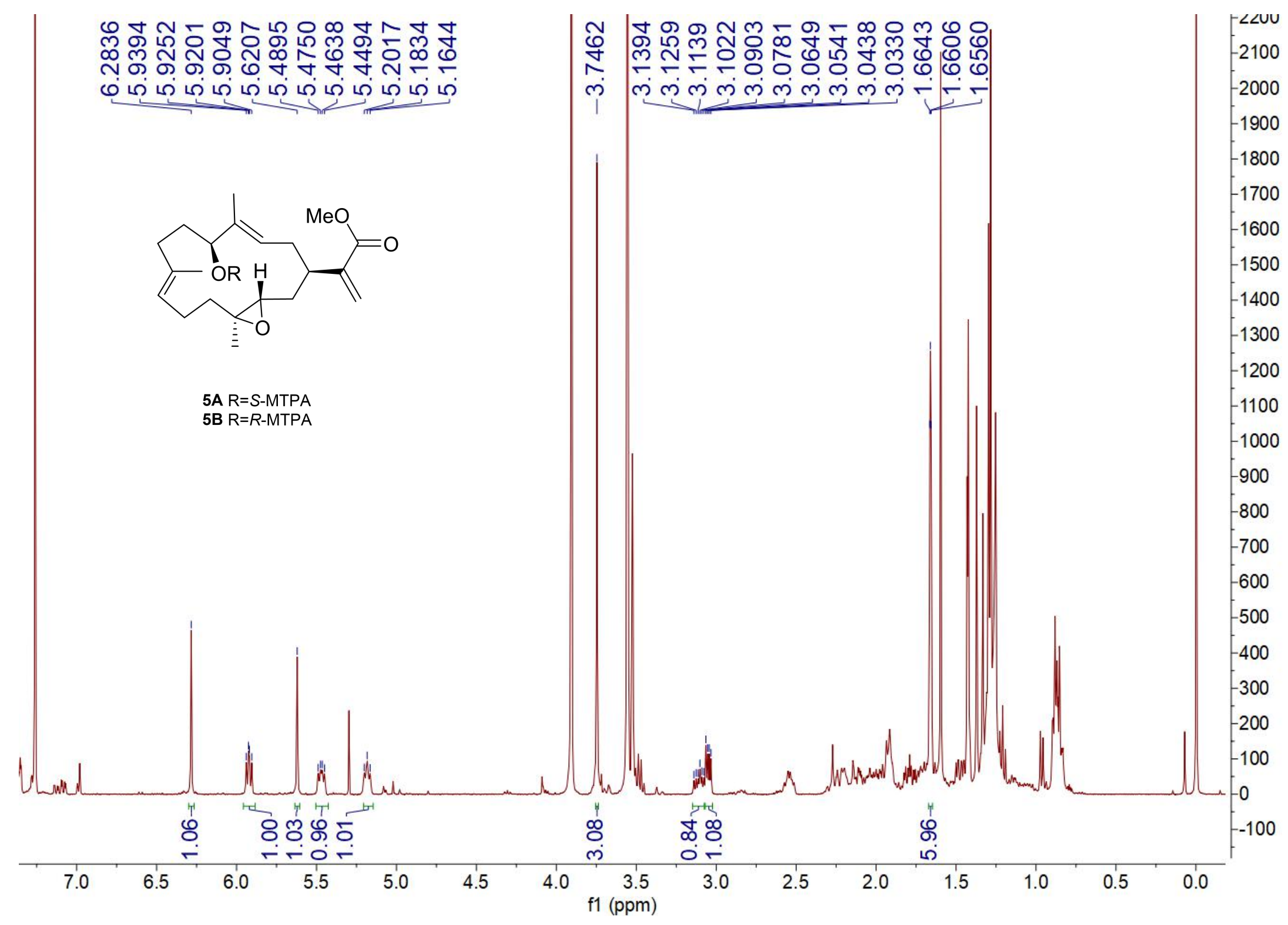

Figure S36. ${ }^{1} \mathrm{H}$ NMR spectrum $(400 \mathrm{MHz})$ of $\mathbf{5 A}$ in $\mathrm{CDCl}_{3}$. 


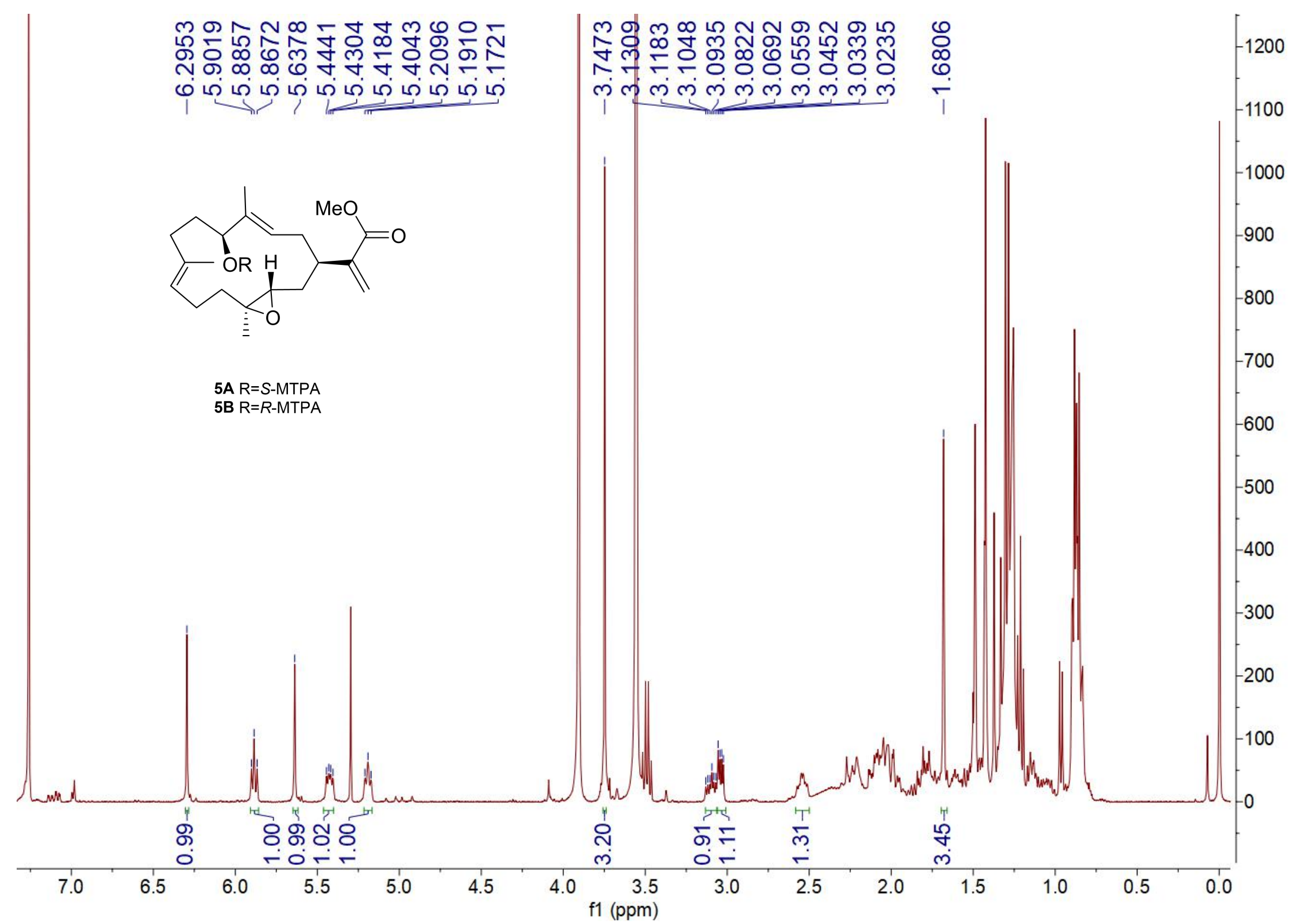

Figure S37. ${ }^{1} \mathrm{H}$ NMR spectrum $(400 \mathrm{MHz})$ of $\mathbf{5 B}$ in $\mathrm{CDCl}_{3}$. 


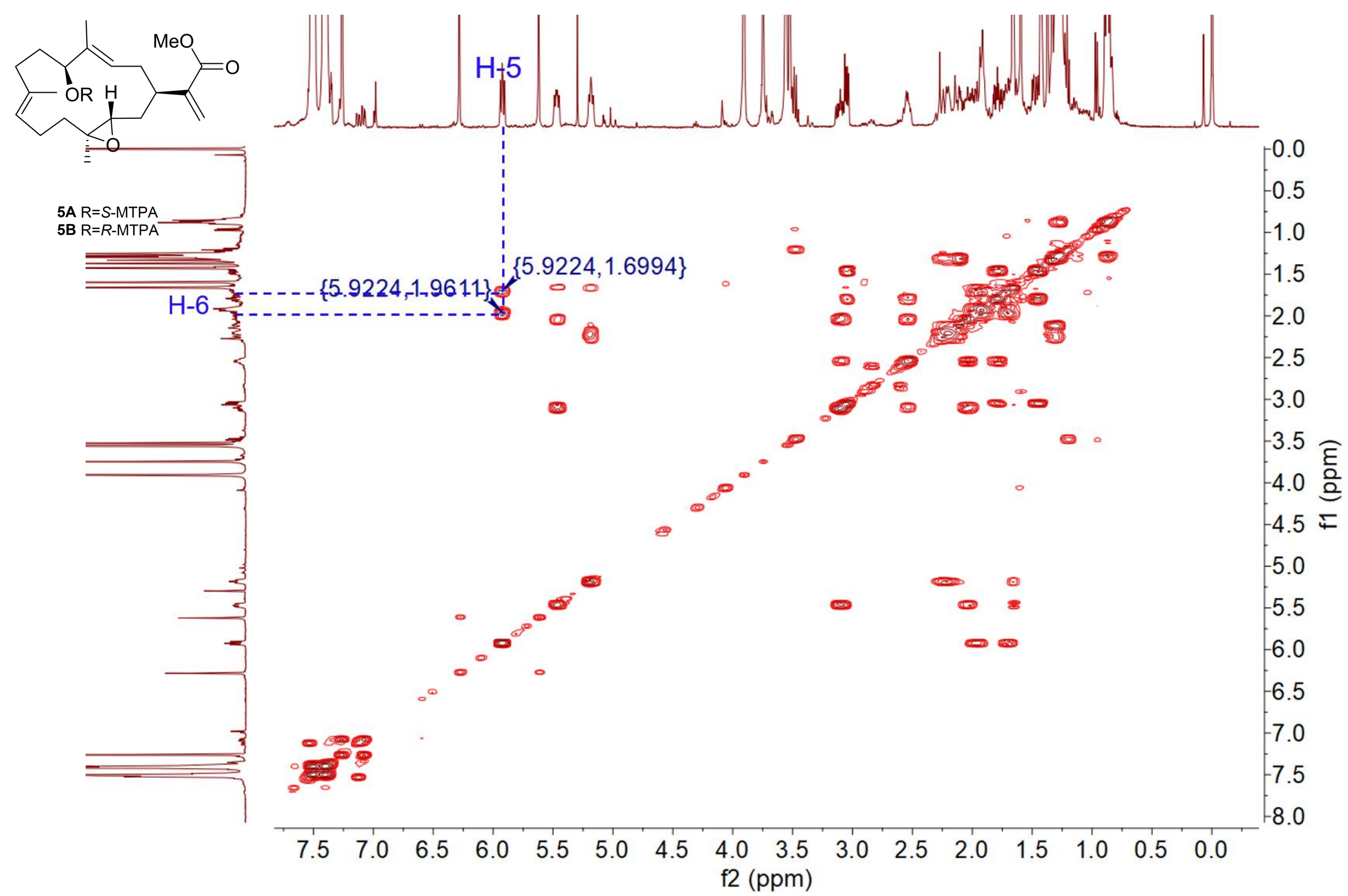

Figure S38. ${ }^{1} \mathrm{H}$ NMR spectrum $(500 \mathrm{MHz})$ of $\mathbf{5 A}$ in $\mathrm{CDCl}_{3}$. 


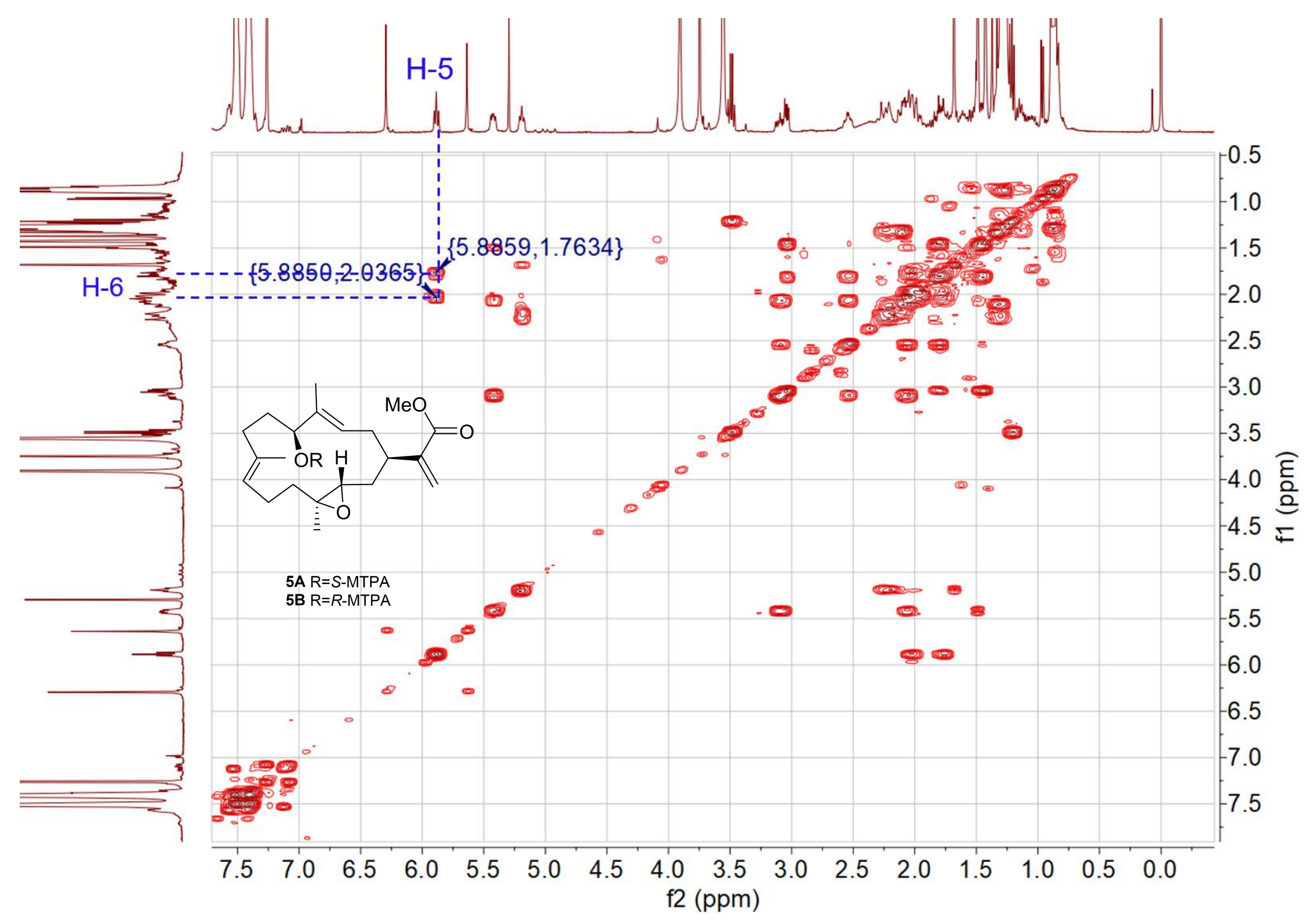

Figure S39. ${ }^{1} \mathrm{H}$ NMR spectrum $(500 \mathrm{MHz})$ of $\mathbf{5 B}$ in $\mathrm{CDCl}_{3}$. 


\section{Computational Section}

\subsection{QM-NMR calculation for compounds 1 and 3}

Theoretical calculation of all theoretical stereoisomers was carried out to determine the relative configuration of 1 and 3 , based on the alignment of its $1 \mathrm{D}$ NMR chemical shifts $\left({ }^{13} \mathrm{C}\right.$ NMR chemical shifts herein) and calculation-generated chemical shifts. Confab was used to search the conformational space of $\mathbf{1 a}-\mathbf{1 d}$ and $\mathbf{3 a}-\mathbf{3 h}$. Conformational searches were carried out using the torsional sampling (MCMM) method and OPLS_2005 force field in the Macromodel 9.9.223 software applying an energy window of $21 \mathrm{~kJ} / \mathrm{mol}$. Conformers above $1 \%$ population were re-optimized with Gaussian 09 at the B3LYP/6-311G(d,p) level with IEFPCM (Polarizable Continuum Model using the Integral Equation Formalism variant) solvent model for acetonitrile. The initial torsional sampling (MCMM) and OPLS_2005 force field conformational searches of 1a-1d, afforded 87, 63, 54 and 45 conformers; 3a-3h, afforded 233, 180, 49, 173, 133, 220, 133 and 141 conformers within the $21 \mathrm{~kJ} / \mathrm{mol}$ energy window, respectively. The Boltzmann populations of the conformers were obtained based on the potential energy provided by the OPLS_2005 force field, leading to $16,8,10$, and 6 conformers for $1 \mathbf{a}-1 \mathbf{d} ; 20,18,10,17,17,18,12$ and 12 conformers for 3a-3h; above $1 \%$ population for further re-optimization, respectively. The obtained conformers were subjected to optimization and frequency calculations on B3LYP/6-311G(d,p) $\left(\mathrm{CH}_{3} \mathrm{CN}\right)$ level of theory. GIAO DFT ${ }^{13} \mathrm{C}$ NMR calculations were calculated on mPW1PW91/6-31G* $\left(\mathrm{CHCl}_{3}\right)$ level of theory, and the calculated shielding tensors were Boltzmann averaged according to Gibbs free energy and then converted into chemical shifts following MSTD protocol. The experimental ${ }^{13} \mathrm{C}$ NMR data of $\mathbf{1}$ and $\mathbf{3}$ were compared with the calculated NMR chemical shifts of $\mathbf{1 a}-\mathbf{1 d}$ and $\mathbf{3 a}-\mathbf{3 h}$ using the mean absolute error (MAE) values, maximum deviation (MD) values, correlation coefficient $\left(R^{2}\right)$, and DP4+ probability analysis.

\subsubsection{QM-NMR calculation for compound 1}

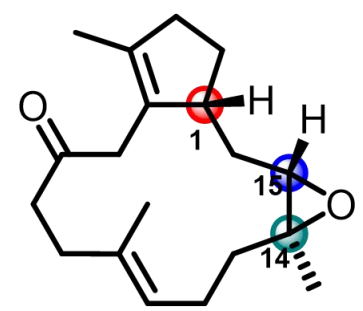

1a $1 R^{*}, 14 S^{*}, 15 S^{*}$

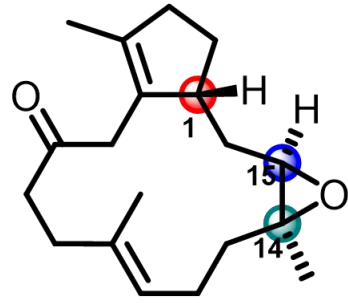

1b $1 R^{*}, 14 S^{*}, 15 R^{*}$

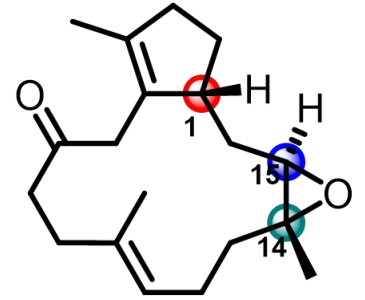

$1 \mathrm{c} 1 R^{*}, 14 R^{*}, 15 R^{*}$

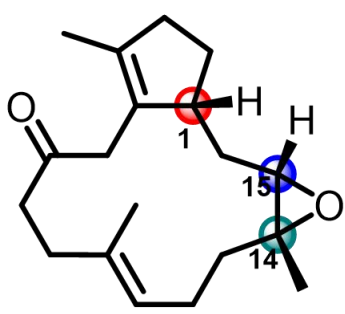

$1 \mathrm{~d} 1 R^{*}, 14 R^{*}, 15 S^{*}$

Figure S40. Structures of four stereoisomers 1a-1d. Three chiral centers resulted four models for 1. 
Table S5. Cartesian coordinates of all conformers of isomers 1a-1d used after optimization at the B3LYP/6-311G(d,p) level of theory as required for DP4+ analysis.

\begin{tabular}{|c|c|c|c|c|c|c|c|}
\hline \multicolumn{4}{|c|}{ 1a_1 } & \multicolumn{4}{|c|}{$1 \mathrm{a} \_2$} \\
\hline $\mathrm{C}$ & 0.787306 & 2.980546 & 0.459776 & $\mathrm{C}$ & -1.88128 & -2.85446 & 0.498344 \\
\hline C & 1.81368 & 1.936853 & 0.870145 & $\mathrm{C}$ & -2.80965 & -1.70341 & 0.151073 \\
\hline C & -0.59121 & 2.375765 & 0.132812 & $\mathrm{C}$ & -0.60048 & -2.88168 & -0.35839 \\
\hline C & 2.664739 & 1.440332 & -0.03682 & $\mathrm{C}$ & -2.78087 & -0.56301 & 0.85436 \\
\hline C & 3.696692 & 0.355393 & 0.115636 & $\mathrm{C}$ & -3.60143 & 0.681008 & 0.609735 \\
\hline C & 3.370398 & -0.89846 & -0.73318 & $\mathrm{C}$ & -2.97357 & 1.672378 & -0.39846 \\
\hline C & 2.219423 & -1.73942 & -0.20039 & $\mathrm{C}$ & -1.62392 & 2.257275 & -0.00787 \\
\hline C & 0.838544 & -1.35442 & -0.54816 & $\mathrm{C}$ & -0.39951 & 1.559077 & -0.44181 \\
\hline C & -0.37399 & -1.66914 & 0.297768 & $\mathrm{C}$ & 0.958565 & 1.664399 & 0.217402 \\
\hline C & -0.67299 & 1.719912 & -1.23654 & $\mathrm{C}$ & 0.450632 & -1.87285 & 0.092975 \\
\hline C & -1.88606 & 0.832714 & -1.54698 & $\mathrm{C}$ & 1.431881 & -1.38983 & -0.97888 \\
\hline C & -2.34289 & -0.18435 & -0.53039 & $\mathrm{C}$ & 2.407699 & -0.33598 & -0.53533 \\
\hline C & -1.73758 & -1.59052 & -0.44317 & $\mathrm{C}$ & 2.119535 & 1.156359 & -0.68662 \\
\hline C & -2.84333 & -2.3655 & 0.321751 & $\mathrm{C}$ & 3.49323 & 1.799291 & -0.34583 \\
\hline C & -3.58989 & -1.29557 & 1.141615 & $\mathrm{C}$ & 4.278151 & 0.728769 & 0.441011 \\
\hline C & -3.34537 & -0.02727 & 0.350964 & $\mathrm{C}$ & 3.574547 & -0.56227 & 0.085184 \\
\hline C & -4.17456 & 1.191391 & 0.616088 & $\mathrm{C}$ & 4.169629 & -1.87932 & 0.479656 \\
\hline $\mathrm{O}$ & 0.143669 & 1.946332 & -2.10829 & $\mathrm{O}$ & 0.530469 & -1.51774 & 1.250819 \\
\hline C & 1.75614 & 1.521654 & 2.319662 & $\mathrm{C}$ & -3.71574 & -1.94825 & -1.0309 \\
\hline C & 2.540991 & -2.55875 & 1.031689 & $\mathrm{C}$ & -1.62971 & 3.194225 & 1.179605 \\
\hline $\mathrm{O}$ & 1.484016 & -2.46039 & -1.22783 & $\mathrm{O}$ & -0.86933 & 2.726905 & -1.1597 \\
\hline $\mathrm{H}$ & -1.5954 & -2.01555 & -1.44399 & $\mathrm{H}$ & 1.848029 & 1.393564 & -1.72323 \\
\hline $\mathrm{H}$ & 0.747981 & -0.48256 & -1.1902 & $\mathrm{H}$ & -0.57083 & 0.639742 & -0.99836 \\
\hline $\mathrm{H}$ & 1.138979 & 3.528885 & -0.41615 & $\mathrm{H}$ & -2.41108 & -3.80254 & 0.359992 \\
\hline $\mathrm{H}$ & 0.660366 & 3.707245 & 1.269034 & $\mathrm{H}$ & -1.58431 & -2.79762 & 1.547207 \\
\hline $\mathrm{H}$ & -1.35967 & 3.161304 & 0.131325 & $\mathrm{H}$ & -0.11871 & -3.86557 & -0.27784 \\
\hline $\mathrm{H}$ & -0.91669 & 1.661622 & 0.893708 & $\mathrm{H}$ & -0.81921 & -2.74398 & -1.42063 \\
\hline $\mathrm{H}$ & 2.583779 & 1.834277 & -1.0482 & $\mathrm{H}$ & -2.07324 & -0.50709 & 1.678126 \\
\hline $\mathrm{H}$ & 3.840635 & 0.073542 & 1.160884 & $\mathrm{H}$ & -3.76741 & 1.191208 & 1.56394 \\
\hline $\mathrm{H}$ & 4.663644 & 0.740385 & -0.23009 & $\mathrm{H}$ & -4.59464 & 0.418952 & 0.234812 \\
\hline $\mathrm{H}$ & 4.26299 & -1.53152 & -0.79621 & $\mathrm{H}$ & -3.67072 & 2.505001 & -0.55379 \\
\hline $\mathrm{H}$ & 3.126527 & -0.59178 & -1.75486 & $\mathrm{H}$ & -2.8543 & 1.173654 & -1.36446 \\
\hline $\mathrm{H}$ & -0.25827 & -2.68421 & 0.682506 & $\mathrm{H}$ & 1.135281 & 2.714572 & 0.458712 \\
\hline $\mathrm{H}$ & -0.38978 & -1.00113 & 1.167279 & $\mathrm{H}$ & 0.955242 & 1.104535 & 1.157402 \\
\hline $\mathrm{H}$ & -1.66582 & 0.346559 & -2.50117 & $\mathrm{H}$ & 0.845957 & -1.05109 & -1.84069 \\
\hline $\mathrm{H}$ & -2.70268 & 1.539957 & -1.74125 & $\mathrm{H}$ & 1.952094 & -2.28895 & -1.33375 \\
\hline $\mathrm{H}$ & -3.53489 & -2.80832 & -0.40046 & $\mathrm{H}$ & 4.02265 & 2.026016 & -1.27499 \\
\hline $\mathrm{H}$ & -2.4428 & -3.17744 & 0.932855 & $\mathrm{H}$ & 3.387562 & 2.737534 & 0.203046 \\
\hline $\mathrm{H}$ & -3.19157 & -1.19879 & 2.160839 & $\mathrm{H}$ & 4.237051 & 0.895744 & 1.526035 \\
\hline $\mathrm{H}$ & -4.65772 & -1.51351 & 1.247611 & $\mathrm{H}$ & 5.340902 & 0.70809 & 0.175458 \\
\hline $\mathrm{H}$ & -4.01957 & 1.547255 & 1.641674 & $\mathrm{H}$ & 4.232011 & -1.96096 & 1.571425 \\
\hline $\mathrm{H}$ & -3.94831 & 2.019202 & -0.05768 & $\mathrm{H}$ & 3.592406 & -2.73186 & 0.117417 \\
\hline $\mathrm{H}$ & -5.24153 & 0.959038 & 0.526023 & $\mathrm{H}$ & 5.193598 & -1.97472 & 0.101071 \\
\hline & 1.995359 & 2.372607 & 2.968362 & $\mathrm{H}$ & -4.33468 & -1.08713 & -1.28202 \\
\hline
\end{tabular}




$\begin{array}{lrrrrrrr}\mathrm{H} & 0.746955 & 1.197293 & 2.597102 & \mathrm{H} & -3.13478 & -2.20954 & -1.92255 \\ \mathrm{H} & 2.443534 & 0.711727 & 2.562274 & \mathrm{H} & -4.37837 & -2.79918 & -0.83407 \\ \mathrm{H} & 3.367953 & -3.24052 & 0.810496 & \mathrm{H} & -2.33024 & 4.016092 & 1.001837 \\ \mathrm{H} & 1.69155 & -3.15343 & 1.363941 & \mathrm{H} & -0.64439 & 3.619916 & 1.364683 \\ \mathrm{H} & 2.85255 & -1.91669 & 1.85966 & \mathrm{H} & -1.95127 & 2.67187 & 2.084725\end{array}$

\begin{tabular}{|c|c|c|c|c|c|c|c|}
\hline $1 \mathrm{a} \_3$ & & & & 1a_4 & & & \\
\hline $\mathrm{C}$ & -1.85305 & -2.94781 & -0.11073 & $\mathrm{C}$ & 1.447984 & 2.847106 & 0.814701 \\
\hline $\mathrm{C}$ & -2.74345 & -1.7573 & 0.214802 & $\mathrm{C}$ & 2.483299 & 1.854594 & 0.324268 \\
\hline $\mathrm{C}$ & -0.62657 & -2.67808 & -0.98363 & $\mathrm{C}$ & 0.294968 & 3.122416 & -0.18431 \\
\hline $\mathrm{C}$ & -2.77497 & -0.65084 & -0.53676 & $\mathrm{C}$ & 2.665477 & 0.6922 & 0.966824 \\
\hline $\mathrm{C}$ & -3.61228 & 0.589604 & -0.34227 & $\mathrm{C}$ & 3.645373 & -0.40215 & 0.616715 \\
\hline $\mathrm{C}$ & -2.89239 & 1.879064 & -0.78572 & $\mathrm{C}$ & 3.123366 & -1.42173 & -0.42116 \\
\hline $\mathrm{C}$ & -1.64603 & 2.254055 & 0.003275 & $\mathrm{C}$ & 1.86603 & -2.1829 & -0.03026 \\
\hline $\mathrm{C}$ & -0.32724 & 1.804401 & -0.48076 & $\mathrm{C}$ & 0.554687 & -1.67101 & -0.47213 \\
\hline $\mathrm{C}$ & 0.917034 & 1.642878 & 0.3731 & $\mathrm{C}$ & -0.77348 & -1.97094 & 0.204643 \\
\hline $\mathrm{C}$ & 0.435473 & -1.83167 & -0.29032 & $\mathrm{C}$ & -0.53028 & 1.900044 & -0.55763 \\
\hline $\mathrm{C}$ & 1.485329 & -1.18153 & -1.19903 & $\mathrm{C}$ & -1.20128 & 1.147633 & 0.590449 \\
\hline $\mathrm{C}$ & 2.439084 & -0.2398 & -0.51673 & $\mathrm{C}$ & -2.27375 & 0.168576 & 0.20276 \\
\hline $\mathrm{C}$ & 2.182999 & 1.263709 & -0.45124 & $\mathrm{C}$ & -1.97042 & -1.20485 & -0.39914 \\
\hline $\mathrm{C}$ & 3.512541 & 1.810694 & 0.142891 & $\mathrm{C}$ & -3.31704 & -1.94435 & -0.22323 \\
\hline $\mathrm{C}$ & 4.22753 & 0.600448 & 0.780925 & $\mathrm{C}$ & -4.37872 & -0.83112 & -0.17628 \\
\hline $\mathrm{C}$ & 3.544533 & -0.59418 & 0.153581 & $\mathrm{C}$ & -3.59647 & 0.371549 & 0.312429 \\
\hline $\mathrm{C}$ & 4.087928 & -1.97301 & 0.371888 & $\mathrm{C}$ & -4.32177 & 1.594638 & 0.784625 \\
\hline $\mathrm{O}$ & 0.472021 & -1.72948 & 0.918163 & $\mathrm{O}$ & -0.64332 & 1.548153 & -1.71622 \\
\hline $\mathrm{C}$ & -3.59798 & -1.97203 & 1.439581 & $\mathrm{C}$ & 3.265121 & 2.273544 & -0.89676 \\
\hline $\mathrm{C}$ & -1.86524 & 2.616855 & 1.455276 & $\mathrm{C}$ & 1.980695 & -3.09137 & 1.173928 \\
\hline $\mathrm{O}$ & -0.77751 & 3.158499 & -0.73214 & $\mathrm{O}$ & 1.192753 & -2.76766 & -1.17482 \\
\hline $\mathrm{H}$ & 2.042197 & 1.669684 & -1.46125 & $\mathrm{H}$ & -1.77211 & -1.06995 & -1.47167 \\
\hline $\mathrm{H}$ & -0.34282 & 1.196736 & -1.38448 & $\mathrm{H}$ & 0.574566 & -0.74894 & -1.04898 \\
\hline $\mathrm{H}$ & -2.46364 & -3.72742 & -0.58368 & $\mathrm{H}$ & 1.93589 & 3.809833 & 1.008386 \\
\hline $\mathrm{H}$ & -1.50664 & -3.37767 & 0.834044 & $\mathrm{H}$ & 1.036922 & 2.510895 & 1.769698 \\
\hline $\mathrm{H}$ & -0.8864 & -2.23529 & -1.94851 & $\mathrm{H}$ & 0.675774 & 3.554022 & -1.11153 \\
\hline $\mathrm{H}$ & -0.1405 & -3.63166 & -1.22943 & $\mathrm{H}$ & -0.38209 & 3.856242 & 0.268218 \\
\hline $\mathrm{H}$ & -2.14124 & -0.61534 & -1.42007 & $\mathrm{H}$ & 2.03784 & 0.498673 & 1.834847 \\
\hline $\mathrm{H}$ & -3.9466 & 0.684303 & 0.693324 & $\mathrm{H}$ & 3.928645 & -0.93018 & 1.532512 \\
\hline $\mathrm{H}$ & -4.52711 & 0.510772 & -0.94484 & $\mathrm{H}$ & 4.57007 & 0.025514 & 0.21891 \\
\hline $\mathrm{H}$ & -3.59565 & 2.717918 & -0.72272 & $\mathrm{H}$ & 3.914549 & -2.15512 & -0.62083 \\
\hline $\mathrm{H}$ & -2.60796 & 1.788918 & -1.83899 & $\mathrm{H}$ & 2.923213 & -0.9061 & -1.36437 \\
\hline $\mathrm{H}$ & 1.091857 & 2.595501 & 0.878095 & $\mathrm{H}$ & -0.95546 & -3.04424 & 0.101819 \\
\hline $\mathrm{H}$ & 0.746168 & 0.890412 & 1.148789 & $\mathrm{H}$ & -0.71431 & -1.77177 & 1.281393 \\
\hline $\mathrm{H}$ & 0.961364 & -0.68848 & -2.02453 & $\mathrm{H}$ & -1.57976 & 1.886725 & 1.302061 \\
\hline $\mathrm{H}$ & 2.023527 & -2.01464 & -1.66952 & $\mathrm{H}$ & -0.39048 & 0.635285 & 1.125021 \\
\hline $\mathrm{H}$ & 4.128721 & 2.212189 & -0.66561 & $\mathrm{H}$ & -3.49219 & -2.68402 & -1.00815 \\
\hline $\mathrm{H}$ & 3.343926 & 2.623712 & 0.852117 & $\mathrm{H}$ & -3.3074 & -2.47526 & 0.735066 \\
\hline $\mathrm{H}$ & 4.107306 & 0.573237 & 1.87246 & $\mathrm{H}$ & -5.22554 & -1.07308 & 0.474836 \\
\hline $\mathrm{H}$ & 5.307037 & 0.605451 & 0.593245 & $\mathrm{H}$ & -4.79976 & -0.62862 & -1.1713 \\
\hline $\mathrm{H}$ & 4.047702 & -2.23813 & 1.435139 & $\mathrm{H}$ & -4.9901 & 1.35309 & 1.619093 \\
\hline $\mathrm{H}$ & 3.538855 & -2.73786 & -0.18009 & $\mathrm{H}$ & -3.65124 & 2.393068 & 1.106146 \\
\hline
\end{tabular}




$\begin{array}{lrlllrll}\mathrm{H} & 5.141701 & -2.02864 & 0.076159 & \mathrm{H} & -4.95655 & 1.995695 & -0.01455 \\ \mathrm{H} & -4.30333 & -1.16191 & 1.621789 & \mathrm{H} & 2.62703 & 2.291253 & -1.78674 \\ \mathrm{H} & -4.17299 & -2.90039 & 1.342783 & \mathrm{H} & 3.659242 & 3.288245 & -0.77268 \\ \mathrm{H} & -2.96971 & -2.08826 & 2.330262 & \mathrm{H} & 4.100831 & 1.606536 & -1.10799 \\ \mathrm{H} & -2.59823 & 3.425826 & 1.532429 & \mathrm{H} & 2.80829 & -3.79416 & 1.036926 \\ \mathrm{H} & -0.94175 & 2.945268 & 1.931083 & \mathrm{H} & 1.066892 & -3.66463 & 1.328167 \\ \mathrm{H} & -2.25149 & 1.760784 & 2.014942 & \mathrm{H} & 2.179002 & -2.51424 & 2.081343\end{array}$

\begin{tabular}{|c|c|c|c|c|c|c|c|}
\hline 1a_5 & & & & 1a & & & \\
\hline $\mathrm{C}$ & 1.344503 & 2.935799 & -0.74591 & $\mathrm{C}$ & -2.14042 & -2.70895 & -0.79401 \\
\hline $\mathrm{C}$ & 2.33628 & 1.950385 & -0.15729 & $\mathrm{C}$ & -2.72886 & -1.56539 & 0.007637 \\
\hline $\mathrm{C}$ & 0.061558 & 3.150245 & 0.099045 & $\mathrm{C}$ & -0.74059 & -3.19225 & -0.32381 \\
\hline $\mathrm{C}$ & 2.734767 & 0.897461 & -0.88406 & $\mathrm{C}$ & -2.86731 & -0.35384 & -0.54779 \\
\hline $\mathrm{C}$ & 3.721019 & -0.18315 & -0.52885 & $\mathrm{C}$ & -3.46533 & 0.888437 & 0.056721 \\
\hline $\mathrm{C}$ & 3.202198 & -1.60371 & -0.83934 & $\mathrm{C}$ & -2.72742 & 2.185338 & -0.3221 \\
\hline C & 2.035096 & -2.07499 & 0.014726 & $\mathrm{C}$ & -1.31933 & 2.379467 & 0.228644 \\
\hline $\mathrm{C}$ & 0.657814 & -1.81259 & -0.44109 & $\mathrm{C}$ & -0.15636 & 2.247894 & -0.68437 \\
\hline $\mathrm{C}$ & -0.55496 & -1.72492 & 0.46848 & $\mathrm{C}$ & 1.303689 & 2.064973 & -0.31869 \\
\hline $\mathrm{C}$ & -0.6919 & 1.867435 & 0.414271 & $\mathrm{C}$ & 0.354217 & -2.18654 & -0.64886 \\
\hline $\mathrm{C}$ & -1.33658 & 1.135454 & -0.76662 & $\mathrm{C}$ & 0.724639 & -1.18521 & 0.443186 \\
\hline $\mathrm{C}$ & -2.29729 & 0.034601 & -0.40333 & $\mathrm{C}$ & 1.961575 & -0.38415 & 0.167685 \\
\hline $\mathrm{C}$ & -1.88358 & -1.43307 & -0.26555 & $\mathrm{C}$ & 1.980295 & 0.76531 & -0.8378 \\
\hline $\mathrm{C}$ & -3.11039 & -2.05139 & 0.444503 & $\mathrm{C}$ & 3.502492 & 0.953217 & -1.07766 \\
\hline $\mathrm{C}$ & -4.29137 & -1.13599 & 0.077185 & $\mathrm{C}$ & 4.182511 & 0.417645 & 0.197316 \\
\hline $\mathrm{C}$ & -3.6189 & 0.187109 & -0.22673 & $\mathrm{C}$ & 3.159158 & -0.55344 & 0.750174 \\
\hline $\mathrm{C}$ & -4.43549 & 1.438092 & -0.33816 & $\mathrm{C}$ & 3.550963 & -1.51991 & 1.826374 \\
\hline $\mathrm{O}$ & -0.76989 & 1.455323 & 1.555448 & $\mathrm{O}$ & 0.877939 & -2.17864 & -1.74627 \\
\hline $\mathrm{C}$ & 2.824227 & 2.268108 & 1.235048 & $\mathrm{C}$ & -3.16524 & -1.91762 & 1.408824 \\
\hline $\mathrm{C}$ & 2.339572 & -2.31082 & 1.477866 & $\mathrm{C}$ & -1.17168 & 2.274575 & 1.72974 \\
\hline $\mathrm{O}$ & 1.254063 & -3.12164 & -0.62193 & $\mathrm{O}$ & -0.69721 & 3.548355 & -0.36339 \\
\hline $\mathrm{H}$ & -1.80609 & -1.86062 & -1.27783 & $\mathrm{H}$ & 1.48554 & 0.473215 & -1.76929 \\
\hline $\mathrm{H}$ & 0.566489 & -1.27503 & -1.38374 & $\mathrm{H}$ & -0.38957 & 1.934738 & -1.70372 \\
\hline $\mathrm{H}$ & 1.071111 & 2.620584 & -1.75601 & $\mathrm{H}$ & -2.07214 & -2.43225 & -1.84945 \\
\hline $\mathrm{H}$ & 1.82281 & 3.918195 & -0.84373 & $\mathrm{H}$ & -2.81381 & -3.57169 & -0.73342 \\
\hline $\mathrm{H}$ & 0.303696 & 3.625059 & 1.051146 & $\mathrm{H}$ & -0.49653 & -4.11227 & -0.86018 \\
\hline $\mathrm{H}$ & -0.60248 & 3.823938 & -0.45235 & $\mathrm{H}$ & -0.75702 & -3.41545 & 0.745158 \\
\hline $\mathrm{H}$ & 2.314669 & 0.799767 & -1.8853 & $\mathrm{H}$ & -2.54467 & -0.24008 & -1.5827 \\
\hline $\mathrm{H}$ & 4.032424 & -0.1169 & 0.515483 & $\mathrm{H}$ & -3.54565 & 0.80722 & 1.142638 \\
\hline $\mathrm{H}$ & 4.63132 & -0.03694 & -1.12507 & $\mathrm{H}$ & -4.49543 & 0.994467 & -0.3095 \\
\hline $\mathrm{H}$ & 4.025541 & -2.31705 & -0.71625 & $\mathrm{H}$ & -3.32052 & 3.03603 & 0.034678 \\
\hline $\mathrm{H}$ & 2.895486 & -1.65586 & -1.88873 & $\mathrm{H}$ & -2.68332 & 2.275445 & -1.41215 \\
\hline $\mathrm{H}$ & -0.64426 & -2.68723 & 0.97964 & $\mathrm{H}$ & 1.814676 & 2.915548 & -0.78514 \\
\hline $\mathrm{H}$ & -0.39995 & -0.96676 & 1.241472 & $\mathrm{H}$ & 1.456712 & 2.172727 & 0.757723 \\
\hline $\mathrm{H}$ & -0.51346 & 0.753015 & -1.3823 & $\mathrm{H}$ & 0.796867 & -1.73152 & 1.387528 \\
\hline $\mathrm{H}$ & -1.82089 & 1.893149 & -1.39124 & $\mathrm{H}$ & -0.15854 & -0.54044 & 0.553811 \\
\hline $\mathrm{H}$ & -3.26573 & -3.09843 & 0.173201 & $\mathrm{H}$ & 3.806134 & 0.336704 & -1.92837 \\
\hline $\mathrm{H}$ & -2.94616 & -2.01136 & 1.526676 & $\mathrm{H}$ & 3.768785 & 1.986854 & -1.30983 \\
\hline $\mathrm{H}$ & -5.03529 & -1.05613 & 0.877003 & $\mathrm{H}$ & 4.391111 & 1.212896 & 0.926359 \\
\hline & -4.82788 & -1.49874 & -0.81114 & $\mathrm{H}$ & 5.141916 & -0.06795 & -0.01083 \\
\hline
\end{tabular}




$\begin{array}{lllll}\mathrm{H} & -4.91244 & 1.675352 & 0.619983 & \mathrm{H} \\ \mathrm{H} & -3.84711 & 2.305876 & -0.64097 & \mathrm{H} \\ \mathrm{H} & -5.24638 & 1.307314 & -1.06435 & \mathrm{H} \\ \mathrm{H} & 3.623338 & 1.605121 & 1.564269 & \mathrm{H} \\ \mathrm{H} & 3.200062 & 3.296643 & 1.281755 & \mathrm{H} \\ \mathrm{H} & 2.008533 & 2.197609 & 1.961615 & \mathrm{H} \\ \mathrm{H} & 3.161286 & -3.02671 & 1.577409 & \mathrm{H} \\ \mathrm{H} & 1.474275 & -2.70593 & 2.008931 & \mathrm{H} \\ \mathrm{H} & 2.644153 & -1.38167 & 1.966926 & \mathrm{H}\end{array}$

$\begin{array}{llll}\mathrm{H} & 3.967716 & -0.98758 & 2.689539 \\ \mathrm{H} & 2.718115 & -2.13072 & 2.178138 \\ \mathrm{H} & 4.336767 & -2.19696 & 1.471436 \\ \mathrm{H} & -3.54879 & -1.06213 & 1.963109 \\ \mathrm{H} & -3.95543 & -2.67689 & 1.376299 \\ \mathrm{H} & -2.34368 & -2.35044 & 1.988858 \\ \mathrm{H} & -1.91192 & 2.912782 & 2.222093 \\ \mathrm{H} & -0.18191 & 2.593372 & 2.054521 \\ \mathrm{H} & -1.33317 & 1.249258 & 2.072622\end{array}$

\begin{tabular}{|c|c|c|c|c|c|c|c|}
\hline & & & & 1a_8 & & & \\
\hline $\mathrm{C}$ & 1.804272 & 2.946758 & 0.10812 & $\mathrm{C}$ & 0.959785 & 2.830916 & -1.25689 \\
\hline $\mathrm{C}$ & 2.753386 & 1.763001 & 0.225339 & $\mathrm{C}$ & 2.00942 & 2.135563 & -0.40067 \\
\hline $\mathrm{C}$ & 0.539386 & 2.745105 & -0.72831 & $\mathrm{C}$ & -0.42669 & 2.132897 & -1.24036 \\
\hline $\mathrm{C}$ & 2.748868 & 0.740471 & -0.63852 & $\mathrm{C}$ & 2.631865 & 1.046999 & -0.8695 \\
\hline $\mathrm{C}$ & 3.63218 & -0.48242 & -0.64605 & $\mathrm{C}$ & 3.663416 & 0.186405 & -0.19304 \\
\hline $\mathrm{C}$ & 2.881838 & -1.77118 & -1.04106 & $\mathrm{C}$ & 3.392083 & -1.3232 & -0.34432 \\
\hline $\mathrm{C}$ & 1.74138 & -2.17304 & -0.11812 & $\mathrm{C}$ & 2.155161 & -1.87456 & 0.354323 \\
\hline $\mathrm{C}$ & 0.361098 & -1.7973 & -0.47748 & $\mathrm{C}$ & 0.942148 & -2.17719 & -0.44628 \\
\hline $\mathrm{C}$ & -0.77789 & -1.63339 & 0.511521 & $\mathrm{C}$ & -0.47806 & -2.37887 & 0.050878 \\
\hline $\mathrm{C}$ & -0.46815 & 1.798819 & -0.08323 & $\mathrm{C}$ & -1.00664 & 2.059222 & 0.163328 \\
\hline $\mathrm{C}$ & -1.45294 & 1.117075 & -1.03407 & $\mathrm{C}$ & -1.00857 & 0.704703 & 0.863523 \\
\hline $\mathrm{C}$ & -2.44634 & 0.182945 & -0.40078 & $\mathrm{C}$ & -1.997 & -0.25212 & 0.222741 \\
\hline $\mathrm{C}$ & -2.14404 & -1.2938 & -0.12673 & $\mathrm{C}$ & -1.56748 & -1.47755 & -0.58252 \\
\hline $\mathrm{C}$ & -3.32853 & -1.70967 & 0.778159 & $\mathrm{C}$ & -2.90776 & -2.23885 & -0.76395 \\
\hline $\mathrm{C}$ & -4.45627 & -0.71248 & 0.460579 & $\mathrm{C}$ & -4.01577 & -1.18943 & -0.56601 \\
\hline $\mathrm{C}$ & -3.71369 & 0.490164 & -0.08368 & $\mathrm{C}$ & -3.33258 & -0.10741 & 0.241145 \\
\hline $\mathrm{C}$ & -4.42779 & 1.796767 & -0.24947 & $\mathrm{C}$ & -4.13974 & 0.964437 & 0.907658 \\
\hline $\mathrm{O}$ & -0.50809 & 1.642968 & 1.119958 & $\mathrm{O}$ & -1.44249 & 3.058463 & 0.705929 \\
\hline $\mathrm{C}$ & 3.704593 & 1.884286 & 1.390757 & $\mathrm{C}$ & 2.26091 & 2.757525 & 0.951144 \\
\hline $\mathrm{C}$ & 2.120428 & -2.46457 & 1.317309 & $\mathrm{C}$ & 2.095264 & -1.67427 & 1.851705 \\
\hline $\mathrm{O}$ & 0.843008 & -3.14172 & -0.72293 & $\mathrm{O}$ & 1.865302 & -3.22628 & -0.08307 \\
\hline $\mathrm{H}$ & -2.2089 & -1.83919 & -1.08159 & $\mathrm{H}$ & -1.20293 & -1.15103 & -1.56808 \\
\hline $\mathrm{H}$ & 0.254228 & -1.23491 & -1.40323 & $\mathrm{H}$ & 0.997837 & -1.91785 & -1.50468 \\
\hline $\mathrm{H}$ & 2.360817 & 3.807072 & -0.2847 & $\mathrm{H}$ & 1.291646 & 2.872064 & -2.29765 \\
\hline $\mathrm{H}$ & 1.495167 & 3.23261 & 1.118462 & $\mathrm{H}$ & 0.8288 & 3.863758 & -0.92199 \\
\hline $\mathrm{H}$ & 0.020439 & 3.707346 & -0.8314 & $\mathrm{H}$ & -0.33469 & 1.135277 & -1.67285 \\
\hline $\mathrm{H}$ & 0.757324 & 2.425372 & -1.75005 & $\mathrm{H}$ & -1.11877 & 2.713889 & -1.85688 \\
\hline $\mathrm{H}$ & 2.041587 & 0.773196 & -1.46387 & $\mathrm{H}$ & 2.377047 & 0.726532 & -1.88055 \\
\hline $\mathrm{H}$ & 4.125169 & -0.62157 & 0.318231 & $\mathrm{H}$ & 3.769863 & 0.444351 & 0.862605 \\
\hline $\mathrm{H}$ & 4.4388 & -0.34411 & -1.37827 & $\mathrm{H}$ & 4.643392 & 0.379766 & -0.64914 \\
\hline $\mathrm{H}$ & 3.598098 & -2.60062 & -1.07688 & $\mathrm{H}$ & 4.253584 & -1.8744 & 0.051367 \\
\hline $\mathrm{H}$ & 2.477446 & -1.66254 & -2.05246 & $\mathrm{H}$ & 3.323297 & -1.5762 & -1.4071 \\
\hline $\mathrm{H}$ & -0.87728 & -2.57776 & 1.053439 & $\mathrm{H}$ & -0.72026 & -3.41606 & -0.20902 \\
\hline $\mathrm{H}$ & -0.5284 & -0.86513 & 1.249119 & $\mathrm{H}$ & -0.53327 & -2.32578 & 1.141449 \\
\hline $\mathrm{H}$ & -0.86316 & 0.607296 & -1.80484 & $\mathrm{H}$ & -1.24371 & 0.873239 & 1.916907 \\
\hline $\mathrm{H}$ & -1.95956 & 1.926768 & -1.57319 & $\mathrm{H}$ & 0.001062 & 0.289884 & 0.802696 \\
\hline $\mathrm{H}$ & -3.61461 & -2.75432 & 0.635073 & $\mathrm{H}$ & -2.9632 & -2.75683 & -1.72404 \\
\hline $\mathrm{H}$ & -3.02714 & -1.59161 & 1.824427 & $\mathrm{H}$ & -2.98829 & -2.99707 & 0.021517 \\
\hline
\end{tabular}




$\begin{array}{lrrrlrrr}\mathrm{H} & -5.0687 & -0.4681 & 1.335082 & \mathrm{H} & -4.90062 & -1.59381 & -0.06312 \\ \mathrm{H} & -5.14613 & -1.10265 & -0.30115 & \mathrm{H} & -4.36492 & -0.776 & -1.5224 \\ \mathrm{H} & -4.76572 & 2.176263 & 0.721795 & \mathrm{H} & -4.87144 & 0.519544 & 1.591843 \\ \mathrm{H} & -3.80874 & 2.568232 & -0.71028 & \mathrm{H} & -3.52561 & 1.6712 & 1.465359 \\ \mathrm{H} & -5.32727 & 1.670689 & -0.86347 & \mathrm{H} & -4.71492 & 1.533015 & 0.166815 \\ \mathrm{H} & 4.448646 & 1.089499 & 1.426101 & \mathrm{H} & 2.932946 & 2.170693 & 1.576765 \\ \mathrm{H} & 4.23874 & 2.840481 & 1.345777 & \mathrm{H} & 2.703631 & 3.753036 & 0.82727 \\ \mathrm{H} & 3.153586 & 1.881157 & 2.338267 & \mathrm{H} & 1.325838 & 2.906274 & 1.500481 \\ \mathrm{H} & 2.915703 & -3.21565 & 1.350214 & \mathrm{H} & 3.019125 & -2.0403 & 2.310089 \\ \mathrm{H} & 1.270286 & -2.84076 & 1.885221 & \mathrm{H} & 1.261347 & -2.21888 & 2.293529 \\ \mathrm{H} & 2.489447 & -1.56239 & 1.812268 & \mathrm{H} & 1.992115 & -0.61714 & 2.108497\end{array}$

\begin{tabular}{|c|c|c|c|c|c|c|c|}
\hline 1a_9 & & & & 1a_10 & & & \\
\hline $\mathrm{C}$ & 1.280433 & 2.821785 & -1.2278 & $\mathrm{C}$ & -2.03147 & -2.91449 & -0.10757 \\
\hline $\mathrm{C}$ & 2.163601 & 2.01425 & -0.28684 & $\mathrm{C}$ & -2.95417 & -1.70863 & -0.12987 \\
\hline $\mathrm{C}$ & -0.17353 & 2.287433 & -1.37652 & $\mathrm{C}$ & -0.71202 & -2.76504 & 0.650086 \\
\hline $\mathrm{C}$ & 2.737818 & 0.881911 & -0.71301 & $\mathrm{C}$ & -2.68585 & -0.57996 & 0.539052 \\
\hline $\mathrm{C}$ & 3.619225 & -0.06948 & 0.050871 & $\mathrm{C}$ & -3.48633 & 0.694557 & 0.575079 \\
\hline $\mathrm{C}$ & 3.258944 & -1.55387 & -0.15909 & $\mathrm{C}$ & -2.86986 & 1.834429 & -0.26704 \\
\hline $\mathrm{C}$ & 1.917769 & -2.0243 & 0.391231 & $\mathrm{C}$ & -1.50687 & 2.351618 & 0.173137 \\
\hline $\mathrm{C}$ & 0.786776 & -2.23026 & -0.54653 & $\mathrm{C}$ & -0.28282 & 1.895546 & -0.52585 \\
\hline $\mathrm{C}$ & -0.69383 & -2.31244 & -0.22835 & $\mathrm{C}$ & 1.136738 & 1.960232 & 0.029301 \\
\hline $\mathrm{C}$ & -0.902 & 2.212655 & -0.04589 & $\mathrm{C}$ & 0.301384 & -1.8535 & -0.03351 \\
\hline $\mathrm{C}$ & -0.89069 & 0.87813 & 0.696955 & $\mathrm{C}$ & 1.251436 & -1.10664 & 0.897933 \\
\hline $\mathrm{C}$ & -1.92395 & -0.0749 & 0.130378 & $\mathrm{C}$ & 2.364177 & -0.35126 & 0.234814 \\
\hline $\mathrm{C}$ & -1.56272 & -1.18214 & -0.85577 & $\mathrm{C}$ & 2.12781 & 0.931278 & -0.56451 \\
\hline $\mathrm{C}$ & -2.96116 & -1.66329 & -1.33603 & $\mathrm{C}$ & 3.561829 & 1.497267 & -0.70301 \\
\hline $\mathrm{C}$ & -3.95639 & -1.21687 & -0.24558 & $\mathrm{C}$ & 4.489264 & 0.276115 & -0.58003 \\
\hline $\mathrm{C}$ & -3.22383 & -0.09995 & 0.465553 & $\mathrm{C}$ & 3.657814 & -0.71023 & 0.214685 \\
\hline $\mathrm{C}$ & -3.9407 & 0.778671 & 1.443992 & $\mathrm{C}$ & 4.304582 & -1.9252 & 0.806698 \\
\hline $\mathrm{O}$ & -1.46632 & 3.187372 & 0.416081 & $\mathrm{O}$ & 0.359313 & -1.75723 & -1.24277 \\
\hline $\mathrm{C}$ & 2.321567 & 2.59122 & 1.098796 & $\mathrm{C}$ & -4.18022 & -1.92306 & -0.98348 \\
\hline $\mathrm{C}$ & 1.707345 & -1.83795 & 1.876774 & $\mathrm{C}$ & -1.43121 & 2.924581 & 1.571299 \\
\hline $\mathrm{O}$ & 1.58225 & -3.34815 & -0.10034 & $\mathrm{O}$ & -0.88938 & 3.168828 & -0.8512 \\
\hline $\mathrm{H}$ & -1.00616 & -0.7827 & -1.71147 & $\mathrm{H}$ & 1.758081 & 0.644885 & -1.55848 \\
\hline $\mathrm{H}$ & 0.985688 & -1.96372 & -1.586 & $\mathrm{H}$ & -0.43092 & 1.151976 & -1.30815 \\
\hline $\mathrm{H}$ & 1.718029 & 2.837506 & -2.22936 & $\mathrm{H}$ & -1.80325 & -3.18826 & -1.1439 \\
\hline $\mathrm{H}$ & 1.229842 & 3.860001 & -0.88686 & $\mathrm{H}$ & -2.58369 & -3.76758 & 0.305818 \\
\hline $\mathrm{H}$ & -0.14089 & 1.302611 & -1.84653 & $\mathrm{H}$ & -0.22379 & -3.74638 & 0.713153 \\
\hline $\mathrm{H}$ & -0.72865 & 2.967636 & -2.02738 & $\mathrm{H}$ & -0.86891 & -2.44729 & 1.684111 \\
\hline $\mathrm{H}$ & 2.567379 & 0.598149 & -1.75231 & $\mathrm{H}$ & -1.77089 & -0.54153 & 1.122927 \\
\hline $\mathrm{H}$ & 3.630393 & 0.164912 & 1.117192 & $\mathrm{H}$ & -3.58313 & 1.028578 & 1.614572 \\
\hline $\mathrm{H}$ & 4.654635 & 0.055116 & -0.29296 & $\mathrm{H}$ & -4.50428 & 0.530523 & 0.216126 \\
\hline $\mathrm{H}$ & 4.03269 & -2.16894 & 0.315853 & $\mathrm{H}$ & -3.55714 & 2.689627 & -0.25162 \\
\hline $\mathrm{H}$ & 3.289087 & -1.78872 & -1.22777 & $\mathrm{H}$ & -2.79135 & 1.510453 & -1.3088 \\
\hline $\mathrm{H}$ & -1.02489 & -3.2693 & -0.64773 & $\mathrm{H}$ & 1.506401 & 2.962692 & -0.20696 \\
\hline $\mathrm{H}$ & -0.8639 & -2.37831 & 0.848759 & $\mathrm{H}$ & 1.140427 & 1.891081 & 1.122042 \\
\hline $\mathrm{H}$ & -1.08681 & 1.085304 & 1.750446 & $\mathrm{H}$ & 1.634587 & -1.83444 & 1.620783 \\
\hline $\mathrm{H}$ & 0.103773 & 0.436064 & 0.608339 & $\mathrm{H}$ & 0.623208 & -0.43227 & 1.496809 \\
\hline
\end{tabular}




\begin{tabular}{|c|c|c|c|c|c|c|c|}
\hline $\mathrm{H}$ & -3.20764 & -1.15946 & -2.27435 & $\mathrm{H}$ & 3.699017 & 2.060608 & -1.62912 \\
\hline $\mathrm{H}$ & -2.98898 & -2.73781 & -1.52813 & $\mathrm{H}$ & 3.754173 & 2.181088 & 0.130693 \\
\hline $\mathrm{H}$ & -4.19413 & -2.02382 & 0.46096 & $\mathrm{H}$ & 5.442914 & 0.508864 & -0.09425 \\
\hline $\mathrm{H}$ & -4.9124 & -0.88393 & -0.66358 & $\mathrm{H}$ & 4.736446 & -0.14805 & -1.56348 \\
\hline $\mathrm{H}$ & -4.38232 & 0.18027 & 2.249872 & $\mathrm{H}$ & 5.10557 & -1.64031 & 1.498554 \\
\hline $\mathrm{H}$ & -3.28886 & 1.532617 & 1.885709 & $\mathrm{H}$ & 3.603907 & -2.56537 & 1.344951 \\
\hline $\mathrm{H}$ & -4.77072 & 1.301611 & 0.955035 & $\mathrm{H}$ & 4.773688 & -2.5305 & 0.02192 \\
\hline $\mathrm{H}$ & 2.866341 & 1.937725 & 1.779415 & $\mathrm{H}$ & -4.8638 & -1.07501 & -0.97957 \\
\hline $\mathrm{H}$ & 2.861135 & 3.544263 & 1.04685 & $\mathrm{H}$ & -3.89203 & -2.11712 & -2.02292 \\
\hline $\mathrm{H}$ & 1.349398 & 2.816659 & 1.549614 & $\mathrm{H}$ & -4.73394 & -2.80633 & -0.64487 \\
\hline $\mathrm{H}$ & 2.537009 & -2.29393 & 2.425844 & $\mathrm{H}$ & -2.23594 & 3.65016 & 1.72382 \\
\hline $\mathrm{H}$ & 0.782942 & -2.30789 & 2.21081 & $\mathrm{H}$ & -0.48057 & 3.430573 & 1.738309 \\
\hline $\mathrm{H}$ & 1.672467 & -0.7793 & 2.145008 & $\mathrm{H}$ & -1.53959 & 2.140791 & 2.326046 \\
\hline 1a_11 & & & & 1a & & & \\
\hline $\mathrm{C}$ & -1.47991 & -2.83157 & -0.9427 & $\mathrm{C}$ & 1.908805 & 2.892932 & -0.64193 \\
\hline C & -2.38908 & -1.88599 & -0.18129 & $\mathrm{C}$ & 2.754387 & 1.75665 & -0.09851 \\
\hline $\mathrm{C}$ & -0.15039 & -3.1936 & -0.22651 & $\mathrm{C}$ & 0.531237 & 2.990962 & 0.046227 \\
\hline $\mathrm{C}$ & -2.80433 & -0.75481 & -0.76652 & $\mathrm{C}$ & 2.8587 & 0.607291 & -0.77928 \\
\hline $\mathrm{C}$ & -3.72181 & 0.311004 & -0.22728 & $\mathrm{C}$ & 3.638407 & -0.62613 & -0.41386 \\
\hline $\mathrm{C}$ & -3.22349 & 1.741032 & -0.52497 & $\mathrm{C}$ & 2.876744 & -1.94375 & -0.64939 \\
\hline $\mathrm{C}$ & -1.93479 & 2.140127 & 0.177904 & $\mathrm{C}$ & 1.642536 & -2.19993 & 0.208841 \\
\hline $\mathrm{C}$ & -0.64434 & 1.851016 & -0.47251 & $\mathrm{C}$ & 0.296707 & -2.19629 & -0.41854 \\
\hline $\mathrm{C}$ & 0.674128 & 1.666918 & 0.258826 & $\mathrm{C}$ & -1.04915 & -2.07079 & 0.279788 \\
\hline $\mathrm{C}$ & 0.675839 & -1.98137 & 0.176518 & $\mathrm{C}$ & -0.41686 & 1.879231 & -0.39091 \\
\hline $\mathrm{C}$ & 1.489567 & -1.30511 & -0.93082 & $\mathrm{C}$ & -1.13646 & 1.12318 & 0.720959 \\
\hline $\mathrm{C}$ & 2.338823 & -0.13456 & -0.51437 & $\mathrm{C}$ & -2.27477 & 0.254969 & 0.273292 \\
\hline $\mathrm{C}$ & 1.871434 & 1.30889 & -0.67726 & $\mathrm{C}$ & -2.06871 & -1.10966 & -0.3814 \\
\hline $\mathrm{C}$ & 3.17093 & 2.112737 & -0.39456 & $\mathrm{C}$ & -3.505 & -1.69266 & -0.38102 \\
\hline $\mathrm{C}$ & 4.08401 & 1.162468 & 0.408006 & $\mathrm{C}$ & -4.44071 & -0.47356 & -0.32223 \\
\hline $\mathrm{C}$ & 3.53635 & -0.21066 & 0.083577 & $\mathrm{C}$ & -3.5723 & 0.599159 & 0.300845 \\
\hline $\mathrm{C}$ & 4.291477 & -1.44162 & 0.481387 & $\mathrm{C}$ & -4.19079 & 1.875659 & 0.783865 \\
\hline $\mathrm{O}$ & 0.686979 & -1.59094 & 1.327619 & $\mathrm{O}$ & -0.58154 & 1.624875 & -1.56731 \\
\hline $\mathrm{C}$ & -2.7893 & -2.3334 & 1.203158 & $\mathrm{C}$ & 3.434531 & 2.042746 & 1.218403 \\
\hline $\mathrm{C}$ & -2.02751 & 2.318436 & 1.677367 & $\mathrm{C}$ & 1.817292 & -2.01628 & 1.699262 \\
\hline $\mathrm{O}$ & -1.20577 & 3.186594 & -0.51896 & $\mathrm{O}$ & 0.995104 & -3.43754 & -0.17638 \\
\hline $\mathrm{H}$ & 1.5488 & 1.489454 & -1.70997 & $\mathrm{H}$ & -1.74869 & -0.93782 & -1.41833 \\
\hline $\mathrm{H}$ & -0.70215 & 1.346185 & -1.43619 & $\mathrm{H}$ & 0.268807 & -1.93251 & -1.47652 \\
\hline $\mathrm{H}$ & -1.2628 & -2.41315 & -1.92876 & $\mathrm{H}$ & 1.744563 & 2.76618 & -1.71404 \\
\hline $\mathrm{H}$ & -2.00515 & -3.77936 & -1.11506 & $\mathrm{H}$ & 2.434951 & 3.843296 & -0.50231 \\
\hline $\mathrm{H}$ & 0.434642 & -3.8286 & -0.89837 & $\mathrm{H}$ & 0.63052 & 3.003741 & 1.133894 \\
\hline $\mathrm{H}$ & -0.35488 & -3.76215 & 0.682349 & $\mathrm{H}$ & 0.045029 & 3.932544 & -0.23678 \\
\hline $\mathrm{H}$ & -2.4557 & -0.56771 & -1.78209 & $\mathrm{H}$ & 2.335828 & 0.54509 & -1.73308 \\
\hline $\mathrm{H}$ & -3.89312 & 0.187525 & 0.844104 & $\mathrm{H}$ & 3.993434 & -0.58226 & 0.61753 \\
\hline $\mathrm{H}$ & -4.7057 & 0.207461 & -0.70398 & $\mathrm{H}$ & 4.541528 & -0.66958 & -1.03743 \\
\hline $\mathrm{H}$ & -4.00513 & 2.456786 & -0.24475 & $\mathrm{H}$ & 3.564894 & -2.7761 & -0.45809 \\
\hline $\mathrm{H}$ & -3.06733 & 1.852232 & -1.6026 & $\mathrm{H}$ & 2.586848 & -2.01811 & -1.70237 \\
\hline $\mathrm{H}$ & 0.89055 & 2.598341 & 0.787239 & $\mathrm{H}$ & -1.47768 & -3.07864 & 0.253516 \\
\hline $\mathrm{H}$ & 0.564237 & 0.889105 & 1.019113 & $\mathrm{H}$ & -0.93103 & -1.82962 & 1.340051 \\
\hline
\end{tabular}




\begin{tabular}{|c|c|c|c|c|c|c|c|}
\hline $\mathrm{H}$ & 0.787535 & -1.02139 & -1.72407 & $\mathrm{H}$ & -1.45829 & 1.856595 & 1.466771 \\
\hline $\mathrm{H}$ & 2.105727 & -2.09456 & -1.37799 & $\mathrm{H}$ & -0.35512 & 0.537832 & 1.227079 \\
\hline $\mathrm{H}$ & 3.654333 & 2.356579 & -1.34452 & $\mathrm{H}$ & -3.6893 & -2.34595 & -1.23713 \\
\hline $\mathrm{H}$ & 2.975163 & 3.055681 & 0.120369 & $\mathrm{H}$ & -3.63954 & -2.29221 & 0.52541 \\
\hline $\mathrm{H}$ & 4.02792 & 1.345498 & 1.489868 & $\mathrm{H}$ & -5.35535 & -0.6625 & 0.250085 \\
\hline $\mathrm{H}$ & 5.139859 & 1.265115 & 0.134306 & $\mathrm{H}$ & -4.76147 & -0.15704 & -1.32479 \\
\hline $\mathrm{H}$ & 4.39132 & -1.49653 & 1.57204 & $\mathrm{H}$ & -4.96013 & 1.673251 & 1.53775 \\
\hline $\mathrm{H}$ & 3.807721 & -2.36101 & 0.146929 & $\mathrm{H}$ & -3.46489 & 2.565912 & 1.216245 \\
\hline $\mathrm{H}$ & 5.309345 & -1.42418 & 0.075658 & $\mathrm{H}$ & -4.69348 & 2.395171 & -0.04079 \\
\hline $\mathrm{H}$ & -3.54462 & -1.68958 & 1.652475 & $\mathrm{H}$ & 3.93754 & 1.174268 & 1.642611 \\
\hline $\mathrm{H}$ & -3.19221 & -3.35233 & 1.17196 & $\mathrm{H}$ & 4.184055 & 2.832774 & 1.090437 \\
\hline $\mathrm{H}$ & -1.92305 & -2.35581 & 1.871461 & $\mathrm{H}$ & 2.720092 & 2.412046 & 1.961441 \\
\hline $\mathrm{H}$ & -2.79476 & 3.060273 & 1.919457 & $\mathrm{H}$ & 2.701493 & -2.56217 & 2.042621 \\
\hline $\mathrm{H}$ & -1.08097 & 2.652424 & 2.10115 & $\mathrm{H}$ & 0.954519 & -2.39367 & 2.247643 \\
\hline $\mathrm{H}$ & -2.30488 & 1.378892 & 2.162856 & $\mathrm{H}$ & 1.954765 & -0.96166 & 1.951603 \\
\hline 1b_1 & & & & 1b & & & \\
\hline $\mathrm{C}$ & -0.65219 & 3.11706 & -0.05814 & $\mathrm{C}$ & -0.07762 & 3.101496 & -0.0737 \\
\hline $\mathrm{C}$ & -1.46243 & 2.166428 & 0.811055 & $\mathrm{C}$ & -0.92467 & 2.293297 & 0.897032 \\
\hline $\mathrm{C}$ & 0.689744 & 2.518371 & -0.52933 & $\mathrm{C}$ & 1.09651 & 2.295523 & -0.66656 \\
\hline $\mathrm{C}$ & -2.34933 & 1.339548 & 0.241036 & $\mathrm{C}$ & -2.01224 & 1.655851 & 0.444409 \\
\hline $\mathrm{C}$ & -3.22395 & 0.29345 & 0.887222 & $\mathrm{C}$ & -2.98132 & 0.768758 & 1.185355 \\
\hline $\mathrm{C}$ & -2.65088 & -1.14857 & 0.926182 & $\mathrm{C}$ & -2.72556 & -0.75966 & 1.080027 \\
\hline $\mathrm{C}$ & -2.12802 & -1.71576 & -0.39076 & $\mathrm{C}$ & -2.48291 & -1.31235 & -0.32099 \\
\hline $\mathrm{C}$ & -0.71086 & -2.12197 & -0.56254 & $\mathrm{C}$ & -1.20274 & -1.96565 & -0.6901 \\
\hline $\mathrm{C}$ & 0.365699 & -2.0652 & 0.502848 & $\mathrm{C}$ & -0.031 & -2.24583 & 0.234339 \\
\hline $\mathrm{C}$ & 0.520677 & 1.431112 & -1.58576 & $\mathrm{C}$ & 0.654014 & 1.220024 & -1.65321 \\
\hline $\mathrm{C}$ & 1.544178 & 0.295403 & -1.65309 & $\mathrm{C}$ & 1.478885 & -0.07134 & -1.74734 \\
\hline $\mathrm{C}$ & 2.033192 & -0.24914 & -0.33765 & $\mathrm{C}$ & 1.946046 & -0.62993 & -0.43113 \\
\hline $\mathrm{C}$ & 1.070972 & -0.71013 & 0.757788 & $\mathrm{C}$ & 0.960905 & -1.12562 & 0.624128 \\
\hline $\mathrm{C}$ & 2.004172 & -0.79663 & 1.996155 & $\mathrm{C}$ & 1.8922 & -1.63812 & 1.760645 \\
\hline $\mathrm{C}$ & 3.423313 & -0.98491 & 1.425243 & $\mathrm{C}$ & 3.295688 & -1.07785 & 1.460519 \\
\hline $\mathrm{C}$ & 3.315812 & -0.41568 & 0.025839 & $\mathrm{C}$ & 3.210913 & -0.6219 & 0.021647 \\
\hline $\mathrm{C}$ & 4.558961 & -0.14764 & -0.76588 & $\mathrm{C}$ & 4.453928 & -0.16066 & -0.67621 \\
\hline $\mathrm{O}$ & -0.37006 & 1.491141 & -2.4113 & $\mathrm{O}$ & -0.29051 & 1.395032 & -2.39774 \\
\hline $\mathrm{C}$ & -1.16631 & 2.21955 & 2.288704 & $\mathrm{C}$ & -0.43556 & 2.254147 & 2.32265 \\
\hline $\mathrm{C}$ & -2.95551 & -1.43635 & -1.62985 & $\mathrm{C}$ & -3.37197 & -0.78492 & -1.43062 \\
\hline $\mathrm{O}$ & -1.73614 & -3.11199 & -0.31375 & $\mathrm{O}$ & -2.37508 & -2.7579 & -0.37939 \\
\hline $\mathrm{H}$ & 0.285198 & 0.032898 & 0.922209 & $\mathrm{H}$ & 0.368288 & -0.27192 & 0.977783 \\
\hline $\mathrm{H}$ & -0.34859 & -2.14291 & -1.58989 & $\mathrm{H}$ & -0.96845 & -1.9665 & -1.75377 \\
\hline $\mathrm{H}$ & -0.4438 & 4.037579 & 0.495919 & $\mathrm{H}$ & 0.33115 & 3.981366 & 0.433442 \\
\hline $\mathrm{H}$ & -1.22643 & 3.394983 & -0.94398 & $\mathrm{H}$ & -0.69545 & 3.460769 & -0.89901 \\
\hline $\mathrm{H}$ & 1.294148 & 3.30247 & -1.00579 & $\mathrm{H}$ & 1.75406 & 2.967786 & -1.23446 \\
\hline $\mathrm{H}$ & 1.286619 & 2.147688 & 0.305933 & $\mathrm{H}$ & 1.7174 & 1.855838 & 0.115314 \\
\hline $\mathrm{H}$ & -2.45246 & 1.421819 & -0.83686 & $\mathrm{H}$ & -2.23133 & 1.789574 & -0.61046 \\
\hline $\mathrm{H}$ & -4.17929 & 0.274055 & 0.352371 & $\mathrm{H}$ & -3.98672 & 0.977884 & 0.804254 \\
\hline $\mathrm{H}$ & -3.46334 & 0.56879 & 1.917891 & $\mathrm{H}$ & -3.00573 & 1.018086 & 2.249406 \\
\hline $\mathrm{H}$ & -1.86497 & -1.20069 & 1.681681 & $\mathrm{H}$ & -1.88713 & -1.02892 & 1.725078 \\
\hline$H$ & -3.45144 & -1.81579 & 1.267271 & $\mathrm{H}$ & -3.60533 & -1.27419 & 1.48443 \\
\hline
\end{tabular}




\begin{tabular}{|c|c|c|c|c|c|c|c|}
\hline $\mathrm{H}$ & -0.09429 & -2.38376 & 1.441721 & $\mathrm{H}$ & -0.457 & -2.63177 & 1.164794 \\
\hline $\mathrm{H}$ & 1.117413 & -2.82547 & 0.258098 & $\mathrm{H}$ & 0.53673 & -3.07696 & -0.20205 \\
\hline $\mathrm{H}$ & 2.388052 & 0.69563 & -2.2277 & $\mathrm{H}$ & 2.341698 & 0.176099 & -2.37669 \\
\hline $\mathrm{H}$ & 1.091455 & -0.47939 & -2.27995 & $\mathrm{H}$ & 0.87512 & -0.78136 & -2.31803 \\
\hline $\mathrm{H}$ & 1.959753 & 0.146585 & 2.547538 & $\mathrm{H}$ & 1.522842 & -1.35527 & 2.74847 \\
\hline $\mathrm{H}$ & 1.710234 & -1.5913 & 2.685593 & $\mathrm{H}$ & 1.920614 & -2.73121 & 1.730511 \\
\hline $\mathrm{H}$ & 3.72093 & -2.04151 & 1.387146 & $\mathrm{H}$ & 4.089245 & -1.81622 & 1.619799 \\
\hline $\mathrm{H}$ & 4.187148 & -0.47225 & 2.019423 & $\mathrm{H}$ & 3.54359 & -0.22012 & 2.100435 \\
\hline $\mathrm{H}$ & 5.137229 & -1.07036 & -0.89337 & $\mathrm{H}$ & 5.203872 & -0.95956 & -0.68942 \\
\hline $\mathrm{H}$ & 4.35805 & 0.25825 & -1.75838 & $\mathrm{H}$ & 4.279945 & 0.153834 & -1.70614 \\
\hline $\mathrm{H}$ & 5.210999 & 0.55594 & -0.23602 & $\mathrm{H}$ & 4.907176 & 0.681374 & -0.13958 \\
\hline $\mathrm{H}$ & -1.72522 & 1.47918 & 2.861646 & $\mathrm{H}$ & -1.03651 & 1.603965 & 2.959072 \\
\hline $\mathrm{H}$ & -0.10014 & 2.056084 & 2.482259 & $\mathrm{H}$ & 0.601932 & 1.905268 & 2.374256 \\
\hline $\mathrm{H}$ & -1.40366 & 3.211976 & 2.689411 & $\mathrm{H}$ & -0.44451 & 3.26057 & 2.757393 \\
\hline $\mathrm{H}$ & -2.56072 & -2.00294 & -2.47503 & $\mathrm{H}$ & -3.19968 & -1.35696 & -2.34395 \\
\hline $\mathrm{H}$ & -3.99273 & -1.74558 & -1.46631 & $\mathrm{H}$ & -4.42441 & -0.89734 & -1.15143 \\
\hline $\mathrm{H}$ & -2.95399 & -0.3788 & -1.89684 & $\mathrm{H}$ & -3.18774 & 0.267221 & -1.64781 \\
\hline 1b_3 & & & & $1 b$ & & & \\
\hline $\mathrm{C}$ & -2.33201 & 2.535394 & -0.46462 & $\mathrm{C}$ & -2.36074 & -2.56056 & -0.2533 \\
\hline $\mathrm{C}$ & -2.92129 & 1.246922 & 0.086526 & $\mathrm{C}$ & -2.97193 & -1.187 & -0.00473 \\
\hline $\mathrm{C}$ & -0.96498 & 2.892282 & 0.144646 & $\mathrm{C}$ & -1.01143 & -2.60534 & -0.97002 \\
\hline $\mathrm{C}$ & -2.80462 & 0.102757 & -0.60013 & $\mathrm{C}$ & -2.70256 & -0.1259 & -0.77503 \\
\hline $\mathrm{C}$ & -3.30361 & -1.2715 & -0.22816 & $\mathrm{C}$ & -3.19875 & 1.290348 & -0.63427 \\
\hline $\mathrm{C}$ & -2.23228 & -2.21159 & 0.381521 & $\mathrm{C}$ & -2.06422 & 2.343141 & -0.66449 \\
\hline $\mathrm{C}$ & -0.94455 & -2.3205 & -0.41959 & $\mathrm{C}$ & -1.00361 & 2.176357 & 0.413144 \\
\hline $\mathrm{C}$ & 0.281678 & -1.61786 & 0.009677 & $\mathrm{C}$ & 0.331062 & 1.624276 & 0.105325 \\
\hline $\mathrm{C}$ & 0.392937 & -0.73375 & 1.237687 & $\mathrm{C}$ & 0.780077 & 1.138277 & -1.26074 \\
\hline $\mathrm{C}$ & 0.212008 & 2.133613 & -0.45287 & $\mathrm{C}$ & 0.148119 & -2.06021 & -0.1492 \\
\hline $\mathrm{C}$ & 1.533414 & 2.184859 & 0.328356 & $\mathrm{C}$ & 1.509538 & -1.96554 & -0.85517 \\
\hline $\mathrm{C}$ & 2.247209 & 0.863413 & 0.440022 & $\mathrm{C}$ & 2.330853 & -0.75835 & -0.48673 \\
\hline $\mathrm{C}$ & 1.809118 & -0.1562 & 1.481993 & $\mathrm{C}$ & 2.2013 & 0.528667 & -1.28836 \\
\hline $\mathrm{C}$ & 2.947951 & -1.21549 & 1.444766 & $\mathrm{C}$ & 3.310126 & 1.444562 & -0.69262 \\
\hline $\mathrm{C}$ & 3.740419 & -0.94345 & 0.142907 & $\mathrm{C}$ & 3.822858 & 0.731142 & 0.583117 \\
\hline $\mathrm{C}$ & 3.285625 & 0.4345 & -0.29156 & $\mathrm{C}$ & 3.20693 & -0.65025 & 0.521323 \\
\hline $\mathrm{C}$ & 3.97199 & 1.126809 & -1.42854 & $\mathrm{C}$ & 3.586922 & -1.68676 & 1.533389 \\
\hline $\mathrm{O}$ & 0.14257 & 1.563966 & -1.52263 & $\mathrm{O}$ & 0.027 & -1.76956 & 1.02258 \\
\hline $\mathrm{C}$ & -3.61104 & 1.375174 & 1.422405 & $\mathrm{C}$ & -3.92223 & -1.17026 & 1.167362 \\
\hline $\mathrm{C}$ & -1.09323 & -2.71862 & -1.87368 & $\mathrm{C}$ & -1.51011 & 2.098679 & 1.838028 \\
\hline $\mathrm{O}$ & 0.12549 & -3.03915 & 0.249398 & $\mathrm{O}$ & 0.145407 & 3.052695 & 0.263617 \\
\hline $\mathrm{H}$ & 1.771434 & 0.324383 & 2.467632 & $\mathrm{H}$ & 2.41394 & 0.317097 & -2.34413 \\
\hline $\mathrm{H}$ & 0.995451 & -1.39245 & -0.78057 & $\mathrm{H}$ & 0.851945 & 1.166271 & 0.944229 \\
\hline $\mathrm{H}$ & -3.02394 & 3.360962 & -0.26698 & $\mathrm{H}$ & -3.08024 & -3.17223 & -0.81228 \\
\hline $\mathrm{H}$ & -2.21732 & 2.461322 & -1.54776 & $\mathrm{H}$ & -2.24505 & -3.05614 & 0.715577 \\
\hline $\mathrm{H}$ & -0.7393 & 3.956799 & -0.01237 & $\mathrm{H}$ & -0.75306 & -3.64466 & -1.2151 \\
\hline $\mathrm{H}$ & -0.95974 & 2.759763 & 1.230667 & $\mathrm{H}$ & -1.03235 & -2.09479 & -1.93748 \\
\hline $\mathrm{H}$ & -2.27001 & 0.15895 & -1.54542 & $\mathrm{H}$ & -2.03026 & -0.26817 & -1.61779 \\
\hline $\mathrm{H}$ & -3.71286 & -1.74299 & -1.1285 & $\mathrm{H}$ & -3.78873 & 1.415238 & 0.275135 \\
\hline$U$ & -4.13198 & -1.21021 & 0.481703 & $\mathrm{H}$ & -3.87352 & 1.525499 & -1.46741 \\
\hline
\end{tabular}




\begin{tabular}{|c|c|c|c|c|c|c|c|}
\hline $\mathrm{H}$ & -1.99822 & -1.88167 & 1.395085 & $\mathrm{H}$ & -1.58635 & 2.338257 & -1.64692 \\
\hline $\mathrm{H}$ & -2.66173 & -3.21706 & 0.468783 & $\mathrm{H}$ & -2.51088 & 3.336999 & -0.54133 \\
\hline $\mathrm{H}$ & -0.32996 & 0.085503 & 1.168183 & $\mathrm{H}$ & 0.072623 & 0.396667 & -1.6444 \\
\hline $\mathrm{H}$ & 0.100483 & -1.32391 & 2.110365 & $\mathrm{H}$ & 0.74067 & 1.981491 & -1.9558 \\
\hline $\mathrm{H}$ & 1.352228 & 2.597897 & 1.325244 & $\mathrm{H}$ & 1.362989 & -2.01305 & -1.9381 \\
\hline $\mathrm{H}$ & 2.157005 & 2.912474 & -0.20272 & $\mathrm{H}$ & 2.036166 & -2.88709 & -0.5778 \\
\hline $\mathrm{H}$ & 3.601147 & -1.07919 & 2.309679 & $\mathrm{H}$ & 4.123168 & 1.557693 & -1.412 \\
\hline $\mathrm{H}$ & 2.561984 & -2.23573 & 1.491182 & $\mathrm{H}$ & 2.934034 & 2.446905 & -0.477 \\
\hline $\mathrm{H}$ & 3.524896 & -1.68311 & -0.63961 & $\mathrm{H}$ & 3.514036 & 1.247007 & 1.5018 \\
\hline $\mathrm{H}$ & 4.824209 & -0.97846 & 0.301449 & $\mathrm{H}$ & 4.917278 & 0.68095 & 0.61897 \\
\hline $\mathrm{H}$ & 3.896606 & 0.527103 & -2.34306 & $\mathrm{H}$ & 3.314068 & -1.35651 & 2.54241 \\
\hline $\mathrm{H}$ & 3.548768 & 2.109426 & -1.64243 & $\mathrm{H}$ & 3.098199 & -2.64617 & 1.35747 \\
\hline $\mathrm{H}$ & 5.041741 & 1.249228 & -1.22348 & $\mathrm{H}$ & 4.67133 & -1.84659 & 1.5396 \\
\hline $\mathrm{H}$ & -4.47124 & 2.050653 & 1.346182 & $\mathrm{H}$ & -3.3869 & -1.3932 & 2.0974 \\
\hline $\mathrm{H}$ & -3.96228 & 0.420529 & 1.813619 & $\mathrm{H}$ & -4.44382 & -0.22207 & 1.29176 \\
\hline $\mathrm{H}$ & -2.94038 & 1.812606 & 2.170337 & $\mathrm{H}$ & -4.67939 & -1.95437 & $1.0503^{7}$ \\
\hline $\mathrm{H}$ & -0.11083 & -2.859 & -2.32794 & $\mathrm{H}$ & -0.67138 & 2.109101 & 2.5362 \\
\hline $\mathrm{H}$ & -1.64658 & -3.66 & -1.9522 & $\mathrm{H}$ & -2.15603 & 2.953909 & 2.06173 \\
\hline $\mathrm{H}$ & -1.6325 & -1.96296 & -2.44952 & $\mathrm{H}$ & -2.08503 & 1.185173 & 2.0046 \\
\hline 1b_5 & & & & $1 b$ & & & \\
\hline $\mathrm{C}$ & 1.855135 & 2.580106 & -0.95344 & $\mathrm{C}$ & 1.854499 & 2.580356 & -0.953 \\
\hline C & 2.649514 & 1.435569 & -0.35409 & $\mathrm{C}$ & 2.649232 & 1.435916 & -0.35423 \\
\hline $\mathrm{C}$ & 0.685168 & 3.097688 & -0.06932 & $\mathrm{C}$ & 0.684677 & 3.097658 & -0.06907 \\
\hline $\mathrm{C}$ & 2.741661 & 0.274305 & -1.01641 & $\mathrm{C}$ & 2.741659 & 0.274797 & $-1.0167 t$ \\
\hline $\mathrm{C}$ & 3.420306 & -1.00396 & -0.60374 & $\mathrm{C}$ & 3.420594 & -1.00338 & -0.6043 \\
\hline $\mathrm{C}$ & 2.448813 & -2.20801 & -0.52487 & $\mathrm{C}$ & 2.449297 & -2.20761 & -0.52518 \\
\hline $\mathrm{C}$ & 1.362624 & -2.08739 & 0.533102 & $\mathrm{C}$ & 1.36341 & -2.08704 & $0.53303 \mathrm{C}$ \\
\hline $\mathrm{C}$ & -0.00863 & -1.65808 & 0.197923 & $\mathrm{C}$ & -0.00801 & -1.65788 & 0.19831 \\
\hline $\mathrm{C}$ & -0.49325 & -1.25967 & -1.18396 & $\mathrm{C}$ & -0.49293 & -1.25969 & $-1.1835^{7}$ \\
\hline $\mathrm{C}$ & -0.31367 & 2.012879 & 0.304936 & $\mathrm{C}$ & -0.31394 & 2.012647 & 0.30532 \\
\hline $\mathrm{C}$ & -1.43381 & 1.740249 & -0.70064 & $\mathrm{C}$ & -1.43417 & 1.73996 & -0.70015 \\
\hline $\mathrm{C}$ & -2.2637 & 0.504724 & -0.4839 & $\mathrm{C}$ & -2.26389 & 0.504298 & $-0.4835 \mathrm{~s}$ \\
\hline $\mathrm{C}$ & -1.9639 & -0.79228 & -1.2284 & $\mathrm{C}$ & -1.96371 & -0.79277 & -1.22792 \\
\hline $\mathrm{C}$ & -2.97415 & -1.80154 & -0.61939 & $\mathrm{C}$ & -2.97355 & -1.80213 & -0.61863 \\
\hline $\mathrm{C}$ & -4.02394 & -0.94996 & 0.130416 & $\mathrm{C}$ & -4.02443 & -0.95052 & 0.12955 \\
\hline $\mathrm{C}$ & -3.37902 & 0.414276 & 0.253747 & $\mathrm{C}$ & -3.37965 & 0.413777 & 0.253407 \\
\hline $\mathrm{C}$ & -4.0312 & 1.481436 & 1.078012 & $\mathrm{C}$ & -4.03257 & 1.480928 & 1.07710 \\
\hline $\mathrm{O}$ & -0.2332 & 1.425135 & 1.365303 & $\mathrm{O}$ & -0.23331 & 1.424942 & 1.36568 \\
\hline $\mathrm{C}$ & 3.29046 & 1.719657 & 0.982262 & $\mathrm{C}$ & 3.290233 & 1.71999 & 0.98208 \\
\hline $\mathrm{C}$ & 1.845221 & -1.91913 & 1.959165 & $\mathrm{C}$ & 1.846368 & -1.91868 & 1.958982 \\
\hline $\mathrm{O}$ & 0.290885 & -3.06117 & 0.411628 & $\mathrm{O}$ & 0.291582 & -3.06091 & 0.412027 \\
\hline $\mathrm{H}$ & -2.1963 & -0.62881 & -2.29122 & $\mathrm{H}$ & -2.19627 & -0.62949 & -2.29073 \\
\hline $\mathrm{H}$ & -0.56701 & -1.20934 & 1.016602 & $\mathrm{H}$ & -0.56608 & -1.20898 & 1.017113 \\
\hline $\mathrm{H}$ & 1.469898 & 2.285384 & -1.93312 & $\mathrm{H}$ & 1.469165 & 2.285521 & -1.93 \\
\hline $\mathrm{H}$ & 2.517666 & 3.437847 & -1.12212 & $\mathrm{H}$ & 2.516904 & 3.438164 & -1.12225 \\
\hline $\mathrm{H}$ & 1.076507 & 3.517762 & 0.859024 & $\mathrm{H}$ & 1.076051 & 3.5178 & 0.85922 \\
\hline $\mathrm{H}$ & 0.174919 & 3.896462 & -0.61563 & $\mathrm{H}$ & 0.174102 & 3.896365 & $-0.6151 \mathrm{C}$ \\
\hline & 2.243582 & 0.217652 & -1.98356 & $\mathrm{H}$ & 2.243543 & 0.218186 & -1.98 \\
\hline
\end{tabular}




\begin{tabular}{|c|c|c|c|c|c|c|c|}
\hline $\mathrm{H}$ & 3.945649 & -0.89019 & 0.346283 & $\mathrm{H}$ & 3.946231 & -0.8896 & 0.34551 \\
\hline $\mathrm{H}$ & 4.185764 & -1.2585 & -1.34721 & $\mathrm{H}$ & 4.185815 & -1.25782 & -1.3481 \\
\hline $\mathrm{H}$ & 1.986438 & -2.36856 & -1.50165 & $\mathrm{H}$ & 1.986724 & -2.36831 & -1.50184 \\
\hline $\mathrm{H}$ & 3.031567 & -3.11013 & -0.30356 & $\mathrm{H}$ & 3.032311 & -3.10957 & -0.30392 \\
\hline $\mathrm{H}$ & 0.141878 & -0.46502 & -1.58445 & $\mathrm{H}$ & 0.141911 & -0.46491 & -1.58422 \\
\hline $\mathrm{H}$ & -0.37054 & -2.11557 & -1.85356 & $\mathrm{H}$ & -0.37002 & -2.11568 & -1.85302 \\
\hline $\mathrm{H}$ & -0.99764 & 1.74563 & -1.70646 & $\mathrm{H}$ & -0.99813 & 1.745611 & -1.70602 \\
\hline $\mathrm{H}$ & -2.0621 & 2.64028 & -0.66853 & $\mathrm{H}$ & -2.0626 & 2.639881 & -0.66775 \\
\hline $\mathrm{H}$ & -3.42028 & -2.44252 & -1.38269 & $\mathrm{H}$ & -3.41862 & -2.44443 & -1.38144 \\
\hline $\mathrm{H}$ & -2.45975 & -2.45809 & 0.086435 & $\mathrm{H}$ & -2.45908 & -2.45736 & 0.088382 \\
\hline $\mathrm{H}$ & -4.29063 & -1.37318 & 1.105478 & $\mathrm{H}$ & -4.29251 & -1.37359 & 1.104277 \\
\hline $\mathrm{H}$ & -4.96377 & -0.86577 & -0.43228 & $\mathrm{H}$ & -4.9634 & -0.8664 & -0.43458 \\
\hline $\mathrm{H}$ & -4.06948 & 1.184502 & 2.132513 & $\mathrm{H}$ & -4.07311 & 1.18332 & 2.131325 \\
\hline $\mathrm{H}$ & -3.50885 & 2.438024 & 1.022184 & $\mathrm{H}$ & -3.50938 & 2.437144 & 1.022886 \\
\hline $\mathrm{H}$ & -5.06897 & 1.638807 & 0.761266 & $\mathrm{H}$ & -5.06962 & 1.639318 & 0.758498 \\
\hline $\mathrm{H}$ & 2.531943 & 1.800943 & 1.767139 & $\mathrm{H}$ & 4.003275 & 0.952137 & 1.280875 \\
\hline $\mathrm{H}$ & 4.003567 & 0.951863 & 1.281054 & $\mathrm{H}$ & 3.82187 & 2.677639 & 0.953002 \\
\hline $\mathrm{H}$ & 3.822007 & 2.677357 & 0.953232 & $\mathrm{H}$ & 2.531781 & 1.801366 & 1.76701 \\
\hline $\mathrm{H}$ & 1.000791 & -1.96093 & 2.649163 & $\mathrm{H}$ & 1.002163 & -1.9613 & 2.6492 \\
\hline $\mathrm{H}$ & 2.545309 & -2.71918 & 2.221043 & $\mathrm{H}$ & 2.547195 & -2.71819 & 2.220534 \\
\hline $\mathrm{H}$ & 2.35456 & -0.96367 & 2.100753 & $\mathrm{H}$ & 2.354963 & -0.96283 & 2.100583 \\
\hline 1b_7 & & & & $1 b$ & & & \\
\hline $\mathrm{C}$ & -0.13556 & 3.152088 & 0.102185 & $\mathrm{C}$ & 1.81566 & -2.75848 & 0.27831 \\
\hline $\mathrm{C}$ & -1.23221 & 2.300719 & 0.711459 & $\mathrm{C}$ & 2.65267 & -1.51204 & 0.502716 \\
\hline $\mathrm{C}$ & 1.303512 & 2.545812 & 0.150779 & $\mathrm{C}$ & 0.860511 & -2.78784 & -0.92894 \\
\hline $\mathrm{C}$ & -2.12071 & 1.671179 & -0.06919 & $\mathrm{C}$ & 2.639381 & -0.47021 & -0.33872 \\
\hline $\mathrm{C}$ & -3.30507 & 0.832929 & 0.3513 & $\mathrm{C}$ & 3.380561 & 0.836887 & -0.21621 \\
\hline $\mathrm{C}$ & -3.05659 & -0.6887 & 0.510486 & $\mathrm{C}$ & 2.492847 & 2.010707 & 0.269654 \\
\hline $\mathrm{C}$ & -2.42175 & -1.39294 & -0.68216 & $\mathrm{C}$ & 1.225341 & 2.225634 & -0.5429 \\
\hline $\mathrm{C}$ & -1.09893 & -2.05684 & -0.58614 & $\mathrm{C}$ & -0.08179 & 1.734158 & -0.0629 \\
\hline $\mathrm{C}$ & -0.22964 & -2.18541 & 0.648719 & $\mathrm{C}$ & -0.30552 & 0.974394 & 1.233659 \\
\hline $\mathrm{C}$ & 1.655387 & 1.751958 & -1.09883 & $\mathrm{C}$ & -0.28668 & -1.78196 & -0.9287 \\
\hline $\mathrm{C}$ & 1.141872 & 0.32029 & -1.24374 & $\mathrm{C}$ & -1.29588 & -1.86788 & 0.219634 \\
\hline $\mathrm{C}$ & 1.63829 & -0.58108 & -0.13474 & $\mathrm{C}$ & -2.16276 & -0.65909 & 0.441946 \\
\hline $\mathrm{C}$ & 0.733674 & -1.0256 & 1.007196 & $\mathrm{C}$ & -1.7398 & 0.432353 & 1.418891 \\
\hline $\mathrm{C}$ & 1.758831 & -1.48304 & 2.076656 & $\mathrm{C}$ & -2.85473 & 1.503936 & 1.274331 \\
\hline $\mathrm{C}$ & 3.01996 & -1.87268 & 1.273646 & $\mathrm{C}$ & -4.00861 & 0.817152 & 0.507097 \\
\hline $\mathrm{C}$ & 2.883024 & -1.06598 & -0.00248 & $\mathrm{C}$ & -3.38973 & -0.44618 & -0.05283 \\
\hline $\mathrm{C}$ & 4.039595 & -0.90762 & -0.94066 & $\mathrm{C}$ & -4.17191 & -1.30999 & -0.99388 \\
\hline $\mathrm{O}$ & 2.329608 & 2.258461 & -1.97704 & $\mathrm{O}$ & -0.43537 & -1.00257 & -1.8491 \\
\hline $\mathrm{C}$ & -1.25978 & 2.270843 & 2.219495 & $\mathrm{C}$ & 3.480587 & -1.58123 & 1.762955 \\
\hline $\mathrm{C}$ & -2.94179 & -1.00957 & -2.05382 & $\mathrm{C}$ & 1.418341 & 2.465765 & -2.02622 \\
\hline $\mathrm{O}$ & -2.318 & -2.83274 & -0.534 & $\mathrm{O}$ & 0.268045 & 3.136894 & 0.056599 \\
\hline $\mathrm{H}$ & 0.126668 & -0.19107 & 1.369824 & $\mathrm{H}$ & -1.76783 & 0.002041 & 2.430874 \\
\hline $\mathrm{H}$ & -0.56294 & -2.17455 & -1.52826 & $\mathrm{H}$ & -0.82107 & 1.540951 & -0.83722 \\
\hline $\mathrm{H}$ & -0.09636 & 4.102195 & 0.645377 & $\mathrm{H}$ & 1.252867 & -2.96311 & 1.197311 \\
\hline $\mathrm{H}$ & -0.38375 & 3.401148 & -0.9333 & $\mathrm{H}$ & 2.495288 & -3.61471 & 0.179622 \\
\hline 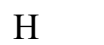 & 2.029252 & 3.358883 & 0.207749 & $\mathrm{H}$ & 1.408574 & -2.65195 & -1.86354 \\
\hline
\end{tabular}




$\begin{array}{lrrrrrrr}\mathrm{H} & 1.422464 & 1.924827 & 1.041776 & \mathrm{H} & 0.405044 & -3.78515 & -0.96186 \\ \mathrm{H} & -1.99933 & 1.796407 & -1.14323 & \mathrm{H} & 1.996309 & -0.53446 & -1.21031 \\ \mathrm{H} & -4.10157 & 0.980801 & -0.38471 & \mathrm{H} & 3.801451 & 1.093733 & -1.19461 \\ \mathrm{H} & -3.7075 & 1.19292 & 1.301916 & \mathrm{H} & 4.22911 & 0.754153 & 0.465728 \\ \mathrm{H} & -2.45291 & -0.86583 & 1.40263 & \mathrm{H} & 2.225208 & 1.850894 & 1.315935 \\ \mathrm{H} & -4.02644 & -1.16727 & 0.691418 & \mathrm{H} & 3.081924 & 2.935044 & 0.230266 \\ \mathrm{H} & -0.88016 & -2.36877 & 1.508146 & \mathrm{H} & 0.403932 & 0.144597 & 1.306916 \\ \mathrm{H} & 0.362195 & -3.09923 & 0.51378 & \mathrm{H} & -0.07316 & 1.647313 & 2.064107 \\ \mathrm{H} & 1.449657 & -0.03028 & -2.23117 & \mathrm{H} & -0.76921 & -2.12993 & 1.142573 \\ \mathrm{H} & 0.049284 & 0.354129 & -1.22391 & \mathrm{H} & -1.9091 & -2.74732 & -0.0167 \\ \mathrm{H} & 1.996092 & -0.64223 & 2.734392 & \mathrm{H} & -3.17096 & 1.89303 & 2.24438 \\ \mathrm{H} & 1.379704 & -2.29401 & 2.703282 & \mathrm{H} & -2.48149 & 2.35475 & 0.699073 \\ \mathrm{H} & 3.057792 & -2.94752 & 1.050529 & \mathrm{H} & -4.42946 & 1.455004 & -0.27851 \\ \mathrm{H} & 3.94577 & -1.639 & 1.81 & \mathrm{H} & -4.84575 & 0.557962 & 1.16936 \\ \mathrm{H} & 4.413334 & -1.88765 & -1.25985 & \mathrm{H} & -4.40821 & -0.76137 & -1.91294 \\ \mathrm{H} & 3.781578 & -0.3326 & -1.83112 & \mathrm{H} & -3.63738 & -2.2179 & -1.27838 \\ \mathrm{H} & 4.876922 & -0.40328 & -0.44443 & \mathrm{H} & -5.13125 & -1.60147 & -0.55022 \\ \mathrm{H} & -2.01505 & 1.593426 & 2.617184 & \mathrm{H} & 4.110525 & -2.47811 & 1.760245 \\ \mathrm{H} & -0.2909 & 1.964652 & 2.62812 & \mathrm{H} & 4.127594 & -0.71552 & 1.899276 \\ \mathrm{H} & -1.4576 & 3.27392 & 2.615334 & \mathrm{H} & 2.833307 & -1.65886 & 2.644178 \\ \mathrm{H} & -2.50158 & -1.65804 & -2.81318 & \mathrm{H} & 0.461249 & 2.693305 & -2.49865 \\ \mathrm{H} & -4.02898 & -1.13172 & -2.08957 & \mathrm{H} & 2.093297 & 3.31242 & -2.18848 \\ \mathrm{H} & -2.71132 & 0.025751 & -2.31139 & \mathrm{H} & 1.846816 & 1.59344 & -2.52564 \\ & & & & & & & \\ & & & & & \\ \mathrm{H} & & & & & & \\ \mathrm{H} & & & & & & \\ \mathrm{H} & & & & & \end{array}$

\begin{tabular}{|c|c|c|c|c|c|c|c|}
\hline \multicolumn{4}{|l|}{ 1c_1 } & \multicolumn{4}{|c|}{ 1c_2 } \\
\hline $\mathrm{C}$ & 1.626559 & 2.7923 & 0.619134 & $\mathrm{C}$ & -1.52622 & 2.887878 & 0.348341 \\
\hline $\mathrm{C}$ & 2.471621 & 1.541965 & 0.375349 & $\mathrm{C}$ & -2.25594 & 1.792428 & -0.40925 \\
\hline $\mathrm{C}$ & 0.106819 & 2.574607 & 0.569065 & $\mathrm{C}$ & 0.008541 & 2.767124 & 0.297562 \\
\hline $\mathrm{C}$ & 2.410054 & 0.51736 & 1.23473 & $\mathrm{C}$ & -2.72505 & 0.710072 & 0.225227 \\
\hline $\mathrm{C}$ & 3.140121 & -0.79942 & 1.200272 & $\mathrm{C}$ & -3.45017 & -0.46636 & -0.38917 \\
\hline $\mathrm{C}$ & 2.19652 & -2.02249 & 1.254771 & $\mathrm{C}$ & -2.52732 & -1.54901 & -0.99806 \\
\hline $\mathrm{C}$ & 1.349963 & -2.23614 & 0.008287 & $\mathrm{C}$ & -1.50825 & -2.14564 & -0.04078 \\
\hline $\mathrm{C}$ & 0.076019 & -1.50493 & -0.10787 & $\mathrm{C}$ & -0.1793 & -1.51445 & 0.034008 \\
\hline $\mathrm{C}$ & -0.59911 & -1.12137 & -1.4052 & $\mathrm{C}$ & 0.743608 & -1.54487 & 1.229571 \\
\hline $\mathrm{C}$ & -0.458 & 2.259676 & -0.80457 & $\mathrm{C}$ & 0.61046 & 1.888223 & 1.3842 \\
\hline $\mathrm{C}$ & -1.94854 & 1.908418 & -0.87499 & $\mathrm{C}$ & 2.089816 & 1.516426 & 1.231108 \\
\hline $\mathrm{C}$ & -2.31478 & 0.526013 & -0.38094 & $\mathrm{C}$ & 2.337789 & 0.30489 & 0.356849 \\
\hline $\mathrm{C}$ & -2.08562 & -0.70835 & -1.24944 & $\mathrm{C}$ & 2.197491 & -1.11208 & 0.908999 \\
\hline $\mathrm{C}$ & -2.95769 & -1.78784 & -0.55085 & $\mathrm{C}$ & 2.877592 & -1.98121 & -0.18599 \\
\hline $\mathrm{C}$ & -3.18332 & -1.26906 & 0.886523 & $\mathrm{C}$ & 2.922646 & -1.09827 & -1.45273 \\
\hline $\mathrm{C}$ & -2.90994 & 0.217875 & 0.783199 & $\mathrm{C}$ & 2.740355 & 0.30981 & -0.92464 \\
\hline $\mathrm{C}$ & -3.29518 & 1.127476 & 1.909061 & $\mathrm{C}$ & 3.005533 & 1.480609 & -1.82082 \\
\hline $\mathrm{O}$ & 0.217923 & 2.315617 & -1.81373 & $\mathrm{O}$ & -0.02871 & 1.527996 & 2.352994 \\
\hline $\mathrm{C}$ & 3.355264 & 1.592832 & -0.8451 & $\mathrm{C}$ & -2.39153 & 2.02441 & -1.8944 \\
\hline $\mathrm{C}$ & 2.091229 & -2.7522 & -1.20549 & $\mathrm{C}$ & -2.068 & -2.96893 & 1.097795 \\
\hline $\mathrm{O}$ & 0.084532 & -2.90378 & 0.273184 & $\mathrm{O}$ & -0.36749 & -2.73711 & -0.72313 \\
\hline $\mathrm{H}$ & -2.45481 & -0.51545 & -2.26382 & $\mathrm{H}$ & 2.751029 & -1.18787 & 1.852807 \\
\hline $\mathrm{H}$ & -0.16272 & -0.85073 & 0.728161 & $\mathrm{H}$ & -0.04377 & -0.64592 & -0.60763 \\
\hline $\mathrm{H}$ & 1.891867 & 3.557614 & -0.11413 & $\mathrm{H}$ & -1.83114 & 2.885888 & 1.396549 \\
\hline
\end{tabular}




\begin{tabular}{|c|c|c|c|c|c|c|c|}
\hline $\mathrm{H}$ & 1.869637 & 3.207908 & 1.602306 & $\mathrm{H}$ & -1.81011 & 3.858896 & -0.07021 \\
\hline $\mathrm{H}$ & -0.20692 & 1.786482 & 1.260888 & $\mathrm{H}$ & 0.360903 & 2.417529 & -0.67745 \\
\hline $\mathrm{H}$ & -0.4119 & 3.479315 & 0.914585 & $\mathrm{H}$ & 0.475032 & 3.752781 & 0.432841 \\
\hline $\mathrm{H}$ & 1.743794 & 0.626042 & 2.091886 & $\mathrm{H}$ & -2.55161 & 0.651848 & 1.297248 \\
\hline $\mathrm{H}$ & 3.796017 & -0.87052 & 0.330292 & $\mathrm{H}$ & -4.08551 & -0.92522 & 0.374652 \\
\hline $\mathrm{H}$ & 3.79683 & -0.8602 & 2.077766 & $\mathrm{H}$ & -4.12816 & -0.13083 & -1.17952 \\
\hline $\mathrm{H}$ & 2.792323 & -2.92582 & 1.43016 & $\mathrm{H}$ & -3.14917 & -2.36029 & -1.39616 \\
\hline $\mathrm{H}$ & 1.519479 & -1.92042 & 2.108705 & $\mathrm{H}$ & -1.97975 & -1.1235 & -1.84347 \\
\hline $\mathrm{H}$ & -0.54697 & -1.96099 & -2.10258 & $\mathrm{H}$ & 0.766968 & -2.55384 & 1.648743 \\
\hline $\mathrm{H}$ & -0.04215 & -0.29746 & -1.86261 & $\mathrm{H}$ & 0.337484 & -0.88632 & 2.003011 \\
\hline $\mathrm{H}$ & -2.23963 & 2.010381 & -1.92443 & $\mathrm{H}$ & 2.470765 & 1.323914 & 2.238111 \\
\hline $\mathrm{H}$ & -2.4949 & 2.660892 & -0.29895 & $\mathrm{H}$ & 2.621935 & 2.380744 & 0.825043 \\
\hline $\mathrm{H}$ & -3.91944 & -1.86388 & -1.06459 & $\mathrm{H}$ & 3.8966 & -2.22526 & 0.12479 \\
\hline $\mathrm{H}$ & -2.49198 & -2.77427 & -0.5751 & $\mathrm{H}$ & 2.350032 & -2.92236 & -0.34916 \\
\hline $\mathrm{H}$ & -2.49964 & -1.7361 & 1.607662 & $\mathrm{H}$ & 2.120747 & -1.3476 & -2.16026 \\
\hline $\mathrm{H}$ & -4.19682 & -1.47022 & 1.249997 & $\mathrm{H}$ & 3.862502 & -1.20546 & -2.00504 \\
\hline $\mathrm{H}$ & -4.36158 & 1.024787 & 2.13914 & $\mathrm{H}$ & 4.010965 & 1.412632 & -2.25101 \\
\hline $\mathrm{H}$ & -2.75345 & 0.859734 & 2.823969 & $\mathrm{H}$ & 2.307351 & 1.489566 & -2.66637 \\
\hline $\mathrm{H}$ & -3.09183 & 2.178577 & 1.6989 & $\mathrm{H}$ & 2.92009 & 2.44027 & -1.30882 \\
\hline $\mathrm{H}$ & 3.916069 & 0.673401 & -1.013 & $\mathrm{H}$ & -2.8842 & 1.201548 & -2.41229 \\
\hline $\mathrm{H}$ & 2.749585 & 1.80293 & -1.73092 & $\mathrm{H}$ & -2.96195 & 2.940546 & -2.08784 \\
\hline $\mathrm{H}$ & 4.077975 & 2.41307 & -0.75498 & $\mathrm{H}$ & -1.40951 & 2.168785 & -2.3591 \\
\hline $\mathrm{H}$ & 1.424491 & -2.90787 & -2.05261 & $\mathrm{H}$ & -1.27574 & -3.41774 & $1.69565 \mathrm{C}$ \\
\hline $\mathrm{H}$ & 2.875773 & -2.05499 & -1.51138 & $\mathrm{H}$ & -2.6873 & -2.35352 & 1.756232 \\
\hline $\mathrm{H}$ & 2.569287 & -3.7073 & -0.96661 & $\mathrm{H}$ & -2.69727 & -3.77258 & 0.70274 \\
\hline 1c_3 & & & & 1c & & & \\
\hline $\mathrm{C}$ & -0.24915 & 3.162323 & 0.311448 & $\mathrm{C}$ & -1.5261 & 2.887975 & 0.348077 \\
\hline $\mathrm{C}$ & -1.24737 & 2.20767 & 0.945959 & $\mathrm{C}$ & -2.25587 & 1.792472 & -0.4094 \\
\hline $\mathrm{C}$ & 1.045313 & 2.465469 & -0.14931 & $\mathrm{C}$ & 0.008642 & 2.767005 & 0.29760 \\
\hline $\mathrm{C}$ & -2.26792 & 1.73122 & 0.221203 & $\mathrm{C}$ & -2.72511 & 0.710232 & 0.225177 \\
\hline $\mathrm{C}$ & -3.35397 & 0.765933 & 0.612504 & $\mathrm{C}$ & -3.45027 & -0.46622 & -0.38915 \\
\hline $\mathrm{C}$ & -3.47782 & -0.43177 & -0.35747 & $\mathrm{C}$ & -2.52743 & -1.54876 & -0.99825 \\
\hline $\mathrm{C}$ & -2.41641 & -1.51591 & -0.22679 & $\mathrm{C}$ & -1.50843 & -2.14551 & -0.04097 \\
\hline $\mathrm{C}$ & -1.18508 & -1.43169 & -1.04255 & $\mathrm{C}$ & -0.17935 & -1.51458 & 0.033712 \\
\hline $\mathrm{C}$ & 0.121827 & -2.11257 & -0.69588 & $\mathrm{C}$ & 0.743486 & -1.54514 & 1.229338 \\
\hline $\mathrm{C}$ & 0.857631 & 1.586628 & -1.37704 & $\mathrm{C}$ & 0.610257 & 1.887919 & 1.384276 \\
\hline $\mathrm{C}$ & 1.84029 & 0.432579 & -1.59756 & $\mathrm{C}$ & 2.089616 & 1.516096 & 1.231538 \\
\hline $\mathrm{C}$ & 2.129243 & -0.4363 & -0.40334 & $\mathrm{C}$ & 2.337846 & 0.304698 & 0.357107 \\
\hline $\mathrm{C}$ & 1.054354 & -1.31193 & 0.246548 & $\mathrm{C}$ & 2.19741 & -1.11237 & 0.90889 \\
\hline $\mathrm{C}$ & 1.894748 & -2.23531 & 1.162246 & $\mathrm{C}$ & 2.877461 & -1.98139 & -0.18626 \\
\hline $\mathrm{C}$ & 3.192894 & -1.45883 & 1.440555 & $\mathrm{C}$ & 2.923352 & -1.09796 & -1.45267 \\
\hline $\mathrm{C}$ & 3.295366 & -0.51676 & 0.259082 & $\mathrm{C}$ & 2.740802 & 0.309951 & -0.92425 \\
\hline $\mathrm{C}$ & 4.575628 & 0.217314 & 0.003445 & $\mathrm{C}$ & 3.006214 & 1.480907 & -1.82015 \\
\hline $\mathrm{O}$ & -0.00424 & 1.819223 & -2.20239 & $\mathrm{O}$ & -0.02917 & 1.527664 & 2.352887 \\
\hline $\mathrm{C}$ & -0.97962 & 1.866515 & 2.391412 & $\mathrm{C}$ & -2.3914 & 2.024296 & -1.89458 \\
\hline $\mathrm{C}$ & -2.4843 & -2.32926 & 1.048523 & $\mathrm{C}$ & -2.06833 & -2.96823 & 1.097949 \\
\hline $\mathrm{O}$ & -2.26993 & -2.30192 & -1.44115 & $\mathrm{O}$ & -0.36787 & -2.73735 & -0.72326 \\
\hline & 0.418555 & -0.66898 & 0.873214 & $\mathrm{H}$ & 2.750951 & -1.1884 & 1.85268 \\
\hline
\end{tabular}




$\begin{array}{llllllll}\mathrm{H} & -1.09817 & -0.55182 & -1.67745 & \mathrm{H} & -0.04362 & -0.6462 & -0.60807 \\ \mathrm{H} & 0.019638 & 3.944321 & 1.029584 & \mathrm{H} & -1.83126 & 2.886338 & 1.396219 \\ \mathrm{H} & -0.69993 & 3.656219 & -0.55135 & \mathrm{H} & -1.80974 & 3.858937 & -0.07079 \\ \mathrm{H} & 1.794411 & 3.217956 & -0.43261 & \mathrm{H} & 0.36111 & 2.417461 & -0.67738 \\ \mathrm{H} & 1.503239 & 1.884072 & 0.653722 & \mathrm{H} & 0.47521 & 3.752593 & 0.433087 \\ \mathrm{H} & -2.33707 & 2.07577 & -0.80919 & \mathrm{H} & -2.55179 & 0.652151 & 1.297223 \\ \mathrm{H} & -3.23666 & 0.413572 & 1.639468 & \mathrm{H} & -4.08538 & -0.92522 & 0.374774 \\ \mathrm{H} & -4.31384 & 1.297891 & 0.586191 & \mathrm{H} & -4.12847 & -0.1307 & -1.17933 \\ \mathrm{H} & -4.45367 & -0.90889 & -0.2082 & \mathrm{H} & -3.14929 & -2.35999 & -1.39644 \\ \mathrm{H} & -3.4641 & -0.06447 & -1.38813 & \mathrm{H} & -1.97985 & -1.12314 & -1.8436 \\ \mathrm{H} & 0.650591 & -2.35694 & -1.62482 & \mathrm{H} & 0.766765 & -2.55416 & 1.64839 \\ \mathrm{H} & -0.1104 & -3.07031 & -0.22624 & \mathrm{H} & 0.337331 & -0.88665 & 2.002809 \\ \mathrm{H} & 2.768717 & 0.896191 & -1.95046 & \mathrm{H} & 2.470283 & 1.323385 & 2.238613 \\ \mathrm{H} & 1.445334 & -0.15057 & -2.43459 & \mathrm{H} & 2.621937 & 2.38043 & 0.825777 \\ \mathrm{H} & 1.356131 & -2.52701 & 2.066646 & \mathrm{H} & 3.896215 & -2.22619 & 0.124732 \\ \mathrm{H} & 2.133606 & -3.15079 & 0.610818 & \mathrm{H} & 2.349388 & -2.92215 & -0.35 \\ \mathrm{H} & 4.068407 & -2.10967 & 1.535595 & \mathrm{H} & 2.12196 & -1.347 & -2.16087 \\ \mathrm{H} & 3.131909 & -0.88188 & 2.374073 & \mathrm{H} & 3.863601 & -1.20497 & -2.00435 \\ \mathrm{H} & 4.841921 & 0.836567 & 0.868276 & \mathrm{H} & 4.012103 & 1.413481 & -2.24937 \\ \mathrm{H} & 5.402211 & -0.48782 & -0.13944 & \mathrm{H} & 2.308834 & 1.489385 & -2.66637 \\ \mathrm{H} & 4.532331 & 0.868367 & -0.87108 & \mathrm{H} & 2.919754 & 2.44055 & -1.30829 \\ \mathrm{H} & 0.046028 & 1.508015 & 2.530492 & \mathrm{H} & -2.88491 & 1.201801 & -2.41226 \\ \mathrm{H} & -1.08086 & 2.76137 & 3.01702 & \mathrm{H} & -2.96096 & 2.94093 & -2.08816 \\ \mathrm{H} & -1.654 & 1.106381 & 2.785378 & \mathrm{H} & -1.40929 & 2.167624 & -2.35945 \\ \mathrm{H} & -1.74908 & -3.13218 & 1.063771 & \mathrm{H} & -1.2761 & -3.4191 & 1.694303 \\ \mathrm{H} & -2.31787 & -1.69807 & 1.925135 & \mathrm{H} & -2.68524 & -2.35184 & 1.757721 \\ \mathrm{H} & -3.478 & -2.77768 & 1.144509 & \mathrm{H} & -2.69997 & -3.77029 & 0.703455\end{array}$

\begin{tabular}{llllllll} 
1c_5 & \multicolumn{7}{c}{$\mathbf{1 c \_ 6}$} \\
C & 1.62668 & -2.79237 & -0.61909 & C & 2.107211 & 2.736726 & 0.026036 \\
C & 2.471628 & -1.54197 & -0.37516 & C & 2.775963 & 1.370876 & 0.017621 \\
C & 0.106911 & -2.57477 & -0.56918 & C & 0.792261 & 2.776998 & -0.7737 \\
C & 2.410267 & -0.51744 & -1.23465 & C & 2.631679 & 0.538577 & 1.057409 \\
C & 3.140219 & 0.799382 & -1.20006 & C & 3.177109 & -0.85277 & 1.244621 \\
C & 2.196501 & 2.02239 & -1.25479 & C & 2.075996 & -1.92254 & 1.441816 \\
C & 1.349832 & 2.236182 & -0.00841 & C & 1.235838 & -2.20412 & 0.205192 \\
C & 0.076153 & 1.504556 & 0.10791 & C & 0.022087 & -1.39757 & -0.01637 \\
C & -0.59894 & 1.121111 & 1.405288 & C & -0.56585 & -1.08227 & -1.3719 \\
C & -0.45801 & -2.25971 & 0.804385 & C & -0.40221 & 2.190935 & -0.03603 \\
C & -1.9486 & -1.90865 & 0.87468 & C & -1.67006 & 1.9111 & -0.85887 \\
C & -2.31475 & -0.52611 & 0.380897 & C & -2.33884 & 0.59903 & -0.53304 \\
C & -2.08546 & 0.708105 & 1.249573 & C & -2.05066 & -0.64387 & -1.36342 \\
C & -2.95755 & 1.787728 & 0.551224 & C & -3.03009 & -1.70595 & -0.7822 \\
C & -3.18305 & 1.26927 & -0.88627 & C & -3.61089 & -1.09437 & 0.515682 \\
C & -2.90989 & -0.21773 & -0.78319 & C & -3.19035 & 0.358773 & 0.473184 \\
C & -3.29539 & -1.12709 & -1.90915 & C & -3.70547 & 1.320146 & 1.499323 \\
O & 0.217935 & -2.31526 & 1.813552 & O & -0.38656 & 1.998594 & 1.162151 \\
C & 3.354968 & -1.59275 & 0.845503 & C & 3.574846 & 1.066527 & -1.22626 \\
C & 2.090921 & 2.752998 & 1.205144 & C & 1.959965 & -2.89419 & -0.93122
\end{tabular}




$\begin{array}{llllllll}\mathrm{O} & 0.084156 & 2.903293 & -0.27358 & \mathrm{O} & -0.08086 & -2.74948 & 0.498295 \\ \mathrm{H} & -2.45456 & 0.515055 & 2.263963 & \mathrm{H} & -2.30095 & -0.43958 & -2.41267 \\ \mathrm{H} & -0.16234 & 0.850028 & -0.72793 & \mathrm{H} & -0.18917 & -0.64904 & 0.743871 \\ \mathrm{H} & 1.891925 & -3.55767 & 0.1142 & \mathrm{H} & 2.792745 & 3.476949 & -0.40019 \\ \mathrm{H} & 1.869891 & -3.20794 & -1.60225 & \mathrm{H} & 1.89536 & 3.044591 & 1.05164 \\ \mathrm{H} & -0.20682 & -1.78669 & -1.26108 & \mathrm{H} & 0.517287 & 3.813968 & -1.01365 \\ \mathrm{H} & -0.41174 & -3.47951 & -0.91471 & \mathrm{H} & 0.891875 & 2.279578 & -1.7434 \\ \mathrm{H} & 1.744249 & -0.62623 & -2.09199 & \mathrm{H} & 2.024086 & 0.893694 & 1.88796 \\ \mathrm{H} & 3.795943 & 0.870544 & -0.32996 & \mathrm{H} & 3.835541 & -1.14352 & 0.42351 \\ \mathrm{H} & 3.797096 & 0.860232 & -2.07743 & \mathrm{H} & 3.80025 & -0.86325 & 2.147746 \\ \mathrm{H} & 2.792221 & 2.925746 & -1.43032 & \mathrm{H} & 2.545205 & -2.85955 & 1.764179 \\ \mathrm{H} & 1.519537 & 1.920084 & -2.10877 & \mathrm{H} & 1.404801 & -1.61026 & 2.247779 \\ \mathrm{H} & -0.54678 & 1.960751 & 2.102643 & \mathrm{H} & -0.46457 & -1.94602 & -2.03277 \\ \mathrm{H} & -0.04197 & 0.297202 & 1.862701 & \mathrm{H} & 0.038164 & -0.28229 & -1.81922 \\ \mathrm{H} & -2.23985 & -2.01084 & 1.924052 & \mathrm{H} & -1.4418 & 1.973291 & -1.92598 \\ \mathrm{H} & -2.49485 & -2.66098 & 0.298362 & \mathrm{H} & -2.34389 & 2.747324 & -0.63606 \\ \mathrm{H} & -3.91933 & 1.863562 & 1.064932 & \mathrm{H} & -3.83265 & -1.89753 & -1.49796 \\ \mathrm{H} & -2.49188 & 2.774171 & 0.575743 & \mathrm{H} & -2.52695 & -2.65621 & -0.59804 \\ \mathrm{H} & -2.49913 & 1.736335 & -1.60718 & \mathrm{H} & -3.21325 & -1.57828 & 1.417542 \\ \mathrm{H} & -4.19645 & 1.470698 & -1.24991 & \mathrm{H} & -4.70069 & -1.19808 & 0.572808 \\ \mathrm{H} & -4.36199 & -1.0248 & -2.13851 & \mathrm{H} & -4.80173 & 1.328429 & 1.508062 \\ \mathrm{H} & -2.75439 & -0.85873 & -2.8243 & \mathrm{H} & -3.38658 & 1.018829 & 2.503787 \\ \mathrm{H} & -3.09144 & -2.17818 & -1.69952 & \mathrm{H} & -3.35113 & 2.338281 & 1.334443 \\ \mathrm{H} & 3.915203 & -0.67306 & 1.013897 & \mathrm{H} & 3.978708 & 0.054849 & -1.24021 \\ \mathrm{H} & 2.749175 & -1.80353 & 1.731085 & \mathrm{H} & 2.965347 & 1.194254 & -2.12759 \\ \mathrm{H} & 4.078186 & -2.41254 & 0.755256 & \mathrm{H} & 4.414615 & 1.765299 & -1.31956 \\ \mathrm{H} & 1.424419 & 2.907675 & 2.052632 & \mathrm{H} & 1.280689 & -3.15672 & -1.74076 \\ \mathrm{H} & 2.87652 & 2.056747 & 1.510506 & \mathrm{H} & 2.750433 & -2.25945 & -1.33943 \\ \mathrm{H} & 2.56763 & 3.708759 & 0.966176 & \mathrm{H} & 2.426416 & -3.81473 & -0.56668\end{array}$

\begin{tabular}{|c|c|c|c|c|c|c|c|}
\hline \multicolumn{4}{|l|}{ 1c_ 7} & \multicolumn{4}{|l|}{ 1c_8 } \\
\hline $\mathrm{C}$ & -1.23063 & -2.95863 & -0.54807 & $\mathrm{C}$ & 0.089966 & 3.254368 & 0.347756 \\
\hline $\mathrm{C}$ & -2.36099 & -1.94867 & -0.67855 & $\mathrm{C}$ & -0.7662 & 2.533324 & -0.67593 \\
\hline $\mathrm{C}$ & 0.169193 & -2.40821 & -0.27509 & $\mathrm{C}$ & 1.33529 & 2.444992 & 0.758174 \\
\hline $\mathrm{C}$ & -2.13885 & -0.6432 & -0.87013 & $\mathrm{C}$ & -1.91995 & 1.963126 & -0.3071 \\
\hline $\mathrm{C}$ & -3.13447 & 0.483878 & -0.9319 & $\mathrm{C}$ & -2.84808 & 1.10816 & -1.13361 \\
\hline $\mathrm{C}$ & -2.94419 & 1.508979 & 0.21225 & $\mathrm{C}$ & -3.50298 & -0.02913 & -0.32968 \\
\hline $\mathrm{C}$ & -1.62672 & 2.273168 & 0.200325 & $\mathrm{C}$ & -2.6331 & -1.21495 & 0.077343 \\
\hline $\mathrm{C}$ & -0.49871 & 1.776576 & 1.019954 & $\mathrm{C}$ & -1.15856 & -1.14048 & 0.087608 \\
\hline $\mathrm{C}$ & 0.961754 & 2.063074 & 0.742957 & $\mathrm{C}$ & -0.25842 & -1.95661 & 0.987823 \\
\hline $\mathrm{C}$ & 0.324543 & -1.76986 & 1.095964 & $\mathrm{C}$ & 1.054201 & 1.250377 & 1.659274 \\
\hline $\mathrm{C}$ & 1.615048 & -0.99937 & 1.405726 & $\mathrm{C}$ & 2.109613 & 0.139635 & 1.679596 \\
\hline $\mathrm{C}$ & 2.255186 & -0.1955 & 0.307748 & $\mathrm{C}$ & 2.059684 & -0.79947 & 0.491847 \\
\hline $\mathrm{C}$ & 1.631502 & 1.088363 & -0.2541 & $\mathrm{C}$ & 1.206906 & -2.0708 & 0.512788 \\
\hline $\mathrm{C}$ & 2.826276 & 1.731041 & -0.99759 & $\mathrm{C}$ & 1.367353 & -2.62421 & -0.92571 \\
\hline $\mathrm{C}$ & 3.743844 & 0.553934 & -1.36655 & $\mathrm{C}$ & 2.649078 & -1.9688 & -1.47665 \\
\hline $\mathrm{C}$ & 3.408365 & -0.48652 & -0.31804 & $\mathrm{C}$ & 2.860451 & -0.76285 & -0.58635 \\
\hline $\mathrm{C}$ & 4.306786 & -1.67021 & -0.13186 & $\mathrm{C}$ & 3.942044 & 0.217869 & -0.92208 \\
\hline , & -0.50868 & -1.91493 & 1.96878 & $\mathrm{O}$ & 0.067623 & 1.1949 & 2.365973 \\
\hline
\end{tabular}




\begin{tabular}{|c|c|c|c|c|c|c|c|}
\hline $\mathrm{C}$ & -3.72817 & -2.57615 & -0.56168 & C & -0.20527 & 2.482978 & -2.07632 \\
\hline $\mathrm{C}$ & -1.41411 & 3.180086 & -0.99308 & $\mathrm{C}$ & -3.3745 & -2.21104 & 0.946718 \\
\hline $\mathrm{O}$ & -1.31072 & 2.877798 & 1.483699 & $\mathrm{O}$ & -1.85697 & -1.80887 & -0.99624 \\
\hline $\mathrm{H}$ & 0.872413 & 0.808939 & -1.00012 & $\mathrm{H}$ & 1.692622 & -2.75734 & 1.223551 \\
\hline $\mathrm{H}$ & -0.69244 & 0.859949 & 1.574824 & $\mathrm{H}$ & -0.72836 & -0.19496 & -0.22508 \\
\hline $\mathrm{H}$ & -1.19912 & -3.57426 & -1.45607 & $\mathrm{H}$ & -0.49731 & 3.46778 & 1.243074 \\
\hline $\mathrm{H}$ & -1.49233 & -3.64655 & 0.262818 & $\mathrm{H}$ & 0.428507 & 4.214451 & -0.058 \\
\hline $\mathrm{H}$ & 0.900386 & -3.22725 & -0.31355 & $\mathrm{H}$ & 1.904808 & 2.117001 & -0.11371 \\
\hline $\mathrm{H}$ & 0.503288 & -1.7081 & -1.0448 & $\mathrm{H}$ & 2.022574 & 3.083372 & 1.331073 \\
\hline $\mathrm{H}$ & -1.10658 & -0.31764 & -0.95266 & $\mathrm{H}$ & -2.2244 & 2.088899 & 0.730806 \\
\hline $\mathrm{H}$ & -3.04683 & 1.002974 & -1.89422 & $\mathrm{H}$ & -3.66188 & 1.727674 & -1.53262 \\
\hline $\mathrm{H}$ & -4.15908 & 0.111314 & -0.88167 & $\mathrm{H}$ & -2.33578 & 0.685532 & -2.00142 \\
\hline $\mathrm{H}$ & -3.75588 & 2.245 & 0.163883 & $\mathrm{H}$ & -3.95038 & 0.385945 & 0.581258 \\
\hline $\mathrm{H}$ & -3.03554 & 0.99633 & 1.174096 & $\mathrm{H}$ & -4.33627 & -0.43795 & -0.9138 \\
\hline $\mathrm{H}$ & 1.512541 & 2.064359 & 1.691147 & $\mathrm{H}$ & -0.66057 & -2.96662 & 1.095162 \\
\hline $\mathrm{H}$ & 1.044114 & 3.077011 & 0.345728 & $\mathrm{H}$ & -0.27931 & -1.50149 & 1.98443 \\
\hline $\mathrm{H}$ & 2.317503 & -1.76282 & 1.760048 & $\mathrm{H}$ & 1.960479 & -0.42527 & 2.603677 \\
\hline $\mathrm{H}$ & 1.390328 & -0.37606 & 2.276355 & $\mathrm{H}$ & 3.093544 & 0.615796 & 1.731511 \\
\hline $\mathrm{H}$ & 2.510068 & 2.327902 & -1.85612 & $\mathrm{H}$ & 1.404932 & -3.71577 & -0.94423 \\
\hline $\mathrm{H}$ & 3.352564 & 2.397171 & -0.30555 & $\mathrm{H}$ & 0.510234 & -2.32114 & -1.52973 \\
\hline $\mathrm{H}$ & 4.805684 & 0.821066 & -1.36618 & $\mathrm{H}$ & 2.568052 & -1.69991 & -2.53558 \\
\hline $\mathrm{H}$ & 3.521331 & 0.163123 & -2.36955 & $\mathrm{H}$ & 3.519036 & -2.63557 & -1.39559 \\
\hline $\mathrm{H}$ & 4.38556 & -2.24104 & -1.06466 & $\mathrm{H}$ & 4.882587 & -0.30873 & -1.12015 \\
\hline $\mathrm{H}$ & 5.323105 & -1.34726 & 0.120719 & $\mathrm{H}$ & 3.698747 & 0.770972 & -1.83684 \\
\hline $\mathrm{H}$ & 3.964982 & -2.35323 & 0.647282 & $\mathrm{H}$ & 4.124571 & 0.946705 & -0.13077 \\
\hline $\mathrm{H}$ & -4.54223 & -1.88273 & -0.76977 & $\mathrm{H}$ & -0.83901 & 1.925502 & -2.76549 \\
\hline $\mathrm{H}$ & -3.81999 & -3.42017 & -1.25484 & $\mathrm{H}$ & -0.09263 & 3.498524 & -2.47371 \\
\hline $\mathrm{H}$ & -3.87765 & -2.98341 & 0.445048 & $\mathrm{H}$ & 0.791477 & 2.029198 & -2.09667 \\
\hline $\mathrm{H}$ & -0.50615 & 3.773089 & -0.89522 & $\mathrm{H}$ & -2.78715 & -3.10928 & 1.130495 \\
\hline $\mathrm{H}$ & -1.34894 & 2.604331 & -1.91996 & $\mathrm{H}$ & -3.63268 & -1.76093 & 1.909839 \\
\hline $\mathrm{H}$ & -2.25988 & 3.868189 & -1.08663 & $\mathrm{H}$ & -4.30686 & -2.50958 & 0.457587 \\
\hline 1c_9 & & & & & & & \\
\hline $\mathrm{C}$ & -2.02362 & 2.836462 & -0.12611 & $\mathrm{C}$ & -0.61049 & 3.146395 & 0.385812 \\
\hline $\mathrm{C}$ & -2.78358 & 1.523087 & -0.0346 & $\mathrm{C}$ & -1.55626 & 2.080226 & 0.915446 \\
\hline $\mathrm{C}$ & -0.71406 & 2.835428 & 0.682734 & $\mathrm{C}$ & 0.78609 & 2.603721 & 0.026463 \\
\hline $\mathrm{C}$ & -2.6978 & 0.625145 & -1.02472 & $\mathrm{C}$ & -2.44928 & 1.508185 & 0.096724 \\
\hline $\mathrm{C}$ & -3.33128 & -0.73545 & -1.13485 & $\mathrm{C}$ & -3.4707 & 0.442223 & 0.389859 \\
\hline $\mathrm{C}$ & -2.30116 & -1.86547 & -1.36375 & $\mathrm{C}$ & -3.39396 & -0.76537 & -0.57387 \\
\hline $\mathrm{C}$ & -1.41394 & -2.19013 & -0.17113 & $\mathrm{C}$ & -2.23522 & -1.72521 & -0.34217 \\
\hline $\mathrm{C}$ & -0.12073 & -1.49341 & -0.02942 & $\mathrm{C}$ & -0.96704 & -1.51725 & -1.07173 \\
\hline $\mathrm{C}$ & 0.593869 & -1.26636 & 1.285743 & $\mathrm{C}$ & 0.387924 & -1.99217 & -0.59247 \\
\hline $\mathrm{C}$ & 0.421967 & 2.068617 & 0.018935 & $\mathrm{C}$ & 0.802744 & 1.777727 & -1.252 \\
\hline $\mathrm{C}$ & 1.623056 & 1.712756 & 0.904356 & $\mathrm{C}$ & 1.927266 & 0.761324 & -1.44497 \\
\hline $\mathrm{C}$ & 2.378233 & 0.473417 & 0.505778 & $\mathrm{C}$ & 2.198342 & -0.16794 & -0.2901 \\
\hline $\mathrm{C}$ & 2.082875 & -0.87384 & 1.160639 & $\mathrm{C}$ & 1.074082 & -0.96703 & 0.364255 \\
\hline $\mathrm{C}$ & 2.938822 & -1.86481 & 0.328585 & $\mathrm{C}$ & 1.78193 & -1.60498 & 1.593376 \\
\hline $\mathrm{C}$ & 3.990471 & -0.99783 & -0.39692 & $\mathrm{C}$ & 3.29364 & -1.53648 & 1.294573 \\
\hline$C$ & 3.416407 & 0.402283 & -0.33878 & $\mathrm{C}$ & 3.40185 & -0.48031 & 0.216117 \\
\hline
\end{tabular}




\begin{tabular}{|c|c|c|c|c|c|c|c|}
\hline $\mathrm{C}$ & 4.05202 & 1.510907 & -1.11933 & $\mathrm{C}$ & 4.750971 & 0.030721 & -0.18751 \\
\hline $\mathrm{O}$ & 0.414274 & 1.807413 & -1.16632 & $\mathrm{O}$ & -0.01704 & 1.953864 & -2.13286 \\
\hline $\mathrm{C}$ & -3.59727 & 1.345399 & 1.224309 & $\mathrm{C}$ & -1.39405 & 1.753464 & 2.379863 \\
\hline $\mathrm{C}$ & -2.12122 & -2.80806 & 1.016388 & $\mathrm{C}$ & -2.30007 & -2.51644 & 0.946888 \\
\hline $\mathrm{O}$ & -0.17251 & -2.84723 & -0.54611 & $\mathrm{O}$ & -1.90827 & -2.51755 & -1.51884 \\
\hline $\mathrm{H}$ & 2.46638 & -0.83063 & 2.191534 & $\mathrm{H}$ & 0.286054 & -0.29282 & 0.716372 \\
\hline $\mathrm{H}$ & 0.109712 & -0.75969 & -0.79907 & $\mathrm{H}$ & -0.93679 & -0.64632 & -1.72579 \\
\hline $\mathrm{H}$ & -2.65671 & 3.649976 & 0.244272 & $\mathrm{H}$ & -0.4904 & 3.933887 & 1.137408 \\
\hline $\mathrm{H}$ & -1.78523 & 3.059738 & -1.16763 & $\mathrm{H}$ & -1.03427 & 3.612029 & -0.50578 \\
\hline $\mathrm{H}$ & -0.34699 & 3.862508 & 0.817646 & $\mathrm{H}$ & 1.477241 & 3.439589 & -0.15105 \\
\hline $\mathrm{H}$ & -0.86671 & 2.449594 & 1.694678 & $\mathrm{H}$ & 1.227761 & 2.029851 & 0.844738 \\
\hline $\mathrm{H}$ & -2.0734 & 0.893419 & -1.87519 & $\mathrm{H}$ & -2.45617 & 1.852816 & -0.93592 \\
\hline $\mathrm{H}$ & -3.95455 & -0.96524 & -0.26832 & $\mathrm{H}$ & -3.41931 & 0.102244 & 1.426325 \\
\hline $\mathrm{H}$ & -4.00639 & -0.73721 & -2.0002 & $\mathrm{H}$ & -4.46909 & 0.882375 & 0.268883 \\
\hline $\mathrm{H}$ & -2.83503 & -2.78023 & -1.64764 & $\mathrm{H}$ & -4.32361 & -1.34094 & -0.49297 \\
\hline $\mathrm{H}$ & -1.65575 & -1.60229 & -2.20732 & $\mathrm{H}$ & -3.33805 & -0.40384 & -1.60529 \\
\hline $\mathrm{H}$ & 0.524 & -2.17179 & 1.893109 & $\mathrm{H}$ & 1.021628 & -2.18909 & -1.46403 \\
\hline $\mathrm{H}$ & 0.062006 & -0.48449 & 1.842096 & $\mathrm{H}$ & 0.267331 & -2.94871 & -0.08202 \\
\hline $\mathrm{H}$ & 1.298267 & 1.647638 & 1.94666 & $\mathrm{H}$ & 2.828047 & 1.341132 & -1.6768 \\
\hline $\mathrm{H}$ & 2.277145 & 2.593142 & 0.853868 & $\mathrm{H}$ & 1.675515 & 0.201056 & -2.35197 \\
\hline $\mathrm{H}$ & 3.385972 & -2.64414 & 0.949815 & $\mathrm{H}$ & 1.562287 & -1.00784 & 2.482145 \\
\hline $\mathrm{H}$ & 2.304414 & -2.36421 & -0.40652 & $\mathrm{H}$ & 1.433664 & -2.62007 & 1.793386 \\
\hline $\mathrm{H}$ & 4.175807 & -1.33136 & -1.42402 & $\mathrm{H}$ & 3.690421 & -2.49171 & 0.925185 \\
\hline $\mathrm{H}$ & 4.963995 & -1.01942 & 0.112468 & $\mathrm{H}$ & 3.884045 & -1.28246 & 2.181559 \\
\hline $\mathrm{H}$ & 5.12545 & 1.572546 & -0.90503 & $\mathrm{H}$ & 5.272655 & 0.464511 & 0.673003 \\
\hline $\mathrm{H}$ & 3.958726 & 1.326575 & -2.19583 & $\mathrm{H}$ & 5.379977 & -0.79116 & -0.54961 \\
\hline $\mathrm{H}$ & 3.604532 & 2.48417 & -0.91104 & $\mathrm{H}$ & 4.707414 & 0.787444 & -0.97218 \\
\hline $\mathrm{H}$ & -4.0643 & 0.363725 & 1.299452 & $\mathrm{H}$ & -2.00496 & 0.910582 & 2.702108 \\
\hline $\mathrm{H}$ & -2.98033 & 1.490047 & 2.117892 & $\mathrm{H}$ & -0.35064 & 1.522184 & 2.620378 \\
\hline $\mathrm{H}$ & -4.39185 & 2.099487 & 1.272443 & $\mathrm{H}$ & -1.66684 & 2.619797 & 2.994206 \\
\hline $\mathrm{H}$ & -1.41868 & -3.10977 & 1.79175 & $\mathrm{H}$ & -1.49466 & -3.24581 & 1.017358 \\
\hline $\mathrm{H}$ & -2.83585 & -2.1079 & 1.456784 & $\mathrm{H}$ & -2.24252 & -1.85824 & 1.817421 \\
\hline $\mathrm{H}$ & -2.67711 & -3.69455 & 0.695705 & $\mathrm{H}$ & -3.25082 & -3.056 & 0.999069 \\
\hline 1d_1 & & & & 1d & & & \\
\hline $\mathrm{C}$ & 0.636135 & 2.993274 & 0.000091 & $\mathrm{C}$ & -0.2548 & 3.184748 & 0.16045 \\
\hline $\mathrm{C}$ & 1.56507 & 2.117717 & 0.826798 & $\mathrm{C}$ & -1.28963 & 2.398848 & -0.62609 \\
\hline $\mathrm{C}$ & -0.7004 & 2.314586 & -0.35658 & $\mathrm{C}$ & 1.154142 & 2.549398 & 0.183758 \\
\hline $\mathrm{C}$ & 2.512682 & 1.388071 & 0.223716 & $\mathrm{C}$ & -2.23866 & 1.682959 & -0.00652 \\
\hline $\mathrm{C}$ & 3.494545 & 0.422407 & 0.842464 & $\mathrm{C}$ & -3.32219 & 0.851328 & -0.65624 \\
\hline $\mathrm{C}$ & 3.001176 & -1.04694 & 0.919749 & $\mathrm{C}$ & -2.92606 & -0.60384 & -1.01502 \\
\hline $\mathrm{C}$ & 2.384727 & -1.59056 & -0.36089 & $\mathrm{C}$ & -2.6494 & -1.53715 & 0.155301 \\
\hline $\mathrm{C}$ & 0.91863 & -1.7087 & -0.50149 & $\mathrm{C}$ & -1.29207 & -1.72974 & 0.701136 \\
\hline $\mathrm{C}$ & -0.07459 & -1.30497 & 0.573688 & $\mathrm{C}$ & -0.0845 & -0.93908 & 0.260914 \\
\hline $\mathrm{C}$ & -0.59603 & 1.280408 & -1.46912 & $\mathrm{C}$ & 1.436751 & 1.666626 & 1.393161 \\
\hline $\mathrm{C}$ & -1.76222 & 0.303344 & -1.66153 & $\mathrm{C}$ & 2.617616 & 0.696345 & 1.289877 \\
\hline $\mathrm{C}$ & -2.31389 & -0.39826 & -0.44852 & $\mathrm{C}$ & 2.396969 & -0.51015 & 0.405859 \\
\hline $\mathrm{C}$ & -1.56584 & -1.54203 & 0.242066 & $\mathrm{C}$ & 1.280206 & -1.51069 & 0.695635 \\
\hline$C$ & -2.40798 & -1.7639 & 1.522839 & $\mathrm{C}$ & 1.710192 & -2.75028 & -0.12795 \\
\hline
\end{tabular}




\begin{tabular}{|c|c|c|c|c|c|c|c|}
\hline $\mathrm{C}$ & -3.80175 & -1.19592 & 1.205221 & $\mathrm{C}$ & 2.627388 & -2.18951 & -1.23728 \\
\hline $\mathrm{C}$ & -3.5349 & -0.2162 & 0.082221 & $\mathrm{C}$ & 3.114375 & -0.86959 & -0.67059 \\
\hline $\mathrm{C}$ & -4.60883 & 0.741272 & -0.33436 & $\mathrm{C}$ & 4.250936 & -0.15075 & -1.32974 \\
\hline $\mathrm{O}$ & 0.335059 & 1.26948 & -2.25027 & $\mathrm{O}$ & 0.799279 & 1.760697 & 2.424217 \\
\hline $\mathrm{C}$ & 1.318722 & 2.132659 & 2.315186 & $\mathrm{C}$ & -1.15622 & 2.49586 & -2.12643 \\
\hline $\mathrm{C}$ & 3.24501 & -1.51234 & -1.60478 & $\mathrm{C}$ & -3.83735 & -1.85577 & 1.038357 \\
\hline $\mathrm{O}$ & 1.726711 & -2.87676 & -0.21353 & $\mathrm{O}$ & -1.85951 & -2.70988 & -0.19735 \\
\hline $\mathrm{H}$ & -1.63243 & -2.43427 & -0.39927 & $\mathrm{H}$ & 1.230599 & -1.74186 & 1.767232 \\
\hline $\mathrm{H}$ & 0.536859 & -1.70227 & -1.52125 & $\mathrm{H}$ & -1.24114 & -2.08192 & 1.732844 \\
\hline $\mathrm{H}$ & 1.128669 & 3.285048 & -0.92935 & $\mathrm{H}$ & -0.58355 & 3.309569 & 1.194034 \\
\hline $\mathrm{H}$ & 0.415869 & 3.912521 & 0.552767 & $\mathrm{H}$ & -0.17388 & 4.186151 & -0.27581 \\
\hline $\mathrm{H}$ & -1.41537 & 3.065625 & -0.72105 & $\mathrm{H}$ & 1.919967 & 3.335704 & 0.231659 \\
\hline $\mathrm{H}$ & -1.18065 & 1.865139 & 0.516191 & $\mathrm{H}$ & 1.375641 & 1.990384 & -0.72922 \\
\hline $\mathrm{H}$ & 2.567403 & 1.470683 & -0.85829 & $\mathrm{H}$ & -2.2311 & 1.681405 & 1.081809 \\
\hline $\mathrm{H}$ & 3.766851 & 0.731323 & 1.855316 & $\mathrm{H}$ & -3.65694 & 1.32802 & -1.58177 \\
\hline $\mathrm{H}$ & 4.420244 & 0.453683 & 0.258685 & $\mathrm{H}$ & -4.19657 & 0.833606 & 0.001665 \\
\hline $\mathrm{H}$ & 2.277367 & -1.13972 & 1.730708 & $\mathrm{H}$ & -2.05627 & -0.58982 & -1.67339 \\
\hline $\mathrm{H}$ & 3.851775 & -1.68659 & 1.184557 & $\mathrm{H}$ & -3.74662 & -1.04699 & -1.59278 \\
\hline $\mathrm{H}$ & 0.077549 & -0.25713 & 0.847696 & $\mathrm{H}$ & -0.2099 & 0.057174 & 0.685442 \\
\hline $\mathrm{H}$ & 0.170603 & -1.88931 & 1.464268 & $\mathrm{H}$ & -0.09248 & -0.81682 & -0.82462 \\
\hline $\mathrm{H}$ & -1.42863 & -0.42059 & -2.41144 & $\mathrm{H}$ & 2.828113 & 0.366607 & 2.312493 \\
\hline $\mathrm{H}$ & -2.55537 & 0.895485 & -2.1328 & $\mathrm{H}$ & 3.483283 & 1.26312 & 0.934113 \\
\hline $\mathrm{H}$ & -2.42346 & -2.81135 & 1.832375 & $\mathrm{H}$ & 2.283776 & -3.43062 & 0.507394 \\
\hline $\mathrm{H}$ & -1.96198 & -1.18821 & 2.34054 & $\mathrm{H}$ & 0.854973 & -3.305 & -0.51914 \\
\hline $\mathrm{H}$ & -4.27811 & -0.72423 & 2.071207 & $\mathrm{H}$ & 2.088464 & -2.02383 & -2.17999 \\
\hline $\mathrm{H}$ & -4.49334 & -1.97607 & 0.857854 & $\mathrm{H}$ & 3.457244 & -2.86346 & -1.47515 \\
\hline $\mathrm{H}$ & -5.52614 & 0.199743 & -0.59345 & $\mathrm{H}$ & 5.151987 & -0.77473 & -1.33477 \\
\hline $\mathrm{H}$ & -4.86782 & 1.411931 & 0.49297 & $\mathrm{H}$ & 4.014255 & 0.060442 & -2.37907 \\
\hline $\mathrm{H}$ & -4.32956 & 1.359449 & -1.18909 & $\mathrm{H}$ & 4.497512 & 0.795611 & -0.84634 \\
\hline $\mathrm{H}$ & 0.283944 & 1.85581 & 2.547337 & $\mathrm{H}$ & -0.17798 & 2.130993 & -2.45872 \\
\hline $\mathrm{H}$ & 1.973435 & 1.45253 & 2.860565 & $\mathrm{H}$ & -1.91636 & 1.926744 & -2.66093 \\
\hline $\mathrm{H}$ & 1.464707 & 3.1424 & 2.716448 & $\mathrm{H}$ & -1.22199 & 3.541469 & -2.44906 \\
\hline $\mathrm{H}$ & 3.471237 & -0.47909 & -1.87712 & $\mathrm{H}$ & -4.19708 & -0.96245 & 1.556015 \\
\hline $\mathrm{H}$ & 4.19376 & -2.03297 & -1.43925 & $\mathrm{H}$ & -4.66275 & -2.2542 & 0.439981 \\
\hline $\mathrm{H}$ & 2.736846 & -1.98486 & -2.44728 & $\mathrm{H}$ & -3.56551 & -2.59924 & 1.789598 \\
\hline 1d_3 & & & & 1d_4 & & & \\
\hline $\mathrm{C}$ & -1.21929 & 2.840076 & -0.35922 & $\mathrm{C}$ & -1.03466 & 2.790526 & -0.80537 \\
\hline $\mathrm{C}$ & -2.27027 & 1.78792 & -0.68886 & $\mathrm{C}$ & -2.21727 & 1.8222 & -0.7816 \\
\hline $\mathrm{C}$ & 0.195887 & 2.335568 & -0.06959 & $\mathrm{C}$ & 0.316639 & 2.167776 & -0.43026 \\
\hline $\mathrm{C}$ & -1.9611 & 0.638649 & -1.30013 & $\mathrm{C}$ & -2.1557 & 0.649268 & -1.42387 \\
\hline $\mathrm{C}$ & -2.86178 & -0.52617 & -1.61974 & $\mathrm{C}$ & -3.15814 & -0.47438 & -1.43492 \\
\hline $\mathrm{C}$ & -2.26635 & -1.88141 & -1.16771 & $\mathrm{C}$ & -2.56138 & -1.82098 & -0.95697 \\
\hline $\mathrm{C}$ & -1.84507 & -1.93435 & 0.293144 & $\mathrm{C}$ & -1.9944 & -1.81579 & 0.455906 \\
\hline $\mathrm{C}$ & -0.41869 & -1.86838 & 0.680968 & $\mathrm{C}$ & -0.53753 & -1.7557 & 0.702786 \\
\hline $\mathrm{C}$ & 0.754918 & -1.74702 & -0.28282 & $\mathrm{C}$ & 0.520912 & -1.66011 & -0.3847 \\
\hline $\mathrm{C}$ & 0.318933 & 1.535593 & 1.21859 & $\mathrm{C}$ & 0.359402 & 1.584149 & 0.969948 \\
\hline $\mathrm{C}$ & 1.692904 & 0.943974 & 1.564031 & $\mathrm{C}$ & 1.628036 & 0.848211 & 1.421436 \\
\hline $\mathrm{C}$ & 2.388595 & 0.102102 & 0.522338 & $\mathrm{C}$ & 2.394588 & -0.00163 & 0.440995 \\
\hline
\end{tabular}




\begin{tabular}{|c|c|c|c|c|c|c|c|}
\hline $\mathrm{C}$ & 2.116498 & -1.39776 & 0.377925 & $\mathrm{C}$ & 1.973519 & -1.43197 & 0.086798 \\
\hline $\mathrm{C}$ & 3.324193 & -1.86736 & -0.47871 & $\mathrm{C}$ & 2.976194 & -1.81337 & -1.0319 \\
\hline $\mathrm{C}$ & 3.822279 & -0.60918 & -1.21668 & $\mathrm{C}$ & 4.166344 & -0.8512 & -0.87825 \\
\hline $\mathrm{C}$ & 3.317064 & 0.525408 & -0.35142 & $\mathrm{C}$ & 3.591847 & 0.304055 & -0.0882 \\
\hline $\mathrm{C}$ & 3.844867 & 1.912223 & -0.5547 & $\mathrm{C}$ & 4.39826 & 1.555904 & 0.076087 \\
\hline $\mathrm{O}$ & -0.60534 & 1.426219 & 1.999329 & $\mathrm{O}$ & -0.54676 & 1.750478 & 1.763185 \\
\hline $\mathrm{C}$ & -3.66612 & 2.191114 & -0.28375 & $\mathrm{C}$ & -3.40504 & 2.311834 & 0.005983 \\
\hline $\mathrm{C}$ & -2.90108 & -1.54408 & 1.305379 & $\mathrm{C}$ & -2.94093 & -1.37242 & 1.551037 \\
\hline $\mathrm{O}$ & -1.12103 & -3.13526 & 0.662485 & $\mathrm{O}$ & -1.24898 & -3.01358 & 0.798123 \\
\hline $\mathrm{H}$ & 2.140362 & -1.88607 & 1.359932 & $\mathrm{H}$ & 2.142524 & -2.06675 & 0.970255 \\
\hline $\mathrm{H}$ & -0.22256 & -1.51668 & 1.693132 & $\mathrm{H}$ & -0.24165 & -1.3752 & 1.679195 \\
\hline $\mathrm{H}$ & -1.56525 & 3.39769 & 0.516299 & $\mathrm{H}$ & -1.24028 & 3.612393 & -0.11526 \\
\hline $\mathrm{H}$ & -1.1729 & 3.571556 & -1.17581 & $\mathrm{H}$ & -0.94161 & 3.243081 & -1.79906 \\
\hline $\mathrm{H}$ & 0.876786 & 3.190775 & 0.031183 & $\mathrm{H}$ & 1.113221 & 2.922376 & -0.48473 \\
\hline $\mathrm{H}$ & 0.613249 & 1.750036 & -0.89278 & $\mathrm{H}$ & 0.62399 & 1.39631 & -1.14061 \\
\hline $\mathrm{H}$ & -0.92353 & 0.481103 & -1.58338 & $\mathrm{H}$ & -1.2524 & 0.438331 & -1.99466 \\
\hline $\mathrm{H}$ & -3.0248 & -0.58996 & -2.70323 & $\mathrm{H}$ & -3.52358 & -0.63282 & -2.45743 \\
\hline $\mathrm{H}$ & -3.84892 & -0.39283 & -1.1734 & $\mathrm{H}$ & -4.03634 & -0.22758 & -0.83564 \\
\hline $\mathrm{H}$ & -1.41097 & -2.13572 & -1.79798 & $\mathrm{H}$ & -1.78579 & -2.14383 & -1.65544 \\
\hline $\mathrm{H}$ & -3.0149 & -2.66571 & -1.33054 & $\mathrm{H}$ & -3.34894 & -2.58309 & -0.99387 \\
\hline $\mathrm{H}$ & 0.542886 & -1.02408 & -1.07564 & $\mathrm{H}$ & 0.259899 & -0.8839 & -1.10863 \\
\hline $\mathrm{H}$ & 0.850232 & -2.71928 & -0.77347 & $\mathrm{H}$ & 0.493167 & -2.60613 & -0.93329 \\
\hline $\mathrm{H}$ & 1.556649 & 0.376352 & 2.48792 & $\mathrm{H}$ & 1.33762 & 0.26096 & 2.297889 \\
\hline $\mathrm{H}$ & 2.322898 & 1.806958 & 1.812497 & $\mathrm{H}$ & 2.279354 & 1.645498 & 1.799512 \\
\hline $\mathrm{H}$ & 4.11362 & -2.22909 & 0.185574 & $\mathrm{H}$ & 3.260973 & -2.86684 & -0.98827 \\
\hline $\mathrm{H}$ & 3.065032 & -2.68616 & -1.15321 & $\mathrm{H}$ & 2.498873 & -1.64646 & -2.00289 \\
\hline $\mathrm{H}$ & 3.408915 & -0.53006 & -2.23137 & $\mathrm{H}$ & 4.586694 & -0.5368 & -1.83953 \\
\hline $\mathrm{H}$ & 4.911676 & -0.5887 & -1.32625 & $\mathrm{H}$ & 4.992948 & -1.30506 & -0.31421 \\
\hline $\mathrm{H}$ & 4.939627 & 1.916825 & -0.51452 & $\mathrm{H}$ & 5.387982 & 1.322873 & 0.485835 \\
\hline $\mathrm{H}$ & 3.56799 & 2.290455 & -1.54611 & $\mathrm{H}$ & 4.569398 & 2.033157 & -0.89565 \\
\hline $\mathrm{H}$ & 3.474469 & 2.623408 & 0.185266 & $\mathrm{H}$ & 3.926925 & 2.291819 & 0.729182 \\
\hline $\mathrm{H}$ & -3.91692 & 3.165011 & -0.7204 & $\mathrm{H}$ & -3.74805 & 3.274748 & -0.39107 \\
\hline $\mathrm{H}$ & -4.43188 & 1.48184 & -0.59576 & $\mathrm{H}$ & -4.25278 & 1.627085 & -0.01044 \\
\hline $\mathrm{H}$ & -3.73025 & 2.313653 & 0.80346 & $\mathrm{H}$ & -3.11261 & 2.485542 & 1.046239 \\
\hline $\mathrm{H}$ & -3.17385 & -0.49181 & 1.207788 & $\mathrm{H}$ & -3.20705 & -0.31916 & 1.444765 \\
\hline $\mathrm{H}$ & -3.80343 & -2.14767 & 1.163254 & $\mathrm{H}$ & -3.86268 & -1.96224 & 1.518205 \\
\hline $\mathrm{H}$ & -2.53177 & -1.71107 & 2.318739 & $\mathrm{H}$ & -2.47803 & -1.51173 & 2.529542 \\
\hline 1d_5 & & & & 1d_6 & & & \\
\hline $\mathrm{C}$ & 0.021646 & 2.51507 & -0.87021 & $\mathrm{C}$ & 0.254694 & 3.184909 & -0.15977 \\
\hline $\mathrm{C}$ & -1.38767 & 1.964123 & -0.94572 & $\mathrm{C}$ & 1.289266 & 2.398291 & 0.626362 \\
\hline $\mathrm{C}$ & 0.577264 & 2.708384 & 0.570588 & $\mathrm{C}$ & -1.1544 & 2.549994 & -0.1839 \\
\hline $\mathrm{C}$ & -1.60672 & 0.816849 & -1.60091 & $\mathrm{C}$ & 2.238789 & 1.683306 & 0.006533 \\
\hline $\mathrm{C}$ & -2.86857 & 0.006026 & -1.71216 & $\mathrm{C}$ & 3.32237 & 0.851542 & 0.656084 \\
\hline $\mathrm{C}$ & -2.67762 & -1.4371 & -1.18222 & $\mathrm{C}$ & 2.926168 & -0.60356 & 1.015006 \\
\hline $\mathrm{C}$ & -2.29078 & -1.53459 & 0.286272 & $\mathrm{C}$ & 2.649554 & -1.53693 & -0.1553 \\
\hline $\mathrm{C}$ & -0.88874 & -1.74598 & 0.707935 & $\mathrm{C}$ & 1.292148 & -1.72973 & -0.70084 \\
\hline $\mathrm{C}$ & 0.303867 & -1.88423 & -0.22949 & $\mathrm{C}$ & 0.084538 & -0.9392 & -0.26047 \\
\hline $\mathrm{C}$ & 0.505736 & 1.477862 & 1.468126 & $\mathrm{C}$ & -1.43637 & 1.666714 & -1.39304 \\
\hline
\end{tabular}




\begin{tabular}{|c|c|c|c|c|c|c|c|}
\hline $\mathrm{C}$ & 1.76665 & 0.637136 & 1.691137 & $\mathrm{C}$ & -2.61736 & 0.696537 & -1.28999 \\
\hline $\mathrm{C}$ & 2.204122 & -0.26732 & 0.556524 & $\mathrm{C}$ & -2.39686 & -0.51004 & -0.40606 \\
\hline $\mathrm{C}$ & 1.703538 & -1.71209 & 0.41894 & $\mathrm{C}$ & -1.28009 & -1.51061 & -0.69566 \\
\hline $\mathrm{C}$ & 2.808969 & -2.34215 & -0.47181 & $\mathrm{C}$ & -1.71048 & -2.75028 & 0.127612 \\
\hline $\mathrm{C}$ & 3.402922 & -1.16624 & -1.26975 & $\mathrm{C}$ & -2.62772 & -2.18953 & 1.23688 \\
\hline $\mathrm{C}$ & 3.128778 & 0.024179 & -0.37462 & $\mathrm{C}$ & -3.11451 & -0.86953 & 0.67023 \\
\hline $\mathrm{C}$ & 3.867243 & 1.308932 & -0.59182 & $\mathrm{C}$ & -4.25131 & -0.1508 & 1.32909 \\
\hline $\mathrm{O}$ & -0.52514 & 1.201971 & 2.052319 & $\mathrm{O}$ & -0.79836 & 1.760385 & -2.4238 \\
\hline $\mathrm{C}$ & -2.45202 & 2.778248 & -0.25319 & $\mathrm{C}$ & 1.155461 & 2.49396 & 2.126766 \\
\hline $\mathrm{C}$ & -3.26925 & -0.92045 & 1.26554 & $\mathrm{C}$ & 3.837374 & -1.8553 & -1.03859 \\
\hline $\mathrm{O}$ & -1.83152 & -2.84838 & 0.696311 & $\mathrm{O}$ & 1.859903 & -2.70972 & 0.197548 \\
\hline $\mathrm{H}$ & 1.680269 & -2.20027 & 1.400898 & $\mathrm{H}$ & -1.23023 & -1.74165 & -1.76727 \\
\hline $\mathrm{H}$ & -0.65124 & -1.40724 & 1.71398 & $\mathrm{H}$ & 1.24111 & -2.08191 & -1.73254 \\
\hline $\mathrm{H}$ & 0.061309 & 3.502988 & -1.3458 & $\mathrm{H}$ & 0.583699 & 3.310499 & -1.19319 \\
\hline $\mathrm{H}$ & 0.697377 & 1.864695 & -1.43006 & $\mathrm{H}$ & 0.173896 & 4.185996 & 0.277273 \\
\hline $\mathrm{H}$ & 0.00072 & 3.487187 & 1.076297 & $\mathrm{H}$ & -1.9198 & 3.336657 & -0.23301 \\
\hline $\mathrm{H}$ & 1.607802 & 3.059337 & 0.491214 & $\mathrm{H}$ & -1.37696 & 1.991725 & 0.729278 \\
\hline $\mathrm{H}$ & -0.74769 & 0.36215 & -2.09098 & $\mathrm{H}$ & 2.231757 & 1.682799 & -1.0818 \\
\hline $\mathrm{H}$ & -3.16655 & -0.07342 & -2.76507 & $\mathrm{H}$ & 3.657396 & 1.328318 & 1.581476 \\
\hline $\mathrm{H}$ & -3.70091 & 0.485318 & -1.19338 & $\mathrm{H}$ & 4.19659 & 0.833682 & -0.00202 \\
\hline $\mathrm{H}$ & -1.92654 & -1.95062 & -1.78749 & $\mathrm{H}$ & 2.05636 & -0.58951 & 1.673333 \\
\hline $\mathrm{H}$ & -3.61622 & -1.98743 & -1.31788 & $\mathrm{H}$ & 3.746697 & -1.04672 & 1.592809 \\
\hline $\mathrm{H}$ & 0.217581 & -1.20036 & -1.07603 & $\mathrm{H}$ & 0.210039 & 0.057238 & -0.68453 \\
\hline $\mathrm{H}$ & 0.248823 & -2.89536 & -0.64264 & $\mathrm{H}$ & 0.092333 & -0.81742 & 0.825129 \\
\hline $\mathrm{H}$ & 1.57684 & 0.033139 & 2.581975 & $\mathrm{H}$ & -2.82775 & 0.366903 & -2.31267 \\
\hline $\mathrm{H}$ & 2.578325 & 1.332902 & 1.926743 & $\mathrm{H}$ & -3.48302 & 1.263331 & -0.93427 \\
\hline $\mathrm{H}$ & 3.587129 & -2.76552 & 0.169555 & $\mathrm{H}$ & -2.28411 & -3.43035 & -0.50798 \\
\hline $\mathrm{H}$ & 2.430356 & -3.14787 & -1.10451 & $\mathrm{H}$ & -0.85545 & -3.30531 & 0.518815 \\
\hline $\mathrm{H}$ & 2.917558 & -1.03826 & -2.24688 & $\mathrm{H}$ & -2.08884 & -2.02393 & 2.179635 \\
\hline $\mathrm{H}$ & 4.472234 & -1.28916 & -1.47053 & $\mathrm{H}$ & -3.45764 & -2.86343 & 1.474663 \\
\hline $\mathrm{H}$ & 4.945281 & 1.125246 & -0.65542 & $\mathrm{H}$ & -5.15274 & -0.77427 & 1.332409 \\
\hline $\mathrm{H}$ & 3.573861 & 1.772049 & -1.5415 & $\mathrm{H}$ & -4.0156 & 0.058941 & 2.37892 \\
\hline $\mathrm{H}$ & 3.693431 & 2.039926 & 0.199421 & $\mathrm{H}$ & -4.49684 & 0.796291 & 0.846595 \\
\hline $\mathrm{H}$ & -2.38999 & 3.826571 & -0.56729 & $\mathrm{H}$ & 0.176622 & 2.130102 & 2.458362 \\
\hline $\mathrm{H}$ & -3.46008 & 2.425707 & -0.47079 & $\mathrm{H}$ & 1.914675 & 1.923291 & 2.66093 \\
\hline $\mathrm{H}$ & -2.31027 & 2.7588 & 0.831082 & $\mathrm{H}$ & 1.222562 & 3.539167 & 2.450456 \\
\hline $\mathrm{H}$ & -3.34975 & 0.159401 & 1.129288 & $\mathrm{H}$ & 4.196424 & -0.96203 & -1.5568 \\
\hline $\mathrm{H}$ & -4.26365 & -1.35779 & 1.126724 & $\mathrm{H}$ & 4.663215 & -2.25303 & -0.44036 \\
\hline 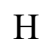 & -2.949 & -1.11085 & 2.290997 & $\mathrm{H}$ & 3.565631 & -2.59926 & -1.7894 \\
\hline
\end{tabular}

Table S6. SCF energies (Hartree) computed at the PCM/ mPW1PW91/6-31G* level of theory using coordinate files incorporated in Table S5.

1a_1_DP4+ 1a_2_DP4+ 1a_3_DP4+ 1a_4_DP4+ 1a_5_DP4+ 1a_6_DP4+ 1a_7_DP4+
SCF Energy (PCM/mPW1PW91/6-31G*) =-891.08846931

SCF Energy (PCM/mPW1PW91/6-31G*) $=-891.08953519$

SCF Energy (PCM/mPW1PW91/6-31G*) $=-891.08735574$

SCF Energy (PCM/mPW1PW91/6-31G*) =-891.09005001

SCF Energy (PCM/mPW1PW91/6-31G*) =-891.08959197

SCF Energy $\left(\mathrm{PCM} / \mathrm{mPW} 1 \mathrm{PW} 91 / 6-31 \mathrm{G}^{*}\right)=-891.08848152$

SCF Energy $\left(\mathrm{PCM} / \mathrm{mPW} 1 \mathrm{PW} 91 / 6-31 \mathrm{G}^{*}\right)=-891.08976960$ 
1a_8_DP4+

1a_9_DP4+

1a_10_DP4+

1a_11_DP4+

1a_12_DP4+

1a_13_DP4+

1a_14_DP4+

1a_15_DP4+

1a_16_DP4+

1b_1_DP4+

1b_2_DP4+

1b_3_DP4+

1b_4_DP4+

1b_5_DP4+

1b_6_DP4+

1b_7_DP4+

1b_8_DP4+

1c_1_DP4+

1c_2_DP4+

1c_3_DP4+

1c_4_DP4+

1c_5_DP4+

1c_6_DP4+

1c_7_DP4+

1c_8_DP4+

1c 9 DP4+

1c_10_DP4+

1d_1_DP4+

1d_2_DP4+

1d_3_DP4+

1d_4_DP4+

1d_5_DP4+

1d_6_DP4+
SCF Energy (PCM/mPW1PW91/6-31G*) =-891.08640584

SCF Energy (PCM/mPW1PW91/6-31G*) $=-891.08530990$

SCF Energy $\left(\mathrm{PCM} / \mathrm{mPW} 1 \mathrm{PW} 91 / 6-31 \mathrm{G}^{*}\right)=-891.08899357$

SCF Energy $\left(\mathrm{PCM} / \mathrm{mPW} 1 \mathrm{PW} 91 / 6-31 \mathrm{G}^{*}\right)=-891.08673750$

SCF Energy (PCM/mPW1PW91/6-31G*) $=-891.08857022$

SCF Energy $\left(\mathrm{PCM} / \mathrm{mPW} 1 \mathrm{PW} 91 / 6-31 \mathrm{G}^{*}\right)=-891.08547229$

SCF Energy (PCM/mPW1PW91/6-31G*) $=-891.09080101$

SCF Energy (PCM/mPW1PW91/6-31G*) $=-891.08614952$

SCF Energy (PCM/mPW1PW91/6-31G*) $=-891.08614895$

SCF Energy (PCM/mPW1PW91/6-31G*) $=-891.08518597$

SCF Energy (PCM/mPW1PW91/6-31G*) $=-891.08375051$

SCF Energy $\left(\mathrm{PCM} / \mathrm{mPW} 1 \mathrm{PW} 91 / 6-31 \mathrm{G}^{*}\right)=-891.08375051$

SCF Energy (PCM/mPW1PW91/6-31G*) $=-891.08946754$

SCF Energy (PCM/mPW1PW91/6-31G*) $=-891.09084810$

SCF Energy (PCM/mPW1PW91/6-31G*) $=-891.09084881$

SCF Energy (PCM/mPW1PW91/6-31G*) $=-891.08381851$

SCF Energy (PCM/mPW1PW91/6-31G*) $=-891.08890652$

SCF Energy (PCM/mPW1PW91/6-31G*) $=-891.09175513$

SCF Energy (PCM/mPW1PW91/6-31G*) $=-891.09194945$

SCF Energy $\left(\mathrm{PCM} / \mathrm{mPW} 1 \mathrm{PW} 91 / 6-31 \mathrm{G}^{*}\right)=-891.09102940$

SCF Energy (PCM/mPW1PW91/6-31G*) $=-891.09194865$

SCF Energy (PCM/mPW1PW91/6-31G*) $=-891.09175522$

SCF Energy (PCM/mPW1PW91/6-31G*) =-891.09232659

SCF Energy (PCM/mPW1PW91/6-31G*) $=-891.08964073$

SCF Energy (PCM/mPW1PW91/6-31G*) $=-891.09099453$

SCF Energy (PCM/mPW1PW91/6-31G*) $=-891.09261536$

SCF Energy (PCM/mPW1PW91/6-31G*) $=-891.08898723$

SCF Energy $\left(\mathrm{PCM} / \mathrm{mPW} 1 \mathrm{PW} 91 / 6-31 \mathrm{G}^{*}\right)=-891.08741492$

SCF Energy (PCM/mPW1PW91/6-31G*) $=-891.08902751$

SCF Energy (PCM/mPW1PW91/6-31G*) $=-891.08498457$

SCF Energy (PCM/mPW1PW91/6-31G*) $=-891.08583342$

SCF Energy (PCM/mPW1PW91/6-31G*) $=-891.08226843$

SCF Energy (PCM/mPW1PW91/6-31G*) =-891.08902855

Table S7. Imaginary frequencies and absolute energy values of all conformers of isomers $\mathbf{1 a}-\mathbf{1 d}$ used after optimization at the B3LYP/6-311G(d,p) level of theory as required for DP4+.

1a_1_DP4+ Imaginary Freq $=0$

Zero-point correction=

Thermal correction to Energy=

Thermal correction to Enthalpy=

Thermal correction to Gibbs Free Energy=

Sum of electronic and zero-point Energies=

Sum of electronic and thermal Energies $=$

Sum of electronic and thermal Enthalpies=

Sum of electronic and thermal Free Energies=

1a_2_DP4+ Imaginary Freq $=0$

Zero-point correction=

Thermal correction to Energy=

0.430255 (Hartree/Particle)
0.452056
0.453000
0.380141
-891.086234
-891.064433
-891.063489
-891.136348

0.430311 (Hartree/Particle)

0.452155 
Thermal correction to Enthalpy=

Thermal correction to Gibbs Free Energy=

Sum of electronic and zero-point Energies=

Sum of electronic and thermal Energies=

Sum of electronic and thermal Enthalpies=

Sum of electronic and thermal Free Energies=

1a_3_DP4+ Imaginary Freq $=0$

Zero-point correction=

Thermal correction to Energy=

Thermal correction to Enthalpy=

Thermal correction to Gibbs Free Energy=

Sum of electronic and zero-point Energies=

Sum of electronic and thermal Energies=

Sum of electronic and thermal Enthalpies=

Sum of electronic and thermal Free Energies=

1a_4_DP4+ Imaginary Freq $=0$

Zero-point correction=

Thermal correction to Energy=

Thermal correction to Enthalpy=

Thermal correction to Gibbs Free Energy=

Sum of electronic and zero-point Energies=

Sum of electronic and thermal Energies=

Sum of electronic and thermal Enthalpies=

Sum of electronic and thermal Free Energies=

1a_5_DP4+ Imaginary Freq $=0$

Zero-point correction=

Thermal correction to Energy=

Thermal correction to Enthalpy=

Thermal correction to Gibbs Free Energy=

Sum of electronic and zero-point Energies=

Sum of electronic and thermal Energies=

Sum of electronic and thermal Enthalpies=

Sum of electronic and thermal Free Energies=

1a_6_DP4+ Imaginary Freq $=0$

Zero-point correction=

Thermal correction to Energy=

Thermal correction to Enthalpy=

Thermal correction to Gibbs Free Energy=

Sum of electronic and zero-point Energies=

Sum of electronic and thermal Energies $=$

Sum of electronic and thermal Enthalpies=

Sum of electronic and thermal Free Energies=

1a_7_DP4+ Imaginary Freq $=0$

Zero-point correction=

Thermal correction to Energy=

Thermal correction to Enthalpy=

Thermal correction to Gibbs Free Energy=

Sum of electronic and zero-point Energies=

Sum of electronic and thermal Energies=
0.453099

0.380105

$-891.088348$

$-891.066504$

$-891.065560$

$-891.138554$

0.429784 (Hartree/Particle)

0.451800

0.452744

0.378702

$-891.087479$

$-891.065463$

$-891.064518$

$-891.138561$

0.430415 (Hartree/Particle)

0.452217

0.453162

0.379985

$-891.088245$

$-891.066443$

$-891.065498$

$-891.138675$

0.430520 (Hartree/Particle)

0.452222

0.453166

0.380538

$-891.088067$

$-891.066366$

$-891.065421$

$-891.138049$

0.430232 (Hartree/Particle)

0.452148

0.453092

0.378869

$-891.087247$

$-891.065331$

$-891.064387$

$-891.138610$

0.430193 (Hartree/Particle)

0.451943

0.452887

0.380168

$-891.089064$

$-891.067315$ 
Sum of electronic and thermal Enthalpies=

Sum of electronic and thermal Free Energies=

1a_8_DP4+ Imaginary Freq $=0$

Zero-point correction=

Thermal correction to Energy=

Thermal correction to Enthalpy=

Thermal correction to Gibbs Free Energy=

Sum of electronic and zero-point Energies=

Sum of electronic and thermal Energies=

Sum of electronic and thermal Enthalpies=

Sum of electronic and thermal Free Energies=

1a_9_DP4+ Imaginary Freq $=0$

Zero-point correction $=$

Thermal correction to Energy=

Thermal correction to Enthalpy=

Thermal correction to Gibbs Free Energy=

Sum of electronic and zero-point Energies=

Sum of electronic and thermal Energies=

Sum of electronic and thermal Enthalpies=

Sum of electronic and thermal Free Energies=

1a_10_DP4 + Imaginary Freq $=0$

Zero-point correction $=$

Thermal correction to Energy=

Thermal correction to Enthalpy=

Thermal correction to Gibbs Free Energy=

Sum of electronic and zero-point Energies=

Sum of electronic and thermal Energies=

Sum of electronic and thermal Enthalpies=

Sum of electronic and thermal Free Energies=

1a_11_DP4+ Imaginary Freq $=0$

Zero-point correction $=$

Thermal correction to Energy=

Thermal correction to Enthalpy=

Thermal correction to Gibbs Free Energy=

Sum of electronic and zero-point Energies=

Sum of electronic and thermal Energies=

Sum of electronic and thermal Enthalpies=

Sum of electronic and thermal Free Energies=

1a_12_DP4+ Imaginary Freq $=0$

Zero-point correction=

Thermal correction to Energy=

Thermal correction to Enthalpy=

Thermal correction to Gibbs Free Energy=

Sum of electronic and zero-point Energies=

Sum of electronic and thermal Energies=

Sum of electronic and thermal Enthalpies=

Sum of electronic and thermal Free Energies=

1a_13_DP4+ Imaginary Freq $=0$

Zero-point correction $=$
$-891.066370$

$-891.139089$

0.430626 (Hartree/Particle)

0.452413

0.453357

0.380453

$-891.083596$

$-891.061809$

$-891.060865$

$-891.133769$

0.430825 (Hartree/Particle)

0.452674

0.453618

0.380222

$-891.082727$

$-891.060877$

$-891.059933$

$-891.133329$

0.429842 (Hartree/Particle)

0.451769

0.452713

0.378765

$-891.088382$

$-891.066455$

$-891.065510$

$-891.139459$

0.430146 (Hartree/Particle)

0.452065

0.453009

0.379310

$-891.086144$

$-891.064225$

$-891.063281$

$-891.136980$

0.430084 (Hartree/Particle)

0.451918

0.452862

0.379246

$-891.087612$

$-891.065778$

$-891.064834$

$-891.138451$

0.431029 (Hartree/Particle) 
Thermal correction to Energy=

Thermal correction to Enthalpy=

Thermal correction to Gibbs Free Energy=

Sum of electronic and zero-point Energies=

Sum of electronic and thermal Energies=

Sum of electronic and thermal Enthalpies=

Sum of electronic and thermal Free Energies=

1a_14_DP4+ Imaginary Freq $=0$

Zero-point correction=

Thermal correction to Energy=

Thermal correction to Enthalpy=

Thermal correction to Gibbs Free Energy=

Sum of electronic and zero-point Energies=

Sum of electronic and thermal Energies=

Sum of electronic and thermal Enthalpies=

Sum of electronic and thermal Free Energies=

1a_15_DP4+ Imaginary Freq $=0$

Zero-point correction=

Thermal correction to Energy=

Thermal correction to Enthalpy=

Thermal correction to Gibbs Free Energy=

Sum of electronic and zero-point Energies=

Sum of electronic and thermal Energies=

Sum of electronic and thermal Enthalpies=

Sum of electronic and thermal Free Energies=

1a_16_DP4+ Imaginary Freq $=0$

Zero-point correction=

Thermal correction to Energy=

Thermal correction to Enthalpy=

Thermal correction to Gibbs Free Energy=

Sum of electronic and zero-point Energies=

Sum of electronic and thermal Energies=

Sum of electronic and thermal Enthalpies=

Sum of electronic and thermal Free Energies=

1b_1_DP4+ Imaginary Freq $=0$

Zero-point correction=

Thermal correction to Energy=

Thermal correction to Enthalpy=

Thermal correction to Gibbs Free Energy=

Sum of electronic and zero-point Energies=

Sum of electronic and thermal Energies=

Sum of electronic and thermal Enthalpies=

Sum of electronic and thermal Free Energies=

1b_2_DP4+ Imaginary Freq $=0$

Zero-point correction=

Thermal correction to Energy=

Thermal correction to Enthalpy=

Thermal correction to Gibbs Free Energy=

Sum of electronic and zero-point Energies=
0.452470

0.453414

0.382199

$-891.082369$

$-891.060928$

$-891.059984$

$-891.131199$

0.430256 (Hartree/Particle)

0.452070

0.453014

0.380064

$-891.089595$

$-891.067781$

$-891.066837$

$-891.139786$

0.430201 (Hartree/Particle)

0.451993

0.452938

0.380044

$-891.083952$

$-891.062160$

$-891.061215$

$-891.134109$

0.430201 (Hartree/Particle)

0.451993

0.452937

0.380045

$-891.083952$

$-891.062160$

$-891.061216$

$-891.134108$

0.431052 (Hartree/Particle)

0.452579

0.453523

0.382048

$-891.081710$

$-891.060183$

$-891.059239$

$-891.130714$

0.430935 (Hartree/Particle)

0.452557

0.453502

0.381537

$-891.080629$ 
Sum of electronic and thermal Energies=

Sum of electronic and thermal Enthalpies=

Sum of electronic and thermal Free Energies=

1b_3_DP4+ Imaginary Freq $=0$

Zero-point correction=

Thermal correction to Energy=

Thermal correction to Enthalpy=

Thermal correction to Gibbs Free Energy=

Sum of electronic and zero-point Energies=

Sum of electronic and thermal Energies=

Sum of electronic and thermal Enthalpies=

Sum of electronic and thermal Free Energies=

1b_4_DP4+ Imaginary Freq $=0$

Zero-point correction $=$

Thermal correction to Energy=

Thermal correction to Enthalpy=

Thermal correction to Gibbs Free Energy=

Sum of electronic and zero-point Energies=

Sum of electronic and thermal Energies=

Sum of electronic and thermal Enthalpies=

Sum of electronic and thermal Free Energies=

1b_5_DP4+ Imaginary Freq $=0$

Zero-point correction $=$

Thermal correction to Energy=

Thermal correction to Enthalpy=

Thermal correction to Gibbs Free Energy=

Sum of electronic and zero-point Energies=

Sum of electronic and thermal Energies=

Sum of electronic and thermal Enthalpies=

Sum of electronic and thermal Free Energies=

1b_6_DP4+ Imaginary Freq $=0$

Zero-point correction=

Thermal correction to Energy=

Thermal correction to Enthalpy=

Thermal correction to Gibbs Free Energy=

Sum of electronic and zero-point Energies=

Sum of electronic and thermal Energies=

Sum of electronic and thermal Enthalpies=

Sum of electronic and thermal Free Energies=

1b_7_DP4+ Imaginary Freq $=0$

Zero-point correction=

Thermal correction to Energy=

Thermal correction to Enthalpy=

Thermal correction to Gibbs Free Energy=

Sum of electronic and zero-point Energies=

Sum of electronic and thermal Energies=

Sum of electronic and thermal Enthalpies=

Sum of electronic and thermal Free Energies=

1b_8_DP4+ Imaginary Freq $=0$
$-891.059007$

$-891.058063$

$-891.130027$

0.430305 (Hartree/Particle)

0.452128

0.453072

0.379751

$-891.088068$

$-891.066245$

$-891.065300$

$-891.138622$

0.430453 (Hartree/Particle)

0.452148

0.453092

0.380719

$-891.087975$

$-891.066280$

$-891.065336$

$-891.137709$

0.430720 (Hartree/Particle)

0.452325

0.453269

0.381281

$-891.088809$

$-891.067204$

$-891.066259$

$-891.138247$

0.430722 (Hartree/Particle)

0.452326

0.453270

0.381288

$-891.088807$

$-891.067202$

$-891.066258$

$-891.138241$

0.431138 (Hartree/Particle)

0.452814

0.453758

0.381329

$-891.080320$

$-891.058645$

$-891.057700$

$-891.130130$ 
Zero-point correction=

Thermal correction to Energy=

Thermal correction to Enthalpy=

Thermal correction to Gibbs Free Energy=

Sum of electronic and zero-point Energies=

Sum of electronic and thermal Energies=

Sum of electronic and thermal Enthalpies=

Sum of electronic and thermal Free Energies=

1c_1_DP4+ Imaginary Freq $=0$

Zero-point correction $=$

Thermal correction to Energy=

Thermal correction to Enthalpy=

Thermal correction to Gibbs Free Energy=

Sum of electronic and zero-point Energies=

Sum of electronic and thermal Energies=

Sum of electronic and thermal Enthalpies=

Sum of electronic and thermal Free Energies=

1c_2_DP4+ Imaginary Freq $=0$

Zero-point correction $=$

Thermal correction to Energy=

Thermal correction to Enthalpy=

Thermal correction to Gibbs Free Energy=

Sum of electronic and zero-point Energies=

Sum of electronic and thermal Energies=

Sum of electronic and thermal Enthalpies=

Sum of electronic and thermal Free Energies=

1c_3_DP4+ Imaginary Freq $=0$

Zero-point correction=

Thermal correction to Energy=

Thermal correction to Enthalpy=

Thermal correction to Gibbs Free Energy=

Sum of electronic and zero-point Energies=

Sum of electronic and thermal Energies=

Sum of electronic and thermal Enthalpies=

Sum of electronic and thermal Free Energies=

1c_4_DP4+ Imaginary Freq $=0$

Zero-point correction $=$

Thermal correction to Energy=

Thermal correction to Enthalpy=

Thermal correction to Gibbs Free Energy=

Sum of electronic and zero-point Energies=

Sum of electronic and thermal Energies=

Sum of electronic and thermal Enthalpies=

Sum of electronic and thermal Free Energies=

1c_5_DP4+ Imaginary Freq $=0$

Zero-point correction=

Thermal correction to Energy=

Thermal correction to Enthalpy=

Thermal correction to Gibbs Free Energy=
0.430524 (Hartree/Particle)

0.452327

0.453271

0.379947

$-891.086656$

$-891.064852$

$-891.063908$

$-891.137232$

0.430761 (Hartree/Particle)

0.452404

0.453348

0.380930

$-891.088940$

$-891.067297$

$-891.066353$

$-891.138771$

0.430786 (Hartree/Particle)

0.452488

0.453432

0.380982

$-891.089339$

$-891.067638$

$-891.066693$

$-891.139144$

0.430462 (Hartree/Particle)

0.452165

0.453109

0.380441

$-891.088514$

$-891.066811$

$-891.065866$

$-891.138535$

0.430785 (Hartree/Particle)

0.452487

0.453431

0.380980

$-891.089341$

$-891.067639$

$-891.066694$

$-891.139146$

0.430767 (Hartree/Particle)

0.452406

0.453350

0.380956 
Sum of electronic and zero-point Energies=

Sum of electronic and thermal Energies=

Sum of electronic and thermal Enthalpies=

Sum of electronic and thermal Free Energies=

1c_6_DP4+ Imaginary Freq $=0$

Zero-point correction=

Thermal correction to Energy=

Thermal correction to Enthalpy=

Thermal correction to Gibbs Free Energy=

Sum of electronic and zero-point Energies=

Sum of electronic and thermal Energies=

Sum of electronic and thermal Enthalpies=

Sum of electronic and thermal Free Energies=

1c_7_DP4+ Imaginary Freq $=0$

Zero-point correction $=$

Thermal correction to Energy=

Thermal correction to Enthalpy=

Thermal correction to Gibbs Free Energy=

Sum of electronic and zero-point Energies=

Sum of electronic and thermal Energies=

Sum of electronic and thermal Enthalpies=

Sum of electronic and thermal Free Energies=

1c_8_DP4+ Imaginary Freq $=0$

Zero-point correction=

Thermal correction to Energy=

Thermal correction to Enthalpy=

Thermal correction to Gibbs Free Energy=

Sum of electronic and zero-point Energies=

Sum of electronic and thermal Energies=

Sum of electronic and thermal Enthalpies=

Sum of electronic and thermal Free Energies=

1c_9_DP4+ Imaginary Freq $=0$

Zero-point correction=

Thermal correction to Energy=

Thermal correction to Enthalpy=

Thermal correction to Gibbs Free Energy=

Sum of electronic and zero-point Energies=

Sum of electronic and thermal Energies=

Sum of electronic and thermal Enthalpies=

Sum of electronic and thermal Free Energies=

1c_10_DP4+ Imaginary Freq $=0$

Zero-point correction $=$

Thermal correction to Energy=

Thermal correction to Enthalpy=

Thermal correction to Gibbs Free Energy=

Sum of electronic and zero-point Energies=

Sum of electronic and thermal Energies=

Sum of electronic and thermal Enthalpies=

Sum of electronic and thermal Free Energies=
$-891.088934$

$-891.067295$

$-891.066351$

$-891.138745$

0.430410 (Hartree/Particle)

0.452180

0.453124

0.380397

$-891.090641$

$-891.068871$

$-891.067927$

$-891.140654$

0.430454 (Hartree/Particle)

0.452103

0.453047

0.380644

$-891.087330$

$-891.065681$

$-891.064737$

$-891.137140$

0.430194 (Hartree/Particle)

0.452135

0.453079

0.378962

$-891.088724$

$-891.066783$

$-891.065838$

$-891.139956$

0.430305 (Hartree/Particle)

0.452066

0.453010

0.380378

$-891.091280$

$-891.069519$

$-891.068575$

$-891.141207$

0.430461 (Hartree/Particle)

0.452234

0.453178

0.380172

$-891.086453$

$-891.064680$

$-891.063736$

$-891.136742$ 
1d_1_DP4+

1d_2_DP4+

1d_3_DP4+

1d_4_DP4+

1d_5_DP4+

1d_6_DP4+
Imaginary Freq $=0$

Zero-point correction=

Thermal correction to Energy=

Thermal correction to Enthalpy=

Thermal correction to Gibbs Free Energy=

Sum of electronic and zero-point Energies=

Sum of electronic and thermal Energies=

Sum of electronic and thermal Enthalpies=

Sum of electronic and thermal Free Energies=

Imaginary Freq $=0$

Zero-point correction=

Thermal correction to Energy=

Thermal correction to Enthalpy=

Thermal correction to Gibbs Free Energy=

Sum of electronic and zero-point Energies=

Sum of electronic and thermal Energies=

Sum of electronic and thermal Enthalpies=

Sum of electronic and thermal Free Energies=

Imaginary Freq $=0$

Zero-point correction=

Thermal correction to Energy=

Thermal correction to Enthalpy=

Thermal correction to Gibbs Free Energy=

Sum of electronic and zero-point Energies=

Sum of electronic and thermal Energies=

Sum of electronic and thermal Enthalpies=

Sum of electronic and thermal Free Energies=

Imaginary Freq $=0$

Zero-point correction=

Thermal correction to Energy=

Thermal correction to Enthalpy=

Thermal correction to Gibbs Free Energy=

Sum of electronic and zero-point Energies=

Sum of electronic and thermal Energies=

Sum of electronic and thermal Enthalpies=

Sum of electronic and thermal Free Energies=

Imaginary Freq $=0$

Zero-point correction=

Thermal correction to Energy=

Thermal correction to Enthalpy=

Thermal correction to Gibbs Free Energy=

Sum of electronic and zero-point Energies=

Sum of electronic and thermal Energies=

Sum of electronic and thermal Enthalpies=

Sum of electronic and thermal Free Energies=

Imaginary Freq $=0$

Zero-point correction=

Thermal correction to Energy=

Thermal correction to Enthalpy=
0.430529 (Hartree/Particle)

0.452168

0.453112

0.381280

$-891.084410$

$-891.062771$

$-891.061826$

$-891.133659$

0.430376 (Hartree/Particle)

0.452230

0.453174

0.379510

$-891.086886$

$-891.065032$

$-891.064088$

$-891.137752$

0.430364 (Hartree/Particle)

0.452036

0.452980

0.380764

$-891.082856$

$-891.061183$

$-891.060239$

$-891.132456$

0.430287 (Hartree/Particle)

0.451903

0.452847

0.381375

$-891.083328$

$-891.061712$

$-891.060767$

$-891.132240$

0.430973 (Hartree/Particle)

0.452626

0.453570

0.381300

$-891.079411$

$-891.057758$

$-891.056814$

$-891.129084$

0.430376 (Hartree/Particle)

0.452230

0.453175 
Thermal correction to Gibbs Free Energy=

Sum of electronic and zero-point Energies=

Sum of electronic and thermal Energies=

Sum of electronic and thermal Enthalpies=

Sum of electronic and thermal Free Energies=
0.379509

$-891.086886$

$-891.065032$

$-891.064087$

$-891.137753$

Table S8. Experiment and calculated ${ }^{13} \mathrm{C}$ NMR chemical shifts of stereoisomers 1a-1d.

\begin{tabular}{|c|c|c|c|c|c|}
\hline \multirow[b]{2}{*}{ No. } & \multirow[b]{2}{*}{$\delta_{\text {exp. }}$} & \multicolumn{4}{|c|}{$\delta_{\text {calc. }}$} \\
\hline & & $1 a$ & $1 b$ & $1 \mathrm{c}$ & $1 d$ \\
\hline 1 & 131.5 & 133.0 & 132.0 & 130.1 & 133.8 \\
\hline 2 & 42.4 & 43.9 & 43.9 & 44.8 & 45.2 \\
\hline 3 & 208.7 & 208.1 & 208.3 & 208.1 & 207.7 \\
\hline 4 & 40.4 & 38.8 & 38.7 & 41.0 & 37.3 \\
\hline 5 & 33.7 & 32.7 & 33.2 & 30.7 & 31.1 \\
\hline 6 & 134.6 & 133.4 & 134.5 & 135.1 & 134.8 \\
\hline 7 & 125.3 & 125.1 & 125.3 & 125.5 & 123.9 \\
\hline 8 & 23.4 & 24.9 & 25.0 & 26.0 & 24.8 \\
\hline 9 & 38.2 & 39.1 & 32.1 & 40.0 & 33.2 \\
\hline 10 & 60.3 & 59.1 & 59.8 & 58.3 & 59.0 \\
\hline 11 & 63.6 & 62.5 & 61.6 & 59.9 & 63.2 \\
\hline 12 & 35.5 & 35.8 & 36.6 & 31.9 & 36.1 \\
\hline 13 & 46.8 & 48.5 & 47.1 & 49.9 & 49.1 \\
\hline 14 & 32.2 & 33.5 & 30.4 & 29.5 & 31.6 \\
\hline 15 & 137.3 & 138.2 & 138.7 & 139.7 & 138.3 \\
\hline 16 & 14.5 & 14.6 & 15.3 & 15.8 & 15.1 \\
\hline 17 & 36.6 & 36.3 & 37.2 & 37.8 & 36.4 \\
\hline 18 & 16.6 & 15.7 & 16.4 & 18.1 & 16.6 \\
\hline 19 & 17.1 & 15.6 & 22.6 & 16.7 & 21.7 \\
\hline
\end{tabular}

Table S9. Mean absolute error (MAE) values, maximum deviation (MD) values and correlation coefficient $\left(R^{2}\right)$ analysis of four stereoisomers $\mathbf{1 a}-\mathbf{1 d}$.

\begin{tabular}{llll}
\hline Stereoisomers & MAE $(\mathrm{ppm})$ & MD $(\mathrm{ppm})$ & $R^{2}$ \\
\hline $\mathbf{1 a}$ & 1.0 & 1.6 & 0.9996 \\
$\mathbf{1 b}$ & 1.4 & 6.1 & 0.9984 \\
\hline
\end{tabular}




\begin{tabular}{llll}
\hline $\mathbf{1 c}$ & 1.8 & 3.5 & 0.9984 \\
$\mathbf{1 d}$ & 1.7 & 5.0 & 0.9983 \\
\hline
\end{tabular}

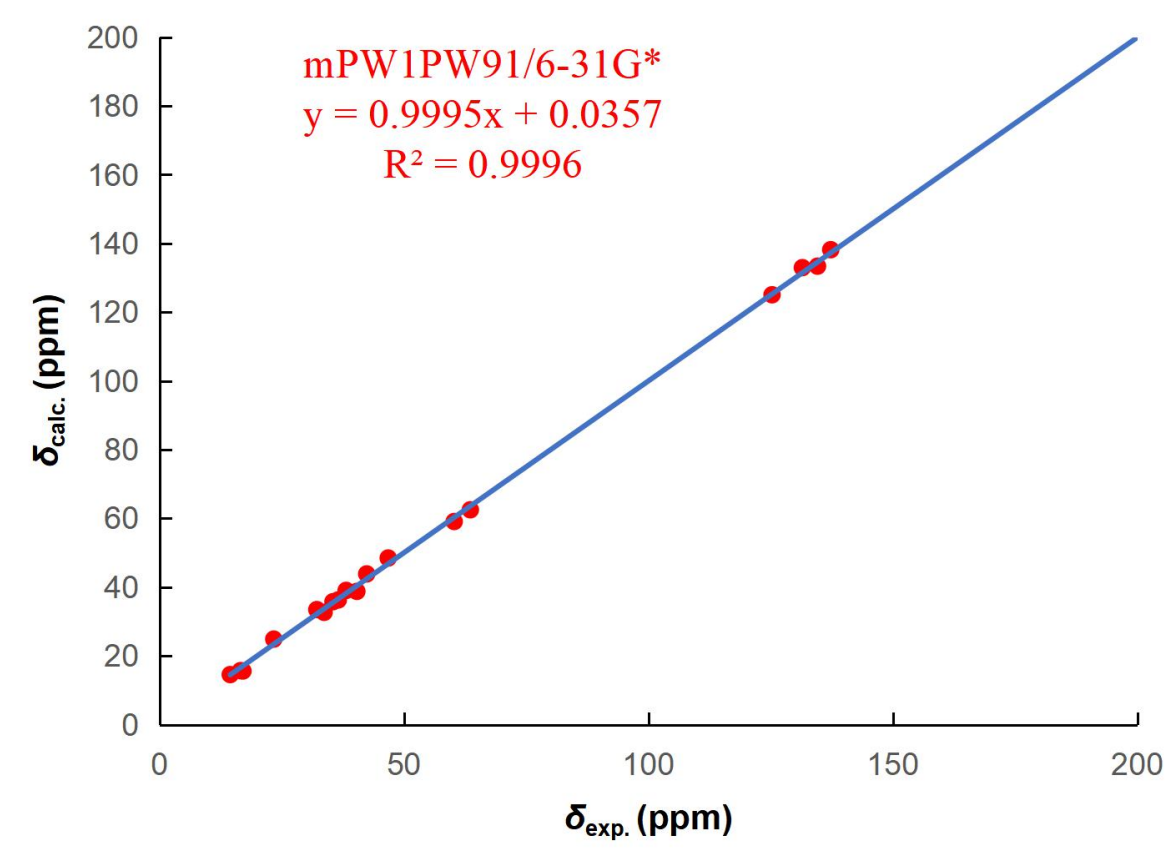

Figure S41. Regression analysis of experimental versus calculated ${ }^{13} \mathrm{C}$ NMR chemical shifts at mPW1PW91/6-31G* level using GIAO method of 1a; linear fitting is shown as a line (blue).

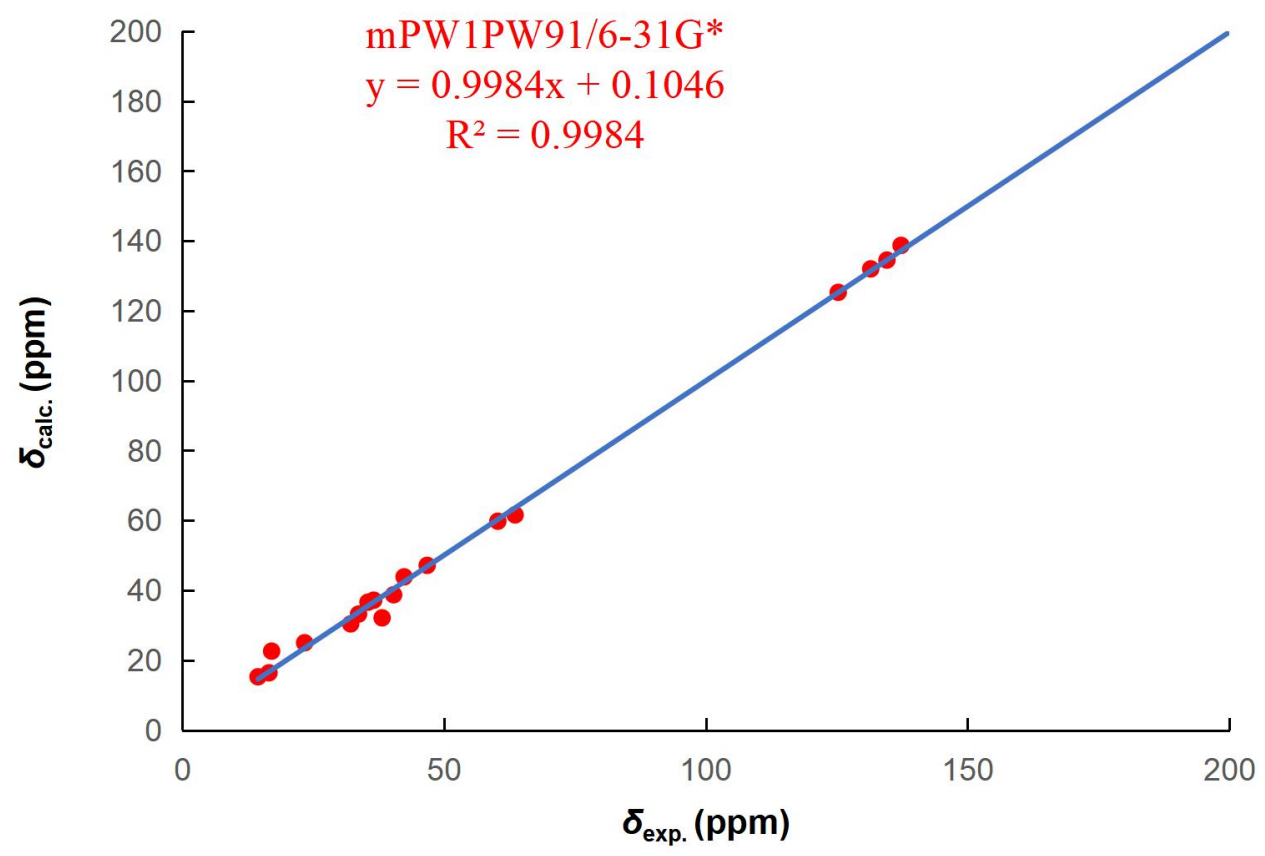

Figure S42. Regression analysis of experimental versus calculated ${ }^{13} \mathrm{C}$ NMR chemical shifts at mPW1PW91/6-31G* level using GIAO method of $\mathbf{1 b}$; linear fitting is shown as a line (blue). 


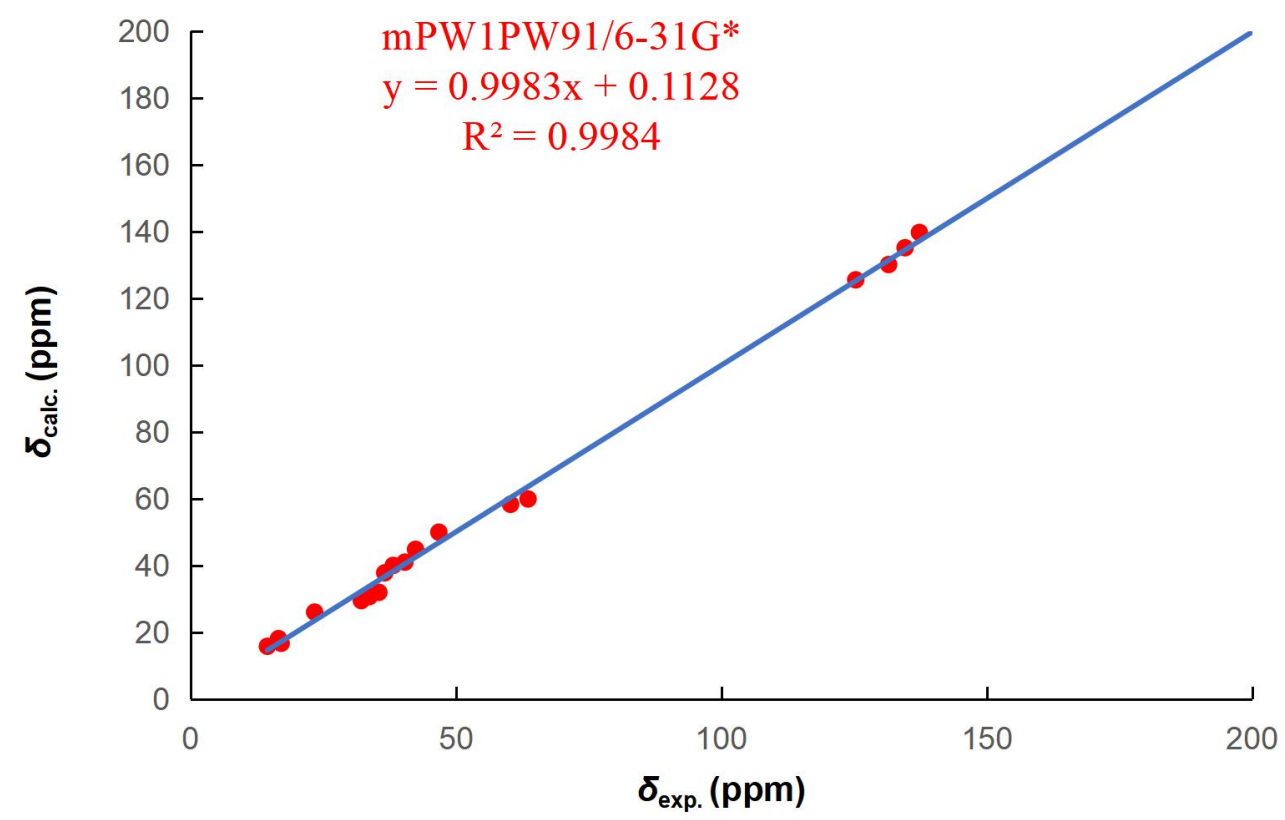

Figure S43. Regression analysis of experimental versus calculated ${ }^{13} \mathrm{C}$ NMR chemical shifts at mPW1PW91/6-31G* level using GIAO method of $\mathbf{1 c}$; linear fitting is shown as a line (blue).

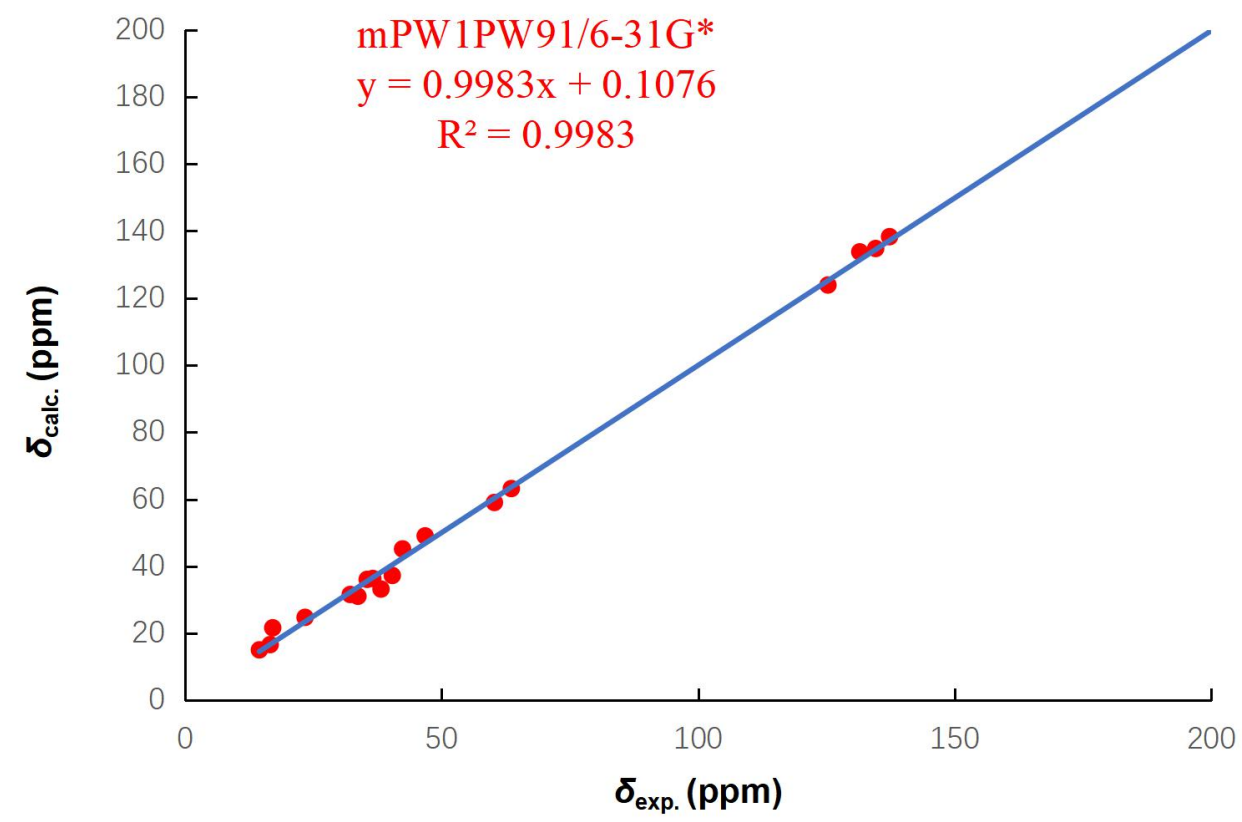

Figure S44. Regression analysis of experimental versus calculated ${ }^{13} \mathrm{C}$ NMR chemical shifts at mPW1PW91/6-31G* level using GIAO method of $\mathbf{1 d}$; linear fitting is shown as a line (blue). 


\begin{tabular}{|c|c|c|c|c|c|c|c|}
\hline \multicolumn{2}{|c|}{$\begin{array}{c}\text { Functional } \\
\text { mPW1PW91 }\end{array}$} & \multicolumn{2}{|c|}{ Solvent? } & \multicolumn{2}{|c|}{$\begin{array}{l}\text { Basis Set } \\
6-31 G(d)\end{array}$} & \multicolumn{2}{|c|}{ Type of Data } \\
\hline & & DP4+ & $100.00 \%$ & जी $0.00 \%$ & ज्ञll $0.00 \%$ & जी $0.00 \%$ & - \\
\hline Nuclei & sp2? & xperimenta & Isomer 1 & Isomer 2 & Isomer 3 & Isomer 4 & Isomer 5 \\
\hline $\mathrm{C}$ & $\mathrm{x}$ & 131.5 & 63. 1475956 & 63. 9403113 & 65. 9552277 & 62. 4025942 & \\
\hline $\mathrm{C}$ & & 42.4 & 149. 769144 & 150.407167 & 149. 552169 & 148. 867599 & \\
\hline $\mathrm{C}$ & $\mathrm{x}$ & 208.7 & -9.9404386 & -10.960981 & -10.485597 & -9.7113721 & \\
\hline $\mathrm{C}$ & & 40.4 & 154. 708873 & 155.43984 & 153. 319403 & 156. 59211 & \\
\hline $\mathrm{C}$ & & 33.7 & 160. 637878 & 160.862481 & 163. 426455 & 162. 648006 & \\
\hline $\mathrm{C}$ & $\mathrm{x}$ & 134.6 & 62.731657 & 61. 4727891 & 61.0245768 & 61. 4273961 & \\
\hline $\mathrm{C}$ & $\mathrm{x}$ & 125.3 & 70.8193026 & 70.5287821 & 70.4431362 & 72. 0531498 & \\
\hline C & & 23.4 & 168. 206608 & 168. 915872 & 167.974257 & 168. 787133 & \\
\hline $\mathrm{C}$ & & 38.2 & 154. 431052 & 161. 922491 & 154. 269643 & 160.551769 & \\
\hline $\mathrm{C}$ & & 60.3 & 134. 948106 & 134. 786182 & 136. 332028 & 135.352936 & \\
\hline $\mathrm{C}$ & & 63.6 & 131. 630879 & 133. 011442 & 134. 769467 & 131. 301049 & \\
\hline $\mathrm{C}$ & & 35.5 & 157.631496 & 157.522178 & 162.181126 & 157. 727301 & \\
\hline $\mathrm{C}$ & & 46.8 & 145. 270119 & 147.186483 & 144. 516039 & 145.077777 & \\
\hline C & & 32.2 & 159. 861044 & 163. 615719 & 164.600616 & 162. 12692 & \\
\hline $\mathrm{C}$ & $\mathrm{x}$ & 137.3 & 58.0749695 & 57.3438045 & 56. 5344109 & 57.9796406 & \\
\hline $\mathrm{C}$ & & 14.5 & 178. 243223 & 178. 469455 & 178. 013331 & 178. 247632 & \\
\hline $\mathrm{C}$ & & 36.6 & 157. 122 & 156. 965754 & 156. 446263 & 157.478121 & \\
\hline $\mathrm{C}$ & & 16. 6 & 177. 126247 & 177.348083 & 175. 767467 & 176. 719805 & \\
\hline $\mathrm{C}$ & & 17.1 & 177. 220727 & 171.265389 & 177. 133221 & 171.825895 & \\
\hline
\end{tabular}

\begin{tabular}{|c|c|c|c|c|c|c|}
\hline Functional & \multicolumn{2}{|c|}{ Solvent? } & \multicolumn{2}{|c|}{ Basis Set } & \multicolumn{2}{|c|}{ Type of Data } \\
\hline mPW1PW91 & \multicolumn{2}{|c|}{ PCM } & \multicolumn{2}{|c|}{$6-31 G(d)$} & \multicolumn{2}{|c|}{ Shielding Tensors } \\
\hline & Isomer 1 & Isomer 2 & Isomer 3 & Isomer 4 & Isomer 5 & Isomer 6 \\
\hline sDP4+ (H data) & - & - & - & - & - & - \\
\hline sDP4+ (C data) & 에 $99.78 \%$ & 메 $0.22 \%$ & $0.00 \%$ & जी $0.00 \%$ & - & - \\
\hline sDP4+ (a11 data) & - $99.78 \%$ & 메 $0.22 \%$ & - $0.00 \%$ & जी $0.00 \%$ & - & - \\
\hline uDP4+ (H data $)$ & - & - & - & - & - & - \\
\hline uDP4+ (C data) & $100.00 \%$ & 페 $0.00 \%$ & - $0.00 \%$ & जी $0.00 \%$ & - & - \\
\hline uDP4+ (a11 data) & $100.00 \%$ & 페 $0.00 \%$ & $0.00 \%$ & जी $0.00 \%$ & - & - \\
\hline DP4+ (H data) & - & - & - & - & - & - \\
\hline $\mathrm{DP} 4+(\mathrm{C}$ data $)$ & $100.00 \%$ & 페 $0.00 \%$ & जी $0.00 \%$ & - $0.00 \%$ & - & - \\
\hline DP4+ (a11 data) & $100.00 \%$ & 政 $0.00 \%$ & जी $0.00 \%$ & जी $0.00 \%$ & - & - \\
\hline
\end{tabular}

Figure S45. DP4+ evaluation of theoretical and experimental data of $\mathbf{1}$.

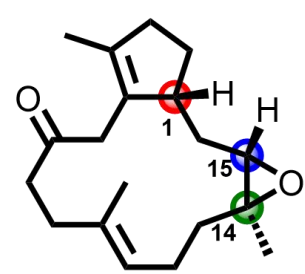

1a $1 R^{*}, 14 S^{*}, 15 S^{*}$

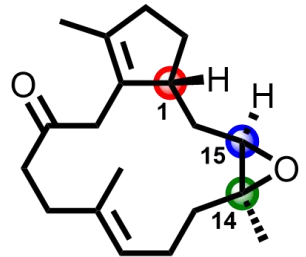

1b $1 R^{*}, 14 S^{*}, 15 R^{*}$

1.4

6.1

0.9984

$0.00 \%$

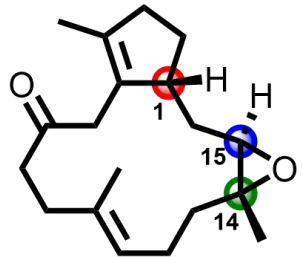

$1 \mathrm{c} 1 R^{*}, 14 R^{*}, 15 R^{*}$

1.8

3.5

0.9984

$0.00 \%$

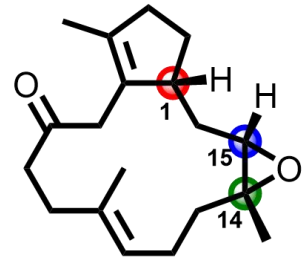

$1 \mathrm{~d} 1 R^{*}, 14 R^{*}, 15 S^{*}$

1.7

5.0

0.9983

$0.00 \%$

Figure S46. Correlated analysis of experimental vs calculated ${ }^{13} \mathrm{C}$ NMR chemical shifts of four possible diastereoisomers models (1a-1d) for compound 1 at the PCM/mPW1PW91/6-31+G** level using DP4+ method. 


\subsubsection{QM-NMR calculation for compound 3}

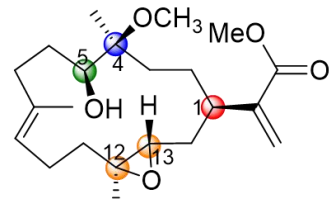

3a $1 R^{*}, 4 S^{*}, 5 S^{*}, 12 S^{*}, 13 S^{*}$

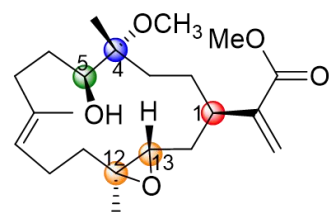

3e $1 R^{*}, 4 R^{*}, 5 S^{*}, 12 S^{*}, 13 S^{*}$

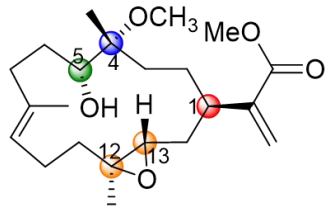

3b $1 R^{*}, 4 R^{\star}, 5 R^{*}, 12 S^{*}, 13 S^{*}$

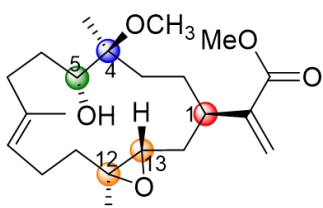

3f $1 R^{*}, 4 S^{*}, 5 R^{*}, 12 S^{*}, 13 S^{*}$

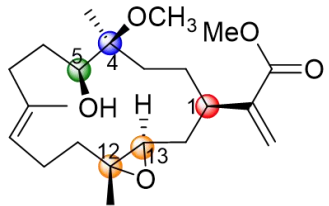

3c $1 R^{*}, 4 S^{*}, 5 S^{*}, 12 R^{*}, 13 R^{*}$

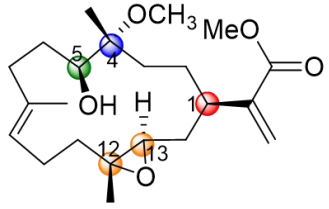

3 g $1 R^{*}, 4 R^{*}, 5 S^{*}, 12 R^{*}, 13 R^{*}$

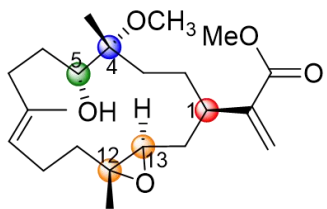

3d $1 R^{\star}, 4 R^{\star}, 5 R^{*}, 12 R^{*}, 13 R^{*}$

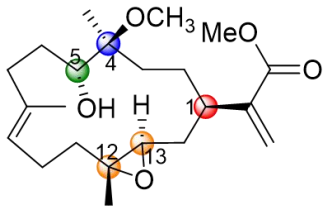

3h $1 R^{*}, 4 S^{*}, 5 R^{*}, 12 R^{*}, 13 R^{*}$

Figure S47. Structures of four stereoisomers 3a-3h. Five chiral centers were distributed into four regions, resulting eight models for 3 .

Table S10. Cartesian coordinates of all conformers of isomers $\mathbf{3 a}-\mathbf{3 h}$ used after optimization at the $\mathrm{B} 3 \mathrm{LYP} / 6-311 \mathrm{G}(\mathrm{d}, \mathrm{p})$ level of theory as required for DP4+ analysis.

$\begin{array}{lrrr}\text { 3a_1 } & & & \\ \text { C } & -3.77247 & 0.699458 & 1.303435 \\ \text { C } & -3.22245 & 2.01685 & 0.774192 \\ \text { C } & -2.8527 & -0.52502 & 1.423877 \\ \text { C } & -2.68511 & -1.28182 & 0.106731 \\ \text { C } & -1.91601 & 2.304758 & 0.678613 \\ \text { C } & -1.29991 & 3.638121 & 0.330905 \\ \text { C } & -0.43448 & 3.69213 & -0.94785 \\ \text { C } & -1.67139 & -2.46448 & 0.128889 \\ \text { C } & -0.21109 & -1.99964 & 0.316693 \\ \text { C } & 0.895948 & 2.950123 & -0.92802 \\ \text { C } & 1.048906 & 1.720539 & -1.7364 \\ \text { C } & 0.346187 & -1.11208 & -0.80422 \\ \text { C } & 2.080138 & 0.635519 & -1.50876 \\ \text { C } & 1.675485 & -0.42098 & -0.44646 \\ \text { C } & 2.841245 & -1.37867 & -0.23398 \\ \text { C } & 3.929735 & -1.01114 & 0.734604 \\ \text { O } & 4.903074 & -1.69245 & 0.979136 \\ \text { C } & 3.005214 & -2.52783 & -0.89525 \\ \text { C } & -4.33178 & 2.991809 & 0.450894 \\ \text { C } & 1.813287 & 3.258465 & 0.234943 \\ \text { O } & -3.97783 & -1.77982 & -0.26297 \\ \text { O } & 1.542251 & 2.983922 & -2.23043 \\ \text { H } & 0.155562 & 1.394388 & -2.26627 \\ \text { C } & -2.04422 & -3.49312 & 1.202384 \\ \text { O } & 3.712408 & 0.180803 & 1.320869 \\ \text { C } & 4.707399 & 0.615965 & 2.270392 \\ \end{array}$

$\begin{array}{lrrr}\text { 3a_2 } & & & \\ \text { C } & 3.777229 & 1.638031 & 0.583203 \\ \text { C } & 4.0438 & 0.196291 & 0.172685 \\ \text { C } & 2.365722 & 2.068196 & 1.009702 \\ \text { C } & 1.449259 & 2.378994 & -0.1733 \\ \text { C } & 3.205868 & -0.82399 & 0.409518 \\ \text { C } & 3.474124 & -2.29341 & 0.190673 \\ \text { C } & 2.536668 & -3.03657 & -0.78756 \\ \text { C } & -0.03286 & 2.698178 & 0.182634 \\ \mathrm{C} & -0.7855 & 1.481263 & 0.761976 \\ \mathrm{C} & 1.095476 & -3.25931 & -0.3455 \\ \mathrm{C} & 0.009606 & -2.50272 & -1.00602 \\ \mathrm{C} & -0.90524 & 0.277623 & -0.17957 \\ \mathrm{C} & -1.36346 & -2.23783 & -0.42477 \\ \mathrm{C} & -1.40683 & -1.00757 & 0.517122 \\ \mathrm{C} & -2.7635 & -0.86598 & 1.195098 \\ \mathrm{C} & -4.02178 & -0.55591 & 0.432408 \\ \mathrm{O} & -5.122 & -0.44818 & 0.931114 \\ \mathrm{C} & -2.90744 & -1.02929 & 2.514525 \\ \mathrm{C} & 5.408332 & 0.044371 & -0.46049 \\ \mathrm{C} & 0.909043 & -3.89648 & 1.014758 \\ \mathrm{O} & 2.017072 & 3.498533 & -0.86671 \\ \mathrm{O} & 0.29762 & -3.86002 & -1.40298 \\ \mathrm{H} & 0.330819 & -1.78092 & -1.75494 \\ \mathrm{C} & -0.12993 & 3.889132 & 1.142371 \\ \mathrm{O} & -3.80982 & -0.4052 & -0.88777 \\ \mathrm{C} & -4.97251 & -0.11369 & -1.69194\end{array}$




\begin{tabular}{|c|c|c|c|c|c|c|c|}
\hline $\mathrm{O}$ & -1.86033 & -3.01084 & -1.20647 & $\mathrm{O}$ & -0.55699 & 3.045955 & -1.12991 \\
\hline $\mathrm{C}$ & -1.24264 & -4.2525 & -1.52158 & $\mathrm{C}$ & -1.88683 & 3.54179 & -1.2179 \\
\hline $\mathrm{H}$ & -4.63649 & 0.413948 & 0.693913 & $\mathrm{H}$ & 4.099485 & 2.296274 & -0.23076 \\
\hline $\mathrm{H}$ & -4.19058 & 0.917546 & 2.295795 & $\mathrm{H}$ & 4.468928 & 1.855073 & 1.408674 \\
\hline $\mathrm{H}$ & -1.87832 & -0.23998 & 1.827123 & $\mathrm{H}$ & 1.910876 & 1.314861 & 1.656875 \\
\hline $\mathrm{H}$ & -3.29962 & -1.20993 & 2.149932 & $\mathrm{H}$ & 2.458018 & 2.977456 & 1.610293 \\
\hline $\mathrm{H}$ & -2.35215 & -0.58396 & -0.67051 & $\mathrm{H}$ & 1.439604 & 1.522484 & -0.85717 \\
\hline $\mathrm{H}$ & -1.20151 & 1.526573 & 0.936071 & $\mathrm{H}$ & 2.246507 & -0.59969 & 0.869547 \\
\hline $\mathrm{H}$ & -0.68672 & 3.965536 & 1.179766 & $\mathrm{H}$ & 3.430924 & -2.79885 & 1.163612 \\
\hline $\mathrm{H}$ & -2.07494 & 4.397912 & 0.223554 & $\mathrm{H}$ & 4.493151 & -2.44349 & -0.16785 \\
\hline $\mathrm{H}$ & -1.0184 & 3.340626 & -1.80369 & $\mathrm{H}$ & 2.532443 & -2.52692 & -1.75572 \\
\hline $\mathrm{H}$ & -0.19648 & 4.745428 & -1.14279 & $\mathrm{H}$ & 2.96251 & -4.03285 & -0.96077 \\
\hline $\mathrm{H}$ & -0.14725 & -1.46519 & 1.270035 & $\mathrm{H}$ & -0.28013 & 1.181025 & 1.685964 \\
\hline $\mathrm{H}$ & 0.421411 & -2.8835 & 0.439734 & $\mathrm{H}$ & -1.78212 & 1.808189 & 1.075378 \\
\hline $\mathrm{H}$ & 0.444712 & -1.68652 & -1.73004 & $\mathrm{H}$ & -1.55103 & 0.521631 & -1.02423 \\
\hline $\mathrm{H}$ & -0.37728 & -0.32656 & -1.02482 & $\mathrm{H}$ & 0.077322 & 0.054443 & -0.59754 \\
\hline $\mathrm{H}$ & 3.025465 & 1.097066 & -1.21875 & $\mathrm{H}$ & -1.69796 & -3.12088 & 0.123996 \\
\hline $\mathrm{H}$ & 2.259887 & 0.127297 & -2.46356 & $\mathrm{H}$ & -2.06377 & -2.09388 & -1.25175 \\
\hline $\mathrm{H}$ & 1.519343 & 0.114626 & 0.493388 & $\mathrm{H}$ & -0.70355 & -1.21449 & 1.331807 \\
\hline $\mathrm{H}$ & 3.880386 & -3.14031 & -0.71904 & $\mathrm{H}$ & -3.87953 & -0.93851 & 2.982848 \\
\hline $\mathrm{H}$ & 2.283678 & -2.88228 & -1.62039 & $\mathrm{H}$ & -2.05931 & -1.25774 & 3.149265 \\
\hline $\mathrm{H}$ & -3.97987 & 3.972855 & 0.135916 & $\mathrm{H}$ & 5.663786 & -0.98193 & -0.71864 \\
\hline $\mathrm{H}$ & -4.96425 & 2.589539 & -0.34856 & $\mathrm{H}$ & 5.466858 & 0.645054 & -1.37514 \\
\hline $\mathrm{H}$ & -4.98473 & 3.13038 & 1.320493 & $\mathrm{H}$ & 6.186451 & 0.426967 & 0.210419 \\
\hline $\mathrm{H}$ & 1.963313 & 4.339892 & 0.312368 & $\mathrm{H}$ & 1.506319 & -4.81116 & 1.0816 \\
\hline $\mathrm{H}$ & 1.383684 & 2.912025 & 1.178168 & $\mathrm{H}$ & 1.233756 & -3.22663 & 1.814865 \\
\hline $\mathrm{H}$ & 2.786508 & 2.786123 & 0.112669 & $\mathrm{H}$ & -0.13282 & -4.16056 & 1.191064 \\
\hline $\mathrm{H}$ & -3.80919 & -2.36292 & -1.01587 & $\mathrm{H}$ & 1.347869 & 3.747896 & -1.5187 \\
\hline $\mathrm{H}$ & -3.08292 & -3.80707 & 1.087727 & $\mathrm{H}$ & 0.415208 & 4.746511 & 0.74464 \\
\hline $\mathrm{H}$ & -1.40092 & -4.37316 & 1.1482 & $\mathrm{H}$ & -1.16901 & 4.17777 & 1.31102 \\
\hline $\mathrm{H}$ & -1.92108 & -3.06371 & 2.198625 & $\mathrm{H}$ & 0.295285 & 3.629421 & 2.113867 \\
\hline $\mathrm{H}$ & 4.778862 & -0.09363 & 3.095096 & $\mathrm{H}$ & -5.6997 & -0.92202 & -1.6124 \\
\hline $\mathrm{H}$ & 5.67933 & 0.708963 & 1.785193 & $\mathrm{H}$ & -5.43062 & 0.821542 & -1.36981 \\
\hline $\mathrm{H}$ & 4.364361 & 1.583445 & 2.627469 & $\mathrm{H}$ & -4.60533 & -0.02877 & -2.71133 \\
\hline $\mathrm{H}$ & -0.16554 & -4.24151 & -1.32289 & $\mathrm{H}$ & -2.61624 & 2.843877 & -0.79288 \\
\hline $\mathrm{H}$ & -1.39687 & -4.406 & -2.59019 & $\mathrm{H}$ & -2.09707 & 3.657197 & -2.28176 \\
\hline $\mathrm{H}$ & -1.69372 & -5.08957 & -0.97914 & $\mathrm{H}$ & -2.00054 & 4.516101 & -0.73213 \\
\hline
\end{tabular}

\begin{tabular}{|c|c|c|c|c|c|c|c|}
\hline 3a_3 & & & & 3a_4 & & & \\
\hline $\mathrm{C}$ & -3.42262 & 1.695632 & 1.21105 & $\mathrm{C}$ & 3.790144 & 1.644249 & 0.726795 \\
\hline $\mathrm{C}$ & -2.57618 & 2.532849 & 0.257053 & $\mathrm{C}$ & 4.088447 & 0.207574 & 0.319942 \\
\hline $\mathrm{C}$ & -2.91975 & 0.253958 & 1.447392 & $\mathrm{C}$ & 2.35274 & 2.064109 & 1.069483 \\
\hline $\mathrm{C}$ & -3.04005 & -0.66296 & 0.233804 & $\mathrm{C}$ & 1.509634 & 2.381707 & -0.16468 \\
\hline $\mathrm{C}$ & -1.44826 & 3.109107 & 0.6956 & $\mathrm{C}$ & 3.243617 & -0.81898 & 0.497925 \\
\hline $\mathrm{C}$ & -0.51101 & 4.040976 & -0.03561 & $\mathrm{C}$ & 3.531314 & -2.28492 & 0.280645 \\
\hline $\mathrm{C}$ & 0.961016 & 3.934505 & 0.40457 & $\mathrm{C}$ & 2.645564 & -3.02522 & -0.74693 \\
\hline $\mathrm{C}$ & -2.28906 & -2.0214 & 0.343668 & $\mathrm{C}$ & 0.007964 & 2.696404 & 0.101268 \\
\hline $\mathrm{C}$ & -0.75799 & -1.84784 & 0.462094 & $\mathrm{C}$ & -0.77233 & 1.481007 & 0.646312 \\
\hline $\mathrm{C}$ & 1.834649 & 2.8747 & -0.25946 & $\mathrm{C}$ & 1.186339 & -3.25895 & -0.37543 \\
\hline
\end{tabular}




\begin{tabular}{|c|c|c|c|c|c|c|c|}
\hline $\mathrm{C}$ & 1.250951 & 1.779342 & -1.06123 & $\mathrm{C}$ & 0.12968 & -2.49865 & -1.0777 \\
\hline $\mathrm{C}$ & -0.10724 & -0.98928 & -0.62973 & $\mathrm{C}$ & -0.82888 & 0.268945 & -0.28983 \\
\hline $\mathrm{C}$ & 1.895737 & 0.43601 & -1.33124 & $\mathrm{C}$ & -1.26968 & -2.2428 & -0.55938 \\
\hline $\mathrm{C}$ & 1.388434 & -0.69968 & -0.39874 & $\mathrm{C}$ & -1.36482 & -1.01518 & 0.382264 \\
\hline $\mathrm{C}$ & 2.288009 & -1.91888 & -0.55763 & $\mathrm{C}$ & -2.76525 & -0.87068 & 0.959934 \\
\hline $\mathrm{C}$ & 3.561683 & -2.003 & 0.235946 & $\mathrm{C}$ & -3.88752 & -0.54402 & 0.022845 \\
\hline $\mathrm{O}$ & 4.355048 & -2.91911 & 0.182886 & $\mathrm{O}$ & -3.75844 & -0.38653 & -1.17518 \\
\hline $\mathrm{C}$ & 2.055964 & -2.9281 & -1.40194 & $\mathrm{C}$ & -3.01109 & -1.04109 & 2.263754 \\
\hline $\mathrm{C}$ & -3.12318 & 2.702288 & -1.13951 & $\mathrm{C}$ & 5.48834 & 0.068801 & -0.23422 \\
\hline $\mathrm{C}$ & 3.207001 & 2.760314 & 0.369743 & $\mathrm{C}$ & 0.939763 & -3.91149 & 0.967944 \\
\hline $\mathrm{O}$ & -4.43416 & -0.8966 & 0.000325 & $\mathrm{O}$ & 2.116568 & 3.505787 & -0.81639 \\
\hline $\mathrm{O}$ & 1.836539 & 2.929808 & -1.71119 & $\mathrm{O}$ & 0.440982 & -3.85088 & -1.47509 \\
\hline $\mathrm{H}$ & 0.16804 & 1.777384 & -1.13886 & $\mathrm{H}$ & 0.483793 & -1.76826 & -1.80298 \\
\hline $\mathrm{C}$ & -2.82153 & -2.8629 & 1.507937 & $\mathrm{C}$ & -0.15166 & 3.895894 & 1.042116 \\
\hline $\mathrm{O}$ & 3.740352 & -0.9345 & 1.033982 & $\mathrm{O}$ & -5.07798 & -0.43636 & 0.639647 \\
\hline $\mathrm{C}$ & 4.938705 & -0.93234 & 1.837025 & $\mathrm{C}$ & -6.20787 & -0.12596 & -0.20067 \\
\hline $\mathrm{O}$ & -2.63176 & -2.62006 & -0.93846 & $\mathrm{O}$ & -0.43626 & 3.03163 & -1.24338 \\
\hline $\mathrm{C}$ & -2.33248 & -3.99487 & -1.14503 & $\mathrm{C}$ & -1.76761 & 3.497505 & -1.42261 \\
\hline $\mathrm{H}$ & -4.45267 & 1.651885 & 0.845508 & $\mathrm{H}$ & 4.158482 & 2.31132 & -0.05998 \\
\hline $\mathrm{H}$ & -3.45826 & 2.193937 & 2.184546 & $\mathrm{H}$ & 4.428932 & 1.85686 & 1.595006 \\
\hline $\mathrm{H}$ & -1.87848 & 0.299533 & 1.775747 & $\mathrm{H}$ & 1.862878 & 1.301967 & 1.679506 \\
\hline $\mathrm{H}$ & -3.49561 & -0.18589 & 2.268096 & $\mathrm{H}$ & 2.403661 & 2.967118 & 1.684519 \\
\hline $\mathrm{H}$ & -2.62668 & -0.15963 & -0.64601 & $\mathrm{H}$ & 1.54227 & 1.529542 & -0.85333 \\
\hline $\mathrm{H}$ & -1.18617 & 2.948246 & 1.742344 & $\mathrm{H}$ & 2.257925 & -0.60377 & 0.903436 \\
\hline $\mathrm{H}$ & -0.83071 & 5.071132 & 0.17024 & $\mathrm{H}$ & 3.445514 & -2.79867 & 1.2465 \\
\hline $\mathrm{H}$ & -0.57858 & 3.927963 & -1.12092 & $\mathrm{H}$ & 4.56703 & -2.42623 & -0.03058 \\
\hline $\mathrm{H}$ & 1.457601 & 4.893785 & 0.216466 & $\mathrm{H}$ & 2.683964 & -2.50722 & -1.70988 \\
\hline $\mathrm{H}$ & 1.005146 & 3.783646 & 1.489255 & $\mathrm{H}$ & 3.084947 & -4.01763 & -0.90799 \\
\hline $\mathrm{H}$ & -0.54108 & -1.41128 & 1.442477 & $\mathrm{H}$ & -0.32199 & 1.192109 & 1.601813 \\
\hline $\mathrm{H}$ & -0.29998 & -2.84148 & 0.480294 & $\mathrm{H}$ & -1.78734 & 1.80624 & 0.896034 \\
\hline $\mathrm{H}$ & -0.26645 & -1.44427 & -1.61133 & $\mathrm{H}$ & -1.42839 & 0.499458 & -1.17167 \\
\hline $\mathrm{H}$ & -0.61749 & -0.02762 & -0.66602 & $\mathrm{H}$ & 0.177702 & 0.049972 & -0.648 \\
\hline $\mathrm{H}$ & 2.979814 & 0.52271 & -1.24892 & $\mathrm{H}$ & -1.6258 & -3.12959 & -0.03068 \\
\hline $\mathrm{H}$ & 1.682125 & 0.157037 & -2.37051 & $\mathrm{H}$ & -1.93216 & -2.09476 & -1.41644 \\
\hline $\mathrm{H}$ & 1.500606 & -0.34566 & 0.629794 & $\mathrm{H}$ & -0.71551 & -1.22152 & 1.240607 \\
\hline $\mathrm{H}$ & 2.762598 & -3.7443 & -1.48398 & $\mathrm{H}$ & -4.00857 & -0.95041 & 2.671995 \\
\hline $\mathrm{H}$ & 1.171873 & -2.96832 & -2.02505 & $\mathrm{H}$ & -2.20846 & -1.27814 & 2.953352 \\
\hline $\mathrm{H}$ & -2.40777 & 3.156444 & -1.8261 & $\mathrm{H}$ & 5.766803 & -0.95436 & -0.48078 \\
\hline $\mathrm{H}$ & -3.4433 & 1.74342 & -1.55839 & $\mathrm{H}$ & 5.594722 & 0.673797 & -1.14171 \\
\hline $\mathrm{H}$ & -4.01538 & 3.34044 & -1.11975 & $\mathrm{H}$ & 6.223395 & 0.455002 & 0.481664 \\
\hline $\mathrm{H}$ & 3.680422 & 3.74645 & 0.402321 & $\mathrm{H}$ & 1.543653 & -4.81997 & 1.056242 \\
\hline $\mathrm{H}$ & 3.128559 & 2.39219 & 1.396387 & $\mathrm{H}$ & 1.216425 & -3.24578 & 1.789346 \\
\hline $\mathrm{H}$ & 3.859166 & 2.091234 & -0.18891 & $\mathrm{H}$ & -0.10643 & -4.1893 & 1.089334 \\
\hline $\mathrm{H}$ & -4.45892 & -1.55517 & -0.70701 & $\mathrm{H}$ & 1.491791 & 3.753075 & -1.51171 \\
\hline $\mathrm{H}$ & -3.89833 & -3.01058 & 1.412366 & $\mathrm{H}$ & 0.41497 & 4.75076 & 0.670189 \\
\hline $\mathrm{H}$ & -2.33303 & -3.83834 & 1.550883 & $\mathrm{H}$ & -1.20021 & 4.182638 & 1.142295 \\
\hline $\mathrm{H}$ & -2.62745 & -2.35942 & 2.457011 & $\mathrm{H}$ & 0.212392 & 3.647863 & 2.041237 \\
\hline $\mathrm{H}$ & 4.940605 & -1.78601 & 2.515324 & $\mathrm{H}$ & -6.06804 & 0.841048 & -0.68504 \\
\hline $\mathrm{H}$ & 5.822395 & -0.97244 & 1.19973 & $\mathrm{H}$ & -6.34155 & -0.89708 & -0.95996 \\
\hline
\end{tabular}




$\begin{array}{lrlllllr}\mathrm{H} & 4.912144 & -0.00063 & 2.395885 & \mathrm{H} & -7.06477 & -0.09629 & 0.467416 \\ \mathrm{H} & -1.27832 & -4.22429 & -0.95565 & \mathrm{H} & -2.50921 & 2.776207 & -1.06292 \\ \mathrm{H} & -2.54725 & -4.19776 & -2.19487 & \mathrm{H} & -1.90144 & 3.623308 & -2.4976 \\ \mathrm{H} & -2.95421 & -4.65117 & -0.52744 & \mathrm{H} & -1.94131 & 4.461989 & -0.93482\end{array}$

\begin{tabular}{|c|c|c|c|c|c|c|c|}
\hline 3a_5 & & & & 3a_6 & & & \\
\hline $\mathrm{C}$ & 3.562728 & 1.812824 & 0.531882 & $\mathrm{C}$ & -2.82769 & 2.237899 & 1.399539 \\
\hline $\mathrm{C}$ & 3.583051 & 0.302112 & 0.685708 & $\mathrm{C}$ & -1.42347 & 2.796095 & 1.25272 \\
\hline $\mathrm{C}$ & 2.211708 & 2.490651 & 0.867214 & $\mathrm{C}$ & -2.91125 & 0.694629 & 1.498128 \\
\hline $\mathrm{C}$ & 1.288631 & 2.572382 & -0.34689 & $\mathrm{C}$ & -3.0376 & 0.036209 & 0.125872 \\
\hline $\mathrm{C}$ & 3.621293 & -0.50599 & -0.38426 & $\mathrm{C}$ & -0.98983 & 3.291888 & 0.084378 \\
\hline $\mathrm{C}$ & 3.816259 & -2.0006 & -0.42518 & $\mathrm{C}$ & 0.294492 & 4.026777 & -0.20246 \\
\hline $\mathrm{C}$ & 2.817859 & -2.79477 & -1.2959 & $\mathrm{C}$ & 1.086366 & 3.553653 & -1.44075 \\
\hline $\mathrm{C}$ & -0.22025 & 2.799006 & -0.04257 & $\mathrm{C}$ & -2.70668 & -1.48319 & 0.066816 \\
\hline $\mathrm{C}$ & -0.86971 & 1.554214 & 0.602491 & $\mathrm{C}$ & -1.19623 & -1.75183 & 0.257747 \\
\hline $\mathrm{C}$ & 1.44707 & -3.09887 & -0.7022 & $\mathrm{C}$ & 1.94034 & 2.298575 & -1.29937 \\
\hline $\mathrm{C}$ & 0.254782 & -2.38301 & -1.20776 & $\mathrm{C}$ & 1.512529 & 1.041461 & -1.95353 \\
\hline $\mathrm{C}$ & -0.94331 & 0.3188 & -0.30634 & $\mathrm{C}$ & -0.2978 & -1.23397 & -0.87669 \\
\hline $\mathrm{C}$ & -1.06672 & -2.23947 & -0.48085 & $\mathrm{C}$ & 1.992867 & -0.35081 & -1.59619 \\
\hline $\mathrm{C}$ & -1.1414 & -1.00922 & 0.458815 & $\mathrm{C}$ & 1.181187 & -1.0647 & -0.48317 \\
\hline $\mathrm{C}$ & -2.38692 & -1.04547 & 1.334711 & $\mathrm{C}$ & 1.88306 & -2.36814 & -0.12179 \\
\hline $\mathrm{C}$ & -3.77726 & -0.94795 & 0.770325 & $\mathrm{C}$ & 3.013393 & -2.34799 & 0.868115 \\
\hline $\mathrm{O}$ & -4.79427 & -0.99569 & 1.429375 & $\mathrm{O}$ & 3.646092 & -3.32083 & 1.221486 \\
\hline $\mathrm{C}$ & -2.3062 & -1.1824 & 2.662543 & $\mathrm{C}$ & 1.600092 & -3.55521 & -0.66546 \\
\hline $\mathrm{C}$ & 3.631929 & -0.17843 & 2.11709 & $\mathrm{C}$ & -0.61774 & 2.796838 & 2.530453 \\
\hline $\mathrm{C}$ & 1.44835 & -3.78932 & 0.644501 & $\mathrm{C}$ & 2.93523 & 2.303131 & -0.15966 \\
\hline $\mathrm{O}$ & 1.780612 & 3.635475 & -1.17296 & $\mathrm{O}$ & -4.38275 & 0.254413 & -0.31814 \\
\hline $\mathrm{O}$ & 0.57058 & -3.70642 & -1.69123 & $\mathrm{O}$ & 2.49103 & 1.896371 & -2.58382 \\
\hline $\mathrm{H}$ & 0.45038 & -1.61516 & -1.95456 & $\mathrm{H}$ & 0.548123 & 1.077786 & -2.45744 \\
\hline $\mathrm{C}$ & -0.42813 & 4.03975 & 0.831277 & $\mathrm{C}$ & -3.53326 & -2.26772 & 1.090072 \\
\hline $\mathrm{O}$ & -3.79009 & -0.79931 & -0.56695 & $\mathrm{O}$ & 3.261734 & -1.11148 & 1.337626 \\
\hline $\mathrm{C}$ & -5.09058 & -0.70881 & -1.18669 & $\mathrm{C}$ & 4.335631 & -0.99656 & 2.293913 \\
\hline $\mathrm{O}$ & -0.74895 & 3.009957 & -1.38025 & $\mathrm{O}$ & -3.10806 & -1.80848 & -1.29208 \\
\hline $\mathrm{C}$ & -2.11214 & 3.392375 & -1.51183 & $\mathrm{C}$ & -3.1494 & -3.17783 & -1.67297 \\
\hline $\mathrm{H}$ & 3.849084 & 2.092382 & -0.48527 & $\mathrm{H}$ & -3.45464 & 2.566905 & 0.566867 \\
\hline $\mathrm{H}$ & 4.331026 & 2.226405 & 1.19603 & $\mathrm{H}$ & -3.26625 & 2.6674 & 2.307996 \\
\hline $\mathrm{H}$ & 1.724813 & 1.958587 & 1.687497 & $\mathrm{H}$ & -2.03876 & 0.309304 & 2.031047 \\
\hline $\mathrm{H}$ & 2.391221 & 3.511378 & 1.215893 & $\mathrm{H}$ & -3.79026 & 0.412603 & 2.084128 \\
\hline $\mathrm{H}$ & 1.363585 & 1.635627 & -0.91172 & $\mathrm{H}$ & -2.35849 & 0.541089 & -0.57185 \\
\hline $\mathrm{H}$ & 3.600787 & -0.03299 & -1.36625 & $\mathrm{H}$ & -1.67786 & 3.250331 & -0.76016 \\
\hline $\mathrm{H}$ & 3.857252 & -2.42702 & 0.578641 & $\mathrm{H}$ & 0.947771 & 4.049182 & 0.671552 \\
\hline $\mathrm{H}$ & 4.809622 & -2.18002 & -0.85774 & $\mathrm{H}$ & 0.027392 & 5.075017 & -0.3925 \\
\hline $\mathrm{H}$ & 2.677157 & -2.28683 & -2.2551 & $\mathrm{H}$ & 0.405196 & 3.419159 & -2.28698 \\
\hline $\mathrm{H}$ & 3.269979 & -3.76746 & -1.52531 & $\mathrm{H}$ & 1.776974 & 4.356708 & -1.72578 \\
\hline $\mathrm{H}$ & -0.30733 & 1.312578 & 1.509994 & $\mathrm{H}$ & -0.89041 & -1.29277 & 1.203313 \\
\hline $\mathrm{H}$ & -1.87254 & 1.821267 & 0.950635 & $\mathrm{H}$ & -1.0452 & -2.82583 & 0.397484 \\
\hline $\mathrm{H}$ & -1.73494 & 0.440372 & -1.04679 & $\mathrm{H}$ & -0.38909 & -1.87683 & -1.75724 \\
\hline $\mathrm{H}$ & -0.01187 & 0.229537 & -0.86557 & $\mathrm{H}$ & -0.6592 & -0.25639 & -1.19546 \\
\hline $\mathrm{H}$ & -1.25384 & -3.14476 & 0.10023 & $\mathrm{H}$ & 3.042126 & -0.29606 & -1.30229 \\
\hline
\end{tabular}




$\begin{array}{lrrrrrrr}\mathrm{H} & -1.86036 & -2.17711 & -1.23031 & \mathrm{H} & 1.956561 & -0.96402 & -2.5049 \\ \mathrm{H} & -0.30139 & -1.0958 & 1.157597 & \mathrm{H} & 1.21238 & -0.42328 & 0.401277 \\ \mathrm{H} & -3.20028 & -1.21625 & 3.272493 & \mathrm{H} & 2.1679 & -4.43364 & -0.38593 \\ \mathrm{H} & -1.34849 & -1.26415 & 3.162955 & \mathrm{H} & 0.809178 & -3.68171 & -1.39353 \\ \mathrm{H} & 3.541731 & -1.25974 & 2.214217 & \mathrm{H} & 0.422548 & 3.087074 & 2.387175 \\ \mathrm{H} & 4.575086 & 0.124094 & 2.587566 & \mathrm{H} & -1.06165 & 3.485171 & 3.259652 \\ \mathrm{H} & 2.831411 & 0.275454 & 2.710424 & \mathrm{H} & -0.6252 & 1.806453 & 2.997193 \\ \mathrm{H} & 2.123499 & -4.65031 & 0.621823 & \mathrm{H} & 3.5317 & 3.220306 & -0.19012 \\ \mathrm{H} & 1.790885 & -3.11569 & 1.43371 & \mathrm{H} & 2.424974 & 2.265807 & 0.805768 \\ \mathrm{H} & 0.454603 & -4.14779 & 0.909329 & \mathrm{H} & 3.612938 & 1.453056 & -0.21519 \\ \mathrm{H} & 1.102911 & 3.752313 & -1.85308 & \mathrm{H} & -4.47131 & -0.29445 & -1.10968 \\ \mathrm{H} & 0.045962 & 4.909612 & 0.373119 & \mathrm{H} & -4.59708 & -2.06275 & 0.957132 \\ \mathrm{H} & -1.48909 & 4.251368 & 0.977117 & \mathrm{H} & -3.36595 & -3.34244 & 0.998116 \\ \mathrm{H} & 0.011218 & 3.88747 & 1.819325 & \mathrm{H} & -3.25088 & -1.98056 & 2.104965 \\ \mathrm{H} & -5.65852 & -1.62216 & -1.008 & \mathrm{H} & 4.119548 & -1.59489 & 3.179352 \\ \mathrm{H} & -5.64075 & 0.145006 & -0.79101 & \mathrm{H} & 5.274673 & -1.32893 & 1.85058 \\ \mathrm{H} & -4.89656 & -0.5809 & -2.24837 & \mathrm{H} & 4.385692 & 0.058742 & 2.54889 \\ \mathrm{H} & -2.78801 & 2.685087 & -1.01912 & \mathrm{H} & -2.20071 & -3.69067 & -1.48028 \\ \mathrm{H} & -2.32663 & 3.389707 & -2.58105 & \mathrm{H} & -3.33739 & -3.18902 & -2.74714 \\ \mathrm{H} & -2.30109 & 4.396914 & -1.1198 & \mathrm{H} & -3.95506 & -3.72308 & -1.17098\end{array}$

\begin{tabular}{lrrrlrrr} 
3a_7 & \multicolumn{7}{c}{ 3a_8 } \\
C & 3.788626 & 1.647231 & 0.726677 & C & -1.46526 & -2.52745 & -1.96614 \\
C & 4.088211 & 0.210796 & 0.319897 & C & -0.00686 & -2.60291 & -1.55106 \\
C & 2.350872 & 2.065815 & 1.069456 & C & -2.13863 & -1.14374 & -1.80081 \\
C & 1.507413 & 2.382648 & -0.16465 & C & -2.62394 & -0.89927 & -0.37415 \\
C & 3.244265 & -0.81648 & 0.497859 & C & 0.352923 & -3.22922 & -0.42114 \\
C & 3.533249 & -2.28219 & 0.280688 & C & 1.734972 & -3.53984 & 0.094169 \\
C & 2.648244 & -3.0234 & -0.74686 & C & 2.045991 & -3.01519 & 1.514979 \\
C & 0.005472 & 2.695953 & 0.101342 & C & -3.12465 & 0.541828 & -0.06304 \\
C & -0.77371 & 1.479836 & 0.646389 & C & -1.97113 & 1.569396 & -0.0403 \\
C & 1.189232 & -3.25852 & -0.37539 & C & 2.450164 & -1.55152 & 1.625127 \\
C & 0.131877 & -2.49901 & -1.0775 & C & 1.438123 & -0.54808 & 2.021846 \\
C & -0.82921 & 0.267761 & -0.2898 & C & -0.93369 & 1.363419 & 1.08631 \\
C & -1.2677 & -2.2445 & -0.55915 & C & 1.501672 & 0.937069 & 1.737893 \\
C & -1.36406 & -1.01682 & 0.382278 & C & 0.53153 & 1.393027 & 0.614322 \\
C & -2.76467 & -0.87341 & 0.959751 & C & 0.969415 & 2.752609 & 0.084359 \\
C & -3.88687 & -0.5464 & 0.022673 & C & 2.079326 & 2.825334 & -0.92816 \\
O & -3.75812 & -0.39101 & -1.17566 & O & 2.555484 & 3.861395 & -1.37408 \\
C & -3.0108 & -1.04547 & 2.263301 & C & 0.459176 & 3.922868 & 0.477507 \\
C & 5.488281 & 0.073246 & -0.23413 & C & 0.961872 & -1.9841 & -2.52965 \\
C & 0.943292 & -3.91173 & 0.967759 & C & 3.725653 & -1.17103 & 0.905895 \\
O & 2.113273 & 3.507302 & -0.81635 & O & -3.68228 & -1.83345 & -0.12474 \\
O & 0.44435 & -3.85086 & -1.47516 & O & 2.373429 & -1.06171 & 2.993002 \\
H & 0.485338 & -1.76815 & -1.80263 & H & 0.432248 & -0.93624 & 2.174781 \\
C & -0.15524 & 3.895254 & 1.042225 & C & -4.217 & 0.976358 & -1.04473 \\
O & -5.07687 & -0.43583 & 0.639852 & O & 2.504116 & 1.604793 & -1.31275 \\
C & -6.20678 & -0.12574 & -0.2006 & C & 3.568659 & 1.580545 & -2.28621 \\
O & -0.43913 & 3.0307 & -1.24332 & O & -3.66819 & 0.365021 & 1.274403
\end{tabular}




\begin{tabular}{|c|c|c|c|c|c|c|c|}
\hline $\mathrm{C}$ & -1.77065 & 3.496147 & -1.42233 & C & -4.35921 & 1.454395 & 1.873367 \\
\hline $\mathrm{H}$ & 4.156299 & 2.314583 & -0.06017 & $\mathrm{H}$ & -2.05099 & -3.26056 & -1.40737 \\
\hline $\mathrm{H}$ & 4.427312 & 1.860453 & 1.594812 & $\mathrm{H}$ & -1.53127 & -2.81199 & -3.02311 \\
\hline $\mathrm{H}$ & 1.861717 & 1.303252 & 1.679509 & $\mathrm{H}$ & -1.4483 & -0.35322 & -2.10582 \\
\hline $\mathrm{H}$ & 2.401035 & 2.968866 & 1.684502 & $\mathrm{H}$ & -3.00124 & -1.08897 & -2.47053 \\
\hline $\mathrm{H}$ & 1.540812 & 1.530515 & -0.85332 & $\mathrm{H}$ & -1.80085 & -1.11071 & 0.320009 \\
\hline $\mathrm{H}$ & 2.258363 & -0.6021 & 0.903292 & $\mathrm{H}$ & -0.44916 & -3.64124 & 0.191552 \\
\hline $\mathrm{H}$ & 3.447834 & -2.79594 & 1.246572 & $\mathrm{H}$ & 2.507062 & -3.21126 & -0.60382 \\
\hline $\mathrm{H}$ & 4.569112 & -2.4226 & -0.03044 & $\mathrm{H}$ & 1.823775 & -4.63296 & 0.137085 \\
\hline $\mathrm{H}$ & 2.68614 & -2.50545 & -1.70986 & $\mathrm{H}$ & 1.185228 & -3.1885 & 2.168087 \\
\hline $\mathrm{H}$ & 3.088627 & -4.01539 & -0.90785 & $\mathrm{H}$ & 2.873954 & -3.60361 & 1.928149 \\
\hline $\mathrm{H}$ & -0.32305 & 1.191301 & 1.60185 & $\mathrm{H}$ & -1.47985 & 1.541627 & -1.01697 \\
\hline $\mathrm{H}$ & -1.78899 & 1.804163 & 0.896191 & $\mathrm{H}$ & -2.40655 & 2.569995 & 0.026716 \\
\hline $\mathrm{H}$ & -1.42888 & 0.497815 & -1.17165 & $\mathrm{H}$ & -1.08068 & 2.107494 & 1.875111 \\
\hline $\mathrm{H}$ & 0.177568 & 0.049699 & -0.64798 & $\mathrm{H}$ & -1.12029 & 0.406086 & 1.573174 \\
\hline $\mathrm{H}$ & -1.62289 & -3.13151 & -0.03019 & $\mathrm{H}$ & 2.524071 & 1.219897 & 1.485358 \\
\hline $\mathrm{H}$ & -1.93039 & -2.09738 & -1.4162 & $\mathrm{H}$ & 1.249158 & 1.475775 & 2.65967 \\
\hline $\mathrm{H}$ & -0.71466 & -1.22253 & 1.240717 & $\mathrm{H}$ & 0.629424 & 0.679209 & -0.20824 \\
\hline $\mathrm{H}$ & -4.00841 & -0.95567 & 2.671396 & $\mathrm{H}$ & 0.849667 & 4.849684 & 0.076506 \\
\hline $\mathrm{H}$ & -2.20826 & -1.28312 & 2.952807 & $\mathrm{H}$ & -0.34737 & 3.989762 & 1.195814 \\
\hline $\mathrm{H}$ & 5.767614 & -0.94965 & -0.48079 & $\mathrm{H}$ & 1.99583 & -2.0127 & -2.18806 \\
\hline $\mathrm{H}$ & 5.594297 & 0.678439 & -1.14153 & $\mathrm{H}$ & 0.910194 & -2.4998 & -3.49579 \\
\hline $\mathrm{H}$ & 6.222928 & 0.459932 & 0.481907 & $\mathrm{H}$ & 0.706877 & -0.93698 & -2.72343 \\
\hline $\mathrm{H}$ & 1.546909 & -4.82049 & 1.055012 & $\mathrm{H}$ & 4.532045 & -1.84473 & 1.211857 \\
\hline $\mathrm{H}$ & 1.2208 & -3.24668 & 1.789415 & $\mathrm{H}$ & 3.613528 & -1.25145 & -0.17739 \\
\hline $\mathrm{H}$ & -0.10291 & -4.18921 & 1.089782 & $\mathrm{H}$ & 4.031187 & -0.15304 & 1.14157 \\
\hline $\mathrm{H}$ & 1.488362 & 3.753826 & -1.51182 & $\mathrm{H}$ & -4.06809 & -1.54858 & 0.715177 \\
\hline $\mathrm{H}$ & 0.410433 & 4.750741 & 0.670257 & $\mathrm{H}$ & -5.01488 & 0.232459 & -1.07956 \\
\hline $\mathrm{H}$ & -1.20407 & 4.180894 & 1.142642 & $\mathrm{H}$ & -4.64442 & 1.939957 & -0.76181 \\
\hline $\mathrm{H}$ & 0.209286 & 3.647554 & 2.04126 & $\mathrm{H}$ & -3.80337 & 1.087423 & -2.04906 \\
\hline $\mathrm{H}$ & -6.06653 & 0.840689 & -0.68599 & $\mathrm{H}$ & 3.252925 & 2.076233 & -3.20445 \\
\hline $\mathrm{H}$ & -6.34101 & -0.89759 & -0.95903 & $\mathrm{H}$ & 4.454474 & 2.077932 & -1.89052 \\
\hline $\mathrm{H}$ & -7.06353 & -0.09488 & 0.467639 & $\mathrm{H}$ & 3.770657 & 0.52878 & -2.47009 \\
\hline $\mathrm{H}$ & -2.51194 & 2.775103 & -1.06149 & $\mathrm{H}$ & -4.576 & 1.149802 & 2.897764 \\
\hline $\mathrm{H}$ & -1.90509 & 3.620795 & -2.49739 & $\mathrm{H}$ & -5.30421 & 1.674807 & 1.366741 \\
\hline $\mathrm{H}$ & -1.94424 & 4.461112 & -0.93545 & $\mathrm{H}$ & -3.74985 & 2.364127 & 1.901872 \\
\hline 3a_9 & & & & & & & \\
\hline $\mathrm{C}$ & 3.777359 & 1.637288 & 0.583889 & $\mathrm{C}$ & -3.84073 & -0.04986 & -1.42351 \\
\hline $\mathrm{C}$ & 4.043603 & 0.195627 & 0.172868 & $\mathrm{C}$ & -3.55621 & -1.46409 & -0.9376 \\
\hline $\mathrm{C}$ & 2.365822 & 2.067726 & 1.010001 & $\mathrm{C}$ & -2.71802 & 0.998376 & -1.44727 \\
\hline $\mathrm{C}$ & 1.449728 & 2.378684 & -0.17322 & $\mathrm{C}$ & -2.46852 & 1.650956 & -0.08794 \\
\hline $\mathrm{C}$ & 3.205797 & -0.82465 & 0.410117 & $\mathrm{C}$ & -2.32593 & -1.97106 & -0.76937 \\
\hline $\mathrm{C}$ & 3.473857 & -2.29405 & 0.190966 & $\mathrm{C}$ & -1.96511 & -3.40222 & -0.45387 \\
\hline $\mathrm{C}$ & 2.536308 & -3.03678 & -0.78748 & $\mathrm{C}$ & -1.15363 & -3.64378 & 0.838315 \\
\hline $\mathrm{C}$ & -0.03238 & 2.698378 & 0.182446 & $\mathrm{C}$ & -1.28065 & 2.658032 & -0.03073 \\
\hline $\mathrm{C}$ & -0.78544 & 1.481691 & 0.76172 & $\mathrm{C}$ & 0.091179 & 1.97673 & -0.22791 \\
\hline $\mathrm{C}$ & 1.095049 & -3.2593 & -0.34557 & $\mathrm{C}$ & 0.28988 & -3.1554 & 0.860377 \\
\hline$C$ & 0.009378 & -2.50249 & -1.00612 & $\mathrm{C}$ & 0.642014 & -1.98185 & 1.689465 \\
\hline
\end{tabular}




\begin{tabular}{|c|c|c|c|c|c|c|c|}
\hline $\mathrm{C}$ & -0.9055 & 0.278132 & -0.17987 & $\mathrm{C}$ & 0.488592 & 0.973648 & 0.863984 \\
\hline $\mathrm{C}$ & -1.36371 & -2.23732 & -0.42505 & $\mathrm{C}$ & 1.854991 & -1.0963 & 1.496215 \\
\hline $\mathrm{C}$ & -1.40693 & -1.00712 & 0.516899 & $\mathrm{C}$ & 1.650505 & 0.04583 & 0.466269 \\
\hline $\mathrm{C}$ & -2.76342 & -0.86545 & 1.195152 & $\mathrm{C}$ & 2.977068 & 0.757897 & 0.244133 \\
\hline $\mathrm{C}$ & -4.0219 & -0.55563 & 0.432693 & $\mathrm{C}$ & 3.866662 & 0.138999 & -0.79082 \\
\hline $\mathrm{O}$ & -5.12204 & -0.44808 & 0.931574 & $\mathrm{O}$ & 3.568409 & -0.84487 & -1.43796 \\
\hline $\mathrm{C}$ & -2.90702 & -1.02835 & 2.514674 & $\mathrm{C}$ & 3.387607 & 1.82827 & 0.930884 \\
\hline $\mathrm{C}$ & 5.407706 & 0.043685 & -0.46118 & $\mathrm{C}$ & -4.83292 & -2.25069 & -0.74526 \\
\hline $\mathrm{C}$ & 0.908293 & -3.89642 & 1.014665 & $\mathrm{C}$ & 1.163252 & -3.615 & -0.28652 \\
\hline $\mathrm{O}$ & 2.018004 & 3.497991 & -0.86665 & $\mathrm{O}$ & -3.67524 & 2.328003 & 0.287642 \\
\hline $\mathrm{O}$ & 0.297197 & -3.8598 & -1.40316 & $\mathrm{O}$ & 0.888242 & -3.3182 & 2.175931 \\
\hline $\mathrm{H}$ & 0.330829 & -1.7807 & -1.75496 & $\mathrm{H}$ & -0.19133 & -1.50636 & 2.203856 \\
\hline $\mathrm{C}$ & -0.12917 & 3.889391 & 1.142129 & $\mathrm{C}$ & -1.46366 & 3.788709 & -1.04869 \\
\hline $\mathrm{O}$ & -3.8102 & -0.40502 & -0.88756 & $\mathrm{O}$ & 5.038965 & 0.778337 & -0.94262 \\
\hline $\mathrm{C}$ & -4.9731 & -0.1138 & -1.69154 & $\mathrm{C}$ & 5.941237 & 0.231698 & -1.92575 \\
\hline $\mathrm{O}$ & -0.55613 & 3.046247 & -1.13017 & $\mathrm{O}$ & -1.4102 & 3.155583 & 1.330575 \\
\hline $\mathrm{C}$ & -1.88605 & 3.541801 & -1.21856 & $\mathrm{C}$ & -0.60359 & 4.257843 & 1.726383 \\
\hline $\mathrm{H}$ & 4.100115 & 2.295762 & -0.22968 & $\mathrm{H}$ & -4.67147 & 0.357725 & -0.83763 \\
\hline $\mathrm{H}$ & 4.468799 & 1.853784 & 1.40973 & $\mathrm{H}$ & -4.23963 & -0.149 & -2.44245 \\
\hline $\mathrm{H}$ & 1.91065 & 1.314469 & 1.657041 & $\mathrm{H}$ & -1.79337 & 0.565654 & -1.83586 \\
\hline $\mathrm{H}$ & 2.458132 & 2.976947 & 1.61065 & $\mathrm{H}$ & -3.01098 & 1.783718 & -2.14978 \\
\hline $\mathrm{H}$ & 1.439893 & 1.522124 & -0.857 & $\mathrm{H}$ & -2.26844 & 0.870718 & 0.65578 \\
\hline $\mathrm{H}$ & 2.24671 & -0.60043 & 0.870777 & $\mathrm{H}$ & -1.4759 & -1.31316 & -0.93335 \\
\hline $\mathrm{H}$ & 3.430482 & -2.79967 & 1.163814 & $\mathrm{H}$ & -1.39878 & -3.80964 & -1.30088 \\
\hline $\mathrm{H}$ & 4.492896 & -2.44426 & -0.1675 & $\mathrm{H}$ & -2.8648 & -4.01503 & -0.38582 \\
\hline $\mathrm{H}$ & 2.532255 & -2.5269 & -1.75552 & $\mathrm{H}$ & -1.68271 & -3.21236 & 1.693313 \\
\hline $\mathrm{H}$ & 2.961881 & -4.03313 & -0.9609 & $\mathrm{H}$ & -1.11498 & -4.72704 & 1.008488 \\
\hline $\mathrm{H}$ & -0.28022 & 1.181257 & 1.685726 & $\mathrm{H}$ & 0.079515 & 1.471883 & -1.1992 \\
\hline $\mathrm{H}$ & -1.78198 & 1.808924 & 1.075091 & $\mathrm{H}$ & 0.855456 & 2.754098 & -0.31795 \\
\hline $\mathrm{H}$ & -1.5516 & 0.522137 & -1.0243 & $\mathrm{H}$ & 0.71598 & 1.497886 & 1.797001 \\
\hline $\mathrm{H}$ & 0.076915 & 0.054959 & -0.59819 & $\mathrm{H}$ & -0.3692 & 0.339839 & 1.090191 \\
\hline $\mathrm{H}$ & -1.6985 & -3.12034 & 0.123592 & $\mathrm{H}$ & 2.701666 & -1.71389 & 1.191777 \\
\hline $\mathrm{H}$ & -2.06386 & -2.09321 & -1.25213 & $\mathrm{H}$ & 2.119701 & -0.6581 & 2.466029 \\
\hline $\mathrm{H}$ & -0.70349 & -1.21401 & 1.331454 & $\mathrm{H}$ & 1.387192 & -0.4282 & -0.48377 \\
\hline $\mathrm{H}$ & -3.879 & -0.93742 & 2.983205 & $\mathrm{H}$ & 4.357445 & 2.272579 & 0.753133 \\
\hline $\mathrm{H}$ & -2.05873 & -1.25659 & 3.149271 & $\mathrm{H}$ & 2.765859 & 2.288023 & 1.689793 \\
\hline $\mathrm{H}$ & 5.465204 & 0.643405 & -1.37653 & $\mathrm{H}$ & -5.45338 & -1.7777 & 0.024325 \\
\hline $\mathrm{H}$ & 6.186154 & 0.427399 & 0.208702 & $\mathrm{H}$ & -5.42748 & -2.24649 & -1.66635 \\
\hline $\mathrm{H}$ & 5.663432 & -0.98278 & -0.71841 & $\mathrm{H}$ & -4.67367 & -3.28775 & -0.45491 \\
\hline $\mathrm{H}$ & 1.505776 & -4.81093 & 1.081872 & $\mathrm{H}$ & 1.119421 & -4.7054 & -0.37111 \\
\hline $\mathrm{H}$ & 1.232533 & -3.22635 & 1.814796 & $\mathrm{H}$ & 0.823757 & -3.19172 & -1.23498 \\
\hline $\mathrm{H}$ & -0.13358 & -4.16073 & 1.190579 & $\mathrm{H}$ & 2.202694 & -3.32514 & -0.14214 \\
\hline $\mathrm{H}$ & 1.349025 & 3.747503 & -1.51879 & $\mathrm{H}$ & -3.43552 & 2.836849 & 1.074297 \\
\hline $\mathrm{H}$ & 0.416289 & 4.746564 & 0.744389 & $\mathrm{H}$ & -2.44342 & 4.254222 & -0.93131 \\
\hline $\mathrm{H}$ & -1.16817 & 4.178387 & 1.310638 & $\mathrm{H}$ & -0.69257 & 4.552815 & -0.93475 \\
\hline $\mathrm{H}$ & 0.295831 & 3.629587 & 2.113697 & $\mathrm{H}$ & -1.38519 & 3.399712 & -2.06574 \\
\hline $\mathrm{H}$ & -5.70002 & -0.92237 & -1.61194 & $\mathrm{H}$ & 6.220188 & -0.78868 & -1.66056 \\
\hline $\mathrm{H}$ & -5.43145 & 0.821258 & -1.36927 & $\mathrm{H}$ & 5.47754 & 0.237202 & -2.91266 \\
\hline $\mathrm{H}$ & -4.60611 & -0.02871 & -2.71099 & $\mathrm{H}$ & 6.813031 & 0.880717 & -1.91057 \\
\hline
\end{tabular}




$\begin{array}{lrlllrll}\mathrm{H} & -2.6155 & 2.843409 & -0.79438 & \mathrm{H} & 0.4619 & 4.076042 & 1.548502 \\ \mathrm{H} & -2.09571 & 3.657833 & -2.28247 & \mathrm{H} & -0.76032 & 4.379119 & 2.798769 \\ \mathrm{H} & -2.00028 & 4.515765 & -0.73223 & \mathrm{H} & -0.89381 & 5.185557 & 1.223042\end{array}$

\begin{tabular}{|c|c|c|c|c|c|c|c|}
\hline \multicolumn{4}{|c|}{ 3a_11 } & \multicolumn{4}{|c|}{ 3a_12 } \\
\hline $\mathrm{C}$ & 3.776762 & 1.638407 & 0.583567 & $\mathrm{C}$ & 3.595686 & 1.694311 & 0.776061 \\
\hline $\mathrm{C}$ & 4.043619 & 0.196819 & 0.17274 & $\mathrm{C}$ & 3.98288 & 0.319833 & 0.244782 \\
\hline $\mathrm{C}$ & 2.365083 & 2.068263 & 1.009879 & $\mathrm{C}$ & 2.12146 & 2.011796 & 1.072098 \\
\hline $\mathrm{C}$ & 1.448788 & 2.379184 & -0.17319 & $\mathrm{C}$ & 1.337867 & 2.47546 & -0.16692 \\
\hline $\mathrm{C}$ & 3.20609 & -0.82375 & 0.409724 & $\mathrm{C}$ & 3.221075 & -0.77606 & 0.373092 \\
\hline $\mathrm{C}$ & 3.474689 & -2.29305 & 0.190584 & $\mathrm{C}$ & 3.595874 & -2.19863 & 0.037017 \\
\hline $\mathrm{C}$ & 2.537198 & -3.03618 & -0.78764 & $\mathrm{C}$ & 2.641702 & -2.95493 & -0.91223 \\
\hline $\mathrm{C}$ & -0.03338 & 2.698242 & 0.182542 & $\mathrm{C}$ & -0.18568 & 2.729773 & 0.063642 \\
\hline $\mathrm{C}$ & -0.78597 & 1.481233 & 0.761825 & $\mathrm{C}$ & -0.90019 & 1.507112 & 0.686766 \\
\hline $\mathrm{C}$ & 1.096098 & -3.25918 & -0.34547 & $\mathrm{C}$ & 1.234801 & -3.23879 & -0.40059 \\
\hline $\mathrm{C}$ & 0.010069 & -2.50277 & -1.00588 & $\mathrm{C}$ & 0.093774 & -2.50717 & -0.99321 \\
\hline $\mathrm{C}$ & -0.90526 & 0.277489 & -0.17961 & $\mathrm{C}$ & -0.86558 & 0.239286 & -0.17449 \\
\hline $\mathrm{C}$ & -1.36304 & -2.23809 & -0.42468 & $\mathrm{C}$ & -1.25756 & -2.29335 & -0.34584 \\
\hline $\mathrm{C}$ & -1.40665 & -1.00776 & 0.517153 & $\mathrm{C}$ & -1.3215 & -1.04004 & 0.565102 \\
\hline $\mathrm{C}$ & -2.76335 & -0.86642 & 1.195078 & $\mathrm{C}$ & -2.67512 & -0.92711 & 1.254778 \\
\hline $\mathrm{C}$ & -4.02159 & -0.55621 & 0.432402 & $\mathrm{C}$ & -3.94985 & -0.67205 & 0.499591 \\
\hline $\mathrm{O}$ & -5.12173 & -0.44799 & 0.931141 & $\mathrm{O}$ & -5.05154 & -0.61668 & 1.003534 \\
\hline $\mathrm{C}$ & -2.90734 & -1.03007 & 2.514462 & $\mathrm{C}$ & -2.79989 & -1.07067 & 2.578376 \\
\hline $\mathrm{C}$ & 5.40795 & 0.045366 & -0.46096 & $\mathrm{C}$ & 5.370148 & 0.32393 & -0.35486 \\
\hline $\mathrm{C}$ & 0.909781 & -3.89632 & 1.014805 & $\mathrm{C}$ & 1.139131 & -3.90075 & 0.956736 \\
\hline $\mathrm{O}$ & 2.016667 & 3.498868 & -0.86643 & $\mathrm{O}$ & 1.965361 & 3.628621 & -0.7458 \\
\hline $\mathrm{O}$ & 0.298313 & -3.85999 & -1.40292 & $\mathrm{O}$ & 0.402753 & -3.84906 & -1.42537 \\
\hline $\mathrm{H}$ & 0.331175 & -1.78085 & -1.75475 & $\mathrm{H}$ & 0.35445 & -1.76527 & -1.746 \\
\hline $\mathrm{C}$ & -0.13063 & 3.889085 & 1.14243 & $\mathrm{C}$ & -0.41208 & 3.968574 & 0.942542 \\
\hline $\mathrm{O}$ & -3.80969 & -0.40608 & -0.88788 & $\mathrm{O}$ & -3.75036 & -0.50484 & -0.82068 \\
\hline $\mathrm{C}$ & -4.97237 & -0.11445 & -1.69204 & $\mathrm{C}$ & -4.92903 & -0.26485 & -1.61873 \\
\hline $\mathrm{O}$ & -0.55738 & 3.045952 & -1.12997 & $\mathrm{O}$ & -0.66613 & 2.955546 & -1.28196 \\
\hline $\mathrm{C}$ & -1.8873 & 3.541571 & -1.21812 & $\mathrm{C}$ & -2.02498 & 3.332096 & -1.44815 \\
\hline $\mathrm{H}$ & 4.099058 & 2.296882 & -0.23019 & $\mathrm{H}$ & 3.970939 & 2.451277 & 0.080015 \\
\hline $\mathrm{H}$ & 4.468247 & 1.855411 & 1.409225 & $\mathrm{H}$ & 4.176613 & 1.845619 & 1.695997 \\
\hline $\mathrm{H}$ & 1.910185 & 1.314676 & 1.656725 & $\mathrm{H}$ & 1.632584 & 1.157424 & 1.54241 \\
\hline $\mathrm{H}$ & 2.457107 & 2.977335 & 1.610794 & $\mathrm{H}$ & 2.091755 & 2.815534 & 1.817024 \\
\hline $\mathrm{H}$ & 1.439291 & 1.522784 & -0.85721 & $\mathrm{H}$ & 1.413331 & 1.719867 & -0.95115 \\
\hline $\mathrm{H}$ & 2.246821 & -0.59983 & 0.870137 & $\mathrm{H}$ & 2.241514 & -0.6636 & 0.83133 \\
\hline $\mathrm{H}$ & 3.431773 & -2.7987 & 1.163436 & $\mathrm{H}$ & 3.670913 & -2.76555 & 0.974126 \\
\hline $\mathrm{H}$ & 4.493688 & -2.44285 & -0.16816 & $\mathrm{H}$ & 4.591112 & -2.23927 & -0.40744 \\
\hline $\mathrm{H}$ & 2.532803 & -2.52635 & -1.7557 & $\mathrm{H}$ & 2.565793 & -2.42057 & -1.86391 \\
\hline $\mathrm{H}$ & 2.96312 & -4.03238 & -0.96109 & $\mathrm{H}$ & 3.096062 & -3.92852 & -1.13469 \\
\hline $\mathrm{H}$ & -0.28083 & 1.181217 & 1.686009 & $\mathrm{H}$ & -0.46324 & 1.308825 & 1.669891 \\
\hline $\mathrm{H}$ & -1.78274 & 1.808069 & 1.074892 & $\mathrm{H}$ & -1.93837 & 1.786777 & 0.890234 \\
\hline $\mathrm{H}$ & -1.55103 & 0.521233 & -1.02437 & $\mathrm{H}$ & -1.46775 & 0.384255 & -1.07226 \\
\hline $\mathrm{H}$ & 0.077407 & 0.054476 & -0.59743 & $\mathrm{H}$ & 0.157266 & 0.063875 & -0.51331 \\
\hline $\mathrm{H}$ & -1.69745 & -3.12114 & 0.124138 & $\mathrm{H}$ & -1.52349 & -3.17554 & 0.240307 \\
\hline $\mathrm{H}$ & -2.06334 & -2.0943 & -1.25169 & $\mathrm{H}$ & -2.00263 & -2.20427 & -1.14114 \\
\hline
\end{tabular}




$\begin{array}{lrrrrrrr}\mathrm{H} & -0.70335 & -1.21447 & 1.331874 & \mathrm{H} & -0.60403 & -1.20284 & 1.377376 \\ \mathrm{H} & -3.87947 & -0.93946 & 2.982743 & \mathrm{H} & -3.76939 & -1.00319 & 3.055938 \\ \mathrm{H} & -2.05922 & -1.25858 & 3.149192 & \mathrm{H} & -1.93806 & -1.25927 & 3.207767 \\ \mathrm{H} & 5.664241 & -0.98102 & -0.71794 & \mathrm{H} & 5.716264 & -0.65819 & -0.67255 \\ \mathrm{H} & 5.465434 & 0.644963 & -1.3764 & \mathrm{H} & 5.404149 & 0.991521 & -1.22328 \\ \mathrm{H} & 6.186043 & 0.429556 & 0.20906 & \mathrm{H} & 6.095562 & 0.718843 & 0.366189 \\ \mathrm{H} & 1.507418 & -4.81074 & 1.081875 & \mathrm{H} & 1.768469 & -4.79594 & 0.979378 \\ \mathrm{H} & 1.234139 & -3.22617 & 1.814826 & \mathrm{H} & 1.481604 & -3.23169 & 1.750043 \\ \mathrm{H} & -0.132 & -4.16078 & 1.191018 & \mathrm{H} & 0.116605 & -4.20187 & 1.180313 \\ \mathrm{H} & 1.347587 & 3.748197 & -1.51854 & \mathrm{H} & 2.077351 & 4.289236 & -0.05282 \\ \mathrm{H} & 0.414132 & 4.74669 & 0.744658 & \mathrm{H} & -0.01871 & 4.864894 & 0.458229 \\ \mathrm{H} & -1.16976 & 4.177351 & 1.311367 & \mathrm{H} & -1.47488 & 4.128732 & 1.132974 \\ \mathrm{H} & 0.294923 & 3.62938 & 2.113786 & \mathrm{H} & 0.074484 & 3.852001 & 1.913568 \\ \mathrm{H} & -5.69952 & -0.92285 & -1.61266 & \mathrm{H} & -5.61637 & -1.10747 & -1.54058 \\ \mathrm{H} & -5.43053 & 0.820688 & -1.36975 & \mathrm{H} & -5.43008 & 0.645661 & -1.2898 \\ \mathrm{H} & -4.60516 & -0.02936 & -2.71142 & \mathrm{H} & -4.57067 & -0.15713 & -2.63906 \\ \mathrm{H} & -2.61662 & 2.843608 & -0.79303 & \mathrm{H} & -2.72143 & 2.583101 & -1.05352 \\ \mathrm{H} & -2.09744 & 3.656783 & -2.28202 & \mathrm{H} & -2.18514 & 3.416625 & -2.524 \\ \mathrm{H} & -2.00117 & 4.515943 & -0.73251 & \mathrm{H} & -2.25091 & 4.30075 & -0.98891\end{array}$

\begin{tabular}{lrrrlrrr} 
3a_13 & \multicolumn{7}{c}{ 3a_14 } \\
C & -2.82579 & 2.23993 & 1.399055 & C & 2.666786 & 1.523141 & 1.46785 \\
C & -1.42111 & 2.797036 & 1.252573 & C & 3.425713 & 0.530171 & 0.601911 \\
C & -2.91056 & 0.696724 & 1.49786 & C & 2.203615 & 2.833473 & 0.799978 \\
C & -3.03782 & 0.038147 & 0.125778 & C & 1.184136 & 2.739361 & -0.34402 \\
C & -0.98686 & 3.292528 & 0.084327 & C & 3.087523 & -0.76769 & 0.628557 \\
C & 0.298065 & 4.02646 & -0.20221 & C & 3.79144 & -1.94682 & 0.008063 \\
C & 1.089629 & 3.55305 & -1.44058 & C & 2.973277 & -2.73931 & -1.038 \\
C & -2.7078 & -1.48143 & 0.066759 & C & -0.33456 & 2.735152 & 0.032034 \\
C & -1.1975 & -1.75103 & 0.25751 & C & -0.80876 & 1.427703 & 0.704873 \\
C & 1.942528 & 2.297253 & -1.29949 & C & 1.599407 & -3.20711 & -0.57934 \\
C & 1.513468 & 1.040579 & -1.95365 & C & 0.417047 & -2.47104 & -1.0733 \\
C & -0.29879 & -1.23354 & -0.87689 & C & -0.73032 & 0.178505 & -0.18072 \\
C & 1.992559 & -0.35214 & -1.59645 & C & -0.9295 & -2.37568 & -0.39314 \\
C & 1.180304 & -1.06532 & -0.48339 & C & -1.04945 & -1.1475 & 0.550781 \\
C & 1.881264 & -2.36926 & -0.12192 & C & -2.37585 & -1.15774 & 1.30041 \\
C & 3.011386 & -2.34988 & 0.868175 & C & -3.70035 & -1.00021 & 0.605862 \\
O & 3.643747 & -3.32306 & 1.221249 & O & -4.77922 & -1.047 & 1.157694 \\
C & 1.597658 & -3.55608 & -0.66583 & C & -2.42911 & -1.33249 & 2.625089 \\
C & -0.61567 & 2.797282 & 2.530486 & C & 4.599839 & 1.091018 & -0.16193 \\
C & 2.937497 & 2.300744 & -0.15982 & C & 1.563533 & -4.04516 & 0.68029 \\
O & -4.38305 & 0.257025 & -0.3177 & O & 1.435527 & 3.874913 & -1.1865 \\
O & 2.492704 & 1.894658 & -2.58402 & O & 0.788575 & -3.73513 & -1.66434 \\
H & 0.549047 & 1.077849 & -2.45746 & H & 0.637616 & -1.63874 & -1.73828 \\
C & -3.53477 & -2.2655 & 1.090054 & C & -0.69342 & 3.943088 & 0.905239 \\
O & 3.259914 & -1.11366 & 1.338355 & O & -3.57456 & -0.79611 & -0.7184 \\
C & 4.333438 & -0.99947 & 2.295114 & C & -4.80311 & -0.64699 & -1.46148 \\
O & -3.10945 & -1.8065 & -1.2921 & O & -0.93666 & 2.86198 & -1.28943 \\
C & -3.15207 & -3.17588 & -1.67275 & C & -2.34313 & 3.053027 & -1.38337
\end{tabular}




\begin{tabular}{|c|c|c|c|c|c|c|c|}
\hline $\mathrm{H}$ & -3.4522 & 2.569313 & 0.56613 & $\mathrm{H}$ & 3.338334 & 1.820849 & 2.285363 \\
\hline $\mathrm{H}$ & -3.26433 & 2.669907 & 2.307296 & $\mathrm{H}$ & 1.828672 & 1.01129 & 1.94624 \\
\hline $\mathrm{H}$ & -2.03824 & 0.310755 & 2.030587 & $\mathrm{H}$ & 1.836567 & 3.508463 & 1.576923 \\
\hline $\mathrm{H}$ & -3.7896 & 0.415437 & 2.084171 & $\mathrm{H}$ & 3.074666 & 3.332701 & 0.36774 \\
\hline $\mathrm{H}$ & -2.35872 & 0.542471 & -0.57237 & $\mathrm{H}$ & 1.37878 & 1.829489 & -0.92468 \\
\hline $\mathrm{H}$ & -1.67476 & 3.251496 & -0.76035 & $\mathrm{H}$ & 2.21919 & -1.02809 & 1.231457 \\
\hline & 0.951291 & 4.048035 & 0.671858 & $\mathrm{H}$ & 4.070781 & -2.63414 & 0.816821 \\
\hline $\mathrm{H}$ & 0.031856 & 5.074988 & -0.39194 & $\mathrm{H}$ & 4.728621 & -1.64442 & -0.46291 \\
\hline $\mathrm{H}$ & 0.40839 & 3.419323 & -2.28688 & $\mathrm{H}$ & 2.845646 & -2.13364 & -1.93993 \\
\hline 1 & 1.780882 & 4.355639 & -1.72536 & $\mathrm{H}$ & 3.552082 & -3.62427 & -1.3291 \\
\hline & -0.89128 & -1.29243 & 1.203167 & $\mathrm{H}$ & -0.22506 & 1.280835 & 1.618731 \\
\hline $\mathrm{H}$ & -1.04713 & -2.82516 & 0.396961 & $\mathrm{H}$ & -1.83873 & 1.575344 & 1.045808 \\
\hline $\mathrm{H}$ & -0.39054 & -1.87624 & -1.75751 & $\mathrm{H}$ & -1.39173 & 0.291437 & -1.04012 \\
\hline $\mathrm{H}$ & -0.6596 & -0.2557 & -1.19553 & $\mathrm{H}$ & 0.28296 & 0.092356 & -0.57455 \\
\hline $\mathrm{H}$ & 3.041899 & -0.29837 & -1.30266 & $\mathrm{H}$ & -1.12307 & -3.28622 & 0.177885 \\
\hline H & 1.955616 & -0.96528 & -2.50518 & $\mathrm{H}$ & -1.69905 & -2.31516 & -1.16754 \\
\hline $\mathrm{H}$ & 1.212007 & -0.42388 & 0.401013 & $\mathrm{H}$ & -0.28695 & -1.27076 & 1.327972 \\
\hline $\mathrm{H}$ & 2.164883 & -4.4349 & -0.38635 & $\mathrm{H}$ & -3.37881 & -1.35516 & 3.14481 \\
\hline $\mathrm{H}$ & 0.806769 & -3.68197 & -1.39403 & $\mathrm{H}$ & -1.52729 & -1.45697 & 3.213026 \\
\hline $\mathrm{H}$ & 0.425062 & 3.085907 & 2.387234 & $\mathrm{H}$ & 5.197806 & 0.319445 & -0.64558 \\
\hline $\mathrm{H}$ & -1.05871 & 3.486655 & 3.259229 & $\mathrm{H}$ & 4.268082 & 1.787284 & -0.93938 \\
\hline $\mathrm{H}$ & -0.62471 & 1.807151 & 2.997733 & $\mathrm{H}$ & 5.256212 & 1.661135 & 0.505874 \\
\hline $\mathrm{H}$ & 3.534206 & 3.217784 & -0.18958 & $\mathrm{H}$ & 2.246914 & -4.89375 & 0.576911 \\
\hline $\mathrm{H}$ & 2.42726 & 2.262794 & 0.805602 & $\mathrm{H}$ & 1.876804 & -3.4704 & 1.555412 \\
\hline $\mathrm{H}$ & 3.615001 & 1.450556 & -0.21605 & $\mathrm{H}$ & 0.565112 & -4.43894 & 0.866989 \\
\hline $\mathrm{H}$ & -4.47199 & -0.29122 & -1.10961 & $\mathrm{H}$ & 0.677495 & 3.903981 & -1.7879 \\
\hline $\mathrm{H}$ & -4.59847 & -2.05993 & 0.957141 & $\mathrm{H}$ & -0.31793 & 4.864468 & 0.456951 \\
\hline $\mathrm{H}$ & -3.36803 & -3.34031 & 0.998071 & $\mathrm{H}$ & -1.77354 & 4.028244 & 1.035073 \\
\hline $\mathrm{H}$ & -3.25219 & -1.97846 & 2.104925 & $\mathrm{H}$ & -0.25976 & 3.839739 & 1.901481 \\
\hline $\mathrm{H}$ & 4.117793 & -1.59972 & 3.179353 & $\mathrm{H}$ & -5.4131 & -1.5456 & -1.36746 \\
\hline $\mathrm{H}$ & 5.272934 & -1.33005 & 1.851372 & $\mathrm{H}$ & -5.36382 & 0.213905 & -1.09709 \\
\hline $\mathrm{H}$ & 4.382411 & 0.055395 & 2.552083 & $\mathrm{H}$ & -4.50007 & -0.49678 & -2.49431 \\
\hline $\mathrm{H}$ & -2.20409 & -3.68977 & -1.47937 & $\mathrm{H}$ & -2.90135 & 2.258381 & -0.87797 \\
\hline & -3.33938 & -3.18707 & -2.74704 & $\mathrm{H}$ & -2.58121 & 3.021269 & -2.44714 \\
\hline & -3.95867 & -3.7201 & -1.17113 & $\mathrm{H}$ & -2.65874 & 4.021694 & -0.98362 \\
\hline
\end{tabular}

\begin{tabular}{lrrllllrr} 
3a_15 & \multicolumn{7}{c}{ 3a_16 } \\
C & 3.045464 & 2.411701 & 0.276058 & C & 3.555691 & 0.885472 & 1.506945 \\
C & 3.531361 & 0.99416 & 0.514141 & C & 3.740374 & -0.4025 & 0.729431 \\
C & 1.589858 & 2.662048 & 0.766281 & C & 2.851156 & 2.055001 & 0.77459 \\
C & 0.603206 & 2.863707 & -0.39335 & C & 1.450134 & 1.660215 & 0.283011 \\
C & 3.649724 & 0.124271 & -0.49871 & C & 3.050512 & -1.50374 & 1.05903 \\
C & 4.19749 & -1.27918 & -0.46904 & C & 3.134255 & -2.86645 & 0.412038 \\
C & 3.372133 & -2.32243 & -1.25021 & C & 2.221109 & -3.11077 & -0.81418 \\
C & -0.89646 & 2.707795 & -0.02977 & C & 0.417008 & 2.805459 & 0.014289 \\
C & -1.19007 & 1.337689 & 0.628531 & C & -1.04316 & 2.282331 & 0.092954 \\
C & 2.09142 & -2.8373 & -0.60406 & C & 0.711069 & -3.09585 & -0.59913 \\
C & 0.780024 & -2.35714 & -1.09438 & C & -0.09202 & -1.98881 & -1.16929 \\
C & -0.8266 & 0.114417 & -0.22335 & C & -1.43462 & 1.011199 & -0.69525
\end{tabular}




\begin{tabular}{|c|c|c|c|c|c|c|c|}
\hline $\mathrm{C}$ & -0.53671 & -2.43942 & -0.34918 & $\mathrm{C}$ & -1.47734 & -1.57368 & -0.72278 \\
\hline $\mathrm{C}$ & -0.86507 & -1.22272 & 0.552208 & $\mathrm{C}$ & -1.45917 & -0.29118 & 0.153593 \\
\hline $\mathrm{C}$ & -2.14757 & -1.46455 & 1.339298 & $\mathrm{C}$ & -2.57054 & -0.28679 & 1.193789 \\
\hline $\mathrm{C}$ & -3.49255 & -1.58226 & 0.677709 & $\mathrm{C}$ & -4.02442 & -0.31718 & 0.810107 \\
\hline $\mathrm{O}$ & -4.52763 & -1.83455 & 1.257288 & $\mathrm{O}$ & -4.94835 & -0.26559 & 1.594551 \\
\hline $\mathrm{C}$ & -2.13793 & -1.60583 & 2.668913 & $\mathrm{C}$ & -2.31378 & -0.26277 & 2.506263 \\
\hline $\mathrm{C}$ & 3.886686 & 0.691611 & 1.94984 & $\mathrm{C}$ & 4.739169 & -0.33843 & -0.39769 \\
\hline $\mathrm{C}$ & 2.249292 & -3.46581 & 0.763374 & $\mathrm{C}$ & 0.1985 & -3.96673 & 0.526854 \\
\hline $\mathrm{O}$ & 0.778016 & 4.175383 & -0.95386 & $\mathrm{O}$ & 1.576789 & 0.866392 & -0.90112 \\
\hline $\mathrm{O}$ & 1.311542 & -3.62211 & -1.54728 & $\mathrm{O}$ & 0.002135 & -3.25454 & -1.86053 \\
\hline $\mathrm{H}$ & 0.817906 & -1.59079 & -1.86699 & $\mathrm{H}$ & 0.481288 & -1.19412 & -1.64041 \\
\hline $\mathrm{C}$ & -1.35773 & 3.845596 & 0.890917 & $\mathrm{C}$ & 0.558564 & 3.967711 & 1.006165 \\
\hline $\mathrm{O}$ & -3.44104 & -1.37296 & -0.65062 & $\mathrm{O}$ & -4.20868 & -0.41822 & -0.51828 \\
\hline $\mathrm{C}$ & -4.69216 & -1.47692 & -1.36269 & $\mathrm{C}$ & -5.57725 & -0.46632 & -0.97258 \\
\hline $\mathrm{O}$ & -1.54241 & 2.781573 & -1.32351 & $\mathrm{O}$ & 0.772211 & 3.241949 & -1.32263 \\
\hline $\mathrm{C}$ & -2.9614 & 2.78594 & -1.35962 & $\mathrm{C}$ & -0.00993 & 4.252202 & -1.94744 \\
\hline $\mathrm{H}$ & 3.127386 & 2.644402 & -0.79066 & $\mathrm{H}$ & 4.541042 & 1.242992 & 1.83358 \\
\hline $\mathrm{H}$ & 3.724194 & 3.101477 & 0.78938 & $\mathrm{H}$ & 2.986164 & 0.670752 & 2.417566 \\
\hline $\mathrm{H}$ & 1.282196 & 1.822691 & 1.39112 & $\mathrm{H}$ & 2.799152 & 2.883675 & 1.483984 \\
\hline $\mathrm{H}$ & 1.548876 & 3.555125 & 1.395415 & $\mathrm{H}$ & 3.442198 & 2.403427 & -0.07785 \\
\hline $\mathrm{H}$ & 0.80664 & 2.123957 & -1.17531 & $\mathrm{H}$ & 1.008703 & 1.013834 & 1.04685 \\
\hline $\mathrm{H}$ & 3.384458 & 0.479255 & -1.49474 & $\mathrm{H}$ & 2.345529 & -1.41911 & 1.88463 \\
\hline $\mathrm{H}$ & 4.365843 & -1.6233 & 0.553101 & $\mathrm{H}$ & 2.922081 & -3.62852 & 1.169075 \\
\hline $\mathrm{H}$ & 5.190523 & -1.25637 & -0.93783 & $\mathrm{H}$ & 4.158919 & -3.05949 & 0.080624 \\
\hline $\mathrm{H}$ & 3.124104 & -1.92783 & -2.24065 & $\mathrm{H}$ & 2.464656 & -2.39049 & -1.59972 \\
\hline $\mathrm{H}$ & 4.005087 & -3.20222 & -1.41821 & $\mathrm{H}$ & 2.464127 & -4.10407 & -1.21371 \\
\hline $\mathrm{H}$ & -0.67368 & 1.292491 & 1.591563 & $\mathrm{H}$ & -1.26106 & 2.130434 & 1.155222 \\
\hline $\mathrm{H}$ & -2.25471 & 1.308084 & 0.879082 & $\mathrm{H}$ & -1.69577 & 3.106381 & -0.20846 \\
\hline $\mathrm{H}$ & -1.48315 & 0.063204 & -1.09317 & $\mathrm{H}$ & -2.4273 & 1.149372 & -1.12845 \\
\hline $\mathrm{H}$ & 0.190409 & 0.225995 & -0.60484 & $\mathrm{H}$ & -0.74929 & 0.867157 & -1.5297 \\
\hline $\mathrm{H}$ & -0.54277 & -3.34441 & 0.261717 & $\mathrm{H}$ & -1.94918 & -2.39222 & -0.17627 \\
\hline $\mathrm{H}$ & -1.32702 & -2.56044 & -1.09561 & $\mathrm{H}$ & -2.09029 & -1.40532 & -1.61296 \\
\hline $\mathrm{H}$ & -0.07562 & -1.1653 & 1.310204 & $\mathrm{H}$ & -0.53297 & -0.32678 & 0.732934 \\
\hline $\mathrm{H}$ & -3.05469 & -1.79265 & 3.214085 & $\mathrm{H}$ & -3.11952 & -0.26243 & 3.22987 \\
\hline $\mathrm{H}$ & -1.21675 & -1.53761 & 3.235703 & $\mathrm{H}$ & -1.29712 & -0.24813 & 2.881078 \\
\hline $\mathrm{H}$ & 4.105175 & -0.36055 & 2.129339 & $\mathrm{H}$ & 4.910106 & -1.30576 & -0.87084 \\
\hline $\mathrm{H}$ & 4.765814 & 1.273441 & 2.251817 & $\mathrm{H}$ & 4.388866 & 0.352299 & -1.17097 \\
\hline $\mathrm{H}$ & 3.075458 & 0.985477 & 2.624198 & $\mathrm{H}$ & 5.703069 & 0.043403 & -0.04147 \\
\hline $\mathrm{H}$ & 3.035578 & -4.22659 & 0.734965 & $\mathrm{H}$ & 0.630446 & -4.96958 & 0.450855 \\
\hline $\mathrm{H}$ & 2.533106 & -2.71901 & 1.508989 & $\mathrm{H}$ & 0.47729 & -3.55582 & 1.501 \\
\hline $\mathrm{H}$ & 1.328438 & -3.94464 & 1.093449 & $\mathrm{H}$ & -0.88575 & -4.06551 & 0.493598 \\
\hline $\mathrm{H}$ & 1.629005 & 4.19043 & -1.40289 & $\mathrm{H}$ & 1.52046 & 1.509654 & -1.62416 \\
\hline $\mathrm{H}$ & -1.22205 & 4.80904 & 0.39891 & $\mathrm{H}$ & 1.484375 & 4.518648 & 0.83413 \\
\hline $\mathrm{H}$ & -2.40792 & 3.731876 & 1.1671 & $\mathrm{H}$ & -0.27733 & 4.665209 & 0.914186 \\
\hline $\mathrm{H}$ & -0.77602 & 3.845782 & 1.815488 & $\mathrm{H}$ & 0.561255 & 3.596911 & 2.034226 \\
\hline $\mathrm{H}$ & -5.10943 & -2.47772 & -1.24942 & $\mathrm{H}$ & -6.08636 & -1.33285 & -0.55006 \\
\hline $\mathrm{H}$ & -5.40382 & -0.74092 & -0.98819 & $\mathrm{H}$ & -6.10502 & 0.442725 & -0.68315 \\
\hline $\mathrm{H}$ & -4.44967 & -1.27922 & -2.40349 & $\mathrm{H}$ & -5.52043 & -0.54702 & -2.05496 \\
\hline $\mathrm{H}$ & -3.39846 & 1.879548 & -0.92426 & $\mathrm{H}$ & -1.03303 & 3.91925 & -2.15009 \\
\hline
\end{tabular}


2.313467

$-1.68084$

2.131719

\subsection{8}

$-2.73696$

1.22021

C

$\mathrm{C}$

3.025395

$-0.48431$

1.45122

2.091974

0.263715

0.486412

0.391933

$-2.89098$

1.128411

$\mathrm{C}$

$\mathrm{C}$

$\mathrm{C}$

$\mathrm{C}$

C

C

C

C

C

C

C

$\mathrm{O}$

C

C

C

O

O

$\mathrm{H}$

C

$\mathrm{O}$

C

$\mathrm{O}$

C

$\mathrm{H}$

$\mathrm{H}$

$\mathrm{H}$

$\mathrm{H}$

$\mathrm{H}$

$\mathrm{H}$

$\mathrm{H}$

$\mathrm{H}$

$\mathrm{H}$

$\mathrm{H}$

$\mathrm{H}$

$\mathrm{H}$

$\mathrm{H}$

$\mathrm{H}$

$\mathrm{H}$

$\mathrm{H}$

$\mathrm{H}$

$\begin{array}{llll}-0.35818 & -3.9025 & 0.294597\end{array}$

$\begin{array}{lll}-0.7162 & -3.4682 & -1.14688\end{array}$

$\begin{array}{lll}2.408961 & 1.769224 & 0.1707\end{array}$

$\begin{array}{lll}1.119765 & 2.540419 & -0.22425\end{array}$

$\begin{array}{lll}-1.68608 & -2.30485 & -1.31956\end{array}$

$\begin{array}{lll}-1.16777 & -0.99862 & -1.78993\end{array}$

$\begin{array}{lll}0.16443 & 1.901388 & -1.26042\end{array}$

$\begin{array}{lll}-1.84734 & 0.345163 & -1.62739\end{array}$

$\begin{array}{llll}-1.11258 & 1.292603 & -0.63918\end{array}$

$\begin{array}{lll}-2.08788 & 2.339739 & -0.11544\end{array}$

$\begin{array}{lll}-3.05963 & 1.953076 & 0.965959\end{array}$

$\begin{array}{lll}-3.95572 & 2.657489 & 1.380955\end{array}$

$\begin{array}{lll}-2.17854 & 3.599123 & -0.55292\end{array}$

$\begin{array}{llll}2.723638 & -3.56808 & 0.461972\end{array}$

$\begin{array}{lll}-3.00858 & -2.44063 & -0.59967\end{array}$

$\begin{array}{lll}2.010507 & -0.4646 & -0.74518\end{array}$

$\begin{array}{lll}-1.79324 & -1.91503 & -2.71659\end{array}$

$\begin{array}{lll}-0.08745 & -0.94435 & -1.90095\end{array}$

$\begin{array}{llll}3.054737 & 2.502444 & 1.353004\end{array}$

$\begin{array}{lll}-2.82309 & 0.720756 & 1.453289\end{array}$

$\begin{array}{lll}-3.69929 & 0.276355 & 2.50961\end{array}$

3.355643

1.671

$-0.92426$

$\begin{array}{llll}3.779615 & 2.859948 & -1.57987\end{array}$

3.037908

$-2.16156$

1.519929

$-1.27787$

2.802131

3.370709

0.171375

2.770054

3.914557

$-0.81046$

2.253067

1.090974

0.243068

0.90314

$-0.2291$

$-2.21267$

0.925605

$-1.27694$

$-4.18229$

1.711338

0.224357

$-4.82434$

0.820721

0.200606

$-3.23519$

0.20918

$-1.17302$

$-4.32952$

$-1.69465$

0.572805

2.71818

$-1.65156$

1.423793

3.534624

0.707203

$-0.12098$

2.64470

$-0.56249$

0.689795

1.119772

$-2.01025$

$-2.88304$

0.20475

$-1.80498$

$-1.88712$

0.829327

$-1.31433$

$-0.81675$

0.686462
$-2.61137$

0.220292 3a_18

$\mathrm{C}$

C

C

C

C

C

C

C

C

C

C

C

C

C

C

C

$\mathrm{O}$

C

C

C

$\mathrm{O}$

$\mathrm{O}$

$\mathrm{H}$

C

$\mathrm{O}$

C

$\mathrm{O}$

C

$\mathrm{H}$

$\mathrm{H}$

$\mathrm{H}$

$\mathrm{H}$

$\mathrm{H}$

$\mathrm{H}$

$\mathrm{H}$

$\mathrm{H}$

$\mathrm{H}$

$\mathrm{H}$

$\mathrm{H}$

$\mathrm{H}$

$\mathrm{H}$

$\mathrm{H}$

$\mathrm{H}$

$\mathrm{H}$

$\mathrm{H}$

$-3.84076$

$-3.55618$

$-0.04997$

$-1.42347$

$-2.71808$

$-1.46422$

$-0.93765$

$-2.46859$

0.998288

$-1.44723$

$-2.32589$

650904

$-0.08791$

$-1.96503$

$-1.9711$

$-0.76924$

$-1.15352$

$-0.45375$

$-1.28075$

$-3.64377$

0.838418

0.091107

1.976769

$-0.03074$

0.289978

$-3.15534$

$-0.22794$

0.642049

$-1.98174$

0.860458

0.488617

0.973765

1.689507

1.855016

$-1.09618$

0.863988

1.650527

0.045943

1.496226

2.977104

0.757974

0.466263

3.866696

0.13898

0.244077

3.568507

$-0.84505$

$-0.79081$

3.387687

1.828353

$-1.43774$

$-4.83286$

$-2.25094$

0.930792

1.163313

$-3.61489$

$-0.74553$

$-3.67533$

0.888345

$-3.318$

$-0.28648$

$-0.19129$

$-1.50628$

$-1.46382$

3.788688

2.20392

5.038904

0.778439

$-1.0487$

5.941212

0.231693

$-0.94282$

$-1.41029$

3.155592

$-1.92584$

$-0.60369$

4.257883

1.330558

$-4.67149$

0.357553

1.726339

$-4.23973$

$-0.14906$

$-0.83751$

$-1.79342$

0.565565

$-2.44239$

$-3.01104$

1.783628

$-1.83581$

$-2.26847$

0.870696

$-2.14975$

$-1.47589$

$-1.31313$ 


$\begin{array}{lrrrlrrr}\mathrm{H} & -2.92582 & 4.264818 & -0.1398 & \mathrm{H} & 4.35755 & 2.272612 & 0.753037 \\ \mathrm{H} & -1.52313 & 3.997195 & -1.316 & \mathrm{H} & 2.76596 & 2.288139 & 1.689698 \\ \mathrm{H} & 2.266231 & -4.38534 & -0.09621 & \mathrm{H} & -4.67356 & -3.28801 & -0.45522 \\ \mathrm{H} & 3.26388 & -2.93863 & -0.2518 & \mathrm{H} & -5.45346 & -1.77805 & 0.024 \\ \mathrm{H} & 3.469855 & -3.99426 & 1.142471 & \mathrm{H} & -5.4273 & -2.24675 & -1.6667 \\ \mathrm{H} & -3.45227 & -3.41703 & -0.81866 & \mathrm{H} & 1.119513 & -4.70529 & -0.37109 \\ \mathrm{H} & -2.87673 & -2.36736 & 0.482621 & \mathrm{H} & 0.823742 & -3.19159 & -1.23492 \\ \mathrm{H} & -3.7147 & -1.67269 & -0.91249 & \mathrm{H} & 2.202757 & -3.325 & -0.14216 \\ \mathrm{H} & 2.677322 & -0.046 & -1.31166 & \mathrm{H} & -3.43564 & 2.836784 & 1.074303 \\ \mathrm{H} & 4.065669 & 2.1355 & 1.53617 & \mathrm{H} & -2.44368 & 4.254028 & -0.93143 \\ \mathrm{H} & 3.10915 & 3.576302 & 1.159511 & \mathrm{H} & -0.69286 & 4.552915 & -0.93464 \\ \mathrm{H} & 2.464099 & 2.365049 & 2.261982 & \mathrm{H} & -1.38516 & 3.399724 & -2.06575 \\ \mathrm{H} & -3.63753 & 0.952789 & 3.362345 & \mathrm{H} & 6.220392 & -0.78855 & -1.66038 \\ \mathrm{H} & -4.72906 & 0.232353 & 2.154068 & \mathrm{H} & 5.477453 & 0.236797 & -2.91274 \\ \mathrm{H} & -3.34678 & -0.71483 & 2.782467 & \mathrm{H} & 6.812879 & 0.88089 & -1.91092 \\ \mathrm{H} & 2.968415 & 3.333182 & -2.14234 & \mathrm{H} & 0.46178 & 4.076183 & 1.548273 \\ \mathrm{H} & 4.558544 & 2.557271 & -2.28018 & \mathrm{H} & -0.76027 & 4.379043 & 2.798762 \\ \mathrm{H} & 4.203302 & 3.587401 & -0.88013 & \mathrm{H} & -0.89407 & 5.185617 & 1.223121\end{array}$

\begin{tabular}{|c|c|c|c|c|c|c|c|}
\hline 3a_19 & & & & 3a_20 & & & \\
\hline $\mathrm{C}$ & -3.42181 & 1.697475 & 1.21106 & $\mathrm{C}$ & 3.410508 & 1.926421 & 0.585684 \\
\hline $\mathrm{C}$ & -2.57499 & 2.534067 & 0.256876 & $\mathrm{C}$ & 3.546246 & 0.685444 & -0.29052 \\
\hline $\mathrm{C}$ & -2.91982 & 0.255485 & 1.447382 & $\mathrm{C}$ & 1.973436 & 2.233729 & 1.064771 \\
\hline $\mathrm{C}$ & -3.0405 & -0.66132 & 0.233759 & $\mathrm{C}$ & 1.042878 & 2.789635 & -0.02148 \\
\hline $\mathrm{C}$ & -1.44671 & 3.109774 & 0.695253 & $\mathrm{C}$ & 3.617491 & -0.52537 & 0.279955 \\
\hline $\mathrm{C}$ & -0.50896 & 4.040917 & -0.03622 & $\mathrm{C}$ & 3.81246 & -1.87268 & -0.3748 \\
\hline $\mathrm{C}$ & 0.962979 & 3.933755 & 0.404108 & $\mathrm{C}$ & 3.150269 & -3.04165 & 0.377515 \\
\hline $\mathrm{C}$ & -2.29053 & -2.02031 & 0.343761 & $\mathrm{C}$ & -0.46792 & 2.858777 & 0.366212 \\
\hline $\mathrm{C}$ & -0.75934 & -1.84791 & 0.462411 & $\mathrm{C}$ & -0.99933 & 1.503033 & 0.890992 \\
\hline $\mathrm{C}$ & 1.836033 & 2.873257 & -0.25957 & $\mathrm{C}$ & 1.670224 & -3.30833 & 0.122829 \\
\hline $\mathrm{C}$ & 1.251736 & 1.777978 & -1.06106 & $\mathrm{C}$ & 0.808801 & -2.31823 & -0.55575 \\
\hline $\mathrm{C}$ & -0.10776 & -0.99011 & -0.62953 & $\mathrm{C}$ & -0.83649 & 0.330496 & -0.0842 \\
\hline $\mathrm{C}$ & 1.895762 & 0.434255 & -1.33091 & $\mathrm{C}$ & -0.69431 & -2.20665 & -0.41601 \\
\hline $\mathrm{C}$ & 1.387962 & -0.70105 & -0.3982 & $\mathrm{C}$ & -1.1145 & -1.05736 & 0.539738 \\
\hline $\mathrm{C}$ & 2.287178 & -1.92057 & -0.55652 & $\mathrm{C}$ & -2.53714 & -1.22547 & 1.055185 \\
\hline $\mathrm{C}$ & 3.561622 & -2.00379 & 0.235941 & $\mathrm{C}$ & -3.73077 & -1.2219 & 0.140741 \\
\hline $\mathrm{O}$ & 4.35516 & -2.91974 & 0.182847 & $\mathrm{O}$ & -4.87841 & -1.37549 & 0.501824 \\
\hline $\mathrm{C}$ & 2.054166 & -2.93093 & -1.39918 & $\mathrm{C}$ & -2.79782 & -1.39469 & 2.355794 \\
\hline $\mathrm{C}$ & -3.12197 & 2.703565 & -1.13967 & $\mathrm{C}$ & 3.644751 & 0.924207 & -1.77728 \\
\hline $\mathrm{C}$ & 3.20836 & 2.758405 & 0.369581 & $\mathrm{C}$ & 1.095224 & -4.37409 & 1.032291 \\
\hline $\mathrm{O}$ & -4.43475 & -0.89394 & $-2.3 \mathrm{E}-05$ & $\mathrm{O}$ & 1.524402 & 4.059728 & -0.48302 \\
\hline $\mathrm{O}$ & 1.837968 & 2.927934 & -1.71133 & $\mathrm{O}$ & 1.323053 & -3.45828 & -1.28026 \\
\hline $\mathrm{H}$ & 0.168813 & 1.776622 & -1.13862 & $\mathrm{H}$ & 1.296051 & -1.39364 & -0.85137 \\
\hline $\mathrm{C}$ & -2.82381 & -2.86135 & 1.508001 & $\mathrm{C}$ & -0.72229 & 3.96152 & 1.402045 \\
\hline $\mathrm{O}$ & 3.74073 & -0.93468 & 1.033068 & $\mathrm{O}$ & -3.39851 & -1.02786 & -1.14828 \\
\hline $\mathrm{C}$ & 4.939741 & -0.93168 & 1.835107 & $\mathrm{C}$ & -4.48793 & -1.01781 & -2.09476 \\
\hline $\mathrm{O}$ & -2.63343 & -2.61874 & -0.93839 & $\mathrm{O}$ & -1.09135 & 3.180698 & -0.90004 \\
\hline $\mathrm{C}$ & -2.33553 & -3.99389 & -1.14471 & $\mathrm{C}$ & -2.48973 & 3.424715 & -0.91272 \\
\hline $\mathrm{H}$ & -4.45194 & 1.654362 & 0.845676 & $\mathrm{H}$ & 3.801245 & 2.799533 & 0.054863 \\
\hline
\end{tabular}




\begin{tabular}{|c|c|c|c|c|c|c|c|}
\hline $\mathrm{H}$ & -3.45696 & 2.195815 & 2.184554 & $\mathrm{H}$ & 4.032232 & 1.798328 & 1.476599 \\
\hline $\mathrm{H}$ & -1.87854 & 0.300467 & 1.775803 & $\mathrm{H}$ & 1.557901 & 1.322605 & 1.498187 \\
\hline $\mathrm{H}$ & -3.49599 & -0.18406 & 2.26803 & $\mathrm{H}$ & 2.028726 & 2.965693 & 1.880887 \\
\hline $\mathrm{H}$ & -2.62661 & -0.15826 & -0.64597 & $\mathrm{H}$ & 1.089224 & 2.157882 & -0.90912 \\
\hline $\mathrm{H}$ & -1.18466 & 2.948933 & 1.742006 & $\mathrm{H}$ & 3.584774 & -0.56097 & 1.369875 \\
\hline $\mathrm{H}$ & -0.82813 & 5.071318 & 0.169246 & $\mathrm{H}$ & 4.889275 & -2.08461 & -0.40936 \\
\hline & -0.57653 & 3.927575 & -1.1215 & $\mathrm{H}$ & 3.480174 & -1.87559 & -1.41625 \\
\hline & 1.460135 & 4.892704 & 0.215812 & $\mathrm{H}$ & 3.673982 & -3.97012 & 0.12079 \\
\hline $\mathrm{H}$ & 1.00687 & 3.783161 & 1.488832 & $\mathrm{H}$ & 3.292787 & -2.90688 & 1.455883 \\
\hline $\mathrm{H}$ & -0.54225 & -1.41127 & 1.442718 & $\mathrm{H}$ & -0.50129 & 1.275626 & 1.838303 \\
\hline & -0.3021 & -2.8419 & 0.480951 & $\mathrm{H}$ & -2.05568 & 1.626206 & 1.148467 \\
\hline $\mathrm{H}$ & -0.26683 & -1.44541 & -1.611 & $\mathrm{H}$ & -1.47779 & 0.476767 & -0.95421 \\
\hline $\mathrm{H}$ & -0.61755 & -0.02823 & -0.66643 & $\mathrm{H}$ & 0.187911 & 0.314514 & -0.45872 \\
\hline $\mathrm{H}$ & 2.979886 & 0.520458 & -1.24881 & $\mathrm{H}$ & -1.10804 & -3.15082 & -0.05768 \\
\hline $\mathrm{H}$ & 1.681835 & 0.155273 & -2.37012 & $\mathrm{H}$ & -1.12024 & -2.03191 & -1.40786 \\
\hline $\mathrm{H}$ & 1.50005 & -0.3468 & 0.630271 & $\mathrm{H}$ & -0.48033 & -1.13979 & 1.430364 \\
\hline $\mathrm{H}$ & 2.76064 & -3.74732 & -1.48078 & $\mathrm{H}$ & -3.81459 & -1.51939 & 2.706814 \\
\hline $\mathrm{H}$ & 1.169441 & -2.97197 & -2.02133 & $\mathrm{H}$ & -2.00253 & -1.41201 & 3.091704 \\
\hline $\mathrm{H}$ & -3.4434 & 1.744939 & -1.55808 & $\mathrm{H}$ & 3.609453 & 0.002873 & -2.35938 \\
\hline $\mathrm{H}$ & -4.01334 & 3.342888 & -1.12004 & $\mathrm{H}$ & 2.846526 & 1.584471 & -2.12946 \\
\hline H & -2.40612 & 3.15655 & -1.82658 & $\mathrm{H}$ & 4.587917 & 1.431545 & -2.01438 \\
\hline $\mathrm{H}$ & 3.683326 & 3.743894 & 0.399286 & $\mathrm{H}$ & 1.072744 & -4.02491 & 2.068681 \\
\hline $\mathrm{H}$ & 3.129502 & 2.393333 & 1.39728 & $\mathrm{H}$ & 0.086283 & -4.6603 & 0.738652 \\
\hline $\mathrm{H}$ & 3.859388 & 2.086668 & -0.18718 & $\mathrm{H}$ & 1.723123 & -5.26956 & 0.995415 \\
\hline $\mathrm{H}$ & -4.45987 & -1.55237 & -0.70747 & $\mathrm{H}$ & 1.701515 & 4.609968 & 0.288232 \\
\hline $\mathrm{H}$ & -3.90075 & -3.00795 & 1.412416 & $\mathrm{H}$ & -0.47058 & 4.94083 & 0.98999 \\
\hline $\mathrm{H}$ & -2.33626 & -3.83727 & 1.550912 & $\mathrm{H}$ & -1.76831 & 3.982201 & 1.713199 \\
\hline $\mathrm{H}$ & -2.62922 & -2.35809 & 2.457088 & $\mathrm{H}$ & -0.12195 & 3.794212 & 2.299332 \\
\hline $\mathrm{H}$ & 4.942154 & -1.78459 & 2.514352 & $\mathrm{H}$ & -5.00715 & -1.97643 & -2.08398 \\
\hline $\mathrm{H}$ & 5.822912 & -0.97254 & 1.197145 & $\mathrm{H}$ & -5.19101 & -0.22014 & -1.85446 \\
\hline $\mathrm{r}$ & 4.913686 & 0.000647 & 2.392972 & $\mathrm{H}$ & -4.02762 & -0.84331 & -3.0637 \\
\hline & -1.28191 & -4.22462 & -0.95395 & $\mathrm{H}$ & -3.07259 & 2.555552 & -0.58552 \\
\hline t & -2.54915 & -4.19644 & -2.19484 & $\mathrm{H}$ & -2.74911 & 3.640565 & -1.95018 \\
\hline & -2.95886 & -4.64949 & -0.52797 & $\mathrm{H}$ & -2.77082 & 4.288203 & -0.29983 \\
\hline
\end{tabular}

\begin{tabular}{lrrrllllr} 
3b_1 & \multicolumn{7}{c}{ 3b_2 } \\
C & 2.46082 & 1.874998 & 1.6787 & C & 3.386111 & 2.219238 & -0.05092 \\
C & 1.57851 & 2.876017 & 0.946688 & C & 3.756314 & 0.831037 & 0.444341 \\
C & 2.229934 & 0.372511 & 1.446505 & C & 2.034597 & 2.417575 & -0.75728 \\
C & 2.588061 & -0.08154 & 0.033089 & C & 0.830553 & 2.113215 & 0.135866 \\
C & 0.335654 & 2.614864 & 0.520566 & C & 3.291487 & -0.30207 & -0.09551 \\
C & -0.58196 & 3.593667 & -0.18644 & C & 3.663479 & -1.70792 & 0.331931 \\
C & -2.00268 & 3.077753 & -0.47424 & C & 2.942456 & -2.82345 & -0.44091 \\
C & 2.365343 & -1.58953 & -0.27287 & C & -0.5597 & 2.503844 & -0.4394 \\
C & 0.894345 & -2.02997 & -0.1136 & C & -0.93758 & 1.768046 & -1.744 \\
C & -2.14457 & 1.908735 & -1.44673 & C & 1.435782 & -2.94374 & -0.24178 \\
C & -2.68518 & 0.619164 & -0.95053 & C & 0.528309 & -2.52354 & -1.33384 \\
C & -0.13435 & -1.13272 & -0.80643 & C & -0.66083 & 0.258516 & -1.88309 \\
C & -2.52776 & -0.73733 & -1.60613 & C & -0.94205 & -2.1888 & -1.1993
\end{tabular}




\begin{tabular}{|c|c|c|c|c|c|c|c|}
\hline $\mathrm{C}$ & -1.54695 & -1.74096 & -0.93408 & $\mathrm{C}$ & -1.28322 & -0.71222 & -0.85735 \\
\hline $\mathrm{C}$ & -2.12236 & -2.41939 & 0.309922 & $\mathrm{C}$ & -2.79492 & -0.5968 & -0.72643 \\
\hline $\mathrm{C}$ & -2.24581 & -1.77831 & 1.664132 & $\mathrm{C}$ & -3.43795 & -0.87451 & 0.601164 \\
\hline $\mathrm{O}$ & -2.85312 & -2.25878 & 2.597202 & $\mathrm{O}$ & -4.60862 & -0.68798 & 0.860761 \\
\hline $\mathrm{C}$ & -2.57522 & -3.67704 & 0.239986 & $\mathrm{C}$ & -3.6226 & -0.31766 & -1.73717 \\
\hline $\mathrm{C}$ & 2.230564 & 4.227052 & 0.773649 & $\mathrm{C}$ & 4.738104 & 0.845471 & 1.590877 \\
\hline $\mathrm{C}$ & -1.33347 & 2.006567 & -2.71957 & $\mathrm{C}$ & 0.960531 & -3.05556 & 1.189569 \\
\hline $\mathrm{O}$ & 3.963116 & 0.252068 & -0.20226 & $\mathrm{O}$ & 1.022252 & 2.789933 & 1.384769 \\
\hline $\mathrm{O}$ & -3.53826 & 1.55269 & -1.64859 & $\mathrm{O}$ & 0.864171 & -3.91765 & -1.1558 \\
\hline $\mathrm{H}$ & -2.89553 & 0.585475 & 0.114679 & $\mathrm{H}$ & 1.014117 & -2.09185 & -2.20785 \\
\hline $\mathrm{C}$ & 3.277835 & -2.48234 & 0.575509 & $\mathrm{C}$ & -0.66537 & 4.018736 & -0.66151 \\
\hline $\mathrm{O}$ & -1.59337 & -0.60375 & 1.763193 & $\mathrm{O}$ & -2.56711 & -1.37285 & 1.498234 \\
\hline $\mathrm{C}$ & -1.6787 & 0.056706 & 3.045797 & $\mathrm{C}$ & -3.0984 & -1.67428 & 2.804725 \\
\hline $\mathrm{O}$ & 2.755166 & -1.63021 & -1.67703 & $\mathrm{O}$ & -1.43368 & 2.078149 & 0.652177 \\
\hline $\mathrm{C}$ & 2.932483 & -2.89757 & -2.29736 & $\mathrm{C}$ & -2.74419 & 2.629267 & 0.724629 \\
\hline $\mathrm{H}$ & 2.379977 & 2.077962 & 2.755227 & $\mathrm{H}$ & 4.181804 & 2.566192 & -0.72419 \\
\hline $\mathrm{H}$ & 3.501825 & 2.094368 & 1.422947 & $\mathrm{H}$ & 3.41444 & 2.899052 & 0.805137 \\
\hline $\mathrm{H}$ & 2.850839 & -0.17366 & 2.163334 & $\mathrm{H}$ & 1.993438 & 3.460009 & -1.08598 \\
\hline $\mathrm{H}$ & 1.194462 & 0.100984 & 1.663766 & $\mathrm{H}$ & 1.976451 & 1.807591 & -1.66297 \\
\hline $\mathrm{H}$ & 1.973368 & 0.472822 & -0.68415 & $\mathrm{H}$ & 0.799543 & 1.036127 & 0.331742 \\
\hline $\mathrm{H}$ & -0.07177 & 1.622859 & 0.686767 & $\mathrm{H}$ & 2.600887 & -0.2317 & -0.93134 \\
\hline $\mathrm{H}$ & -0.12445 & 3.926448 & -1.12526 & $\mathrm{H}$ & 3.489532 & -1.83942 & 1.405387 \\
\hline $\mathrm{H}$ & -0.68899 & 4.502934 & 0.415953 & $\mathrm{H}$ & 4.740273 & -1.86407 & 0.194159 \\
\hline $\mathrm{H}$ & -2.49939 & 2.813235 & 0.464746 & $\mathrm{H}$ & 3.139451 & -2.71694 & -1.51259 \\
\hline $\mathrm{H}$ & -2.57823 & 3.905992 & -0.90479 & $\mathrm{H}$ & 3.375168 & -3.78543 & -0.14087 \\
\hline $\mathrm{H}$ & 0.672797 & -2.09462 & 0.955475 & $\mathrm{H}$ & -0.4092 & 2.264201 & -2.565 \\
\hline $\mathrm{H}$ & 0.807914 & -3.05439 & -0.49252 & $\mathrm{H}$ & -1.99785 & 1.965089 & -1.92678 \\
\hline $\mathrm{H}$ & 0.210793 & -0.91276 & -1.82147 & $\mathrm{H}$ & 0.419826 & 0.097224 & -1.87011 \\
\hline $\mathrm{H}$ & -0.19831 & -0.18021 & -0.28519 & $\mathrm{H}$ & -0.97782 & -0.03426 & -2.89118 \\
\hline $\mathrm{H}$ & -2.20822 & -0.60623 & -2.64258 & $\mathrm{H}$ & -1.38529 & -2.83893 & -0.44371 \\
\hline $\mathrm{H}$ & -3.52167 & -1.19683 & -1.64812 & $\mathrm{H}$ & -1.42434 & -2.44449 & -2.15072 \\
\hline $\mathrm{H}$ & -1.46069 & -2.56167 & -1.65363 & $\mathrm{H}$ & -0.85892 & -0.49129 & 0.121837 \\
\hline $\mathrm{H}$ & -3.00731 & -4.1615 & 1.106333 & $\mathrm{H}$ & -4.69317 & -0.28841 & -1.57771 \\
\hline $\mathrm{H}$ & -2.52236 & -4.24432 & -0.68195 & $\mathrm{H}$ & -3.26091 & -0.11637 & -2.73785 \\
\hline $\mathrm{H}$ & 1.550648 & 4.987208 & 0.389419 & $\mathrm{H}$ & 5.601094 & 1.476683 & 1.348902 \\
\hline $\mathrm{H}$ & 2.634999 & 4.583278 & 1.728148 & $\mathrm{H}$ & 4.274668 & 1.283094 & 2.483053 \\
\hline $\mathrm{H}$ & 3.081173 & 4.153769 & 0.085475 & $\mathrm{H}$ & 5.108059 & -0.14571 & 1.852464 \\
\hline $\mathrm{H}$ & -1.62101 & 1.237327 & -3.43505 & $\mathrm{H}$ & -0.10832 & -3.25685 & 1.242519 \\
\hline $\mathrm{H}$ & -1.49759 & 2.980846 & -3.19059 & $\mathrm{H}$ & 1.488982 & -3.8694 & 1.695821 \\
\hline $\mathrm{H}$ & -0.26427 & 1.908356 & -2.51556 & $\mathrm{H}$ & 1.160627 & -2.13224 & 1.739057 \\
\hline $\mathrm{H}$ & 4.16311 & -0.12625 & -1.06883 & $\mathrm{H}$ & 0.193752 & 2.652181 & 1.863892 \\
\hline $\mathrm{H}$ & 4.325233 & -2.21871 & 0.423149 & $\mathrm{H}$ & -0.46547 & 4.559845 & 0.264113 \\
\hline $\mathrm{H}$ & 3.045247 & -2.36229 & 1.635324 & $\mathrm{H}$ & 0.058942 & 4.342845 & -1.41011 \\
\hline $\mathrm{H}$ & 3.139177 & -3.53732 & 0.330524 & $\mathrm{H}$ & -1.65516 & 4.297399 & -1.02809 \\
\hline $\mathrm{H}$ & -1.1212 & 0.982693 & 2.93365 & $\mathrm{H}$ & -2.26202 & -2.07188 & 3.373633 \\
\hline $\mathrm{H}$ & -2.71951 & 0.263382 & 3.294542 & $\mathrm{H}$ & -3.48437 & -0.76921 & 3.27461 \\
\hline $\mathrm{H}$ & -1.23548 & -0.56767 & 3.821469 & $\mathrm{H}$ & -3.89633 & -2.41337 & 2.728749 \\
\hline $\mathrm{H}$ & 2.04242 & -3.53064 & -2.21389 & $\mathrm{H}$ & -3.26099 & 2.081774 & 1.513212 \\
\hline $\mathrm{H}$ & 3.11311 & -2.69461 & -3.35361 & $\mathrm{H}$ & -2.73027 & 3.691506 & 0.987711 \\
\hline
\end{tabular}




\begin{tabular}{|c|c|c|c|c|c|c|c|}
\hline 3b_3 & & & & 3b_4 & & & \\
\hline $\mathrm{C}$ & 2.459409 & 3.05113 & 0.062578 & $\mathrm{C}$ & 3.422585 & -0.17915 & -1.86643 \\
\hline $\mathrm{C}$ & 2.982878 & 1.753488 & 0.669831 & $\mathrm{C}$ & 3.243359 & -1.3663 & -0.9262 \\
\hline $\mathrm{C}$ & 1.104216 & 2.954317 & -0.67475 & $\mathrm{C}$ & 2.478641 & 1.017275 & -1.60435 \\
\hline $\mathrm{C}$ & -0.00856 & 2.318059 & 0.150081 & $\mathrm{C}$ & 2.465664 & 1.500062 & -0.15927 \\
\hline $\mathrm{C}$ & 3.576538 & 0.830239 & -0.09893 & $\mathrm{C}$ & 2.264519 & -2.26144 & -1.12292 \\
\hline $\mathrm{C}$ & 4.24658 & -0.44976 & 0.328229 & $\mathrm{C}$ & 2.012398 & -3.53486 & -0.355 \\
\hline $\mathrm{C}$ & 3.874808 & -1.6988 & -0.49499 & $\mathrm{C}$ & 0.534769 & -3.90457 & -0.11609 \\
\hline $\mathrm{C}$ & -1.39328 & 2.210544 & -0.54403 & $\mathrm{C}$ & 1.386531 & 2.559975 & 0.190248 \\
\hline $\mathrm{C}$ & -1.39667 & 1.365815 & -1.83781 & $\mathrm{C}$ & -0.05042 & 2.056381 & -0.07151 \\
\hline $\mathrm{C}$ & 2.487712 & -2.29501 & -0.28556 & $\mathrm{C}$ & -0.25834 & -3.07266 & 0.888472 \\
\hline $\mathrm{C}$ & 1.466667 & -2.14925 & -1.34696 & $\mathrm{C}$ & -1.4126 & -2.27058 & 0.416484 \\
\hline $\mathrm{C}$ & -0.5902 & 0.05496 & -1.91281 & $\mathrm{C}$ & -0.36108 & 0.645229 & 0.442619 \\
\hline $\mathrm{C}$ & -0.02854 & -2.32803 & -1.18604 & $\mathrm{C}$ & -2.06978 & -1.10747 & 1.129598 \\
\hline $\mathrm{C}$ & -0.8503 & -1.04935 & -0.86657 & $\mathrm{C}$ & -1.85607 & 0.276999 & 0.456881 \\
\hline $\mathrm{C}$ & -2.31257 & -1.44628 & -0.72803 & $\mathrm{C}$ & -2.54913 & 0.335208 & -0.89874 \\
\hline $\mathrm{C}$ & -2.84411 & -1.81814 & 0.624002 & $\mathrm{C}$ & -4.02728 & 0.607911 & -0.95058 \\
\hline $\mathrm{O}$ & -4.01845 & -1.95747 & 0.897016 & $\mathrm{O}$ & -4.689 & 0.665017 & -1.96557 \\
\hline $\mathrm{C}$ & -3.1662 & -1.54673 & -1.75055 & $\mathrm{C}$ & -1.95193 & 0.1297 & -2.07623 \\
\hline $\mathrm{C}$ & 2.816894 & 1.641839 & 2.166711 & $\mathrm{C}$ & 4.270104 & -1.45516 & 0.178843 \\
\hline $\mathrm{C}$ & 2.110251 & -2.6118 & 1.144203 & $\mathrm{C}$ & 0.429513 & -2.78788 & 2.204221 \\
\hline $\mathrm{O}$ & -0.15184 & 3.066109 & 1.364833 & $\mathrm{O}$ & 3.767882 & 2.008178 & 0.157407 \\
\hline $\mathrm{O}$ & 2.239457 & -3.36572 & -1.23679 & $\mathrm{O}$ & -1.61895 & -3.56837 & 1.01362 \\
\hline $\mathrm{H}$ & 1.763699 & -1.54673 & -2.204 & $\mathrm{H}$ & -1.57785 & -2.27839 & -0.66022 \\
\hline $\mathrm{C}$ & -1.96954 & 3.600523 & -0.8478 & $\mathrm{C}$ & 1.626087 & 3.882695 & -0.54499 \\
\hline $\mathrm{O}$ & -1.86293 & -2.00066 & 1.526996 & $\mathrm{O}$ & -4.55769 & 0.78756 & 0.272714 \\
\hline $\mathrm{C}$ & -2.27956 & -2.36622 & 2.858399 & $\mathrm{C}$ & -5.97476 & 1.053099 & 0.321929 \\
\hline $\mathrm{O}$ & -2.16894 & 1.539136 & 0.497507 & $\mathrm{O}$ & 1.619 & 2.703848 & 1.621659 \\
\hline $\mathrm{C}$ & -3.58842 & 1.639719 & 0.451476 & $\mathrm{C}$ & 0.996622 & 3.783701 & 2.306339 \\
\hline $\mathrm{H}$ & 3.192087 & 3.441097 & -0.65089 & $\mathrm{H}$ & 3.266998 & -0.50132 & -2.90063 \\
\hline $\mathrm{H}$ & 2.368161 & 3.798174 & 0.855098 & $\mathrm{H}$ & 4.457031 & 0.169266 & -1.80078 \\
\hline $\mathrm{H}$ & 0.810832 & 3.963472 & -0.98018 & $\mathrm{H}$ & 2.766146 & 1.842459 & -2.26374 \\
\hline $\mathrm{H}$ & 1.23977 & 2.371567 & -1.58946 & $\mathrm{H}$ & 1.46482 & 0.725181 & -1.88669 \\
\hline $\mathrm{H}$ & 0.295713 & 1.296284 & 0.406221 & $\mathrm{H}$ & 2.260871 & 0.6442 & 0.493122 \\
\hline $\mathrm{H}$ & 3.654877 & 1.041756 & -1.16649 & $\mathrm{H}$ & 1.608101 & -2.10895 & -1.98063 \\
\hline $\mathrm{H}$ & 4.092032 & -0.64543 & 1.390868 & $\mathrm{H}$ & 2.549406 & -3.53317 & 0.595515 \\
\hline $\mathrm{H}$ & 5.330439 & -0.315 & 0.210154 & $\mathrm{H}$ & 2.443657 & -4.36582 & -0.93041 \\
\hline $\mathrm{H}$ & 4.003218 & -1.48923 & -1.56176 & $\mathrm{H}$ & -0.00397 & -3.9116 & -1.06911 \\
\hline $\mathrm{H}$ & 4.589962 & -2.49329 & -0.24855 & $\mathrm{H}$ & 0.507841 & -4.93481 & 0.259323 \\
\hline $\mathrm{H}$ & -1.03227 & 2.005502 & -2.64849 & $\mathrm{H}$ & -0.24479 & 2.122501 & -1.14475 \\
\hline $\mathrm{H}$ & -2.44377 & 1.165296 & -2.08181 & $\mathrm{H}$ & -0.74333 & 2.768385 & 0.390851 \\
\hline $\mathrm{H}$ & 0.475462 & 0.293487 & -1.87088 & $\mathrm{H}$ & -0.00267 & 0.563214 & 1.473367 \\
\hline $\mathrm{H}$ & -0.75205 & -0.35861 & -2.91569 & $\mathrm{H}$ & 0.197794 & -0.10069 & -0.12869 \\
\hline $\mathrm{H}$ & -0.21762 & -3.07388 & -0.41255 & $\mathrm{H}$ & -1.72219 & -1.04682 & 2.162986 \\
\hline $\mathrm{H}$ & -0.40531 & -2.75324 & -2.12456 & $\mathrm{H}$ & -3.14324 & -1.31808 & 1.175198 \\
\hline $\mathrm{H}$ & -0.52584 & -0.68055 & 0.106025 & $\mathrm{H}$ & -2.36354 & 1.000961 & 1.099374 \\
\hline $\mathrm{H}$ & -4.18657 & -1.86974 & -1.58631 & $\mathrm{H}$ & -2.52486 & 0.165709 & -2.99425 \\
\hline
\end{tabular}




\begin{tabular}{|c|c|c|c|c|c|c|c|}
\hline $\mathrm{H}$ & -2.87343 & -1.31624 & -2.76775 & $\mathrm{H}$ & -0.89328 & -0.08023 & -2.15608 \\
\hline $\mathrm{H}$ & 3.461237 & 2.372071 & 2.672314 & $\mathrm{H}$ & 4.00886 & -2.16977 & 0.959718 \\
\hline $\mathrm{H}$ & 1.789947 & 1.888388 & 2.449381 & $\mathrm{H}$ & 5.240857 & -1.75645 & -0.2349 \\
\hline $\mathrm{H}$ & 3.062777 & 0.655294 & 2.559657 & $\mathrm{H}$ & 4.421968 & -0.4739 & 0.636607 \\
\hline $\mathrm{H}$ & 1.174926 & -3.16689 & 1.198092 & $\mathrm{H}$ & -0.26306 & -2.36532 & 2.931111 \\
\hline $\mathrm{H}$ & 2.893454 & -3.21553 & 1.613219 & $\mathrm{H}$ & 0.832939 & -3.71504 & 2.623083 \\
\hline $\mathrm{H}$ & 1.997192 & -1.69517 & 1.728315 & $\mathrm{H}$ & 1.259815 & -2.0901 & 2.069893 \\
\hline $\mathrm{H}$ & -0.92028 & 2.675141 & 1.802932 & $\mathrm{H}$ & 3.686599 & 2.357587 & 1.055044 \\
\hline $\mathrm{H}$ & -2.04983 & 4.197105 & 0.061663 & $\mathrm{H}$ & 2.617205 & 4.276505 & -0.31605 \\
\hline $\mathrm{H}$ & -1.32062 & 4.130856 & -1.54652 & $\mathrm{H}$ & 1.559509 & 3.730091 & -1.624 \\
\hline $\mathrm{H}$ & -2.95519 & 3.5285 & -1.31149 & $\mathrm{H}$ & 0.876821 & 4.629614 & -0.27437 \\
\hline $\mathrm{H}$ & -1.36117 & -2.49416 & 3.425432 & $\mathrm{H}$ & -6.21345 & 1.158131 & 1.377039 \\
\hline $\mathrm{H}$ & -2.89025 & -1.57498 & 3.294315 & $\mathrm{H}$ & -6.20619 & 1.972363 & -0.21677 \\
\hline $\mathrm{H}$ & -2.84938 & -3.29543 & 2.836701 & $\mathrm{H}$ & -6.53087 & 0.224189 & -0.11663 \\
\hline $\mathrm{H}$ & -3.96641 & 0.961657 & 1.216456 & $\mathrm{H}$ & -0.09052 & 3.790903 & 2.172033 \\
\hline $\mathrm{H}$ & -3.93608 & 2.652985 & 0.675494 & $\mathrm{H}$ & 1.211901 & 3.63521 & 3.365148 \\
\hline $\mathrm{H}$ & -3.99607 & 1.326731 & -0.51545 & $\mathrm{H}$ & 1.398361 & 4.755173 & 2.00063 \\
\hline 3b_5 & & & & $\mathbf{3 b}$ & & & \\
\hline $\mathrm{C}$ & 1.897576 & 1.601607 & 2.325427 & $\mathrm{C}$ & 1.92964 & 2.107628 & 1.975129 \\
\hline $\mathrm{C}$ & 1.200154 & 2.620392 & 1.43006 & $\mathrm{C}$ & 1.063456 & 2.893735 & 0.996885 \\
\hline $\mathrm{C}$ & 1.995538 & 0.172146 & 1.743688 & $\mathrm{C}$ & 2.107806 & 0.609272 & 1.638069 \\
\hline $\mathrm{C}$ & 2.600928 & 0.11228 & 0.346542 & $\mathrm{C}$ & 2.608504 & 0.344977 & 0.223649 \\
\hline $\mathrm{C}$ & -0.13777 & 2.680471 & 1.370803 & $\mathrm{C}$ & -0.27396 & 2.847183 & 1.075367 \\
\hline $\mathrm{C}$ & -0.98561 & 3.671046 & 0.613059 & $\mathrm{C}$ & -1.28217 & 3.621394 & 0.26574 \\
\hline $\mathrm{C}$ & -2.2859 & 3.11297 & 0.000425 & $\mathrm{C}$ & -2.54716 & 2.847089 & -0.15689 \\
\hline $\mathrm{C}$ & 2.560375 & -1.27333 & -0.35078 & $\mathrm{C}$ & 2.629671 & -1.14228 & -0.21897 \\
\hline $\mathrm{C}$ & 1.124146 & -1.81811 & -0.51061 & $\mathrm{C}$ & 1.230167 & -1.79416 & -0.18624 \\
\hline $\mathrm{C}$ & -2.16797 & 2.173608 & -1.19709 & $\mathrm{C}$ & -2.41255 & 1.788099 & -1.2486 \\
\hline $\mathrm{C}$ & -2.61875 & 0.767392 & -1.06262 & $\mathrm{C}$ & -2.69524 & 0.367452 & -0.9263 \\
\hline $\mathrm{C}$ & 0.089187 & -0.81852 & -1.03797 & $\mathrm{C}$ & 0.100598 & -0.96134 & -0.80189 \\
\hline $\mathrm{C}$ & -2.25142 & -0.3886 & -1.97116 & $\mathrm{C}$ & -2.27362 & -0.84002 & -1.74001 \\
\hline $\mathrm{C}$ & -1.26365 & -1.45409 & -1.42065 & $\mathrm{C}$ & -1.17268 & -1.76323 & -1.14541 \\
\hline $\mathrm{C}$ & -1.88436 & -2.38177 & -0.37619 & $\mathrm{C}$ & -1.69574 & -2.72932 & -0.08179 \\
\hline $\mathrm{C}$ & -1.99724 & -1.942 & 1.050258 & $\mathrm{C}$ & -2.0146 & -2.35754 & 1.338656 \\
\hline $\mathrm{O}$ & -1.66331 & -0.84883 & 1.463566 & $\mathrm{O}$ & -2.5909 & -3.07984 & 2.124241 \\
\hline $\mathrm{C}$ & -2.33955 & -3.59109 & -0.72576 & $\mathrm{C}$ & -1.91456 & -4.01324 & -0.39067 \\
\hline $\mathrm{C}$ & 2.130436 & 3.544855 & 0.680085 & $\mathrm{C}$ & 1.831657 & 3.731814 & 0.002141 \\
\hline $\mathrm{C}$ & -1.22207 & 2.615872 & -2.29114 & $\mathrm{C}$ & -1.60243 & 2.190811 & -2.46042 \\
\hline $\mathrm{O}$ & 3.955223 & 0.579527 & 0.418462 & $\mathrm{O}$ & 3.92085 & 0.90825 & 0.098195 \\
\hline $\mathrm{O}$ & -3.48078 & 1.760828 & -1.66418 & $\mathrm{O}$ & -3.70287 & 1.193638 & -1.5514 \\
\hline $\mathrm{H}$ & -2.94266 & 0.478771 & -0.06711 & $\mathrm{H}$ & -2.912 & 0.161699 & 0.118382 \\
\hline $\mathrm{C}$ & 3.441475 & -2.30034 & 0.368449 & $\mathrm{C}$ & 3.62203 & -1.97017 & 0.603798 \\
\hline $\mathrm{O}$ & -2.50631 & -2.88951 & 1.857982 & $\mathrm{O}$ & -1.57618 & -1.1313 & 1.680766 \\
\hline $\mathrm{C}$ & -2.64992 & -2.53589 & 3.248516 & $\mathrm{C}$ & -1.83656 & -0.73036 & 3.043604 \\
\hline $\mathrm{O}$ & 3.116641 & -0.926 & -1.65275 & $\mathrm{O}$ & 3.081477 & -0.99726 & -1.59769 \\
\hline $\mathrm{C}$ & 3.492567 & -1.97611 & -2.53431 & $\mathrm{C}$ & 3.505754 & -2.15888 & -2.29978 \\
\hline $\mathrm{H}$ & 1.371229 & 1.532645 & 3.282509 & $\mathrm{H}$ & 1.499115 & 2.170944 & 2.979246 \\
\hline $\mathrm{H}$ & 2.906389 & 1.959849 & 2.54897 & $\mathrm{H}$ & 2.916729 & 2.575244 & 2.0287 \\
\hline
\end{tabular}




\begin{tabular}{|c|c|c|c|c|c|c|c|}
\hline $\mathrm{H}$ & 2.590254 & -0.44301 & 2.427453 & $\mathrm{H}$ & 2.802998 & 0.168867 & $2.36034 \mathrm{C}$ \\
\hline $\mathrm{H}$ & 0.993066 & -0.25964 & 1.711848 & $\mathrm{H}$ & 1.147184 & 0.106778 & 1.77123 \\
\hline $\mathrm{H}$ & 2.037383 & 0.787989 & -0.30626 & $\mathrm{H}$ & 1.944179 & 0.855906 & -0.4820 \\
\hline $\mathrm{H}$ & -0.6991 & 1.973348 & 1.981511 & $\mathrm{H}$ & -0.70766 & 2.233255 & $1.8650 \mathrm{~s}$ \\
\hline $\mathrm{H}$ & -0.40388 & 4.179963 & -0.15851 & $\mathrm{H}$ & -0.81843 & 4.079868 & -0.6099 \\
\hline $\mathrm{H}$ & -1.29373 & 4.460825 & 1.312444 & $\mathrm{H}$ & -1.63297 & 4.461387 & 0.88129 \\
\hline $\mathrm{H}$ & -2.87635 & 2.616901 & 0.777467 & $\mathrm{H}$ & -3.01137 & 2.388806 & 0.7224 \\
\hline $\mathrm{H}$ & -2.8838 & 3.965985 & -0.34348 & $\mathrm{H}$ & -3.26998 & 3.578238 & -0.539 \\
\hline $\mathrm{H}$ & 0.80389 & -2.2123 & 0.458179 & $\mathrm{H}$ & 0.988143 & -2.03695 & 0.85248 \\
\hline $\mathrm{H}$ & 1.167372 & -2.68744 & -1.17663 & $\mathrm{H}$ & 1.301274 & -2.75819 & -0.702( \\
\hline $\mathrm{H}$ & 0.485427 & -0.33694 & -1.93761 & $\mathrm{H}$ & 0.453956 & -0.52026 & -1.7393 \\
\hline $\mathrm{H}$ & -0.08039 & -0.03176 & -0.30421 & $\mathrm{H}$ & -0.15567 & -0.13397 & -0.14 \\
\hline $\mathrm{H}$ & -1.83317 & -0.00052 & -2.90293 & $\mathrm{H}$ & -1.9325 & -0.51199 & -2.7245 \\
\hline $\mathrm{H}$ & -3.18643 & -0.89224 & -2.24222 & $\mathrm{H}$ & -3.17357 & -1.44041 & -1.914 \\
\hline $\mathrm{H}$ & -1.06851 & -2.11282 & -2.27365 & $\mathrm{H}$ & -0.89783 & -2.42552 & -1.9729 \\
\hline $\mathrm{H}$ & -2.80007 & -4.25639 & -0.00838 & $\mathrm{H}$ & -2.30898 & -4.7032 & 0.3444 \\
\hline $\mathrm{H}$ & -2.26192 & -3.93907 & -1.75007 & $\mathrm{H}$ & -1.70423 & -4.39751 & -1.3819 \\
\hline $\mathrm{H}$ & 2.588636 & 4.261571 & 1.373591 & $\mathrm{H}$ & 2.286934 & 4.592107 & 0.50900 \\
\hline $\mathrm{H}$ & 2.952999 & 2.976793 & 0.237157 & $\mathrm{H}$ & 2.65674 & 3.154819 & -0.42412 \\
\hline $\mathrm{H}$ & 1.635529 & 4.117257 & -0.1051 & $\mathrm{H}$ & 1.215473 & 4.116659 & -0.81097 \\
\hline $\mathrm{H}$ & -1.33536 & 2.006349 & -3.18673 & $\mathrm{H}$ & -1.72196 & 1.47759 & -3.27475 \\
\hline $\mathrm{H}$ & -1.42761 & 3.655773 & -2.56382 & $\mathrm{H}$ & -1.93186 & 3.170077 & -2.8215 \\
\hline $\mathrm{H}$ & -0.18262 & 2.553833 & -1.95958 & $\mathrm{H}$ & -0.53968 & 2.261972 & -2.2153 \\
\hline $\mathrm{H}$ & 4.306064 & 0.461387 & -0.47444 & $\mathrm{H}$ & 4.216447 & 0.651449 & -0.7856 \\
\hline $\mathrm{H}$ & 4.473054 & -1.95039 & 0.426647 & $\mathrm{H}$ & 4.627262 & -1.5526 & 0.531511 \\
\hline $\mathrm{H}$ & 3.078409 & -2.46152 & 1.385453 & $\mathrm{H}$ & 3.327761 & -1.97331 & 1.65516 \\
\hline $\mathrm{H}$ & 3.423669 & -3.26624 & -0.14084 & $\mathrm{H}$ & 3.645659 & -3.00928 & 0.26879 \\
\hline $\mathrm{H}$ & -3.08835 & -3.40946 & 3.724056 & $\mathrm{H}$ & -1.41018 & 0.263922 & 3.14166 \\
\hline $\mathrm{H}$ & -1.67689 & -2.31145 & 3.686677 & $\mathrm{H}$ & -2.90994 & -0.70714 & 3.23179 \\
\hline $\mathrm{H}$ & -3.30624 & -1.67191 & 3.356801 & $\mathrm{H}$ & -1.35931 & -1.42178 & 3.73788 \\
\hline $\mathrm{H}$ & 2.66792 & -2.66939 & -2.7339 & $\mathrm{H}$ & 2.747982 & -2.94982 & -2.29483 \\
\hline $\mathrm{H}$ & 3.773947 & -1.49733 & -3.47305 & $\mathrm{H}$ & 3.670245 & -1.84602 & -3.33158 \\
\hline $\mathrm{H}$ & 4.350987 & -2.54419 & -2.16113 & $\mathrm{H}$ & 4.442725 & -2.5633 & -1.90295 \\
\hline 3b_7 & & & & $3 b$ & & & \\
\hline $\mathrm{C}$ & -3.65545 & -1.74389 & -0.34102 & $\mathrm{C}$ & -3.24671 & 0.363906 & -1.9442 \\
\hline $\mathrm{C}$ & -3.9038 & -0.37109 & 0.262665 & $\mathrm{C}$ & -3.04766 & 1.560133 & $-1.0175 \&$ \\
\hline $\mathrm{C}$ & -2.27049 & -2.057 & -0.93091 & $\mathrm{C}$ & -2.37126 & -0.87052 & -1.62297 \\
\hline $\mathrm{C}$ & -1.12344 & -2.00715 & 0.096419 & $\mathrm{C}$ & -2.52607 & -1.3968 & -0.18976 \\
\hline $\mathrm{C}$ & -3.11007 & 0.686108 & 0.048621 & $\mathrm{C}$ & -2.0015 & 2.382883 & -1.17939 \\
\hline $\mathrm{C}$ & -3.28324 & 2.081226 & 0.607697 & $\mathrm{C}$ & -1.69584 & 3.651738 & -0.42377 \\
\hline $\mathrm{C}$ & -2.48623 & 3.162664 & -0.14272 & $\mathrm{C}$ & -0.20616 & 3.924057 & -0.13492 \\
\hline $\mathrm{C}$ & 0.25384 & -2.5035 & -0.41862 & $\mathrm{C}$ & -1.50277 & -2.48651 & 0.236242 \\
\hline $\mathrm{C}$ & 0.676792 & -1.82995 & -1.74744 & $\mathrm{C}$ & -0.04813 & -2.05521 & -0.06385 \\
\hline $\mathrm{C}$ & -0.96534 & 3.090446 & -0.05176 & $\mathrm{C}$ & 0.497076 & 3.038935 & 0.89105 \\
\hline $\mathrm{C}$ & -0.19071 & 2.605579 & -1.21782 & $\mathrm{C}$ & 1.612005 & 2.166418 & 0.450941 \\
\hline $\mathrm{C}$ & 0.609105 & -0.29671 & -1.89115 & $\mathrm{C}$ & 0.35248 & -0.67057 & $0.46256 \mathrm{~S}$ \\
\hline $\mathrm{C}$ & 1.232971 & 2.087007 & -1.19688 & $\mathrm{C}$ & 2.168381 & 0.958611 & 1.17528 \\
\hline & 1.411425 & 0.572432 & -0.90116 & $\mathrm{C}$ & 1.86938 & -0.40629 & 0.494 \\
\hline
\end{tabular}




\begin{tabular}{|c|c|c|c|c|c|c|c|}
\hline $\mathrm{C}$ & 2.901763 & 0.262087 & -0.88298 & $\mathrm{C}$ & 2.578099 & -0.51187 & -0.84959 \\
\hline $\mathrm{C}$ & 3.662911 & 0.3874 & 0.403784 & $\mathrm{C}$ & 4.033244 & -0.89115 & -0.8782 \\
\hline $\mathrm{O}$ & 4.814735 & 0.042895 & 0.568584 & $\mathrm{O}$ & 4.705939 & -0.99375 & -1.8824 \\
\hline $\mathrm{C}$ & 3.618321 & -0.06407 & -1.96213 & $\mathrm{C}$ & 2.016606 & -0.26335 & -2.03625 \\
\hline $\mathrm{C}$ & -5.14388 & -0.31326 & 1.122185 & $\mathrm{C}$ & -4.12454 & 1.750346 & 0.024661 \\
\hline $\mathrm{C}$ & -0.38418 & 3.087689 & 1.344906 & $\mathrm{C}$ & -0.25276 & 2.7923 & 2.180242 \\
\hline $\mathrm{O}$ & -1.46563 & -2.79826 & 1.252314 & $\mathrm{O}$ & -3.84641 & -1.9403 & 0.000741 \\
\hline $\mathrm{O}$ & -0.33913 & 4.022121 & -0.9729 & $\mathrm{O}$ & 1.881585 & 3.445168 & 1.064363 \\
\hline $\mathrm{H}$ & -0.78362 & 2.271815 & -2.06824 & $\mathrm{H}$ & 1.81285 & 2.169495 & -0.61968 \\
\hline $\mathrm{C}$ & 0.268044 & -4.02518 & -0.62427 & $\mathrm{C}$ & -1.77989 & -3.83268 & -0.44339 \\
\hline $\mathrm{O}$ & 2.930191 & 0.953321 & 1.38153 & $\mathrm{O}$ & 4.528597 & -1.11286 & 0.35282 \\
\hline $\mathrm{C}$ & 3.590549 & 1.132102 & 2.650541 & $\mathrm{C}$ & 5.920602 & -1.48453 & 0.424407 \\
\hline $\mathrm{O}$ & 1.271697 & -2.08476 & 0.525762 & $\mathrm{O}$ & -1.50865 & -2.60741 & 1.680339 \\
\hline $\mathrm{C}$ & 1.535215 & -2.86934 & 1.685639 & $\mathrm{C}$ & -2.57412 & -3.28955 & 2.336235 \\
\hline $\mathrm{H}$ & -4.4083 & -1.91088 & -1.12303 & $\mathrm{H}$ & -3.02844 & 0.658599 & -2.97481 \\
\hline $\mathrm{H}$ & -3.88179 & -2.49623 & 0.423476 & $\mathrm{H}$ & -4.3056 & 0.079865 & -1.94112 \\
\hline $\mathrm{H}$ & -2.32059 & -3.06254 & -1.35983 & $\mathrm{H}$ & -2.61706 & -1.67223 & -2.32708 \\
\hline $\mathrm{H}$ & -2.05612 & -1.37959 & -1.76105 & $\mathrm{H}$ & -1.33224 & -0.59481 & -1.80458 \\
\hline $\mathrm{H}$ & -0.97019 & -0.97619 & 0.422786 & $\mathrm{H}$ & -2.38213 & -0.56599 & 0.510057 \\
\hline $\mathrm{H}$ & -2.23876 & 0.550169 & -0.58293 & $\mathrm{H}$ & -1.31345 & 2.162594 & -1.99643 \\
\hline $\mathrm{H}$ & -3.00401 & 2.099967 & 1.668659 & $\mathrm{H}$ & -2.26534 & 3.703978 & 0.505843 \\
\hline $\mathrm{H}$ & -4.3378 & 2.372046 & 0.582697 & $\mathrm{H}$ & -2.04796 & 4.498121 & -1.02946 \\
\hline $\mathrm{H}$ & -2.76746 & 3.160473 & -1.20066 & $\mathrm{H}$ & 0.36262 & 3.897315 & -1.06994 \\
\hline $\mathrm{H}$ & -2.77693 & 4.142704 & 0.254119 & $\mathrm{H}$ & -0.1253 & 4.949866 & 0.244634 \\
\hline $\mathrm{H}$ & 0.065854 & -2.25343 & -2.55059 & $\mathrm{H}$ & 0.130578 & -2.12547 & -1.13865 \\
\hline $\mathrm{H}$ & 1.700058 & -2.16499 & -1.93327 & $\mathrm{H}$ & 0.588778 & -2.81233 & 0.405216 \\
\hline $\mathrm{H}$ & -0.43426 & 0.021617 & -1.83715 & $\mathrm{H}$ & -0.00576 & -0.57621 & 1.491159 \\
\hline $\mathrm{H}$ & 0.920598 & -0.05757 & -2.91507 & $\mathrm{H}$ & -0.14978 & 0.118078 & -0.10411 \\
\hline $\mathrm{H}$ & 1.804492 & 2.660322 & -0.46505 & $\mathrm{H}$ & 1.794028 & 0.921425 & 2.200505 \\
\hline $\mathrm{H}$ & 1.675275 & 2.30684 & -2.17634 & $\mathrm{H}$ & 3.252958 & 1.091667 & 1.245185 \\
\hline $\mathrm{H}$ & 1.033163 & 0.3789 & 0.101371 & $\mathrm{H}$ & 2.315349 & -1.16452 & 1.143372 \\
\hline $\mathrm{H}$ & 4.684151 & -0.23704 & -1.88146 & $\mathrm{H}$ & 2.600347 & -0.33945 & -2.94498 \\
\hline $\mathrm{H}$ & 3.168778 & -0.16651 & -2.9421 & $\mathrm{H}$ & 0.977257 & 0.021966 & -2.13291 \\
\hline $\mathrm{H}$ & -6.00293 & -0.7351 & 0.588225 & $\mathrm{H}$ & -3.90836 & 2.551431 & 0.730555 \\
\hline $\mathrm{H}$ & -5.0122 & -0.92005 & 2.02588 & $\mathrm{H}$ & -5.08109 & 1.981178 & -0.45925 \\
\hline $\mathrm{H}$ & -5.40123 & 0.698445 & 1.435066 & $\mathrm{H}$ & -4.2852 & 0.833614 & 0.602565 \\
\hline $\mathrm{H}$ & 0.702996 & 3.146112 & 1.327035 & $\mathrm{H}$ & 0.387023 & 2.325745 & 2.928224 \\
\hline $\mathrm{H}$ & -0.76518 & 3.94484 & 1.90884 & $\mathrm{H}$ & -0.61323 & 3.740877 & 2.590174 \\
\hline $\mathrm{H}$ & -0.66725 & 2.179817 & 1.883577 & $\mathrm{H}$ & -1.11848 & 2.14649 & 2.013088 \\
\hline $\mathrm{H}$ & -2.00607 & -2.25232 & 1.83172 & $\mathrm{H}$ & -4.48825 & -1.27005 & -0.2546 \\
\hline $\mathrm{H}$ & -0.04374 & -4.55595 & 0.274319 & $\mathrm{H}$ & -2.8009 & -4.17029 & -0.27125 \\
\hline $\mathrm{H}$ & -0.41148 & -4.30527 & -1.43143 & $\mathrm{H}$ & -1.63469 & -3.74634 & -1.52258 \\
\hline $\mathrm{H}$ & 1.272037 & -4.35616 & -0.90149 & $\mathrm{H}$ & -1.08618 & -4.58884 & -0.06705 \\
\hline $\mathrm{H}$ & 2.852884 & 1.598033 & 3.298777 & $\mathrm{H}$ & 6.133286 & -1.60866 & 1.483011 \\
\hline $\mathrm{H}$ & 3.901743 & 0.168971 & 3.055965 & $\mathrm{H}$ & 6.09087 & -2.41804 & -0.11264 \\
\hline $\mathrm{H}$ & 4.462514 & 1.777147 & 2.537639 & $\mathrm{H}$ & 6.545033 & -0.69966 & -0.00304 \\
\hline $\mathrm{H}$ & 2.293697 & -2.31991 & 2.246093 & $\mathrm{H}$ & -2.65102 & -4.33582 & 2.019978 \\
\hline $\mathrm{H}$ & 0.647441 & -2.99875 & 2.306632 & $\mathrm{H}$ & -2.32461 & -3.27167 & 3.398765 \\
\hline $\mathrm{H}$ & 1.944589 & -3.85398 & 1.43281 & $\mathrm{H}$ & -3.53729 & -2.80056 & 2.1794 \\
\hline
\end{tabular}




\begin{tabular}{|c|c|c|c|c|c|c|c|}
\hline 3b_9 & & & & 3b_10 & & & \\
\hline $\mathrm{C}$ & 2.461186 & 1.875257 & 1.678029 & $\mathrm{C}$ & -3.5738 & -1.87501 & -0.27544 \\
\hline $\mathrm{C}$ & 1.578644 & 2.876441 & 0.946546 & $\mathrm{C}$ & -3.86766 & -0.499 & 0.296586 \\
\hline $\mathrm{C}$ & 2.230029 & 0.37277 & 1.446084 & $\mathrm{C}$ & -2.19332 & -2.15093 & -0.89298 \\
\hline $\mathrm{C}$ & 2.588164 & -0.08179 & 0.032835 & $\mathrm{C}$ & -1.02557 & -2.08437 & 0.108605 \\
\hline $\mathrm{C}$ & 0.336157 & 2.615032 & 0.51953 & $\mathrm{C}$ & -3.12829 & 0.589025 & 0.047943 \\
\hline $\mathrm{C}$ & -0.58153 & 3.593837 & -0.18735 & $\mathrm{C}$ & -3.36769 & 1.983629 & 0.586298 \\
\hline $\mathrm{C}$ & -2.00228 & 3.077908 & -0.47497 & $\mathrm{C}$ & -2.61335 & 3.093651 & -0.1657 \\
\hline $\mathrm{C}$ & 2.365168 & -1.58989 & -0.27266 & $\mathrm{C}$ & 0.369704 & -2.48512 & -0.46595 \\
\hline $\mathrm{C}$ & 0.894132 & -2.03006 & -0.11323 & $\mathrm{C}$ & 0.721474 & -1.75555 & -1.78554 \\
\hline $\mathrm{C}$ & -2.14421 & 1.908601 & -1.44717 & $\mathrm{C}$ & -1.09283 & 3.088293 & -0.05588 \\
\hline $\mathrm{C}$ & -2.68512 & 0.619304 & -0.95056 & $\mathrm{C}$ & -0.28589 & 2.637138 & -1.21243 \\
\hline $\mathrm{C}$ & -0.13448 & -1.13297 & -0.80634 & $\mathrm{C}$ & 0.588026 & -0.22444 & -1.88578 \\
\hline $\mathrm{C}$ & -2.52793 & -0.73743 & -1.60579 & $\mathrm{C}$ & 1.152855 & 2.166164 & -1.17146 \\
\hline $\mathrm{C}$ & -1.54719 & -1.74112 & -0.93375 & $\mathrm{C}$ & 1.367593 & 0.654401 & -0.8854 \\
\hline $\mathrm{C}$ & -2.12236 & -2.41935 & 0.310399 & $\mathrm{C}$ & 2.864627 & 0.380494 & -0.86278 \\
\hline $\mathrm{C}$ & -2.24628 & -1.77774 & 1.664324 & $\mathrm{C}$ & 3.610546 & 0.481661 & 0.434589 \\
\hline $\mathrm{O}$ & -2.85431 & -2.25762 & 2.597236 & $\mathrm{O}$ & 4.764568 & 0.146342 & 0.603109 \\
\hline $\mathrm{C}$ & -2.57451 & -3.6773 & 0.240969 & $\mathrm{C}$ & 3.596786 & 0.107975 & -1.9462 \\
\hline $\mathrm{C}$ & 2.230172 & 4.227907 & 0.774904 & $\mathrm{C}$ & -5.09376 & -0.47719 & 1.17787 \\
\hline $\mathrm{C}$ & -1.33279 & 2.005897 & -2.71983 & $\mathrm{C}$ & -0.52879 & 3.107952 & 1.347659 \\
\hline $\mathrm{O}$ & 3.96328 & 0.251494 & -0.20246 & $\mathrm{O}$ & -1.33625 & -2.83972 & 1.292206 \\
\hline $\mathrm{O}$ & -3.53794 & 1.552764 & -1.64902 & $\mathrm{O}$ & -0.49238 & 4.046188 & -0.96802 \\
\hline $\mathrm{H}$ & -2.8956 & 0.585952 & 0.114636 & $\mathrm{H}$ & -0.85452 & 2.282315 & -2.0708 \\
\hline $\mathrm{C}$ & 3.277557 & -2.48259 & 0.575926 & $\mathrm{C}$ & 0.46723 & -3.99608 & -0.72727 \\
\hline $\mathrm{O}$ & -1.59324 & -0.60352 & 1.763275 & $\mathrm{O}$ & 2.861288 & 1.01579 & 1.417644 \\
\hline $\mathrm{C}$ & -1.67879 & 0.057453 & 3.04559 & $\mathrm{C}$ & 3.505286 & 1.171358 & 2.697995 \\
\hline $\mathrm{O}$ & 2.754997 & -1.63105 & -1.67685 & $\mathrm{O}$ & 1.393246 & -2.04388 & 0.459781 \\
\hline $\mathrm{C}$ & 2.931666 & -2.89865 & -2.29685 & $\mathrm{C}$ & 1.69262 & -2.82171 & 1.616033 \\
\hline $\mathrm{H}$ & 2.380927 & 2.07827 & 2.754588 & $\mathrm{H}$ & -4.33727 & -2.09379 & -1.0345 \\
\hline $\mathrm{H}$ & 3.50209 & 2.094536 & 1.42175 & $\mathrm{H}$ & -3.74952 & -2.60718 & 0.519977 \\
\hline $\mathrm{H}$ & 2.85082 & -0.17334 & 2.163061 & $\mathrm{H}$ & -2.22953 & -3.15376 & -1.33443 \\
\hline $\mathrm{H}$ & 1.194509 & 0.101486 & 1.663407 & $\mathrm{H}$ & -2.00854 & -1.46599 & -1.72363 \\
\hline $\mathrm{H}$ & 1.973652 & 0.472425 & -0.68467 & $\mathrm{H}$ & -0.92434 & -1.06369 & 0.478251 \\
\hline $\mathrm{H}$ & -0.07081 & 1.62268 & 0.684655 & $\mathrm{H}$ & -2.25742 & 0.48177 & -0.59024 \\
\hline $\mathrm{H}$ & -0.12417 & 3.926542 & -1.12628 & $\mathrm{H}$ & -3.10266 & 2.027742 & 1.650103 \\
\hline $\mathrm{H}$ & -0.68845 & 4.503152 & 0.414969 & $\mathrm{H}$ & -4.43404 & 2.226276 & 0.545759 \\
\hline $\mathrm{H}$ & -2.49901 & 2.81365 & 0.46408 & $\mathrm{H}$ & -2.88239 & 3.070478 & -1.22664 \\
\hline $\mathrm{H}$ & -2.5778 & 3.906058 & -0.90574 & $\mathrm{H}$ & -2.9528 & 4.062535 & 0.219796 \\
\hline $\mathrm{H}$ & 0.672591 & -2.09423 & 0.955878 & $\mathrm{H}$ & 0.105879 & -2.1838 & -2.58256 \\
\hline $\mathrm{H}$ & 0.807525 & -3.05464 & -0.49168 & $\mathrm{H}$ & 1.751945 & -2.04047 & -2.0111 \\
\hline $\mathrm{H}$ & 0.2106 & -0.9133 & -1.82145 & $\mathrm{H}$ & -0.46665 & 0.048576 & -1.80796 \\
\hline $\mathrm{H}$ & -0.19834 & -0.1803 & -0.28536 & $\mathrm{H}$ & 0.874254 & 0.052852 & -2.9076 \\
\hline $\mathrm{H}$ & -2.20857 & -0.6066 & -2.64233 & $\mathrm{H}$ & 1.695764 & 2.748971 & -0.42531 \\
\hline $\mathrm{H}$ & -3.52193 & -1.19678 & -1.64752 & $\mathrm{H}$ & 1.604855 & 2.406194 & -2.14157 \\
\hline $\mathrm{H}$ & -1.46116 & -2.5619 & -1.65324 & $\mathrm{H}$ & 0.988371 & 0.445405 & 0.113694 \\
\hline $\mathrm{H}$ & -3.00638 & -4.16162 & 1.107496 & $\mathrm{H}$ & 4.665855 & -0.04262 & -1.86348 \\
\hline $\mathrm{H}$ & -2.52123 & -4.24495 & -0.68071 & $\mathrm{H}$ & 3.156182 & 0.027989 & -2.9325 \\
\hline
\end{tabular}




$\begin{array}{lrrrrrrr}\mathrm{H} & 3.080844 & 4.15568 & 0.086691 & \mathrm{H} & -5.93555 & -0.97239 & 0.680808 \\ \mathrm{H} & 1.549991 & 4.988182 & 0.39137 & \mathrm{H} & -4.9075 & -1.03503 & 2.103333 \\ \mathrm{H} & 2.634419 & 4.583345 & 1.72977 & \mathrm{H} & -5.40645 & 0.529807 & 1.453372 \\ \mathrm{H} & -1.62072 & 1.236852 & -3.43537 & \mathrm{H} & 0.553688 & 3.225774 & 1.342028 \\ \mathrm{H} & -1.49606 & 2.980279 & -3.19091 & \mathrm{H} & -0.96111 & 3.940507 & 1.911478 \\ \mathrm{H} & -0.26372 & 1.906874 & -2.51553 & \mathrm{H} & -0.76843 & 2.183206 & 1.87859 \\ \mathrm{H} & 4.163253 & -0.12693 & -1.069 & \mathrm{H} & -1.56722 & -3.73753 & 1.027654 \\ \mathrm{H} & 4.324986 & -2.21923 & 0.423353 & \mathrm{H} & 0.266596 & -4.58968 & 0.165846 \\ \mathrm{H} & 3.045131 & -2.36213 & 1.635731 & \mathrm{H} & -0.24247 & -4.29868 & -1.4998 \\ \mathrm{H} & 3.138633 & -3.53761 & 0.331319 & \mathrm{H} & 1.470777 & -4.24937 & -1.07715 \\ \mathrm{H} & -1.11994 & 0.98265 & 2.933624 & \mathrm{H} & 2.757836 & 1.62138 & 3.346265 \\ \mathrm{H} & -2.71953 & 0.265627 & 3.293352 & \mathrm{H} & 3.814768 & 0.20137 & 3.0884 \\ \mathrm{H} & -1.23711 & -0.56726 & 3.821857 & \mathrm{H} & 4.376362 & 1.82131 & 2.609127 \\ \mathrm{H} & 2.041388 & -3.53136 & -2.21296 & \mathrm{H} & 2.451057 & -2.25804 & 2.161804 \\ \mathrm{H} & 3.112114 & -2.6961 & -3.35321 & \mathrm{H} & 0.819223 & -2.96581 & 2.253931 \\ \mathrm{H} & 3.79222 & -3.44174 & -1.89286 & \mathrm{H} & 2.116297 & -3.79867 & 1.356812\end{array}$

\begin{tabular}{|c|c|c|c|c|c|c|c|}
\hline 3b_11 & & & & 3b_12 & & & \\
\hline $\mathrm{C}$ & -3.85263 & 1.384865 & -0.05662 & $\mathrm{C}$ & -3.4172 & 0.075091 & -1.8353 \\
\hline $\mathrm{C}$ & -3.70052 & -0.08254 & 0.299841 & $\mathrm{C}$ & -3.22741 & 1.293723 & -0.93952 \\
\hline $\mathrm{C}$ & -2.61928 & 2.258704 & 0.282478 & $\mathrm{C}$ & -2.46126 & -1.10606 & -1.5554 \\
\hline $\mathrm{C}$ & -1.42118 & 2.002366 & -0.64395 & $\mathrm{C}$ & -2.46364 & -1.59365 & -0.10888 \\
\hline $\mathrm{C}$ & -3.39957 & -0.98654 & -0.64286 & $\mathrm{C}$ & -2.26708 & 2.192375 & -1.19979 \\
\hline $\mathrm{C}$ & -3.23067 & -2.47885 & -0.5133 & $\mathrm{C}$ & -2.00452 & 3.498238 & -0.49321 \\
\hline $\mathrm{C}$ & -1.98499 & -3.03626 & -1.2317 & $\mathrm{C}$ & -0.52202 & 3.88371 & -0.32065 \\
\hline $\mathrm{C}$ & -0.08485 & 2.645111 & -0.19598 & $\mathrm{C}$ & -1.38388 & -2.65793 & 0.230974 \\
\hline $\mathrm{C}$ & 0.326808 & 2.229753 & 1.239807 & $\mathrm{C}$ & 0.069621 & -2.12923 & 0.115621 \\
\hline $\mathrm{C}$ & -0.66488 & -3.0331 & -0.46592 & $\mathrm{C}$ & 0.298818 & 3.113553 & 0.709099 \\
\hline $\mathrm{C}$ & -0.46606 & -2.19006 & 0.732189 & $\mathrm{C}$ & 1.411165 & 2.245015 & 0.254528 \\
\hline $\mathrm{C}$ & 0.241992 & 0.741559 & 1.630665 & $\mathrm{C}$ & 0.352111 & -0.65425 & 0.452202 \\
\hline $\mathrm{C}$ & 0.862692 & -1.713 & 1.27647 & $\mathrm{C}$ & 2.071787 & 1.130068 & 1.038677 \\
\hline $\mathrm{C}$ & 1.204771 & -0.23716 & 0.92582 & $\mathrm{C}$ & 1.851064 & -0.29791 & 0.466133 \\
\hline $\mathrm{C}$ & 2.672396 & 0.010104 & 1.250921 & $\mathrm{C}$ & 2.568075 & -0.4643 & -0.86759 \\
\hline $\mathrm{C}$ & 3.723123 & -0.41554 & 0.261872 & $\mathrm{C}$ & 4.047922 & -0.73565 & -0.86813 \\
\hline $\mathrm{O}$ & 4.93058 & -0.3268 & 0.439013 & $\mathrm{O}$ & 4.731485 & -0.85909 & -1.86233 \\
\hline $\mathrm{C}$ & 3.125808 & 0.549103 & 2.386289 & $\mathrm{C}$ & 1.994687 & -0.36221 & -2.07009 \\
\hline $\mathrm{C}$ & -3.9335 & -0.41201 & 1.754651 & $\mathrm{C}$ & -4.22095 & 1.411464 & 0.192722 \\
\hline $\mathrm{C}$ & 0.497959 & -3.53515 & -1.29483 & $\mathrm{C}$ & -0.33413 & 2.951871 & 2.073075 \\
\hline $\mathrm{O}$ & -1.70264 & 2.496591 & -1.96404 & $\mathrm{O}$ & -3.76406 & -2.12136 & 0.180255 \\
\hline $\mathrm{O}$ & -0.74925 & -3.60213 & 0.867305 & $\mathrm{O}$ & 1.674279 & 3.581383 & 0.733026 \\
\hline $\mathrm{H}$ & -1.31203 & -1.57477 & 1.020634 & $\mathrm{H}$ & 1.529283 & 2.159384 & -0.82513 \\
\hline $\mathrm{C}$ & -0.15758 & 4.176353 & -0.28199 & $\mathrm{C}$ & -1.53433 & -3.91898 & -0.62514 \\
\hline $\mathrm{O}$ & 3.189549 & -0.91379 & -0.87098 & $\mathrm{O}$ & 4.551102 & -0.83252 & 0.375659 \\
\hline $\mathrm{C}$ & 4.125051 & -1.34613 & -1.88008 & $\mathrm{C}$ & 5.966732 & -1.09281 & 0.474 \\
\hline $\mathrm{O}$ & 0.860729 & 2.105896 & -1.15471 & $\mathrm{O}$ & -1.69874 & -3.15081 & 1.569517 \\
\hline $\mathrm{C}$ & 2.126677 & 2.735175 & -1.27477 & $\mathrm{C}$ & -1.3743 & -2.35428 & 2.704721 \\
\hline $\mathrm{H}$ & -4.71422 & 1.795888 & 0.48122 & $\mathrm{H}$ & -3.28406 & 0.364941 & -2.88213 \\
\hline $\mathrm{H}$ & -4.0935 & 1.48448 & -1.12048 & $\mathrm{H}$ & -4.44741 & -0.27933 & -1.73883 \\
\hline H & -2.90257 & 3.311685 & 0.194576 & $\mathrm{H}$ & -2.73529 & -1.93787 & -2.21113 \\
\hline
\end{tabular}




\begin{tabular}{|c|c|c|c|c|c|c|c|}
\hline $\mathrm{H}$ & -2.34336 & 2.092636 & 1.326345 & $\mathrm{H}$ & -1.44726 & -0.8069 & -1.83085 \\
\hline $\mathrm{H}$ & -1.23946 & 0.924121 & -0.70832 & $\mathrm{H}$ & -2.2839 & -0.73162 & 0.54450 \\
\hline $\mathrm{H}$ & -3.28978 & -0.6204 & -1.66419 & $\mathrm{H}$ & -1.63669 & 2.0144 & -2.0719 \\
\hline $\mathrm{H}$ & -4.10594 & -2.95689 & -0.97139 & $\mathrm{H}$ & -2.51128 & 3.533788 & 0.473 \\
\hline $\mathrm{H}$ & -3.22501 & -2.80678 & 0.528488 & $\mathrm{H}$ & -2.46071 & 4.300346 & -1.089 \\
\hline $\mathrm{H}$ & -2.17391 & -4.08099 & -1.50577 & $\mathrm{H}$ & -0.01089 & 3.830898 & -1.287 \\
\hline $\mathrm{H}$ & -1.83618 & -2.50067 & -2.1761 & $\mathrm{H}$ & -0.48417 & 4.935019 & -0.010 \\
\hline $\mathrm{H}$ & -0.29445 & 2.793375 & 1.94269 & $\mathrm{H}$ & 0.401787 & -2.31997 & -0.907 \\
\hline $\mathrm{H}$ & 1.344706 & 2.594844 & 1.403091 & $\mathrm{H}$ & 0.690766 & -2.77399 & 0.74815 \\
\hline $\mathrm{H}$ & -0.77791 & 0.390678 & 1.466613 & $\mathrm{H}$ & -0.03503 & -0.408 & 1.4429 \\
\hline $\mathrm{H}$ & 0.390394 & 0.671014 & 2.714 & $\mathrm{H}$ & -0.17866 & 0.004821 & -0.238 \\
\hline $\mathrm{H}$ & 1.659732 & -2.36701 & 0.921293 & $\mathrm{H}$ & 1.734541 & 1.140804 & 2.0772 \\
\hline $\mathrm{H}$ & 0.838337 & -1.8191 & 2.368592 & $\mathrm{H}$ & 3.145839 & 1.340094 & 1.0609 \\
\hline $\mathrm{H}$ & 1.090001 & -0.11071 & -0.15061 & $\mathrm{H}$ & 2.339542 & -0.97577 & 1.1708 \\
\hline $\mathrm{H}$ & 4.190516 & 0.656423 & 2.551186 & $\mathrm{H}$ & 2.588305 & -0.47129 & $-2.968 \mathrm{~s}$ \\
\hline $\mathrm{H}$ & 2.462158 & 0.89326 & 3.168689 & $\mathrm{H}$ & 0.936479 & -0.17096 & -2.192 \\
\hline $\mathrm{H}$ & -3.63578 & -1.42691 & 2.017998 & $\mathrm{H}$ & -3.93889 & 2.152003 & 0.9414 \\
\hline $\mathrm{H}$ & -3.39313 & 0.279527 & 2.409211 & $\mathrm{H}$ & -5.20575 & 1.694863 & -0.199 \\
\hline $\mathrm{H}$ & -4.99653 & -0.30315 & 2.000542 & $\mathrm{H}$ & -4.3546 & 0.445708 & 0.6879 \\
\hline $\mathrm{H}$ & 1.42768 & -3.56292 & -0.72944 & $\mathrm{H}$ & 0.389922 & 2.597187 & 2.8055 \\
\hline $\mathrm{H}$ & 0.285457 & -4.54841 & -1.64951 & $\mathrm{H}$ & -0.72166 & 3.914067 & 2.4218 \\
\hline $\mathrm{H}$ & 0.64477 & -2.89704 & -2.17042 & $\mathrm{H}$ & -1.16767 & 2.245664 & 2.03920 \\
\hline $\mathrm{H}$ & -2.48583 & 2.040345 & -2.28637 & $\mathrm{H}$ & -3.65197 & -2.63768 & 0.990957 \\
\hline $\mathrm{H}$ & -0.36071 & 4.491856 & -1.30539 & $\mathrm{H}$ & -2.54167 & -4.32575 & -0.52702 \\
\hline $\mathrm{H}$ & -0.95728 & 4.553185 & 0.358631 & $\mathrm{H}$ & -1.34549 & -3.70802 & -1.6788 \\
\hline $\mathrm{H}$ & 0.773986 & 4.636379 & 0.054074 & $\mathrm{H}$ & -0.81939 & -4.67362 & -0.290 \\
\hline $\mathrm{H}$ & 3.516439 & -1.71919 & -2.69955 & $\mathrm{H}$ & 6.181863 & -1.12597 & 1.53882 \\
\hline $\mathrm{H}$ & 4.739314 & -0.508 & -2.2102 & $\mathrm{H}$ & 6.209462 & -2.04624 & 0.004095 \\
\hline $\mathrm{H}$ & 4.767026 & -2.1358 & -1.48892 & $\mathrm{H}$ & 6.532729 & -0.29493 & -0.0073 \\
\hline $\mathrm{H}$ & 2.718141 & 2.099425 & -1.9351 & $\mathrm{H}$ & -1.8373 & -1.36172 & 2.673035 \\
\hline $\mathrm{H}$ & 2.054182 & 3.730999 & -1.72595 & $\mathrm{H}$ & -1.768 & -2.89081 & 3.56881 \\
\hline $\mathrm{H}$ & 2.653688 & 2.819996 & -0.31699 & $\mathrm{H}$ & -0.29294 & -2.24168 & 2.83037 \\
\hline 3b_13 & & & & & & & \\
\hline $\mathrm{C}$ & 3.149387 & 2.359077 & 0.497892 & $\mathrm{C}$ & -3.14339 & 0.264738 & $1.75308 \mathrm{C}$ \\
\hline $\mathrm{C}$ & 3.643864 & 0.942621 & 0.261834 & $\mathrm{C}$ & -3.04458 & 1.590874 & 1.022902 \\
\hline $\mathrm{C}$ & 1.840631 & 2.750917 & -0.22906 & $\mathrm{C}$ & -3.01816 & -0.98321 & 0.85504 \\
\hline $\mathrm{C}$ & 0.599116 & 2.189475 & 0.459592 & $\mathrm{C}$ & -1.57926 & -1.21725 & 0.37643 \\
\hline $\mathrm{C}$ & 3.298253 & -0.05946 & 1.083007 & $\mathrm{C}$ & -1.90529 & 2.298733 & 1.02083 \\
\hline $\mathrm{C}$ & 3.801239 & -1.48862 & 1.053526 & $\mathrm{C}$ & -1.66231 & 3.656849 & 0.40756 \\
\hline $\mathrm{C}$ & 3.240651 & -2.44334 & -0.02319 & $\mathrm{C}$ & -0.91751 & 3.67085 & -0.94695 \\
\hline $\mathrm{C}$ & -0.76151 & 2.51317 & -0.21917 & $\mathrm{C}$ & -1.38236 & -2.46238 & -0.5297 \\
\hline $\mathrm{C}$ & -0.93828 & 1.855841 & -1.60529 & $\mathrm{C}$ & 0.088537 & -2.58018 & -0.9999 \\
\hline $\mathrm{C}$ & 1.742402 & -2.72289 & -0.03508 & $\mathrm{C}$ & 0.488591 & 3.090863 & -0.97505 \\
\hline $\mathrm{C}$ & 0.942571 & -2.25753 & -1.19177 & $\mathrm{C}$ & 0.731989 & 1.836117 & -1.72125 \\
\hline $\mathrm{C}$ & -0.49093 & 0.395203 & -1.80453 & $\mathrm{C}$ & 0.675967 & -1.33769 & -1.70848 \\
\hline $\mathrm{C}$ & -0.56117 & -2.101 & -1.25048 & $\mathrm{C}$ & 1.90272 & 0.896804 & -1.53422 \\
\hline $\mathrm{C}$ & -1.12336 & -0.69404 & -0.91233 & $\mathrm{C}$ & 1.54518 & -0.4325 & -0.8122 \\
\hline & -2.64172 & -0.76247 & -0.99797 & $\mathrm{C}$ & 2.830142 & -1.11823 & -0.3580 \\
\hline
\end{tabular}




\begin{tabular}{|c|c|c|c|c|c|c|c|}
\hline $\mathrm{C}$ & -3.42578 & -1.20554 & 0.203475 & $\mathrm{C}$ & 3.478326 & -0.68352 & 0.927885 \\
\hline $\mathrm{O}$ & -4.63652 & -1.20078 & 0.285505 & $\mathrm{O}$ & 4.507626 & -1.14049 & 1.375628 \\
\hline $\mathrm{C}$ & -3.34957 & -0.51069 & -2.10276 & $\mathrm{C}$ & 3.474762 & -2.06549 & -1.03983 \\
\hline $\mathrm{C}$ & 4.568883 & 0.776147 & -0.91885 & $\mathrm{C}$ & -4.31819 & 2.020313 & 0.330245 \\
\hline $\mathrm{C}$ & 1.109868 & -3.06622 & 1.293652 & $\mathrm{C}$ & 1.436065 & 3.586669 & 0.093982 \\
\hline $\mathrm{O}$ & 0.582773 & 2.695962 & 1.800782 & $\mathrm{O}$ & -0.69439 & -1.32086 & 1.49979 \\
\hline $\mathrm{O}$ & 1.408407 & -3.62328 & -1.12671 & $\mathrm{O}$ & 1.031926 & 3.11229 & -2.32381 \\
\hline $\mathrm{H}$ & 1.488903 & -1.66945 & -1.92753 & $\mathrm{H}$ & -0.15789 & 1.378748 & -2.15666 \\
\hline $\mathrm{C}$ & -0.98322 & 4.026719 & -0.33069 & $\mathrm{C}$ & -2.35053 & -2.46557 & -1.71904 \\
\hline $\mathrm{O}$ & -2.63354 & -1.62841 & 1.205806 & $\mathrm{O}$ & 2.780706 & 0.286579 & 1.546336 \\
\hline $\mathrm{C}$ & -3.30219 & -2.08078 & 2.400922 & $\mathrm{C}$ & 3.323581 & 0.786639 & 2.786065 \\
\hline $\mathrm{O}$ & -1.69408 & 1.92055 & 0.736673 & $\mathrm{O}$ & -1.69463 & -3.54938 & 0.379909 \\
\hline $\mathrm{C}$ & -3.05235 & 2.344159 & 0.714462 & $\mathrm{C}$ & -1.52088 & -4.88455 & -0.07776 \\
\hline $\mathrm{H}$ & 3.933376 & 3.054025 & 0.178513 & $\mathrm{H}$ & -4.11472 & 0.222756 & 2.259043 \\
\hline $\mathrm{H}$ & 2.997268 & 2.526579 & 1.565758 & $\mathrm{H}$ & -2.37496 & 0.20245 & 2.526408 \\
\hline $\mathrm{H}$ & 1.771841 & 3.84234 & -0.24276 & $\mathrm{H}$ & -3.33787 & -1.87034 & 1.411056 \\
\hline $\mathrm{H}$ & 1.870841 & 2.419511 & -1.2715 & $\mathrm{H}$ & -3.68704 & -0.88921 & -0.00737 \\
\hline $\mathrm{H}$ & 0.683625 & 1.097688 & 0.502808 & $\mathrm{H}$ & -1.24445 & -0.34006 & -0.18131 \\
\hline $\mathrm{H}$ & 2.630728 & 0.185274 & 1.906866 & $\mathrm{H}$ & -1.05458 & 1.870052 & 1.549805 \\
\hline $\mathrm{H}$ & 3.625718 & -1.93119 & 2.038354 & $\mathrm{H}$ & -1.09883 & 4.260699 & 1.127641 \\
\hline $\mathrm{H}$ & 4.889415 & -1.48629 & 0.925228 & $\mathrm{H}$ & -2.60757 & 4.182509 & 0.256982 \\
\hline $\mathrm{H}$ & 3.526621 & -2.09376 & -1.01877 & $\mathrm{H}$ & -1.51716 & 3.152709 & -1.70089 \\
\hline $\mathrm{H}$ & 3.732653 & -3.41466 & 0.114266 & $\mathrm{H}$ & -0.83371 & 4.714544 & -1.2764 \\
\hline $\mathrm{H}$ & -0.38806 & 2.462352 & -2.33206 & $\mathrm{H}$ & 0.159251 & -3.43978 & -1.67307 \\
\hline $\mathrm{H}$ & -1.99129 & 1.962742 & -1.88116 & $\mathrm{H}$ & 0.698459 & -2.8253 & -0.12828 \\
\hline $\mathrm{H}$ & 0.592371 & 0.341668 & -1.67178 & $\mathrm{H}$ & -0.13232 & -0.7353 & -2.1275 \\
\hline $\mathrm{H}$ & -0.66184 & 0.142545 & -2.85785 & $\mathrm{H}$ & 1.264472 & -1.65711 & -2.57308 \\
\hline $\mathrm{H}$ & -1.02193 & -2.83707 & -0.59003 & $\mathrm{H}$ & 2.705742 & 1.407917 & -1.00113 \\
\hline $\mathrm{H}$ & -0.87139 & -2.36232 & -2.2701 & $\mathrm{H}$ & 2.293588 & 0.653779 & -2.5305 \\
\hline $\mathrm{H}$ & -0.87244 & -0.47338 & 0.124841 & $\mathrm{H}$ & 0.977549 & -0.18745 & 0.086992 \\
\hline $\mathrm{H}$ & -4.42709 & -0.6168 & -2.09938 & $\mathrm{H}$ & 4.399192 & -2.47842 & -0.65762 \\
\hline $\mathrm{H}$ & -2.88113 & -0.19875 & -3.02779 & $\mathrm{H}$ & 3.105163 & -2.45414 & -1.97908 \\
\hline $\mathrm{H}$ & 4.920159 & -0.24732 & -1.04762 & $\mathrm{H}$ & -4.19533 & 2.900307 & -0.29807 \\
\hline $\mathrm{H}$ & 4.072402 & 1.0786 & -1.84822 & $\mathrm{H}$ & -4.70381 & 1.214842 & -0.30395 \\
\hline $\mathrm{H}$ & 5.444642 & 1.426658 & -0.81129 & $\mathrm{H}$ & -5.10276 & 2.233243 & 1.066381 \\
\hline $\mathrm{H}$ & 0.069213 & -3.36562 & 1.177105 & $\mathrm{H}$ & 2.46229 & 3.286526 & -0.11318 \\
\hline $\mathrm{H}$ & 1.652792 & -3.89169 & 1.764643 & $\mathrm{H}$ & 1.404616 & 4.679236 & 0.134295 \\
\hline $\mathrm{H}$ & 1.145751 & -2.21035 & 1.972589 & $\mathrm{H}$ & 1.158949 & 3.20722 & 1.081716 \\
\hline $\mathrm{H}$ & -0.2786 & 2.426406 & 2.148572 & $\mathrm{H}$ & -0.80274 & -2.22741 & 1.819889 \\
\hline $\mathrm{H}$ & -0.9159 & 4.499507 & 0.650465 & $\mathrm{H}$ & -3.38437 & -2.57182 & -1.38434 \\
\hline $\mathrm{H}$ & -0.22647 & 4.472484 & -0.97786 & $\mathrm{H}$ & -2.27431 & -1.53535 & -2.28757 \\
\hline $\mathrm{H}$ & -1.95776 & 4.254846 & -0.76652 & $\mathrm{H}$ & -2.12869 & -3.28497 & -2.40701 \\
\hline $\mathrm{H}$ & -2.50926 & -2.37887 & 3.081856 & $\mathrm{H}$ & 2.638754 & 1.566188 & 3.109697 \\
\hline $\mathrm{H}$ & -3.89415 & -1.27331 & 2.83264 & $\mathrm{H}$ & 3.371559 & -0.01127 & 3.527673 \\
\hline $\mathrm{H}$ & -3.9516 & -2.92706 & 2.175164 & $\mathrm{H}$ & 4.321616 & 1.19728 & 2.628571 \\
\hline $\mathrm{H}$ & -3.50598 & 2.234676 & -0.27605 & $\mathrm{H}$ & -0.46993 & -5.1246 & -0.26896 \\
\hline $\mathrm{H}$ & -3.58358 & 1.693399 & 1.409553 & $\mathrm{H}$ & -1.88533 & -5.53178 & 0.720844 \\
\hline $\mathrm{H}$ & -3.16712 & 3.379699 & 1.049401 & $\mathrm{H}$ & -2.10324 & -5.08517 & -0.98269 \\
\hline
\end{tabular}




\begin{tabular}{|c|c|c|c|c|c|c|c|}
\hline \multirow{2}{*}{$\begin{array}{l}\text { 3b_15 } \\
\mathrm{C}\end{array}$} & \multirow[b]{2}{*}{-3.23055} & \multirow[b]{2}{*}{0.361078} & \multirow[b]{2}{*}{-1.97497} & \multicolumn{2}{|l|}{ 3b_16 } & \multirow[b]{2}{*}{1.601693} & \multirow[b]{2}{*}{2.325422} \\
\hline & & & & $\mathrm{C}$ & 1.897432 & & \\
\hline $\mathrm{C}$ & -3.0426 & 1.553216 & -1.04264 & $\mathrm{C}$ & 1.199965 & 2.620445 & 1.430051 \\
\hline $\mathrm{C}$ & -2.36363 & -0.87165 & -1.63301 & $\mathrm{C}$ & 1.995484 & 0.172243 & 1.743672 \\
\hline $\mathrm{C}$ & -2.51963 & -1.37097 & -0.19103 & $\mathrm{C}$ & 2.600896 & 0.112423 & 0.346538 \\
\hline $\mathrm{C}$ & -1.99115 & 2.373711 & -1.18151 & $\mathrm{C}$ & -0.13797 & 2.680441 & 1.370774 \\
\hline $\mathrm{C}$ & -1.69915 & 3.637122 & -0.4112 & $\mathrm{C}$ & -0.98586 & 3.67096 & 0.613027 \\
\hline $\mathrm{C}$ & -0.21264 & 3.920025 & -0.11701 & $\mathrm{C}$ & -2.28611 & 3.112795 & 0.000367 \\
\hline $\mathrm{C}$ & -1.50782 & -2.4736 & 0.249085 & $\mathrm{C}$ & 2.560453 & -1.27319 & -0.35078 \\
\hline $\mathrm{C}$ & -0.04658 & -2.06151 & -0.04635 & $\mathrm{C}$ & 1.12427 & -1.8181 & -0.51063 \\
\hline $\mathrm{C}$ & 0.496322 & 3.031884 & 0.902189 & $\mathrm{C}$ & -2.16809 & 2.173423 & -1.19713 \\
\hline $\mathrm{C}$ & 1.612708 & 2.16446 & 0.4555 & $\mathrm{C}$ & -2.6188 & 0.767186 & -1.06267 \\
\hline $\mathrm{C}$ & 0.357144 & -0.67276 & 0.467034 & $\mathrm{C}$ & 0.089249 & -0.81859 & -1.03803 \\
\hline $\mathrm{C}$ & 2.174878 & 0.956014 & 1.174425 & $\mathrm{C}$ & -2.25138 & -0.3888 & -1.97119 \\
\hline $\mathrm{C}$ & 1.873994 & -0.40892 & 0.494862 & $\mathrm{C}$ & -1.26357 & -1.45424 & -1.42065 \\
\hline $\mathrm{C}$ & 2.578116 & -0.51479 & -0.85199 & $\mathrm{C}$ & -1.88425 & -2.38186 & -0.37613 \\
\hline $\mathrm{C}$ & 4.033508 & -0.89276 & -0.88538 & $\mathrm{C}$ & -1.99708 & -1.94202 & 1.050305 \\
\hline $\mathrm{O}$ & 4.702729 & -0.99591 & -1.89184 & $\mathrm{O}$ & -1.6632 & -0.84881 & 1.463532 \\
\hline $\mathrm{C}$ & 2.01219 & -0.26769 & -2.03685 & $\mathrm{C}$ & -2.33945 & -3.5912 & -0.72562 \\
\hline $\mathrm{C}$ & -4.138 & 1.73619 & -0.01842 & $\mathrm{C}$ & 2.130199 & 3.544984 & 0.680117 \\
\hline $\mathrm{C}$ & -0.24987 & 2.777246 & 2.191959 & $\mathrm{C}$ & -1.22217 & 2.615727 & -2.29116 \\
\hline $\mathrm{O}$ & -3.88151 & -1.76191 & 0.056145 & $\mathrm{O}$ & 3.955161 & 0.579753 & 0.418468 \\
\hline $\mathrm{O}$ & 1.880292 & 3.441308 & 1.073782 & $\mathrm{O}$ & -3.48086 & 1.760569 & -1.66427 \\
\hline $\mathrm{H}$ & 1.81089 & 2.172202 & -0.61564 & $\mathrm{H}$ & -2.94274 & 0.478547 & -0.06718 \\
\hline $\mathrm{C}$ & -1.79535 & -3.82409 & -0.41707 & $\mathrm{C}$ & 3.441636 & -2.30013 & 0.368456 \\
\hline $\mathrm{O}$ & 4.533449 & -1.11248 & 0.344134 & $\mathrm{O}$ & -2.50605 & -2.88953 & 1.858099 \\
\hline $\mathrm{C}$ & 5.92622 & -1.48203 & 0.411315 & $\mathrm{C}$ & -2.64964 & -2.53584 & 3.248624 \\
\hline $\mathrm{O}$ & -1.53155 & -2.57586 & 1.694503 & $\mathrm{O}$ & 3.116727 & -0.92581 & -1.65274 \\
\hline $\mathrm{C}$ & -2.57999 & -3.29309 & 2.341055 & $\mathrm{C}$ & 3.49264 & -1.97589 & -2.53435 \\
\hline $\mathrm{H}$ & -2.99312 & 0.655505 & -3.00188 & $\mathrm{H}$ & 1.371075 & 1.532697 & 3.282497 \\
\hline $\mathrm{H}$ & -4.28429 & 0.069018 & -1.97106 & $\mathrm{H}$ & 2.906219 & 1.959996 & 2.548986 \\
\hline $\mathrm{H}$ & -2.61188 & -1.68191 & -2.33 & $\mathrm{H}$ & 2.590219 & -0.44289 & 2.427445 \\
\hline $\mathrm{H}$ & -1.32011 & -0.61663 & -1.82138 & $\mathrm{H}$ & 0.993032 & -0.25959 & 1.711817 \\
\hline $\mathrm{H}$ & -2.35882 & -0.53507 & 0.491946 & $\mathrm{H}$ & 2.037318 & 0.788093 & -0.30628 \\
\hline $\mathrm{H}$ & -1.28889 & 2.157685 & -1.98771 & $\mathrm{H}$ & -0.69926 & 1.973281 & 1.981472 \\
\hline $\mathrm{H}$ & -2.27155 & 3.671547 & 0.517857 & $\mathrm{H}$ & -0.40417 & 4.179934 & -0.15852 \\
\hline $\mathrm{H}$ & -2.05927 & 4.488283 & -1.00566 & $\mathrm{H}$ & -1.29406 & 4.460705 & 1.312418 \\
\hline $\mathrm{H}$ & 0.358045 & 3.905723 & -1.05122 & $\mathrm{H}$ & -2.87654 & 2.616702 & 0.777401 \\
\hline $\mathrm{H}$ & -0.14014 & 4.943122 & 0.271644 & $\mathrm{H}$ & -2.88405 & 3.96577 & -0.34357 \\
\hline $\mathrm{H}$ & 0.138389 & -2.14477 & -1.11932 & $\mathrm{H}$ & 0.804027 & -2.21227 & 0.458169 \\
\hline $\mathrm{H}$ & 0.582827 & -2.81678 & 0.435995 & $\mathrm{H}$ & 1.167574 & -2.68745 & -1.17662 \\
\hline $\mathrm{H}$ & 0.000354 & -0.56906 & 1.495297 & $\mathrm{H}$ & 0.485443 & -0.33705 & -1.93771 \\
\hline $\mathrm{H}$ & -0.14535 & 0.111307 & -0.10585 & $\mathrm{H}$ & -0.08035 & -0.0318 & -0.30431 \\
\hline $\mathrm{H}$ & 1.806523 & 0.91745 & 2.201746 & $\mathrm{H}$ & -1.83311 & -0.00069 & -2.90295 \\
\hline $\mathrm{H}$ & 3.259649 & 1.090513 & 1.23835 & $\mathrm{H}$ & -3.18635 & -0.89247 & -2.24228 \\
\hline $\mathrm{H}$ & 2.322129 & -1.16696 & 1.142092 & $\mathrm{H}$ & -1.06843 & -2.11301 & -2.27361 \\
\hline $\mathrm{H}$ & 2.592795 & -0.34397 & -2.94757 & $\mathrm{H}$ & -2.79997 & -4.25646 & -0.0082 \\
\hline $\mathrm{H}$ & 0.972336 & 0.016879 & -2.13 & $\mathrm{H}$ & -2.26188 & -3.93923 & -1.74992 \\
\hline $\mathrm{H}$ & -3.88997 & 2.459601 & 0.758604 & $\mathrm{H}$ & 2.588372 & 4.261686 & 1.373656 \\
\hline
\end{tabular}




$\begin{array}{lrrrlrrr}\mathrm{H} & -5.05657 & 2.080322 & -0.51042 & \mathrm{H} & 2.952788 & 2.97699 & 0.237147 \\ \mathrm{H} & -4.38266 & 0.7817 & 0.455525 & \mathrm{H} & 1.635265 & 4.117405 & -0.10504 \\ \mathrm{H} & 0.39212 & 2.308162 & 2.936473 & \mathrm{H} & -1.33539 & 2.006174 & -3.18674 \\ \mathrm{H} & -0.61138 & 3.72311 & 2.607226 & \mathrm{H} & -1.42778 & 3.655606 & -2.56388 \\ \mathrm{H} & -1.11484 & 2.130876 & 2.023159 & \mathrm{H} & -0.18273 & 2.553776 & -1.95956 \\ \mathrm{H} & -4.15442 & -2.37075 & -0.63973 & \mathrm{H} & 4.306018 & 0.46164 & -0.47443 \\ \mathrm{H} & -2.81109 & -4.1728 & -0.22279 & \mathrm{H} & 4.473166 & -1.95004 & 0.426728 \\ \mathrm{H} & -1.66496 & -3.75028 & -1.49903 & \mathrm{H} & 3.078525 & -2.4614 & 1.38543 \\ \mathrm{H} & -1.10145 & -4.58179 & -0.04489 & \mathrm{H} & 3.423987 & -3.26601 & -0.14088 \\ \mathrm{H} & 6.14305 & -1.60356 & 1.469379 & \mathrm{H} & -3.08801 & -3.40942 & 3.724218 \\ \mathrm{H} & 6.095804 & -2.41648 & -0.12433 & \mathrm{H} & -1.67662 & -2.31131 & 3.686745 \\ \mathrm{H} & 6.547919 & -0.69721 & -0.02018 & \mathrm{H} & -3.30602 & -1.6719 & 3.356876 \\ \mathrm{H} & -2.5697 & -4.35867 & 2.084411 & \mathrm{H} & 2.667967 & -2.66911 & -2.734 \\ \mathrm{H} & -2.38418 & -3.19985 & 3.410833 & \mathrm{H} & 3.774069 & -1.49705 & -3.47305 \\ \mathrm{H} & -3.56435 & -2.88014 & 2.114376 & \mathrm{H} & 4.351024 & -2.54402 & -2.16118\end{array}$

\begin{tabular}{|c|c|c|c|c|c|c|c|}
\hline 3b_17 & & & & 3b_18 & & & \\
\hline $\mathrm{C}$ & 2.583891 & 1.785825 & 1.749457 & $\mathrm{C}$ & 1.880931 & 1.21983 & 2.399731 \\
\hline $\mathrm{C}$ & 3.333003 & 0.689395 & 1.015728 & $\mathrm{C}$ & 1.295787 & 2.374885 & 1.591031 \\
\hline $\mathrm{C}$ & 1.711338 & 2.67575 & 0.834053 & $\mathrm{C}$ & 1.882436 & -0.15142 & 1.681453 \\
\hline $\mathrm{C}$ & 0.407817 & 1.966063 & 0.444861 & $\mathrm{C}$ & 2.605078 & -0.14826 & 0.328896 \\
\hline $\mathrm{C}$ & 2.961503 & -0.59361 & 1.142638 & $\mathrm{C}$ & -0.03121 & 2.515769 & 1.465351 \\
\hline $\mathrm{C}$ & 3.614959 & -1.80633 & 0.524343 & $\mathrm{C}$ & -0.77978 & 3.626632 & 0.770928 \\
\hline $\mathrm{C}$ & 3.079379 & -2.23633 & -0.86016 & $\mathrm{C}$ & -2.11239 & 3.220584 & 0.11147 \\
\hline $\mathrm{C}$ & -0.5111 & 2.745381 & -0.53489 & $\mathrm{C}$ & 2.455188 & -1.44875 & -0.50996 \\
\hline $\mathrm{C}$ & -1.71886 & 1.86527 & -0.93896 & $\mathrm{C}$ & 0.976011 & -1.88221 & -0.63157 \\
\hline $\mathrm{C}$ & 1.633419 & -2.71504 & -0.94026 & $\mathrm{C}$ & -2.05315 & 2.33213 & -1.12831 \\
\hline $\mathrm{C}$ & 0.648601 & -1.90966 & -1.70125 & $\mathrm{C}$ & -2.5994 & 0.955858 & -1.05833 \\
\hline $\mathrm{C}$ & -1.35449 & 0.567524 & -1.69824 & $\mathrm{C}$ & 0.005536 & -0.79712 & -1.11227 \\
\hline $\mathrm{C}$ & -0.85715 & -1.95394 & -1.55042 & $\mathrm{C}$ & -2.30521 & -0.18178 & -2.01481 \\
\hline $\mathrm{C}$ & -1.46347 & -0.71315 & -0.84299 & $\mathrm{C}$ & -1.3872 & -1.32906 & -1.51023 \\
\hline C & -2.89687 & -1.02312 & -0.42966 & $\mathrm{C}$ & -2.06365 & -2.25544 & -0.49977 \\
\hline $\mathrm{C}$ & -3.16557 & -1.59562 & 0.933639 & $\mathrm{C}$ & -2.14066 & -1.86806 & 0.943875 \\
\hline $\mathrm{O}$ & -4.26442 & -1.7734 & 1.405406 & $\mathrm{O}$ & -1.74205 & -0.81295 & 1.397132 \\
\hline $\mathrm{C}$ & -3.96066 & -0.8642 & -1.22253 & $\mathrm{C}$ & -2.59878 & -3.41722 & -0.89463 \\
\hline $\mathrm{C}$ & 4.534431 & 1.144213 & 0.223499 & $\mathrm{C}$ & 2.309265 & 3.329418 & 1.005621 \\
\hline $\mathrm{C}$ & 1.216603 & -3.74762 & 0.082931 & $\mathrm{C}$ & -1.07017 & 2.757572 & -2.19592 \\
\hline $\mathrm{O}$ & -0.34095 & 1.604694 & 1.611305 & $\mathrm{O}$ & 4.015166 & 0.09164 & 0.511403 \\
\hline $\mathrm{O}$ & 1.268555 & -3.06115 & -2.30582 & $\mathrm{O}$ & -3.38753 & 2.032116 & -1.61853 \\
\hline $\mathrm{H}$ & 1.035965 & -0.97705 & -2.10895 & $\mathrm{H}$ & -2.94929 & 0.646394 & -0.07793 \\
\hline $\mathrm{C}$ & 0.263121 & 3.241328 & -1.76895 & $\mathrm{C}$ & 3.273598 & -2.60677 & 0.074123 \\
\hline $\mathrm{O}$ & -2.03518 & -1.90252 & 1.594525 & $\mathrm{O}$ & -2.6962 & -2.817 & 1.718603 \\
\hline $\mathrm{C}$ & -2.19842 & -2.43148 & 2.925585 & $\mathrm{C}$ & -2.81041 & -2.51113 & 3.123092 \\
\hline $\mathrm{O}$ & -0.96274 & 3.855486 & 0.277954 & $\mathrm{O}$ & 2.824243 & -1.17788 & -1.88503 \\
\hline $\mathrm{C}$ & -1.8352 & 4.809398 & -0.31307 & $\mathrm{C}$ & 4.197924 & -1.04865 & -2.24 \\
\hline $\mathrm{H}$ & 3.305209 & 2.419823 & 2.279382 & $\mathrm{H}$ & 1.31522 & 1.099819 & 3.328305 \\
\hline $\mathrm{H}$ & 1.930593 & 1.33774 & 2.501563 & $\mathrm{H}$ & 2.899229 & 1.479383 & 2.712729 \\
\hline $\mathrm{H}$ & 1.477292 & 3.620445 & 1.333634 & $\mathrm{H}$ & 2.352991 & -0.89228 & 2.336952 \\
\hline $\mathrm{H}$ & 2.268646 & 2.926617 & -0.0727 & $\mathrm{H}$ & 0.843988 & -0.45304 & 1.544321 \\
\hline
\end{tabular}




$\begin{array}{lrrrrrrr}\mathrm{H} & 0.676799 & 1.020461 & -0.02968 & \mathrm{H} & 2.189376 & 0.6539 & -0.29097 \\ \mathrm{H} & 2.095624 & -0.7972 & 1.771174 & \mathrm{H} & -0.66262 & 1.77705 & 1.95784 \\ \mathrm{H} & 3.527317 & -2.64884 & 1.21882 & \mathrm{H} & -0.14659 & 4.132889 & 0.03911 \\ \mathrm{H} & 4.688052 & -1.63234 & 0.406859 & \mathrm{H} & -1.0298 & 4.39201 & 1.518691 \\ \mathrm{H} & 3.212287 & -1.41304 & -1.56946 & \mathrm{H} & -2.76 & 2.741735 & 0.852894 \\ \mathrm{H} & 3.702749 & -3.06359 & -1.22222 & \mathrm{H} & -2.62473 & 4.140039 & -0.1973 \\ \mathrm{H} & -2.39332 & 2.470441 & -1.54981 & \mathrm{H} & 0.647397 & -2.28681 & 0.329347 \\ \mathrm{H} & -2.26312 & 1.617776 & -0.02586 & \mathrm{H} & 0.96544 & -2.71744 & -1.33991 \\ \mathrm{H} & -0.33008 & 0.641781 & -2.06764 & \mathrm{H} & 0.429469 & -0.31499 & -1.99739 \\ \mathrm{H} & -1.97255 & 0.463652 & -2.59445 & \mathrm{H} & -0.10917 & -0.02554 & -0.35168 \\ \mathrm{H} & -1.14388 & -2.85469 & -1.005 & \mathrm{H} & -1.86212 & 0.218 & -2.93006 \\ \mathrm{H} & -1.29952 & -2.04603 & -2.55095 & \mathrm{H} & -3.26986 & -0.61385 & -2.30501 \\ \mathrm{H} & -0.89969 & -0.55403 & 0.075955 & \mathrm{H} & -1.23711 & -1.96541 & -2.38902 \\ \mathrm{H} & -4.94883 & -1.13747 & -0.87326 & \mathrm{H} & -3.09868 & -4.08016 & -0.20184 \\ \mathrm{H} & -3.87832 & -0.46582 & -2.22688 & \mathrm{H} & -2.54886 & -3.7268 & -1.93289 \\ \mathrm{H} & 5.031173 & 0.331494 & -0.30627 & \mathrm{H} & 1.866006 & 4.094315 & 0.36852 \\ \mathrm{H} & 4.257822 & 1.901002 & -0.51868 & \mathrm{H} & 2.856079 & 3.839862 & 1.80753 \\ \mathrm{H} & 5.267123 & 1.617938 & 0.888175 & \mathrm{H} & 3.060695 & 2.7976 & 0.411174 \\ \mathrm{H} & 0.185277 & -4.06859 & -0.06098 & \mathrm{H} & -1.2145 & 2.196195 & -3.11803 \\ \mathrm{H} & 1.858359 & -4.63013 & -0.00822 & \mathrm{H} & -1.20465 & 3.819617 & -2.42403 \\ \mathrm{H} & 1.317992 & -3.36284 & 1.100401 & \mathrm{H} & -0.04021 & 2.612923 & -1.8603 \\ \mathrm{H} & -0.72028 & 2.431875 & 1.938086 & \mathrm{H} & 4.117195 & 0.894843 & 1.031715 \\ \mathrm{H} & 0.834278 & 4.139862 & -1.52286 & \mathrm{H} & 4.321537 & -2.3386 & 0.20072 \\ \mathrm{H} & 0.969662 & 2.487058 & -2.12386 & \mathrm{H} & 2.881428 & -2.88846 & 1.053948 \\ \mathrm{H} & -0.40972 & 3.47851 & -2.59651 & \mathrm{H} & 3.205371 & -3.47836 & -0.58194 \\ \mathrm{H} & -1.20639 & -2.74779 & 3.236789 & \mathrm{H} & -3.29157 & -3.37813 & 3.568399 \\ \mathrm{H} & -2.57627 & -1.6548 & 3.592125 & \mathrm{H} & -1.8236 & -2.35619 & 3.560481 \\ \mathrm{H} & -2.88897 & -3.27543 & 2.919328 & \mathrm{H} & -3.41879 & -1.61841 & 3.271114 \\ \mathrm{H} & -2.83577 & 4.401873 & -0.49444 & \mathrm{H} & 4.764774 & -1.96442 & -2.03746 \\ \mathrm{H} & -1.91874 & 5.6299 & 0.40118 & \mathrm{H} & 4.208745 & -0.87214 & -3.31722 \\ \mathrm{H} & -1.43663 & 5.206141 & -1.25384 & \mathrm{H} & 4.67965 & -0.21377 & -1.72805\end{array}$

\begin{tabular}{lrrrlrrr} 
3c_1 & \multicolumn{7}{c}{ 3c_2 } \\
C & 3.748116 & -1.43524 & -0.87809 & C & 3.497717 & 1.06295 & 1.692072 \\
C & 3.777432 & -0.20157 & 0.015491 & C & 3.722868 & -0.1246 & 0.76418 \\
C & 2.333322 & -1.9656 & -1.19664 & C & 2.104153 & 1.718049 & 1.577094 \\
C & 1.49594 & -2.28746 & 0.03531 & C & 1.747503 & 2.183326 & 0.170335 \\
C & 3.546649 & 1.014402 & -0.49871 & C & 3.245615 & -1.33529 & 1.084668 \\
C & 3.618888 & 2.347939 & 0.199224 & C & 3.43575 & -2.63595 & 0.347862 \\
C & 2.561474 & 3.381787 & -0.23398 & C & 2.216257 & -3.578 & 0.342167 \\
C & 0.021475 & -2.68337 & -0.24552 & C & 0.295289 & 2.700025 & -0.01288 \\
C & -0.76885 & -1.56404 & -0.9563 & C & -0.76031 & 1.616948 & 0.295242 \\
C & 1.124866 & 3.183054 & 0.23993 & C & 1.045955 & -3.2313 & -0.5733 \\
C & 0.061252 & 2.870232 & -0.74593 & C & -0.25876 & -2.83926 & 0.013058 \\
C & -0.74043 & -0.18813 & -0.28089 & C & -0.58453 & 0.279218 & -0.43404 \\
C & -1.31554 & 2.301778 & -0.46057 & C & -1.40201 & -2.13287 & -0.68821 \\
C & -1.54217 & 0.888017 & -1.0473 & C & -1.71774 & -0.72271 & -0.13572 \\
C & -3.02316 & 0.567118 & -1.20096 & C & -3.07182 & -0.25901 & -0.66468 \\
C & -3.95222 & 0.464681 & -0.02309 & C & -4.27137 & -0.23103 & 0.236665
\end{tabular}




\begin{tabular}{|c|c|c|c|c|c|c|c|}
\hline $\mathrm{O}$ & -5.13561 & 0.209408 & -0.0976 & $\mathrm{O}$ & -5.3786 & 0.092813 & -0.10217 \\
\hline $\mathrm{C}$ & -3.5802 & 0.373732 & -2.40106 & $\mathrm{C}$ & -3.27836 & 0.104409 & -1.93446 \\
\hline $\mathrm{C}$ & 4.113223 & -0.46887 & 1.463396 & $\mathrm{C}$ & 4.532809 & 0.175353 & -0.47494 \\
\hline $\mathrm{C}$ & 0.946537 & 2.907741 & 1.715889 & $\mathrm{C}$ & 1.401337 & -2.90419 & -2.00629 \\
\hline $\mathrm{O}$ & 2.150588 & -3.33509 & 0.762566 & $\mathrm{O}$ & 2.679027 & 3.202522 & -0.21457 \\
\hline $\mathrm{O}$ & 0.264478 & 4.224451 & -0.29399 & $\mathrm{O}$ & -0.02804 & -4.19663 & -0.41606 \\
\hline $\mathrm{H}$ & 0.398354 & 2.7115 & -1.77213 & $\mathrm{H}$ & -0.27984 & -2.73538 & 1.099393 \\
\hline $\mathrm{C}$ & -0.07351 & -3.98752 & -1.04542 & $\mathrm{C}$ & 0.02928 & 3.954319 & 0.826634 \\
\hline $\mathrm{O}$ & -3.33469 & 0.690073 & 1.150735 & $\mathrm{O}$ & -3.9839 & -0.61034 & 1.492316 \\
\hline $\mathrm{C}$ & -4.16182 & 0.619103 & 2.331279 & $\mathrm{C}$ & -5.08354 & -0.611 & 2.426013 \\
\hline $\mathrm{O}$ & -0.4651 & -2.87082 & 1.114207 & $\mathrm{O}$ & 0.303068 & 3.014729 & -1.43447 \\
\hline $\mathrm{C}$ & -1.75568 & -3.43769 & 1.301285 & $\mathrm{C}$ & -0.80457 & 3.720608 & -1.98009 \\
\hline $\mathrm{H}$ & 4.326903 & -2.23435 & -0.40694 & $\mathrm{H}$ & 4.260461 & 1.821645 & 1.496485 \\
\hline $\mathrm{H}$ & 4.239249 & -1.21083 & -1.82992 & $\mathrm{H}$ & 3.629088 & 0.746324 & 2.731296 \\
\hline $\mathrm{H}$ & 1.810391 & -1.2135 & -1.79264 & $\mathrm{H}$ & 1.354088 & 0.99744 & 1.912696 \\
\hline $\mathrm{H}$ & 2.425523 & -2.86183 & -1.81856 & $\mathrm{H}$ & 2.060516 & 2.569913 & 2.263463 \\
\hline $\mathrm{H}$ & 1.452349 & -1.39873 & 0.674886 & $\mathrm{H}$ & 1.856005 & 1.338542 & -0.51892 \\
\hline $\mathrm{H}$ & 3.348955 & 1.07572 & -1.56989 & $\mathrm{H}$ & 2.70669 & -1.42673 & 2.028883 \\
\hline $\mathrm{H}$ & 4.595849 & 2.796143 & -0.02915 & $\mathrm{H}$ & 4.247362 & -3.18815 & 0.841568 \\
\hline $\mathrm{H}$ & 3.60231 & 2.229972 & 1.284819 & $\mathrm{H}$ & 3.781116 & -2.46742 & -0.67438 \\
\hline $\mathrm{H}$ & 2.559791 & 3.472702 & -1.32506 & $\mathrm{H}$ & 1.838075 & -3.69997 & 1.362349 \\
\hline $\mathrm{H}$ & 2.869908 & 4.360068 & 0.154715 & $\mathrm{H}$ & 2.55927 & -4.56911 & 0.021092 \\
\hline $\mathrm{H}$ & -0.3918 & -1.47997 & -1.98108 & $\mathrm{H}$ & -0.7694 & 1.444188 & 1.376318 \\
\hline $\mathrm{H}$ & -1.80554 & -1.89889 & -1.06086 & $\mathrm{H}$ & -1.74541 & 2.03215 & 0.060179 \\
\hline $\mathrm{H}$ & -1.11342 & -0.26837 & 0.741451 & $\mathrm{H}$ & -0.51041 & 0.452846 & -1.51191 \\
\hline $\mathrm{H}$ & 0.289849 & 0.170647 & -0.21223 & $\mathrm{H}$ & 0.357348 & -0.18708 & -0.13331 \\
\hline $\mathrm{H}$ & -1.52795 & 2.305974 & 0.607965 & $\mathrm{H}$ & -1.22839 & -2.07684 & -1.76558 \\
\hline $\mathrm{H}$ & -2.03061 & 2.99108 & -0.92514 & $\mathrm{H}$ & -2.28528 & -2.76764 & -0.5518 \\
\hline $\mathrm{H}$ & -1.15076 & 0.907737 & -2.07184 & $\mathrm{H}$ & -1.80134 & -0.81322 & 0.949119 \\
\hline $\mathrm{H}$ & -4.63725 & 0.157725 & -2.49411 & $\mathrm{H}$ & -4.26456 & 0.400828 & -2.26836 \\
\hline $\mathrm{H}$ & -2.9928 & 0.428459 & -3.31008 & $\mathrm{H}$ & -2.47727 & 0.116312 & -2.66372 \\
\hline $\mathrm{H}$ & 3.916613 & 0.380481 & 2.118341 & $\mathrm{H}$ & 4.491953 & -0.61757 & -1.22241 \\
\hline $\mathrm{H}$ & 3.553118 & -1.33328 & 1.830877 & $\mathrm{H}$ & 4.196316 & 1.109139 & -0.93375 \\
\hline $\mathrm{H}$ & 5.175594 & -0.72536 & 1.562371 & $\mathrm{H}$ & 5.586614 & 0.325587 & -0.20811 \\
\hline $\mathrm{H}$ & -0.09901 & 2.987745 & 2.010645 & $\mathrm{H}$ & 0.517338 & -2.89948 & -2.64268 \\
\hline $\mathrm{H}$ & 1.302732 & 1.90715 & 1.974104 & $\mathrm{H}$ & 1.883258 & -1.92557 & -2.07661 \\
\hline $\mathrm{H}$ & 1.519845 & 3.631408 & 2.303441 & $\mathrm{H}$ & 2.096527 & -3.65172 & -2.40037 \\
\hline $\mathrm{H}$ & 1.538804 & -3.56082 & 1.476228 & $\mathrm{H}$ & 2.358012 & 3.521446 & -1.06884 \\
\hline $\mathrm{H}$ & 0.463454 & -4.78938 & -0.53681 & $\mathrm{H}$ & 0.75227 & 4.735645 & 0.588262 \\
\hline $\mathrm{H}$ & -1.11267 & -4.29132 & -1.18583 & $\mathrm{H}$ & -0.97784 & 4.339887 & 0.656349 \\
\hline $\mathrm{H}$ & 0.363473 & -3.85763 & -2.03745 & $\mathrm{H}$ & 0.112674 & 3.722589 & 1.890215 \\
\hline $\mathrm{H}$ & -3.49461 & 0.822303 & 3.1648 & $\mathrm{H}$ & -4.66109 & -0.94269 & 3.370949 \\
\hline $\mathrm{H}$ & -4.95423 & 1.366367 & 2.283553 & $\mathrm{H}$ & -5.49868 & 0.392557 & 2.522791 \\
\hline $\mathrm{H}$ & -4.6025 & -0.3735 & 2.425732 & $\mathrm{H}$ & -5.86306 & -1.29653 & 2.092602 \\
\hline $\mathrm{H}$ & -1.80048 & -4.48416 & 0.982109 & $\mathrm{H}$ & -0.87175 & 4.745067 & -1.59992 \\
\hline $\mathrm{H}$ & -2.53656 & -2.87389 & 0.77961 & $\mathrm{H}$ & -1.75505 & 3.208686 & -1.79489 \\
\hline $\mathrm{H}$ & -1.95396 & -3.39219 & 2.372764 & $\mathrm{H}$ & -0.63729 & 3.759542 & -3.05712 \\
\hline
\end{tabular}




\begin{tabular}{|c|c|c|c|c|c|c|c|}
\hline $\mathrm{C}$ & 3.711067 & 0.163178 & 1.678523 & $\mathrm{C}$ & -3.71223 & -1.69174 & 0.813711 \\
\hline $\mathrm{C}$ & 3.619775 & -0.99599 & 0.693525 & $\mathrm{C}$ & -3.81469 & -0.461 & -0.07885 \\
\hline $\mathrm{C}$ & 2.524134 & 1.14891 & 1.626182 & $\mathrm{C}$ & -2.27089 & -2.11232 & 1.173026 \\
\hline $\mathrm{C}$ & 2.287907 & 1.765955 & 0.252939 & $\mathrm{C}$ & -1.37649 & -2.3745 & -0.0327 \\
\hline $\mathrm{C}$ & 2.848217 & -2.05693 & 0.967292 & $\mathrm{C}$ & -3.69518 & 0.767315 & 0.443709 \\
\hline $\mathrm{C}$ & 2.692876 & -3.32685 & 0.171429 & $\mathrm{C}$ & -3.86114 & 2.095257 & -0.24862 \\
\hline $\mathrm{C}$ & 1.273105 & -3.92523 & 0.158365 & $\mathrm{C}$ & -2.89435 & 3.206056 & 0.206214 \\
\hline $\mathrm{C}$ & 1.021668 & 2.655185 & 0.129638 & $\mathrm{C}$ & 0.117048 & -2.64374 & 0.292697 \\
\hline $\mathrm{C}$ & -0.28135 & 1.876529 & 0.410096 & $\mathrm{C}$ & 0.794579 & -1.4468 & 0.99346 \\
\hline $\mathrm{C}$ & 0.217224 & -3.24685 & -0.70852 & $\mathrm{C}$ & -1.44643 & 3.137568 & -0.26906 \\
\hline $\mathrm{C}$ & -0.92485 & -2.54784 & -0.0692 & $\mathrm{C}$ & -0.35957 & 2.883255 & 0.708143 \\
\hline $\mathrm{C}$ & -0.48525 & 0.586092 & -0.3956 & $\mathrm{C}$ & 0.650957 & -0.09186 & 0.289974 \\
\hline $\mathrm{C}$ & -1.861 & -1.54218 & -0.71128 & $\mathrm{C}$ & 1.056327 & 2.433565 & 0.404443 \\
\hline $\mathrm{C}$ & -1.81502 & -0.12496 & -0.08665 & $\mathrm{C}$ & 1.413993 & 1.050186 & 0.997218 \\
\hline $\mathrm{C}$ & -3.05493 & 0.645664 & -0.5226 & $\mathrm{C}$ & 2.919866 & 0.834359 & 1.038981 \\
\hline $\mathrm{C}$ & -4.33758 & 0.458874 & 0.23886 & $\mathrm{C}$ & 3.656584 & 0.755227 & -0.26329 \\
\hline $\mathrm{O}$ & -5.38367 & 1.020088 & -0.01028 & $\mathrm{O}$ & 3.154053 & 0.958434 & -1.35043 \\
\hline $\mathrm{C}$ & -3.11358 & 1.455217 & -1.58374 & $\mathrm{C}$ & 3.590282 & 0.741185 & 2.192577 \\
\hline $\mathrm{C}$ & 4.475318 & -0.85615 & -0.54284 & $\mathrm{C}$ & -4.09277 & -0.74825 & -1.53497 \\
\hline $\mathrm{C}$ & 0.614094 & -2.97529 & -2.14205 & $\mathrm{C}$ & -1.24595 & 2.921996 & -1.75218 \\
\hline $\mathrm{O}$ & 3.450283 & 2.524787 & -0.10364 & $\mathrm{O}$ & -1.9217 & -3.47957 & -0.76515 \\
\hline $\mathrm{O}$ & -1.06999 & -3.90019 & -0.54814 & $\mathrm{O}$ & -0.67219 & 4.229313 & 0.295109 \\
\hline $\mathrm{H}$ & -0.89557 & -2.48432 & 1.020123 & $\mathrm{H}$ & -0.68076 & 2.669471 & 1.729464 \\
\hline $\mathrm{C}$ & 1.10558 & 3.888704 & 1.036019 & $\mathrm{C}$ & 0.296704 & -3.91834 & 1.124547 \\
\hline $\mathrm{O}$ & -4.20722 & -0.41867 & 1.250539 & $\mathrm{O}$ & 4.954715 & 0.432509 & -0.11786 \\
\hline $\mathrm{C}$ & -5.39088 & -0.66969 & 2.035971 & $\mathrm{C}$ & 5.736383 & 0.368222 & -1.32803 \\
\hline $\mathrm{O}$ & 1.094794 & 3.030988 & -1.27572 & $\mathrm{O}$ & 0.654637 & -2.81571 & -1.04958 \\
\hline $\mathrm{C}$ & 0.248547 & 4.073813 & -1.74384 & $\mathrm{C}$ & 1.980143 & -3.3105 & -1.19221 \\
\hline $\mathrm{H}$ & 4.636487 & 0.716613 & 1.497859 & $\mathrm{H}$ & -4.21311 & -2.53216 & 0.325425 \\
\hline $\mathrm{H}$ & 3.772257 & -0.22711 & 2.699059 & $\mathrm{H}$ & -4.24683 & -1.51 & 1.751124 \\
\hline $\mathrm{H}$ & 1.620169 & 0.618619 & 1.936248 & $\mathrm{H}$ & -1.82212 & -1.32279 & 1.780878 \\
\hline $\mathrm{H}$ & 2.697298 & 1.944401 & 2.358102 & $\mathrm{H}$ & -2.31384 & -3.01182 & 1.795728 \\
\hline $\mathrm{H}$ & 2.163155 & 0.960181 & -0.47891 & $\mathrm{H}$ & -1.38809 & -1.49148 & -0.68133 \\
\hline $\mathrm{H}$ & 2.308106 & -2.05116 & 1.915205 & $\mathrm{H}$ & -3.53069 & 0.838906 & 1.519886 \\
\hline $\mathrm{H}$ & 3.343332 & -4.08964 & 0.621407 & $\mathrm{H}$ & -4.87341 & 2.463084 & -0.03065 \\
\hline $\mathrm{H}$ & 3.056276 & -3.20348 & -0.85108 & $\mathrm{H}$ & -3.82141 & 1.986469 & -1.33451 \\
\hline $\mathrm{H}$ & 0.892455 & -3.99301 & 1.182665 & $\mathrm{H}$ & -2.90096 & 3.276246 & 1.298829 \\
\hline $\mathrm{H}$ & 1.343981 & -4.95524 & -0.21196 & $\mathrm{H}$ & -3.28335 & 4.162215 & -0.1648 \\
\hline $\mathrm{H}$ & -0.31595 & 1.644544 & 1.479633 & $\mathrm{H}$ & 0.399086 & -1.37755 & 2.012424 \\
\hline $\mathrm{H}$ & -1.1213 & 2.555094 & 0.234323 & $\mathrm{H}$ & 1.855818 & -1.68644 & 1.114158 \\
\hline $\mathrm{H}$ & -0.40052 & 0.795332 & -1.4661 & $\mathrm{H}$ & 0.991159 & -0.17189 & -0.74411 \\
\hline $\mathrm{H}$ & 0.315286 & -0.12145 & -0.16573 & $\mathrm{H}$ & -0.4028 & 0.196951 & 0.253096 \\
\hline $\mathrm{H}$ & -1.68608 & -1.46844 & -1.78755 & $\mathrm{H}$ & 1.253912 & 2.439525 & -0.66693 \\
\hline $\mathrm{H}$ & -2.87031 & -1.94874 & -0.58824 & $\mathrm{H}$ & 1.718535 & 3.18469 & 0.851066 \\
\hline $\mathrm{H}$ & -1.87833 & -0.24898 & 0.998495 & $\mathrm{H}$ & 1.091938 & 1.05802 & 2.045815 \\
\hline $\mathrm{H}$ & -4.04496 & 1.938229 & -1.85088 & $\mathrm{H}$ & 4.662542 & 0.601216 & 2.218746 \\
\hline $\mathrm{H}$ & -2.25028 & 1.653697 & -2.20573 & $\mathrm{H}$ & 3.072061 & 0.809379 & 3.142812 \\
\hline $\mathrm{H}$ & 4.239372 & -1.58549 & -1.31832 & $\mathrm{H}$ & -3.94928 & 0.117181 & -2.18246 \\
\hline $\mathrm{H}$ & 4.377806 & 0.14853 & -0.96373 & $\mathrm{H}$ & -3.45644 & -1.56311 & -1.89164 \\
\hline
\end{tabular}




$\begin{array}{lrrrlrrr}\mathrm{H} & 5.534277 & -0.97908 & -0.2827 & \mathrm{H} & -5.12831 & -1.08899 & -1.66048 \\ \mathrm{H} & -0.247 & -2.69058 & -2.74523 & \mathrm{H} & -0.21264 & 3.108453 & -2.04201 \\ \mathrm{H} & 1.357045 & -2.17573 & -2.20023 & \mathrm{H} & -1.50665 & 1.90057 & -2.04113 \\ \mathrm{H} & 1.052976 & -3.87497 & -2.58427 & \mathrm{H} & -1.88478 & 3.606103 & -2.3192 \\ \mathrm{H} & 3.215302 & 2.962141 & -0.93309 & \mathrm{H} & -1.27244 & -3.66 & -1.4584 \\ \mathrm{H} & 2.010307 & 4.460429 & 0.82447 & \mathrm{H} & -0.15711 & -4.77279 & 0.620305 \\ \mathrm{H} & 0.237877 & 4.537886 & 0.903809 & \mathrm{H} & 1.353009 & -4.13172 & 1.300054 \\ \mathrm{H} & 1.128632 & 3.586793 & 2.084852 & \mathrm{H} & -0.1784 & -3.80451 & 2.100893 \\ \mathrm{H} & -5.09163 & -1.39595 & 2.787152 & \mathrm{H} & 6.7424 & 0.108901 & -1.0083 \\ \mathrm{H} & -5.73413 & 0.251132 & 2.508224 & \mathrm{H} & 5.339702 & -0.3957 & -1.99748 \\ \mathrm{H} & -6.18424 & -1.07394 & 1.406719 & \mathrm{H} & 5.733456 & 1.333407 & -1.83554 \\ \mathrm{H} & 0.36466 & 4.095018 & -2.82811 & \mathrm{H} & 2.065947 & -4.35946 & -0.8898 \\ \mathrm{H} & 0.53518 & 5.051179 & -1.34232 & \mathrm{H} & 2.708547 & -2.71826 & -0.62813 \\ \mathrm{H} & -0.80599 & 3.890336 & -1.51146 & \mathrm{H} & 2.220055 & -3.23287 & -2.25326\end{array}$

\begin{tabular}{|c|c|c|c|c|c|c|c|}
\hline \multicolumn{4}{|c|}{ 3c_5 } & \multicolumn{4}{|c|}{ 3c_6 } \\
\hline $\mathrm{C}$ & 3.025884 & 1.182978 & 1.484258 & $\mathrm{C}$ & 3.688751 & -1.09191 & -1.04841 \\
\hline $\mathrm{C}$ & 3.545813 & 0.113428 & 0.542333 & $\mathrm{C}$ & 3.717807 & 0.097856 & -0.09526 \\
\hline $\mathrm{C}$ & 2.61582 & 2.511163 & 0.814357 & $\mathrm{C}$ & 2.28403 & -1.68221 & -1.30602 \\
\hline $\mathrm{C}$ & 1.589393 & 2.429287 & -0.32713 & $\mathrm{C}$ & 1.57991 & -2.21831 & -0.05211 \\
\hline $\mathrm{C}$ & 3.077769 & -1.13896 & 0.620589 & $\mathrm{C}$ & 3.376217 & 1.317398 & -0.53271 \\
\hline $\mathrm{C}$ & 3.491874 & -2.32859 & -0.21561 & $\mathrm{C}$ & 3.406323 & 2.621522 & 0.221143 \\
\hline $\mathrm{C}$ & 2.513317 & -3.51219 & -0.12231 & $\mathrm{C}$ & 2.276866 & 3.607044 & -0.13368 \\
\hline $\mathrm{C}$ & 0.089753 & 2.655614 & 0.035588 & $\mathrm{C}$ & 0.110024 & -2.67222 & -0.26897 \\
\hline $\mathrm{C}$ & -0.51994 & 1.508893 & 0.872512 & $\mathrm{C}$ & -0.7296 & -1.578 & -0.96558 \\
\hline $\mathrm{C}$ & 1.083778 & -3.23226 & -0.56759 & $\mathrm{C}$ & 0.865864 & 3.274768 & 0.340612 \\
\hline $\mathrm{C}$ & 0.044658 & -3.02273 & 0.467417 & $\mathrm{C}$ & -0.17979 & 2.928236 & -0.65262 \\
\hline $\mathrm{C}$ & -0.60526 & 0.145657 & 0.17218 & $\mathrm{C}$ & -0.76217 & -0.21094 & -0.27102 \\
\hline $\mathrm{C}$ & -1.28719 & -2.32347 & 0.303764 & $\mathrm{C}$ & -1.50445 & 2.241123 & -0.38698 \\
\hline $\mathrm{C}$ & -1.34707 & -0.93595 & 0.994471 & $\mathrm{C}$ & -1.62295 & 0.832927 & -1.01877 \\
\hline $\mathrm{C}$ & -2.77543 & -0.56217 & 1.371241 & $\mathrm{C}$ & -3.07686 & 0.416445 & -1.20167 \\
\hline $\mathrm{C}$ & -3.85909 & -0.38751 & 0.343476 & $\mathrm{C}$ & -4.00121 & 0.184774 & -0.0387 \\
\hline $\mathrm{O}$ & -5.0159 & -0.12528 & 0.596552 & $\mathrm{O}$ & -5.14519 & -0.20722 & -0.13372 \\
\hline $\mathrm{C}$ & -3.14713 & -0.39302 & 2.644464 & $\mathrm{C}$ & -3.61252 & 0.247008 & -2.41494 \\
\hline $\mathrm{C}$ & 4.628095 & 0.536208 & -0.42092 & $\mathrm{C}$ & 4.186343 & -0.20078 & 1.308741 \\
\hline $\mathrm{C}$ & 0.916353 & -2.73432 & -1.98513 & $\mathrm{C}$ & 0.727385 & 2.910302 & 1.801097 \\
\hline $\mathrm{O}$ & 1.979948 & 3.419126 & -1.29236 & $\mathrm{O}$ & 2.303753 & -3.34115 & 0.487312 \\
\hline $\mathrm{O}$ & 0.176247 & -4.30064 & -0.18942 & $\mathrm{O}$ & -0.07905 & 4.270328 & -0.13322 \\
\hline $\mathrm{H}$ & 0.396032 & -3.04671 & 1.50034 & $\mathrm{H}$ & 0.156935 & 2.848724 & -1.68794 \\
\hline $\mathrm{C}$ & -0.11824 & 4.000156 & 0.74227 & $\mathrm{C}$ & 0.026686 & -3.96479 & -1.09102 \\
\hline $\mathrm{O}$ & -3.41491 & -0.55449 & -0.91553 & $\mathrm{O}$ & -3.42981 & 0.464415 & 1.146488 \\
\hline $\mathrm{C}$ & -4.39757 & -0.41778 & -1.96354 & $\mathrm{C}$ & -4.25381 & 0.26874 & 2.314805 \\
\hline $\mathrm{O}$ & -0.50902 & 2.675715 & -1.29345 & $\mathrm{O}$ & -0.53637 & -2.81306 & 1.019919 \\
\hline $\mathrm{C}$ & -1.86089 & 3.097897 & -1.42695 & $\mathrm{C}$ & -0.32158 & -3.98858 & 1.796679 \\
\hline $\mathrm{H}$ & 3.815829 & 1.429162 & 2.206982 & $\mathrm{H}$ & 4.359394 & -1.87496 & -0.67663 \\
\hline $\mathrm{H}$ & 2.19804 & 0.771578 & 2.067877 & $\mathrm{H}$ & 4.09906 & -0.79182 & -2.01696 \\
\hline $\mathrm{H}$ & 2.283765 & 3.205522 & 1.589565 & $\mathrm{H}$ & 1.675416 & -0.89989 & -1.76304 \\
\hline $\mathrm{H}$ & 3.504738 & 2.967405 & 0.372078 & $\mathrm{H}$ & 2.370467 & -2.49301 & -2.03687 \\
\hline $\mathrm{H}$ & 1.656518 & 1.441861 & -0.79923 & $\mathrm{H}$ & 1.541163 & -1.42768 & 0.704934 \\
\hline
\end{tabular}




$\begin{array}{lrrrlrrr}\mathrm{H} & 2.322256 & -1.34065 & 1.377302 & \mathrm{H} & 3.094847 & 1.409627 & -1.58245 \\ \mathrm{H} & 4.476184 & -2.6919 & 0.106874 & \mathrm{H} & 4.348493 & 3.133564 & -0.0182 \\ \mathrm{H} & 3.617041 & -2.03655 & -1.26258 & \mathrm{H} & 3.429969 & 2.457585 & 1.300546 \\ \mathrm{H} & 2.482237 & -3.88135 & 0.907786 & \mathrm{H} & 2.252174 & 3.765315 & -1.21668 \\ \mathrm{H} & 2.89496 & -4.33541 & -0.73762 & \mathrm{H} & 2.523976 & 4.577429 & 0.313648 \\ \mathrm{H} & 0.050769 & 1.421724 & 1.803398 & \mathrm{H} & -0.38354 & -1.46299 & -1.99739 \\ \mathrm{H} & -1.52354 & 1.818983 & 1.180783 & \mathrm{H} & -1.74677 & -1.97485 & -1.02821 \\ \mathrm{H} & -1.09604 & 0.262529 & -0.79434 & \mathrm{H} & -1.12848 & -0.32974 & 0.748843 \\ \mathrm{H} & 0.400408 & -0.23037 & -0.03056 & \mathrm{H} & 0.249239 & 0.198524 & -0.19769 \\ \mathrm{H} & -1.55745 & -2.23175 & -0.74777 & \mathrm{H} & -1.71473 & 2.192372 & 0.680736 \\ \mathrm{H} & -2.03639 & -2.97868 & 0.763715 & \mathrm{H} & -2.27338 & 2.886567 & -0.82806 \\ \mathrm{H} & -0.82164 & -1.03176 & 1.952516 & \mathrm{H} & -1.22259 & 0.908415 & -2.03739 \\ \mathrm{H} & -4.17068 & -0.14356 & 2.895052 & \mathrm{H} & -4.65069 & -0.03918 & -2.52867 \\ \mathrm{H} & -2.4388 & -0.50117 & 3.457492 & \mathrm{H} & -3.02595 & 0.392241 & -3.31456 \\ \mathrm{H} & 5.04966 & -0.30548 & -0.97016 & \mathrm{H} & 4.113121 & 0.654669 & 1.979673 \\ \mathrm{H} & 4.255379 & 1.262324 & -1.15134 & \mathrm{H} & 3.613744 & -1.02114 & 1.755895 \\ \mathrm{H} & 5.445473 & 1.029994 & 0.117618 & \mathrm{H} & 5.233105 & -0.52707 & 1.296127 \\ \mathrm{H} & -0.13442 & -2.68027 & -2.26694 & \mathrm{H} & -0.31788 & 2.886039 & 2.106435 \\ \mathrm{H} & 1.358034 & -1.7422 & -2.10812 & \mathrm{H} & 1.169297 & 1.931046 & 2.002779 \\ \mathrm{H} & 1.419467 & -3.4151 & -2.67878 & \mathrm{H} & 1.244481 & 3.648345 & 2.422011 \\ \mathrm{H} & 1.237007 & 3.460397 & -1.91151 & \mathrm{H} & 3.180327 & -3.03935 & 0.745494 \\ \mathrm{H} & 0.350594 & 4.806773 & 0.176243 & \mathrm{H} & 0.646255 & -4.7549 & -0.66889 \\ \mathrm{H} & -1.17981 & 4.223002 & 0.861435 & \mathrm{H} & -1.00904 & -4.31043 & -1.13716 \\ \mathrm{H} & 0.317283 & 3.979862 & 1.742584 & \mathrm{H} & 0.366502 & -3.78331 & -2.11305 \\ \mathrm{H} & -3.85906 & -0.59815 & -2.89019 & \mathrm{H} & -3.62819 & 0.54425 & 3.159751 \\ \mathrm{H} & -5.194 & -1.15076 & -1.83356 & \mathrm{H} & -5.13663 & 0.906681 & 2.267136 \\ \mathrm{H} & -4.82162 & 0.586514 & -1.95613 & \mathrm{H} & -4.56183 & -0.77435 & 2.39001 \\ \mathrm{H} & -2.14784 & 2.874356 & -2.45514 & \mathrm{H} & 0.729268 & -4.12358 & 2.058427 \\ \mathrm{H} & -1.97646 & 4.173022 & -1.2575 & \mathrm{H} & -0.68249 & -4.8902 & 1.288955 \\ \mathrm{H} & -2.53308 & 2.557406 & -0.75305 & \mathrm{H} & -0.90933 & -3.85184 & 2.706317\end{array}$

3c $\_7$

$\begin{array}{lrrrl}\mathrm{C} & 3.755377 & -1.09764 & -0.97057 & \mathrm{C} \\ \mathrm{C} & 3.708793 & 0.10873 & -0.04266 & \mathrm{C} \\ \mathrm{C} & 2.385493 & -1.75456 & -1.24742 & \mathrm{C} \\ \mathrm{C} & 1.645891 & -2.2256 & 0.002091 & \mathrm{C} \\ \mathrm{C} & 3.370716 & 1.314732 & -0.51919 & \mathrm{C} \\ \mathrm{C} & 3.342266 & 2.630086 & 0.214502 & \mathrm{C} \\ \mathrm{C} & 2.215548 & 3.58962 & -0.21189 & \mathrm{C} \\ \mathrm{C} & 0.215755 & -2.77283 & -0.25537 & \mathrm{C} \\ \mathrm{C} & -0.76725 & -1.70825 & -0.8047 & \mathrm{C} \\ \mathrm{C} & 0.79239 & 3.250998 & 0.218332 & \mathrm{C} \\ \mathrm{C} & -0.20798 & 2.844216 & -0.79833 & \mathrm{C} \\ \mathrm{C} & -0.71253 & -0.26483 & -0.27273 & \mathrm{C} \\ \mathrm{C} & -1.53225 & 2.150871 & -0.54818 & \mathrm{C} \\ \mathrm{C} & -1.61225 & 0.700554 & -1.082 & \mathrm{C} \\ \mathrm{C} & -3.05868 & 0.242379 & -1.22325 & \mathrm{C} \\ \mathrm{C} & -3.97586 & 0.086955 & -0.0424 & \mathrm{C} \\ \mathrm{O} & -5.13883 & -0.24956 & -0.11068 & \mathrm{O}\end{array}$

$\begin{array}{llll}\text { 3c_8 } & & & \\ \text { C } & 3.704167 & -1.06215 & -1.07265 \\ \text { C } & 3.716839 & 0.117131 & -0.10804 \\ \text { C } & 2.306008 & -1.66872 & -1.32027 \\ \text { C } & 1.603456 & -2.17777 & -0.05539 \\ \text { C } & 3.352739 & 1.336736 & -0.52736 \\ \text { C } & 3.367978 & 2.628855 & 0.248082 \\ \text { C } & 2.241262 & 3.617313 & -0.10678 \\ \text { C } & 0.134297 & -2.65558 & -0.26555 \\ \text { C } & -0.72125 & -1.57906 & -0.9715 \\ \text { C } & 0.826085 & 3.277929 & 0.349219 \\ \text { C } & -0.20697 & 2.930864 & -0.65681 \\ \text { C } & -0.75355 & -0.20364 & -0.29317 \\ \text { C } & -1.53109 & 2.236698 & -0.40745 \\ \text { C } & -1.63458 & 0.826195 & -1.03653 \\ \text { C } & -3.08447 & 0.390665 & -1.20674 \\ \text { C } & -3.99362 & 0.138925 & -0.03581 \\ \text { O } & -5.13123 & -0.27323 & -0.12164\end{array}$




\begin{tabular}{|c|c|c|c|c|c|c|c|}
\hline $\mathrm{C}$ & -3.59609 & -0.0266 & -2.41762 & $\mathrm{C}$ & -3.63044 & 0.221019 & -2.41537 \\
\hline $\mathrm{C}$ & 4.102462 & -0.16444 & 1.389723 & $\mathrm{C}$ & 4.193259 & -0.19837 & 1.289591 \\
\hline $\mathrm{C}$ & 0.603389 & 2.943641 & 1.686717 & $\mathrm{C}$ & 0.672692 & 2.907489 & 1.806825 \\
\hline $\mathrm{O}$ & 2.438245 & -3.23715 & 0.63697 & $\mathrm{O}$ & 2.403608 & -3.18048 & 0.595891 \\
\hline $\mathrm{O}$ & -0.14911 & 4.208953 & -0.33395 & $\mathrm{O}$ & -0.11797 & 4.271467 & -0.13138 \\
\hline $\mathrm{H}$ & 0.167768 & 2.72616 & -1.81631 & $\mathrm{H}$ & 0.141262 & 2.856833 & -1.68872 \\
\hline $\mathrm{C}$ & 0.226853 & -3.97393 & -1.20832 & $\mathrm{C}$ & 0.059295 & -3.9588 & -1.07095 \\
\hline $\mathrm{O}$ & -3.37197 & 0.363705 & 1.128475 & $\mathrm{O}$ & -3.4163 & 0.424654 & 1.144994 \\
\hline $\mathrm{C}$ & -4.19249 & 0.251908 & 2.311057 & $\mathrm{C}$ & -4.22476 & 0.208322 & 2.320498 \\
\hline $\mathrm{O}$ & -0.22837 & -3.37739 & 0.99639 & $\mathrm{O}$ & -0.50123 & -2.7894 & 1.029295 \\
\hline $\mathrm{C}$ & -0.66909 & -2.54272 & 2.063628 & $\mathrm{C}$ & -0.28444 & -3.96401 & 1.807211 \\
\hline $\mathrm{H}$ & 4.427548 & -1.851 & -0.55017 & $\mathrm{H}$ & 4.373651 & -1.84165 & -0.69867 \\
\hline $\mathrm{H}$ & 4.179965 & -0.8019 & -1.93481 & $\mathrm{H}$ & 4.102638 & -0.74763 & -2.04208 \\
\hline $\mathrm{H}$ & 1.758055 & -1.03912 & -1.78586 & $\mathrm{H}$ & 1.685963 & -0.90941 & -1.80049 \\
\hline $\mathrm{H}$ & 2.538647 & -2.60999 & -1.91205 & $\mathrm{H}$ & 2.401514 & -2.4924 & -2.03892 \\
\hline $\mathrm{H}$ & 1.551018 & -1.37596 & 0.689021 & $\mathrm{H}$ & 1.552995 & -1.36955 & 0.675953 \\
\hline $\mathrm{H}$ & 3.145721 & 1.386168 & -1.58422 & $\mathrm{H}$ & 3.065893 & 1.440589 & -1.57464 \\
\hline $\mathrm{H}$ & 4.286942 & 3.154564 & 0.014684 & $\mathrm{H}$ & 4.312475 & 3.147726 & 0.033255 \\
\hline $\mathrm{H}$ & 3.316509 & 2.481378 & 1.296308 & $\mathrm{H}$ & 3.376547 & 2.445493 & 1.324874 \\
\hline $\mathrm{H}$ & 2.230239 & 3.715286 & -1.29938 & $\mathrm{H}$ & 2.227128 & 3.787301 & -1.18826 \\
\hline $\mathrm{H}$ & 2.430917 & 4.576689 & 0.215061 & $\mathrm{H}$ & 2.481896 & 4.583362 & 0.35343 \\
\hline $\mathrm{H}$ & -0.61284 & -1.66917 & -1.8883 & $\mathrm{H}$ & -0.3852 & -1.47565 & -2.00804 \\
\hline $\mathrm{H}$ & -1.77455 & -2.11632 & -0.67151 & $\mathrm{H}$ & -1.73694 & -1.98224 & -1.0188 \\
\hline $\mathrm{H}$ & -0.99688 & -0.21712 & 0.777208 & $\mathrm{H}$ & -1.10148 & -0.31373 & 0.734066 \\
\hline $\mathrm{H}$ & 0.306945 & 0.122871 & -0.33903 & $\mathrm{H}$ & 0.255626 & 0.214727 & -0.2424 \\
\hline $\mathrm{H}$ & -1.7884 & 2.174438 & 0.510473 & $\mathrm{H}$ & -1.75454 & 2.188942 & 0.657614 \\
\hline $\mathrm{H}$ & -2.29003 & 2.750768 & -1.06616 & $\mathrm{H}$ & -2.29841 & 2.876166 & -0.85995 \\
\hline $\mathrm{H}$ & -1.22141 & 0.714735 & -2.10665 & $\mathrm{H}$ & -1.24317 & 0.905317 & -2.05837 \\
\hline $\mathrm{H}$ & -4.63017 & -0.33619 & -2.50364 & $\mathrm{H}$ & -4.66575 & -0.07869 & -2.51987 \\
\hline $\mathrm{H}$ & -3.01544 & 0.057891 & -3.32869 & $\mathrm{H}$ & -3.05546 & 0.379586 & -3.32022 \\
\hline $\mathrm{H}$ & 5.187006 & -0.31722 & 1.458595 & $\mathrm{H}$ & 4.013419 & 0.608514 & 2.000566 \\
\hline $\mathrm{H}$ & 3.840045 & 0.644397 & 2.072633 & $\mathrm{H}$ & 3.715265 & -1.10817 & 1.663225 \\
\hline $\mathrm{H}$ & 3.639753 & -1.08892 & 1.746238 & $\mathrm{H}$ & 5.272074 & -0.39889 & 1.281532 \\
\hline $\mathrm{H}$ & -0.45174 & 2.918377 & 1.955591 & $\mathrm{H}$ & -0.37569 & 2.877911 & 2.100694 \\
\hline $\mathrm{H}$ & 1.048459 & 1.978729 & 1.942625 & $\mathrm{H}$ & 1.116478 & 1.929395 & 2.009843 \\
\hline $\mathrm{H}$ & 1.089385 & 3.711835 & 2.295986 & $\mathrm{H}$ & 1.180021 & 3.645334 & 2.435995 \\
\hline $\mathrm{H}$ & 1.833254 & -3.68353 & 1.246182 & $\mathrm{H}$ & 2.648923 & -3.84558 & -0.05744 \\
\hline $\mathrm{H}$ & 0.891861 & -4.75159 & -0.83066 & $\mathrm{H}$ & 0.65768 & -4.75714 & -0.62832 \\
\hline $\mathrm{H}$ & -0.78177 & -4.38583 & -1.28402 & $\mathrm{H}$ & -0.97538 & -4.3053 & -1.1276 \\
\hline $\mathrm{H}$ & 0.558995 & -3.6878 & -2.20723 & $\mathrm{H}$ & 0.414847 & -3.79732 & -2.09084 \\
\hline $\mathrm{H}$ & -3.54132 & 0.513005 & 3.141097 & $\mathrm{H}$ & -3.59745 & 0.495817 & 3.160177 \\
\hline $\mathrm{H}$ & -5.03468 & 0.941628 & 2.252816 & $\mathrm{H}$ & -5.122 & 0.826454 & 2.282618 \\
\hline $\mathrm{H}$ & -4.56294 & -0.76755 & 2.419504 & $\mathrm{H}$ & -4.50858 & -0.84161 & 2.396142 \\
\hline $\mathrm{H}$ & -1.62007 & -2.05477 & 1.831898 & $\mathrm{H}$ & 0.771146 & -4.11691 & 2.038288 \\
\hline $\mathrm{H}$ & 0.067688 & -1.77972 & 2.3377 & $\mathrm{H}$ & -0.67973 & -4.86151 & 1.317707 \\
\hline $\mathrm{H}$ & -0.81703 & -3.20342 & 2.918898 & $\mathrm{H}$ & -0.84013 & -3.81043 & 2.734071 \\
\hline
\end{tabular}




\begin{tabular}{|c|c|c|c|c|c|c|c|}
\hline $\mathrm{C}$ & 3.796901 & 0.622244 & 0.08768 & $\mathrm{C}$ & -3.81437 & -0.46246 & -0.07928 \\
\hline $\mathrm{C}$ & 2.134042 & 2.549356 & 0.56257 & $\mathrm{C}$ & -2.27017 & -2.11328 & 1.172809 \\
\hline $\mathrm{C}$ & 0.965802 & 2.54961 & -0.42005 & $\mathrm{C}$ & -1.37528 & -2.37499 & -0.03267 \\
\hline $\mathrm{C}$ & 3.510601 & -0.30119 & -0.84137 & $\mathrm{C}$ & -3.69585 & 0.765819 & 0.44359 \\
\hline $\mathrm{C}$ & 3.907984 & -1.7637 & -0.83264 & $\mathrm{C}$ & -3.86228 & 2.093843 & -0.24845 \\
\hline $\mathrm{C}$ & 3.091392 & -2.75634 & 0.025576 & $\mathrm{C}$ & -2.89577 & 3.204885 & 0.206402 \\
\hline $\mathrm{C}$ & -0.45058 & 2.685572 & 0.20114 & $\mathrm{C}$ & 0.118297 & -2.64353 & 0.293091 \\
\hline $\mathrm{C}$ & -0.84674 & 1.456001 & 1.044711 & $\mathrm{C}$ & 0.795049 & -1.44621 & 0.99399 \\
\hline $\mathrm{C}$ & 1.615223 & -2.96152 & -0.30416 & $\mathrm{C}$ & -1.44785 & 3.136951 & -0.26897 \\
\hline $\mathrm{C}$ & 0.599845 & -2.68838 & 0.74272 & $\mathrm{C}$ & -0.36081 & 2.883274 & 0.708225 \\
\hline $\mathrm{C}$ & -0.71462 & 0.105121 & 0.331665 & $\mathrm{C}$ & 0.650903 & -0.09139 & 0.290392 \\
\hline $\mathrm{C}$ & -0.88288 & -2.43835 & 0.545494 & $\mathrm{C}$ & 1.055286 & 2.434194 & 0.404565 \\
\hline $\mathrm{C}$ & -1.33328 & -1.07329 & 1.116199 & $\mathrm{C}$ & 1.413637 & 1.051021 & 0.997417 \\
\hline $\mathrm{C}$ & -2.845 & -0.98271 & 1.272668 & $\mathrm{C}$ & 2.919585 & 0.835758 & 1.038878 \\
\hline $\mathrm{C}$ & -3.78301 & -1.0201 & 0.097436 & $\mathrm{C}$ & 3.656043 & 0.756512 & -0.26354 \\
\hline $\mathrm{O}$ & -4.98799 & -0.90394 & 0.173712 & $\mathrm{O}$ & 3.153378 & 0.959992 & -1.35057 \\
\hline $\mathrm{C}$ & -3.42191 & -0.87179 & 2.474023 & $\mathrm{C}$ & 3.590332 & 0.743214 & 2.192333 \\
\hline $\mathrm{C}$ & 4.507152 & 0.310395 & 1.382413 & $\mathrm{C}$ & -4.09167 & -0.74957 & -1.53558 \\
\hline $\mathrm{C}$ & 1.25186 & -3.02599 & -1.76936 & $\mathrm{C}$ & -1.24739 & 2.921388 & -1.75209 \\
\hline $\mathrm{O}$ & 1.192089 & 3.626285 & -1.33931 & $\mathrm{O}$ & -1.91977 & -3.48027 & -0.76532 \\
\hline $\mathrm{O}$ & 1.052182 & -4.03391 & 0.499058 & $\mathrm{O}$ & -0.67406 & 4.229126 & 0.295037 \\
\hline $\mathrm{H}$ & 0.989681 & -2.28008 & 1.677225 & $\mathrm{H}$ & -0.68186 & 2.669481 & 1.729591 \\
\hline $\mathrm{C}$ & -0.58334 & 3.971221 & 1.02352 & $\mathrm{C}$ & 0.29839 & -3.91803 & 1.125008 \\
\hline $\mathrm{O}$ & -3.14857 & -1.201 & -1.07494 & $\mathrm{O}$ & 4.954042 & 0.433186 & -0.11837 \\
\hline $\mathrm{C}$ & -3.98357 & -1.25668 & -2.25082 & $\mathrm{C}$ & 5.73551 & 0.368784 & -1.32864 \\
\hline $\mathrm{O}$ & -1.26991 & 2.746719 & -1.00219 & $\mathrm{O}$ & 0.65626 & -2.81528 & -1.04907 \\
\hline $\mathrm{C}$ & -2.6375 & 3.115406 & -0.87515 & $\mathrm{C}$ & 1.982041 & -3.30942 & -1.19147 \\
\hline $\mathrm{H}$ & 3.389968 & 2.316367 & -1.17896 & $\mathrm{H}$ & -4.21188 & -2.5339 & 0.324464 \\
\hline $\mathrm{H}$ & 4.280011 & 2.685566 & 0.283373 & $\mathrm{H}$ & -4.24658 & -1.51202 & 1.750351 \\
\hline $\mathrm{H}$ & 1.90977 & 1.911173 & 1.421181 & $\mathrm{H}$ & -1.82193 & -1.3236 & 1.780865 \\
\hline $\mathrm{H}$ & 2.250999 & 3.567349 & 0.944209 & $\mathrm{H}$ & -2.3129 & -3.01285 & 1.795425 \\
\hline $\mathrm{H}$ & 0.97193 & 1.6086 & -0.983 & $\mathrm{H}$ & -1.38713 & -1.49194 & -0.68126 \\
\hline $\mathrm{H}$ & 3.00142 & 0.040052 & -1.74066 & $\mathrm{H}$ & -3.53193 & 0.837278 & 1.519864 \\
\hline $\mathrm{H}$ & 4.945832 & -1.85156 & -0.49389 & $\mathrm{H}$ & -4.8746 & 2.461349 & -0.03016 \\
\hline $\mathrm{H}$ & 3.909803 & -2.1194 & -1.86715 & $\mathrm{H}$ & -3.82277 & 1.985292 & -1.33437 \\
\hline $\mathrm{H}$ & 3.177486 & -2.4941 & 1.083688 & $\mathrm{H}$ & -2.90233 & 3.274963 & 1.299025 \\
\hline $\mathrm{H}$ & 3.564792 & -3.73991 & -0.08637 & $\mathrm{H}$ & -3.28515 & 4.160947 & -0.16448 \\
\hline $\mathrm{H}$ & -0.24588 & 1.45245 & 1.960383 & $\mathrm{H}$ & 0.399244 & -1.37714 & 2.012845 \\
\hline $\mathrm{H}$ & -1.87991 & 1.597957 & 1.377603 & $\mathrm{H}$ & 1.856374 & -1.6853 & 1.114977 \\
\hline $\mathrm{H}$ & -1.16712 & 0.164483 & -0.65926 & $\mathrm{H}$ & 0.991031 & -0.17142 & -0.74372 \\
\hline $\mathrm{H}$ & 0.343293 & -0.12453 & 0.179931 & $\mathrm{H}$ & -0.40296 & 0.197049 & 0.253599 \\
\hline $\mathrm{H}$ & -1.16641 & -2.52187 & -0.50346 & $\mathrm{H}$ & 1.252874 & 2.440159 & -0.66681 \\
\hline $\mathrm{H}$ & -1.41177 & -3.23475 & 1.081669 & $\mathrm{H}$ & 1.717149 & 3.185673 & 0.851107 \\
\hline $\mathrm{H}$ & -0.93952 & -1.01987 & 2.13883 & $\mathrm{H}$ & 1.091775 & 1.05879 & 2.046074 \\
\hline $\mathrm{H}$ & -4.49904 & -0.81487 & 2.569741 & $\mathrm{H}$ & 4.662651 & 0.603635 & 2.218276 \\
\hline $\mathrm{H}$ & -2.83049 & -0.83816 & 3.381454 & $\mathrm{H}$ & 3.072338 & 0.811573 & 3.14268 \\
\hline $\mathrm{H}$ & 4.74813 & -0.74623 & 1.495666 & $\mathrm{H}$ & -3.94858 & 0.11611 & -2.18281 \\
\hline $\mathrm{H}$ & 5.4388 & 0.883569 & 1.45377 & $\mathrm{H}$ & -3.4546 & -1.56388 & -1.8922 \\
\hline $\mathrm{H}$ & 3.89565 & 0.606719 & 2.242594 & $\mathrm{H}$ & -5.1269 & -1.09108 & -1.66156 \\
\hline
\end{tabular}




\begin{tabular}{|c|c|c|c|c|c|c|c|}
\hline $\mathrm{H}$ & 0.220695 & -3.35038 & -1.90551 & $\mathrm{H}$ & -0.21395 & 3.107147 & -2.04189 \\
\hline $\mathrm{H}$ & 1.375818 & -2.05246 & -2.25059 & $\mathrm{H}$ & -1.50878 & 1.90017 & -2.04112 \\
\hline $\mathrm{H}$ & 1.900913 & -3.74087 & -2.28438 & $\mathrm{H}$ & -1.88575 & 3.605984 & -2.31905 \\
\hline $\mathrm{H}$ & 0.386007 & 3.665997 & -1.87192 & $\mathrm{H}$ & -1.27023 & -3.66041 & -1.45838 \\
\hline $\mathrm{H}$ & -0.33562 & 4.841597 & 0.413653 & $\mathrm{H}$ & -0.15488 & -4.7727 & 0.620646 \\
\hline $\mathrm{H}$ & -1.59509 & 4.091045 & 1.415962 & $\mathrm{H}$ & 1.354764 & -4.13088 & 1.300735 \\
\hline $\mathrm{H}$ & 0.095959 & 3.946158 & 1.877841 & $\mathrm{H}$ & -0.17699 & -3.80447 & 2.101246 \\
\hline $\mathrm{H}$ & -3.30057 & -1.39707 & -3.0845 & $\mathrm{H}$ & 6.741252 & 0.108069 & -1.00918 \\
\hline $\mathrm{H}$ & -4.67999 & -2.09255 & -2.18097 & $\mathrm{H}$ & 5.337872 & -0.3942 & -1.9986 \\
\hline $\mathrm{H}$ & -4.54105 & -0.32675 & -2.36376 & $\mathrm{H}$ & 5.733695 & 1.334323 & -1.8355 \\
\hline $\mathrm{H}$ & -2.75809 & 4.160722 & -0.57254 & $\mathrm{H}$ & 2.068194 & -4.35849 & -0.88955 \\
\hline $\mathrm{H}$ & -3.17726 & 2.476682 & -0.16799 & $\mathrm{H}$ & 2.710002 & -2.71715 & -0.62686 \\
\hline $\mathrm{H}$ & -3.07901 & 2.985568 & -1.8639 & $\mathrm{H}$ & 2.222305 & -3.23117 & -2.2524 \\
\hline 3d_1 & & & & 3d & & & \\
\hline $\mathrm{C}$ & -3.60193 & 2.010385 & -0.32189 & $\mathrm{C}$ & -3.40009 & 2.00121 & 0.581684 \\
\hline C & -3.83571 & 0.558102 & 0.04842 & $\mathrm{C}$ & -3.69485 & 0.512467 & 0.599833 \\
\hline $\mathrm{C}$ & -2.3099 & 2.62396 & 0.265076 & $\mathrm{C}$ & -1.94957 & 2.402995 & 0.9553 \\
\hline $\mathrm{C}$ & -1.06775 & 2.24306 & -0.53542 & $\mathrm{C}$ & -1.02511 & 2.484411 & -0.25947 \\
\hline $\mathrm{C}$ & -3.61732 & -0.42064 & -0.8412 & $\mathrm{C}$ & -3.89655 & -0.16404 & -0.53988 \\
\hline $\mathrm{C}$ & -3.88675 & -1.89552 & -0.70363 & $\mathrm{C}$ & -4.28282 & -1.60913 & -0.72816 \\
\hline $\mathrm{C}$ & -2.72561 & -2.81778 & -1.12732 & $\mathrm{C}$ & -3.23068 & -2.45945 & -1.47866 \\
\hline $\mathrm{C}$ & 0.291469 & 2.549884 & 0.148931 & $\mathrm{C}$ & 0.492083 & 2.665652 & 0.060169 \\
\hline $\mathrm{C}$ & 0.527876 & 1.705051 & 1.42325 & $\mathrm{C}$ & 1.12815 & 1.353153 & 0.578926 \\
\hline $\mathrm{C}$ & -1.55274 & -2.96985 & -0.16532 & $\mathrm{C}$ & -2.00339 & -2.82575 & -0.65737 \\
\hline $\mathrm{C}$ & -0.23466 & -2.38366 & -0.50855 & $\mathrm{C}$ & -0.89179 & -1.86033 & -0.60834 \\
\hline $\mathrm{C}$ & 0.308534 & 0.180116 & 1.354563 & $\mathrm{C}$ & 1.379284 & 0.285464 & -0.50104 \\
\hline $\mathrm{C}$ & 0.923646 & -2.12141 & 0.434424 & $\mathrm{C}$ & 0.114353 & -1.72809 & 0.509261 \\
\hline $\mathrm{C}$ & 1.344251 & -0.63466 & 0.554617 & $\mathrm{C}$ & 1.471693 & -1.17849 & 0.016986 \\
\hline $\mathrm{C}$ & 2.752979 & -0.57805 & 1.132285 & $\mathrm{C}$ & 2.579455 & -1.33273 & 1.046617 \\
\hline $\mathrm{C}$ & 3.925198 & -0.79679 & 0.21601 & $\mathrm{C}$ & 4.010492 & -1.31609 & 0.585236 \\
\hline $\mathrm{O}$ & 5.087945 & -0.80686 & 0.56194 & $\mathrm{O}$ & 4.977763 & -1.31098 & 1.317157 \\
\hline $\mathrm{C}$ & 3.046106 & -0.39474 & 2.423115 & $\mathrm{C}$ & 2.389225 & -1.48571 & 2.360671 \\
\hline $\mathrm{C}$ & -4.36024 & 0.340692 & 1.447556 & $\mathrm{C}$ & -3.79437 & -0.09332 & 1.979447 \\
\hline $\mathrm{C}$ & -1.90739 & -3.34702 & 1.255866 & $\mathrm{C}$ & -2.22368 & -3.86661 & 0.418258 \\
\hline $\mathrm{O}$ & -1.14377 & 2.93519 & -1.7888 & $\mathrm{O}$ & -1.48121 & 3.587504 & -1.0503 \\
\hline $\mathrm{O}$ & -0.50404 & -3.77865 & -0.76099 & $\mathrm{O}$ & -0.80459 & -3.0278 & -1.45869 \\
\hline $\mathrm{H}$ & -0.20048 & -1.7879 & -1.42206 & $\mathrm{H}$ & -1.05759 & -0.94804 & -1.17613 \\
\hline $\mathrm{C}$ & 0.420089 & 4.041227 & 0.481779 & $\mathrm{C}$ & 0.717464 & 3.811291 & 1.051387 \\
\hline $\mathrm{O}$ & 3.547854 & -0.98484 & -1.06214 & $\mathrm{O}$ & 4.115418 & -1.32325 & -0.75622 \\
\hline $\mathrm{C}$ & 4.607512 & -1.21109 & -2.01431 & $\mathrm{C}$ & 5.4548 & -1.32742 & -1.29218 \\
\hline $\mathrm{O}$ & 1.224296 & 2.172191 & -0.90586 & $\mathrm{O}$ & 1.026143 & 3.00191 & -1.24969 \\
\hline $\mathrm{C}$ & 2.575752 & 2.605032 & -0.80479 & $\mathrm{C}$ & 2.376057 & 3.442907 & -1.3341 \\
\hline $\mathrm{H}$ & -4.45505 & 2.599885 & 0.034008 & $\mathrm{H}$ & -4.07932 & 2.486076 & 1.293117 \\
\hline $\mathrm{H}$ & -3.57218 & 2.122541 & -1.40828 & $\mathrm{H}$ & -3.63065 & 2.418434 & -0.40101 \\
\hline $\mathrm{H}$ & -2.39701 & 3.714095 & 0.257988 & $\mathrm{H}$ & -1.96635 & 3.390069 & 1.424319 \\
\hline $\mathrm{H}$ & -2.19541 & 2.322092 & 1.309248 & $\mathrm{H}$ & -1.54573 & 1.708571 & 1.69624 \\
\hline $\mathrm{H}$ & -1.08589 & 1.165455 & -0.73521 & $\mathrm{H}$ & -1.12468 & 1.563039 & -0.8494 \\
\hline$H$ & -3.25746 & -0.12431 & -1.82675 & $\mathrm{H}$ & -3.80622 & 0.396485 & -1.47052 \\
\hline
\end{tabular}




$\begin{array}{lrrrlrrr}\mathrm{H} & -4.73075 & -2.13919 & -1.36278 & \mathrm{H} & -5.20278 & -1.63507 & -1.32507 \\ \mathrm{H} & -4.21985 & -2.15266 & 0.303517 & \mathrm{H} & -4.53267 & -2.08339 & 0.223145 \\ \mathrm{H} & -3.12916 & -3.82796 & -1.26846 & \mathrm{H} & -3.70348 & -3.38585 & -1.82443 \\ \mathrm{H} & -2.33978 & -2.50188 & -2.10174 & \mathrm{H} & -2.89645 & -1.92305 & -2.37179 \\ \mathrm{H} & -0.12988 & 2.104633 & 2.201063 & \mathrm{H} & 0.479571 & 0.962108 & 1.368059 \\ \mathrm{H} & 1.542292 & 1.912655 & 1.776783 & \mathrm{H} & 2.071836 & 1.590938 & 1.078523 \\ \mathrm{H} & -0.68012 & -0.03466 & 0.940583 & \mathrm{H} & 2.305185 & 0.517814 & -1.02954 \\ \mathrm{H} & 0.271019 & -0.19952 & 2.381743 & \mathrm{H} & 0.597773 & 0.328823 & -1.26085 \\ \mathrm{H} & 0.72332 & -2.52434 & 1.430744 & \mathrm{H} & -0.3142 & -1.07767 & 1.279487 \\ \mathrm{H} & 1.767103 & -2.69351 & 0.03419 & \mathrm{H} & 0.274471 & -2.70288 & 0.972572 \\ \mathrm{H} & 1.391413 & -0.21985 & -0.45289 & \mathrm{H} & 1.760441 & -1.78551 & -0.84644 \\ \mathrm{H} & 4.076373 & -0.40743 & 2.755939 & \mathrm{H} & 3.237782 & -1.57619 & 3.027235 \\ \mathrm{H} & 2.281582 & -0.22999 & 3.171184 & \mathrm{H} & 1.401767 & -1.52773 & 2.801316 \\ \mathrm{H} & -4.42239 & -0.71029 & 1.727823 & \mathrm{H} & -3.9736 & -1.16762 & 1.966843 \\ \mathrm{H} & -3.72934 & 0.846067 & 2.18629 & \mathrm{H} & -2.87818 & 0.082433 & 2.55288 \\ \mathrm{H} & -5.36227 & 0.774463 & 1.548455 & \mathrm{H} & -4.60752 & 0.378332 & 2.543755 \\ \mathrm{H} & -2.36654 & -2.50891 & 1.785911 & \mathrm{H} & -2.93896 & -3.51427 & 1.165835 \\ \mathrm{H} & -1.02582 & -3.66342 & 1.811827 & \mathrm{H} & -1.29751 & -4.12741 & 0.928545 \\ \mathrm{H} & -2.62152 & -4.17613 & 1.254588 & \mathrm{H} & -2.63181 & -4.77733 & -0.03048 \\ \mathrm{H} & -0.29207 & 2.765524 & -2.21404 & \mathrm{H} & -0.76952 & 3.741792 & -1.68764 \\ \mathrm{H} & 0.288534 & 4.644377 & -0.41806 & \mathrm{H} & 0.220963 & 4.71999 & 0.705178 \\ \mathrm{H} & -0.34243 & 4.334419 & 1.205371 & \mathrm{H} & 0.317883 & 3.549955 & 2.033068 \\ \mathrm{H} & 1.392691 & 4.270343 & 0.921449 & \mathrm{H} & 1.781206 & 4.018369 & 1.180758 \\ \mathrm{H} & 4.112682 & -1.3393 & -2.97347 & \mathrm{H} & 5.334567 & -1.33086 & -2.37236 \\ \mathrm{H} & 5.280994 & -0.35416 & -2.04177 & \mathrm{H} & 5.995684 & -0.43759 & -0.96901 \\ \mathrm{H} & 5.169105 & -2.10746 & -1.74986 & \mathrm{H} & 5.991031 & -2.21815 & -0.96373 \\ \mathrm{H} & 3.115924 & 2.100578 & -1.60628 & \mathrm{H} & 2.624346 & 3.461477 & -2.39589 \\ \mathrm{H} & 2.673038 & 3.686474 & -0.94242 & \mathrm{H} & 2.507765 & 4.449984 & -0.92577 \\ \mathrm{H} & 3.030574 & 2.325173 & 0.151294 & \mathrm{H} & 3.065795 & 2.760424 & -0.82631\end{array}$

\begin{tabular}{|c|c|c|c|c|c|c|c|}
\hline 3d_3 & & & & 3d_4 & & & \\
\hline $\mathrm{C}$ & -3.60172 & 2.010595 & -0.32237 & $\mathrm{C}$ & -3.65933 & 2.033762 & -0.18195 \\
\hline $\mathrm{C}$ & -3.83586 & 0.558399 & 0.048054 & $\mathrm{C}$ & -3.88983 & 0.573784 & 0.158974 \\
\hline $\mathrm{C}$ & -2.30973 & 2.623986 & 0.264898 & $\mathrm{C}$ & -2.33763 & 2.621792 & 0.363652 \\
\hline $\mathrm{C}$ & -1.06746 & 2.24307 & -0.53541 & $\mathrm{C}$ & -1.13409 & 2.249905 & -0.49792 \\
\hline $\mathrm{C}$ & -3.61709 & -0.42054 & -0.84126 & $\mathrm{C}$ & -3.73012 & -0.38088 & -0.76855 \\
\hline $\mathrm{C}$ & -3.88676 & -1.89536 & -0.70348 & $\mathrm{C}$ & -4.01082 & -1.85599 & -0.65867 \\
\hline $\mathrm{C}$ & -2.72579 & -2.81785 & -1.12713 & $\mathrm{C}$ & -2.8797 & -2.77988 & -1.15429 \\
\hline $\mathrm{C}$ & 0.291651 & 2.549793 & 0.149187 & $\mathrm{C}$ & 0.255658 & 2.525196 & 0.136742 \\
\hline $\mathrm{C}$ & 0.527878 & 1.704821 & 1.423443 & $\mathrm{C}$ & 0.536303 & 1.643437 & 1.376433 \\
\hline $\mathrm{C}$ & -1.55289 & -2.96988 & -0.16519 & $\mathrm{C}$ & -1.66848 & -2.96793 & -0.24786 \\
\hline $\mathrm{C}$ & -0.23479 & -2.38384 & -0.50856 & $\mathrm{C}$ & -0.36056 & -2.38496 & -0.63232 \\
\hline $\mathrm{C}$ & 0.308498 & 0.179891 & 1.354593 & $\mathrm{C}$ & 0.294004 & 0.123756 & 1.277062 \\
\hline $\mathrm{C}$ & 0.92361 & -2.12157 & 0.434269 & $\mathrm{C}$ & 0.841068 & -2.15817 & 0.26436 \\
\hline $\mathrm{C}$ & 1.34417 & -0.63482 & 0.554553 & $\mathrm{C}$ & 1.279348 & -0.67889 & 0.406292 \\
\hline $\mathrm{C}$ & 2.752919 & -0.57827 & 1.132199 & $\mathrm{C}$ & 2.716052 & -0.64834 & 0.909053 \\
\hline $\mathrm{C}$ & 3.925124 & -0.79694 & 0.215874 & $\mathrm{C}$ & 3.759548 & -0.81404 & -0.15435 \\
\hline $\mathrm{O}$ & 5.087869 & -0.80722 & 0.56181 & $\mathrm{O}$ & 3.505454 & -0.96115 & -1.33312 \\
\hline $\mathrm{C}$ & 3.046084 & -0.39506 & 2.423032 & $\mathrm{C}$ & 3.078017 & -0.54019 & 2.191197 \\
\hline
\end{tabular}




\begin{tabular}{|c|c|c|c|c|c|c|c|}
\hline $\mathrm{C}$ & -4.36112 & 0.341349 & 1.446977 & $\mathrm{C}$ & -4.34252 & 0.319861 & 1.577014 \\
\hline $\mathrm{C}$ & -1.90748 & -3.34682 & 1.256083 & $\mathrm{C}$ & -1.96636 & -3.37852 & 1.177113 \\
\hline $\mathrm{O}$ & -1.14325 & 2.935292 & -1.78875 & $\mathrm{O}$ & -1.25437 & 2.975771 & -1.72856 \\
\hline $\mathrm{O}$ & -0.50428 & -3.77885 & -0.76082 & $\mathrm{O}$ & -0.65478 & -3.77061 & -0.9086 \\
\hline $\mathrm{H}$ & -0.20061 & -1.78819 & -1.42216 & $\mathrm{H}$ & -0.3599 & -1.76597 & -1.5309 \\
\hline $\mathrm{C}$ & 0.420273 & 4.041092 & 0.482166 & $\mathrm{C}$ & 0.413651 & 4.0057 & 0.503806 \\
\hline $\mathrm{O}$ & 3.547775 & -0.98462 & -1.06232 & $\mathrm{O}$ & 5.018058 & -0.784 & 0.317605 \\
\hline $\mathrm{C}$ & 4.607411 & -1.21077 & -2.01454 & $\mathrm{C}$ & 6.069127 & -0.94892 & -0.65575 \\
\hline $\mathrm{O}$ & 1.224601 & 2.172164 & -0.90551 & $\mathrm{O}$ & 1.14019 & 2.167909 & -0.96536 \\
\hline $\mathrm{C}$ & 2.576034 & 2.605025 & -0.80429 & $\mathrm{C}$ & 2.497695 & 2.590401 & -0.91141 \\
\hline $\mathrm{H}$ & -4.45483 & 2.600288 & 0.033218 & $\mathrm{H}$ & -4.49105 & 2.619804 & 0.226658 \\
\hline $\mathrm{H}$ & -3.57163 & 2.122627 & -1.40877 & $\mathrm{H}$ & -3.67613 & 2.174674 & -1.2653 \\
\hline $\mathrm{H}$ & -2.39674 & 3.71413 & 0.257901 & $\mathrm{H}$ & -2.41453 & 3.712513 & 0.38597 \\
\hline $\mathrm{H}$ & -2.19547 & 2.321998 & 1.309062 & $\mathrm{H}$ & -2.18152 & 2.294405 & 1.394637 \\
\hline $\mathrm{H}$ & -1.0856 & 1.165486 & -0.73529 & $\mathrm{H}$ & -1.17233 & 1.178194 & -0.72479 \\
\hline $\mathrm{H}$ & -3.25673 & -0.12442 & -1.8267 & $\mathrm{H}$ & -3.41726 & -0.06031 & -1.76248 \\
\hline $\mathrm{H}$ & -4.73089 & -2.139 & -1.36249 & $\mathrm{H}$ & -4.88434 & -2.07264 & -1.28812 \\
\hline $\mathrm{H}$ & -4.21978 & -2.15228 & 0.303746 & $\mathrm{H}$ & -4.30541 & -2.13575 & 0.354413 \\
\hline $\mathrm{H}$ & -3.12949 & -3.82799 & -1.26808 & $\mathrm{H}$ & -3.30081 & -3.7819 & -1.30241 \\
\hline $\mathrm{H}$ & -2.34 & -2.50216 & -2.10163 & $\mathrm{H}$ & -2.53224 & -2.4442 & -2.13653 \\
\hline $\mathrm{H}$ & -0.12993 & 2.104324 & 2.201256 & $\mathrm{H}$ & -0.08035 & 2.029636 & 2.193645 \\
\hline $\mathrm{H}$ & 1.54227 & 1.91236 & 1.777081 & $\mathrm{H}$ & 1.568526 & 1.827977 & 1.689282 \\
\hline $\mathrm{H}$ & -0.68017 & -0.0348 & 0.940613 & $\mathrm{H}$ & -0.71636 & -0.06852 & 0.906864 \\
\hline $\mathrm{H}$ & 0.27099 & -0.19984 & 2.38174 & $\mathrm{H}$ & 0.302485 & -0.28301 & 2.294655 \\
\hline $\mathrm{H}$ & 0.723446 & -2.52463 & 1.430569 & $\mathrm{H}$ & 0.683201 & -2.58922 & 1.256614 \\
\hline $\mathrm{H}$ & 1.767052 & -2.69357 & 0.033861 & $\mathrm{H}$ & 1.661338 & -2.72267 & -0.19136 \\
\hline $\mathrm{H}$ & 1.39132 & -0.21996 & -0.45293 & $\mathrm{H}$ & 1.288323 & -0.24059 & -0.59292 \\
\hline $\mathrm{H}$ & 4.076366 & -0.40777 & 2.755808 & $\mathrm{H}$ & 4.118601 & -0.56353 & 2.485166 \\
\hline $\mathrm{H}$ & 2.281591 & -0.23035 & 3.17114 & $\mathrm{H}$ & 2.34602 & -0.42641 & 2.98153 \\
\hline $\mathrm{H}$ & -4.42335 & -0.70955 & 1.727543 & $\mathrm{H}$ & -4.38901 & -0.73817 & 1.832773 \\
\hline $\mathrm{H}$ & -3.73067 & 0.847028 & 2.185888 & $\mathrm{H}$ & -3.67461 & 0.806835 & 2.295102 \\
\hline $\mathrm{H}$ & -5.36325 & 0.775062 & 1.547184 & $\mathrm{H}$ & -5.33828 & 0.74897 & 1.740709 \\
\hline $\mathrm{H}$ & -2.36647 & -2.50855 & 1.786024 & $\mathrm{H}$ & -2.39302 & -2.54975 & 1.747494 \\
\hline $\mathrm{H}$ & -1.02591 & -3.66325 & 1.812015 & $\mathrm{H}$ & -1.06568 & -3.71835 & 1.686741 \\
\hline $\mathrm{H}$ & -2.62171 & -4.17583 & 1.254986 & $\mathrm{H}$ & -2.68945 & -4.1998 & 1.185409 \\
\hline $\mathrm{H}$ & -0.29149 & 2.765643 & -2.21388 & $\mathrm{H}$ & -0.42293 & 2.808665 & -2.19312 \\
\hline $\mathrm{H}$ & 0.288828 & 4.644313 & -0.41765 & $\mathrm{H}$ & 0.252255 & 4.634187 & -0.37359 \\
\hline $\mathrm{H}$ & -0.34232 & 4.334244 & 1.2057 & $\mathrm{H}$ & -0.31544 & 4.287022 & 1.265496 \\
\hline $\mathrm{H}$ & 1.392829 & 4.270163 & 0.921959 & $\mathrm{H}$ & 1.405988 & 4.212942 & 0.9089 \\
\hline $\mathrm{H}$ & 4.112577 & -1.33874 & -2.97372 & $\mathrm{H}$ & 6.996555 & -0.89848 & -0.09105 \\
\hline $\mathrm{H}$ & 5.280975 & -0.3539 & -2.04182 & $\mathrm{H}$ & 5.977692 & -1.91418 & -1.15488 \\
\hline $\mathrm{H}$ & 5.168929 & -2.10724 & -1.75027 & $\mathrm{H}$ & 6.029907 & -0.15085 & -1.39778 \\
\hline $\mathrm{H}$ & 3.116206 & 2.100925 & -1.606 & $\mathrm{H}$ & 3.002138 & 2.094319 & -1.7407 \\
\hline $\mathrm{H}$ & 2.673282 & 3.686525 & -0.94148 & $\mathrm{H}$ & 2.596693 & 3.673434 & -1.03492 \\
\hline $\mathrm{H}$ & 3.030884 & 2.324784 & 0.151672 & $\mathrm{H}$ & 2.988264 & 2.292059 & 0.021296 \\
\hline
\end{tabular}




\begin{tabular}{|c|c|c|c|c|c|c|c|}
\hline $\mathrm{C}$ & -2.34306 & 1.826878 & 1.299285 & $\mathrm{C}$ & 1.999133 & 2.518443 & -0.67471 \\
\hline $\mathrm{C}$ & -1.54741 & 2.049637 & 0.013012 & $\mathrm{C}$ & 0.605996 & 2.64678 & -0.05961 \\
\hline $\mathrm{C}$ & -3.10248 & -1.07762 & 0.739391 & $\mathrm{C}$ & 3.590584 & 0.079235 & 0.712301 \\
\hline $\mathrm{C}$ & -3.25825 & -2.53565 & 0.391304 & $\mathrm{C}$ & 3.568026 & -1.28665 & 1.352804 \\
\hline $\mathrm{C}$ & -2.40665 & -3.02968 & -0.79926 & $\mathrm{C}$ & 3.574446 & -2.4675 & 0.360395 \\
\hline $\mathrm{C}$ & -0.10399 & 2.599819 & 0.198638 & $\mathrm{C}$ & -0.57559 & 2.188484 & -0.95993 \\
\hline $\mathrm{C}$ & 0.829785 & 1.646422 & 0.979989 & $\mathrm{C}$ & -0.60651 & 0.652474 & -1.15874 \\
\hline $\mathrm{C}$ & -0.89481 & -3.07212 & -0.61218 & $\mathrm{C}$ & 2.421256 & -2.54428 & -0.6419 \\
\hline $\mathrm{C}$ & -0.03304 & -2.11641 & -1.34962 & $\mathrm{C}$ & 1.064819 & -2.8679 & -0.14526 \\
\hline $\mathrm{C}$ & 0.856465 & 0.149837 & 0.615143 & $\mathrm{C}$ & -0.63544 & -0.16774 & 0.142388 \\
\hline $\mathrm{C}$ & 1.398744 & -1.7462 & -1.0158 & $\mathrm{C}$ & -0.25526 & -2.5475 & -0.8218 \\
\hline $\mathrm{C}$ & 1.598404 & -0.24713 & -0.68196 & $\mathrm{C}$ & -1.16228 & -1.6272 & 0.018194 \\
\hline $\mathrm{C}$ & 3.079494 & 0.113918 & -0.68837 & $\mathrm{C}$ & -2.61171 & -1.64847 & -0.43855 \\
\hline $\mathrm{C}$ & 4.034999 & -0.34463 & 0.378744 & $\mathrm{C}$ & -3.68262 & -1.22105 & 0.526867 \\
\hline $\mathrm{O}$ & 5.169902 & 0.063308 & 0.508598 & $\mathrm{O}$ & -4.83906 & -1.00482 & 0.230445 \\
\hline $\mathrm{C}$ & 3.623529 & 0.826123 & -1.6806 & $\mathrm{C}$ & -3.03217 & -2.01703 & -1.65262 \\
\hline $\mathrm{C}$ & -5.37626 & -0.29192 & -0.01417 & $\mathrm{C}$ & 2.61139 & 1.375797 & 2.630583 \\
\hline $\mathrm{C}$ & -0.4025 & -3.791 & 0.624702 & $\mathrm{C}$ & 2.636852 & -1.85381 & -1.97143 \\
\hline $\mathrm{O}$ & -2.29187 & 2.959697 & -0.80705 & $\mathrm{O}$ & 0.4357 & 4.019881 & 0.313048 \\
\hline $\mathrm{O}$ & -0.22474 & -3.45942 & -1.84055 & $\mathrm{O}$ & 1.869231 & -3.88354 & -0.77835 \\
\hline $\mathrm{H}$ & -0.55512 & -1.37088 & -1.95207 & $\mathrm{H}$ & 0.974522 & -3.01953 & 0.931951 \\
\hline $\mathrm{C}$ & -0.11848 & 3.972957 & 0.883912 & $\mathrm{C}$ & -0.56822 & 2.910419 & -2.31055 \\
\hline $\mathrm{O}$ & 3.509871 & -1.28401 & 1.187044 & $\mathrm{O}$ & -3.22415 & -1.11347 & 1.786802 \\
\hline $\mathrm{C}$ & 4.373501 & -1.78632 & 2.228409 & $\mathrm{C}$ & -4.18827 & -0.7216 & 2.78624 \\
\hline $\mathrm{O}$ & 0.322293 & 2.714916 & -1.19298 & $\mathrm{O}$ & -1.71219 & 2.603703 & -0.15056 \\
\hline $\mathrm{C}$ & 1.397465 & 3.594615 & -1.50252 & $\mathrm{C}$ & -3.00972 & 2.582798 & -0.73395 \\
\hline $\mathrm{H}$ & -4.35027 & 1.463022 & 1.983618 & $\mathrm{H}$ & 4.092136 & 2.562339 & -0.17408 \\
\hline $\mathrm{H}$ & -4.24476 & 2.006695 & 0.333437 & $\mathrm{H}$ & 3.072684 & 3.379892 & 0.998863 \\
\hline $\mathrm{H}$ & -2.41474 & 2.77727 & 1.835756 & $\mathrm{H}$ & 2.146037 & 3.366258 & -1.35129 \\
\hline $\mathrm{H}$ & -1.80004 & 1.142989 & 1.956888 & $\mathrm{H}$ & 2.061975 & 1.610997 & -1.27982 \\
\hline $\mathrm{H}$ & -1.45762 & 1.099566 & -0.52599 & $\mathrm{H}$ & 0.54581 & 2.032336 & 0.843874 \\
\hline $\mathrm{H}$ & -2.148 & -0.82064 & 1.19095 & $\mathrm{H}$ & 4.030276 & 0.119483 & -0.28304 \\
\hline $\mathrm{H}$ & -4.29949 & -2.76627 & 0.159833 & $\mathrm{H}$ & 2.700917 & -1.38071 & 2.013155 \\
\hline $\mathrm{H}$ & -3.01147 & -3.13711 & 1.274391 & $\mathrm{H}$ & 4.44712 & -1.41324 & 1.999256 \\
\hline $\mathrm{H}$ & -2.71368 & -4.05839 & -1.02685 & $\mathrm{H}$ & 4.517856 & -2.46618 & -0.19773 \\
\hline $\mathrm{H}$ & -2.63464 & -2.4314 & -1.68626 & $\mathrm{H}$ & 3.55521 & -3.39622 & 0.93697 \\
\hline $\mathrm{H}$ & 0.550828 & 1.724726 & 2.035622 & $\mathrm{H}$ & 0.248307 & 0.368011 & -1.77827 \\
\hline $\mathrm{H}$ & 1.844431 & 2.056307 & 0.92213 & $\mathrm{H}$ & -1.4914 & 0.420953 & -1.75966 \\
\hline $\mathrm{H}$ & -0.16448 & -0.22977 & 0.540144 & $\mathrm{H}$ & -1.26466 & 0.351573 & 0.867984 \\
\hline $\mathrm{H}$ & 1.310017 & -0.38883 & 1.449784 & $\mathrm{H}$ & 0.366649 & -0.21319 & 0.577392 \\
\hline $\mathrm{H}$ & 1.784067 & -2.36475 & -0.20557 & $\mathrm{H}$ & -0.08833 & -2.1382 & -1.81975 \\
\hline $\mathrm{H}$ & 1.996669 & -1.98199 & -1.90372 & $\mathrm{H}$ & -0.76803 & -3.50551 & -0.9624 \\
\hline $\mathrm{H}$ & 1.157627 & 0.329798 & -1.49985 & $\mathrm{H}$ & -1.1582 & -2.02525 & 1.038028 \\
\hline $\mathrm{H}$ & 4.682311 & 1.05315 & -1.68773 & $\mathrm{H}$ & -4.08684 & -1.9891 & -1.89634 \\
\hline $\mathrm{H}$ & 3.023786 & 1.187969 & -2.50662 & $\mathrm{H}$ & -2.35067 & -2.35632 & -2.42176 \\
\hline $\mathrm{H}$ & -5.60803 & -1.32844 & -0.25518 & $\mathrm{H}$ & 2.459889 & 0.418283 & 3.130029 \\
\hline $\mathrm{H}$ & -6.14823 & 0.077176 & 0.671051 & $\mathrm{H}$ & 3.325484 & 1.95211 & 3.232109 \\
\hline $\mathrm{H}$ & -5.47213 & 0.297119 & -0.93346 & $\mathrm{H}$ & 1.670592 & 1.933273 & 2.659539 \\
\hline $\mathrm{H}$ & -0.609 & -3.21329 & 1.529494 & $\mathrm{H}$ & 2.72271 & -0.77261 & -1.85068 \\
\hline
\end{tabular}




$\begin{array}{lrrrlrrr}\mathrm{H} & 0.669542 & -3.9759 & 0.572266 & \mathrm{H} & 1.825864 & -2.06322 & -2.66815 \\ \mathrm{H} & -0.90764 & -4.75702 & 0.72039 & \mathrm{H} & 3.567205 & -2.21419 & -2.42049 \\ \mathrm{H} & -1.70266 & 3.154332 & -1.54863 & \mathrm{H} & -0.49683 & 4.09207 & 0.559838 \\ \mathrm{H} & -0.74656 & 4.673794 & 0.332296 & \mathrm{H} & -0.5804 & 3.992068 & -2.1635 \\ \mathrm{H} & -0.51309 & 3.88559 & 1.897271 & \mathrm{H} & 0.330028 & 2.650543 & -2.87404 \\ \mathrm{H} & 0.888426 & 4.386614 & 0.963978 & \mathrm{H} & -1.42879 & 2.628399 & -2.92028 \\ \mathrm{H} & 3.780305 & -2.52068 & 2.767057 & \mathrm{H} & -3.6375 & -0.68799 & 3.722568 \\ \mathrm{H} & 5.25841 & -2.25241 & 1.794567 & \mathrm{H} & -4.60224 & 0.258721 & 2.549099 \\ \mathrm{H} & 4.676652 & -0.97655 & 2.892375 & \mathrm{H} & -4.99436 & -1.4536 & 2.841646 \\ \mathrm{H} & 1.63505 & 3.423776 & -2.55304 & \mathrm{H} & -3.7058 & 2.828677 & 0.0687 \\ \mathrm{H} & 1.119378 & 4.645418 & -1.37471 & \mathrm{H} & -3.11669 & 3.326125 & -1.53021 \\ \mathrm{H} & 2.291229 & 3.385632 & -0.90578 & \mathrm{H} & -3.27163 & 1.596166 & -1.12982\end{array}$

\section{3d_7}

$\mathrm{C}$

C

C

C

C

C

C

C

C

C

C

C

C

C

C

C

$\mathrm{O}$

C

C

$\mathrm{C}$

$\mathrm{O}$

$\mathrm{O}$

$\mathrm{H}$

$\mathrm{C}$

$\mathrm{O}$

C

$\mathrm{O}$

C

$\mathrm{H}$

$\mathrm{H}$

$\mathrm{H}$

$\mathrm{H}$

$\mathrm{H}$

$\mathrm{H}$

$\mathrm{H}$
$-3.66764 \quad 1.698483$

$-4.06052 \quad 0.302411$

$-0.68823$

$-2.16993 \quad 2.022726$

$-1.47553 \quad 2.343948$

$-3.2392-0.75633$

$-3.54053 \quad-2.17656$

$-2.54683$

0.032786

$-3.21576$

2.707591

0.853666

1.695292

$-1.10193$

$-3.12602$

$-0.03544$

$-2.71609$

0.895241

0.247458

1.35365

$-2.20635$

$-0.72752$

1.583059

3.053689

$-0.41673$

$-0.5203$

$-0.21534$

$-0.82061$

0.511165

$-0.28681$

0.137121

$-0.41097$

0.364756

$-0.46871$

4.087583

$-0.25735$

5.263667

3.50544

$-0.04288$

0.07564

$-0.87293$

0.042693

$-0.53959$

$-0.94061$

$-1.18448$

$-0.09772$

$-0.23528$

$-5.47419$

0.216732

$-2.38621$

0.304096

$-0.91058$

$-3.04073$

1.572925

$-1.605$

1.3029291 .489291

$-0.26846$

$-4.11032$

$-0.58859$

$-0.37673$

$-2.4295$

$-1.86938$

0.64093

2.922626

1.755992

3.575049

$-0.94793$

1.070425

4.505724

$-1.08466$

2.164668

$-0.04672$

3.962523

$-0.35875$

1.150678

4.695049

$-0.57627$

$-4.13128$

2.431779

$-0.01536$

$-4.15299$

1.862465

$-1.65904$

$-1.65588$

1.214366

$-1.34526$

$-2.05872$

2.909223

$-1.44829$

$-1.94897$

3.251755

0.913211

$-2.24293$

$-0.60463$

$-0.68909$

$-4.5416 \quad-2.46852$

$-0.19473$

\section{3d_8}

$\mathrm{C}$

C

C

C

C

C

C

C

C

C

C

C

C

C

C

C

$\mathrm{O}$

C

C

C

$\mathrm{O}$

$\mathrm{O}$

$\mathrm{H}$

C

$\mathrm{O}$

C

$\mathrm{O}$

$\mathrm{C}$

$\mathrm{H}$

$\mathrm{H}$

$\mathrm{H}$

$\mathrm{H}$

$\mathrm{H}$

$\mathrm{H}$

$\mathrm{H}$ $\begin{array}{lll}-3.7632 & 1.98742 & 0.083924\end{array}$

$\begin{array}{lll}-4.1344 & 0.545496 & -0.22787\end{array}$

$\begin{array}{lll}-2.40767 & 2.29423 & 0.738816\end{array}$

$\begin{array}{lll}-1.22125 & 2.138731 & -0.21223\end{array}$

$\begin{array}{lll}-3.53009 & -0.51197 & 0.331027\end{array}$

$\begin{array}{lll}-3.87548 & -1.96897 & 0.15717\end{array}$

$\begin{array}{lll}-2.85736 & -2.803 & -0.65271\end{array}$

$\begin{array}{lll}0.175796 & 2.454415 & 0.395435\end{array}$

$\begin{array}{lll}0.58215 & 1.501091 & 1.542753\end{array}$

$\begin{array}{lll}-1.4904 & -3.04719 & -0.02451\end{array}$

$\begin{array}{lll}-0.2944 & -2.37577 & -0.58811\end{array}$

$\begin{array}{lll}0.446786 & -0.01862 & 1.33186\end{array}$

$\begin{array}{lll}1.040445 & -2.16908 & 0.099834\end{array}$

$\begin{array}{lll}1.411227 & -0.68235 & 0.331292\end{array}$

$\begin{array}{llll}2.877555 & -0.60205 & 0.729034\end{array}$

$\begin{array}{llll}3.845801 & -0.62269 & -0.41534\end{array}$

$\begin{array}{lll}3.512052 & -0.65308 & -1.583\end{array}$

$\begin{array}{lll}3.329181 & -0.55796 & 1.986385\end{array}$

$\begin{array}{lll}-5.29267 & 0.449172 & -1.19289\end{array}$

$\begin{array}{lll}-1.49827 & -3.6112 & 1.379159\end{array}$

$-1.44944 \quad 3.001434 \quad-1.33403$

$\begin{array}{lll}-0.60507 & -3.73788 & -0.94559\end{array}$

$\begin{array}{lll}-0.48259 & -1.67348 & -1.40189\end{array}$

$\begin{array}{lll}0.256845 & 3.906823 & 0.88312\end{array}$

$\begin{array}{lll}5.133508 & -0.60285 & -0.02975\end{array}$

$\begin{array}{lll}6.117028 & -0.62162 & -1.08418\end{array}$

$\begin{array}{lll}1.025808 & 2.255828 & -0.77308\end{array}$

$\begin{array}{lll}2.355318 & 2.762021 & -0.74386\end{array}$

$\begin{array}{lll}-4.55684 & 2.393375 & 0.726133\end{array}$

$\begin{array}{lll}-3.82544 & 2.564896 & -0.84381\end{array}$

$\begin{array}{lll}-2.43984 & 3.330491 & 1.08693\end{array}$

$-2.25606 \quad 1.675503 \quad 1.626905$

$\begin{array}{lll}-1.18321 & 1.106089 & -0.57729\end{array}$

$\begin{array}{lll}-2.7085 & -0.32123 & 1.015883\end{array}$

$\begin{array}{lll}-4.8438 & -2.07742 & -0.33427\end{array}$ 


\begin{tabular}{|c|c|c|c|c|c|c|c|}
\hline $\mathrm{H}$ & -3.56643 & -2.24525 & 1.231463 & $\mathrm{H}$ & -3.99425 & -2.42305 & 1.148128 \\
\hline $\mathrm{H}$ & -2.90501 & -4.21262 & -0.12806 & $\mathrm{H}$ & -3.29577 & -3.79431 & -0.82368 \\
\hline $\mathrm{H}$ & -2.54691 & -3.18561 & -1.50529 & $\mathrm{H}$ & -2.71352 & -2.34958 & -1.63778 \\
\hline $\mathrm{H}$ & 1.879025 & 2.07129 & -0.53523 & $\mathrm{H}$ & -0.02182 & 1.771959 & 2.414531 \\
\hline $\mathrm{H}$ & 0.463417 & 1.708681 & -1.49165 & $\mathrm{H}$ & 1.611767 & 1.740867 & 1.825438 \\
\hline $\mathrm{H}$ & -0.11907 & -0.10933 & 0.215901 & $\mathrm{H}$ & -0.5702 & -0.25293 & 1.011829 \\
\hline $\mathrm{H}$ & 1.400724 & 0.20022 & 1.009294 & $\mathrm{H}$ & 0.551706 & -0.50329 & 2.309102 \\
\hline $\mathrm{H}$ & 1.587181 & -2.35288 & 0.514476 & $\mathrm{H}$ & 1.08066 & -2.70887 & 1.049458 \\
\hline $\mathrm{H}$ & 2.050315 & -2.83686 & -1.10468 & $\mathrm{H}$ & 1.797053 & -2.62196 & -0.54955 \\
\hline $\mathrm{H}$ & 1.102487 & -0.58818 & -1.91681 & $\mathrm{H}$ & 1.317915 & -0.16824 & -0.62673 \\
\hline $\mathrm{H}$ & 4.555619 & 0.169562 & -2.54323 & $\mathrm{H}$ & 4.388873 & -0.53527 & 2.201007 \\
\hline $\mathrm{H}$ & 2.836981 & 0.055809 & -3.23349 & $\mathrm{H}$ & 2.654628 & -0.54254 & 2.833753 \\
\hline $\mathrm{H}$ & -5.79307 & -0.80432 & 0.512026 & $\mathrm{H}$ & -5.64547 & -0.56842 & -1.35481 \\
\hline $\mathrm{H}$ & -5.5783 & 0.798327 & 1.227244 & $\mathrm{H}$ & -6.13837 & 1.048296 & -0.83568 \\
\hline $\mathrm{H}$ & -6.1724 & 0.65375 & -0.41868 & $\mathrm{H}$ & -5.00864 & 0.865217 & -2.16625 \\
\hline $\mathrm{H}$ & -1.25305 & -2.07739 & 1.959559 & $\mathrm{H}$ & -1.83128 & -2.86637 & 2.106633 \\
\hline $\mathrm{H}$ & 0.136251 & -3.16771 & 1.845954 & $\mathrm{H}$ & -0.50789 & -3.95799 & 1.671202 \\
\hline $\mathrm{H}$ & -1.48805 & -3.8272 & 2.068457 & $\mathrm{H}$ & -2.18362 & -4.46262 & 1.433964 \\
\hline $\mathrm{H}$ & -2.36101 & 0.751391 & 1.250157 & $\mathrm{H}$ & -0.62867 & 2.959788 & -1.84359 \\
\hline $\mathrm{H}$ & 0.136583 & 3.750228 & 2.262937 & $\mathrm{H}$ & -0.00296 & 4.596253 & 0.078622 \\
\hline $\mathrm{H}$ & 0.519132 & 2.026196 & 2.362288 & $\mathrm{H}$ & -0.43426 & 4.070275 & 1.711668 \\
\hline $\mathrm{H}$ & 1.707321 & 3.150705 & 1.695196 & $\mathrm{H}$ & 1.258402 & 4.147144 & 1.244695 \\
\hline $\mathrm{H}$ & 3.913981 & -1.43532 & 3.006178 & $\mathrm{H}$ & 7.080411 & -0.61618 & -0.58079 \\
\hline $\mathrm{H}$ & 5.281515 & -1.80837 & 1.913693 & $\mathrm{H}$ & 6.007773 & -1.5211 & -1.69084 \\
\hline $\mathrm{H}$ & 4.965539 & -0.12303 & 2.393613 & $\mathrm{H}$ & 6.013125 & 0.25957 & -1.71821 \\
\hline $\mathrm{H}$ & 0.857779 & 5.598217 & -1.11348 & $\mathrm{H}$ & 2.852821 & 2.354536 & -1.62406 \\
\hline $\mathrm{H}$ & 1.635172 & 4.991623 & 0.360335 & $\mathrm{H}$ & 2.381043 & 3.855036 & -0.79765 \\
\hline $\mathrm{H}$ & 1.874313 & 4.147913 & -1.19048 & $\mathrm{H}$ & 2.904151 & 2.436355 & 0.146029 \\
\hline 3d_9 & & & & 3d_10 & & & \\
\hline $\mathrm{C}$ & -4.19746 & 1.049769 & -0.23106 & $\mathrm{C}$ & 3.761921 & 1.772687 & -0.30951 \\
\hline $\mathrm{C}$ & -3.98647 & -0.3486 & 0.323571 & $\mathrm{C}$ & 4.073533 & 0.360587 & 0.165746 \\
\hline $\mathrm{C}$ & -3.13186 & 2.068571 & 0.239752 & $\mathrm{C}$ & 2.379793 & 2.07506 & -0.90674 \\
\hline $\mathrm{C}$ & -1.77828 & 1.770209 & -0.41128 & $\mathrm{C}$ & 1.241143 & 2.083642 & 0.129894 \\
\hline $\mathrm{C}$ & -3.4367 & -1.3152 & -0.42771 & $\mathrm{C}$ & 3.383769 & -0.7165 & -0.23427 \\
\hline $\mathrm{C}$ & -3.22594 & -2.76459 & -0.06596 & $\mathrm{C}$ & 3.650011 & -2.1636 & 0.088752 \\
\hline $\mathrm{C}$ & -2.00197 & -3.45202 & -0.7051 & $\mathrm{C}$ & 2.510579 & -2.89809 & 0.828356 \\
\hline $\mathrm{C}$ & -0.53276 & 2.480925 & 0.186826 & $\mathrm{C}$ & -0.15929 & 2.452416 & -0.43567 \\
\hline $\mathrm{C}$ & 0.73789 & 1.826244 & -0.40736 & $\mathrm{C}$ & -0.5691 & 1.551579 & -1.62537 \\
\hline $\mathrm{C}$ & -0.60846 & -3.18107 & -0.13347 & $\mathrm{C}$ & 1.200786 & -3.10274 & 0.074339 \\
\hline $\mathrm{C}$ & 0.447684 & -2.5982 & -1.00283 & $\mathrm{C}$ & -0.01637 & -2.37798 & 0.514425 \\
\hline $\mathrm{C}$ & 0.98829 & 0.384601 & 0.070844 & $\mathrm{C}$ & -0.5212 & 0.021862 & -1.44804 \\
\hline $\mathrm{C}$ & 1.760313 & -1.96491 & -0.57631 & $\mathrm{C}$ & -1.27314 & -2.12024 & -0.29331 \\
\hline $\mathrm{C}$ & 1.852048 & -0.45367 & -0.89714 & $\mathrm{C}$ & -1.56992 & -0.61599 & -0.5163 \\
\hline $\mathrm{C}$ & 3.286944 & 0.039914 & -1.01339 & $\mathrm{C}$ & -3.00465 & -0.4539 & -0.99667 \\
\hline $\mathrm{C}$ & 4.247669 & -0.00413 & 0.142229 & $\mathrm{C}$ & -4.1166 & -0.44181 & 0.01519 \\
\hline $\mathrm{O}$ & 5.392099 & 0.39559 & 0.105069 & $\mathrm{O}$ & -5.29456 & -0.31273 & -0.24579 \\
\hline $\mathrm{C}$ & 3.769819 & 0.538271 & -2.1564 & $\mathrm{C}$ & -3.37917 & -0.35534 & -2.27555 \\
\hline & -4.45287 & -0.5456 & 1.746081 & $\mathrm{C}$ & 5.27584 & 0.311621 & 1.078666 \\
\hline
\end{tabular}




\begin{tabular}{|c|c|c|c|c|c|c|c|}
\hline $\mathrm{C}$ & -0.49874 & -3.15804 & 1.373824 & $\mathrm{C}$ & 1.318775 & -3.68631 & -1.31633 \\
\hline $\mathrm{O}$ & -1.8401 & 2.033523 & -1.82069 & $\mathrm{O}$ & 1.552815 & 3.02777 & 1.175283 \\
\hline $\mathrm{O}$ & 0.370839 & -4.02222 & -0.79881 & $\mathrm{O}$ & 0.206169 & -3.74619 & 0.91322 \\
\hline $\mathrm{H}$ & 0.121367 & -2.29641 & -1.99995 & $\mathrm{H}$ & 0.122972 & -1.67443 & 1.337133 \\
\hline $\mathrm{C}$ & -0.51271 & 2.457917 & 1.718793 & $\mathrm{C}$ & -0.21567 & 3.917326 & -0.89142 \\
\hline $\mathrm{O}$ & 3.70739 & -0.54589 & 1.249191 & $\mathrm{O}$ & -3.66766 & -0.59684 & 1.274602 \\
\hline $\mathrm{C}$ & 4.569681 & -0.63462 & 2.403009 & $\mathrm{C}$ & -4.66948 & -0.60832 & 2.311916 \\
\hline $\mathrm{O}$ & -0.67798 & 3.8352 & -0.31203 & $\mathrm{O}$ & -1.15338 & 2.175311 & 0.583016 \\
\hline $\mathrm{C}$ & 0.263215 & 4.818458 & 0.09867 & $\mathrm{C}$ & -1.44282 & 3.14448 & 1.587 \\
\hline $\mathrm{H}$ & -5.18755 & 1.408358 & 0.073416 & $\mathrm{H}$ & 4.525647 & 2.042704 & -1.05145 \\
\hline $\mathrm{H}$ & -4.1873 & 1.025673 & -1.32349 & $\mathrm{H}$ & 3.934264 & 2.463867 & 0.523266 \\
\hline $\mathrm{H}$ & -3.44448 & 3.08412 & -0.02289 & $\mathrm{H}$ & 2.431431 & 3.066226 & -1.36757 \\
\hline $\mathrm{H}$ & -3.05105 & 2.032753 & 1.328858 & $\mathrm{H}$ & 2.153441 & 1.373927 & -1.71343 \\
\hline $\mathrm{H}$ & -1.61337 & 0.696809 & -0.32804 & $\mathrm{H}$ & 1.146537 & 1.09192 & 0.580212 \\
\hline $\mathrm{H}$ & -3.17152 & -1.06069 & -1.45385 & $\mathrm{H}$ & 2.545472 & -0.55588 & -0.90653 \\
\hline $\mathrm{H}$ & -4.10216 & -3.3279 & -0.41581 & $\mathrm{H}$ & 4.550034 & -2.26638 & 0.697001 \\
\hline $\mathrm{H}$ & -3.20834 & -2.90621 & 1.016751 & $\mathrm{H}$ & 3.86147 & -2.69907 & -0.8454 \\
\hline $\mathrm{H}$ & -2.14439 & -4.53423 & -0.60012 & $\mathrm{H}$ & 2.872986 & -3.90039 & 1.08877 \\
\hline $\mathrm{H}$ & -1.98645 & -3.2515 & -1.78135 & $\mathrm{H}$ & 2.295379 & -2.38802 & 1.772074 \\
\hline $\mathrm{H}$ & 1.608851 & 2.443169 & -0.17124 & $\mathrm{H}$ & 0.066878 & 1.810497 & -2.47647 \\
\hline $\mathrm{H}$ & 0.623503 & 1.838225 & -1.49456 & $\mathrm{H}$ & -1.58273 & 1.854194 & -1.90163 \\
\hline $\mathrm{H}$ & 0.037545 & -0.14327 & 0.173224 & $\mathrm{H}$ & 0.463658 & -0.2734 & -1.08018 \\
\hline $\mathrm{H}$ & 1.445672 & 0.381195 & 1.062002 & $\mathrm{H}$ & -0.59659 & -0.43423 & -2.44174 \\
\hline $\mathrm{H}$ & 1.960292 & -2.14138 & 0.480092 & $\mathrm{H}$ & -1.24148 & -2.63759 & -1.25603 \\
\hline $\mathrm{H}$ & 2.542765 & -2.49251 & -1.13465 & $\mathrm{H}$ & -2.09968 & -2.56293 & 0.27203 \\
\hline $\mathrm{H}$ & 1.425575 & -0.31989 & -1.89833 & $\mathrm{H}$ & -1.49963 & -0.11796 & 0.449765 \\
\hline $\mathrm{H}$ & 4.793861 & 0.884294 & -2.22188 & $\mathrm{H}$ & -4.42789 & -0.27323 & -2.53208 \\
\hline $\mathrm{H}$ & 3.153973 & 0.607988 & -3.04524 & $\mathrm{H}$ & -2.66427 & -0.35223 & -3.08819 \\
\hline $\mathrm{H}$ & -4.14633 & -1.50082 & 2.171905 & $\mathrm{H}$ & 5.570385 & -0.6981 & 1.360781 \\
\hline $\mathrm{H}$ & -4.07686 & 0.249788 & 2.397591 & $\mathrm{H}$ & 6.13686 & 0.792619 & 0.600054 \\
\hline $\mathrm{H}$ & -5.54732 & -0.4947 & 1.795391 & $\mathrm{H}$ & 5.078716 & 0.875547 & 1.997528 \\
\hline $\mathrm{H}$ & -0.99266 & -2.27595 & 1.789736 & $\mathrm{H}$ & 1.753093 & -2.96659 & -2.01532 \\
\hline $\mathrm{H}$ & 0.542213 & -3.15487 & 1.694716 & $\mathrm{H}$ & 0.345975 & -3.99305 & -1.69864 \\
\hline $\mathrm{H}$ & -0.98038 & -4.04449 & 1.797913 & $\mathrm{H}$ & 1.966731 & -4.56799 & -1.29714 \\
\hline $\mathrm{H}$ & -1.67361 & 2.983378 & -1.90117 & $\mathrm{H}$ & 2.021395 & 2.558479 & 1.871329 \\
\hline $\mathrm{H}$ & -1.28473 & 3.111186 & 2.128848 & $\mathrm{H}$ & 0.115743 & 4.596664 & -0.10658 \\
\hline $\mathrm{H}$ & -0.68849 & 1.447069 & 2.093475 & $\mathrm{H}$ & 0.425754 & 4.069336 & -1.76142 \\
\hline $\mathrm{H}$ & 0.455349 & 2.786533 & 2.10435 & $\mathrm{H}$ & -1.23671 & 4.179932 & -1.17886 \\
\hline $\mathrm{H}$ & 3.965512 & -1.09296 & 3.181578 & $\mathrm{H}$ & -4.12345 & -0.751 & 3.240843 \\
\hline $\mathrm{H}$ & 5.439298 & -1.25277 & 2.178916 & $\mathrm{H}$ & -5.21031 & 0.338312 & 2.327292 \\
\hline $\mathrm{H}$ & 4.898281 & 0.35922 & 2.707734 & $\mathrm{H}$ & -5.37263 & -1.42606 & 2.15134 \\
\hline 11 & -0.0215 & 5.741249 & -0.40781 & $\mathrm{H}$ & -2.19038 & 2.681229 & 2.233618 \\
\hline $\mathrm{H}$ & 0.232221 & 4.990271 & 1.179773 & $\mathrm{H}$ & -0.56245 & 3.403597 & 2.176821 \\
\hline $\mathrm{H}$ & 1.288103 & 4.564284 & -0.19131 & $\mathrm{H}$ & -1.87636 & 4.058209 & 1.165371 \\
\hline
\end{tabular}

$\begin{array}{lrrr}\text { 3d_11 } & & & \\ \text { C } & -3.62031 & 1.949358 & -0.17013 \\ \text { C } & -3.82131 & 0.47695 & 0.134171 \\ \text { C } & -2.30189 & 2.540693 & 0.380226\end{array}$

3d_12

$\begin{array}{llll}\text { C } & -3.83412 & 1.878906 & -0.38154\end{array}$

$\begin{array}{llll}\text { C } & -3.98665 & 0.461686 & 0.144969\end{array}$

$\begin{array}{llll}\text { C } & -2.59241 & 2.638225 & 0.140122\end{array}$ 


\begin{tabular}{|c|c|c|c|c|c|c|c|}
\hline C & -1.1111 & 2.292738 & -0.55583 & $\mathrm{C}$ & -1.29227 & 1.885217 & -0.16576 \\
\hline $\mathrm{C}$ & -3.63045 & -0.45214 & -0.8131 & $\mathrm{C}$ & -3.87569 & -0.57604 & -0.69577 \\
\hline $\mathrm{C}$ & -3.87386 & -1.93587 & -0.73797 & $\mathrm{C}$ & -4.02739 & -2.05189 & -0.43257 \\
\hline $\mathrm{C}$ & -2.69215 & -2.82029 & -1.18536 & $\mathrm{C}$ & -2.89927 & -2.91094 & -1.05335 \\
\hline $\mathrm{C}$ & 0.287651 & 2.576395 & 0.064542 & $\mathrm{C}$ & 0.042694 & 2.55395 & 0.326334 \\
\hline $\mathrm{C}$ & 0.537519 & 1.752665 & 1.354094 & $\mathrm{C}$ & 1.100248 & 1.467818 & 0.645128 \\
\hline $\mathrm{C}$ & -1.52272 & -2.97627 & -0.22023 & $\mathrm{C}$ & -1.60996 & -3.01703 & -0.24879 \\
\hline $\mathrm{C}$ & -0.21473 & -2.35383 & -0.53613 & $\mathrm{C}$ & -0.5519 & -2.00333 & -0.42907 \\
\hline $\mathrm{C}$ & 0.30155 & 0.228875 & 1.302984 & $\mathrm{C}$ & 1.44801 & 0.470832 & -0.4791 \\
\hline $\mathrm{C}$ & 0.926258 & -2.09016 & 0.428038 & $\mathrm{C}$ & 0.505076 & -1.67563 & 0.603888 \\
\hline $\mathrm{C}$ & 1.351707 & -0.60449 & 0.541021 & $\mathrm{C}$ & 1.748715 & -0.97825 & 0.005756 \\
\hline $\mathrm{C}$ & 2.746251 & -0.54853 & 1.152043 & $\mathrm{C}$ & 2.946357 & -1.0088 & 0.941169 \\
\hline $\mathrm{C}$ & 3.938623 & -0.78835 & 0.267329 & $\mathrm{C}$ & 4.324693 & -0.83459 & 0.365357 \\
\hline $\mathrm{O}$ & 5.092881 & -0.79919 & 0.640798 & $\mathrm{O}$ & 5.342793 & -0.7279 & 1.01632 \\
\hline $\mathrm{C}$ & 3.010013 & -0.34606 & 2.446379 & $\mathrm{C}$ & 2.88262 & -1.17192 & 2.266443 \\
\hline $\mathrm{C}$ & -4.27992 & 0.182094 & 1.542343 & $\mathrm{C}$ & -4.2795 & 0.339454 & 1.620398 \\
\hline $\mathrm{C}$ & -1.87821 & -3.39725 & 1.188606 & $\mathrm{C}$ & -1.7084 & -3.8608 & 1.004528 \\
\hline $\mathrm{O}$ & -1.29637 & 3.0158 & -1.78101 & $\mathrm{O}$ & -1.21537 & 1.620939 & -1.57261 \\
\hline $\mathrm{O}$ & -0.45301 & -3.74713 & -0.82795 & $\mathrm{O}$ & -0.44746 & -3.29265 & -1.07927 \\
\hline $\mathrm{H}$ & -0.18435 & -1.73437 & -1.43377 & $\mathrm{H}$ & -0.78543 & -1.19914 & -1.12423 \\
\hline $\mathrm{C}$ & 0.456401 & 4.071331 & 0.373272 & $\mathrm{C}$ & -0.16025 & 3.415004 & 1.580307 \\
\hline $\mathrm{O}$ & 3.590431 & -0.99486 & -1.01618 & $\mathrm{O}$ & 4.319731 & -0.82352 & -0.98014 \\
\hline $\mathrm{C}$ & 4.670509 & -1.24012 & -1.94006 & $\mathrm{C}$ & 5.602418 & -0.67383 & -1.62264 \\
\hline $\mathrm{O}$ & 1.191928 & 2.152486 & -0.9837 & $\mathrm{O}$ & 0.42191 & 3.392536 & -0.79202 \\
\hline $\mathrm{C}$ & 2.546394 & 2.571404 & -0.90671 & $\mathrm{C}$ & 1.678918 & 4.0569 & -0.74629 \\
\hline $\mathrm{H}$ & -4.4585 & 2.508804 & 0.261105 & $\mathrm{H}$ & -4.72362 & 2.461372 & -0.10887 \\
\hline $\mathrm{H}$ & -3.64914 & 2.120119 & -1.24889 & $\mathrm{H}$ & -3.78934 & 1.851515 & -1.47155 \\
\hline $\mathrm{H}$ & -2.42085 & 3.623149 & 0.506592 & $\mathrm{H}$ & -2.56336 & 3.628753 & -0.32654 \\
\hline $\mathrm{H}$ & -2.10451 & 2.139847 & 1.376505 & $\mathrm{H}$ & -2.68332 & 2.796308 & 1.217667 \\
\hline $\mathrm{H}$ & -1.10584 & 1.246787 & -0.86918 & $\mathrm{H}$ & -1.35764 & 0.90881 & 0.32196 \\
\hline $\mathrm{H}$ & -3.31532 & -0.10216 & -1.79626 & $\mathrm{H}$ & -3.67324 & -0.33937 & -1.73997 \\
\hline $\mathrm{H}$ & -4.70724 & -2.16735 & -1.41444 & $\mathrm{H}$ & -4.96851 & -2.38216 & -0.89178 \\
\hline $\mathrm{H}$ & -4.21105 & -2.2384 & 0.255012 & $\mathrm{H}$ & -4.1264 & -2.26724 & 0.633528 \\
\hline $\mathrm{H}$ & -3.07433 & -3.83355 & -1.36027 & $\mathrm{H}$ & -3.27257 & -3.93046 & -1.20411 \\
\hline $\mathrm{H}$ & -2.30785 & -2.46584 & -2.14703 & $\mathrm{H}$ & -2.6508 & -2.52119 & -2.04502 \\
\hline $\mathrm{H}$ & -0.09163 & 2.171694 & 2.144494 & $\mathrm{H}$ & 0.707721 & 0.932124 & 1.514621 \\
\hline $\mathrm{H}$ & 1.563816 & 1.949298 & 1.6776 & $\mathrm{H}$ & 2.010304 & 1.95626 & 1.005276 \\
\hline $\mathrm{H}$ & -0.67731 & 0.015965 & 0.864537 & $\mathrm{H}$ & 2.313861 & 0.832545 & -1.03851 \\
\hline $\mathrm{H}$ & 0.232934 & -0.13846 & 2.333006 & $\mathrm{H}$ & 0.630509 & 0.420748 & -1.19598 \\
\hline $\mathrm{H}$ & 0.704167 & -2.48184 & 1.424334 & $\mathrm{H}$ & 0.051917 & -1.05717 & 1.386215 \\
\hline $\mathrm{H}$ & 1.774955 & -2.66961 & 0.050492 & $\mathrm{H}$ & 0.820524 & -2.6031 & 1.08388 \\
\hline $\mathrm{H}$ & 1.425121 & -0.2027 & -0.46999 & $\mathrm{H}$ & 2.032567 & -1.54373 & -0.88691 \\
\hline $\mathrm{H}$ & 4.032136 & -0.35918 & 2.803404 & $\mathrm{H}$ & 3.78775 & -1.16174 & 2.860732 \\
\hline $\mathrm{H}$ & 2.229212 & -0.16408 & 3.17336 & $\mathrm{H}$ & 1.945593 & -1.3211 & 2.786605 \\
\hline $\mathrm{H}$ & -4.31674 & -0.88227 & 1.771852 & $\mathrm{H}$ & -4.39492 & -0.6936 & 1.948058 \\
\hline $\mathrm{H}$ & -3.62131 & 0.658316 & 2.276256 & $\mathrm{H}$ & -3.48441 & 0.793001 & 2.222119 \\
\hline $\mathrm{H}$ & -5.28091 & 0.59695 & 1.710423 & $\mathrm{H}$ & -5.20177 & 0.876422 & 1.871805 \\
\hline $\mathrm{H}$ & -2.34203 & -2.57744 & 1.742793 & $\mathrm{H}$ & -2.43346 & -3.43635 & 1.704095 \\
\hline $\mathrm{H}$ & -0.99515 & -3.72487 & 1.735632 & $\mathrm{H}$ & -0.75263 & -3.95062 & 1.518539 \\
\hline
\end{tabular}




$\begin{array}{lrrrlrrr}\mathrm{H} & -2.58757 & -4.22996 & 1.162151 & \mathrm{H} & -2.04902 & -4.86723 & 0.742806 \\ \mathrm{H} & -1.48623 & 3.934051 & -1.5586 & \mathrm{H} & -0.65011 & 2.327301 & -1.92184 \\ \mathrm{H} & 0.396422 & 4.666569 & -0.54088 & \mathrm{H} & -0.79981 & 4.273291 & 1.369491 \\ \mathrm{H} & -0.31966 & 4.411487 & 1.061679 & \mathrm{H} & -0.61733 & 2.830136 & 2.381905 \\ \mathrm{H} & 1.41992 & 4.274729 & 0.844185 & \mathrm{H} & 0.797458 & 3.783952 & 1.954547 \\ \mathrm{H} & 4.196839 & -1.38583 & -2.90736 & \mathrm{H} & 5.394472 & -0.67883 & -2.68943 \\ \mathrm{H} & 5.345476 & -0.38438 & -1.96899 & \mathrm{H} & 6.06739 & 0.26693 & -1.3268 \\ \mathrm{H} & 5.225106 & -2.13176 & -1.64647 & \mathrm{H} & 6.258302 & -1.50316 & -1.3564 \\ \mathrm{H} & 3.0749 & 2.034543 & -1.69529 & \mathrm{H} & 1.740281 & 4.652876 & -1.65706 \\ \mathrm{H} & 2.657511 & 3.646708 & -1.08394 & \mathrm{H} & 1.757793 & 4.726611 & 0.1159 \\ \mathrm{H} & 3.014674 & 2.323199 & 0.053093 & \mathrm{H} & 2.516721 & 3.352263 & -0.73016\end{array}$

3d_13

\begin{tabular}{|c|c|c|c|c|c|c|c|}
\hline $\mathrm{C}$ & 3.622512 & 1.9907 & 0.025604 & $\mathrm{C}$ & -3.58888 & 1.760486 & 0.96160 \\
\hline $\mathrm{C}$ & 3.837227 & 0.709574 & 0.816206 & $\mathrm{C}$ & -3.61853 & 0.247724 & 1.07265 \\
\hline $\mathrm{C}$ & 2.343498 & 2.124034 & -0.81489 & $\mathrm{C}$ & -2.18331 & 2.389093 & 1.10219 \\
\hline $\mathrm{C}$ & 1.09479 & 2.450193 & 0.008607 & $\mathrm{C}$ & -1.36902 & 2.290233 & -0.1849 \\
\hline $\mathrm{C}$ & 3.012662 & -0.34018 & 0.718477 & $\mathrm{C}$ & -3.76261 & -0.51612 & -0.019 \\
\hline $\mathrm{C}$ & 3.060558 & -1.66745 & 1.42421 & $\mathrm{C}$ & -3.91824 & -2.01065 & -0.1119 \\
\hline $\mathrm{C}$ & 3.016703 & -2.89239 & 0.480152 & $\mathrm{C}$ & -2.99147 & -2.70664 & -1.1302 \\
\hline $\mathrm{C}$ & -0.26156 & 2.291669 & -0.74233 & $\mathrm{C}$ & 0.142872 & 2.614457 & -0.050 \\
\hline $\mathrm{C}$ & -0.64212 & 0.811341 & -0.99891 & $\mathrm{C}$ & 0.904769 & 1.598497 & 0.8338 \\
\hline $\mathrm{C}$ & 1.879776 & -2.89675 & -0.5449 & $\mathrm{C}$ & -1.5407 & -2.93913 & -0.724 \\
\hline $\mathrm{C}$ & 0.480067 & -2.90459 & -0.05591 & $\mathrm{C}$ & -0.45323 & -2.16275 & -1.367 \\
\hline $\mathrm{C}$ & -0.88388 & -0.03428 & 0.260701 & $\mathrm{C}$ & 0.68651 & 0.089763 & 0.59922 \\
\hline $\mathrm{C}$ & -0.75276 & -2.40961 & -0.79328 & $\mathrm{C}$ & 0.967007 & -1.99737 & -0.8595 \\
\hline $\mathrm{C}$ & -1.58541 & -1.40519 & 0.029697 & $\mathrm{C}$ & 1.39656 & -0.5277 & -0.6276 \\
\hline $\mathrm{C}$ & -2.98926 & -1.21243 & -0.52054 & $\mathrm{C}$ & 2.915075 & -0.42469 & -0.5558 \\
\hline C & -4.06414 & -0.70266 & 0.399569 & $\mathrm{C}$ & 3.610272 & -0.99346 & 0.64287 \\
\hline $\mathrm{O}$ & -5.16181 & -0.32731 & 0.044526 & $\mathrm{O}$ & 3.098889 & -1.75679 & 1.43763 \\
\hline $\mathrm{C}$ & -3.36354 & -1.45641 & -1.78012 & $\mathrm{C}$ & 3.641204 & 0.079101 & -1.559 \\
\hline $\mathrm{C}$ & 5.063186 & 0.752182 & 1.695296 & $\mathrm{C}$ & -3.51697 & -0.28306 & 2.482288 \\
\hline $\mathrm{C}$ & 2.248579 & -2.41735 & -1.93172 & $\mathrm{C}$ & -1.32939 & -3.64403 & 0.59778 \\
\hline $\mathrm{O}$ & 1.239878 & 3.805543 & 0.452885 & $\mathrm{O}$ & -1.96856 & 3.180686 & -1.13586 \\
\hline $\mathrm{O}$ & 1.086696 & -4.11322 & -0.55541 & $\mathrm{O}$ & -0.77868 & -3.4911 & -1.8295 \\
\hline $\mathrm{H}$ & 0.356947 & -2.9248 & 1.028154 & $\mathrm{H}$ & -0.77022 & -1.38544 & -2.06527 \\
\hline $\mathrm{C}$ & -0.25792 & 3.075683 & -2.05895 & $\mathrm{C}$ & 0.355608 & 4.034092 & 0.49007 \\
\hline $\mathrm{O}$ & -3.6837 & -0.71757 & 1.6897 & $\mathrm{O}$ & 4.884781 & -0.57719 & 0.75896 \\
\hline C & -4.65939 & -0.26046 & 2.649357 & $\mathrm{C}$ & 5.638082 & -1.12765 & 1.858827 \\
\hline $\mathrm{O}$ & -1.18282 & 2.884594 & 0.214226 & $\mathrm{O}$ & 0.574371 & 2.512347 & $-1.4407 \mathrm{C}$ \\
\hline $\mathrm{C}$ & -2.51865 & 3.146132 & -0.20038 & $\mathrm{C}$ & 1.781861 & 3.160689 & -1.82319 \\
\hline $\mathrm{H}$ & 4.489979 & 2.117849 & -0.63571 & $\mathrm{H}$ & -4.23226 & 2.178142 & 1.744935 \\
\hline $\mathrm{H}$ & 3.671298 & 2.838934 & 0.719588 & $\mathrm{H}$ & -4.0111 & 2.075863 & 0.004631 \\
\hline $\mathrm{H}$ & 2.487237 & 2.943226 & -1.52456 & $\mathrm{H}$ & -2.28982 & 3.447496 & $1.3567 \mathrm{C}$ \\
\hline $\mathrm{H}$ & 2.188501 & 1.223591 & -1.41417 & $\mathrm{H}$ & -1.64418 & 1.918282 & 1.928342 \\
\hline $\mathrm{H}$ & 1.052307 & 1.800411 & 0.890614 & $\mathrm{H}$ & -1.44054 & 1.269805 & -0.5780 \\
\hline $\mathrm{H}$ & 2.15969 & -0.2359 & 0.058533 & $\mathrm{H}$ & -3.84456 & -0.00083 & $-0.9767 \varsigma$ \\
\hline F & 2.197236 & -1.73033 & 2.099648 & $\mathrm{H}$ & -4.94635 & -2.21406 & -0.43999 \\
\hline & 3.945156 & -1.75531 & 2.058139 & $\mathrm{H}$ & -3.82391 & -2.4868 & 0.86566 \\
\hline
\end{tabular}




\begin{tabular}{|c|c|c|c|c|c|c|c|}
\hline $\mathrm{H}$ & 3.970331 & -2.97394 & -0.05185 & $\mathrm{H}$ & -3.40495 & -3.70052 & -1.34105 \\
\hline $\mathrm{H}$ & 2.913083 & -3.79559 & 1.087944 & $\mathrm{H}$ & -3.00678 & -2.16005 & -2.0786 \\
\hline $\mathrm{H}$ & 0.13235 & 0.363795 & -1.62839 & $\mathrm{H}$ & 0.640728 & 1.817536 & 1.87300 \\
\hline $\mathrm{H}$ & -1.54828 & 0.808409 & -1.61284 & $\mathrm{H}$ & 1.9736 & 1.829225 & 0.7586 \\
\hline $\mathrm{H}$ & -1.49333 & 0.543235 & 0.95805 & $\mathrm{H}$ & -0.38122 & -0.12815 & 0.52201 \\
\hline $\mathrm{H}$ & 0.059243 & -0.22669 & 0.778329 & $\mathrm{H}$ & 1.031379 & -0.4401 & 1.49035 \\
\hline $\mathrm{H}$ & -0.48689 & -2.00056 & -1.77029 & $\mathrm{H}$ & 1.135128 & -2.57898 & 0.046 \\
\hline $\mathrm{H}$ & -1.37261 & -3.29147 & -0.98761 & $\mathrm{H}$ & 1.616354 & -2.42592 & -1.6315 \\
\hline $\mathrm{H}$ & -1.70534 & -1.84067 & 1.02662 & $\mathrm{H}$ & 1.101936 & 0.046506 & -1.5102 \\
\hline $\mathrm{H}$ & -4.38559 & -1.27786 & -2.09014 & $\mathrm{H}$ & 4.721661 & 0.11676 & -1.518 \\
\hline $\mathrm{H}$ & -2.676 & -1.83844 & -2.52336 & $\mathrm{H}$ & 3.162285 & 0.45392 & -2.456 \\
\hline $\mathrm{H}$ & 5.26714 & -0.1931 & 2.197418 & $\mathrm{H}$ & -3.44462 & -1.369 & 2.53207 \\
\hline $\mathrm{H}$ & 5.947887 & 1.020394 & 1.106715 & $\mathrm{H}$ & -2.64386 & 0.133339 & 2.99551 \\
\hline $\mathrm{H}$ & 4.955982 & 1.526167 & 2.464232 & $\mathrm{H}$ & -4.3934 & 0.02194 & 3.0664 \\
\hline $\mathrm{H}$ & 2.681086 & -1.4149 & -1.90361 & $\mathrm{H}$ & -1.55928 & -2.98597 & 1.43962 \\
\hline $\mathrm{H}$ & 1.393414 & -2.41419 & -2.60717 & $\mathrm{H}$ & -0.30021 & -3.98532 & 0.7005 \\
\hline $\mathrm{H}$ & 3.00493 & -3.08981 & -2.34842 & $\mathrm{H}$ & -1.98395 & -4.51847 & 0.6646 \\
\hline $\mathrm{H}$ & 0.374576 & 4.035941 & 0.8189 & $\mathrm{H}$ & -1.36579 & 3.170638 & -1.8918 \\
\hline $\mathrm{H}$ & 0.017645 & 4.116613 & -1.88044 & $\mathrm{H}$ & -0.14843 & 4.765595 & -0.14346 \\
\hline $\mathrm{H}$ & 0.462071 & 2.642836 & -2.75604 & $\mathrm{H}$ & -0.05278 & 4.117281 & 1.498603 \\
\hline $\mathrm{H}$ & -1.23571 & 3.047123 & -2.54298 & $\mathrm{H}$ & 1.416304 & 4.286234 & 0.546465 \\
\hline $\mathrm{H}$ & -4.16957 & -0.33217 & 3.616894 & $\mathrm{H}$ & 6.62272 & -0.67221 & 1.79123 \\
\hline $\mathrm{H}$ & -4.9441 & 0.770548 & 2.438491 & $\mathrm{H}$ & 5.164437 & -0.87543 & 2.808021 \\
\hline $\mathrm{H}$ & -5.54464 & -0.89628 & 2.620169 & $\mathrm{H}$ & 5.711426 & -2.2118 & 1.76605 \\
\hline $\mathrm{H}$ & -3.05716 & 3.459178 & 0.694713 & $\mathrm{H}$ & 1.983751 & 2.845606 & -2.84763 \\
\hline $\mathrm{H}$ & -2.57139 & 3.950835 & -0.94033 & $\mathrm{H}$ & 1.686695 & 4.250906 & -1.80595 \\
\hline $\mathrm{H}$ & -3.00905 & 2.256037 & -0.60832 & $\mathrm{H}$ & 2.628548 & 2.864876 & -1.19558 \\
\hline 3d_15 & & & & & & & \\
\hline $\mathrm{C}$ & -2.90066 & 2.222044 & -0.90669 & $\mathrm{C}$ & -3.77786 & 1.198574 & -0.74368 \\
\hline $\mathrm{C}$ & -3.86793 & 1.117699 & -0.53317 & $\mathrm{C}$ & -4.0159 & -0.2152 & -0.225 \\
\hline $\mathrm{C}$ & -1.79315 & 2.379713 & 0.169351 & $\mathrm{C}$ & -2.32782 & 1.690931 & -0.88726 \\
\hline $\mathrm{C}$ & -0.42968 & 2.740589 & -0.40844 & $\mathrm{C}$ & -1.68348 & 2.162826 & 0.424642 \\
\hline $\mathrm{C}$ & -3.7613 & -0.08229 & -1.11969 & $\mathrm{C}$ & -3.05316 & -1.14755 & -0.1706 \\
\hline $\mathrm{C}$ & -4.51389 & -1.34776 & -0.81062 & $\mathrm{C}$ & -3.17659 & -2.57799 & 0.29833 \\
\hline $\mathrm{C}$ & -3.61506 & -2.60047 & -0.77676 & $\mathrm{C}$ & -2.09939 & -3.51179 & -0.2833 \\
\hline $\mathrm{C}$ & 0.7802 & 2.397626 & 0.501049 & $\mathrm{C}$ & -0.2327 & 2.703861 & 0.253135 \\
\hline $\mathrm{C}$ & 0.890145 & 0.882016 & 0.794886 & $\mathrm{C}$ & 0.7054 & 1.766741 & -0.5399 \\
\hline $\mathrm{C}$ & -2.48932 & -2.62806 & 0.254626 & $\mathrm{C}$ & -0.65519 & -3.25994 & 0.143648 \\
\hline $\mathrm{C}$ & -1.07528 & -2.63518 & -0.19994 & $\mathrm{C}$ & 0.315948 & -2.71306 & -0.83837 \\
\hline $\mathrm{C}$ & 0.869272 & -0.03264 & -0.43812 & $\mathrm{C}$ & 0.918514 & 0.347436 & 0.004135 \\
\hline $\mathrm{C}$ & 0.158446 & -2.2898 & 0.626614 & $\mathrm{C}$ & 1.649483 & -2.05116 & -0.5447 \\
\hline $\mathrm{C}$ & 1.233341 & -1.52152 & -0.16932 & $\mathrm{C}$ & 1.709506 & -0.56107 & -0.96546 \\
\hline $\mathrm{C}$ & 2.624908 & -1.64214 & 0.426739 & $\mathrm{C}$ & 3.135393 & -0.09122 & -1.21941 \\
\hline $\mathrm{C}$ & 3.743843 & -1.40604 & -0.54233 & $\mathrm{C}$ & 4.176455 & -0.05855 & -0.13517 \\
\hline $\mathrm{O}$ & 3.597833 & -1.35975 & -1.74254 & $\mathrm{O}$ & 5.316932 & 0.326866 & -0.2837 \\
\hline $\mathrm{C}$ & 2.89878 & -1.95131 & 1.698116 & $\mathrm{C}$ & 3.539501 & 0.312398 & -2.4284 \\
\hline $\mathrm{C}$ & -4.84459 & 1.459117 & 0.564106 & $\mathrm{C}$ & -5.44606 & -0.47 & 0.18213 \\
\hline$C$ & -2.85896 & -2.21983 & 1.66259 & $\mathrm{C}$ & -0.41021 & -3.17474 & 1.63363 \\
\hline
\end{tabular}




\begin{tabular}{|c|c|c|c|c|c|c|c|}
\hline $\mathrm{O}$ & -0.43196 & 4.135572 & -0.73023 & $\mathrm{O}$ & -1.66169 & 1.161182 & 1.453947 \\
\hline $\mathrm{O}$ & -1.73731 & -3.86431 & 0.165939 & $\mathrm{O}$ & 0.258935 & -4.12926 & -0.57248 \\
\hline $\mathrm{H}$ & -0.92475 & -2.5512 & -1.27699 & $\mathrm{H}$ & -0.09582 & -2.45282 & -1.81539 \\
\hline $\mathrm{C}$ & 0.74242 & 3.191104 & 1.811228 & $\mathrm{C}$ & 0.37221 & 3.051711 & 1.617688 \\
\hline $\mathrm{O}$ & 4.940312 & -1.25399 & 0.049568 & $\mathrm{O}$ & 3.715592 & -0.51146 & 1.045034 \\
\hline $\mathrm{C}$ & 6.070091 & -1.07994 & -0.83044 & $\mathrm{C}$ & 4.657319 & -0.51687 & 2.138329 \\
\hline $\mathrm{O}$ & 1.881461 & 2.833336 & -0.34385 & $\mathrm{O}$ & -0.30116 & 3.867724 & -0.61221 \\
\hline $\mathrm{C}$ & 3.188839 & 2.879958 & 0.213975 & $\mathrm{C}$ & -0.75505 & 5.100652 & -0.06744 \\
\hline $\mathrm{H}$ & -3.42735 & 3.172165 & -1.04599 & $\mathrm{H}$ & -4.33017 & 1.898165 & -0.10144 \\
\hline $\mathrm{H}$ & -2.43293 & 1.975412 & -1.86416 & $\mathrm{H}$ & -4.27272 & 1.270105 & -1.72104 \\
\hline $\mathrm{H}$ & -2.07416 & 3.133194 & 0.910103 & $\mathrm{H}$ & -1.71772 & 0.926449 & -1.37285 \\
\hline $\mathrm{H}$ & -1.70606 & 1.430715 & 0.702087 & $\mathrm{H}$ & -2.32158 & 2.552423 & -1.55941 \\
\hline $\mathrm{H}$ & -0.27871 & 2.173774 & -1.33539 & $\mathrm{H}$ & -2.29045 & 3.003191 & 0.792822 \\
\hline $\mathrm{H}$ & -3.02399 & -0.18056 & -1.91668 & $\mathrm{H}$ & -2.0564 & -0.86726 & -0.49218 \\
\hline $\mathrm{H}$ & -5.27192 & -1.52514 & -1.58519 & $\mathrm{H}$ & -4.15438 & -2.98798 & 0.031073 \\
\hline $\mathrm{H}$ & -5.06224 & -1.26211 & 0.129854 & $\mathrm{H}$ & -3.13264 & -2.61884 & 1.393818 \\
\hline $\mathrm{H}$ & -4.2478 & -3.47158 & -0.56824 & $\mathrm{H}$ & -2.33588 & -4.53856 & 0.02012 \\
\hline $\mathrm{H}$ & -3.17941 & -2.76561 & -1.76742 & $\mathrm{H}$ & -2.1455 & -3.49262 & -1.37677 \\
\hline $\mathrm{H}$ & 0.091206 & 0.610177 & 1.490208 & $\mathrm{H}$ & 1.66666 & 2.285193 & -0.61327 \\
\hline $\mathrm{H}$ & 1.818796 & 0.722714 & 1.350011 & $\mathrm{H}$ & 0.322999 & 1.709741 & -1.56381 \\
\hline $\mathrm{H}$ & 1.567793 & 0.360852 & -1.18026 & $\mathrm{H}$ & -0.04637 & -0.12111 & 0.19417 \\
\hline $\mathrm{H}$ & -0.11935 & -0.01658 & -0.90648 & $\mathrm{H}$ & 1.432304 & 0.379358 & 0.966844 \\
\hline $\mathrm{H}$ & -0.11457 & -1.75784 & 1.54138 & $\mathrm{H}$ & 1.921324 & -2.16176 & 0.504455 \\
\hline $\mathrm{H}$ & 0.582719 & -3.24711 & 0.944349 & $\mathrm{H}$ & 2.397001 & -2.60837 & -1.12176 \\
\hline $\mathrm{H}$ & 1.295882 & -1.98898 & -1.15792 & $\mathrm{H}$ & 1.214047 & -0.49321 & -1.94163 \\
\hline $\mathrm{H}$ & 3.919072 & -2.01641 & 2.051296 & $\mathrm{H}$ & 4.558489 & 0.640077 & -2.59246 \\
\hline $\mathrm{H}$ & 2.114214 & -2.15388 & 2.416693 & $\mathrm{H}$ & 2.862119 & 0.32243 & -3.27438 \\
\hline $\mathrm{H}$ & -5.50249 & 0.631853 & 0.829284 & $\mathrm{H}$ & -5.64396 & -1.51458 & 0.420907 \\
\hline $\mathrm{H}$ & -4.32039 & 1.779897 & 1.471452 & $\mathrm{H}$ & -5.70706 & 0.130885 & 1.060879 \\
\hline $\mathrm{H}$ & -5.47368 & 2.302616 & 0.256795 & $\mathrm{H}$ & -6.13082 & -0.16551 & -0.61736 \\
\hline $\mathrm{H}$ & -3.09618 & -1.15416 & 1.711462 & $\mathrm{H}$ & -0.84519 & -2.26464 & 2.05465 \\
\hline $\mathrm{H}$ & -2.0467 & -2.4265 & 2.35942 & $\mathrm{H}$ & 0.655134 & -3.18259 & 1.860449 \\
\hline $\mathrm{H}$ & -3.73975 & -2.77796 & 1.9948 & $\mathrm{H}$ & -0.86965 & -4.03113 & 2.136879 \\
\hline $\mathrm{H}$ & 0.471092 & 4.326847 & -1.01705 & $\mathrm{H}$ & -2.42495 & 0.583069 & 1.331103 \\
\hline $\mathrm{H}$ & 0.731668 & 4.262944 & 1.607574 & $\mathrm{H}$ & -0.27775 & 3.713476 & 2.194802 \\
\hline $\mathrm{H}$ & -0.15677 & 2.941557 & 2.378236 & $\mathrm{H}$ & 0.51898 & 2.146255 & 2.204745 \\
\hline $\mathrm{H}$ & 1.602469 & 2.959876 & 2.442928 & $\mathrm{H}$ & 1.339404 & 3.543255 & 1.482888 \\
\hline $\mathrm{H}$ & 6.93167 & -0.97233 & -0.17657 & $\mathrm{H}$ & 4.105551 & -0.8971 & 2.994056 \\
\hline $\mathrm{H}$ & 6.186993 & -1.95083 & -1.47627 & $\mathrm{H}$ & 5.501615 & -1.16689 & 1.907403 \\
\hline $\mathrm{H}$ & 5.940659 & -0.18709 & -1.44263 & $\mathrm{H}$ & 5.018292 & 0.493313 & 2.332592 \\
\hline $\mathrm{H}$ & 3.855216 & 3.164768 & -0.60097 & $\mathrm{H}$ & -0.8221 & 5.792811 & -0.90809 \\
\hline $\mathrm{H}$ & 3.273374 & 3.625148 & 1.01145 & $\mathrm{H}$ & -1.74515 & 5.025402 & 0.39609 \\
\hline $\mathrm{H}$ & 3.510834 & 1.907149 & 0.601151 & $\mathrm{H}$ & -0.05445 & 5.512872 & 0.666469 \\
\hline 3d_17 & & & & $3 \mathbf{e}$ & & & \\
\hline $\mathrm{C}$ & -2.25062 & 2.897033 & -0.13807 & $\mathrm{C}$ & 3.563927 & 1.61575 & 0.710416 \\
\hline $\mathrm{C}$ & -3.27268 & 1.873271 & 0.313134 & $\mathrm{C}$ & 3.963934 & 0.211851 & 0.272871 \\
\hline $\mathrm{C}$ & -0.87262 & 2.673894 & 0.534883 & $\mathrm{C}$ & 2.077992 & 1.958068 & 0.885194 \\
\hline $\mathrm{C}$ & 0.311637 & 3.122619 & -0.32452 & $\mathrm{C}$ & 1.389899 & 2.342361 & -0.43579 \\
\hline
\end{tabular}




\begin{tabular}{|c|c|c|c|c|c|c|c|}
\hline $\mathrm{C}$ & -3.61999 & 0.876776 & -0.5132 & $\mathrm{C}$ & 3.172984 & -0.86345 & 0.401085 \\
\hline $\mathrm{C}$ & -4.55388 & -0.27596 & -0.26016 & $\mathrm{C}$ & 3.534459 & -2.30752 & 0.153701 \\
\hline $\mathrm{C}$ & -4.05896 & -1.6164 & -0.83962 & $\mathrm{C}$ & 2.571071 & -3.10708 & -0.74932 \\
\hline $\mathrm{C}$ & 1.632858 & 2.320832 & -0.12226 & $\mathrm{C}$ & -0.13028 & 2.660788 & -0.30741 \\
\hline $\mathrm{C}$ & 1.60739 & 0.943047 & -0.82876 & $\mathrm{C}$ & -0.93142 & 1.539652 & 0.385471 \\
\hline $\mathrm{C}$ & -2.80102 & -2.22992 & -0.23169 & $\mathrm{C}$ & 1.154098 & -3.32531 & -0.23171 \\
\hline $\mathrm{C}$ & -1.56339 & -2.3353 & -1.04663 & $\mathrm{C}$ & 0.030623 & -2.62693 & -0.89522 \\
\hline $\mathrm{C}$ & 0.68002 & -0.16229 & -0.29654 & $\mathrm{C}$ & -0.92556 & 0.197916 & -0.36206 \\
\hline $\mathrm{C}$ & -0.15124 & -2.57808 & -0.54048 & $\mathrm{C}$ & -1.32517 & -2.33968 & -0.28644 \\
\hline $\mathrm{C}$ & 0.887739 & -1.52532 & -1.00011 & $\mathrm{C}$ & -1.38629 & -1.00297 & 0.496847 \\
\hline $\mathrm{C}$ & 2.30809 & -2.06866 & -0.90576 & $\mathrm{C}$ & -2.73933 & -0.81246 & 1.169561 \\
\hline $\mathrm{C}$ & 2.940289 & -2.45137 & 0.403639 & $\mathrm{C}$ & -4.01172 & -0.62944 & 0.389981 \\
\hline $\mathrm{O}$ & 4.057304 & -2.90803 & 0.523658 & $\mathrm{O}$ & -5.11335 & -0.51504 & 0.883809 \\
\hline $\mathrm{C}$ & 3.0648 & -2.24915 & -1.99326 & $\mathrm{C}$ & -2.8648 & -0.81008 & 2.500805 \\
\hline $\mathrm{C}$ & -3.78968 & 2.047641 & 1.719155 & $\mathrm{C}$ & 5.387261 & 0.162191 & -0.23251 \\
\hline $\mathrm{C}$ & -2.76528 & -2.30921 & 1.277511 & $\mathrm{C}$ & 1.034097 & -3.87331 & 1.173891 \\
\hline $\mathrm{O}$ & 0.529932 & 4.510566 & -0.05756 & $\mathrm{O}$ & 2.048052 & 3.477334 & -1.01486 \\
\hline $\mathrm{O}$ & -2.45254 & -3.4633 & -0.9123 & $\mathrm{O}$ & 0.322277 & -4.004 & -1.21268 \\
\hline $\mathrm{H}$ & -1.60747 & -1.8809 & -2.03814 & $\mathrm{H}$ & 0.312491 & -1.9542 & -1.70334 \\
\hline $\mathrm{C}$ & 2.033113 & 2.244032 & 1.352404 & $\mathrm{C}$ & -0.70598 & 2.979131 & -1.69538 \\
\hline $\mathrm{O}$ & 2.131744 & -2.23477 & 1.457156 & $\mathrm{O}$ & -3.80935 & -0.59912 & -0.93994 \\
\hline $\mathrm{C}$ & 2.664331 & -2.582 & 2.752929 & $\mathrm{C}$ & -4.98351 & -0.42304 & -1.76078 \\
\hline $\mathrm{O}$ & 2.569652 & 3.150276 & -0.87602 & $\mathrm{O}$ & -0.11931 & 3.842504 & 0.53714 \\
\hline $\mathrm{C}$ & 3.966717 & 2.967283 & -0.67863 & $\mathrm{C}$ & -1.31734 & 4.599086 & 0.662932 \\
\hline $\mathrm{H}$ & -2.60556 & 3.913571 & 0.067161 & $\mathrm{H}$ & 3.994191 & 2.33616 & 0.00692 \\
\hline $\mathrm{H}$ & -2.13148 & 2.816931 & -1.22233 & $\mathrm{H}$ & 4.083425 & 1.805659 & 1.659834 \\
\hline $\mathrm{H}$ & -0.81347 & 3.206818 & 1.487348 & $\mathrm{H}$ & 1.549954 & 1.135197 & 1.370853 \\
\hline $\mathrm{H}$ & -0.77998 & 1.615213 & 0.768573 & $\mathrm{H}$ & 1.989697 & 2.812854 & 1.561962 \\
\hline $\mathrm{H}$ & 0.055055 & 3.001721 & -1.38746 & $\mathrm{H}$ & 1.518272 & 1.537459 & -1.16304 \\
\hline $\mathrm{H}$ & -3.18573 & 0.889261 & -1.51308 & $\mathrm{H}$ & 2.169706 & -0.71075 & 0.790931 \\
\hline $\mathrm{H}$ & -5.52008 & -0.0678 & -0.73924 & $\mathrm{H}$ & 3.601553 & -2.81872 & 1.123127 \\
\hline $\mathrm{H}$ & -4.76922 & -0.38793 & 0.804777 & $\mathrm{H}$ & 4.529492 & -2.3864 & -0.2864 \\
\hline $\mathrm{H}$ & -4.85529 & -2.35901 & -0.70791 & $\mathrm{H}$ & 2.514456 & -2.63911 & -1.73681 \\
\hline $\mathrm{H}$ & -3.90808 & -1.51502 & -1.91925 & $\mathrm{H}$ & 3.00507 & -4.10336 & -0.89985 \\
\hline $\mathrm{H}$ & 2.633235 & 0.563655 & -0.80744 & $\mathrm{H}$ & -1.96409 & 1.880301 & 0.504962 \\
\hline $\mathrm{H}$ & 1.37759 & 1.129367 & -1.884 & $\mathrm{H}$ & -0.53847 & 1.407382 & 1.397277 \\
\hline $\mathrm{H}$ & -0.36425 & 0.112877 & -0.45847 & $\mathrm{H}$ & 0.09064 & -0.02105 & -0.69624 \\
\hline $\mathrm{H}$ & 0.813535 & -0.2961 & 0.778777 & $\mathrm{H}$ & -1.54314 & 0.263136 & -1.2594 \\
\hline $\mathrm{H}$ & -0.13127 & -2.67157 & 0.54515 & $\mathrm{H}$ & -1.60473 & -3.15728 & 0.381228 \\
\hline $\mathrm{H}$ & 0.147946 & -3.55257 & -0.94318 & $\mathrm{H}$ & -2.0617 & -2.32459 & -1.0944 \\
\hline $\mathrm{H}$ & 0.719008 & -1.35992 & -2.07112 & $\mathrm{H}$ & -0.66993 & -1.08932 & 1.32144 \\
\hline $\mathrm{H}$ & 4.067968 & -2.6487 & -1.9115 & $\mathrm{H}$ & -3.83407 & -0.68357 & 2.966781 \\
\hline $\mathrm{H}$ & 2.697371 & -2.00216 & -2.98236 & $\mathrm{H}$ & -2.00428 & -0.9333 & 3.147856 \\
\hline $\mathrm{H}$ & -4.48119 & 1.262428 & 2.02408 & $\mathrm{H}$ & 5.734908 & -0.84119 & -0.47323 \\
\hline $\mathrm{H}$ & -2.96595 & 2.072517 & 2.441012 & $\mathrm{H}$ & 5.491932 & 0.781816 & -1.13039 \\
\hline $\mathrm{H}$ & -4.31068 & 3.007883 & 1.811632 & $\mathrm{H}$ & 6.070574 & 0.583972 & 0.513982 \\
\hline $\mathrm{H}$ & -2.66166 & -1.31488 & 1.719423 & $\mathrm{H}$ & 0.006211 & -4.14907 & 1.405855 \\
\hline $\mathrm{H}$ & -1.93841 & -2.92916 & 1.622062 & $\mathrm{H}$ & 1.656185 & -4.76762 & 1.278701 \\
\hline $\mathrm{H}$ & -3.69547 & -2.74898 & 1.650371 & $\mathrm{H}$ & 1.370162 & -3.14402 & 1.91535 \\
\hline
\end{tabular}




$\begin{array}{lrrrlrrr}\mathrm{H} & 1.381429 & 4.705586 & -0.47513 & \mathrm{H} & 1.735269 & 4.232795 & -0.49719 \\ \mathrm{H} & 2.137856 & 3.250412 & 1.762978 & \mathrm{H} & -0.2168 & 3.856033 & -2.11998 \\ \mathrm{H} & 1.27376 & 1.719773 & 1.933811 & \mathrm{H} & -0.53888 & 2.140042 & -2.37396 \\ \mathrm{H} & 2.975386 & 1.709258 & 1.487205 & \mathrm{H} & -1.78234 & 3.158701 & -1.65081 \\ \mathrm{H} & 1.878524 & -2.33901 & 3.463311 & \mathrm{H} & -4.62351 & -0.42936 & -2.78624 \\ \mathrm{H} & 2.901326 & -3.64538 & 2.792 & \mathrm{H} & -5.68645 & -1.23981 & -1.59595 \\ \mathrm{H} & 3.56284 & -2.0008 & 2.960951 & \mathrm{H} & -5.46757 & 0.526161 & -1.53012 \\ \mathrm{H} & 4.4605 & 3.583544 & -1.43081 & \mathrm{H} & -1.10218 & 5.388003 & 1.384473 \\ \mathrm{H} & 4.290989 & 3.297208 & 0.313581 & \mathrm{H} & -1.61636 & 5.061985 & -0.28303 \\ \mathrm{H} & 4.276355 & 1.927288 & -0.82606 & \mathrm{H} & -2.15009 & 3.998502 & 1.044282\end{array}$

\begin{tabular}{|c|c|c|c|c|c|c|c|}
\hline 3e_2 & & & & 3e $\_3$ & & & \\
\hline $\mathrm{C}$ & 3.065066 & 2.328864 & 0.359075 & $\mathrm{C}$ & -3.81414 & 0.728658 & 1.096433 \\
\hline $\mathrm{C}$ & 3.499849 & 0.895567 & 0.604203 & $\mathrm{C}$ & -3.17888 & 2.07338 & 0.771146 \\
\hline $\mathrm{C}$ & 1.561569 & 2.578906 & 0.655079 & $\mathrm{C}$ & -2.95673 & -0.54354 & 1.123226 \\
\hline $\mathrm{C}$ & 0.731787 & 2.701796 & -0.6321 & $\mathrm{C}$ & -2.74937 & -1.17157 & -0.25848 \\
\hline $\mathrm{C}$ & 3.67323 & 0.036166 & -0.41013 & $\mathrm{C}$ & -1.857 & 2.301068 & 0.753079 \\
\hline $\mathrm{C}$ & 4.182175 & -1.38138 & -0.36005 & $\mathrm{C}$ & -1.16351 & 3.634518 & 0.609716 \\
\hline $\mathrm{C}$ & 3.331203 & -2.42473 & -1.11473 & $\mathrm{C}$ & -0.23969 & 3.81234 & -0.61611 \\
\hline $\mathrm{C}$ & -0.80856 & 2.640812 & -0.42867 & $\mathrm{C}$ & -1.76293 & -2.3788 & -0.26945 \\
\hline $\mathrm{C}$ & -1.26193 & 1.357158 & 0.299673 & $\mathrm{C}$ & -0.31021 & -1.93379 & 0.02703 \\
\hline $\mathrm{C}$ & 2.036788 & -2.89395 & -0.45948 & $\mathrm{C}$ & 1.054313 & 3.008634 & -0.64784 \\
\hline $\mathrm{C}$ & 0.734451 & -2.46817 & -1.02038 & $\mathrm{C}$ & 1.185141 & 1.886494 & -1.60295 \\
\hline $\mathrm{C}$ & -0.92517 & 0.04857 & -0.43536 & $\mathrm{C}$ & 0.328812 & -0.98736 & -0.99968 \\
\hline $\mathrm{C}$ & -0.60065 & -2.49812 & -0.3041 & $\mathrm{C}$ & 2.159715 & 0.733251 & -1.48291 \\
\hline $\mathrm{C}$ & -0.95979 & -1.20459 & 0.469716 & $\mathrm{C}$ & 1.685731 & -0.41273 & -0.54977 \\
\hline $\mathrm{C}$ & -2.25344 & -1.37652 & 1.256237 & $\mathrm{C}$ & 2.794829 & -1.45025 & -0.43328 \\
\hline $\mathrm{C}$ & -3.58596 & -1.57411 & 0.588161 & $\mathrm{C}$ & 3.916276 & -1.22105 & 0.540931 \\
\hline $\mathrm{O}$ & -4.63301 & -1.75022 & 1.174183 & $\mathrm{O}$ & 4.86203 & -1.96364 & 0.700168 \\
\hline $\mathrm{C}$ & -2.26537 & -1.37502 & 2.593328 & $\mathrm{C}$ & 2.881068 & -2.55781 & -1.1756 \\
\hline $\mathrm{C}$ & 3.72684 & 0.559291 & 2.05882 & $\mathrm{C}$ & -4.22557 & 3.142285 & 0.551625 \\
\hline $\mathrm{C}$ & 2.161548 & -3.40855 & 0.958065 & $\mathrm{C}$ & 1.938782 & 3.123222 & 0.574289 \\
\hline $\mathrm{O}$ & 1.077387 & 3.917891 & -1.30866 & $\mathrm{O}$ & -4.05426 & -1.56807 & -0.71654 \\
\hline $\mathrm{O}$ & 1.286989 & -3.75974 & -1.3556 & $\mathrm{O}$ & 1.751446 & 3.177101 & -1.91319 \\
\hline $\mathrm{H}$ & 0.787924 & -1.76835 & -1.85301 & $\mathrm{H}$ & 0.299072 & 1.674556 & -2.19892 \\
\hline $\mathrm{C}$ & -1.51672 & 2.786267 & -1.78374 & $\mathrm{C}$ & -1.85599 & -3.10689 & -1.61915 \\
\hline $\mathrm{O}$ & -3.50756 & -1.53305 & -0.75454 & $\mathrm{O}$ & 3.762919 & -0.08169 & 1.240565 \\
\hline $\mathrm{C}$ & -4.74598 & -1.71317 & -1.47349 & $\mathrm{C}$ & 4.793129 & 0.217605 & 2.205236 \\
\hline $\mathrm{O}$ & -1.04871 & 3.7975 & 0.414168 & $\mathrm{O}$ & -2.21748 & -3.22422 & 0.810566 \\
\hline $\mathrm{C}$ & -2.39026 & 4.222869 & 0.617615 & $\mathrm{C}$ & -1.70766 & -4.54707 & 0.891811 \\
\hline $\mathrm{H}$ & 3.277698 & 2.621039 & -0.67238 & $\mathrm{H}$ & -4.64569 & 0.56003 & 0.403687 \\
\hline $\mathrm{H}$ & 3.670876 & 2.985234 & 0.993636 & $\mathrm{H}$ & -4.29084 & 0.84611 & 2.079596 \\
\hline $\mathrm{H}$ & 1.180467 & 1.770624 & 1.282064 & $\mathrm{H}$ & -1.9958 & -0.35513 & 1.605733 \\
\hline $\mathrm{H}$ & 1.423226 & 3.500768 & 1.22573 & $\mathrm{H}$ & -3.46085 & -1.29387 & 1.735967 \\
\hline $\mathrm{H}$ & 1.017499 & 1.907269 & -1.32485 & $\mathrm{H}$ & -2.3557 & -0.41574 & -0.94564 \\
\hline $\mathrm{H}$ & 3.49397 & 0.412098 & -1.41766 & $\mathrm{H}$ & -1.19133 & 1.458342 & 0.924023 \\
\hline $\mathrm{H}$ & 4.349277 & -1.71046 & 0.667107 & $\mathrm{H}$ & -0.57471 & 3.814831 & 1.517797 \\
\hline $\mathrm{H}$ & 5.17223 & -1.39162 & -0.83513 & $\mathrm{H}$ & -1.89574 & 4.442244 & 0.575449 \\
\hline $\mathrm{H}$ & 3.098977 & -2.05548 & -2.11869 & $\mathrm{H}$ & -0.80038 & 3.608946 & -1.53338 \\
\hline
\end{tabular}




\begin{tabular}{|c|c|c|c|c|c|c|c|}
\hline $\mathrm{H}$ & 3.943624 & -3.32449 & -1.25113 & $\mathrm{H}$ & 0.054657 & 4.868596 & -0.65793 \\
\hline $\mathrm{H}$ & -2.34356 & 1.412943 & 0.454089 & $\mathrm{H}$ & 0.304213 & -2.83323 & 0.115147 \\
\hline $\mathrm{H}$ & -0.81672 & 1.349776 & 1.297995 & $\mathrm{H}$ & -0.29248 & -1.45902 & 1.012536 \\
\hline $\mathrm{H}$ & 0.085302 & 0.110417 & -0.8437 & $\mathrm{H}$ & -0.33909 & -0.14097 & -1.16659 \\
\hline $\mathrm{H}$ & -1.59902 & -0.09231 & -1.282 & $\mathrm{H}$ & 0.431802 & -1.4798 & -1.97155 \\
\hline $\mathrm{H}$ & -0.61437 & -3.34062 & 0.390191 & $\mathrm{H}$ & 3.118545 & 1.116131 & -1.13005 \\
\hline $\mathrm{H}$ & -1.37139 & -2.69702 & -1.05392 & $\mathrm{H}$ & 2.334393 & 0.325251 & -2.48554 \\
\hline $\mathrm{H}$ & -0.18302 & -1.06077 & 1.22903 & $\mathrm{H}$ & 1.541589 & 0.022 & 0.443056 \\
\hline $\mathrm{H}$ & -3.19001 & -1.51059 & 3.14029 & $\mathrm{H}$ & 3.722277 & -3.22899 & -1.05684 \\
\hline $\mathrm{H}$ & -1.35431 & -1.23977 & 3.164342 & $\mathrm{H}$ & 2.129603 & -2.82351 & -1.90809 \\
\hline $\mathrm{H}$ & 3.904494 & -0.5005 & 2.239055 & $\mathrm{H}$ & -3.81347 & 4.13244 & 0.365071 \\
\hline $\mathrm{H}$ & 4.589113 & 1.115544 & 2.445505 & $\mathrm{H}$ & -4.86063 & 2.877059 & -0.30114 \\
\hline $\mathrm{H}$ & 2.866971 & 0.862677 & 2.665713 & $\mathrm{H}$ & -4.88977 & 3.211153 & 1.421102 \\
\hline $\mathrm{H}$ & 1.245441 & -3.89696 & 1.287437 & $\mathrm{H}$ & 2.887107 & 2.607216 & 0.433961 \\
\hline $\mathrm{H}$ & 2.975087 & -4.13784 & 1.020908 & $\mathrm{H}$ & 2.148456 & 4.177271 & 0.782094 \\
\hline $\mathrm{H}$ & 2.385382 & -2.59537 & 1.652897 & $\mathrm{H}$ & 1.449885 & 2.700311 & 1.455312 \\
\hline $\mathrm{H}$ & 0.655709 & 4.618705 & -0.79267 & $\mathrm{H}$ & -4.05578 & -1.55506 & -1.67808 \\
\hline $\mathrm{H}$ & -1.27962 & 3.748306 & -2.23829 & $\mathrm{H}$ & -2.83008 & -3.58725 & -1.72165 \\
\hline $\mathrm{H}$ & -1.18741 & 2.001037 & -2.46788 & $\mathrm{H}$ & -1.73198 & -2.40497 & -2.44763 \\
\hline $\mathrm{H}$ & -2.60035 & 2.699502 & -1.67866 & $\mathrm{H}$ & -1.08098 & -3.86987 & -1.71769 \\
\hline $\mathrm{H}$ & -4.48149 & -1.65028 & -2.52578 & $\mathrm{H}$ & 4.500482 & 1.160478 & 2.659658 \\
\hline $\mathrm{H}$ & -5.17827 & -2.68741 & -1.24442 & $\mathrm{H}$ & 4.847005 & -0.56974 & 2.957472 \\
\hline $\mathrm{H}$ & -5.45487 & -0.92851 & -1.20859 & $\mathrm{H}$ & 5.759478 & 0.314303 & 1.709727 \\
\hline $\mathrm{H}$ & -2.34247 & 5.041183 & 1.337017 & $\mathrm{H}$ & -2.10414 & -4.96747 & 1.817143 \\
\hline $\mathrm{H}$ & -2.85189 & 4.594115 & -0.3033 & $\mathrm{H}$ & -2.04005 & -5.17356 & 0.056637 \\
\hline $\mathrm{H}$ & -3.01942 & 3.4301 & 1.036225 & $\mathrm{H}$ & -0.61313 & -4.57862 & 0.941192 \\
\hline 3e_4 & & & & $3 \mathbf{e}$ & & & \\
\hline $\mathrm{C}$ & -3.81382 & 0.728485 & 1.097234 & $\mathrm{C}$ & -3.61294 & -0.04515 & -1.4523 \\
\hline $\mathrm{C}$ & -3.17885 & 2.073265 & 0.771463 & $\mathrm{C}$ & -3.41791 & -1.49684 & -1.03353 \\
\hline $\mathrm{C}$ & -2.95625 & -0.54359 & 1.123646 & $\mathrm{C}$ & -2.48491 & 0.969278 & -1.21819 \\
\hline $\mathrm{C}$ & -2.74922 & -1.17142 & -0.2582 & $\mathrm{C}$ & -2.4965 & 1.570391 & 0.198143 \\
\hline $\mathrm{C}$ & -1.857 & 2.30112 & 0.753018 & $\mathrm{C}$ & -2.22034 & -2.05404 & -0.80282 \\
\hline $\mathrm{C}$ & -1.16372 & 3.634642 & 0.609273 & $\mathrm{C}$ & -1.9161 & -3.50952 & -0.54764 \\
\hline $\mathrm{C}$ & -0.23981 & 3.812298 & -0.6165 & $\mathrm{C}$ & -1.06066 & -3.8153 & 0.699923 \\
\hline $\mathrm{C}$ & -1.76284 & -2.37872 & -0.26959 & $\mathrm{C}$ & -1.34786 & 2.585591 & 0.48072 \\
\hline $\mathrm{C}$ & -0.31005 & -1.93389 & 0.026836 & $\mathrm{C}$ & 0.056177 & 2.013282 & 0.194485 \\
\hline $\mathrm{C}$ & 1.054239 & 3.008626 & -0.64808 & $\mathrm{C}$ & 0.374078 & -3.3009 & 0.69911 \\
\hline $\mathrm{C}$ & 1.185142 & 1.886433 & -1.60312 & $\mathrm{C}$ & 0.731776 & -2.17764 & 1.593434 \\
\hline $\mathrm{C}$ & 0.328963 & -0.9874 & -0.99981 & $\mathrm{C}$ & 0.466717 & 0.83877 & 1.098428 \\
\hline $\mathrm{C}$ & 2.159796 & 0.733267 & -1.48299 & $\mathrm{C}$ & 1.915154 & -1.24901 & 1.428325 \\
\hline $\mathrm{C}$ & 1.685817 & -0.41267 & -0.54983 & $\mathrm{C}$ & 1.637484 & 0.000799 & 0.55134 \\
\hline $\mathrm{C}$ & 2.79493 & -1.45017 & -0.43317 & $\mathrm{C}$ & 2.935371 & 0.776477 & 0.377168 \\
\hline $\mathrm{C}$ & 3.916097 & -1.22111 & 0.541361 & $\mathrm{C}$ & 3.800218 & 0.323427 & -0.75903 \\
\hline $\mathrm{O}$ & 4.861464 & -1.96407 & 0.701231 & $\mathrm{O}$ & 3.504426 & -0.58098 & -1.51435 \\
\hline $\mathrm{C}$ & 2.881359 & -2.55758 & -1.17568 & $\mathrm{C}$ & 3.341259 & 1.761796 & 1.183212 \\
\hline $\mathrm{C}$ & -4.2258 & 3.141923 & 0.551963 & $\mathrm{C}$ & -4.72596 & -2.2534 & -1.00208 \\
\hline $\mathrm{C}$ & 1.938675 & 3.12342 & 0.574034 & $\mathrm{C}$ & 1.210263 & -3.65833 & -0.51024 \\
\hline$\Omega$ & -4.05421 & -1.5677 & -0.71602 & $\mathrm{O}$ & -3.75176 & 2.221405 & 0.43841 \\
\hline
\end{tabular}




\begin{tabular}{|c|c|c|c|c|c|c|c|}
\hline $\mathrm{O}$ & 1.751391 & 3.177076 & -1.91343 & $\mathrm{O}$ & 1.028087 & -3.53754 & 1.975688 \\
\hline $\mathrm{H}$ & 0.299089 & 1.674407 & -2.19909 & $\mathrm{H}$ & -0.09229 & -1.76264 & 2.170995 \\
\hline $\mathrm{C}$ & -1.85607 & -3.10644 & -1.6195 & $\mathrm{C}$ & -1.45596 & 3.098896 & 1.924125 \\
\hline $\mathrm{O}$ & 3.762984 & -0.08144 & 1.240554 & $\mathrm{O}$ & 4.945825 & 1.016563 & -0.87047 \\
\hline $\mathrm{C}$ & 4.792946 & 0.217716 & 2.205511 & $\mathrm{C}$ & 5.824509 & 0.628744 & -1.94615 \\
\hline $\mathrm{O}$ & -2.21721 & -3.22437 & 0.81029 & $\mathrm{O}$ & -1.64113 & 3.642027 & -0.47241 \\
\hline $\mathrm{C}$ & -1.70801 & -4.54751 & 0.890673 & $\mathrm{C}$ & -0.98618 & 4.895692 & -0.32177 \\
\hline $\mathrm{H}$ & -4.64571 & 0.559736 & 0.404941 & $\mathrm{H}$ & -4.51771 & 0.339179 & -0.96963 \\
\hline $\mathrm{H}$ & -4.28997 & 0.845985 & 2.080653 & $\mathrm{H}$ & -3.85314 & -0.06232 & -2.52433 \\
\hline $\mathrm{H}$ & -1.99519 & -0.3551 & 1.605868 & $\mathrm{H}$ & -1.51513 & 0.519829 & -1.44013 \\
\hline $\mathrm{H}$ & -3.46009 & -1.29404 & 1.736478 & $\mathrm{H}$ & -2.60167 & 1.796499 & -1.9238 \\
\hline $\mathrm{H}$ & -2.35562 & -0.41547 & -0.94529 & $\mathrm{H}$ & -2.44673 & 0.768948 & 0.938656 \\
\hline $\mathrm{H}$ & -1.19118 & 1.458511 & 0.923958 & $\mathrm{H}$ & -1.34274 & -1.41555 & -0.86578 \\
\hline $\mathrm{H}$ & -0.57505 & 3.815356 & 1.517354 & $\mathrm{H}$ & -1.39982 & -3.91482 & -1.42778 \\
\hline $\mathrm{H}$ & -1.8961 & 4.442218 & 0.574686 & $\mathrm{H}$ & -2.83707 & -4.08738 & -0.45879 \\
\hline $\mathrm{H}$ & -0.8004 & 3.608795 & -1.5338 & $\mathrm{H}$ & -1.56467 & -3.44019 & 1.59585 \\
\hline $\mathrm{H}$ & 0.054515 & 4.868556 & -0.65841 & $\mathrm{H}$ & -1.00167 & -4.9056 & 0.806794 \\
\hline $\mathrm{H}$ & 0.304252 & -2.83342 & 0.11483 & $\mathrm{H}$ & 0.782565 & 2.822648 & 0.300001 \\
\hline $\mathrm{H}$ & -0.29223 & -1.45925 & 1.012398 & $\mathrm{H}$ & 0.097289 & 1.704537 & -0.85326 \\
\hline $\mathrm{H}$ & -0.33899 & -0.14106 & -1.16675 & $\mathrm{H}$ & -0.38493 & 0.165677 & 1.214136 \\
\hline $\mathrm{H}$ & 0.432061 & -1.47982 & -1.97168 & $\mathrm{H}$ & 0.698181 & 1.197133 & 2.106676 \\
\hline $\mathrm{H}$ & 3.118583 & 1.116227 & -1.13011 & $\mathrm{H}$ & 2.753576 & -1.80631 & 1.007362 \\
\hline $\mathrm{H}$ & 2.334558 & 0.325227 & -2.48559 & $\mathrm{H}$ & 2.228837 & -0.91825 & 2.425799 \\
\hline $\mathrm{H}$ & 1.541558 & 0.022118 & 0.442962 & $\mathrm{H}$ & 1.352422 & -0.35923 & -0.44161 \\
\hline $\mathrm{H}$ & 3.722511 & -3.22882 & -1.05684 & $\mathrm{H}$ & 4.291838 & 2.255568 & 1.034201 \\
\hline $\mathrm{H}$ & 2.130091 & -2.8231 & -1.90844 & $\mathrm{H}$ & 2.734551 & 2.101389 & 2.014504 \\
\hline $\mathrm{H}$ & -3.81396 & 4.132287 & 0.365939 & $\mathrm{H}$ & -4.61757 & -3.31332 & -0.7773 \\
\hline $\mathrm{H}$ & -4.86045 & 2.876808 & -0.30114 & $\mathrm{H}$ & -5.39799 & -1.81601 & -0.25503 \\
\hline $\mathrm{H}$ & -4.89034 & 3.210238 & 1.421218 & $\mathrm{H}$ & -5.23919 & -2.16586 & -1.96701 \\
\hline $\mathrm{H}$ & 2.886657 & 2.606645 & 0.434224 & $\mathrm{H}$ & 2.253413 & -3.37806 & -0.37213 \\
\hline $\mathrm{H}$ & 2.149042 & 4.177478 & 0.781089 & $\mathrm{H}$ & 1.167681 & -4.7379 & -0.68524 \\
\hline $\mathrm{H}$ & 1.449373 & 2.701477 & 1.455295 & $\mathrm{H}$ & 0.839905 & -3.15863 & -1.40877 \\
\hline $\mathrm{H}$ & -4.05559 & -1.55577 & -1.67757 & $\mathrm{H}$ & -3.69544 & 3.053723 & -0.05206 \\
\hline $\mathrm{H}$ & -2.83014 & -3.58684 & -1.72205 & $\mathrm{H}$ & -2.40131 & 3.620489 & 2.074552 \\
\hline $\mathrm{H}$ & -1.73215 & -2.40426 & -2.44777 & $\mathrm{H}$ & -1.419 & 2.263635 & 2.626504 \\
\hline $\mathrm{H}$ & -1.08107 & -3.86938 & -1.71834 & $\mathrm{H}$ & -0.63443 & 3.775528 & 2.169695 \\
\hline $\mathrm{H}$ & 4.500422 & 1.160741 & 2.659695 & $\mathrm{H}$ & 6.675771 & 1.301469 & -1.88077 \\
\hline $\mathrm{H}$ & 4.846375 & -0.56953 & 2.957887 & $\mathrm{H}$ & 6.145087 & -0.40608 & -1.82118 \\
\hline $\mathrm{H}$ & 5.759489 & 0.314073 & 1.710309 & $\mathrm{H}$ & 5.321689 & 0.740446 & -2.90725 \\
\hline $\mathrm{H}$ & -2.10379 & -4.96796 & 1.816283 & $\mathrm{H}$ & -1.27526 & 5.493723 & -1.18672 \\
\hline $\mathrm{H}$ & -2.04157 & -5.17358 & 0.055649 & $\mathrm{H}$ & -1.29931 & 5.420192 & 0.586901 \\
\hline $\mathrm{H}$ & -0.61345 & -4.57965 & 0.938944 & $\mathrm{H}$ & 0.10453 & 4.797547 & -0.31719 \\
\hline
\end{tabular}

\begin{tabular}{lllllllll} 
3e_6 & & \multicolumn{7}{c}{ 3e_7 } \\
C & 3.064239 & 2.329597 & 0.357509 & C & 3.769403 & 1.611005 & 0.579142 \\
C & 3.499763 & 0.896707 & 0.603491 & C & 4.060213 & 0.142537 & 0.300854 \\
C & 1.560726 & 2.579076 & 0.654233 & C & 2.328634 & 2.081283 & 0.818159 \\
C & 0.730452 & 2.702577 & -0.63254 & C & 1.530173 & 2.288879 & -0.47209 \\
C & 3.673438 & 0.036802 & -0.41037 & C & 3.193417 & -0.85573 & 0.528391
\end{tabular}




\begin{tabular}{|c|c|c|c|c|c|c|c|}
\hline $\mathrm{C}$ & 4.182809 & -1.38057 & -0.35949 & $\mathrm{C}$ & 3.466846 & -2.33796 & 0.442699 \\
\hline $\mathrm{C}$ & 3.33233 & -2.42435 & -1.11409 & $\mathrm{C}$ & 2.585117 & -3.15664 & -0.52636 \\
\hline $\mathrm{C}$ & -0.8098 & 2.640943 & -0.42853 & $\mathrm{C}$ & 0.029964 & 2.651888 & -0.24759 \\
\hline $\mathrm{C}$ & -1.26243 & 1.357027 & 0.299794 & $\mathrm{C}$ & -0.7567 & 1.475775 & 0.3791 \\
\hline $\mathrm{C}$ & 2.037881 & -2.89368 & -0.45899 & $\mathrm{C}$ & 1.114398 & -3.32743 & -0.16477 \\
\hline $\mathrm{C}$ & 0.735578 & -2.4678 & -1.01991 & $\mathrm{C}$ & 0.084162 & -2.64914 & -0.98167 \\
\hline $\mathrm{C}$ & -0.92492 & 0.04856 & -0.43515 & $\mathrm{C}$ & -0.8681 & 0.209687 & -0.48132 \\
\hline $\mathrm{C}$ & -0.59963 & -2.49804 & -0.30386 & $\mathrm{C}$ & -1.3231 & -2.31932 & -0.52954 \\
\hline $\mathrm{C}$ & -0.95933 & -1.20463 & 0.469951 & $\mathrm{C}$ & -1.41528 & -1.01031 & 0.29501 \\
\hline $\mathrm{C}$ & -2.2531 & -1.37701 & 1.256142 & $\mathrm{C}$ & -2.81702 & -0.80064 & 0.848666 \\
\hline $\mathrm{C}$ & -3.5853 & -1.5757 & 0.587763 & $\mathrm{C}$ & -3.92879 & -0.54328 & -0.12184 \\
\hline $\mathrm{O}$ & -4.63225 & -1.75324 & 1.173506 & $\mathrm{O}$ & -3.79869 & -0.53951 & -1.32994 \\
\hline $\mathrm{C}$ & -2.26543 & -1.37497 & 2.593235 & $\mathrm{C}$ & -3.07392 & -0.8614 & 2.159937 \\
\hline $\mathrm{C}$ & 3.727035 & 0.561403 & 2.058299 & $\mathrm{C}$ & 5.477702 & -0.05971 & -0.18524 \\
\hline $\mathrm{C}$ & 2.162542 & -3.4084 & 0.958519 & $\mathrm{C}$ & 0.825034 & -3.80741 & 1.240908 \\
\hline $\mathrm{O}$ & 1.075388 & 3.919204 & -1.30848 & $\mathrm{O}$ & 2.206022 & 3.330219 & -1.199 \\
\hline $\mathrm{O}$ & 1.288148 & -3.75928 & -1.35528 & $\mathrm{O}$ & 0.388497 & -4.04217 & -1.20315 \\
\hline $\mathrm{H}$ & 0.789137 & -1.7678 & -1.85239 & $\mathrm{H}$ & 0.463548 & -2.01622 & -1.78231 \\
\hline $\mathrm{C}$ & -1.51849 & 2.786396 & -1.78331 & $\mathrm{C}$ & -0.59551 & 3.087541 & -1.58166 \\
\hline $\mathrm{O}$ & -3.50667 & -1.53403 & -0.75493 & $\mathrm{O}$ & -5.11067 & -0.31025 & 0.476486 \\
\hline $\mathrm{C}$ & -4.74479 & -1.71543 & -1.47417 & $\mathrm{C}$ & -6.23139 & -0.06429 & -0.39689 \\
\hline $\mathrm{O}$ & -1.05002 & 3.797438 & 0.41457 & $\mathrm{O}$ & 0.06233 & 3.744455 & 0.696971 \\
\hline $\mathrm{C}$ & -2.39168 & 4.221987 & 0.619008 & $\mathrm{C}$ & -1.12533 & 4.495143 & 0.899953 \\
\hline $\mathrm{H}$ & 3.276084 & 2.62097 & -0.67433 & $\mathrm{H}$ & 4.186191 & 2.207694 & -0.23912 \\
\hline $\mathrm{H}$ & 3.670136 & 2.986806 & 0.991122 & $\mathrm{H}$ & 4.371391 & 1.881829 & 1.457822 \\
\hline $\mathrm{H}$ & 1.180126 & 1.770188 & 1.280725 & $\mathrm{H}$ & 1.8021 & 1.396114 & 1.485431 \\
\hline $\mathrm{H}$ & 1.422317 & 3.500444 & 1.225654 & $\mathrm{H}$ & 2.35664 & 3.044758 & 1.331976 \\
\hline $\mathrm{H}$ & 1.016137 & 1.908588 & -1.32591 & $\mathrm{H}$ & 1.563141 & 1.371484 & -1.06826 \\
\hline $\mathrm{H}$ & 3.493893 & 0.412111 & -1.41807 & $\mathrm{H}$ & 2.197849 & -0.59542 & 0.880034 \\
\hline $\mathrm{H}$ & 4.349595 & -1.70916 & 0.667872 & $\mathrm{H}$ & 3.364718 & -2.76528 & 1.44842 \\
\hline $\mathrm{H}$ & 5.173045 & -1.39074 & -0.83418 & $\mathrm{H}$ & 4.504205 & -2.51706 & 0.15738 \\
\hline $\mathrm{H}$ & 3.100215 & -2.05546 & -2.1182 & $\mathrm{H}$ & 2.651777 & -2.73498 & -1.53379 \\
\hline $\mathrm{H}$ & 3.944966 & -3.32403 & -1.2501 & $\mathrm{H}$ & 3.007093 & -4.16801 & -0.58197 \\
\hline $\mathrm{H}$ & -2.34411 & 1.412142 & 0.454136 & $\mathrm{H}$ & -1.763 & 1.833165 & 0.616403 \\
\hline $\mathrm{H}$ & -0.81729 & 1.349983 & 1.298143 & $\mathrm{H}$ & -0.29331 & 1.227314 & 1.338734 \\
\hline $\mathrm{H}$ & 0.085624 & 0.110756 & -0.84322 & $\mathrm{H}$ & 0.121137 & -0.06023 & -0.85501 \\
\hline $\mathrm{H}$ & -1.59851 & -0.09262 & -1.28194 & $\mathrm{H}$ & -1.49484 & 0.392659 & -1.3561 \\
\hline $\mathrm{H}$ & -0.61329 & -3.34056 & 0.390395 & $\mathrm{H}$ & -1.70917 & -3.14696 & 0.069577 \\
\hline $\mathrm{H}$ & -1.37021 & -2.69707 & -1.05382 & $\mathrm{H}$ & -1.9617 & -2.24126 & -1.41317 \\
\hline $\mathrm{H}$ & -0.18279 & -1.06061 & 1.229451 & $\mathrm{H}$ & -0.77158 & -1.14277 & 1.171666 \\
\hline $\mathrm{H}$ & -3.19017 & -1.51085 & 3.13995 & $\mathrm{H}$ & -4.073 & -0.72574 & 2.551605 \\
\hline $\mathrm{H}$ & -1.35463 & -1.23893 & 3.16446 & $\mathrm{H}$ & -2.27974 & -1.05342 & 2.872928 \\
\hline $\mathrm{H}$ & 3.904675 & -0.49827 & 2.239201 & $\mathrm{H}$ & 5.746995 & -1.10265 & -0.3432 \\
\hline $\mathrm{H}$ & 4.589384 & 1.117895 & 2.444449 & $\mathrm{H}$ & 5.630925 & 0.47371 & -1.13022 \\
\hline $\mathrm{H}$ & 2.86727 & 0.865186 & 2.665131 & $\mathrm{H}$ & 6.191244 & 0.368181 & 0.528773 \\
\hline $\mathrm{H}$ & 1.246561 & -3.89727 & 1.287545 & $\mathrm{H}$ & -0.23025 & -4.04352 & 1.370893 \\
\hline $\mathrm{H}$ & 2.97641 & -4.13731 & 1.02153 & $\mathrm{H}$ & 1.403156 & -4.71245 & 1.452209 \\
\hline $\mathrm{H}$ & 2.385789 & -2.5952 & 1.653523 & $\mathrm{H}$ & 1.102261 & -3.05388 & 1.982271 \\
\hline $\mathrm{H}$ & 0.65348 & 4.619528 & -0.79201 & $\mathrm{H}$ & 2.035472 & 3.20548 & -2.1371 \\
\hline
\end{tabular}




$\begin{array}{lrrrlrrr}\mathrm{H} & -1.28159 & 3.748443 & -2.23795 & \mathrm{H} & -0.16239 & 4.032872 & -1.91164 \\ \mathrm{H} & -1.18946 & 2.001134 & -2.46756 & \mathrm{H} & -0.41245 & 2.337057 & -2.35474 \\ \mathrm{H} & -2.60208 & 2.699561 & -1.67784 & \mathrm{H} & -1.67727 & 3.211912 & -1.49791 \\ \mathrm{H} & -4.47997 & -1.65318 & -2.52641 & \mathrm{H} & -7.08128 & 0.094587 & 0.26183 \\ \mathrm{H} & -5.17659 & -2.68972 & -1.24445 & \mathrm{H} & -6.05079 & 0.820893 & -1.00766 \\ \mathrm{H} & -5.45418 & -0.93093 & -1.21009 & \mathrm{H} & -6.40553 & -0.92374 & -1.0451 \\ \mathrm{H} & -2.34385 & 5.04048 & 1.338198 & \mathrm{H} & -0.90285 & 5.201885 & 1.700766 \\ \mathrm{H} & -2.8543 & 4.592744 & -0.30161 & \mathrm{H} & -1.41778 & 5.062368 & 0.009212 \\ \mathrm{H} & -3.01997 & 3.428882 & 1.038271 & \mathrm{H} & -1.97235 & 3.874826 & 1.215234\end{array}$

\begin{tabular}{|c|c|c|c|c|c|c|c|}
\hline \multicolumn{4}{|c|}{ 3e_8 } & \multicolumn{4}{|c|}{ 3e_9 } \\
\hline $\mathrm{C}$ & 3.56825 & 1.663995 & 0.812954 & $\mathrm{C}$ & 3.770053 & 1.610063 & 0.577905 \\
\hline C & 4.009977 & 0.269645 & 0.385598 & $\mathrm{C}$ & 4.059967 & 0.141322 & 0.300088 \\
\hline C & 2.070594 & 1.979848 & 0.923743 & $\mathrm{C}$ & 2.32968 & 2.081177 & 0.817695 \\
\hline C & 1.4318 & 2.351999 & -0.42518 & $\mathrm{C}$ & 1.530705 & 2.289026 & -0.47219 \\
\hline C & 3.233053 & -0.81961 & 0.476372 & $\mathrm{C}$ & 3.193074 & -0.85651 & 0.529141 \\
\hline C & 3.629914 & -2.25581 & 0.237905 & $\mathrm{C}$ & 3.465929 & -2.33889 & 0.444119 \\
\hline C & 2.718304 & -3.06786 & -0.70636 & $\mathrm{C}$ & 2.584454 & -3.15758 & -0.52518 \\
\hline C & -0.09762 & 2.643929 & -0.35775 & $\mathrm{C}$ & 0.030681 & 2.652302 & -0.24708 \\
\hline C & -0.9069 & 1.503647 & 0.293419 & $\mathrm{C}$ & -0.756 & 1.476155 & 0.379527 \\
\hline C & 1.284429 & -3.31071 & -0.24954 & $\mathrm{C}$ & 1.113518 & -3.32779 & -0.16428 \\
\hline C & 0.178044 & -2.63555 & -0.96387 & $\mathrm{C}$ & 0.083922 & -2.64898 & -0.98154 \\
\hline C & -0.84985 & 0.168727 & -0.46441 & $\mathrm{C}$ & -0.86768 & 0.210245 & -0.48114 \\
\hline C & -1.2102 & -2.37544 & -0.41969 & $\mathrm{C}$ & -1.32338 & -2.31862 & -0.5299 \\
\hline C & -1.34182 & -1.04234 & 0.361239 & $\mathrm{C}$ & -1.41536 & -1.00974 & 0.294862 \\
\hline C & -2.74738 & -0.87351 & 0.919488 & $\mathrm{C}$ & -2.81714 & -0.79982 & 0.848322 \\
\hline C & -3.87315 & -0.68153 & -0.04982 & $\mathrm{C}$ & -3.9288 & -0.5425 & -0.12234 \\
\hline $\mathrm{O}$ & -3.74461 & -0.67493 & -1.25807 & $\mathrm{O}$ & -3.7983 & -0.53767 & -1.3304 \\
\hline C & -2.99347 & -0.90882 & 2.233674 & $\mathrm{C}$ & -3.07416 & -0.86018 & 2.159591 \\
\hline C & 5.453543 & 0.246914 & -0.06086 & $\mathrm{C}$ & 5.476856 & -0.0617 & -0.18743 \\
\hline C & 1.113947 & -3.85733 & 1.15134 & $\mathrm{C}$ & 0.823333 & -3.80785 & 1.241203 \\
\hline C & 2.093896 & 3.498127 & -0.9776 & $\mathrm{O}$ & 2.20653 & 3.33025 & -1.1993 \\
\hline C & 0.509924 & -4.00763 & -1.26391 & $\mathrm{O}$ & 0.387722 & -4.04206 & -1.20306 \\
\hline $\mathrm{H}$ & 0.482079 & -1.95918 & -1.76095 & $\mathrm{H}$ & 0.463891 & -2.01607 & -1.78191 \\
\hline C & -0.62328 & 2.964791 & -1.76454 & $\mathrm{C}$ & -0.59523 & 3.0885 & -1.58077 \\
\hline $\mathrm{O}$ & -5.06524 & -0.51177 & 0.549306 & $\mathrm{O}$ & -5.11105 & -0.31085 & 0.475773 \\
\hline C & -6.19851 & -0.32897 & -0.32341 & $\mathrm{C}$ & -6.23161 & -0.06476 & -0.39779 \\
\hline $\mathrm{O}$ & -0.14054 & 3.818296 & 0.496244 & $\mathrm{O}$ & 0.063604 & 3.744629 & 0.697753 \\
\hline C & -1.35294 & 4.557882 & 0.576293 & $\mathrm{C}$ & -1.12392 & 4.495324 & 0.901556 \\
\hline $\mathrm{H}$ & 4.016864 & 2.395763 & 0.133051 & $\mathrm{H}$ & 4.1866 & 2.206194 & -0.24089 \\
\hline $\mathrm{H}$ & 4.042226 & 1.856248 & 1.785417 & $\mathrm{H}$ & 4.372822 & 1.880932 & 1.456039 \\
\hline $\mathrm{H}$ & 1.537236 & 1.14733 & 1.38666 & $\mathrm{H}$ & 1.803107 & 1.3964 & 1.485329 \\
\hline $\mathrm{H}$ & 1.937671 & 2.8326 & 1.595685 & $\mathrm{H}$ & 2.358484 & 3.044699 & 1.331369 \\
\hline $\mathrm{H}$ & 1.604255 & 1.549643 & -1.14604 & $\mathrm{H}$ & 1.563287 & 1.371648 & -1.06842 \\
\hline H & 2.211702 & -0.6868 & 0.824728 & $\mathrm{H}$ & 2.197945 & -0.59569 & 0.881628 \\
\hline $\mathrm{H}$ & 3.666822 & -2.77033 & 1.207188 & $\mathrm{H}$ & 3.362999 & -2.76576 & 1.449938 \\
\hline $\mathrm{H}$ & 4.643124 & -2.31518 & -0.16193 & $\mathrm{H}$ & 4.503392 & -2.5185 & 0.159505 \\
\hline $\mathrm{H}$ & 2.695551 & -2.59844 & -1.69456 & $\mathrm{H}$ & 2.651744 & -2.7362 & -1.53269 \\
\hline $\mathrm{H}$ & 3.174223 & -4.05666 & -0.84049 & $\mathrm{H}$ & 3.006158 & -4.16909 & -0.5803 \\
\hline
\end{tabular}




$\begin{array}{lrrrrrrr}\mathrm{H} & -1.9487 & 1.827437 & 0.375503 & \mathrm{H} & -1.76224 & 1.833564 & 0.617061 \\ \mathrm{H} & -0.55176 & 1.368056 & 1.318805 & \mathrm{H} & -0.29248 & 1.227439 & 1.33903 \\ \mathrm{H} & 0.183593 & -0.03624 & -0.75107 & \mathrm{H} & 0.121535 & -0.05991 & -0.85472 \\ \mathrm{H} & -1.42661 & 0.233363 & -1.38873 & \mathrm{H} & -1.49424 & 0.393586 & -1.35597 \\ \mathrm{H} & -1.50538 & -3.20028 & 0.232265 & \mathrm{H} & -1.71002 & -3.14621 & 0.068896 \\ \mathrm{H} & -1.9068 & -2.3718 & -1.26258 & \mathrm{H} & -1.9616 & -2.24014 & -1.41378 \\ \mathrm{H} & -0.6865 & -1.11721 & 1.236248 & \mathrm{H} & -0.77185 & -1.14255 & 1.171608 \\ \mathrm{H} & -3.9947 & -0.80106 & 2.628487 & \mathrm{H} & -4.07326 & -0.72433 & 2.551143 \\ \mathrm{H} & -2.18816 & -1.05023 & 2.946027 & \mathrm{H} & -2.28004 & -1.05203 & 2.872702 \\ \mathrm{H} & 5.829203 & -0.74948 & -0.28814 & \mathrm{H} & 5.745915 & -1.1049 & -0.34416 \\ \mathrm{H} & 5.583463 & 0.869986 & -0.95304 & \mathrm{H} & 5.628909 & 0.47027 & -1.13342 \\ \mathrm{H} & 6.097666 & 0.679849 & 0.713536 & \mathrm{H} & 6.191276 & 0.367257 & 0.525048 \\ \mathrm{H} & 0.082139 & -4.15098 & 1.340014 & \mathrm{H} & -0.23214 & -4.04335 & 1.370759 \\ \mathrm{H} & 1.746836 & -4.73999 & 1.28607 & \mathrm{H} & 1.40085 & -4.71326 & 1.452546 \\ \mathrm{H} & 1.404877 & -3.11991 & 1.903789 & \mathrm{H} & 1.100704 & -3.05462 & 1.982821 \\ \mathrm{H} & 1.75187 & 4.247873 & -0.47038 & \mathrm{H} & 2.03513 & 3.205922 & -2.1373 \\ \mathrm{H} & -0.13365 & 3.854081 & -2.16186 & \mathrm{H} & -0.162 & 4.033817 & -1.91066 \\ \mathrm{H} & -0.41495 & 2.13484 & -2.44288 & \mathrm{H} & -0.41274 & 2.3382 & -2.35416 \\ \mathrm{H} & -1.70353 & 3.125128 & -1.75981 & \mathrm{H} & -1.67691 & 3.213186 & -1.49649 \\ \mathrm{H} & -7.0551 & -0.21464 & 0.335857 & \mathrm{H} & -7.08188 & 0.092715 & 0.260779 \\ \mathrm{H} & -6.0669 & 0.562914 & -0.93693 & \mathrm{H} & -6.05143 & 0.821252 & -1.00747 \\ \mathrm{H} & -6.32631 & -1.19854 & -0.96892 & \mathrm{H} & -6.40482 & -0.92362 & -1.04702 \\ \mathrm{H} & -1.18064 & 5.341375 & 1.31509 & \mathrm{H} & -0.90115 & 5.201505 & 1.702784 \\ \mathrm{H} & -1.61575 & 5.027523 & -0.37711 & \mathrm{H} & -1.41653 & 5.063181 & 0.011264 \\ \mathrm{H} & -2.19396 & 3.942642 & 0.913587 & \mathrm{H} & -1.97093 & 3.874905 & 1.216638 \\ & & & & & & & \end{array}$

\begin{tabular}{lrrrlrrrr} 
3e_10 & \multicolumn{7}{c}{ 3e_11 } \\
C & 3.671077 & 1.489125 & 0.430336 & C & 3.307101 & 2.022343 & 0.567635 \\
C & 3.495603 & 0.01234 & 0.738982 & C & 3.566952 & 0.544718 & 0.797131 \\
C & 2.403689 & 2.358189 & 0.5926 & C & 1.822134 & 2.430894 & 0.758449 \\
C & 1.524845 & 2.343568 & -0.65922 & C & 1.09844 & 2.626054 & -0.58233 \\
C & 3.529063 & -0.90895 & -0.23572 & C & 3.731316 & -0.30271 & -0.2289 \\
C & 3.56287 & -2.41141 & -0.11015 & C & 4.093272 & -1.76523 & -0.18719 \\
C & 2.564582 & -3.1917 & -0.99405 & C & 3.206938 & -2.70141 & -1.03628 \\
C & 0.04591 & 2.764757 & -0.4147 & C & -0.45114 & 2.714396 & -0.48509 \\
C & -0.71192 & 1.629186 & 0.316967 & C & -1.07438 & 1.468347 & 0.181349 \\
C & 1.130587 & -3.31501 & -0.49357 & C & 1.828762 & -3.06211 & -0.49286 \\
C & 0.058891 & -2.52007 & -1.13183 & C & 0.619249 & -2.49638 & -1.1322 \\
C & -0.97671 & 0.364822 & -0.51754 & C & -0.84841 & 0.152431 & -0.58258 \\
C & -1.28146 & -2.18749 & -0.50873 & C & -0.76201 & -2.41394 & -0.51479 \\
C & -1.26569 & -0.88997 & 0.338824 & C & -1.0478 & -1.11464 & 0.279793 \\
C & -2.50581 & -0.76312 & 1.213552 & C & -2.40665 & -1.18341 & 0.962155 \\
C & -3.88777 & -0.61639 & 0.639695 & C & -3.62956 & -1.21554 & 0.097911 \\
O & -4.90045 & -0.50744 & 1.298088 & O & -3.61141 & -1.21163 & -1.11701 \\
C & -2.42807 & -0.78874 & 2.54829 & C & -2.5253 & -1.23147 & 2.293469 \\
C & 3.367282 & -0.3108 & 2.208845 & C & 3.643739 & 0.150032 & 2.252803 \\
C & 0.964021 & -3.90887 & 0.888851 & C & 1.796926 & -3.6268 & 0.910418 \\
O & 2.187025 & 3.19883 & -1.60757 & O & 1.60767 & 3.798387 & -1.23288 \\
O & 0.264341 & -3.89872 & -1.50669 & O & 1.070171 & -3.82598 & -1.47062
\end{tabular}




\begin{tabular}{|c|c|c|c|c|c|c|c|}
\hline $\mathrm{H}$ & 0.385383 & -1.83035 & -1.90864 & $\mathrm{H}$ & 0.803931 & -1.78245 & -1.93331 \\
\hline $\mathrm{C}$ & -0.62988 & 3.12199 & -1.74587 & $\mathrm{C}$ & -1.04818 & 2.95035 & -1.87999 \\
\hline $\mathrm{O}$ & -3.89797 & -0.6193 & -0.70576 & $\mathrm{O}$ & -4.77537 & -1.24912 & 0.801588 \\
\hline $\mathrm{C}$ & -5.19038 & -0.49009 & -1.33496 & $\mathrm{C}$ & -5.99494 & -1.29096 & 0.033335 \\
\hline $\mathrm{O}$ & 0.130874 & 3.917833 & 0.451736 & $\mathrm{O}$ & -0.63676 & 3.873933 & 0.367376 \\
\hline $\mathrm{C}$ & -1.04032 & 4.694679 & 0.653656 & $\mathrm{C}$ & -1.93928 & 4.434493 & 0.475471 \\
\hline $\mathrm{H}$ & 4.050751 & 1.617702 & -0.58687 & $\mathrm{H}$ & 3.630623 & 2.314694 & -0.43459 \\
\hline $\mathrm{H}$ & 4.444947 & 1.879705 & 1.102757 & $\mathrm{H}$ & 3.928327 & 2.591573 & 1.268134 \\
\hline $\mathrm{H}$ & 1.835248 & 2.041247 & 1.468355 & $\mathrm{H}$ & 1.315019 & 1.674887 & 1.360958 \\
\hline $\mathrm{H}$ & 2.684896 & 3.398518 & 0.771022 & $\mathrm{H}$ & 1.744488 & 3.368594 & 1.314614 \\
\hline $\mathrm{H}$ & 1.506668 & 1.326827 & -1.06481 & $\mathrm{H}$ & 1.353621 & 1.803271 & -1.25364 \\
\hline $\mathrm{H}$ & 3.646085 & -0.54689 & -1.25745 & $\mathrm{H}$ & 3.670962 & 0.114408 & -1.2344 \\
\hline $\mathrm{H}$ & 3.469461 & -2.72971 & 0.929625 & $\mathrm{H}$ & 4.149472 & -2.13455 & 0.838494 \\
\hline $\mathrm{H}$ & 4.565436 & -2.73264 & -0.42275 & $\mathrm{H}$ & 5.111344 & -1.86127 & -0.58774 \\
\hline $\mathrm{H}$ & 2.547449 & -2.76271 & -2.00091 & $\mathrm{H}$ & 3.085598 & -2.28421 & -2.04095 \\
\hline $\mathrm{H}$ & 2.937357 & -4.21749 & -1.10191 & $\mathrm{H}$ & 3.741662 & -3.65124 & -1.15976 \\
\hline $\mathrm{H}$ & -1.66417 & 2.027729 & 0.677216 & $\mathrm{H}$ & -2.14923 & 1.639444 & 0.29161 \\
\hline $\mathrm{H}$ & -0.14089 & 1.36232 & 1.211408 & $\mathrm{H}$ & -0.67829 & 1.383995 & 1.196686 \\
\hline $\mathrm{H}$ & -0.09968 & 0.141598 & -1.12784 & $\mathrm{H}$ & 0.175931 & 0.121425 & -0.95794 \\
\hline $\mathrm{H}$ & -1.80156 & 0.529969 & -1.21281 & $\mathrm{H}$ & -1.50401 & 0.104309 & -1.45393 \\
\hline $\mathrm{H}$ & -1.59829 & -3.02278 & 0.118968 & $\mathrm{H}$ & -0.90911 & -3.27216 & 0.144213 \\
\hline $\mathrm{H}$ & -2.02071 & -2.09776 & -1.3089 & $\mathrm{H}$ & -1.49288 & -2.5082 & -1.32291 \\
\hline $\mathrm{H}$ & -0.43152 & -0.98714 & 1.042853 & $\mathrm{H}$ & -0.31447 & -1.06397 & 1.092342 \\
\hline $\mathrm{H}$ & -3.31914 & -0.70508 & 3.157873 & $\mathrm{H}$ & -3.49053 & -1.29344 & 2.777698 \\
\hline $\mathrm{H}$ & -1.47619 & -0.8957 & 3.054928 & $\mathrm{H}$ & -1.6474 & -1.21263 & 2.929742 \\
\hline $\mathrm{H}$ & 3.151115 & -1.36083 & 2.402585 & $\mathrm{H}$ & 3.700728 & -0.9265 & 2.40964 \\
\hline $\mathrm{H}$ & 4.293034 & -0.05435 & 2.737767 & $\mathrm{H}$ & 4.523684 & 0.605486 & 2.722637 \\
\hline $\mathrm{H}$ & 2.572394 & 0.282613 & 2.671962 & $\mathrm{H}$ & 2.773043 & 0.523602 & 2.802349 \\
\hline $\mathrm{H}$ & -0.07888 & -4.14058 & 1.100123 & $\mathrm{H}$ & 0.812508 & -4.02059 & 1.159678 \\
\hline $\mathrm{H}$ & 1.539419 & -4.83642 & 0.967085 & $\mathrm{H}$ & 2.521111 & -4.44239 & 1.001227 \\
\hline $\mathrm{H}$ & 1.325026 & -3.22466 & 1.660366 & $\mathrm{H}$ & 2.057126 & -2.86355 & 1.647842 \\
\hline $\mathrm{H}$ & 1.911278 & 2.942991 & -2.49304 & $\mathrm{H}$ & 1.235801 & 4.541985 & -0.73927 \\
\hline $\mathrm{H}$ & -0.1826 & 4.024482 & -2.16507 & $\mathrm{H}$ & -0.69742 & 3.896535 & -2.29208 \\
\hline $\mathrm{H}$ & -0.51194 & 2.311841 & -2.46969 & $\mathrm{H}$ & -0.74256 & 2.153141 & -2.561 \\
\hline $\mathrm{H}$ & -1.70148 & 3.290641 & -1.61977 & $\mathrm{H}$ & -2.13993 & 2.959017 & -1.84947 \\
\hline $\mathrm{H}$ & -4.99497 & -0.51 & -2.4039 & $\mathrm{H}$ & -6.79719 & -1.31579 & 0.766294 \\
\hline $\mathrm{H}$ & -5.83563 & -1.32051 & -1.04749 & $\mathrm{H}$ & -6.07935 & -0.40428 & -0.5958 \\
\hline $\mathrm{H}$ & -5.6591 & 0.451353 & -1.04789 & $\mathrm{H}$ & -6.02061 & -2.18396 & -0.59204 \\
\hline $\mathrm{H}$ & -0.78413 & 5.43714 & 1.410836 & $\mathrm{H}$ & -1.86901 & 5.221022 & 1.227767 \\
\hline $\mathrm{H}$ & -1.35345 & 5.220355 & -0.25535 & $\mathrm{H}$ & -2.27812 & 4.880424 & -0.46559 \\
\hline $\mathrm{H}$ & -1.88469 & 4.102574 & 1.025256 & $\mathrm{H}$ & -2.68236 & 3.701909 & 0.808639 \\
\hline
\end{tabular}

$\begin{array}{lrrr}\text { 3e_12 } & & & \\ \mathrm{C} & 3.814098 & -0.72825 & 1.096709 \\ \mathrm{C} & 3.179147 & -2.07309 & 0.771263 \\ \mathrm{C} & 2.95643 & 0.543758 & 1.123406 \\ \mathrm{C} & 2.749201 & 1.171758 & -0.25834 \\ \mathrm{C} & 1.857312 & -2.30102 & 0.752954 \\ \mathrm{C} & 1.164045 & -3.63456 & 0.60945\end{array}$

$\begin{array}{lrrr}\text { 3e_13 } & & & \\ \text { C } & 3.814215 & -0.72826 & 1.096379 \\ \text { C } & 3.1792 & -2.07312 & 0.771092 \\ \text { C } & 2.956533 & 0.543739 & 1.123211 \\ \text { C } & 2.749222 & 1.171841 & -0.25847 \\ \text { C } & 1.85735 & -2.30097 & 0.752743 \\ \text { C } & 1.164036 & -3.63453 & 0.609475\end{array}$




\begin{tabular}{|c|c|c|c|c|c|c|c|}
\hline $\mathrm{C}$ & 0.240057 & -3.81237 & -0.61625 & $\mathrm{C}$ & 0.240016 & -3.81247 & -0.61617 \\
\hline $\mathrm{C}$ & 1.762657 & 2.378904 & -0.26951 & $\mathrm{C}$ & 1.762602 & 2.378991 & -0.26945 \\
\hline $\mathrm{C}$ & 0.309966 & 1.933786 & 0.027005 & $\mathrm{C}$ & 0.309913 & 1.933809 & 0.026982 \\
\hline $\mathrm{C}$ & -1.05398 & -3.00872 & -0.64786 & $\mathrm{C}$ & -1.05406 & -3.00885 & -0.64779 \\
\hline $\mathrm{C}$ & -1.18498 & -1.88662 & -1.603 & $\mathrm{C}$ & -1.185 & -1.88672 & -1.60289 \\
\hline $\mathrm{C}$ & -0.32893 & 0.987202 & -0.9996 & $\mathrm{C}$ & -0.32891 & 0.987181 & -0.99963 \\
\hline $\mathrm{C}$ & -2.15967 & -0.73348 & -1.48294 & $\mathrm{C}$ & -2.1597 & -0.73359 & -1.48287 \\
\hline $\mathrm{C}$ & -1.68584 & 0.412492 & -0.54973 & $\mathrm{C}$ & -1.68583 & 0.412483 & -0.54978 \\
\hline $\mathrm{C}$ & -2.79498 & 1.449957 & -0.43325 & $\mathrm{C}$ & -2.79501 & 1.449925 & -0.43335 \\
\hline $\mathrm{C}$ & -3.91632 & 1.220853 & 0.541087 & $\mathrm{C}$ & -3.91628 & 1.220908 & 0.541051 \\
\hline $\mathrm{O}$ & -4.8618 & 1.963714 & 0.700702 & $\mathrm{O}$ & -4.86179 & 1.963741 & 0.700602 \\
\hline $\mathrm{C}$ & -2.88131 & 2.557389 & -1.17575 & $\mathrm{C}$ & -2.88139 & 2.557258 & -1.176 \\
\hline $\mathrm{C}$ & 4.226136 & -3.14174 & 0.551831 & $\mathrm{C}$ & 4.226093 & -3.14194 & 0.552051 \\
\hline $\mathrm{C}$ & -1.93839 & -3.12337 & 0.574304 & $\mathrm{C}$ & -1.93844 & -3.12354 & 0.574371 \\
\hline $\mathrm{O}$ & 4.05413 & 1.568382 & -0.71621 & $\mathrm{O}$ & 4.054082 & 1.568468 & -0.71636 \\
\hline $\mathrm{O}$ & -1.75122 & -3.1773 & -1.91316 & $\mathrm{O}$ & -1.75122 & -3.17739 & -1.91311 \\
\hline $\mathrm{H}$ & -0.29896 & -1.67463 & -2.19903 & $\mathrm{H}$ & -0.29896 & -1.6747 & -2.19888 \\
\hline $\mathrm{C}$ & 1.855671 & 3.106779 & -1.61933 & $\mathrm{C}$ & 1.855658 & 3.107088 & -1.61915 \\
\hline $\mathrm{O}$ & -3.76323 & 0.081261 & 1.24041 & $\mathrm{O}$ & -3.76311 & 0.081398 & 1.240495 \\
\hline $\mathrm{C}$ & -4.79332 & -0.2179 & 2.205205 & $\mathrm{C}$ & -4.79318 & -0.21773 & 2.205339 \\
\hline $\mathrm{O}$ & 2.217033 & 3.224499 & 0.810406 & $\mathrm{O}$ & 2.216985 & 3.224459 & 0.810555 \\
\hline $\mathrm{C}$ & 1.707328 & 4.54742 & 0.891233 & $\mathrm{C}$ & 1.707288 & 4.547381 & 0.891498 \\
\hline $\mathrm{H}$ & 4.645704 & -0.55945 & 0.404074 & $\mathrm{H}$ & 4.645677 & -0.55947 & 0.403576 \\
\hline $\mathrm{H}$ & 4.290691 & -0.84565 & 2.079931 & $\mathrm{H}$ & 4.290997 & -0.84565 & 2.079512 \\
\hline $\mathrm{H}$ & 1.995432 & 0.355141 & 1.60572 & $\mathrm{H}$ & 1.995558 & 0.355077 & 1.605539 \\
\hline $\mathrm{H}$ & 3.460275 & 1.294174 & 1.736274 & $\mathrm{H}$ & 3.460381 & 1.294127 & 1.736108 \\
\hline $\mathrm{H}$ & 2.355724 & 0.41587 & -0.94554 & $\mathrm{H}$ & 2.355677 & 0.416013 & -0.94571 \\
\hline $\mathrm{H}$ & 1.191479 & -1.45841 & 0.923857 & $\mathrm{H}$ & 1.191551 & -1.45829 & 0.923394 \\
\hline $\mathrm{H}$ & 0.575419 & -3.81514 & 1.517592 & $\mathrm{H}$ & 0.575458 & -3.81496 & 1.517671 \\
\hline $\mathrm{H}$ & 1.896407 & -4.44215 & 0.574928 & $\mathrm{H}$ & 1.896391 & -4.44213 & 0.575067 \\
\hline $\mathrm{H}$ & 0.800618 & -3.60896 & -1.5336 & $\mathrm{H}$ & 0.800523 & -3.60912 & -1.53356 \\
\hline $\mathrm{H}$ & -0.05424 & -4.86864 & -0.65802 & $\mathrm{H}$ & -0.05427 & -4.86875 & -0.65786 \\
\hline $\mathrm{H}$ & -0.30449 & 2.833209 & 0.115051 & $\mathrm{H}$ & -0.30462 & 2.833184 & 0.114966 \\
\hline $\mathrm{H}$ & 0.292301 & 1.459138 & 1.01257 & $\mathrm{H}$ & 0.292229 & 1.459182 & 1.012554 \\
\hline $\mathrm{H}$ & 0.339034 & 0.140839 & -1.16636 & $\mathrm{H}$ & 0.339053 & 0.140807 & -1.16632 \\
\hline $\mathrm{H}$ & -0.43193 & 1.479508 & -1.97155 & $\mathrm{H}$ & -0.43185 & 1.479454 & -1.9716 \\
\hline $\mathrm{H}$ & -3.11849 & -1.11647 & -1.13017 & $\mathrm{H}$ & -3.11847 & -1.11654 & -1.12993 \\
\hline $\mathrm{H}$ & -2.33433 & -0.32545 & -2.48556 & $\mathrm{H}$ & -2.33448 & -0.32568 & -2.48552 \\
\hline $\mathrm{H}$ & -1.54168 & -0.02227 & 0.44309 & $\mathrm{H}$ & -1.54167 & -0.02222 & 0.443065 \\
\hline $\mathrm{H}$ & -3.7225 & 3.228604 & -1.05702 & $\mathrm{H}$ & -3.72257 & 3.228486 & -1.05736 \\
\hline $\mathrm{H}$ & -2.12993 & 2.822948 & -1.90837 & $\mathrm{H}$ & -2.13001 & 2.822718 & -1.90867 \\
\hline $\mathrm{H}$ & 3.814335 & -4.13212 & 0.365819 & $\mathrm{H}$ & 3.814157 & -4.13199 & 0.364587 \\
\hline $\mathrm{H}$ & 4.860809 & -2.87663 & -0.30126 & $\mathrm{H}$ & 4.861979 & -2.87628 & -0.29995 \\
\hline $\mathrm{H}$ & 4.890646 & -3.21001 & 1.421111 & $\mathrm{H}$ & 4.88945 & -3.21128 & 1.42214 \\
\hline $\mathrm{H}$ & -2.88634 & -2.60653 & 0.434483 & $\mathrm{H}$ & -2.88654 & -2.607 & 0.434432 \\
\hline $\mathrm{H}$ & -2.14881 & -4.1774 & 0.78144 & $\mathrm{H}$ & -2.1486 & -4.17759 & 0.781701 \\
\hline $\mathrm{H}$ & -1.44902 & -2.70139 & 1.455515 & $\mathrm{H}$ & -1.44921 & -2.70127 & 1.455513 \\
\hline $\mathrm{H}$ & 4.05566 & 1.555816 & -1.67774 & $\mathrm{H}$ & 4.05528 & 1.556986 & -1.67791 \\
\hline $\mathrm{H}$ & 2.829667 & 3.587325 & -1.72187 & $\mathrm{H}$ & 2.82966 & 3.587653 & -1.72157 \\
\hline
\end{tabular}




$\begin{array}{lrrrr}\mathrm{H} & 1.73181 & 2.404675 & -2.44767 & \mathrm{H} \\ \mathrm{H} & 1.080529 & 3.869603 & -1.71805 & \mathrm{H} \\ \mathrm{H} & -4.50063 & -1.1607 & 2.659759 & \mathrm{H} \\ \mathrm{H} & -4.84718 & 0.569555 & 2.957327 & \mathrm{H} \\ \mathrm{H} & -5.75972 & -0.31471 & 1.709806 & \mathrm{H} \\ \mathrm{H} & 2.103836 & 4.968062 & 1.816446 & \mathrm{H} \\ \mathrm{H} & 2.039792 & 5.173625 & 0.055875 & \mathrm{H} \\ \mathrm{H} & 0.612799 & 4.579061 & 0.94058 & \mathrm{H}\end{array}$

$\begin{array}{lrrr}\mathrm{H} & 1.731802 & 2.405149 & -2.44763 \\ \mathrm{H} & 1.080561 & 3.869971 & -1.71775 \\ \mathrm{H} & -4.50055 & -1.16057 & 2.659825 \\ \mathrm{H} & -4.84692 & 0.569701 & 2.957499 \\ \mathrm{H} & -5.75961 & -0.31442 & 1.70998 \\ \mathrm{H} & 2.10278 & 4.967539 & 1.817365 \\ \mathrm{H} & 2.040744 & 5.173964 & 0.056809 \\ \mathrm{H} & 0.612718 & 4.57911 & 0.939632\end{array}$

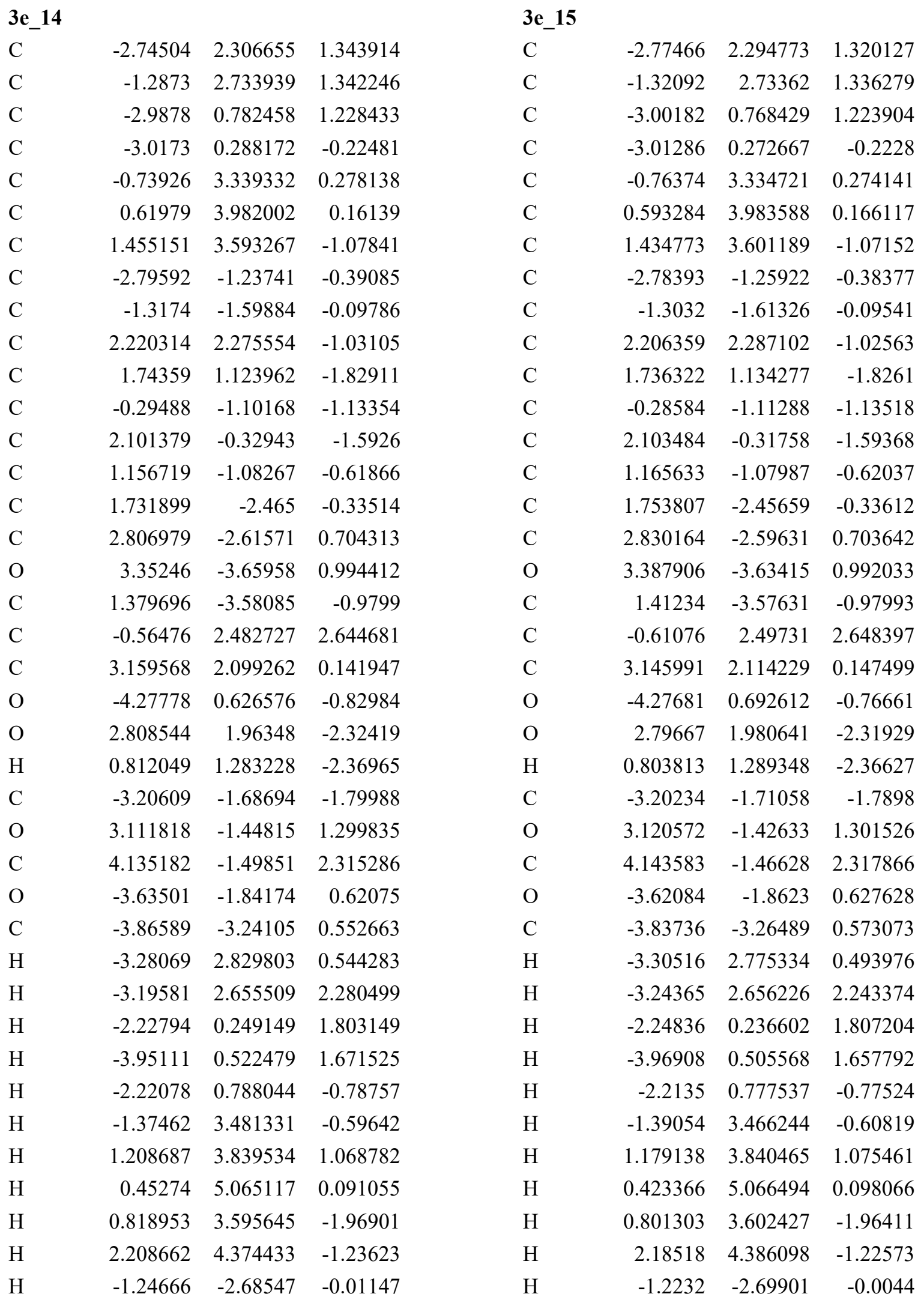




\begin{tabular}{|c|c|c|c|c|c|c|c|}
\hline $\mathrm{H}$ & -1.05405 & -1.19946 & 0.885912 & $\mathrm{H}$ & -1.04046 & -1.20724 & 0.885919 \\
\hline $\mathrm{H}$ & -0.54683 & -0.07957 & -1.4192 & $\mathrm{H}$ & -0.545 & -0.09425 & -1.4265 \\
\hline $\mathrm{H}$ & -0.36206 & -1.68794 & -2.05529 & $\mathrm{H}$ & -0.34863 & -1.70447 & -2.05391 \\
\hline $\mathrm{H}$ & 3.125644 & -0.38604 & -1.22195 & $\mathrm{H}$ & 3.12867 & -0.36802 & -1.22471 \\
\hline $\mathrm{H}$ & 2.091204 & -0.8429 & -2.56179 & $\mathrm{H}$ & 2.095712 & -0.8286 & -2.56419 \\
\hline $\mathrm{H}$ & 1.160208 & -0.53084 & 0.324928 & $\mathrm{H}$ & 1.163416 & -0.52799 & 0.323129 \\
\hline $\mathrm{H}$ & 1.860744 & -4.52173 & -0.74416 & $\mathrm{H}$ & 1.902445 & -4.51227 & -0.74325 \\
\hline $\mathrm{H}$ & 0.615404 & -3.58825 & -1.7463 & $\mathrm{H}$ & 0.648134 & -3.59197 & -1.74626 \\
\hline $\mathrm{H}$ & 0.505945 & 2.67543 & 2.588568 & $\mathrm{H}$ & 0.457635 & 2.706154 & 2.605795 \\
\hline $\mathrm{H}$ & -0.97951 & 3.115619 & 3.438377 & $\mathrm{H}$ & -1.04524 & 3.126136 & 3.434795 \\
\hline $\mathrm{H}$ & -0.69955 & 1.447215 & 2.972958 & $\mathrm{H}$ & -0.73415 & 1.460876 & 2.978538 \\
\hline $\mathrm{H}$ & 3.774981 & 1.208309 & 0.031222 & $\mathrm{H}$ & 3.762795 & 1.223972 & 0.038954 \\
\hline $\mathrm{H}$ & 3.822149 & 2.966934 & 0.219453 & $\mathrm{H}$ & 3.807121 & 2.983175 & 0.223247 \\
\hline $\mathrm{H}$ & 2.607066 & 2.013557 & 1.080463 & $\mathrm{H}$ & 2.593383 & 2.02985 & 1.086078 \\
\hline $\mathrm{H}$ & -4.3317 & 1.586319 & -0.87646 & $\mathrm{H}$ & -4.19061 & 0.759156 & -1.72244 \\
\hline $\mathrm{H}$ & -4.28306 & -1.58667 & -1.92933 & $\mathrm{H}$ & -4.28162 & -1.6087 & -1.91185 \\
\hline $\mathrm{H}$ & -2.71978 & -1.06629 & -2.55478 & $\mathrm{H}$ & -2.71112 & -1.10336 & -2.55413 \\
\hline $\mathrm{H}$ & -2.91838 & -2.72476 & -1.98293 & $\mathrm{H}$ & -2.92864 & -2.75133 & -1.97458 \\
\hline $\mathrm{H}$ & 4.244391 & -0.47643 & 2.668195 & $\mathrm{H}$ & 4.239099 & -0.44388 & 2.673752 \\
\hline $\mathrm{H}$ & 3.827541 & -2.15463 & 3.129914 & $\mathrm{H}$ & 3.843728 & -2.1287 & 3.130282 \\
\hline $\mathrm{H}$ & 5.072247 & -1.86008 & 1.891121 & $\mathrm{H}$ & 5.085657 & -1.81439 & 1.893551 \\
\hline $\mathrm{H}$ & -4.42676 & -3.49924 & 1.452102 & $\mathrm{H}$ & -4.39806 & -3.51972 & 1.473514 \\
\hline $\mathrm{H}$ & -4.46322 & -3.52236 & -0.32172 & $\mathrm{H}$ & -4.42968 & -3.56128 & -0.29982 \\
\hline $\mathrm{H}$ & -2.93823 & -3.82511 & 0.546678 & $\mathrm{H}$ & -2.90373 & -3.83918 & 0.575291 \\
\hline 3e_16 & & & & & & & \\
\hline $\mathrm{C}$ & -2.77414 & 2.295589 & 1.319611 & $\mathrm{C}$ & -3.2971 & 0.404624 & -1.25244 \\
\hline $\mathrm{C}$ & -1.32028 & 2.734036 & 1.336035 & $\mathrm{C}$ & -3.14507 & 1.806982 & -0.6981 \\
\hline $\mathrm{C}$ & -3.00167 & 0.769283 & 1.223639 & $\mathrm{C}$ & -2.97874 & -0.66833 & -0.18082 \\
\hline $\mathrm{C}$ & -3.0131 & 0.273304 & -0.223 & $\mathrm{C}$ & -2.4761 & -2.00035 & -0.762 \\
\hline $\mathrm{C}$ & -0.76262 & 3.334825 & 0.273973 & $\mathrm{C}$ & -2.08636 & 2.549768 & -1.04956 \\
\hline $\mathrm{C}$ & 0.594626 & 3.983289 & 0.166316 & $\mathrm{C}$ & -1.69491 & 3.917563 & -0.55627 \\
\hline $\mathrm{C}$ & 1.436143 & 3.600897 & -1.0713 & $\mathrm{C}$ & -0.17477 & 4.096992 & -0.37274 \\
\hline $\mathrm{C}$ & -2.78438 & -1.25864 & -0.38373 & $\mathrm{C}$ & -1.50634 & -2.7787 & 0.172645 \\
\hline $\mathrm{C}$ & -1.30373 & -1.61293 & -0.09526 & $\mathrm{C}$ & -0.0508 & -2.25393 & 0.020334 \\
\hline $\mathrm{C}$ & 2.207216 & 2.286512 & -1.02557 & $\mathrm{C}$ & 0.514994 & 3.22236 & 0.670109 \\
\hline $\mathrm{C}$ & 1.736558 & 1.133926 & -1.82601 & $\mathrm{C}$ & 1.457857 & 2.164309 & 0.227372 \\
\hline $\mathrm{C}$ & -0.28625 & -1.11287 & -1.13504 & $\mathrm{C}$ & 0.223386 & -0.77572 & 0.334778 \\
\hline $\mathrm{C}$ & 2.103188 & -0.31808 & -1.59367 & $\mathrm{C}$ & 1.950555 & 0.979415 & 1.038107 \\
\hline $\mathrm{C}$ & 1.165252 & -1.08016 & -0.62028 & $\mathrm{C}$ & 1.72121 & -0.42652 & 0.41304 \\
\hline $\mathrm{C}$ & 1.753129 & -2.45697 & -0.33603 & $\mathrm{C}$ & 2.479777 & -0.57518 & -0.90002 \\
\hline $\mathrm{C}$ & 2.829513 & -2.5969 & 0.70372 & $\mathrm{C}$ & 3.964584 & -0.81329 & -0.86265 \\
\hline $\mathrm{O}$ & 3.386835 & -3.6349 & 0.992317 & $\mathrm{O}$ & 4.681885 & -0.88716 & -1.83799 \\
\hline $\mathrm{C}$ & 1.411404 & -3.57666 & -0.97975 & $\mathrm{C}$ & 1.93396 & -0.49649 & -2.11733 \\
\hline $\mathrm{C}$ & -0.61051 & 2.497881 & 2.6484 & $\mathrm{C}$ & -4.21324 & 2.238913 & 0.275056 \\
\hline $\mathrm{C}$ & 3.147005 & 2.113274 & 0.147385 & $\mathrm{C}$ & -0.12206 & 3.199714 & 2.040785 \\
\hline $\mathrm{O}$ & -4.27709 & 0.693298 & -0.76661 & $\mathrm{O}$ & -3.58948 & -2.85022 & -1.0916 \\
\hline $\mathrm{O}$ & 2.797201 & 1.979806 & -2.31935 & $\mathrm{O}$ & 1.947921 & 3.4497 & 0.671167 \\
\hline & 0.804027 & 1.289409 & -2.36603 & $\mathrm{H}$ & 1.548173 & 2.036682 & -0.85063 \\
\hline
\end{tabular}




\begin{tabular}{|c|c|c|c|c|c|c|c|}
\hline $\mathrm{C}$ & -3.20277 & -1.71013 & -1.78972 & C & -1.51157 & -4.27291 & -0.18785 \\
\hline $\mathrm{O}$ & 3.120507 & -1.42688 & 1.301227 & $\mathrm{O}$ & 4.430777 & -0.94736 & 0.392274 \\
\hline $\mathrm{C}$ & 4.143553 & -1.46699 & 2.317515 & $\mathrm{C}$ & 5.84763 & -1.18459 & 0.524868 \\
\hline $\mathrm{O}$ & -3.62145 & -1.86144 & 0.627744 & $\mathrm{O}$ & -2.01114 & -2.56665 & 1.508739 \\
\hline $\mathrm{C}$ & -3.83829 & -3.26399 & 0.573446 & $\mathrm{C}$ & -1.38715 & -3.26523 & 2.576106 \\
\hline $\mathrm{H}$ & -3.30431 & 2.776133 & 0.493236 & $\mathrm{H}$ & -2.6091 & 0.284607 & -2.09525 \\
\hline $\mathrm{H}$ & -3.24322 & 2.657373 & 2.242684 & $\mathrm{H}$ & -4.31067 & 0.260117 & -1.64752 \\
\hline $\mathrm{H}$ & -2.2482 & 0.237379 & 1.806859 & $\mathrm{H}$ & -2.237 & -0.26154 & 0.501567 \\
\hline $\mathrm{H}$ & -3.96891 & 0.506684 & 1.657719 & $\mathrm{H}$ & -3.8574 & -0.88986 & 0.42897 \\
\hline $\mathrm{H}$ & -2.21379 & 0.778016 & -0.77567 & $\mathrm{H}$ & -1.91171 & -1.80347 & -1.68395 \\
\hline $\mathrm{H}$ & -1.3891 & 3.466422 & -0.60858 & $\mathrm{H}$ & -1.40691 & 2.126379 & -1.78937 \\
\hline $\mathrm{H}$ & 1.180298 & 3.839756 & 1.075709 & $\mathrm{H}$ & -2.21169 & 4.16433 & 0.373863 \\
\hline $\mathrm{H}$ & 0.425067 & 5.066267 & 0.098502 & $\mathrm{H}$ & -2.01424 & 4.674809 & -1.285 \\
\hline $\mathrm{H}$ & 0.80275 & 3.602504 & -1.96395 & $\mathrm{H}$ & 0.329718 & 3.950023 & -1.33315 \\
\hline $\mathrm{H}$ & 2.186821 & 4.385592 & -1.22529 & $\mathrm{H}$ & 0.014475 & 5.136997 & -0.08039 \\
\hline $\mathrm{H}$ & -1.224 & -2.69869 & -0.00407 & $\mathrm{H}$ & 0.267496 & -2.4842 & -0.99995 \\
\hline $\mathrm{H}$ & -1.04096 & -1.20678 & 0.88602 & $\mathrm{H}$ & 0.587439 & -2.86471 & 0.66821 \\
\hline $\mathrm{H}$ & -0.54519 & -0.09422 & -1.42646 & $\mathrm{H}$ & -0.21283 & -0.5355 & 1.308886 \\
\hline $\mathrm{H}$ & -0.34915 & -1.70454 & -2.05371 & $\mathrm{H}$ & -0.27141 & -0.12235 & -0.38765 \\
\hline $\mathrm{H}$ & 3.128406 & -0.36887 & -1.22485 & $\mathrm{H}$ & 1.50735 & 0.982088 & 2.036578 \\
\hline $\mathrm{H}$ & 2.095103 & -0.82903 & -2.56422 & $\mathrm{H}$ & 3.026478 & 1.12103 & 1.179047 \\
\hline $\mathrm{H}$ & 1.163238 & -0.52825 & 0.3232 & $\mathrm{H}$ & 2.178017 & -1.12745 & 1.117533 \\
\hline $\mathrm{H}$ & 1.901303 & -4.51271 & -0.743 & $\mathrm{H}$ & 2.553788 & -0.5942 & -2.99976 \\
\hline $\mathrm{H}$ & 0.647173 & -3.59223 & -1.74606 & $\mathrm{H}$ & 0.873545 & -0.33969 & -2.26685 \\
\hline $\mathrm{H}$ & 0.458231 & 2.704946 & 2.60555 & $\mathrm{H}$ & -4.0439 & 3.234429 & 0.684682 \\
\hline $\mathrm{H}$ & -1.04392 & 3.128235 & 3.434178 & $\mathrm{H}$ & -5.19167 & 2.242877 & -0.21972 \\
\hline $\mathrm{H}$ & -0.73555 & 1.461995 & 2.979601 & $\mathrm{H}$ & -4.29309 & 1.53646 & 1.111642 \\
\hline $\mathrm{H}$ & 3.763354 & 1.222697 & 0.038837 & $\mathrm{H}$ & 0.522663 & 2.71043 & 2.770208 \\
\hline $\mathrm{H}$ & 3.808584 & 2.981898 & 0.222921 & $\mathrm{H}$ & -0.30382 & 4.222837 & 2.384343 \\
\hline $\mathrm{H}$ & 2.594557 & 2.02927 & 1.086094 & $\mathrm{H}$ & -1.08145 & 2.676429 & 2.018804 \\
\hline $\mathrm{H}$ & -4.19111 & 0.759578 & -1.72247 & $\mathrm{H}$ & -4.14878 & -2.36373 & -1.70617 \\
\hline $\mathrm{H}$ & -4.28203 & -1.60817 & -1.91186 & $\mathrm{H}$ & -2.47917 & -4.71685 & 0.043361 \\
\hline $\mathrm{H}$ & -2.71147 & -1.10305 & -2.55412 & $\mathrm{H}$ & -1.32454 & -4.40412 & -1.25566 \\
\hline $\mathrm{H}$ & -2.92916 & -2.75092 & -1.97439 & $\mathrm{H}$ & -0.73173 & -4.80953 & 0.356766 \\
\hline $\mathrm{H}$ & 4.238839 & -0.44471 & 2.673804 & $\mathrm{H}$ & 6.031034 & -1.25444 & 1.59385 \\
\hline $\mathrm{H}$ & 3.843928 & -2.12982 & 3.129672 & $\mathrm{H}$ & 6.12363 & -2.11455 & 0.027097 \\
\hline $\mathrm{H}$ & 5.08571 & -1.81466 & 1.893 & $\mathrm{H}$ & 6.412059 & -0.35848 & 0.091684 \\
\hline $\mathrm{H}$ & -4.39948 & -3.51844 & 1.473689 & $\mathrm{H}$ & -1.90081 & -2.94967 & 3.485305 \\
\hline $\mathrm{H}$ & -4.43026 & -3.5605 & -0.29964 & $\mathrm{H}$ & -1.48804 & -4.35181 & 2.480529 \\
\hline $\mathrm{H}$ & -2.9048 & -3.83851 & 0.576281 & $\mathrm{H}$ & -0.32413 & -3.01733 & 2.676864 \\
\hline
\end{tabular}

\begin{tabular}{lrrrrrrrr} 
3f_1 & \multicolumn{7}{c}{ 3f_2 } \\
C & 3.654131 & 1.602328 & -0.0957 & C & 3.142497 & 1.554296 & 0.919977 \\
C & 3.893361 & 0.162169 & 0.324644 & C & 3.825879 & 0.282276 & 0.436949 \\
C & 2.274617 & 2.006237 & -0.6345 & C & 1.761497 & 1.700464 & 0.257835 \\
C & 1.153616 & 1.961259 & 0.419498 & C & 0.995461 & 2.966084 & 0.64491 \\
C & 3.041807 & -0.8394 & 0.070139 & C & 3.395763 & -0.90979 & 0.87115 \\
C & 3.196372 & -2.29266 & 0.458897 & C & 3.840136 & -2.27531 & 0.414543 \\
C & 2.398301 & -3.26854 & -0.42536 & C & 2.997124 & -2.85774 & -0.7447
\end{tabular}




\begin{tabular}{|c|c|c|c|c|c|c|c|}
\hline $\mathrm{C}$ & -0.21818 & 2.513058 & -0.06684 & $\mathrm{C}$ & -0.47803 & 3.00906 & 0.136745 \\
\hline $\mathrm{C}$ & -0.70729 & 1.932353 & -1.41814 & $\mathrm{C}$ & -1.27894 & 1.737204 & 0.540169 \\
\hline $\mathrm{C}$ & 0.878492 & -3.20644 & -0.31954 & $\mathrm{C}$ & 1.518598 & -3.11645 & -0.47631 \\
\hline $\mathrm{C}$ & 0.104615 & -2.54974 & -1.39823 & $\mathrm{C}$ & 0.503724 & -2.19458 & -1.04075 \\
\hline $\mathrm{C}$ & -0.65939 & 0.412802 & -1.6907 & $\mathrm{C}$ & -1.18351 & 0.510783 & -0.39553 \\
\hline $\mathrm{C}$ & -1.31163 & -2.02302 & -1.2882 & $\mathrm{C}$ & -0.93219 & -2.05153 & -0.57867 \\
\hline $\mathrm{C}$ & -1.45828 & -0.55603 & -0.79404 & $\mathrm{C}$ & -1.18883 & -0.84224 & 0.359457 \\
\hline $\mathrm{C}$ & -2.94516 & -0.24134 & -0.69428 & $\mathrm{C}$ & -2.43223 & -1.04691 & 1.216116 \\
\hline $\mathrm{C}$ & -3.68991 & -0.58125 & 0.565264 & $\mathrm{C}$ & -3.80654 & -1.172 & 0.619422 \\
\hline $\mathrm{O}$ & -4.87748 & -0.4087 & 0.739598 & $\mathrm{O}$ & -4.82556 & -1.32849 & 1.258299 \\
\hline $\mathrm{C}$ & -3.67396 & 0.272822 & -1.68901 & $\mathrm{C}$ & -2.3636 & -1.13376 & 2.548653 \\
\hline $\mathrm{C}$ & 5.195706 & -0.02516 & 1.065895 & $\mathrm{C}$ & 4.907156 & 0.480241 & -0.59441 \\
\hline $\mathrm{C}$ & 0.301689 & -3.40824 & 1.064543 & $\mathrm{C}$ & 1.213509 & -4.0285 & 0.691145 \\
\hline $\mathrm{O}$ & 1.549393 & 2.692693 & 1.587902 & $\mathrm{O}$ & 1.6927 & 4.133222 & 0.192801 \\
\hline $\mathrm{O}$ & 0.239811 & -3.98707 & -1.36482 & $\mathrm{O}$ & 0.813297 & -3.43802 & -1.70644 \\
\hline $\mathrm{H}$ & 0.696089 & -2.09971 & -2.19406 & $\mathrm{H}$ & 0.900406 & -1.32727 & -1.56755 \\
\hline $\mathrm{C}$ & -1.2575 & 2.348032 & 1.051555 & $\mathrm{C}$ & -1.16244 & 4.248703 & 0.731655 \\
\hline $\mathrm{O}$ & -2.88651 & -1.10861 & 1.508517 & $\mathrm{O}$ & -3.80027 & -1.09438 & -0.72357 \\
\hline $\mathrm{C}$ & -3.51665 & -1.4665 & 2.756448 & $\mathrm{C}$ & -5.08201 & -1.20803 & -1.37669 \\
\hline $\mathrm{O}$ & 0.084414 & 3.924688 & -0.26151 & $\mathrm{O}$ & -0.31823 & 3.127329 & -1.29506 \\
\hline $\mathrm{C}$ & -0.98956 & 4.829862 & -0.48883 & $\mathrm{C}$ & -1.46672 & 3.458733 & -2.06689 \\
\hline $\mathrm{H}$ & 4.409475 & 1.857848 & -0.85155 & $\mathrm{H}$ & 3.757608 & 2.428937 & 0.693116 \\
\hline $\mathrm{H}$ & 3.882663 & 2.24246 & 0.763413 & $\mathrm{H}$ & 3.013995 & 1.526091 & 2.007891 \\
\hline $\mathrm{H}$ & 2.012348 & 1.387723 & -1.49667 & $\mathrm{H}$ & 1.179783 & 0.824793 & 0.542067 \\
\hline $\mathrm{H}$ & 2.343324 & 3.03273 & -1.00637 & $\mathrm{H}$ & 1.867953 & 1.672686 & -0.83173 \\
\hline $\mathrm{H}$ & 1.006748 & 0.935864 & 0.762837 & $\mathrm{H}$ & 0.964703 & 3.045389 & 1.736616 \\
\hline $\mathrm{H}$ & 2.123622 & -0.60425 & -0.45714 & $\mathrm{H}$ & 2.594293 & -0.90728 & 1.609435 \\
\hline $\mathrm{H}$ & 2.899713 & -2.43553 & 1.505928 & $\mathrm{H}$ & 3.814697 & -2.96797 & 1.262256 \\
\hline $\mathrm{H}$ & 4.247677 & -2.58981 & 0.41344 & $\mathrm{H}$ & 4.880068 & -2.25192 & 0.07809 \\
\hline $\mathrm{H}$ & 2.675926 & -3.12709 & -1.47471 & $\mathrm{H}$ & 3.075368 & -2.19696 & -1.61285 \\
\hline $\mathrm{H}$ & 2.689901 & -4.29209 & -0.16108 & $\mathrm{H}$ & 3.435332 & -3.81973 & -1.03967 \\
\hline $\mathrm{H}$ & -1.72898 & 2.28605 & -1.57693 & $\mathrm{H}$ & -0.94718 & 1.463918 & 1.548477 \\
\hline $\mathrm{H}$ & -0.10048 & 2.405222 & -2.19593 & $\mathrm{H}$ & -2.33125 & 2.011705 & 0.656591 \\
\hline $\mathrm{H}$ & -0.9784 & 0.273749 & -2.73013 & $\mathrm{H}$ & -2.01345 & 0.520709 & -1.10385 \\
\hline $\mathrm{H}$ & 0.381164 & 0.084646 & -1.67101 & $\mathrm{H}$ & -0.27799 & 0.568283 & -0.99783 \\
\hline $\mathrm{H}$ & -1.88283 & -2.68019 & -0.63036 & $\mathrm{H}$ & -1.2381 & -2.97087 & -0.07599 \\
\hline $\mathrm{H}$ & -1.77203 & -2.10162 & -2.28045 & $\mathrm{H}$ & -1.5619 & -1.96331 & -1.46855 \\
\hline $\mathrm{H}$ & -1.04201 & -0.52071 & 0.213543 & $\mathrm{H}$ & -0.35867 & -0.81404 & 1.074715 \\
\hline $\mathrm{H}$ & -4.73756 & 0.430496 & -1.56209 & $\mathrm{H}$ & -3.25712 & -1.28379 & 3.141661 \\
\hline $\mathrm{H}$ & -3.23844 & 0.54458 & -2.64231 & $\mathrm{H}$ & -1.41685 & -1.05856 & 3.070466 \\
\hline $\mathrm{H}$ & 5.151835 & 0.467418 & 2.044546 & $\mathrm{H}$ & 4.518251 & 1.039831 & -1.45327 \\
\hline $\mathrm{H}$ & 5.44978 & -1.07175 & 1.232454 & $\mathrm{H}$ & 5.725337 & 1.080963 & -0.18048 \\
\hline $\mathrm{H}$ & 6.021425 & 0.444124 & 0.519247 & $\mathrm{H}$ & 5.326132 & -0.45489 & -0.96643 \\
\hline $\mathrm{H}$ & 0.581187 & -2.58722 & 1.729502 & $\mathrm{H}$ & 1.484825 & -3.5564 & 1.639148 \\
\hline $\mathrm{H}$ & -0.78516 & -3.47121 & 1.04085 & $\mathrm{H}$ & 0.158 & -4.29381 & 0.730503 \\
\hline $\mathrm{H}$ & 0.688671 & -4.33647 & 1.49628 & $\mathrm{H}$ & 1.791698 & -4.95335 & 0.600897 \\
\hline $\mathrm{H}$ & 1.417626 & 3.621706 & 1.350032 & $\mathrm{H}$ & 1.488078 & 4.191975 & -0.75104 \\
\hline $\mathrm{H}$ & -0.93965 & 2.892009 & 1.941418 & $\mathrm{H}$ & -0.59331 & 5.150933 & 0.504838 \\
\hline $\mathrm{H}$ & -2.23894 & 2.712554 & 0.743814 & $\mathrm{H}$ & -2.18095 & 4.363843 & 0.356434 \\
\hline
\end{tabular}




$\begin{array}{lrlllllr}\mathrm{H} & -1.36609 & 1.298229 & 1.325218 & \mathrm{H} & -1.22307 & 4.145072 & 1.818343 \\ \mathrm{H} & -3.95474 & -0.58484 & 3.224669 & \mathrm{H} & -5.53639 & -2.17357 & -1.15349 \\ \mathrm{H} & -4.29272 & -2.21327 & 2.587536 & \mathrm{H} & -5.74634 & -0.40826 & -1.04861 \\ \mathrm{H} & -2.72229 & -1.87367 & 3.376321 & \mathrm{H} & -4.87372 & -1.12047 & -2.4398 \\ \mathrm{H} & -1.65889 & 4.904923 & 0.374068 & \mathrm{H} & -1.78535 & 4.493959 & -1.90779 \\ \mathrm{H} & -1.57676 & 4.562659 & -1.37366 & \mathrm{H} & -2.3096 & 2.791342 & -1.85761 \\ \mathrm{H} & -0.53135 & 5.804695 & -0.65938 & \mathrm{H} & -1.17855 & 3.338762 & -3.11188\end{array}$

$\mathrm{C}$

C

$\mathrm{C}$

C

C

C

C

C

C

C

$\mathrm{C}$

C

C

C

C

C

$\mathrm{O}$

C

C

C

$\mathrm{O}$

O

$\mathrm{H}$

C

$\mathrm{O}$

C

$\mathrm{O}$

$\mathrm{C}$

$\mathrm{H}$

H

$\mathrm{H}$

$\mathrm{H}$

$\mathrm{H}$

$\mathrm{H}$

$\mathrm{H}$

$\mathrm{H}$

$\mathrm{H}$

$\mathrm{H}$

$\mathrm{H}$

$\mathrm{H}$
3.423771

2.059262

0.689178

3.96158

0.703838

0.24164

1.928985

$2.197572 \quad 1.037432$

1.091129

2.769722

$-0.10818$

3.358385

$-0.46308$

0.504509

3.855067

$-1.85453$

0.205181

2.986936

$-2.67142$

$-0.77819$

$-0.44682$

2.842517

0.168139

$-1.06452$

1.533781

0.708721

$1.577954-3.04122$

$-0.32785$

0.41872

$-2.36066$

$-0.94877$

$\begin{array}{ll}-0.92512 & 0.296607\end{array}$

$-0.18973$

$-0.97803 \quad-2.26664$

$-0.37002$

$-1.21225 \quad-1.03474$

0.541042

$-2.57234$

$-1.10295$

1.224336

$-3.86677-1.01907$

0.463555

$-4.96834$

$-1.10048$

0.964593

$-2.68431$

$-1.2585$

2.54785

5.291709

0.829779

$-0.46269$

1.470394

$-3.74156$

1.008999

$1.364423 \quad 2.029309$

$-1.29927$

0.827393

$-3.66981$

$-1.40247$

0.665188

$-1.58092$

$-1.66737$

$-0.73655$

3.996082

1.13791

$-3.68697$

$-0.83965$

$-0.85803$

$-4.88473$

$-0.75761$

$-1.65933$

$-0.95474$

3.165373

$-1.15407$

$-2.35878$

3.314605

$-1.32387$

3.675337

2.793368

4.021103

2.35638

1.826164

2.87148

$-0.08458$

1.561611

1.52358

1.238409

1.891919

1.410871

3.810321

1.35943

2.400365

$-0.43712$

$-0.27413$

3.930079

$-2.41068$

1.016363

4.867279

$-1.82859$

1.148172

2.917049

$-2.14069$

$-0.20188$

$-1.73226$

3.506611

$-3.61639$

$-0.98129$

$\begin{array}{lll}-0.60626 & 1.333586 & 1.68251\end{array}$

$-2.12085$

1.71908

0.929172

3f_4

$\mathrm{C}$

C

C

C

C

C

C

$\mathrm{C}$

C

C

C

C

C

C

C

C

$\mathrm{O}$

C

C

C

$\mathrm{O}$

$\mathrm{O}$

$\mathrm{H}$

C

$\mathrm{O}$

C

$\mathrm{O}$

C

$\mathrm{H}$

$\mathrm{H}$

$\mathrm{H}$

$\mathrm{H}$

$\mathrm{H}$

$\mathrm{H}$

$\mathrm{H}$

$\mathrm{H}$

H

$\mathrm{H}$
$\mathrm{H}$

$\mathrm{H}$

$\mathrm{H}$

$\mathrm{H}$

$\begin{array}{rrr}3.595149 & 1.729311 & -0.07222 \\ 3.865816 & 0.292581 & 0.343016 \\ 2.209718 & 2.102176 & -0.62096 \\ 1.056696 & 1.904528 & 0.380054 \\ 3.088337 & -0.74006 & -0.00757 \\ 3.289004 & -2.19413 & 0.361765 \\ 2.489804 & -3.18006 & -0.50853 \\ -0.28987 & 2.524031 & -0.07063 \\ -0.78916 & 1.939672 & -1.42338 \\ 0.971532 & -3.15267 & -0.36717 \\ 0.157753 & -2.51746 & -1.42981 \\ -0.70519 & 0.425507 & -1.71218 \\ -1.27049 & -2.03098 & -1.2923 \\ -1.45309 & -0.56817 & -0.79912 \\ -2.94709 & -0.3012 & -0.66962 \\ -3.65503 & -0.66458 & 0.604506 \\ -4.84341 & -0.5282 & 0.8038 \\ -3.71212 & 0.188871 & -1.64922 \\ 5.109774 & 0.139345 & 1.185202 \\ 0.431278 & -3.36582 & 1.029629 \\ 1.396894 & 2.47999 & 1.653758 \\ 0.330345 & -3.95086 & -1.39718 \\ 0.720326 & -2.0539 & -2.23875 \\ -1.32778 & 2.359305 & 1.050675 \\ -2.81695 & -1.16865 & 1.530356 \\ -3.41044 & -1.54625 & 2.790253 \\ 0.011564 & 3.927258 & -0.26586 \\ -1.06321 & 4.810795 & -0.5514 \\ 4.349911 & 2.012932 & -0.81815 \\ 3.804452 & 2.373413 & 0.789874 \\ 2.003128 & 1.542732 & -1.53803 \\ 2.232275 & 3.157678 & -0.90019 \\ 0.875795 & 0.835992 & 0.525839 \\ 2.21484 & -0.53757 & -0.61819 \\ 3.03269 & -2.35507 & 1.416247 \\ 4.346277 & -2.46345 & 0.277802 \\ 2.738718 & -3.02586 & -1.56331 \\ 2.809758 & -4.1989 & -0.25937 \\ -1.8217 & 2.268136 & -1.56278 \\ -0.20755 & 2.435465 & -2.20639\end{array}$

3.595149

1.729311

\section{_ 4}

C.

1.05

3.0

C

C

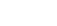

C

C

C

0

C

C -1

$-1$

$-1$

$-3$

O

C

C

.

$\mathrm{O}$

(

C

O

(

$\mathrm{H}$

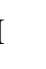




$\begin{array}{lrrrrrrr}\mathrm{H} & -1.5776 & 0.388609 & -1.0601 & \mathrm{H} & -1.04823 & 0.283538 & -2.74357 \\ \mathrm{H} & 0.093583 & 0.256296 & -0.57023 & \mathrm{H} & 0.345367 & 0.128562 & -1.72343 \\ \mathrm{H} & -1.19337 & -3.17545 & 0.19589 & \mathrm{H} & -1.80851 & -2.70361 & -0.62212 \\ \mathrm{H} & -1.68353 & -2.2428 & -1.20535 & \mathrm{H} & -1.74741 & -2.12661 & -2.27524 \\ \mathrm{H} & -0.48418 & -1.10552 & 1.357639 & \mathrm{H} & -1.01915 & -0.51528 & 0.200424 \\ \mathrm{H} & -3.65681 & -1.31779 & 3.020487 & \mathrm{H} & -4.77749 & 0.312074 & -1.50077 \\ \mathrm{H} & -1.80833 & -1.33033 & 3.181869 & \mathrm{H} & -3.3049 & 0.474838 & -2.61085 \\ \mathrm{H} & 5.995588 & 1.408374 & 0.147054 & \mathrm{H} & 4.968654 & 0.607267 & 2.166749 \\ \mathrm{H} & 5.757583 & -0.12657 & -0.69609 & \mathrm{H} & 5.390664 & -0.90076 & 1.349744 \\ \mathrm{H} & 5.168181 & 1.382678 & -1.40112 & \mathrm{H} & 5.957192 & 0.652213 & 0.716386 \\ \mathrm{H} & 1.75803 & -3.07644 & 1.827246 & \mathrm{H} & 0.718399 & -2.54387 & 1.690239 \\ \mathrm{H} & 0.456881 & -4.09439 & 1.19512 & \mathrm{H} & -0.65509 & -3.4403 & 1.033141 \\ \mathrm{H} & 2.139855 & -4.60723 & 1.031021 & \mathrm{H} & 0.839343 & -4.29097 & 1.44843 \\ \mathrm{H} & 0.662913 & 2.287528 & -1.91395 & \mathrm{H} & 1.937207 & 1.837408 & 2.123323 \\ \mathrm{H} & -0.36592 & 4.939235 & 0.729572 & \mathrm{H} & -1.02682 & 2.946105 & 1.917946 \\ \mathrm{H} & -1.8073 & 4.098644 & 1.325476 & \mathrm{H} & -2.31942 & 2.680092 & 0.726946 \\ \mathrm{H} & -0.25453 & 3.823884 & 2.102397 & \mathrm{H} & -1.40311 & 1.316541 & 1.362301 \\ \mathrm{H} & -5.46084 & -1.67916 & -1.57375 & \mathrm{H} & -3.86618 & -0.67885 & 3.268166 \\ \mathrm{H} & -5.49731 & 0.085694 & -1.33986 & \mathrm{H} & -4.16615 & -2.3168 & 2.636415 \\ \mathrm{H} & -4.5406 & -0.61514 & -2.68035 & \mathrm{H} & -2.59158 & -1.92886 & 3.393727 \\ \mathrm{H} & -2.76822 & 4.111377 & -0.69467 & \mathrm{H} & -1.78971 & 4.863177 & 0.26687 \\ \mathrm{H} & -2.90157 & 2.385501 & -1.1221 & \mathrm{H} & -1.59112 & 4.549633 & -1.4758 \\ \mathrm{H} & -2.50981 & 3.586077 & -2.36909 & \mathrm{H} & -0.61679 & 5.798106 & -0.67867 \\ & & & & & & & \end{array}$

\begin{tabular}{lrrrlrrr} 
3f_5 & \multicolumn{7}{l}{ 3f_6 } \\
C & 3.100473 & 1.428352 & 0.995715 & C & 3.062017 & 1.38113 & 1.049673 \\
C & 3.782683 & 0.167167 & 0.489567 & C & 3.758174 & 0.133557 & 0.533351 \\
C & 1.821268 & 1.706017 & 0.184361 & C & 1.859262 & 1.737284 & 0.155225 \\
C & 1.015362 & 2.915192 & 0.657725 & C & 1.020675 & 2.903546 & 0.664376 \\
C & 3.333394 & -1.03168 & 0.883522 & C & 3.300106 & -1.07377 & 0.890314 \\
C & 3.765592 & -2.38908 & 0.393159 & C & 3.740696 & -2.4218 & 0.382671 \\
C & 2.912894 & -2.93342 & -0.77736 & C & 2.891896 & -2.95123 & -0.7974 \\
C & -0.39376 & 3.036294 & 0.012719 & C & -0.3718 & 3.038785 & -0.02839 \\
C & -1.29012 & 1.819402 & 0.415831 & C & -1.29843 & 1.843641 & 0.373006 \\
C & 1.429458 & -3.15915 & -0.50899 & C & 1.407059 & -3.17182 & -0.53386 \\
C & 0.439442 & -2.19713 & -1.04925 & C & 0.425004 & -2.19254 & -1.0568 \\
C & -1.21532 & 0.551938 & -0.46778 & C & -1.22917 & 0.56275 & -0.49237 \\
C & -0.98596 & -2.01504 & -0.5707 & C & -0.99688 & -2.00325 & -0.57114 \\
C & -1.19991 & -0.773 & 0.336085 & C & -1.1987 & -0.75248 & 0.326976 \\
C & -2.41279 & -0.9385 & 1.243117 & C & -2.39787 & -0.90942 & 1.253346 \\
C & -3.80903 & -1.06753 & 0.700726 & C & -3.80193 & -1.04363 & 0.732795 \\
O & -4.80801 & -1.16749 & 1.381307 & O & -4.79153 & -1.12861 & 1.428909 \\
C & -2.29645 & -0.98487 & 2.574415 & C & -2.26191 & -0.94277 & 2.583187 \\
C & 4.880727 & 0.384944 & -0.52008 & C & 4.883578 & 0.373425 & -0.44105 \\
C & 1.104662 & -4.08414 & 0.642994 & C & 1.07564 & -4.1125 & 0.603461 \\
O & 1.750004 & 4.125129 & 0.396909 & O & 1.823917 & 4.082269 & 0.507645 \\
O & 0.707062 & -3.43704 & -1.73948 & O & 0.680484 & -3.42337 & -1.76744 \\
H & 0.858969 & -1.33324 & -1.56384 & H & 0.851964 & -1.32439 & -1.55811 \\
C & -1.05275 & 4.329346 & 0.51779 & C & -1.02043 & 4.352966 & 0.435769
\end{tabular}




\begin{tabular}{|c|c|c|c|c|c|c|c|}
\hline $\mathrm{O}$ & -3.84779 & -1.06578 & -0.6438 & $\mathrm{O}$ & -3.85929 & -1.06568 & -0.61085 \\
\hline $\mathrm{C}$ & -5.15334 & -1.19228 & -1.2455 & $\mathrm{C}$ & -5.1735 & -1.19828 & -1.19227 \\
\hline $\mathrm{O}$ & -0.1547 & 3.081691 & -1.40518 & $\mathrm{O}$ & -0.09671 & 3.061415 & -1.43846 \\
\hline $\mathrm{C}$ & -1.25629 & 3.410637 & -2.23981 & $\mathrm{C}$ & -1.17551 & 3.386338 & -2.30462 \\
\hline $\mathrm{H}$ & 3.784432 & 2.28064 & 0.92965 & $\mathrm{H}$ & 3.756858 & 2.224705 & 1.091804 \\
\hline $\mathrm{H}$ & 2.832023 & 1.310361 & 2.051491 & $\mathrm{H}$ & 2.702028 & 1.20803 & 2.0697 \\
\hline $\mathrm{H}$ & 1.205458 & 0.810895 & 0.25376 & $\mathrm{H}$ & 1.234828 & 0.848984 & 0.085896 \\
\hline $\mathrm{H}$ & 2.063865 & 1.849731 & -0.87194 & $\mathrm{H}$ & 2.189332 & 1.972483 & -0.86041 \\
\hline $\mathrm{H}$ & 0.842314 & 2.829324 & 1.73822 & $\mathrm{H}$ & 0.812112 & 2.752462 & 1.732115 \\
\hline $\mathrm{H}$ & 2.521011 & -1.03889 & 1.609858 & $\mathrm{H}$ & 2.470774 & -1.09476 & 1.596934 \\
\hline $\mathrm{H}$ & 3.733921 & -3.10215 & 1.223636 & $\mathrm{H}$ & 3.708138 & -3.14711 & 1.202631 \\
\hline $\mathrm{H}$ & 4.805093 & -2.36743 & 0.055648 & $\mathrm{H}$ & 4.781125 & -2.39186 & 0.049063 \\
\hline $\mathrm{H}$ & 3.006066 & -2.25949 & -1.63389 & $\mathrm{H}$ & 2.990227 & -2.26812 & -1.64605 \\
\hline $\mathrm{H}$ & 3.329358 & -3.89994 & -1.08834 & $\mathrm{H}$ & 3.305686 & -3.91594 & -1.11732 \\
\hline $\mathrm{H}$ & -1.03903 & 1.566624 & 1.451247 & $\mathrm{H}$ & -1.07081 & 1.599653 & 1.415879 \\
\hline $\mathrm{H}$ & -2.32887 & 2.158505 & 0.45551 & $\mathrm{H}$ & -2.33196 & 2.199845 & 0.389208 \\
\hline $\mathrm{H}$ & -2.06249 & 0.530518 & -1.15528 & $\mathrm{H}$ & -2.08477 & 0.528318 & -1.16878 \\
\hline $\mathrm{H}$ & -0.32596 & 0.589847 & -1.0965 & $\mathrm{H}$ & -0.34845 & 0.596042 & -1.13386 \\
\hline $\mathrm{H}$ & -1.30412 & -2.91318 & -0.0383 & $\mathrm{H}$ & -1.31579 & -2.8957 & -0.02973 \\
\hline $\mathrm{H}$ & -1.62784 & -1.93661 & -1.45267 & $\mathrm{H}$ & -1.64416 & -1.92845 & -1.44944 \\
\hline $\mathrm{H}$ & -0.34461 & -0.72563 & 1.020125 & $\mathrm{H}$ & -0.33339 & -0.69631 & 0.997479 \\
\hline $\mathrm{H}$ & -3.16895 & -1.10733 & 3.203975 & $\mathrm{H}$ & -3.12499 & -1.05932 & 3.226706 \\
\hline $\mathrm{H}$ & -1.33054 & -0.90411 & 3.058916 & $\mathrm{H}$ & -1.28896 & -0.85738 & 3.052542 \\
\hline $\mathrm{H}$ & 5.29369 & -0.54336 & -0.91498 & $\mathrm{H}$ & 5.691582 & 0.936409 & 0.040429 \\
\hline $\mathrm{H}$ & 4.511693 & 0.975171 & -1.36676 & $\mathrm{H}$ & 5.3057 & -0.54592 & -0.84721 \\
\hline $\mathrm{H}$ & 5.700298 & 0.961408 & -0.07557 & $\mathrm{H}$ & 4.540377 & 0.985254 & -1.28308 \\
\hline $\mathrm{H}$ & 1.380767 & -3.63284 & 1.599758 & $\mathrm{H}$ & 1.348347 & -3.67567 & 1.567921 \\
\hline $\mathrm{H}$ & 0.044494 & -4.3313 & 0.673807 & $\mathrm{H}$ & 0.014838 & -4.35781 & 0.625723 \\
\hline $\mathrm{H}$ & 1.666415 & -5.01765 & 0.5393 & $\mathrm{H}$ & 1.635763 & -5.04567 & 0.488738 \\
\hline $\mathrm{H}$ & 2.305375 & 4.302783 & 1.161246 & $\mathrm{H}$ & 1.517517 & 4.746083 & 1.13287 \\
\hline $\mathrm{H}$ & -0.45115 & 5.195811 & 0.245192 & $\mathrm{H}$ & -0.4271 & 5.210927 & 0.114046 \\
\hline $\mathrm{H}$ & -2.06025 & 4.446898 & 0.114534 & $\mathrm{H}$ & -2.03024 & 4.465206 & 0.037747 \\
\hline $\mathrm{H}$ & -1.13766 & 4.298385 & 1.607731 & $\mathrm{H}$ & -1.09734 & 4.368814 & 1.526985 \\
\hline $\mathrm{H}$ & -4.97996 & -1.179 & -2.31834 & $\mathrm{H}$ & -5.63844 & -2.12958 & -0.86839 \\
\hline $\mathrm{H}$ & -5.61978 & -2.13032 & -0.94392 & $\mathrm{H}$ & -5.80262 & -0.35668 & -0.90144 \\
\hline $\mathrm{H}$ & -5.78914 & -0.35765 & -0.94915 & $\mathrm{H}$ & -5.01489 & -1.20376 & -2.26745 \\
\hline $\mathrm{H}$ & -0.92546 & 3.242006 & -3.26565 & $\mathrm{H}$ & -1.47032 & 4.438065 & -2.22118 \\
\hline $\mathrm{H}$ & -1.55288 & 4.460288 & -2.13859 & $\mathrm{H}$ & -2.05652 & 2.758632 & -2.12784 \\
\hline $\mathrm{H}$ & -2.13055 & 2.777952 & -2.04746 & $\mathrm{H}$ & -0.81929 & 3.206586 & -3.31993 \\
\hline
\end{tabular}

\begin{tabular}{lrrrllrrr} 
3f_7 & \multicolumn{7}{c}{ 3f_8 } \\
C & 3.462829 & 1.72089 & -1.09379 & C & -2.16533 & 2.279694 & 0.133464 \\
C & 3.967623 & 0.376767 & -0.59461 & C & -3.22656 & 1.199365 & 0.233567 \\
C & 1.950709 & 1.987605 & -1.11757 & C & -1.33927 & 2.336978 & -1.15622 \\
C & 1.318097 & 2.058995 & 0.282885 & C & -0.08437 & 3.226522 & -1.099 \\
C & 3.18193 & -0.69054 & -0.40209 & C & -3.28683 & 0.145619 & -0.59107 \\
C & 3.596902 & -2.05517 & 0.100306 & C & -4.21771 & -1.03892 & -0.49042 \\
C & 2.635626 & -3.19106 & -0.29651 & C & -3.70119 & -2.1493 & 0.453223 \\
C & -0.19484 & 2.426354 & 0.308167 & C & 1.162007 & 2.684271 & -0.32629
\end{tabular}




\begin{tabular}{|c|c|c|c|c|c|c|c|}
\hline $\mathrm{C}$ & -1.07222 & 1.594731 & -0.66257 & $\mathrm{C}$ & 1.497262 & 1.209301 & -0.68171 \\
\hline $\mathrm{C}$ & 1.254204 & -3.19391 & 0.350739 & $\mathrm{C}$ & -2.34878 & -2.75908 & 0.108245 \\
\hline $\mathrm{C}$ & 0.076429 & -2.77366 & -0.44411 & $\mathrm{C}$ & -1.14105 & -2.25993 & 0.803143 \\
\hline $\mathrm{C}$ & -0.91704 & 0.061192 & -0.74292 & $\mathrm{C}$ & 0.803454 & 0.105911 & 0.136854 \\
\hline $\mathrm{C}$ & -1.26899 & -2.33373 & 0.099057 & $\mathrm{C}$ & 0.29068 & -2.42191 & 0.337027 \\
\hline $\mathrm{C}$ & -1.41068 & -0.82081 & 0.428431 & $\mathrm{C}$ & 0.847844 & -1.28122 & -0.55378 \\
\hline $\mathrm{C}$ & -2.82838 & -0.53324 & 0.911482 & $\mathrm{C}$ & 2.213469 & -1.67036 & -1.11062 \\
\hline $\mathrm{C}$ & -4.01475 & -0.51471 & -0.01312 & $\mathrm{C}$ & 3.422826 & -1.8431 & -0.23483 \\
\hline $\mathrm{O}$ & -5.1385 & -0.19808 & 0.314972 & $\mathrm{O}$ & 4.511565 & -2.20981 & -0.62356 \\
\hline $\mathrm{C}$ & -3.10142 & -0.33331 & 2.204921 & $\mathrm{C}$ & 2.398569 & -1.90291 & -2.41416 \\
\hline $\mathrm{C}$ & 5.454826 & 0.367172 & -0.33268 & $\mathrm{C}$ & -4.16366 & 1.397481 & 1.401259 \\
\hline $\mathrm{C}$ & 1.230841 & -3.19877 & 1.863529 & $\mathrm{C}$ & -2.27204 & -3.48326 & -1.21745 \\
\hline $\mathrm{O}$ & 2.029723 & 3.005607 & 1.091874 & $\mathrm{O}$ & -0.408 & 4.534605 & -0.61314 \\
\hline $\mathrm{O}$ & 0.3835 & -4.17452 & -0.27456 & $\mathrm{O}$ & -1.7846 & -3.48101 & 1.236303 \\
\hline $\mathrm{H}$ & 0.291086 & -2.42375 & -1.45281 & $\mathrm{H}$ & -1.30531 & -1.43385 & 1.492967 \\
\hline $\mathrm{C}$ & -0.69713 & 2.368015 & 1.757415 & $\mathrm{C}$ & 2.360295 & 3.574864 & -0.69617 \\
\hline $\mathrm{O}$ & -3.70959 & -0.89856 & -1.26585 & $\mathrm{O}$ & 3.179829 & -1.54089 & 1.053793 \\
\hline $\mathrm{C}$ & -4.79768 & -0.91239 & -2.21432 & $\mathrm{C}$ & 4.291613 & -1.68296 & 1.963222 \\
\hline $\mathrm{O}$ & -0.17398 & 3.80423 & -0.16195 & $\mathrm{O}$ & 0.802773 & 2.842267 & 1.0667 \\
\hline $\mathrm{C}$ & -1.36487 & 4.575995 & -0.06307 & $\mathrm{C}$ & 1.839679 & 2.753169 & 2.038432 \\
\hline $\mathrm{H}$ & 3.860237 & 1.871907 & -2.10682 & $\mathrm{H}$ & -1.49605 & 2.163304 & 0.993782 \\
\hline $\mathrm{H}$ & 3.932529 & 2.499772 & -0.48339 & $\mathrm{H}$ & -2.64464 & 3.254591 & 0.281401 \\
\hline $\mathrm{H}$ & 1.444931 & 1.236589 & -1.72927 & $\mathrm{H}$ & -1.97274 & 2.724674 & -1.96165 \\
\hline $\mathrm{H}$ & 1.781452 & 2.948069 & -1.61372 & $\mathrm{H}$ & -1.03225 & 1.34012 & -1.47544 \\
\hline $\mathrm{H}$ & 1.440964 & 1.101359 & 0.791029 & $\mathrm{H}$ & 0.247802 & 3.369209 & -2.1318 \\
\hline $\mathrm{H}$ & 2.122344 & -0.58593 & -0.60803 & $\mathrm{H}$ & -2.56481 & 0.082798 & -1.39901 \\
\hline $\mathrm{H}$ & 3.700933 & -2.03832 & 1.19276 & $\mathrm{H}$ & -4.37731 & -1.45656 & -1.48952 \\
\hline $\mathrm{H}$ & 4.589086 & -2.31464 & -0.27958 & $\mathrm{H}$ & -5.2051 & -0.73548 & -0.13173 \\
\hline $\mathrm{H}$ & 2.505108 & -3.20056 & -1.38326 & $\mathrm{H}$ & -3.63897 & -1.75478 & 1.471279 \\
\hline $\mathrm{H}$ & 3.10377 & -4.14661 & -0.03114 & $\mathrm{H}$ & -4.43425 & -2.96568 & 0.473568 \\
\hline $\mathrm{H}$ & -2.12074 & 1.837113 & -0.46272 & $\mathrm{H}$ & 1.280638 & 1.076247 & -1.74802 \\
\hline $\mathrm{H}$ & -0.8632 & 1.980935 & -1.6644 & $\mathrm{H}$ & 2.579445 & 1.073145 & -0.59534 \\
\hline $\mathrm{H}$ & -1.43771 & -0.25557 & -1.64852 & $\mathrm{H}$ & 1.2654 & 0.036875 & 1.122669 \\
\hline $\mathrm{H}$ & 0.133251 & -0.18258 & -0.90524 & $\mathrm{H}$ & -0.24378 & 0.354449 & 0.305363 \\
\hline $\mathrm{H}$ & -1.4966 & -2.90885 & 0.99873 & $\mathrm{H}$ & 0.378174 & -3.36486 & -0.20684 \\
\hline $\mathrm{H}$ & -2.02087 & -2.60804 & -0.64668 & $\mathrm{H}$ & 0.916917 & -2.51795 & 1.228856 \\
\hline $\mathrm{H}$ & -0.76433 & -0.62966 & 1.290367 & $\mathrm{H}$ & 0.196537 & -1.21364 & -1.43275 \\
\hline $\mathrm{H}$ & -4.11759 & -0.16279 & 2.537559 & $\mathrm{H}$ & 3.369156 & -2.19728 & -2.79342 \\
\hline $\mathrm{H}$ & -2.31984 & -0.33979 & 2.955204 & $\mathrm{H}$ & 1.586219 & -1.80401 & -3.12459 \\
\hline $\mathrm{H}$ & 5.693853 & 0.997822 & 0.531898 & $\mathrm{H}$ & -4.909 & 0.608145 & 1.495522 \\
\hline $\mathrm{H}$ & 5.851165 & -0.62903 & -0.13674 & $\mathrm{H}$ & -3.60103 & 1.44154 & 2.341143 \\
\hline $\mathrm{H}$ & 5.999087 & 0.789855 & -1.18469 & $\mathrm{H}$ & -4.69015 & 2.354538 & 1.310708 \\
\hline $\mathrm{H}$ & 1.623611 & -2.26142 & 2.265317 & $\mathrm{H}$ & -2.42834 & -2.79182 & -2.0497 \\
\hline $\mathrm{H}$ & 0.2212 & -3.33959 & 2.247424 & $\mathrm{H}$ & -1.30944 & -3.97375 & -1.35662 \\
\hline $\mathrm{H}$ & 1.854952 & -4.01357 & 2.243502 & $\mathrm{H}$ & -3.05337 & -4.24793 & -1.26762 \\
\hline $\mathrm{H}$ & 1.696261 & 3.868669 & 0.807259 & $\mathrm{H}$ & -0.33288 & 4.470357 & 0.349174 \\
\hline $\mathrm{H}$ & -0.14682 & 3.078508 & 2.374992 & $\mathrm{H}$ & 2.131299 & 4.626058 & -0.51544 \\
\hline $\mathrm{H}$ & -1.76386 & 2.585622 & 1.8265 & $\mathrm{H}$ & 3.253803 & 3.301393 & -0.13226 \\
\hline $\mathrm{H}$ & -0.53769 & 1.372057 & 2.172279 & $\mathrm{H}$ & 2.5925 & 3.450565 & -1.75693 \\
\hline
\end{tabular}




$\begin{array}{lrrrllll}\mathrm{H} & -5.57253 & -1.60788 & -1.89126 & \mathrm{H} & 3.903919 & -1.40539 & 2.939874 \\ \mathrm{H} & -5.22339 & 0.086008 & -2.31638 & \mathrm{H} & 4.645704 & -2.71398 & 1.969753 \\ \mathrm{H} & -4.35829 & -1.23869 & -3.15331 & \mathrm{H} & 5.106296 & -1.01995 & 1.671412 \\ \mathrm{H} & -1.67064 & 4.736239 & 0.97585 & \mathrm{H} & 1.345158 & 2.704726 & 3.009203 \\ \mathrm{H} & -2.19681 & 4.125391 & -0.61443 & \mathrm{H} & 2.494776 & 3.629782 & 2.021156 \\ \mathrm{H} & -1.1364 & 5.543378 & -0.51174 & \mathrm{H} & 2.447484 & 1.851683 & 1.909736\end{array}$

\begin{tabular}{|c|c|c|c|c|c|c|c|}
\hline 3f_9 & & & & $3 \mathbf{f}$ & & & \\
\hline $\mathrm{C}$ & -2.59508 & 1.363044 & -2.1372 & $\mathrm{C}$ & 3.291539 & 1.630681 & 0.642163 \\
\hline $\mathrm{C}$ & -3.24775 & 0.164374 & -1.47555 & $\mathrm{C}$ & 3.96792 & 0.279099 & 0.449326 \\
\hline $\mathrm{C}$ & -1.92636 & 2.367003 & -1.17105 & $\mathrm{C}$ & 1.813811 & 1.664709 & 0.250844 \\
\hline $\mathrm{C}$ & -0.60096 & 1.840983 & -0.61976 & $\mathrm{C}$ & 1.095587 & 2.9871 & 0.515092 \\
\hline $\mathrm{C}$ & -2.67148 & -1.04644 & -1.51541 & $\mathrm{C}$ & 3.256472 & -0.84826 & 0.320655 \\
\hline $\mathrm{C}$ & -3.23382 & -2.34801 & -0.99472 & $\mathrm{C}$ & 3.720731 & -2.26066 & 0.085601 \\
\hline $\mathrm{C}$ & -2.80648 & -2.77283 & 0.428464 & $\mathrm{C}$ & 2.83357 & -2.99523 & -0.94386 \\
\hline $\mathrm{C}$ & -0.02649 & 2.66214 & 0.574354 & $\mathrm{C}$ & -0.41018 & 2.98292 & 0.090861 \\
\hline $\mathrm{C}$ & 1.262344 & 1.99943 & 1.131064 & $\mathrm{C}$ & -1.17517 & 1.717722 & 0.578968 \\
\hline $\mathrm{C}$ & -1.325 & -3.03741 & 0.665138 & $\mathrm{C}$ & 1.374871 & -3.21369 & -0.54957 \\
\hline $\mathrm{C}$ & -0.56203 & -2.11525 & 1.538131 & $\mathrm{C}$ & 0.328055 & -2.30905 & -1.08624 \\
\hline $\mathrm{C}$ & 1.071544 & 0.608288 & 1.773578 & $\mathrm{C}$ & -1.11945 & 0.473172 & -0.33623 \\
\hline $\mathrm{C}$ & 0.940627 & -1.93835 & 1.552368 & $\mathrm{C}$ & -1.05923 & -2.10359 & -0.5092 \\
\hline $\mathrm{C}$ & 1.411694 & -0.61847 & 0.891501 & $\mathrm{C}$ & -1.21253 & -0.87227 & 0.426089 \\
\hline $\mathrm{C}$ & 2.899323 & -0.68685 & 0.564352 & $\mathrm{C}$ & -2.45705 & -0.9903 & 1.298178 \\
\hline $\mathrm{C}$ & 3.280359 & -0.45297 & -0.86016 & $\mathrm{C}$ & -3.84601 & -1.00509 & 0.722257 \\
\hline $\mathrm{O}$ & 2.52813 & 0.026875 & -1.69377 & $\mathrm{O}$ & -4.86359 & -1.09404 & 1.376119 \\
\hline C & 3.847759 & -0.91565 & 1.478267 & $\mathrm{C}$ & -2.37577 & -1.09716 & 2.62854 \\
\hline $\mathrm{C}$ & -4.57865 & 0.441721 & -0.8191 & $\mathrm{C}$ & 5.475002 & 0.338565 & 0.434064 \\
\hline $\mathrm{C}$ & -0.64455 & -3.94797 & -0.33237 & $\mathrm{C}$ & 1.154098 & -4.03676 & 0.70081 \\
\hline $\mathrm{O}$ & 0.323058 & 1.814065 & -1.70958 & $\mathrm{O}$ & 1.760423 & 4.08153 & -0.12388 \\
\hline $\mathrm{O}$ & -1.06189 & -3.3639 & 2.05793 & $\mathrm{O}$ & 0.561423 & -3.60084 & -1.68865 \\
\hline $\mathrm{H}$ & -1.12147 & -1.25876 & 1.911742 & $\mathrm{H}$ & 0.689321 & -1.49032 & -1.70754 \\
\hline $\mathrm{C}$ & 0.276099 & 4.104675 & 0.149946 & $\mathrm{C}$ & -1.07413 & 4.241946 & 0.665865 \\
\hline $\mathrm{O}$ & 4.532735 & -0.81214 & -1.15271 & $\mathrm{O}$ & -3.85569 & -0.90965 & -0.61966 \\
\hline $\mathrm{C}$ & 4.979876 & -0.56205 & -2.50401 & $\mathrm{C}$ & -5.15271 & -0.92325 & -1.25248 \\
\hline $\mathrm{O}$ & -1.08565 & 2.605322 & 1.554462 & $\mathrm{O}$ & -0.33175 & 3.035629 & -1.35351 \\
\hline $\mathrm{C}$ & -0.91079 & 3.338935 & 2.75877 & $\mathrm{C}$ & -1.51809 & 3.346831 & -2.07532 \\
\hline $\mathrm{H}$ & -3.36094 & 1.897778 & -2.71238 & $\mathrm{H}$ & 3.834488 & 2.381048 & 0.057284 \\
\hline $\mathrm{H}$ & -1.84006 & 1.027099 & -2.8521 & $\mathrm{H}$ & 3.411014 & 1.940606 & 1.690897 \\
\hline $\mathrm{H}$ & -2.59006 & 2.609805 & -0.33988 & $\mathrm{H}$ & 1.282152 & 0.892964 & 0.808204 \\
\hline $\mathrm{H}$ & -1.73229 & 3.297577 & -1.71372 & $\mathrm{H}$ & 1.725953 & 1.417258 & -0.81107 \\
\hline $\mathrm{H}$ & -0.77817 & 0.825145 & -0.25012 & $\mathrm{H}$ & 1.134116 & 3.202091 & 1.588175 \\
\hline $\mathrm{H}$ & -1.70711 & -1.11872 & -2.01583 & $\mathrm{H}$ & 2.178062 & -0.76329 & 0.375629 \\
\hline $\mathrm{H}$ & -2.96328 & -3.14512 & -1.69545 & $\mathrm{H}$ & 3.711995 & -2.82186 & 1.029175 \\
\hline $\mathrm{H}$ & -4.32635 & -2.31134 & -0.9992 & $\mathrm{H}$ & 4.753338 & -2.28882 & -0.2698 \\
\hline $\mathrm{H}$ & -3.14669 & -2.02534 & 1.151083 & $\mathrm{H}$ & 2.852565 & -2.45101 & -1.8931 \\
\hline $\mathrm{H}$ & -3.33207 & -3.70529 & 0.670244 & $\mathrm{H}$ & 3.263581 & -3.98495 & -1.13879 \\
\hline $\mathrm{H}$ & 2.000852 & 1.967883 & 0.325991 & $\mathrm{H}$ & -0.78988 & 1.471864 & 1.575051 \\
\hline $\mathrm{H}$ & 1.685674 & 2.67652 & 1.877841 & $\mathrm{H}$ & -2.22253 & 1.988098 & 0.742307 \\
\hline $\mathrm{H}$ & 1.68753 & 0.542986 & 2.676018 & $\mathrm{H}$ & -1.92814 & 0.519652 & -1.06714 \\
\hline
\end{tabular}




$\begin{array}{lrrrrrrr}\mathrm{H} & 0.035515 & 0.532243 & 2.110908 & \mathrm{H} & -0.19685 & 0.474443 & -0.91301 \\ \mathrm{H} & 1.419093 & -2.78539 & 1.058867 & \mathrm{H} & -1.3508 & -3.00299 & 0.036683 \\ \mathrm{H} & 1.271484 & -1.96126 & 2.597837 & \mathrm{H} & -1.75682 & -2.01014 & -1.346 \\ \mathrm{H} & 0.883697 & -0.53032 & -0.05924 & \mathrm{H} & -0.37487 & -0.90123 & 1.132631 \\ \mathrm{H} & 4.894529 & -0.95753 & 1.209292 & \mathrm{H} & -3.26936 & -1.18684 & 3.233507 \\ \mathrm{H} & 3.600914 & -1.05869 & 2.524327 & \mathrm{H} & -1.41855 & -1.09902 & 3.13651 \\ \mathrm{H} & -5.00073 & -0.42947 & -0.31852 & \mathrm{H} & 5.942188 & -0.64161 & 0.337394 \\ \mathrm{H} & -4.48463 & 1.237608 & -0.07274 & \mathrm{H} & 5.829523 & 0.970364 & -0.38857 \\ \mathrm{H} & -5.30524 & 0.79627 & -1.55981 & \mathrm{H} & 5.846989 & 0.796713 & 1.358135 \\ \mathrm{H} & -0.5456 & -3.46421 & -1.30755 & \mathrm{H} & 1.518452 & -3.51062 & 1.587059 \\ \mathrm{H} & 0.347307 & -4.24135 & 0.008722 & \mathrm{H} & 0.100657 & -4.26859 & 0.850283 \\ \mathrm{H} & -1.23604 & -4.85863 & -0.46832 & \mathrm{H} & 1.701529 & -4.98133 & 0.624451 \\ \mathrm{H} & 1.008499 & 1.143306 & -1.56544 & \mathrm{H} & 1.449061 & 4.053499 & -1.04028 \\ \mathrm{H} & -0.64475 & 4.64677 & -0.07576 & \mathrm{H} & -0.51571 & 5.134875 & 0.380607 \\ \mathrm{H} & 0.814202 & 4.651366 & 0.927424 & \mathrm{H} & -2.10761 & 4.34398 & 0.33007 \\ \mathrm{H} & 0.896667 & 4.092476 & -0.74734 & \mathrm{H} & -1.08836 & 4.178938 & 1.757155 \\ \mathrm{H} & 6.005509 & -0.91933 & -2.53677 & \mathrm{H} & -4.95579 & -0.83258 & -2.31749 \\ \mathrm{H} & 4.359504 & -1.10951 & -3.21346 & \mathrm{H} & -5.66922 & -1.85881 & -1.03664 \\ \mathrm{H} & 4.937006 & 0.504468 & -2.72523 & \mathrm{H} & -5.75497 & -0.08539 & -0.90069 \\ \mathrm{H} & -1.802 & 3.154398 & 3.359998 & \mathrm{H} & -1.29312 & 3.164215 & -3.12671 \\ \mathrm{H} & -0.82844 & 4.416391 & 2.580367 & \mathrm{H} & -1.80964 & 4.39512 & -1.95527 \\ \mathrm{H} & -0.03427 & 3.00814 & 3.327874 & \mathrm{H} & -2.35739 & 2.707961 & -1.78097 \\ & & & & & & & \end{array}$

\begin{tabular}{|c|c|c|c|c|c|c|c|}
\hline \multicolumn{4}{|l|}{ 3f_11 } & \multicolumn{4}{|l|}{ 3f_12 } \\
\hline $\mathrm{C}$ & 3.210632 & 1.39811 & 1.065596 & $\mathrm{C}$ & 3.095722 & -0.83928 & 1.905462 \\
\hline $\mathrm{C}$ & 3.867995 & 0.099979 & 0.614312 & $\mathrm{C}$ & 2.807344 & -2.07828 & 1.058407 \\
\hline $\mathrm{C}$ & 1.878385 & 1.609159 & 0.326353 & $\mathrm{C}$ & 2.228584 & 0.405129 & 1.620229 \\
\hline $\mathrm{C}$ & 1.146526 & 2.90393 & 0.68266 & $\mathrm{C}$ & 2.725676 & 1.26765 & 0.461673 \\
\hline $\mathrm{C}$ & 3.335912 & -1.07483 & 0.976579 & $\mathrm{C}$ & 1.5867 & -2.62991 & 1.046119 \\
\hline $\mathrm{C}$ & 3.745325 & -2.45438 & 0.531254 & $\mathrm{C}$ & 1.152095 & -3.89533 & 0.347502 \\
\hline $\mathrm{C}$ & 2.951449 & -2.98149 & -0.68779 & $\mathrm{C}$ & 0.456886 & -3.71886 & -1.02177 \\
\hline $\mathrm{C}$ & -0.28702 & 3.022582 & 0.08191 & $\mathrm{C}$ & 1.865565 & 2.549703 & 0.190321 \\
\hline $\mathrm{C}$ & -1.172 & 1.785225 & 0.409884 & $\mathrm{C}$ & 0.339565 & 2.303039 & 0.174736 \\
\hline $\mathrm{C}$ & 1.449683 & -3.17947 & -0.51308 & $\mathrm{C}$ & -0.84503 & -2.92706 & -1.05252 \\
\hline $\mathrm{C}$ & 0.511056 & -2.20277 & -1.11584 & $\mathrm{C}$ & -0.86066 & -1.6012 & -1.71262 \\
\hline $\mathrm{C}$ & -1.06774 & 0.566098 & -0.53404 & $\mathrm{C}$ & -0.17759 & 1.310311 & -0.88383 \\
\hline $\mathrm{C}$ & -0.94143 & -2.00312 & -0.73487 & $\mathrm{C}$ & -1.88215 & -0.50332 & -1.50739 \\
\hline $\mathrm{C}$ & -1.20374 & -0.79198 & 0.198845 & $\mathrm{C}$ & -1.48036 & 0.591523 & -0.4825 \\
\hline $\mathrm{C}$ & -2.53139 & -0.93663 & 0.929591 & $\mathrm{C}$ & -2.66913 & 1.521875 & -0.27508 \\
\hline $\mathrm{C}$ & -3.78504 & -0.94619 & 0.109939 & $\mathrm{C}$ & -3.74534 & 1.13813 & 0.700667 \\
\hline $\mathrm{O}$ & -3.81105 & -0.8905 & -1.10332 & $\mathrm{O}$ & -4.74457 & 1.787714 & 0.927036 \\
\hline $\mathrm{C}$ & -2.59812 & -1.06899 & 2.258836 & $\mathrm{C}$ & -2.86124 & 2.661564 & -0.94508 \\
\hline $\mathrm{C}$ & 5.053199 & 0.258992 & -0.30338 & $\mathrm{C}$ & 3.992804 & -2.62053 & 0.303095 \\
\hline $\mathrm{C}$ & 1.037805 & -4.09854 & 0.615702 & $\mathrm{C}$ & -1.8985 & -3.33874 & -0.04878 \\
\hline $\mathrm{O}$ & 1.924641 & 4.042695 & 0.294403 & $\mathrm{O}$ & 2.83014 & 0.458285 & -0.71271 \\
\hline $\mathrm{O}$ & 0.804904 & -3.44668 & -1.7881 & $\mathrm{O}$ & -1.33273 & -2.77252 & -2.41353 \\
\hline $\mathrm{H}$ & 0.976627 & -1.34515 & -1.6002 & $\mathrm{H}$ & 0.101659 & -1.25909 & -2.08865 \\
\hline $\mathrm{C}$ & -0.95083 & 4.284495 & 0.653443 & $\mathrm{C}$ & 2.209375 & 3.617388 & 1.237532 \\
\hline $\mathrm{O}$ & -4.90384 & -1.0247 & 0.852852 & $\mathrm{O}$ & -3.4836 & -0.0286 & 1.319158 \\
\hline
\end{tabular}




\begin{tabular}{|c|c|c|c|c|c|c|c|}
\hline $\mathrm{C}$ & -6.15034 & -1.05074 & 0.128424 & $\mathrm{C}$ & -4.46314 & -0.4757 & 2.278947 \\
\hline $\mathrm{O}$ & -0.03052 & 3.153378 & -1.3347 & $\mathrm{O}$ & 2.351507 & 2.952705 & -1.11595 \\
\hline $\mathrm{C}$ & -1.11221 & 3.546805 & -2.17125 & $\mathrm{C}$ & 1.846779 & 4.153619 & -1.68 \\
\hline $\mathrm{H}$ & 3.874558 & 2.246431 & 0.881115 & $\mathrm{H}$ & 4.151499 & -0.56848 & 1.80357 \\
\hline $\mathrm{H}$ & 3.019111 & 1.371034 & 2.144387 & $\mathrm{H}$ & 2.958714 & -1.10904 & 2.95849 \\
\hline $\mathrm{H}$ & 1.242573 & 0.757582 & 0.564559 & $\mathrm{H}$ & 2.212856 & 1.029467 & 2.5184 \\
\hline $\mathrm{H}$ & 2.048477 & 1.586863 & -0.75526 & $\mathrm{H}$ & 1.199827 & 0.08809 & 1.4405 \\
\hline $\mathrm{H}$ & 1.048665 & 2.971393 & 1.771177 & $\mathrm{H}$ & 3.73326 & 1.631716 & 0.71491 \\
\hline $\mathrm{H}$ & 2.468853 & -1.04194 & 1.635796 & $\mathrm{H}$ & 0.807391 & -2.13331 & 1.62323 \\
\hline $\mathrm{H}$ & 3.632512 & -3.15467 & 1.3657 & $\mathrm{H}$ & 0.479059 & -4.4458 & 1.0145 \\
\hline $\mathrm{H}$ & 4.804464 & -2.47686 & 0.262413 & $\mathrm{H}$ & 2.009891 & -4.55301 & 0.18636 \\
\hline $\mathrm{H}$ & 3.111265 & -2.31056 & -1.53686 & $\mathrm{H}$ & 1.155387 & -3.26168 & -1.728 \\
\hline $\mathrm{H}$ & 3.365504 & -3.95694 & -0.97323 & $\mathrm{H}$ & 0.224061 & -4.718 & $-1.411 \delta$ \\
\hline $\mathrm{H}$ & -0.9258 & 1.485398 & 1.43499 & $\mathrm{H}$ & 0.060507 & 1.950705 & 1.17246 \\
\hline $\mathrm{H}$ & -2.21588 & 2.108724 & 0.455618 & $\mathrm{H}$ & -0.16375 & 3.267835 & $0.06800^{\prime}$ \\
\hline $\mathrm{H}$ & -1.84049 & 0.625181 & -1.3025 & $\mathrm{H}$ & -0.30204 & 1.814373 & -1.8484 \\
\hline $\mathrm{H}$ & -0.1173 & 0.583571 & -1.06509 & $\mathrm{H}$ & 0.584608 & 0.551147 & $-1.0457^{\prime}$ \\
\hline $\mathrm{H}$ & -1.31396 & -2.91214 & -0.2589 & $\mathrm{H}$ & -2.83387 & -0.94589 & -1.209 \\
\hline $\mathrm{H}$ & -1.51762 & -1.87943 & -1.65616 & $\mathrm{H}$ & -2.05373 & -0.0242 & -2.479 \\
\hline $\mathrm{H}$ & -0.43927 & -0.8182 & 0.983896 & $\mathrm{H}$ & -1.29228 & 0.089102 & 0.4704 \\
\hline $\mathrm{H}$ & -3.54323 & -1.17999 & 2.773087 & $\mathrm{H}$ & -3.75072 & 3.254442 & -0.7731 \\
\hline $\mathrm{H}$ & -1.69744 & -1.07215 & 2.862729 & $\mathrm{H}$ & -2.14817 & 3.027244 & -1.672 \\
\hline $\mathrm{H}$ & 5.464756 & -0.69001 & -0.64751 & $\mathrm{H}$ & 4.360539 & -1.85162 & -0.384 \\
\hline $\mathrm{H}$ & 4.776803 & 0.845769 & -1.18749 & $\mathrm{H}$ & 4.816661 & -2.85547 & 0.9875 \\
\hline $\mathrm{H}$ & 5.85286 & 0.816256 & 0.198204 & $\mathrm{H}$ & 3.766365 & -3.5159 & -0.276 \\
\hline $\mathrm{H}$ & 1.270297 & -3.65489 & 1.587507 & $\mathrm{H}$ & -1.57737 & -3.11611 & 0.9718 \\
\hline $\mathrm{H}$ & -0.02794 & -4.32068 & 0.586749 & $\mathrm{H}$ & -2.84335 & -2.8283 & -0.2287 \\
\hline $\mathrm{H}$ & 1.582483 & -5.04491 & 0.541633 & $\mathrm{H}$ & -2.07552 & -4.41668 & -0.1169 \\
\hline $\mathrm{H}$ & 1.783772 & 4.12355 & -0.65942 & $\mathrm{H}$ & 2.913086 & 1.09129 & $-1.4405 t$ \\
\hline $\mathrm{H}$ & -0.32473 & 5.16141 & 0.484869 & $\mathrm{H}$ & 3.269342 & 3.876703 & 1.18264 \\
\hline $\mathrm{H}$ & -1.93401 & 4.456299 & 0.211715 & $\mathrm{H}$ & 1.622413 & 4.525957 & $1.08894^{\prime}$ \\
\hline $\mathrm{H}$ & -1.09209 & 4.165387 & 1.731041 & $\mathrm{H}$ & 1.995449 & 3.255269 & 2.24510 \\
\hline $\mathrm{H}$ & -6.92472 & -1.11682 & 0.888338 & $\mathrm{H}$ & -4.56812 & 0.255274 & 3.081073 \\
\hline $\mathrm{H}$ & -6.26838 & -0.13968 & -0.45913 & $\mathrm{H}$ & -5.42834 & -0.62476 & 1.79418 \\
\hline $\mathrm{H}$ & -6.18869 & -1.91703 & -0.53285 & $\mathrm{H}$ & -4.08127 & -1.41642 & $2.66685 \mathrm{c}$ \\
\hline $\mathrm{H}$ & -0.76744 & 3.420273 & -3.19816 & $\mathrm{H}$ & 2.098612 & 5.031924 & -1.08272 \\
\hline $\mathrm{H}$ & -1.38874 & 4.595391 & -2.02093 & $\mathrm{H}$ & 0.761996 & 4.120477 & -1.8309 \\
\hline $\mathrm{H}$ & -1.9976 & 2.920456 & -2.01934 & $\mathrm{H}$ & 2.325539 & 4.251675 & -2.6602 \\
\hline 3f_13 & & & & & & & \\
\hline $\mathrm{C}$ & 2.907704 & 2.525742 & 0.196528 & $\mathrm{C}$ & -2.26872 & 1.965787 & 0.83794 \\
\hline $\mathrm{C}$ & 3.602012 & 1.216237 & 0.523104 & $\mathrm{C}$ & -1.07202 & 2.866112 & 1.062572 \\
\hline $\mathrm{C}$ & 1.428425 & 2.50483 & 0.672238 & $\mathrm{C}$ & -2.14526 & 0.618598 & 1.579527 \\
\hline $\mathrm{C}$ & 0.436091 & 3.052038 & -0.35774 & $\mathrm{C}$ & -3.16353 & -0.46462 & $1.17476 \mathrm{~S}$ \\
\hline $\mathrm{C}$ & 3.761887 & 0.294103 & -0.4351 & $\mathrm{C}$ & -0.31686 & 3.237191 & 0.02056 \\
\hline $\mathrm{C}$ & 4.349471 & -1.08861 & -0.338 & $\mathrm{C}$ & 0.936588 & 4.073079 & 0.01445 \\
\hline $\mathrm{C}$ & 3.555541 & -2.15235 & -1.12524 & $\mathrm{C}$ & 1.938414 & 3.622535 & -1.06466 \\
\hline $\mathrm{C}$ & -1.06549 & 2.828507 & 0.030368 & $\mathrm{C}$ & -2.91245 & -1.2459 & -0.1528 \\
\hline$C$ & -1.36993 & 1.418063 & 0.583516 & $\mathrm{C}$ & -1.45057 & -1.77203 & -0.2602 \\
\hline
\end{tabular}




\begin{tabular}{|c|c|c|c|c|c|c|c|}
\hline $\mathrm{C}$ & 2.236584 & -2.65036 & -0.54101 & $\mathrm{C}$ & 2.512002 & 2.218231 & -0.92186 \\
\hline $\mathrm{C}$ & 0.948178 & -2.16345 & -1.0906 & $\mathrm{C}$ & 1.901842 & 1.110794 & -1.6925 \\
\hline $\mathrm{C}$ & -1.00853 & 0.222128 & -0.3165 & $\mathrm{C}$ & -0.42538 & -0.88982 & -1.00489 \\
\hline $\mathrm{C}$ & -0.41078 & -2.2865 & -0.42675 & $\mathrm{C}$ & 2.073476 & -0.37115 & -1.43258 \\
\hline $\mathrm{C}$ & -0.84402 & -1.09981 & 0.470395 & $\mathrm{C}$ & 1.032568 & -1.08572 & -0.52922 \\
\hline $\mathrm{C}$ & -2.05291 & -1.472 & 1.320845 & $\mathrm{C}$ & 1.463534 & -2.54635 & -0.42596 \\
\hline $\mathrm{C}$ & -3.39875 & -1.77585 & 0.723091 & $\mathrm{C}$ & 2.359545 & -2.98197 & 0.697153 \\
\hline $\mathrm{O}$ & -4.37874 & -2.10228 & 1.358914 & $\mathrm{O}$ & 2.750349 & -4.11655 & 0.875233 \\
\hline $\mathrm{C}$ & -1.97369 & -1.56202 & 2.652658 & $\mathrm{C}$ & 1.146343 & -3.48109 & -1.3262 \\
\hline $\mathrm{C}$ & 4.001812 & 1.040231 & 1.966393 & $\mathrm{C}$ & -0.81301 & 3.2877 & 2.488496 \\
\hline $\mathrm{C}$ & 2.329103 & -3.28418 & 0.829745 & $\mathrm{C}$ & 3.344914 & 1.968929 & 0.315076 \\
\hline $\mathrm{O}$ & 0.720684 & 2.453551 & -1.62623 & $\mathrm{O}$ & -4.49113 & 0.071735 & 1.167921 \\
\hline $\mathrm{O}$ & 1.504875 & -3.42334 & -1.53049 & $\mathrm{O}$ & 3.102143 & 1.764608 & -2.16867 \\
\hline $\mathrm{H}$ & 1.021711 & -1.38363 & -1.8474 & $\mathrm{H}$ & 1.022157 & 1.371069 & -2.27924 \\
\hline $\mathrm{C}$ & -1.48868 & 3.891788 & 1.052484 & $\mathrm{C}$ & -3.87743 & -2.44337 & -0.1785 \\
\hline $\mathrm{O}$ & -3.41717 & -1.64848 & -0.6163 & $\mathrm{O}$ & 2.698954 & -1.96242 & 1.508223 \\
\hline $\mathrm{C}$ & -4.67293 & -1.93493 & -1.26761 & $\mathrm{C}$ & 3.560447 & -2.29013 & 2.617976 \\
\hline $\mathrm{O}$ & -1.73514 & 3.062047 & -1.23614 & $\mathrm{O}$ & -3.23602 & -0.28768 & -1.1866 \\
\hline $\mathrm{C}$ & -3.15058 & 2.932257 & -1.28978 & $\mathrm{C}$ & -3.47125 & -0.77502 & -2.50358 \\
\hline $\mathrm{H}$ & 2.931007 & 2.681016 & -0.88199 & $\mathrm{H}$ & -2.38767 & 1.786447 & -0.23148 \\
\hline $\mathrm{H}$ & 3.438672 & 3.364636 & 0.659935 & $\mathrm{H}$ & -3.18261 & 2.464644 & 1.183002 \\
\hline $\mathrm{H}$ & 1.313087 & 3.060323 & 1.606577 & $\mathrm{H}$ & -2.28233 & 0.790651 & 2.651676 \\
\hline $\mathrm{H}$ & 1.166508 & 1.468644 & 0.888198 & $\mathrm{H}$ & -1.13139 & 0.224857 & 1.466934 \\
\hline $\mathrm{H}$ & 0.574226 & 4.139518 & -0.45252 & $\mathrm{H}$ & -3.15545 & -1.22012 & 1.966171 \\
\hline $\mathrm{H}$ & 3.4345 & 0.565668 & -1.43866 & $\mathrm{H}$ & -0.63682 & 2.900966 & -0.96501 \\
\hline $\mathrm{H}$ & 4.47961 & -1.4022 & 0.700137 & $\mathrm{H}$ & 1.417059 & 4.076612 & 0.995773 \\
\hline $\mathrm{H}$ & 5.358788 & -1.07169 & -0.77101 & $\mathrm{H}$ & 0.684354 & 5.120413 & -0.19962 \\
\hline $\mathrm{H}$ & 3.360648 & -1.78224 & -2.13679 & $\mathrm{H}$ & 1.462907 & 3.689617 & -2.04839 \\
\hline $\mathrm{H}$ & 4.188466 & -3.04089 & -1.23958 & $\mathrm{H}$ & 2.784173 & 4.320304 & -1.07994 \\
\hline $\mathrm{H}$ & -0.84543 & 1.328867 & 1.539555 & $\mathrm{H}$ & -1.09982 & -1.96373 & 0.760078 \\
\hline $\mathrm{H}$ & -2.43158 & 1.377584 & 0.846173 & $\mathrm{H}$ & -1.47517 & -2.75448 & -0.73557 \\
\hline $\mathrm{H}$ & -1.76325 & 0.096919 & -1.09547 & $\mathrm{H}$ & -0.49511 & -1.08147 & -2.081 \\
\hline $\mathrm{H}$ & -0.06969 & 0.426218 & -0.82908 & $\mathrm{H}$ & -0.67601 & 0.164135 & -0.87828 \\
\hline $\mathrm{H}$ & -0.42049 & -3.201 & 0.169754 & $\mathrm{H}$ & 3.069779 & -0.53784 & -1.0177 \\
\hline $\mathrm{H}$ & -1.14926 & -2.42849 & -1.22105 & $\mathrm{H}$ & 2.066703 & -0.86501 & -2.41209 \\
\hline $\mathrm{H}$ & -0.03381 & -0.93641 & 1.190843 & $\mathrm{H}$ & 1.105233 & -0.65295 & 0.470963 \\
\hline $\mathrm{H}$ & -2.83832 & -1.83814 & 3.243307 & $\mathrm{H}$ & 1.517707 & -4.49285 & -1.2228 \\
\hline $\mathrm{H}$ & -1.04576 & -1.36342 & 3.175929 & $\mathrm{H}$ & 0.516208 & -3.26414 & -2.18043 \\
\hline $\mathrm{H}$ & 3.147025 & 1.208821 & 2.631276 & $\mathrm{H}$ & -1.70576 & 3.768499 & 2.905504 \\
\hline $\mathrm{H}$ & 4.408078 & 0.052505 & 2.184443 & $\mathrm{H}$ & -0.59705 & 2.427095 & 3.129986 \\
\hline $\mathrm{H}$ & 4.759058 & 1.783576 & 2.24252 & $\mathrm{H}$ & 0.01574 & 3.989517 & 2.578783 \\
\hline $\mathrm{H}$ & 2.61069 & -2.54296 & 1.582269 & $\mathrm{H}$ & 2.744405 & 2.105812 & 1.218061 \\
\hline $\mathrm{H}$ & 1.385519 & -3.73582 & 1.132772 & $\mathrm{H}$ & 3.75811 & 0.961379 & 0.331819 \\
\hline $\mathrm{H}$ & 3.094851 & -4.06608 & 0.827493 & $\mathrm{H}$ & 4.175279 & 2.6808 & 0.353806 \\
\hline $\mathrm{H}$ & -0.09081 & 2.575929 & -2.14033 & $\mathrm{H}$ & -4.60131 & 0.456909 & 0.287545 \\
\hline $\mathrm{H}$ & -1.38418 & 4.89122 & 0.62389 & $\mathrm{H}$ & -4.90937 & -2.11635 & -0.04443 \\
\hline $\mathrm{H}$ & -2.52608 & 3.755905 & 1.365381 & $\mathrm{H}$ & -3.79712 & -3.00279 & -1.11209 \\
\hline $\mathrm{H}$ & -0.86853 & 3.834962 & 1.949691 & $\mathrm{H}$ & -3.62547 & -3.12832 & 0.63509 \\
\hline $\mathrm{H}$ & -4.96713 & -2.96847 & -1.08348 & $\mathrm{H}$ & 3.076986 & -3.01839 & 3.269659 \\
\hline
\end{tabular}




\begin{tabular}{|c|c|c|c|c|c|c|c|}
\hline $\mathrm{H}$ & -5.45116 & -1.26457 & -0.9025 & $\mathrm{H}$ & 4.505841 & -2.69613 & 2.257195 \\
\hline $\mathrm{H}$ & -4.49297 & -1.77172 & -2.32694 & $\mathrm{H}$ & 3.722827 & -1.35397 & 3.145702 \\
\hline $\mathrm{H}$ & -3.65282 & 3.617939 & -0.59996 & $\mathrm{H}$ & -4.42001 & -1.31567 & -2.57775 \\
\hline $\mathrm{H}$ & -3.48078 & 1.90945 & -1.08089 & $\mathrm{H}$ & -2.6631 & -1.42582 & -2.85339 \\
\hline $\mathrm{H}$ & -3.43958 & 3.190831 & -2.30876 & $\mathrm{H}$ & -3.51739 & 0.101966 & -3.15025 \\
\hline 3f_15 & & & & $3 f$ & & & \\
\hline $\mathrm{C}$ & 1.732558 & 2.426208 & 0.98349 & $\mathrm{C}$ & 3.456737 & 2.042905 & 0.658512 \\
\hline $\mathrm{C}$ & 3.005524 & 1.815181 & 0.433898 & $\mathrm{C}$ & 3.999137 & 0.674115 & 0.264135 \\
\hline $\mathrm{C}$ & 0.784228 & 3.008795 & -0.08665 & $\mathrm{C}$ & 1.963751 & 2.195958 & 1.008295 \\
\hline $\mathrm{C}$ & -0.61344 & 3.362902 & 0.471515 & $\mathrm{C}$ & 1.120436 & 2.75249 & -0.14093 \\
\hline $\mathrm{C}$ & 3.272878 & 0.524702 & 0.677214 & $\mathrm{C}$ & 3.333274 & -0.47603 & 0.431126 \\
\hline $\mathrm{C}$ & 4.439979 & -0.30845 & 0.222103 & $\mathrm{C}$ & 3.817039 & -1.87835 & 0.163113 \\
\hline $\mathrm{C}$ & 4.060646 & -1.35874 & -0.84917 & $\mathrm{C}$ & 2.941602 & -2.69955 & -0.80924 \\
\hline $\mathrm{C}$ & -1.76198 & 2.375849 & 0.118114 & $\mathrm{C}$ & -0.41458 & 2.841942 & 0.145731 \\
\hline $\mathrm{C}$ & -1.52746 & 0.948464 & 0.709329 & $\mathrm{C}$ & -1.04228 & 1.543437 & 0.700207 \\
\hline $\mathrm{C}$ & 2.912738 & -2.28551 & -0.47448 & $\mathrm{C}$ & 1.532576 & -3.05658 & -0.34835 \\
\hline $\mathrm{C}$ & 1.560523 & -1.96627 & -0.98166 & $\mathrm{C}$ & 0.373838 & -2.3697 & -0.9636 \\
\hline $\mathrm{C}$ & -0.71138 & -0.03871 & -0.13977 & $\mathrm{C}$ & -0.93371 & 0.301933 & -0.19664 \\
\hline $\mathrm{C}$ & 0.247977 & -2.41894 & -0.38163 & $\mathrm{C}$ & -1.01658 & -2.25993 & -0.37194 \\
\hline $\mathrm{C}$ & -0.41956 & -1.3875 & 0.5689 & $\mathrm{C}$ & -1.22663 & -1.02448 & 0.540725 \\
\hline $\mathrm{C}$ & -1.61618 & -2.01531 & 1.274445 & $\mathrm{C}$ & -2.57856 & -1.0763 & 1.24141 \\
\hline $\mathrm{C}$ & -2.85181 & -2.45018 & 0.536804 & $\mathrm{C}$ & -3.88161 & -0.9786 & 0.497203 \\
\hline $\mathrm{O}$ & -3.79675 & -3.01622 & 1.044163 & $\mathrm{O}$ & -4.97752 & -1.04483 & 1.012663 \\
\hline $\mathrm{C}$ & -1.61496 & -2.23783 & 2.592824 & $\mathrm{C}$ & -2.67503 & -1.22875 & 2.566514 \\
\hline $\mathrm{C}$ & 3.89263 & 2.742769 & -0.35819 & $\mathrm{C}$ & 5.406914 & 0.765565 & -0.27743 \\
\hline $\mathrm{C}$ & 3.13468 & -3.15246 & 0.74538 & $\mathrm{C}$ & 1.428921 & -3.75026 & 0.992336 \\
\hline $\mathrm{O}$ & -1.02557 & 4.658966 & 0.005117 & $\mathrm{O}$ & 1.379473 & 1.99066 & -1.32182 \\
\hline $\mathrm{O}$ & 2.361209 & -2.9856 & -1.62166 & $\mathrm{O}$ & 0.768779 & -3.68382 & -1.4143 \\
\hline $\mathrm{H}$ & 1.49102 & -1.06205 & -1.58365 & $\mathrm{H}$ & 0.621047 & -1.59519 & -1.68757 \\
\hline $\mathrm{C}$ & -3.06941 & 2.934669 & 0.706952 & $\mathrm{C}$ & -0.68678 & 4.005254 & 1.108913 \\
\hline $\mathrm{O}$ & -2.8126 & -2.14139 & -0.7726 & $\mathrm{O}$ & -3.71661 & -0.80544 & -0.82714 \\
\hline $\mathrm{C}$ & -3.95887 & -2.53693 & -1.55576 & $\mathrm{C}$ & -4.92357 & -0.71061 & -1.61316 \\
\hline $\mathrm{O}$ & -1.79448 & 2.345669 & -1.32145 & $\mathrm{O}$ & -0.92791 & 3.159306 & -1.17567 \\
\hline $\mathrm{C}$ & -2.94708 & 1.78996 & -1.93844 & $\mathrm{C}$ & -2.33084 & 3.32797 & -1.33613 \\
\hline $\mathrm{H}$ & 1.986419 & 3.234099 & 1.684128 & $\mathrm{H}$ & 3.707943 & 2.746189 & -0.1444 \\
\hline $\mathrm{H}$ & 1.207729 & 1.667671 & 1.569578 & $\mathrm{H}$ & 4.056464 & 2.374831 & 1.516557 \\
\hline $\mathrm{H}$ & 0.68705 & 2.3329 & -0.93793 & $\mathrm{H}$ & 1.870685 & 2.887282 & 1.849762 \\
\hline $\mathrm{H}$ & 1.21578 & 3.930541 & -0.48617 & $\mathrm{H}$ & 1.55485 & 1.246485 & 1.354487 \\
\hline $\mathrm{H}$ & -0.55808 & 3.3853 & 1.568687 & $\mathrm{H}$ & 1.445433 & 3.788016 & -0.32704 \\
\hline $\mathrm{H}$ & 2.542032 & -0.01718 & 1.274619 & $\mathrm{H}$ & 2.322944 & -0.42666 & 0.826512 \\
\hline $\mathrm{H}$ & 4.865878 & -0.82566 & 1.090186 & $\mathrm{H}$ & 3.880424 & -2.4195 & 1.11606 \\
\hline $\mathrm{H}$ & 5.241046 & 0.31282 & -0.18388 & $\mathrm{H}$ & 4.83148 & -1.87207 & -0.23862 \\
\hline $\mathrm{H}$ & 3.793238 & -0.84634 & -1.77785 & $\mathrm{H}$ & 2.87035 & -2.17855 & -1.76862 \\
\hline $\mathrm{H}$ & 4.940202 & -1.97674 & -1.06681 & $\mathrm{H}$ & 3.454716 & -3.64947 & -1.00517 \\
\hline $\mathrm{H}$ & -1.07287 & 1.058935 & 1.700357 & $\mathrm{H}$ & -0.57252 & 1.339556 & 1.667717 \\
\hline $\mathrm{H}$ & -2.50949 & 0.505464 & 0.898972 & $\mathrm{H}$ & -2.09254 & 1.744354 & 0.935583 \\
\hline $\mathrm{H}$ & -1.23263 & -0.233 & -1.07729 & $\mathrm{H}$ & -1.59803 & 0.400298 & -1.0572 \\
\hline $\mathrm{H}$ & 0.25064 & 0.39916 & -0.40396 & $\mathrm{H}$ & 0.078251 & 0.247635 & -0.59273 \\
\hline
\end{tabular}




\begin{tabular}{|c|c|c|c|c|c|c|c|}
\hline $\mathrm{H}$ & 0.39839 & -3.35525 & 0.159831 & $\mathrm{H}$ & -1.23668 & -3.16593 & 0.196608 \\
\hline $\mathrm{H}$ & -0.43628 & -2.63881 & -1.20624 & $\mathrm{H}$ & -1.73034 & -2.22778 & -1.19989 \\
\hline $\mathrm{H}$ & 0.29744 & -1.17746 & 1.370452 & $\mathrm{H}$ & -0.4895 & -1.10204 & 1.348542 \\
\hline $\mathrm{H}$ & -2.46363 & -2.7034 & 3.078219 & $\mathrm{H}$ & -3.64186 & -1.27645 & 3.051912 \\
\hline $\mathrm{H}$ & -0.76875 & -1.95987 & 3.210126 & $\mathrm{H}$ & -1.7917 & -1.30966 & 3.189153 \\
\hline $\mathrm{H}$ & 3.391601 & 3.081274 & -1.27119 & $\mathrm{H}$ & 5.420799 & 1.359578 & -1.19859 \\
\hline $\mathrm{H}$ & 4.119343 & 3.643412 & 0.224179 & $\mathrm{H}$ & 6.058819 & 1.284383 & 0.435156 \\
\hline $\mathrm{H}$ & 4.836659 & 2.282583 & -0.64938 & $\mathrm{H}$ & 5.855541 & -0.20219 & -0.49633 \\
\hline $\mathrm{H}$ & 3.224998 & -2.54694 & 1.650997 & $\mathrm{H}$ & 1.726688 & -3.0838 & 1.805839 \\
\hline $\mathrm{H}$ & 2.3211 & -3.86255 & 0.887924 & $\mathrm{H}$ & 0.414164 & -4.09497 & 1.186549 \\
\hline $\mathrm{H}$ & 4.063018 & -3.72048 & 0.630167 & $\mathrm{H}$ & 2.092357 & -4.62056 & 1.013587 \\
\hline $\mathrm{H}$ & -0.41268 & 5.301574 & 0.377031 & $\mathrm{H}$ & 0.679904 & 2.249847 & -1.93821 \\
\hline $\mathrm{H}$ & -3.28782 & 3.919408 & 0.296793 & $\mathrm{H}$ & -0.30425 & 4.941102 & 0.694741 \\
\hline $\mathrm{H}$ & -3.90978 & 2.266081 & 0.511072 & $\mathrm{H}$ & -1.75578 & 4.123211 & 1.297354 \\
\hline $\mathrm{H}$ & -2.97134 & 3.028173 & 1.792073 & $\mathrm{H}$ & -0.20597 & 3.832158 & 2.073781 \\
\hline $\mathrm{H}$ & -4.08902 & -3.61851 & -1.51518 & $\mathrm{H}$ & -5.50943 & -1.62507 & -1.51824 \\
\hline $\mathrm{H}$ & -4.85884 & -2.04726 & -1.18341 & $\mathrm{H}$ & -5.52193 & 0.1405 & -1.28752 \\
\hline $\mathrm{H}$ & -3.74078 & -2.21574 & -2.57087 & $\mathrm{H}$ & -4.59097 & -0.5742 & -2.63881 \\
\hline $\mathrm{H}$ & -3.82412 & 2.438152 & -1.83403 & $\mathrm{H}$ & -2.72206 & 4.142499 & -0.71815 \\
\hline $\mathrm{H}$ & -3.19516 & 0.796475 & -1.54815 & $\mathrm{H}$ & -2.88672 & 2.411607 & -1.11285 \\
\hline $\mathrm{H}$ & -2.7103 & 1.695703 & -2.99939 & $\mathrm{H}$ & -2.48677 & 3.582864 & -2.3848 \\
\hline 3f_17 & & & & & & & \\
\hline $\mathrm{C}$ & 3.453277 & 1.896808 & 0.681566 & $\mathrm{C}$ & 2.724725 & 1.490264 & -0.53305 \\
\hline $\mathrm{C}$ & 3.943216 & 0.514318 & 0.264261 & $\mathrm{C}$ & 1.876622 & 2.748064 & -0.48958 \\
\hline $\mathrm{C}$ & 1.976654 & 2.090075 & 1.079308 & $\mathrm{C}$ & 2.306526 & 0.39711 & -1.52283 \\
\hline $\mathrm{C}$ & 1.119312 & 2.706214 & -0.03099 & $\mathrm{C}$ & 3.017274 & -0.9582 & -1.35137 \\
\hline $\mathrm{C}$ & 3.271414 & -0.62281 & 0.489196 & $\mathrm{C}$ & 0.649039 & 2.823779 & -1.01976 \\
\hline $\mathrm{C}$ & 3.71717 & -2.03568 & 0.209968 & $\mathrm{C}$ & -0.32257 & 3.97356 & -0.90976 \\
\hline $\mathrm{C}$ & 2.839128 & -2.82253 & -0.78897 & $\mathrm{C}$ & -1.17741 & 3.926627 & 0.377256 \\
\hline $\mathrm{C}$ & -0.40878 & 2.791862 & 0.290883 & $\mathrm{C}$ & 2.559235 & -1.87901 & -0.17481 \\
\hline $\mathrm{C}$ & -1.03713 & 1.472364 & 0.787884 & $\mathrm{C}$ & 1.013308 & -2.00213 & -0.06741 \\
\hline $\mathrm{C}$ & 1.412128 & -3.14824 & -0.36238 & $\mathrm{C}$ & -2.02681 & 2.678864 & 0.580837 \\
\hline $\mathrm{C}$ & 0.285041 & -2.42041 & -0.98843 & $\mathrm{C}$ & -1.49222 & 1.581483 & 1.418022 \\
\hline $\mathrm{C}$ & -0.93424 & 0.262551 & -0.15119 & $\mathrm{C}$ & 0.278943 & -0.96703 & 0.807285 \\
\hline $\mathrm{C}$ & -1.11443 & -2.28606 & -0.42367 & $\mathrm{C}$ & -1.96901 & 0.144375 & 1.424408 \\
\hline $\mathrm{C}$ & -1.30831 & -1.0758 & 0.525605 & $\mathrm{C}$ & -1.22918 & -0.85473 & 0.493331 \\
\hline $\mathrm{C}$ & -2.68736 & -1.09449 & 1.172885 & $\mathrm{C}$ & -1.9774 & -2.18226 & 0.566381 \\
\hline $\mathrm{C}$ & -3.95254 & -0.8787 & 0.389375 & $\mathrm{C}$ & -3.10919 & -2.45127 & -0.38341 \\
\hline $\mathrm{O}$ & -5.06619 & -0.85429 & 0.869584 & $\mathrm{O}$ & -3.77346 & -3.46614 & -0.40377 \\
\hline $\mathrm{C}$ & -2.84424 & -1.3192 & 2.481759 & $\mathrm{C}$ & -1.73704 & -3.12713 & 1.479696 \\
\hline $\mathrm{C}$ & 5.31324 & 0.575444 & -0.3701 & $\mathrm{C}$ & 2.527036 & 3.877238 & 0.273426 \\
\hline $\mathrm{C}$ & 1.262092 & -3.86098 & 0.963867 & $\mathrm{C}$ & -3.12005 & 2.454611 & -0.4399 \\
\hline $\mathrm{O}$ & 1.335188 & 1.993376 & -1.25177 & $\mathrm{O}$ & 4.437432 & -0.78582 & -1.29004 \\
\hline $\mathrm{O}$ & 0.654787 & -3.73674 & -1.45465 & $\mathrm{O}$ & -2.4365 & 2.531296 & 1.966589 \\
\hline $\mathrm{H}$ & 0.566952 & -1.63984 & -1.69297 & $\mathrm{H}$ & -0.4827 & 1.729882 & 1.797158 \\
\hline $\mathrm{C}$ & -0.67595 & 3.887498 & 1.329943 & $\mathrm{C}$ & 3.158045 & -3.27405 & -0.42074 \\
\hline $\mathrm{O}$ & -3.73056 & -0.71182 & -0.92685 & $\mathrm{O}$ & -3.32115 & -1.42862 & -1.23275 \\
\hline & -4.89687 & -0.49702 & -1.74906 & $\mathrm{C}$ & -4.39255 & -1.6014 & -2.18331 \\
\hline
\end{tabular}




\begin{tabular}{|c|c|c|c|c|c|c|c|}
\hline $\mathrm{O}$ & -1.09308 & 3.066407 & -0.96414 & $\mathrm{O}$ & 3.139035 & -1.25765 & 0.996611 \\
\hline $\mathrm{C}$ & -1.02007 & 4.38841 & -1.49841 & $\mathrm{C}$ & 3.217953 & -2.02435 & 2.193854 \\
\hline $\mathrm{H}$ & 3.69867 & 2.596208 & -0.12608 & $\mathrm{H}$ & 2.754995 & 1.077751 & 0.482422 \\
\hline $\mathrm{H}$ & 4.090178 & 2.206856 & 1.520819 & $\mathrm{H}$ & 3.760848 & 1.774767 & -0.75077 \\
\hline $\mathrm{H}$ & 1.928008 & 2.761921 & 1.940572 & $\mathrm{H}$ & 2.519403 & 0.746916 & -2.53898 \\
\hline $\mathrm{H}$ & 1.544175 & 1.146074 & 1.410313 & $\mathrm{H}$ & 1.229868 & 0.222962 & -1.49051 \\
\hline $\mathrm{H}$ & 1.471268 & 3.739558 & -0.17585 & $\mathrm{H}$ & 2.828258 & -1.53003 & -2.26488 \\
\hline $\mathrm{H}$ & 2.290465 & -0.55241 & 0.950186 & $\mathrm{H}$ & 0.261946 & 1.9551 & -1.54232 \\
\hline $\mathrm{H}$ & 3.747572 & -2.58794 & 1.157773 & $\mathrm{H}$ & -0.98026 & 3.975149 & -1.78469 \\
\hline $\mathrm{H}$ & 4.738669 & -2.05365 & -0.17312 & $\mathrm{H}$ & 0.198585 & 4.934679 & -0.92474 \\
\hline $\mathrm{H}$ & 2.80173 & -2.28877 & -1.74321 & $\mathrm{H}$ & -0.52128 & 4.028622 & 1.246146 \\
\hline $\mathrm{H}$ & 3.331572 & -3.78325 & -0.98565 & $\mathrm{H}$ & -1.85334 & 4.790925 & 0.386738 \\
\hline $\mathrm{H}$ & -0.58534 & 1.225463 & 1.753678 & $\mathrm{H}$ & 0.611273 & -1.9727 & -1.08713 \\
\hline $\mathrm{H}$ & -2.08993 & 1.687832 & 0.996529 & $\mathrm{H}$ & 0.774544 & -3.00219 & 0.300709 \\
\hline $\mathrm{H}$ & -1.55839 & 0.424948 & -1.03101 & $\mathrm{H}$ & 0.423289 & -1.20821 & 1.865152 \\
\hline $\mathrm{H}$ & 0.091638 & 0.176815 & -0.50499 & $\mathrm{H}$ & 0.719438 & 0.020301 & 0.671889 \\
\hline $\mathrm{H}$ & -1.37455 & -3.2031 & 0.109403 & $\mathrm{H}$ & -3.03355 & 0.128959 & 1.183263 \\
\hline $\mathrm{H}$ & -1.8077 & -2.20358 & -1.26521 & $\mathrm{H}$ & -1.88569 & -0.2216 & 2.455273 \\
\hline $\mathrm{H}$ & -0.60547 & -1.21225 & 1.355987 & $\mathrm{H}$ & -1.32109 & -0.48368 & -0.53026 \\
\hline $\mathrm{H}$ & -3.83063 & -1.34084 & 2.928118 & $\mathrm{H}$ & -2.33492 & -4.02939 & 1.506178 \\
\hline $\mathrm{H}$ & -1.99203 & -1.48812 & 3.129623 & $\mathrm{H}$ & -0.94869 & -3.02746 & 2.21579 \\
\hline $\mathrm{H}$ & 6.016163 & 1.102378 & 0.285761 & $\mathrm{H}$ & 2.769379 & 3.560597 & 1.294648 \\
\hline $\mathrm{H}$ & 5.735422 & -0.40239 & -0.59663 & $\mathrm{H}$ & 3.476301 & 4.158974 & -0.19654 \\
\hline $\mathrm{H}$ & 5.272026 & 1.148435 & -1.30366 & $\mathrm{H}$ & 1.903691 & 4.768626 & 0.339436 \\
\hline $\mathrm{H}$ & 1.567964 & -3.21879 & 1.793703 & $\mathrm{H}$ & -2.69571 & 2.288338 & -1.43361 \\
\hline $\mathrm{H}$ & 0.232968 & -4.17377 & 1.135113 & $\mathrm{H}$ & -3.74228 & 1.597011 & -0.18832 \\
\hline $\mathrm{H}$ & 1.895494 & -4.75347 & 0.980959 & $\mathrm{H}$ & -3.76254 & 3.338897 & -0.49393 \\
\hline $\mathrm{H}$ & 0.546311 & 2.16239 & -1.78606 & $\mathrm{H}$ & 4.629483 & -0.63196 & -0.35447 \\
\hline $\mathrm{H}$ & -0.20303 & 4.834932 & 1.063963 & $\mathrm{H}$ & 4.237932 & -3.21284 & -0.56288 \\
\hline $\mathrm{H}$ & -1.75082 & 4.053789 & 1.434254 & $\mathrm{H}$ & 2.943586 & -3.95583 & 0.403955 \\
\hline $\mathrm{H}$ & -0.28815 & 3.584876 & 2.304454 & $\mathrm{H}$ & 2.71669 & -3.70463 & -1.32321 \\
\hline $\mathrm{H}$ & -5.57065 & -1.35151 & -1.68198 & $\mathrm{H}$ & -4.20077 & -2.46847 & -2.81575 \\
\hline $\mathrm{H}$ & -5.42116 & 0.40541 & -1.43391 & $\mathrm{H}$ & -5.34214 & -1.73283 & -1.66387 \\
\hline $\mathrm{H}$ & -4.52012 & -0.3859 & -2.76246 & $\mathrm{H}$ & -4.40427 & -0.69065 & -2.7763 \\
\hline $\mathrm{H}$ & 0.011702 & 4.728804 & -1.63915 & $\mathrm{H}$ & 3.979252 & -2.80809 & 2.130345 \\
\hline $\mathrm{H}$ & -1.55182 & 5.111455 & -0.87348 & $\mathrm{H}$ & 2.257924 & -2.48169 & 2.455088 \\
\hline $\mathrm{H}$ & -1.50694 & 4.344203 & -2.47302 & $\mathrm{H}$ & 3.497985 & -1.32733 & 2.984443 \\
\hline
\end{tabular}

\begin{tabular}{lrrrlrrr} 
3g_1 & \multicolumn{7}{c}{$\mathbf{3 g \_ 2}$} \\
C & -3.64586 & 1.305219 & -0.91815 & C & 3.411575 & 0.337534 & 1.795669 \\
C & -3.77395 & 0.03456 & -0.0857 & C & 3.536917 & -0.89971 & 0.914881 \\
C & -2.20193 & 1.830963 & -1.04118 & C & 2.204525 & 1.227775 & 1.443818 \\
C & -1.56064 & 2.187928 & 0.305688 & C & 2.24287 & 1.751301 & 0.004054 \\
C & -3.41372 & -1.14855 & -0.60172 & C & 2.706923 & -1.93732 & 1.084246 \\
C & -3.51635 & -2.51141 & 0.03446 & C & 2.71276 & -3.25424 & 0.349588 \\
C & -2.42285 & -3.50891 & -0.39109 & C & 1.340403 & -3.94788 & 0.268585 \\
C & -0.0725 & 2.624442 & 0.216634 & C & 0.994896 & 2.572834 & -0.42307 \\
C & 0.799655 & 1.625895 & -0.57422 & C & -0.33861 & 1.860482 & -0.11317 \\
C & -1.00444 & -3.27821 & 0.120399 & $\mathrm{C}$ & 0.279712 & -3.34336 & -0.6441
\end{tabular}




\begin{tabular}{|c|c|c|c|c|c|c|c|}
\hline C & 0.060471 & -2.8733 & -0.83012 & $\mathrm{C}$ & -0.89616 & -2.66321 & -0.04467 \\
\hline $\mathrm{C}$ & 0.77656 & 0.177138 & -0.063 & $\mathrm{C}$ & -0.5215 & 0.46549 & -0.73662 \\
\hline $\mathrm{C}$ & 1.410511 & -2.2686 & -0.49748 & $\mathrm{C}$ & -1.86683 & -1.71952 & -0.72509 \\
\hline $\mathrm{C}$ & 1.576807 & -0.79949 & -0.95568 & $\mathrm{C}$ & -1.79874 & -0.24561 & -0.24813 \\
\hline $\mathrm{C}$ & 3.042448 & -0.42066 & -1.1258 & $\mathrm{C}$ & -3.08736 & 0.463151 & -0.65617 \\
\hline $\mathrm{C}$ & 4.007549 & -0.40238 & 0.026639 & $\mathrm{C}$ & -4.28168 & 0.419715 & 0.256166 \\
\hline $\mathrm{O}$ & 5.18076 & -0.10738 & -0.05925 & $\mathrm{O}$ & -5.36037 & 0.923691 & 0.016989 \\
\hline $\mathrm{C}$ & 3.55101 & -0.09602 & -2.31896 & $\mathrm{C}$ & -3.2563 & 1.085538 & -1.82623 \\
\hline $\mathrm{C}$ & -4.34474 & 0.22123 & 1.299287 & $\mathrm{C}$ & 4.647075 & -0.86182 & -0.10625 \\
\hline $\mathrm{C}$ & -0.86404 & -3.07093 & 1.611311 & $\mathrm{C}$ & 0.691532 & -3.10955 & -2.0791 \\
\hline $\mathrm{O}$ & -2.31664 & 3.214127 & 0.962248 & $\mathrm{O}$ & 3.42094 & 2.540056 & -0.20859 \\
\hline $\mathrm{O}$ & -0.09299 & -4.25563 & -0.44849 & $\mathrm{O}$ & -0.98685 & -4.03633 & -0.47772 \\
\hline $\mathrm{H}$ & -0.26746 & -2.67785 & -1.85282 & $\mathrm{H}$ & -0.87977 & -2.55882 & 1.041763 \\
\hline $\mathrm{C}$ & 0.480945 & 2.851051 & 1.630916 & $\mathrm{C}$ & 1.106429 & 2.937694 & -1.91035 \\
\hline $\mathrm{O}$ & 3.434529 & -0.75398 & 1.192079 & $\mathrm{O}$ & -4.03773 & -0.25688 & 1.394087 \\
\hline $\mathrm{C}$ & 4.296933 & -0.76623 & 2.349332 & $\mathrm{C}$ & -5.13123 & -0.35308 & 2.329388 \\
\hline $\mathrm{O}$ & -0.16423 & 3.877234 & -0.51259 & $\mathrm{O}$ & 1.112392 & 3.753684 & 0.414487 \\
\hline $\mathrm{C}$ & 0.992232 & 4.701524 & -0.59295 & $\mathrm{C}$ & 0.321753 & 4.891862 & 0.092101 \\
\hline $\mathrm{H}$ & -4.2719 & 2.089952 & -0.48542 & $\mathrm{H}$ & 4.323639 & 0.934452 & 1.717911 \\
\hline $\mathrm{H}$ & -4.024 & 1.120922 & -1.92867 & $\mathrm{H}$ & 3.316357 & 0.036548 & 2.84399 \\
\hline $\mathrm{H}$ & -2.18869 & 2.718668 & -1.68192 & $\mathrm{H}$ & 2.161235 & 2.079882 & 2.129906 \\
\hline $\mathrm{H}$ & -1.60336 & 1.068025 & -1.54447 & $\mathrm{H}$ & 1.295256 & 0.644122 & 1.603286 \\
\hline $\mathrm{H}$ & -1.61201 & 1.322512 & 0.969444 & $\mathrm{H}$ & 2.334823 & 0.905807 & -0.68046 \\
\hline $\mathrm{H}$ & -3.05461 & -1.15612 & -1.63161 & $\mathrm{H}$ & 1.970565 & -1.86584 & 1.885311 \\
\hline $\mathrm{H}$ & -4.47473 & -2.96092 & -0.26023 & $\mathrm{H}$ & 3.38281 & -3.94698 & 0.876795 \\
\hline $\mathrm{H}$ & -3.55408 & -2.43935 & 1.123979 & $\mathrm{H}$ & 3.137052 & -3.14746 & -0.6518 \\
\hline $\mathrm{H}$ & -2.71393 & -4.5042 & -0.03364 & $\mathrm{H}$ & 1.496629 & -4.97209 & -0.09091 \\
\hline $\mathrm{H}$ & -2.39041 & -3.57276 & -1.48363 & $\mathrm{H}$ & 0.917609 & -4.03585 & 1.274474 \\
\hline $\mathrm{H}$ & 1.831269 & 1.989434 & -0.56056 & $\mathrm{H}$ & -1.15339 & 2.505285 & -0.45439 \\
\hline $\mathrm{H}$ & 0.483506 & 1.651059 & -1.62155 & $\mathrm{H}$ & -0.44186 & 1.787653 & 0.973717 \\
\hline $\mathrm{H}$ & -0.25296 & -0.19035 & -0.02927 & $\mathrm{H}$ & 0.326269 & -0.17737 & -0.48132 \\
\hline $\mathrm{H}$ & 1.162569 & 0.126183 & 0.956739 & $\mathrm{H}$ & -0.52541 & 0.536251 & -1.82824 \\
\hline $\mathrm{H}$ & 1.629809 & -2.36068 & 0.565852 & $\mathrm{H}$ & -1.7613 & -1.75218 & -1.81267 \\
\hline $\mathrm{H}$ & 2.152014 & -2.88258 & -1.02235 & $\mathrm{H}$ & -2.8644 & -2.11626 & -0.50567 \\
\hline $\mathrm{H}$ & 1.150056 & -0.73112 & -1.96367 & $\mathrm{H}$ & -1.76714 & -0.257 & 0.843164 \\
\hline $\mathrm{H}$ & 4.597624 & 0.160959 & -2.4248 & $\mathrm{H}$ & -4.21118 & 1.529296 & -2.07848 \\
\hline $\mathrm{H}$ & 2.933654 & -0.08145 & -3.20947 & $\mathrm{H}$ & -2.45779 & 1.167634 & -2.55369 \\
\hline $\mathrm{H}$ & -4.23807 & -0.66015 & 1.93241 & $\mathrm{H}$ & 4.594014 & -1.67516 & -0.8305 \\
\hline $\mathrm{H}$ & -3.87385 & 1.071404 & 1.801446 & $\mathrm{H}$ & 4.648442 & 0.088834 & -0.64794 \\
\hline $\mathrm{H}$ & -5.41453 & 0.456898 & 1.235954 & $\mathrm{H}$ & 5.620764 & -0.92774 & 0.396982 \\
\hline $\mathrm{H}$ & 0.179642 & -3.1054 & 1.921311 & $\mathrm{H}$ & -0.16408 & -2.8564 & -2.7036 \\
\hline $\mathrm{H}$ & -1.28274 & -2.10787 & 1.914315 & $\mathrm{H}$ & 1.422177 & -2.30005 & -2.15066 \\
\hline $\mathrm{H}$ & -1.40324 & -3.85654 & 2.149551 & $\mathrm{H}$ & 1.153196 & -4.01405 & -2.48728 \\
\hline $\mathrm{H}$ & -2.06338 & 4.033358 & 0.514186 & $\mathrm{H}$ & 3.213633 & 3.401482 & 0.180426 \\
\hline $\mathrm{H}$ & -0.08575 & 3.630316 & 2.141227 & $\mathrm{H}$ & 2.00106 & 3.534346 & -2.09016 \\
\hline $\mathrm{H}$ & 0.397796 & 1.934408 & 2.21862 & $\mathrm{H}$ & 1.180237 & 2.030754 & -2.5144 \\
\hline $\mathrm{H}$ & 1.535157 & 3.134424 & 1.607723 & $\mathrm{H}$ & 0.232244 & 3.493417 & -2.2565 \\
\hline $\mathrm{H}$ & 3.662792 & -1.06446 & 3.18007 & $\mathrm{H}$ & -4.74558 & -0.92408 & 3.170185 \\
\hline $\mathrm{H}$ & 5.107372 & -1.48187 & 2.209382 & $\mathrm{H}$ & -5.4418 & 0.641227 & 2.651787 \\
\hline
\end{tabular}




$\begin{array}{lrrrr}\mathrm{H} & 4.71345 & 0.226391 & 2.521611 & \mathrm{H} \\ \mathrm{H} & 1.291348 & 5.093072 & 0.384869 & \mathrm{H} \\ \mathrm{H} & 1.843049 & 4.178969 & -1.04265 & \mathrm{H} \\ \mathrm{H} & 0.722386 & 5.540192 & -1.23575 & \mathrm{H}\end{array}$

$\begin{array}{lrrr}\mathrm{H} & -5.97599 & -0.86905 & 1.871858 \\ \mathrm{H} & 0.64419 & 5.367682 & -0.83988 \\ \mathrm{H} & -0.74393 & 4.650622 & 0.018831 \\ \mathrm{H} & 0.460529 & 5.598839 & 0.910868\end{array}$

3g_3

C

$\begin{array}{lll}-3.53452 & 0.199843 & -1.68245\end{array}$

$3 g \_4$

$\begin{array}{llll}\text { C } & -3.54286 & -1.04649 & -0.80535\end{array}$

$\mathrm{C}$

$\mathrm{C}$

$\begin{array}{ll}-3.86361 & 1.201207\end{array}$

$-0.69448$

$\begin{array}{llll}\text { C } & -2.35329 & 1.151145 & -1.40531\end{array}$

$\mathrm{C}$

$\begin{array}{llll}\text { C } & -2.32684 & 1.694923 & 0.02871\end{array}$

$\mathrm{C}$

$\begin{array}{llll}\text { C } & -2.70107 & -2.05682 & -1.06236\end{array}$

C

$\begin{array}{lll}-2.59417 & -3.38288 & -0.35371\end{array}$

$\mathrm{C}$

$\begin{array}{lll}-1.17727 & -3.98461 & -0.32115\end{array}$

$\mathrm{C}$

C

$\begin{array}{lll}-1.09253 & 2.577883 & 0.359409\end{array}$

$-4.04372-0.22098$

$-0.18937$

$\begin{array}{lll}-2.44353 & 1.749248 & -0.88028\end{array}$

$\begin{array}{lll}-1.69966 & 2.008577 & 0.43823\end{array}$

C

$\begin{array}{lll}0.246286 & 1.897269 & 0.001403\end{array}$

$\begin{array}{ll}-3.05929 & -1.12884\end{array}$

$-0.1576$

$\begin{array}{lll}-3.15469 & -2.55982 & 0.320926\end{array}$

$\begin{array}{ll}-2.11678 & -3.51035\end{array}$

$-0.30402$

$\mathrm{C}$

$-0.27234$

2.598042

0.260925

C

$\begin{array}{lll}-0.1448 & -3.33477 & 0.593913\end{array}$

0.646936

1.667532

$-0.56692$

C

$-0.66021$

$-3.31325$

0.103593

$\mathrm{C}$

$\begin{array}{lll}0.990238 & -2.58312 & 0.003873\end{array}$

0.308477

$-2.76738$

$-0.87924$

C

$\begin{array}{lll}0.487155 & 0.523429 & 0.64723\end{array}$

0.874983

0.251878

$-0.01843$

C

1.652758

$-2.132$

$-0.58151$

$\begin{array}{llr}1.807631 & -0.137 & 0.20809\end{array}$

1.716564

$-0.63407$

$-0.9679$

C

3.148022

$-0.14758$

$-1.14884$

4.138177

$-0.12138$

$-0.01752$

$\mathrm{O}$

$\begin{array}{rrr}5.35176 & 1.07489 & 0.056701\end{array}$

5.283218

0.266923

$-0.11122$

C

3.604727

0.27108

$-2.33381$

$\begin{array}{lll}-4.56076 & -1.04826 & 0.309375\end{array}$

$-5.45532$

$-0.5084$

0.263892

C

$-0.38685$

$-3.27578$

1.590916

$\begin{array}{llll}-3.52485 & 2.433296 & 0.303677\end{array}$

$-2.47953$

$2.919359 \quad 1.238893$

$\mathrm{O}$

$\begin{array}{lll}1.155814 & -3.95571 & 0.414666\end{array}$

$\mathrm{O}$

$\mathrm{O}$

$\mathrm{H}$

0.221929

$-4.18743 \quad-0.64797$

$-0.11034$

$-2.47408$

$-1.84382$

0.32843

2.888596

1.643721

$\begin{array}{lll}-1.13398 & 2.987563 & 1.839142\end{array}$

3.624283

$-0.58303$

1.137222

$\begin{array}{llll}5.269007 & -0.40506 & -2.14464\end{array}$

4.516204

$-0.59757$

2.271852

$\begin{array}{lll}-1.30161 & 3.727737 & -0.50208\end{array}$

$-0.48356$

3.816452

$-0.48926$

0.585469

4.746097

$-0.57819$

$\mathrm{H}$

$-4.39854$

1.873226

$-0.01389$

$\mathrm{H}$

$-4.39898$

$-2.50691$

1.283353

$-1.65029$

$\mathrm{H}$

$\mathrm{H}$

$-1.85909$

2.701183

$-1.41299$

$\begin{array}{lll}-1.42608 & 0.611998 & -1.61401\end{array}$

$\begin{array}{lll}-2.33712 & 0.858612 & 0.730943\end{array}$

$-1.59446$

1.079121

$-1.51614$

$\mathrm{H}$

$\begin{array}{llll}-2.04255 & -1.95074 & -1.92552\end{array}$

$-2.07493$

1.069808

0.98743

$\mathrm{H}$

$-3.22715 \quad-4.11029 \quad-0.88051$

$-4.14575$

$-0.82592$

$-0.50062$

$\mathrm{H}$

$\mathrm{H}$

$-3.05939$

$-2.96698$

0.103865

$\begin{array}{lll}-3.0001 & -3.32581 & 0.659003\end{array}$

$-2.37895$

$-2.59822 \quad 1.413593$

$\mathrm{H}$

$-2.18224$

$\begin{array}{ll}-4.53645 & -0.01919\end{array}$

$\begin{array}{lll}-0.76861 & -4.01486 & -1.33646\end{array}$

$\mathrm{H}$

$\mathrm{H}$

1.616616

$-3.46454$

$-1.39569$

$\mathrm{H}$

0.23741

2.163858

$-0.66687$

$\begin{array}{llll}0.299691 & 1.799154 & -1.08677\end{array}$

$-0.0842$

1.602753

$-1.57947$

$\mathrm{H}$

$\mathrm{H}$

1.355804

$-0.25019$

0.127749

$\begin{array}{lll}0.456434 & 0.600115 & 1.738482\end{array}$

1.930943

0.288827

0.960791

$\mathrm{H}$

$-2.26834 \quad 0.462875$ 


$\begin{array}{lrrrlrrr}\mathrm{H} & 2.925823 & -1.96316 & 0.521642 & \mathrm{H} & 2.392519 & -2.67787 & -1.1787 \\ \mathrm{H} & 1.814997 & -0.16336 & -0.8855 & \mathrm{H} & 1.262752 & -0.55108 & -1.96276 \\ \mathrm{H} & 4.094534 & 1.809243 & 2.031505 & \mathrm{H} & 4.628509 & 0.605169 & -2.44688 \\ \mathrm{H} & 2.3239 & 1.464379 & 2.448224 & \mathrm{H} & 2.967411 & 0.286658 & -3.21012 \\ \mathrm{H} & -4.4139 & -1.85486 & 1.028556 & \mathrm{H} & -5.6382 & -1.56309 & 0.468178 \\ \mathrm{H} & -4.54963 & -0.09543 & 0.846778 & \mathrm{H} & -5.68424 & 0.053105 & 1.177546 \\ \mathrm{H} & -5.57117 & -1.15388 & -0.10532 & \mathrm{H} & -6.17646 & -0.17681 & -0.49168 \\ \mathrm{H} & 0.280444 & -2.85906 & 2.660233 & \mathrm{H} & 0.680656 & -3.34167 & 1.797252 \\ \mathrm{H} & -1.33462 & -2.37452 & 2.120892 & \mathrm{H} & -0.76965 & -2.35706 & 2.042722 \\ \mathrm{H} & -0.97634 & -4.07863 & 2.428375 & \mathrm{H} & -0.87849 & -4.12135 & 2.081718 \\ \mathrm{H} & -3.39654 & 3.28663 & -0.13365 & \mathrm{H} & -2.80875 & 2.437357 & 2.001634 \\ \mathrm{H} & -2.03595 & 3.563199 & 2.048766 & \mathrm{H} & -0.25233 & 3.660311 & 2.148673 \\ \mathrm{H} & -1.14581 & 2.101441 & 2.477552 & \mathrm{H} & 0.307265 & 1.988645 & 2.262805 \\ \mathrm{H} & -0.26096 & 3.58259 & 2.115321 & \mathrm{H} & 1.368715 & 3.213446 & 1.57085 \\ \mathrm{H} & 4.940887 & -1.06527 & -2.9432 & \mathrm{H} & 5.37081 & -1.2445 & 2.072582 \\ \mathrm{H} & 5.568754 & 0.56307 & -2.54683 & \mathrm{H} & 4.866523 & 0.411162 & 2.491163 \\ \mathrm{H} & 6.103545 & -0.84703 & -1.5998 & \mathrm{H} & 3.927246 & -0.98637 & 3.098399 \\ \mathrm{H} & -0.62066 & 5.343125 & 0.651048 & \mathrm{H} & 0.830175 & 5.193492 & 0.391612 \\ \mathrm{H} & 0.589353 & 4.635881 & -0.45077 & \mathrm{H} & 1.496003 & 4.307764 & -1.00305 \\ \mathrm{H} & -0.76466 & 5.578183 & -1.10203 & \mathrm{H} & 0.243272 & 5.537883 & -1.24631\end{array}$

\begin{tabular}{|c|c|c|c|c|c|c|c|}
\hline \multicolumn{4}{|c|}{ 3g_5 } & \multicolumn{4}{|c|}{$3 g \_6$} \\
\hline $\mathrm{C}$ & -3.68386 & 1.43765 & -0.72908 & $\mathrm{C}$ & -3.63935 & 1.468181 & -0.92599 \\
\hline $\mathrm{C}$ & -3.74717 & 0.11757 & 0.030693 & $\mathrm{C}$ & -3.83955 & 0.218379 & -0.07671 \\
\hline $\mathrm{C}$ & -2.2627 & 1.999877 & -0.93699 & $\mathrm{C}$ & -2.16803 & 1.912202 & -1.04905 \\
\hline $\mathrm{C}$ & -1.47967 & 2.22906 & 0.353628 & $\mathrm{C}$ & -1.5152 & 2.256721 & 0.295527 \\
\hline $\mathrm{C}$ & -3.47039 & -1.03815 & -0.58911 & $\mathrm{C}$ & -3.55048 & -0.99007 & -0.57841 \\
\hline $\mathrm{C}$ & -3.56815 & -2.43642 & -0.03497 & $\mathrm{C}$ & -3.72753 & -2.33696 & 0.074705 \\
\hline $\mathrm{C}$ & -2.49577 & -3.42256 & -0.53556 & $\mathrm{C}$ & -2.69167 & -3.39764 & -0.34131 \\
\hline $\mathrm{C}$ & -0.00412 & 2.66744 & 0.128582 & $\mathrm{C}$ & -0.00479 & 2.608595 & 0.207397 \\
\hline $\mathrm{C}$ & 0.793372 & 1.602091 & -0.66495 & $\mathrm{C}$ & 0.815872 & 1.547269 & -0.55625 \\
\hline $\mathrm{C}$ & -1.07511 & -3.26598 & -0.00301 & $\mathrm{C}$ & -1.2661 & -3.24893 & 0.180316 \\
\hline $\mathrm{C}$ & 0.01021 & -2.84084 & -0.92065 & $\mathrm{C}$ & -0.1733 & -2.89547 & -0.7591 \\
\hline $\mathrm{C}$ & 0.760187 & 0.166745 & -0.12052 & $\mathrm{C}$ & 0.707406 & 0.111477 & -0.0198 \\
\hline $\mathrm{C}$ & 1.370416 & -2.28953 & -0.54088 & $\mathrm{C}$ & 1.206151 & -2.37147 & -0.41122 \\
\hline $\mathrm{C}$ & 1.592398 & -0.8205 & -0.9724 & $\mathrm{C}$ & 1.473837 & -0.92056 & -0.87861 \\
\hline $\mathrm{C}$ & 3.073025 & -0.47132 & -1.05286 & $\mathrm{C}$ & 2.966101 & -0.63624 & -0.97467 \\
\hline $\mathrm{C}$ & 3.969415 & -0.4786 & 0.154373 & $\mathrm{C}$ & 3.77222 & -0.68237 & 0.287061 \\
\hline $\mathrm{O}$ & 5.148999 & -0.19712 & 0.140201 & $\mathrm{O}$ & 3.320957 & -0.96617 & 1.378931 \\
\hline $\mathrm{C}$ & 3.658419 & -0.15212 & -2.21187 & $\mathrm{C}$ & 3.562482 & -0.37017 & -2.14181 \\
\hline $\mathrm{C}$ & -4.17987 & 0.227134 & 1.47332 & $\mathrm{C}$ & -4.3949 & 0.455702 & 1.306889 \\
\hline $\mathrm{C}$ & -0.93871 & -3.15101 & 1.498293 & $\mathrm{C}$ & -1.12471 & -3.06131 & 1.673686 \\
\hline $\mathrm{O}$ & -2.21261 & 3.199218 & 1.118172 & $\mathrm{O}$ & -2.21706 & 3.335007 & 0.928487 \\
\hline $\mathrm{O}$ & -0.18772 & -4.23701 & -0.61904 & $\mathrm{O}$ & -0.40607 & -4.27054 & -0.39023 \\
\hline $\mathrm{H}$ & -0.30154 & -2.58039 & -1.93382 & $\mathrm{H}$ & -0.48183 & -2.67398 & -1.78253 \\
\hline $\mathrm{C}$ & 0.656908 & 2.949423 & 1.488637 & $\mathrm{C}$ & 0.5537 & 2.835613 & 1.619388 \\
\hline $\mathrm{O}$ & 3.326283 & -0.83786 & 1.280384 & $\mathrm{O}$ & 5.068516 & -0.37645 & 0.100021 \\
\hline $\mathrm{C}$ & 4.120445 & -0.87646 & 2.48486 & $\mathrm{C}$ & 5.909225 & -0.4149 & 1.270997 \\
\hline $\mathrm{O}$ & -0.09932 & 3.875639 & -0.65562 & $\mathrm{O}$ & -0.02466 & 3.849087 & -0.54815 \\
\hline
\end{tabular}




\begin{tabular}{|c|c|c|c|c|c|c|c|}
\hline $\mathrm{C}$ & 1.096825 & 4.589354 & -0.933 & $\mathrm{C}$ & 1.179508 & 4.598523 & -0.65502 \\
\hline $\mathrm{H}$ & -4.28313 & 2.184104 & -0.2015 & $\mathrm{H}$ & -4.22338 & 2.291859 & -0.50698 \\
\hline $\mathrm{H}$ & -4.13697 & 1.311278 & -1.71747 & $\mathrm{H}$ & -4.02272 & 1.29034 & -1.93568 \\
\hline $\mathrm{H}$ & -2.32207 & 2.949022 & -1.47656 & $\mathrm{H}$ & -2.10274 & 2.787018 & -1.70411 \\
\hline $\mathrm{H}$ & -1.70546 & 1.307969 & -1.5726 & $\mathrm{H}$ & -1.60953 & 1.109341 & -1.53627 \\
\hline $\mathrm{H}$ & -1.44441 & 1.290523 & 0.917355 & $\mathrm{H}$ & -1.61881 & 1.407778 & 0.974153 \\
\hline $\mathrm{H}$ & -3.2083 & -0.98849 & -1.64687 & $\mathrm{H}$ & -3.19602 & -1.03039 & -1.60916 \\
\hline $\mathrm{H}$ & -4.5365 & -2.85608 & -0.34113 & $\mathrm{H}$ & -4.71035 & -2.73542 & -0.2128 \\
\hline $\mathrm{H}$ & -3.58814 & -2.43058 & 1.056949 & $\mathrm{H}$ & -3.758 & -2.24991 & 1.163387 \\
\hline $\mathrm{H}$ & -2.81131 & -4.43452 & -0.25361 & $\mathrm{H}$ & -3.04245 & -4.37361 & 0.015814 \\
\hline $\mathrm{H}$ & -2.46174 & -3.40593 & -1.62977 & $\mathrm{H}$ & -2.65514 & -3.46588 & -1.43344 \\
\hline $\mathrm{H}$ & 1.834484 & 1.933149 & -0.71367 & $\mathrm{H}$ & 1.865888 & 1.853361 & -0.53861 \\
\hline $\mathrm{H}$ & 0.426839 & 1.606494 & -1.69638 & $\mathrm{H}$ & 0.510539 & 1.570084 & -1.60696 \\
\hline $\mathrm{H}$ & -0.26772 & -0.20527 & -0.11225 & $\mathrm{H}$ & -0.34039 & -0.20013 & 0.002244 \\
\hline $\mathrm{H}$ & 1.1151 & 0.137154 & 0.911743 & $\mathrm{H}$ & 1.074884 & 0.056924 & 1.006804 \\
\hline $\mathrm{H}$ & 1.558061 & -2.40175 & 0.526483 & $\mathrm{H}$ & 1.407013 & -2.46187 & 0.655951 \\
\hline $\mathrm{H}$ & 2.106986 & -2.91791 & -1.05543 & $\mathrm{H}$ & 1.915602 & -3.03566 & -0.91909 \\
\hline $\mathrm{H}$ & 1.226735 & -0.7354 & -2.00295 & $\mathrm{H}$ & 1.095343 & -0.83557 & -1.90444 \\
\hline $\mathrm{H}$ & 4.714625 & 0.083018 & -2.25355 & $\mathrm{H}$ & 4.625443 & -0.18108 & -2.20812 \\
\hline $\mathrm{H}$ & 3.095606 & -0.1208 & -3.13742 & $\mathrm{H}$ & 2.990939 & -0.33858 & -3.06287 \\
\hline $\mathrm{H}$ & -4.03274 & -0.69124 & 2.042304 & $\mathrm{H}$ & -4.33406 & -0.42077 & 1.952887 \\
\hline $\mathrm{H}$ & -3.64515 & 1.038834 & 1.974501 & $\mathrm{H}$ & -3.87741 & 1.286217 & 1.79585 \\
\hline $\mathrm{H}$ & -5.24534 & 0.483832 & 1.527459 & $\mathrm{H}$ & -5.45066 & 0.747835 & 1.242668 \\
\hline $\mathrm{H}$ & 0.100972 & -3.24321 & 1.809806 & $\mathrm{H}$ & -0.08611 & -3.1517 & 1.989061 \\
\hline $\mathrm{H}$ & -1.32323 & -2.19276 & 1.856566 & $\mathrm{H}$ & -1.49517 & -2.08057 & 1.982693 \\
\hline $\mathrm{H}$ & -1.50997 & -3.94651 & 1.986651 & $\mathrm{H}$ & -1.70706 & -3.8226 & 2.201849 \\
\hline $\mathrm{H}$ & -1.91799 & 3.153167 & 2.032788 & $\mathrm{H}$ & -1.91724 & 4.130591 & 0.466837 \\
\hline $\mathrm{H}$ & 0.215035 & 3.834488 & 1.951161 & $\mathrm{H}$ & 0.02557 & 3.653085 & 2.110906 \\
\hline $\mathrm{H}$ & 0.525459 & 2.102155 & 2.166422 & $\mathrm{H}$ & 0.422511 & 1.936549 & 2.225161 \\
\hline $\mathrm{H}$ & 1.730184 & 3.121606 & 1.387511 & $\mathrm{H}$ & 1.620935 & 3.064943 & 1.595134 \\
\hline $\mathrm{H}$ & 3.435987 & -1.17815 & 3.273367 & $\mathrm{H}$ & 6.903414 & -0.14872 & 0.921395 \\
\hline $\mathrm{H}$ & 4.929344 & -1.60001 & 2.381526 & $\mathrm{H}$ & 5.560569 & 0.303158 & 2.013958 \\
\hline $\mathrm{H}$ & 4.537629 & 0.108504 & 2.69522 & $\mathrm{H}$ & 5.911874 & -1.41513 & 1.705276 \\
\hline $\mathrm{H}$ & 1.591322 & 4.946754 & -0.02274 & $\mathrm{H}$ & 1.515683 & 4.984463 & 0.312942 \\
\hline $\mathrm{H}$ & 1.814444 & 4.000909 & -1.51588 & $\mathrm{H}$ & 1.990186 & 4.017715 & -1.10773 \\
\hline$U$ & 0.801194 & 5.45587 & -1.52618 & $\mathrm{H}$ & 0.953753 & 5.44329 & -1.30678 \\
\hline
\end{tabular}

\begin{tabular}{lrrrlrrr} 
3g_7 & \multicolumn{7}{c}{$\mathbf{3 g \_ 8}$} \\
C & -3.67246 & 0.295461 & -1.55721 & C & -3.56498 & 0.660581 & -1.63829 \\
C & -3.59888 & -0.96781 & -0.70798 & C & -3.73547 & -0.59093 & -0.78511 \\
C & -2.49648 & 1.274591 & -1.36256 & C & -2.2588 & 1.431132 & -1.36191 \\
C & -2.29922 & 1.744701 & 0.076842 & C & -2.13127 & 1.929812 & 0.082969 \\
C & -2.79981 & -1.98224 & -1.06669 & C & -3.04566 & -1.70251 & -1.07488 \\
C & -2.66399 & -3.33258 & -0.41074 & C & -3.1068 & -3.04286 & -0.38832 \\
C & -1.24768 & -3.93711 & -0.43406 & C & -1.78383 & -3.83126 & -0.39154 \\
C & -1.035 & 2.627315 & 0.28549 & C & -0.78348 & 2.634235 & 0.401171 \\
C & 0.26141 & 1.862462 & -0.08149 & C & 0.443508 & 1.793372 & -0.01115 \\
C & -0.19934 & -3.34015 & 0.498844 & C & -0.65011 & -3.33407 & 0.499716 \\
C & 0.945669 & -2.58664 & -0.06795 & C & 0.569262 & -2.75413 & -0.11558
\end{tabular}




\begin{tabular}{|c|c|c|c|c|c|c|c|}
\hline $\mathrm{C}$ & 0.476327 & 0.496919 & 0.589537 & $\mathrm{C}$ & 0.521068 & 0.385596 & 0.601855 \\
\hline $\mathrm{C}$ & 1.875632 & -1.64069 & 0.666084 & $\mathrm{C}$ & 1.633726 & -1.91494 & 0.563655 \\
\hline $\mathrm{C}$ & 1.807594 & -0.16824 & 0.190765 & $\mathrm{C}$ & 1.713098 & -0.44196 & 0.089583 \\
\hline $\mathrm{C}$ & 3.047739 & 0.567074 & 0.683945 & $\mathrm{C}$ & 3.076239 & 0.121234 & 0.467617 \\
\hline $\mathrm{C}$ & 4.324049 & 0.455569 & -0.1026 & $\mathrm{C}$ & 4.173078 & -0.1708 & -0.51094 \\
\hline $\mathrm{O}$ & 5.374985 & 0.981784 & 0.19721 & $\mathrm{O}$ & 4.007677 & -0.78547 & -1.54547 \\
\hline $\mathrm{C}$ & 3.111677 & 1.283676 & 1.809568 & $\mathrm{C}$ & 3.340412 & 0.78728 & 1.595382 \\
\hline $\mathrm{C}$ & -4.50429 & -0.98508 & 0.500734 & $\mathrm{C}$ & -4.72491 & -0.47147 & 0.348632 \\
\hline $\mathrm{C}$ & -0.6067 & -3.19591 & 1.947991 & $\mathrm{C}$ & -1.00903 & -3.08103 & 1.946372 \\
\hline $\mathrm{O}$ & -3.49994 & 2.43621 & 0.455131 & $\mathrm{O}$ & -3.21487 & 2.8113 & 0.405152 \\
\hline $\mathrm{O}$ & 1.089946 & -3.9758 & 0.290847 & $\mathrm{O}$ & 0.543547 & -4.13607 & 0.296801 \\
\hline $\mathrm{H}$ & 0.923433 & -2.42722 & -1.14744 & $\mathrm{H}$ & 0.544677 & -2.63309 & -1.19997 \\
\hline $\mathrm{C}$ & -0.99056 & 3.12324 & 1.740522 & $\mathrm{C}$ & -0.73516 & 3.005613 & 1.890392 \\
\hline $\mathrm{O}$ & 4.181261 & -0.30666 & -1.20226 & $\mathrm{O}$ & 5.369556 & 0.31348 & -0.13707 \\
\hline $\mathrm{C}$ & 5.356735 & -0.47408 & -2.02149 & $\mathrm{C}$ & 6.468581 & 0.062864 & -1.0364 \\
\hline $\mathrm{O}$ & -1.21039 & 3.732465 & -0.62631 & $\mathrm{O}$ & -0.85863 & 3.824804 & -0.42685 \\
\hline $\mathrm{C}$ & -0.27042 & 4.796325 & -0.58208 & $\mathrm{C}$ & 0.115178 & 4.844493 & -0.24056 \\
\hline $\mathrm{H}$ & -4.60673 & 0.820408 & -1.34197 & $\mathrm{H}$ & -4.41293 & 1.330906 & -1.47573 \\
\hline $\mathrm{H}$ & -3.70238 & 0.021588 & -2.61658 & $\mathrm{H}$ & -3.57649 & 0.386918 & -2.698 \\
\hline $\mathrm{H}$ & -2.64575 & 2.154426 & -1.99394 & $\mathrm{H}$ & -2.19007 & 2.291502 & -2.03528 \\
\hline $\mathrm{H}$ & -1.58099 & 0.788494 & -1.70721 & $\mathrm{H}$ & -1.41877 & 0.774273 & -1.60063 \\
\hline $\mathrm{H}$ & -2.18041 & 0.867666 & 0.72243 & $\mathrm{H}$ & -2.23421 & 1.084609 & 0.766592 \\
\hline $\mathrm{H}$ & -2.22153 & -1.86426 & -1.98417 & $\mathrm{H}$ & -2.39236 & -1.67064 & -1.94788 \\
\hline $\mathrm{H}$ & -3.30862 & -4.0395 & -0.95132 & $\mathrm{H}$ & -3.84074 & -3.67038 & -0.91264 \\
\hline $\mathrm{H}$ & -3.0466 & -3.31719 & 0.612091 & $\mathrm{H}$ & -3.48293 & -2.94922 & 0.633321 \\
\hline $\mathrm{H}$ & -1.32728 & -4.99511 & -0.15592 & $\mathrm{H}$ & -1.99832 & -4.85464 & -0.06034 \\
\hline $\mathrm{H}$ & -0.85557 & -3.91727 & -1.45615 & $\mathrm{H}$ & -1.40814 & -3.91344 & -1.41657 \\
\hline $\mathrm{H}$ & 1.109091 & 2.50878 & 0.159228 & $\mathrm{H}$ & 1.344441 & 2.348898 & 0.261638 \\
\hline $\mathrm{H}$ & 0.2776 & 1.730887 & -1.16762 & $\mathrm{H}$ & 0.451626 & 1.713288 & -1.10192 \\
\hline $\mathrm{H}$ & -0.32403 & -0.18787 & 0.297672 & $\mathrm{H}$ & -0.38716 & -0.17301 & 0.36059 \\
\hline $\mathrm{H}$ & 0.414406 & 0.582228 & 1.679254 & $\mathrm{H}$ & 0.552896 & 0.444171 & 1.694374 \\
\hline $\mathrm{H}$ & 1.711069 & -1.68145 & 1.745778 & $\mathrm{H}$ & 1.520809 & -1.94093 & 1.650389 \\
\hline $\mathrm{H}$ & 2.888345 & -2.01933 & 0.492642 & $\mathrm{H}$ & 2.586225 & -2.4076 & 0.340499 \\
\hline $\mathrm{H}$ & 1.854021 & -0.17942 & -0.90234 & $\mathrm{H}$ & 1.67297 & -0.45186 & -1.00441 \\
\hline $\mathrm{H}$ & 4.043729 & 1.746125 & 2.108976 & $\mathrm{H}$ & 4.340297 & 1.130958 & 1.82275 \\
\hline $\mathrm{H}$ & 2.252566 & 1.428445 & 2.451855 & $\mathrm{H}$ & 2.566487 & 1.008776 & 2.320513 \\
\hline $\mathrm{H}$ & -4.28204 & -1.79428 & 1.196833 & $\mathrm{H}$ & -5.74586 & -0.41423 & -0.04936 \\
\hline $\mathrm{H}$ & -4.44501 & -0.03461 & 1.03811 & $\mathrm{H}$ & -4.68937 & -1.30981 & 1.045149 \\
\hline $\mathrm{H}$ & -5.54988 & -1.09492 & 0.18653 & $\mathrm{H}$ & -4.56289 & 0.454925 & 0.907679 \\
\hline $\mathrm{H}$ & 0.249731 & -2.962 & 2.579258 & $\mathrm{H}$ & -0.12008 & -2.92173 & 2.55559 \\
\hline $\mathrm{H}$ & -1.35245 & -2.40633 & 2.07088 & $\mathrm{H}$ & -1.65432 & -2.20393 & 2.04135 \\
\hline $\mathrm{H}$ & -1.04568 & -4.13182 & 2.307094 & $\mathrm{H}$ & -1.54856 & -3.9417 & 2.353406 \\
\hline $\mathrm{H}$ & -3.52808 & 2.496083 & 1.414914 & $\mathrm{H}$ & -2.98496 & 3.651827 & -0.01508 \\
\hline $\mathrm{H}$ & -1.80701 & 3.823559 & 1.928793 & $\mathrm{H}$ & -1.55042 & 3.685935 & 2.138207 \\
\hline $\mathrm{H}$ & -1.0836 & 2.287611 & 2.438904 & $\mathrm{H}$ & -0.84499 & 2.111362 & 2.507843 \\
\hline $\mathrm{H}$ & -0.05099 & 3.632193 & 1.963753 & $\mathrm{H}$ & 0.213646 & 3.476486 & 2.156091 \\
\hline $\mathrm{H}$ & 5.049347 & -1.11804 & -2.8413 & $\mathrm{H}$ & 7.334272 & 0.527629 & -0.57158 \\
\hline $\mathrm{H}$ & 5.695555 & 0.491144 & -2.39883 & $\mathrm{H}$ & 6.62745 & -1.00967 & -1.15327 \\
\hline $\mathrm{H}$ & 6.156263 & -0.94104 & -1.44584 & $\mathrm{H}$ & 6.272077 & 0.509876 & -2.01143 \\
\hline
\end{tabular}




$\begin{array}{lrllllll}\mathrm{H} & -0.26567 & 5.309924 & 0.385818 & \mathrm{H} & 0.039659 & 5.315678 & 0.744908 \\ \mathrm{H} & 0.74985 & 4.469324 & -0.81268 & \mathrm{H} & 1.135951 & 4.473595 & -0.38076 \\ \mathrm{H} & -0.58368 & 5.507993 & -1.34725 & \mathrm{H} & -0.08612 & 5.598237 & -1.00263\end{array}$

\begin{tabular}{|c|c|c|c|c|c|c|c|}
\hline \multicolumn{4}{|l|}{ 3g_9 } & \multicolumn{4}{|l|}{ 3g_10 } \\
\hline $\mathrm{C}$ & -3.75129 & 0.496385 & -1.47555 & $\mathrm{C}$ & -3.68908 & 1.563145 & -0.75038 \\
\hline $\mathrm{C}$ & -3.80493 & -0.91856 & -0.92217 & $\mathrm{C}$ & -3.81917 & 0.263581 & 0.035931 \\
\hline $\mathrm{C}$ & -2.47948 & 1.328755 & -1.26704 & $\mathrm{C}$ & -2.24218 & 2.056673 & -0.9556 \\
\hline $\mathrm{C}$ & -2.23793 & 1.743343 & 0.188023 & $\mathrm{C}$ & -1.46001 & 2.274411 & 0.337604 \\
\hline $\mathrm{C}$ & -2.72252 & -1.62718 & -0.57535 & $\mathrm{C}$ & -3.58799 & -0.91645 & -0.55604 \\
\hline $\mathrm{C}$ & -2.70642 & -3.03985 & -0.0316 & $\mathrm{C}$ & -3.75289 & -2.29665 & 0.026978 \\
\hline $\mathrm{C}$ & -1.35823 & -3.76801 & -0.17495 & $\mathrm{C}$ & -2.726 & -3.34034 & -0.45105 \\
\hline $\mathrm{C}$ & -0.95454 & 2.601754 & 0.400065 & $\mathrm{C}$ & 0.035567 & 2.641858 & 0.117402 \\
\hline $\mathrm{C}$ & 0.331457 & 1.840603 & -0.00343 & $\mathrm{C}$ & 0.790175 & 1.527859 & -0.65092 \\
\hline $\mathrm{C}$ & -0.20037 & -3.26332 & 0.679973 & $\mathrm{C}$ & -1.30175 & -3.23961 & 0.085148 \\
\hline $\mathrm{C}$ & 0.937641 & -2.5667 & 0.031963 & $\mathrm{C}$ & -0.1948 & -2.87317 & -0.83206 \\
\hline $\mathrm{C}$ & 0.615353 & 0.517578 & 0.722401 & $\mathrm{C}$ & 0.687637 & 0.105293 & -0.08208 \\
\hline $\mathrm{C}$ & 1.955091 & -1.65962 & 0.694635 & $\mathrm{C}$ & 1.187393 & -2.38141 & -0.45104 \\
\hline $\mathrm{C}$ & 1.885055 & -0.18968 & 0.209748 & $\mathrm{C}$ & 1.487161 & -0.93265 & -0.90373 \\
\hline $\mathrm{C}$ & 3.184535 & 0.522394 & 0.561717 & $\mathrm{C}$ & 2.98434 & -0.66049 & -0.93251 \\
\hline $\mathrm{C}$ & 4.37962 & 0.33782 & -0.33223 & $\mathrm{C}$ & 3.734618 & -0.71355 & 0.36316 \\
\hline $\mathrm{O}$ & 5.471628 & 0.831736 & -0.14617 & $\mathrm{O}$ & 3.233943 & -0.99016 & 1.435163 \\
\hline $\mathrm{C}$ & 3.370984 & 1.287248 & 1.640823 & $\mathrm{C}$ & 3.634295 & -0.40059 & -2.07223 \\
\hline $\mathrm{C}$ & -5.2121 & -1.45654 & -0.81816 & $\mathrm{C}$ & -4.26164 & 0.423148 & 1.470938 \\
\hline $\mathrm{C}$ & -0.48168 & -3.11788 & 2.159013 & $\mathrm{C}$ & -1.1665 & -3.11181 & 1.585442 \\
\hline $\mathrm{O}$ & -3.40708 & 2.462529 & 0.61585 & $\mathrm{O}$ & -2.15441 & 3.290819 & 1.07768 \\
\hline $\mathrm{O}$ & 1.031656 & -3.96498 & 0.366866 & $\mathrm{O}$ & -0.45617 & -4.25595 & -0.51585 \\
\hline $\mathrm{H}$ & 0.841613 & -2.39658 & -1.04196 & $\mathrm{H}$ & -0.48976 & -2.61003 & -1.84955 \\
\hline $\mathrm{C}$ & -0.89098 & 3.063399 & 1.864599 & $\mathrm{C}$ & 0.698519 & 2.918111 & 1.477408 \\
\hline $\mathrm{O}$ & 4.108623 & -0.44874 & -1.38976 & $\mathrm{O}$ & 5.041395 & -0.42348 & 0.231355 \\
\hline $\mathrm{C}$ & 5.195525 & -0.68245 & -2.30907 & $\mathrm{C}$ & 5.830688 & -0.46821 & 1.43738 \\
\hline $\mathrm{O}$ & -1.12507 & 3.725418 & -0.49169 & $\mathrm{O}$ & 0.000563 & 3.83792 & -0.6902 \\
\hline $\mathrm{C}$ & -0.23444 & 4.824544 & -0.3712 & $\mathrm{C}$ & 1.226447 & 4.505532 & -0.95199 \\
\hline $\mathrm{H}$ & -4.59143 & 1.055447 & -1.05168 & $\mathrm{H}$ & -4.25893 & 2.346687 & -0.24448 \\
\hline $\mathrm{H}$ & -3.95813 & 0.439657 & -2.55326 & $\mathrm{H}$ & -4.13842 & 1.436493 & -1.74048 \\
\hline $\mathrm{H}$ & -2.56209 & 2.242237 & -1.86101 & $\mathrm{H}$ & -2.25432 & 2.997128 & -1.51323 \\
\hline $\mathrm{H}$ & -1.60582 & 0.794612 & -1.64812 & $\mathrm{H}$ & -1.7118 & 1.328177 & -1.57302 \\
\hline $\mathrm{H}$ & -2.13723 & 0.845993 & 0.805729 & $\mathrm{H}$ & -1.47212 & 1.345952 & 0.918664 \\
\hline $\mathrm{H}$ & -1.74811 & -1.15954 & -0.68038 & $\mathrm{H}$ & -3.31204 & -0.90143 & -1.61131 \\
\hline $\mathrm{H}$ & -3.4602 & -3.64784 & -0.54026 & $\mathrm{H}$ & -4.73942 & -2.67677 & -0.27271 \\
\hline $\mathrm{H}$ & -3.00179 & -3.04088 & 1.025243 & $\mathrm{H}$ & -3.77423 & -2.26704 & 1.118543 \\
\hline $\mathrm{H}$ & -1.50739 & -4.81868 & 0.101786 & $\mathrm{H}$ & -3.08853 & -4.33164 & -0.15274 \\
\hline $\mathrm{H}$ & -1.04437 & -3.76513 & -1.2237 & $\mathrm{H}$ & -2.68698 & -3.34467 & -1.54525 \\
\hline $\mathrm{H}$ & 1.177857 & 2.514199 & 0.15417 & $\mathrm{H}$ & 1.845544 & 1.811159 & -0.69669 \\
\hline $\mathrm{H}$ & 0.288914 & 1.655141 & -1.08064 & $\mathrm{H}$ & 0.431712 & 1.530947 & -1.68521 \\
\hline $\mathrm{H}$ & -0.21946 & -0.17098 & 0.575225 & $\mathrm{H}$ & -0.35572 & -0.22041 & -0.07909 \\
\hline $\mathrm{H}$ & 0.681382 & 0.671637 & 1.803787 & $\mathrm{H}$ & 1.031244 & 0.076569 & 0.95417 \\
\hline $\mathrm{H}$ & 1.868072 & -1.69079 & 1.783609 & $\mathrm{H}$ & 1.363322 & -2.47991 & 0.619764 \\
\hline $\mathrm{H}$ & 2.939674 & -2.06915 & 0.447213 & $\mathrm{H}$ & 1.896242 & -3.0543 & -0.94803 \\
\hline
\end{tabular}




$\begin{array}{lrrrrrrr}\mathrm{H} & 1.823947 & -0.21792 & -0.88238 & \mathrm{H} & 1.156144 & -0.8454 & -1.94573 \\ \mathrm{H} & 4.339678 & 1.7311 & 1.832617 & \mathrm{H} & 4.700784 & -0.2212 & -2.09143 \\ \mathrm{H} & 2.578711 & 1.491761 & 2.34926 & \mathrm{H} & 3.104244 & -0.36466 & -3.01766 \\ \mathrm{H} & -5.25228 & -2.52029 & -0.58502 & \mathrm{H} & -4.15159 & -0.48581 & 2.062812 \\ \mathrm{H} & -5.76713 & -0.92155 & -0.03828 & \mathrm{H} & -3.70378 & 1.227809 & 1.957764 \\ \mathrm{H} & -5.7572 & -1.29087 & -1.75439 & \mathrm{H} & -5.31807 & 0.717611 & 1.507925 \\ \mathrm{H} & 0.436847 & -2.95617 & 2.721793 & \mathrm{H} & -0.13251 & -3.24056 & 1.902599 \\ \mathrm{H} & -1.15835 & -2.28177 & 2.353433 & \mathrm{H} & -1.51431 & -2.13455 & 1.929583 \\ \mathrm{H} & -0.95534 & -4.02807 & 2.539233 & \mathrm{H} & -1.7708 & -3.87785 & 2.081109 \\ \mathrm{H} & -3.49965 & 2.351971 & 1.566461 & \mathrm{H} & -1.87223 & 3.246285 & 1.996268 \\ \mathrm{H} & -1.70176 & 3.762657 & 2.075528 & \mathrm{H} & 0.291817 & 3.828618 & 1.922529 \\ \mathrm{H} & -0.98802 & 2.211109 & 2.542001 & \mathrm{H} & 0.526738 & 2.088335 & 2.167663 \\ \mathrm{H} & 0.056231 & 3.556646 & 2.092281 & \mathrm{H} & 1.778743 & 3.042843 & 1.380908 \\ \mathrm{H} & 4.78932 & -1.33383 & -3.07847 & \mathrm{H} & 6.843549 & -0.2226 & 1.128682 \\ \mathrm{H} & 5.532878 & 0.2591 & -2.74325 & \mathrm{H} & 5.464083 & 0.261997 & 2.159664 \\ \mathrm{H} & 6.027577 & -1.16619 & -1.79688 & \mathrm{H} & 5.79636 & -1.46481 & 1.878548 \\ \mathrm{H} & -0.32911 & 5.334832 & 0.593791 & \mathrm{H} & 1.705541 & 4.87351 & -0.03774 \\ \mathrm{H} & 0.813989 & 4.540344 & -0.51799 & \mathrm{H} & 1.941955 & 3.878848 & -1.49669 \\ \mathrm{H} & -0.51208 & 5.525767 & -1.15953 & \mathrm{H} & 0.975932 & 5.363304 & -1.57779\end{array}$

3g_11

\begin{tabular}{lrrr}
$\mathrm{C}$ & -2.82456 & 1.08914 & -1.52048 \\
$\mathrm{C}$ & -3.44069 & 0.010382 & -0.65072 \\
$\mathrm{C}$ & -2.49434 & 2.414905 & -0.7998 \\
$\mathrm{C}$ & -1.67803 & 2.347137 & 0.5128 \\
$\mathrm{C}$ & -2.9344 & -1.22898 & -0.66538 \\
$\mathrm{C}$ & -3.39432 & -2.43034 & 0.12975 \\
$\mathrm{C}$ & -2.42608 & -3.623 & 0.028282 \\
$\mathrm{C}$ & -0.14168 & 2.5488 & 0.371727 \\
$\mathrm{C}$ & 0.541764 & 1.511981 & -0.5457 \\
$\mathrm{C}$ & -1.0004 & -3.35479 & 0.49404 \\
$\mathrm{C}$ & 0.037566 & -3.07533 & -0.52519 \\
$\mathrm{C}$ & 0.627424 & 0.083602 & 0.015466 \\
$\mathrm{C}$ & 1.352455 & -2.35308 & -0.32586 \\
$\mathrm{C}$ & 1.361614 & -0.91551 & -0.91203 \\
$\mathrm{C}$ & 2.767582 & -0.47635 & -1.30158 \\
$\mathrm{C}$ & 3.873801 & -0.33245 & -0.29366 \\
$\mathrm{O}$ & 5.009349 & 0.002229 & -0.55693 \\
$\mathrm{C}$ & 3.095721 & -0.21206 & -2.57044 \\
$\mathrm{C}$ & -4.64267 & 0.407259 & 0.17003 \\
$\mathrm{C}$ & -0.84413 & -2.93314 & 1.937419 \\
$\mathrm{O}$ & -2.18014 & 3.338427 & 1.425728 \\
$\mathrm{O}$ & -0.07352 & -4.3869 & 0.064465 \\
$\mathrm{H}$ & -0.31101 & -3.05414 & -1.55912 \\
$\mathrm{C}$ & 0.505772 & 2.566832 & 1.766289 \\
$\mathrm{O}$ & 3.476972 & -0.61885 & 0.95968 \\
$\mathrm{C}$ & 4.484345 & -0.51699 & 1.988123 \\
$\mathrm{O}$ & -0.0645 & 3.862586 & -0.24614 \\
$\mathrm{C}$ & 1.185244 & 4.540313 & -0.25121 \\
& & & \\
\hline
\end{tabular}

3g_12

$\mathrm{C}$

C

C

C

C

C

C

C

C

C

C

C

C

C

C

C

O

C

C

C

O

$\mathrm{O}$

$\mathrm{H}$

C

$\mathrm{O}$

C

O

C

\begin{tabular}{rrr}
-3.86852 & 1.415899 & -0.67251 \\
-4.12191 & 0.004869 & -0.16687 \\
-2.42275 & 1.888769 & -0.8724 \\
-1.64587 & 2.096143 & 0.432058 \\
-3.20472 & -0.97118 & -0.18125 \\
-3.3946 & -2.3951 & 0.294951 \\
-2.40021 & -3.41048 & -0.29673 \\
-0.18093 & 2.586775 & 0.227225 \\
0.670242 & 1.568401 & -0.56893 \\
-0.94734 & -3.30539 & 0.155272 \\
0.083671 & -2.83193 & -0.8003 \\
0.796079 & 0.15664 & 0.020158 \\
1.454018 & -2.27645 & -0.46671 \\
1.62205 & -0.79456 & -0.87864 \\
3.087949 & -0.40079 & -0.98168 \\
3.918617 & -0.45537 & 0.263694 \\
3.502683 & -0.80595 & 1.350218 \\
3.642092 & -0.03037 & -2.14127 \\
-5.53006 & -0.19042 & 0.343132 \\
-0.718 & -3.27452 & 1.649903 \\
-2.39323 & 3.039927 & 1.21819 \\
-0.10055 & -4.24148 & -0.5622 \\
-0.28702 & -2.5221 & -1.77919 \\
0.448042 & 2.883058 & 1.598033 \\
5.193963 & -0.07632 & 0.067786 \\
6.056701 & -0.11513 & 1.222585 \\
-0.31306 & 3.79369 & -0.55641 \\
0.832092 & 4.61671 & -0.72101 \\
& & \\
\hline
\end{tabular}




\begin{tabular}{|c|c|c|c|c|c|c|c|}
\hline $\mathrm{H}$ & -3.52933 & 1.341162 & -2.325 & $\mathrm{H}$ & -4.35843 & 2.113178 & 0.01479 \\
\hline $\mathrm{H}$ & -1.93761 & 0.682021 & -2.01223 & $\mathrm{H}$ & -4.40562 & 1.527296 & -1.62467 \\
\hline $\mathrm{H}$ & -3.43525 & 2.897775 & -0.52511 & $\mathrm{H}$ & -2.44106 & 2.845236 & -1.40039 \\
\hline $\mathrm{H}$ & -1.99929 & 3.090065 & -1.50184 & $\mathrm{H}$ & -1.87921 & 1.194117 & -1.51748 \\
\hline $\mathrm{H}$ & -1.854 & 1.390136 & 1.009054 & $\mathrm{H}$ & -1.59854 & 1.149374 & 0.978318 \\
\hline $\mathrm{H}$ & -2.09148 & -1.40663 & -1.3284 & $\mathrm{H}$ & -2.21521 & -0.73663 & -0.56158 \\
\hline $\mathrm{H}$ & -4.37666 & -2.7696 & -0.2218 & $\mathrm{H}$ & -4.40146 & -2.74322 & 0.047519 \\
\hline & -3.53778 & -2.16103 & 1.18137 & $\mathrm{H}$ & -3.33686 & -2.43717 & 1.389975 \\
\hline $\mathrm{H}$ & -2.82108 & -4.45499 & 0.622602 & $\mathrm{H}$ & -2.73555 & -4.41685 & -0.01833 \\
\hline $\mathrm{H}$ & -2.38338 & -3.97132 & -1.00858 & $\mathrm{H}$ & -2.42996 & -3.36504 & -1.39012 \\
\hline$\pi$ & 1.556978 & 1.870408 & -0.73879 & $\mathrm{H}$ & 1.673922 & 1.988907 & -0.67962 \\
\hline $\mathrm{H}$ & 0.036321 & 1.507879 & -1.51592 & $\mathrm{H}$ & 0.258494 & 1.502723 & -1.58083 \\
\hline $\mathrm{H}$ & -0.37847 & -0.30679 & 0.190876 & $\mathrm{H}$ & -0.19508 & -0.28617 & 0.141753 \\
\hline $\mathrm{H}$ & 1.136171 & 0.092962 & 0.979904 & $\mathrm{H}$ & 1.246042 & 0.186977 & 1.014553 \\
\hline $\mathrm{H}$ & 1.635593 & -2.33182 & 0.726093 & $\mathrm{H}$ & 1.69018 & -2.40207 & 0.589611 \\
\hline $\mathrm{H}$ & 2.113208 & -2.94965 & -0.84278 & $\mathrm{H}$ & 2.178776 & -2.87916 & -1.02651 \\
\hline $\mathrm{H}$ & 0.808007 & -0.94909 & -1.858 & $\mathrm{H}$ & 1.224625 & -0.70218 & -1.89653 \\
\hline $\mathrm{H}$ & 4.103132 & 0.086691 & -2.83226 & $\mathrm{H}$ & 4.687287 & 0.238852 & -2.21245 \\
\hline $\mathrm{H}$ & 2.366963 & -0.28946 & -3.36873 & $\mathrm{H}$ & 3.053114 & 0.008737 & -3.05091 \\
\hline $\mathrm{H}$ & -5.08725 & -0.43933 & 0.693638 & $\mathrm{H}$ & -5.77823 & -1.23288 & 0.541457 \\
\hline $\mathrm{H}$ & -4.38878 & 1.169519 & 0.914099 & $\mathrm{H}$ & -5.67919 & 0.371373 & 1.272965 \\
\hline $\mathrm{H}$ & -5.41341 & 0.848402 & -0.47312 & $\mathrm{H}$ & -6.25745 & 0.203277 & -0.37581 \\
\hline $\mathrm{H}$ & 0.205066 & -2.87479 & 2.224486 & $\mathrm{H}$ & 0.336592 & -3.40511 & 1.889336 \\
\hline $\mathrm{H}$ & -1.30586 & -1.95889 & 2.116679 & $\mathrm{H}$ & -1.05672 & -2.33105 & 2.085489 \\
\hline H & -1.33448 & -3.66237 & 2.58969 & $\mathrm{H}$ & -1.27641 & -4.08467 & 2.128856 \\
\hline $\mathrm{H}$ & -1.85697 & 4.182175 & 1.08154 & $\mathrm{H}$ & -2.21378 & 2.868354 & 2.147045 \\
\hline $\mathrm{H}$ & 0.17488 & 3.438482 & 2.330094 & $\mathrm{H}$ & -0.04112 & 3.741502 & 2.061529 \\
\hline $\mathrm{H}$ & 0.217102 & 1.678222 & 2.330645 & $\mathrm{H}$ & 0.339057 & 2.023996 & 2.264585 \\
\hline $\mathrm{H}$ & 1.596187 & 2.579798 & 1.69983 & $\mathrm{H}$ & 1.515414 & 3.098072 & 1.515301 \\
\hline $\mathrm{H}$ & 3.982408 & -0.78947 & 2.912795 & $\mathrm{H}$ & 7.029861 & 0.213482 & 0.866988 \\
\hline 11 & 5.306372 & -1.20255 & 1.781419 & $\mathrm{H}$ & 5.686253 & 0.556722 & 1.997533 \\
\hline $\mathrm{H}$ & 4.865921 & 0.502638 & 2.044912 & $\mathrm{H}$ & 6.116989 & -1.12922 & 1.618926 \\
\hline $\mathrm{H}$ & 1.513611 & 4.812188 & 0.757402 & $\mathrm{H}$ & 1.17662 & 5.04659 & 0.226204 \\
\hline & 1.975666 & 3.956845 & -0.73563 & $\mathrm{H}$ & 1.668995 & 4.089797 & -1.19345 \\
\hline & 1.031155 & 5.454555 & -0.82559 & $\mathrm{H}$ & 0.526481 & 5.432425 & -1.37787 \\
\hline
\end{tabular}

\section{3h_1}

$\mathrm{C}$

C

C

C

C

C

C

C

C

C

C

C $\begin{array}{ll}-3.57082 & 1.869691\end{array}$

$-0.79566$

$-3.894840 .588938$

$-2.07521$

2.107831

$-1.25849$

2.629695

$\begin{array}{ll}-3.6436 & -0.60676\end{array}$

$-3.9201$

$-1.97015$

$-2.89606$

0.263675

$-3.05696$

2.820738

0.93301

1.60982

$-1.48244$

$-2.9456$

$-0.33684$

$-2.75054$

0.812911

0.254265
$-0.02922$

$-1.09478$

0.08682

$-0.57735$

0.004527

$-0.37295$

$-0.21366$

$-0.90018$

0.19428

$-0.72968$

$-0.19196$ 3h_2

$\mathrm{C}$

C

C

C

C

C

C

C

C

C

C

C

$$
\begin{array}{rrr}
-3.58433 & 1.690084 & -0.82181 \\
-3.83847 & 0.410767 & -0.02686 \\
-2.10844 & 1.977334 & -1.17077 \\
-1.27431 & 2.554499 & -0.02569 \\
-3.57366 & -0.78381 & -0.57149 \\
-3.79305 & -2.14928 & 0.02882 \\
-2.73501 & -3.20404 & -0.34743 \\
0.233685 & 2.769242 & -0.37855 \\
0.91811 & 1.544353 & -1.02088 \\
-1.325 & -3.04927 & 0.2187 \\
-0.18449 & -2.82411 & -0.70488 \\
0.840823 & 0.215763 & -0.25795
\end{array}
$$




\begin{tabular}{|c|c|c|c|c|c|c|c|}
\hline $\mathrm{C}$ & 1.065952 & -2.28686 & -0.38601 & $\mathrm{C}$ & 1.203287 & -2.31743 & -0.35927 \\
\hline $\mathrm{C}$ & 1.441344 & -0.91084 & -0.99035 & $\mathrm{C}$ & 1.547094 & -0.94646 & -0.99185 \\
\hline $\mathrm{C}$ & 2.942213 & -0.77638 & -1.21194 & $\mathrm{C}$ & 3.048766 & -0.74905 & -1.15159 \\
\hline $\mathrm{C}$ & 3.930171 & -0.83936 & -0.08029 & $\mathrm{C}$ & 3.980175 & -0.67793 & 0.026944 \\
\hline $\mathrm{O}$ & 5.134791 & -0.78253 & -0.2099 & $\mathrm{O}$ & 5.17528 & -0.48418 & -0.04822 \\
\hline $\mathrm{C}$ & 3.46318 & -0.61127 & -2.43237 & $\mathrm{C}$ & 3.622237 & -0.63883 & -2.35447 \\
\hline $\mathrm{C}$ & -4.53009 & 0.792517 & 1.321597 & $\mathrm{C}$ & -4.42708 & 0.613564 & 1.345116 \\
\hline $\mathrm{C}$ & -1.37845 & -2.62748 & 1.668117 & $\mathrm{C}$ & -1.23013 & -2.72359 & 1.691463 \\
\hline $\mathrm{O}$ & -1.43735 & 1.766987 & 1.211183 & $\mathrm{O}$ & -1.38783 & 1.722505 & 1.131478 \\
\hline $\mathrm{O}$ & -0.67243 & -4.06754 & -0.24765 & $\mathrm{O}$ & -0.48256 & -4.1487 & -0.21883 \\
\hline $\mathrm{H}$ & -0.59826 & -2.60674 & -1.77984 & $\mathrm{H}$ & -0.44893 & -2.69136 & -1.75582 \\
\hline $\mathrm{C}$ & 0.471318 & 4.080812 & -1.0641 & $\mathrm{C}$ & 0.408573 & 3.978184 & -1.30486 \\
\hline $\mathrm{O}$ & 3.338293 & -0.96827 & 1.121337 & $\mathrm{O}$ & 3.350955 & -0.85817 & 1.202291 \\
\hline $\mathrm{C}$ & 4.218834 & -1.0474 & 2.261876 & $\mathrm{C}$ & 4.178118 & -0.80775 & 2.383535 \\
\hline $\mathrm{O}$ & 0.790114 & 3.037981 & 1.123419 & $\mathrm{O}$ & 0.943286 & 2.957287 & 0.879677 \\
\hline $\mathrm{C}$ & 2.190167 & 3.22637 & 1.28589 & $\mathrm{C}$ & 0.804883 & 4.210797 & 1.549315 \\
\hline $\mathrm{H}$ & -3.97127 & 2.732734 & -0.25353 & $\mathrm{H}$ & -4.00013 & 2.546934 & -0.28128 \\
\hline $\mathrm{H}$ & -4.09862 & 1.846036 & -1.75523 & $\mathrm{H}$ & -4.13956 & 1.631138 & -1.76422 \\
\hline $\mathrm{H}$ & -1.64445 & 1.178888 & -1.47342 & $\mathrm{H}$ & -1.65461 & 1.057926 & -1.54551 \\
\hline $\mathrm{H}$ & -1.99858 & 2.843293 & -1.90148 & $\mathrm{H}$ & -2.08306 & 2.700519 & -1.9922 \\
\hline $\mathrm{H}$ & -1.64151 & 3.627432 & 0.353152 & $\mathrm{H}$ & -1.69354 & 3.543023 & 0.219303 \\
\hline $\mathrm{H}$ & -3.21302 & -0.62167 & -1.57947 & $\mathrm{H}$ & -3.18045 & -0.79717 & -1.58893 \\
\hline $\mathrm{H}$ & -4.89302 & -2.32593 & -0.36249 & $\mathrm{H}$ & -4.75569 & -2.54271 & -0.32668 \\
\hline $\mathrm{H}$ & -4.01937 & -1.92067 & 1.091359 & $\mathrm{H}$ & -3.88588 & -2.09173 & 1.11565 \\
\hline $\mathrm{H}$ & -3.28373 & -4.02048 & -0.02001 & $\mathrm{H}$ & -3.09233 & -4.17839 & 0.007934 \\
\hline $\mathrm{H}$ & -2.82778 & -3.13571 & -1.46299 & $\mathrm{H}$ & -2.66552 & -3.28308 & -1.43738 \\
\hline $\mathrm{H}$ & 0.512293 & 1.531312 & -1.90823 & $\mathrm{H}$ & 0.501863 & 1.409955 & -2.02451 \\
\hline $\mathrm{H}$ & 1.989363 & 1.852772 & -1.05343 & $\mathrm{H}$ & 1.967491 & 1.820763 & -1.16335 \\
\hline $\mathrm{H}$ & 1.268435 & 0.305147 & 0.798837 & $\mathrm{H}$ & 1.273977 & 0.332563 & 0.73627 \\
\hline $\mathrm{H}$ & -0.23929 & 0.019417 & -0.03068 & $\mathrm{H}$ & -0.2026 & -0.06478 & -0.11175 \\
\hline $\mathrm{H}$ & 1.22818 & -2.28285 & 0.691603 & $\mathrm{H}$ & 1.354836 & -2.28454 & 0.719275 \\
\hline $\mathrm{H}$ & 1.738688 & -3.0484 & -0.79839 & $\mathrm{H}$ & 1.900361 & -3.06888 & -0.74907 \\
\hline $\mathrm{H}$ & 1.006307 & -0.87891 & -1.99693 & $\mathrm{H}$ & 1.155616 & -0.96669 & -2.01652 \\
\hline $\mathrm{H}$ & 4.533729 & -0.5279 & -2.57267 & $\mathrm{H}$ & 4.692779 & -0.50581 & -2.44921 \\
\hline $\mathrm{H}$ & 2.831888 & -0.55772 & -3.31157 & $\mathrm{H}$ & 3.034949 & -0.68065 & -3.26427 \\
\hline $\mathrm{H}$ & -5.49142 & 1.309961 & 1.214876 & $\mathrm{H}$ & -5.40954 & 1.09566 & 1.269427 \\
\hline $\mathrm{H}$ & -4.71444 & -0.1384 & 1.85852 & $\mathrm{H}$ & -4.5573 & -0.31462 & 1.902151 \\
\hline $\mathrm{H}$ & -3.88738 & 1.429077 & 1.936249 & $\mathrm{H}$ & -3.78395 & 1.281242 & 1.925296 \\
\hline $\mathrm{H}$ & -0.35914 & -2.7575 & 2.029801 & $\mathrm{H}$ & -0.20804 & -2.82652 & 2.053962 \\
\hline $\mathrm{H}$ & -1.68867 & -1.59843 & 1.866033 & $\mathrm{H}$ & -1.56648 & -1.70211 & 1.88597 \\
\hline $\mathrm{H}$ & -2.02919 & -3.29392 & 2.242636 & $\mathrm{H}$ & -1.86394 & -3.40438 & 2.26807 \\
\hline $\mathrm{H}$ & -0.71465 & 2.000177 & 1.811939 & $\mathrm{H}$ & -0.57641 & 1.880888 & 1.635193 \\
\hline $\mathrm{H}$ & 0.079383 & 4.958452 & -0.54474 & $\mathrm{H}$ & -0.10312 & 4.865895 & -0.92769 \\
\hline $\mathrm{H}$ & 1.529816 & 4.248081 & -1.27491 & $\mathrm{H}$ & 1.469544 & 4.212124 & -1.4225 \\
\hline $\mathrm{H}$ & -0.0389 & 3.991156 & -2.02521 & $\mathrm{H}$ & 0.00339 & 3.757578 & -2.29401 \\
\hline $\mathrm{H}$ & 3.566436 & -1.15131 & 3.124911 & $\mathrm{H}$ & 3.500678 & -0.96655 & 3.21849 \\
\hline $\mathrm{H}$ & 4.877072 & -1.91186 & 2.172671 & $\mathrm{H}$ & 4.935029 & -1.59168 & 2.34979 \\
\hline $\mathrm{H}$ & 4.81794 & -0.14007 & 2.341283 & $\mathrm{H}$ & 4.664378 & 0.164685 & 2.463759 \\
\hline $\mathrm{H}$ & 2.761277 & 2.327246 & 1.033838 & $\mathrm{H}$ & 1.317315 & 4.097878 & 2.505076 \\
\hline
\end{tabular}


3h_3

C

C

C

C

$\mathrm{C}$

$\mathrm{C}$

$\mathrm{C}$

$\mathrm{C}$

$\mathrm{C}$

C

$\mathrm{C}$

$\mathrm{C}$

$\mathrm{C}$

$\mathrm{C}$

$\mathrm{C}$

$\mathrm{C}$

$\mathrm{O}$

C

$\mathrm{C}$

C

$\mathrm{O}$

$\mathrm{O}$

$\mathrm{H}$

$\mathrm{C}$

$\mathrm{O}$

C

$\mathrm{O}$

$\mathrm{C}$

$\mathrm{H}$

$\mathrm{H}$

$\mathrm{H}$

$\mathrm{H}$

$\mathrm{H}$

$\mathrm{H}$

$\mathrm{H}$

$\mathrm{H}$

$\mathrm{H}$

$\mathrm{H}$

$\mathrm{H}$

$\mathrm{H}$

$\mathrm{H}$

$\mathrm{H}$

$\mathrm{H}$

$\mathrm{H}$

$\mathrm{H}$ $\begin{array}{lll}-3.25822 & 1.511004 & -1.69876\end{array}$

$\begin{array}{lll}-3.77994 & 0.322563 & -0.89363\end{array}$

$\begin{array}{lll}-1.75953 & 1.833207 & -1.51297\end{array}$

$\begin{array}{lll}-1.41503 & 2.584757 & -0.22715\end{array}$

$\begin{array}{lll}-3.34557 & -0.91408 & -1.16917\end{array}$

$\begin{array}{llll}-3.75393 & -2.211 & -0.51988\end{array}$

$\begin{array}{lll}-2.61984 & -3.24098 & -0.35714\end{array}$

$\begin{array}{lll}0.112231 & 2.852231 & -0.0255\end{array}$

$\begin{array}{lll}1.006526 & 1.61263 & -0.24554\end{array}$

$\begin{array}{lll}-1.49886 & -2.93498 & 0.634218\end{array}$

$\begin{array}{llll}-0.10952 & -2.76797 & 0.138223\end{array}$

$\begin{array}{lll}0.676604 & 0.358195 & 0.574531\end{array}$

$\begin{array}{lll}1.07239 & -2.15293 & 0.862508\end{array}$

$\begin{array}{llll}1.594962 & -0.83457 & 0.239911\end{array}$

$\begin{array}{lll}3.04297 & -0.59795 & 0.659477\end{array}$

$\begin{array}{lll}4.15796 & -0.75462 & -0.33258\end{array}$

5.322286

3.404148

$-0.62728$

$-0.08056$

$-4.80843$

$-0.28219$

1.907496

$-1.92847$

0.654566

0.156171

$-1.94699$

$-2.41718$

1.987587

$-0.54903$

1.879863

0.895597

0.009574

$-4.03363$

0.671169

0.56547

$-2.78345$

$-0.94675$

$-0.94697$

3.717613

4.728714

3.991969

$-1.56415$

0.143527

$-0566$

$-1.22652$

$-2.57904$

1.393388

3.290853

1.359282

$-3.85168$

3.649422

1.935519

$-3.41901$

2.401996

$-1.46826$

$-1.19627$

1.308286

$-2.76295$

$-1.43355$

0.899801

$-1.56938$

$-1.89362$

2.450977

$-2.35539$

$-2.6286$

3.575671

$-0.27072$

$-4.51803$

$-1.02512$

$-1.98399$

$-4.23421$

$-2.69563$

$-1.14367$

$-3.06814$

$-2.03482$

0.445159

$-2.17175$

$-4.18242$

$-0.01684$

0.969617

$-3.45123$

$-1.33388$

2.044031

1.364721

$-1.31128$

0.737955

1.907881

$-0.05764$

$-0.35607$

0.581863

1.644502

0.392504

$\begin{array}{lll}0.850902 & -2.00387 & 1.922449\end{array}$

$\begin{array}{lll}1.870913 & -2.90248 & 0.818867\end{array}$

$\begin{array}{llll}1.58709 & -0.96482 & -0.84384\end{array}$
$3 h \_4$

$\mathrm{C}$

$\mathrm{C}$

$\mathrm{C}$

C

C

$\mathrm{C}$

C

$\mathrm{C}$

C

C

C

C

C

$\mathrm{C}$

C

$\mathrm{C}$

$\mathrm{O}$

$\mathrm{C}$

C

$\mathrm{C}$

$\mathrm{O}$

$\mathrm{O}$

$\mathrm{H}$

$\mathrm{C}$

$\mathrm{O}$

$\mathrm{C}$

$\mathrm{O}$

$\mathrm{C}$

$\mathrm{H}$

$\mathrm{H}$

$\mathrm{H}$

$\mathrm{H}$

$\mathrm{H}$

$\mathrm{H}$

$\mathrm{H}$

$\mathrm{H}$

$\mathrm{H}$

$\mathrm{H}$

$\mathrm{H}$

$\mathrm{H}$

$\mathrm{H}$

$\mathrm{H}$

$\mathrm{H}$

$\mathrm{H}$

$\mathrm{H}$ $\begin{array}{rrr}3.66032 & 0.790307 & 1.606134 \\ 3.841117 & -0.46251 & 0.751395\end{array}$

$\begin{array}{llll}2.261717 & 1.44065 & 1.546318\end{array}$

$\begin{array}{lll}2.009002 & 2.294596 & 0.304519\end{array}$

$\begin{array}{lll}3.134825 & -1.56779 & 1.021675\end{array}$

$\begin{array}{lll}3.197561 & -2.90482 & 0.329161\end{array}$

$\begin{array}{llll}1.849975 & -3.64276 & 0.216621\end{array}$

$\begin{array}{lll}0.57356 & 2.903543 & 0.213365\end{array}$

$\begin{array}{lll}-0.56672 & 1.886025 & 0.440638\end{array}$

$\begin{array}{lll}0.783773 & -3.0651 & -0.71181\end{array}$

$\begin{array}{lll}-0.50269 & -2.58843 & -0.14424\end{array}$

$\begin{array}{lll}-0.57183 & 0.633298 & -0.4471\end{array}$

$\begin{array}{lll}-1.54066 & -1.70374 & -0.80805\end{array}$

$\begin{array}{lll}-1.74189 & -0.31938 & -0.14136\end{array}$

$\begin{array}{lll}-3.1096 & 0.226271 & -0.53536\end{array}$

$\begin{array}{lll}-4.31873 & -0.17804 & 0.26089\end{array}$

$\begin{array}{lll}-5.45043 & 0.205903 & 0.052395\end{array}$

$\begin{array}{lll}-3.33957 & 1.010581 & -1.59224\end{array}$

$\begin{array}{llll}4.868243 & -0.35148 & -0.34532\end{array}$

$\begin{array}{lll}1.255172 & -2.63294 & -2.08135\end{array}$

$\begin{array}{lll}2.300347 & 1.52939 & -0.86611\end{array}$

$\begin{array}{lll}-0.39659 & -3.9117 & -0.70761\end{array}$

$\begin{array}{lll}-0.57092 & -2.59533 & 0.945106\end{array}$

$\begin{array}{lll}0.439271 & 4.065893 & 1.205302\end{array}$

$\begin{array}{lll}-4.01461 & -1.03463 & 1.253289\end{array}$

$\begin{array}{lll}-5.11675 & -1.48439 & 2.068082\end{array}$

$\begin{array}{llll}0.564924 & 3.400614 & -1.15212\end{array}$

$-0.54393 \quad 4.173616 \quad-1.59254$

$\begin{array}{lll}4.415478 & 1.534207 & 1.331638\end{array}$

$\begin{array}{lll}3.859094 & 0.530913 & 2.651555\end{array}$

$\begin{array}{lll}1.509238 & 0.653912 & 1.629427\end{array}$

$\begin{array}{lll}2.143718 & 2.085027 & 2.422653\end{array}$

$\begin{array}{lll}2.697624 & 3.15368 & 0.335369\end{array}$

$\begin{array}{lll}2.444645 & -1.5302 & 1.865698\end{array}$

$\begin{array}{lll}3.862981 & -3.56924 & 0.897961\end{array}$

$\begin{array}{llll}3.651562 & -2.81599 & -0.66044\end{array}$

$2.052088 \quad-4.65406 \quad-0.15707$

$\begin{array}{lll}1.413566 & -3.76702 & 1.213205\end{array}$

$\begin{array}{lll}-0.53169 & 1.579129 & 1.491216\end{array}$

$\begin{array}{lll}-1.51754 & 2.416418 & 0.329996\end{array}$

$\begin{array}{lll}-0.57878 & 0.919804 & -1.50284\end{array}$

$\begin{array}{lll}0.357805 & 0.08303 & -0.3038\end{array}$

$\begin{array}{lll}-1.32475 & -1.56791 & -1.87096\end{array}$

$\begin{array}{lll}-2.48131 & -2.26238 & -0.75379\end{array}$

$\begin{array}{lll}-1.7614 & -0.48303 & 0.940088\end{array}$ 


$\begin{array}{lrrrrrrr}\mathrm{H} & 4.448075 & -0.14959 & 2.161601 & \mathrm{H} & -4.34789 & 1.325306 & -1.82983 \\ \mathrm{H} & 2.675276 & -0.15066 & 2.698232 & \mathrm{H} & -2.54315 & 1.351612 & -2.24105 \\ \mathrm{H} & -5.71117 & 1.063841 & -0.31406 & \mathrm{H} & 5.86162 & -0.16518 & 0.081111 \\ \mathrm{H} & -5.10974 & -0.20573 & 0.754623 & \mathrm{H} & 4.937362 & -1.24541 & -0.96558 \\ \mathrm{H} & -4.41349 & 1.423636 & 0.824977 & \mathrm{H} & 4.626039 & 0.500623 & -0.98624 \\ \mathrm{H} & -1.09796 & -2.41341 & 2.692716 & \mathrm{H} & 0.414968 & -2.42434 & -2.7428 \\ \mathrm{H} & -2.32039 & -1.40009 & 1.907231 & \mathrm{H} & 1.873526 & -1.73431 & -2.01238 \\ \mathrm{H} & -2.71869 & -3.05266 & 2.399056 & \mathrm{H} & 1.857233 & -3.42417 & -2.53857 \\ \mathrm{H} & -1.48258 & 2.253092 & 1.658817 & \mathrm{H} & 1.90455 & 2.032394 & -1.59207 \\ \mathrm{H} & 0.002555 & 4.902941 & -0.73028 & \mathrm{H} & 1.177793 & 4.841558 & 0.988253 \\ \mathrm{H} & 1.629292 & 4.205089 & -0.82269 & \mathrm{H} & -0.5557 & 4.513627 & 1.160835 \\ \mathrm{H} & 0.408332 & 3.730058 & -1.99517 & \mathrm{H} & 0.593337 & 3.720069 & 2.229154 \\ \mathrm{H} & 4.186946 & -1.46255 & -3.49131 & \mathrm{H} & -4.68073 & -2.1626 & 2.796925 \\ \mathrm{H} & 5.302005 & -0.30712 & -2.70054 & \mathrm{H} & -5.5889 & -0.63747 & 2.566702 \\ \mathrm{H} & 5.401126 & -2.04174 & -2.3106 & \mathrm{H} & -5.85434 & -2.00282 & 1.454923 \\ \mathrm{H} & 2.085749 & 2.802908 & 1.987341 & \mathrm{H} & -1.49222 & 3.635068 & -1.49775 \\ \mathrm{H} & 1.173527 & 3.982181 & 2.950345 & \mathrm{H} & -0.3672 & 4.383048 & -2.64796 \\ \mathrm{H} & 1.874422 & 4.471654 & 1.396284 & \mathrm{H} & -0.61993 & 5.124833 & -1.05589\end{array}$

$\begin{array}{lrrrlrrr}\text { 3h_5 } & & & & \text { 3h_6 } & & & \\ \text { C } & -3.78146 & 1.415102 & -0.87544 & \text { C } & 3.619656 & 1.876677 & 0.830926 \\ \text { C } & -4.03973 & 0.053106 & -0.2395 & \text { C } & 3.965331 & 0.610755 & 0.049492 \\ \text { C } & -2.32459 & 1.828114 & -1.15277 & \text { C } & 2.118324 & 2.093858 & 1.117854 \\ \text { C } & -1.56514 & 2.331297 & 0.087971 & \text { C } & 1.308629 & 2.626566 & -0.06383 \\ \text { C } & -3.14993 & -0.95082 & -0.2569 & \text { C } & 3.724819 & -0.59507 & 0.579954 \\ \text { C } & -3.33353 & -2.33527 & 0.324936 & \text { C } & 4.021711 & -1.94736 & -0.01694 \\ \text { C } & -2.28958 & -3.36138 & -0.14828 & \text { C } & 3.001852 & -3.04829 & 0.32987 \\ \text { C } & -0.08813 & 2.742898 & -0.20258 & \text { C } & -0.21906 & 2.793834 & 0.221306 \\ \text { C } & 0.751229 & 1.661297 & -0.91614 & \text { C } & -0.8827 & 1.56172 & 0.874725 \\ \text { C } & -0.84393 & -3.14674 & 0.293904 & \text { C } & 1.596601 & -2.94317 & -0.25899 \\ \text { C } & 0.182484 & -2.7752 & -0.71234 & \text { C } & 0.433198 & -2.77483 & 0.647868 \\ \text { C } & 0.882553 & 0.304986 & -0.21349 & \text { C } & -0.7343 & 0.221148 & 0.143773 \\ \text { C } & 1.544345 & -2.15919 & -0.45254 & \text { C } & -0.96881 & -2.32374 & 0.286081 \\ \text { C } & 1.691808 & -0.72909 & -1.02865 & \text { C } & -1.3757 & -0.9627 & 0.902895 \\ \text { C } & 3.148728 & -0.34146 & -1.24211 & \text { C } & -2.88577 & -0.84424 & 1.049775 \\ \text { C } & 4.122462 & -0.21074 & -0.10384 & \text { C } & -3.71652 & -0.87115 & -0.19658 \\ \text { O } & 5.280767 & 0.130038 & -0.22181 & \text { O } & -3.26751 & -1.00935 & -1.31704 \\ \text { C } & 3.642004 & -0.10005 & -2.46137 & \text { C } & -3.47633 & -0.73641 & 2.245055 \\ \text { C } & -5.41239 & -0.07186 & 0.374074 & \text { C } & 4.607866 & 0.840335 & -1.29371 \\ \text { C } & -0.63083 & -2.90221 & 1.771116 & \text { C } & 1.51324 & -2.60344 & -1.72925 \\ \text { O } & -1.57572 & 1.399701 & 1.176269 & \text { O } & 1.51165 & 1.786659 & -1.20121 \\ \text { O } & 0.030926 & -4.14579 & -0.29045 & \text { O } & 0.792501 & -4.08081 & 0.152567 \\ \text { H } & -0.18855 & -2.60421 & -1.72482 & \text { H } & 0.674678 & -2.64349 & 1.704482 \\ \text { C } & -0.06658 & 4.022184 & -1.06035 & \text { C } & -0.45181 & 4.034024 & 1.094357 \\ \text { O } & 3.578784 & -0.51852 & 1.087383 & \text { O } & -5.03314 & -0.72551 & 0.039238 \\ \text { C } & 4.451428 & -0.41792 & 2.232105 & \text { C } & -5.89529 & -0.7523 & -1.11626 \\ \text { O } & 0.622636 & 2.97791 & 1.031199 & \text { O } & -0.73095 & 3.032528 & -1.11776 \\ \text { C } & 0.05831 & 3.88727 & 1.966558 & \text { C } & -2.13092 & 3.207542 & -1.2948 \\ \text { H } & -4.25311 & 2.182577 & -0.24791 & \text { H } & 4.015954 & 2.751865 & 0.305484\end{array}$




\begin{tabular}{|c|c|c|c|c|c|c|c|}
\hline $\mathrm{H}$ & -4.34006 & 1.443355 & -1.81977 & $\mathrm{H}$ & 4.137718 & 1.844763 & 1.795527 \\
\hline $\mathrm{H}$ & -1.78709 & 1.010144 & -1.63678 & $\mathrm{H}$ & 1.693861 & 1.154234 & 1.476734 \\
\hline $\mathrm{H}$ & -2.34542 & 2.645951 & -1.8774 & $\mathrm{H}$ & 2.025356 & 2.81513 & 1.935591 \\
\hline $\mathrm{H}$ & -2.08582 & 3.240699 & 0.424011 & $\mathrm{H}$ & 1.682322 & 3.633602 & -0.30756 \\
\hline $\mathrm{H}$ & -2.19056 & -0.77856 & -0.73373 & $\mathrm{H}$ & 3.287154 & -0.62822 & 1.5786 \\
\hline $\mathrm{H}$ & -4.32169 & -2.72596 & 0.062795 & $\mathrm{H}$ & 4.992176 & -2.29987 & 0.359646 \\
\hline $\mathrm{H}$ & -3.32285 & -2.28798 & 1.420358 & $\mathrm{H}$ & 4.136595 & -1.88181 & -1.10139 \\
\hline 11 & -2.58349 & -4.346 & 0.234363 & $\mathrm{H}$ & 3.404254 & -4.00343 & -0.02933 \\
\hline $\mathrm{H}$ & -2.31148 & -3.43483 & -1.2403 & $\mathrm{H}$ & 2.91677 & -3.14208 & 1.417541 \\
\hline $\mathrm{H}$ & 0.344332 & 1.515188 & -1.9221 & $\mathrm{H}$ & -0.47589 & 1.469617 & 1.887408 \\
\hline $\mathrm{H}$ & 1.74792 & 2.092592 & -1.05642 & $\mathrm{H}$ & -1.9444 & 1.788201 & 1.016711 \\
\hline $\mathrm{H}$ & 1.336171 & 0.442869 & 0.767842 & $\mathrm{H}$ & -1.16829 & 0.285177 & -0.85624 \\
\hline $\mathrm{H}$ & -0.1072 & -0.11197 & -0.03283 & $\mathrm{H}$ & 0.323242 & -0.00173 & 0.002242 \\
\hline $\mathrm{H}$ & 1.784153 & -2.16614 & 0.610141 & $\mathrm{H}$ & -1.1144 & -2.30253 & -0.79365 \\
\hline $\mathrm{H}$ & 2.274395 & -2.81331 & -0.94404 & $\mathrm{H}$ & -1.63903 & -3.09994 & 0.67465 \\
\hline $\mathrm{H}$ & 1.258157 & -0.75467 & -2.03617 & $\mathrm{H}$ & -0.98435 & -0.94406 & 1.927547 \\
\hline $\mathrm{H}$ & 4.681562 & 0.171382 & -2.59661 & $\mathrm{H}$ & -4.55084 & -0.66262 & 2.345283 \\
\hline $\mathrm{H}$ & 3.017921 & -0.16974 & -3.34463 & $\mathrm{H}$ & -2.88766 & -0.72262 & 3.155693 \\
\hline $\mathrm{H}$ & -6.17987 & 0.239367 & -0.34355 & $\mathrm{H}$ & 5.565545 & 1.361371 & -1.1723 \\
\hline $\mathrm{H}$ & -5.64377 & -1.08469 & 0.702426 & $\mathrm{H}$ & 4.800672 & -0.0805 & -1.84486 \\
\hline $\mathrm{H}$ & -5.50756 & 0.594316 & 1.239225 & $\mathrm{H}$ & 3.965128 & 1.483255 & -1.90149 \\
\hline $\mathrm{H}$ & 0.424609 & -2.96469 & 2.033077 & $\mathrm{H}$ & 0.501051 & -2.73688 & -2.10909 \\
\hline $\mathrm{H}$ & -1.0005 & -1.91631 & 2.064804 & $\mathrm{H}$ & 1.816715 & -1.56842 & -1.90572 \\
\hline $\mathrm{H}$ & -1.17007 & -3.65303 & 2.356791 & $\mathrm{H}$ & 2.179419 & -3.25466 & -2.30354 \\
\hline $\mathrm{H}$ & -2.32788 & 0.804922 & 1.062002 & $\mathrm{H}$ & 0.794766 & 2.023152 & -1.8076 \\
\hline $\mathrm{H}$ & -0.70127 & 4.807614 & -0.64377 & $\mathrm{H}$ & -0.06538 & 4.926578 & 0.5968 \\
\hline $\mathrm{H}$ & 0.956809 & 4.40011 & -1.11466 & $\mathrm{H}$ & -1.51449 & 4.184102 & 1.296808 \\
\hline $\mathrm{H}$ & -0.4082 & 3.824855 & -2.07829 & $\mathrm{H}$ & 0.049192 & 3.931515 & 2.059001 \\
\hline $\mathrm{H}$ & 3.839493 & -0.69151 & 3.087642 & $\mathrm{H}$ & -6.90257 & -0.62548 & -0.7279 \\
\hline $\mathrm{H}$ & 5.293302 & -1.10288 & 2.128117 & $\mathrm{H}$ & -5.64376 & 0.060528 & -1.79834 \\
\hline $\mathrm{H}$ & 4.822593 & 0.601816 & 2.33657 & $\mathrm{H}$ & -5.80389 & -1.70561 & -1.63778 \\
\hline 11 & -0.12614 & 4.877195 & 1.533584 & $\mathrm{H}$ & -2.69384 & 2.294917 & -1.07509 \\
\hline $\mathrm{H}$ & 0.795456 & 3.995839 & 2.763819 & $\mathrm{H}$ & -2.27325 & 3.458446 & -2.3464 \\
\hline$H$ & -0.87071 & 3.502942 & 2.399229 & $\mathrm{H}$ & -2.52515 & 4.026762 & -0.68525 \\
\hline
\end{tabular}

\begin{tabular}{lrrrlrrr} 
3h_7 & \multicolumn{7}{c}{ 3h_8 } \\
C & -3.68623 & 0.183496 & -1.63315 & C & 3.686646 & 0.183351 & 1.632664 \\
C & -3.65743 & -1.07771 & -0.77223 & C & 3.65756 & -1.07785 & 0.771765 \\
C & -2.41741 & 1.060911 & -1.57151 & C & 2.417804 & 1.060759 & 1.571407 \\
C & -2.30381 & 1.948279 & -0.33007 & C & 2.304068 & 1.948189 & 0.330009 \\
C & -2.7768 & -2.05155 & -1.03638 & C & 2.776827 & -2.05157 & 1.035971 \\
C & -2.61527 & -3.37563 & -0.335 & C & 2.615114 & -3.3757 & 0.334707 \\
C & -1.16269 & -3.87453 & -0.21464 & C & 1.162494 & -3.87457 & 0.214879 \\
C & -0.98172 & 2.779356 & -0.25778 & C & 0.981954 & 2.779298 & 0.25792 \\
C & 0.302014 & 1.953755 & -0.48394 & C & -0.30171 & 1.953688 & 0.48419 \\
C & -0.21294 & -3.12438 & 0.717307 & C & 0.212575 & -3.12454 & -0.717 \\
C & 0.983906 & -2.44835 & 0.155349 & C & -0.98406 & -2.44823 & -0.15489 \\
C & 0.519811 & 0.729288 & 0.415311 & C & -0.51971 & 0.729252 & -0.41511 \\
C & 1.85679 & -1.39922 & 0.817834 & C & -1.85694 & -1.39914 & -0.81742
\end{tabular}




\begin{tabular}{|c|c|c|c|c|c|c|c|}
\hline $\mathrm{C}$ & 1.836749 & -0.01109 & 0.127139 & $\mathrm{C}$ & -1.83674 & -0.01092 & -0.12692 \\
\hline $\mathrm{C}$ & 3.090744 & 0.761549 & 0.515374 & $\mathrm{C}$ & -3.09062 & 0.761811 & -0.51538 \\
\hline $\mathrm{C}$ & 4.387549 & 0.435487 & -0.17201 & $\mathrm{C}$ & -4.38761 & 0.435526 & 0.171599 \\
\hline $\mathrm{O}$ & 5.459252 & 0.944234 & 0.081284 & $\mathrm{O}$ & -5.45944 & 0.94359 & -0.0825 \\
\hline $\mathrm{C}$ & 3.147206 & 1.705046 & 1.459552 & $\mathrm{C}$ & -3.14686 & 1.705575 & -1.45929 \\
\hline $\mathrm{C}$ & -4.68945 & -1.13048 & 0.324197 & $\mathrm{C}$ & 4.689499 & -1.13076 & -0.32474 \\
\hline $\mathrm{C}$ & -0.76014 & -2.76915 & 2.080447 & $\mathrm{C}$ & 0.759435 & -2.76979 & -2.0804 \\
\hline $\mathrm{O}$ & -2.44385 & 1.155679 & 0.851809 & $\mathrm{O}$ & 2.443937 & 1.155642 & -0.8519 \\
\hline $\mathrm{O}$ & 1.089839 & -3.76686 & 0.728766 & $\mathrm{O}$ & -1.09029 & -3.76684 & -0.72799 \\
\hline $\mathrm{H}$ & 1.061482 & -2.45128 & -0.93352 & $\mathrm{H}$ & -1.0614 & -2.45094 & 0.934 \\
\hline $\mathrm{C}$ & -1.00929 & 3.937012 & -1.26233 & $\mathrm{C}$ & 1.009641 & 3.93687 & 1.262512 \\
\hline $\mathrm{O}$ & 4.236628 & -0.5005 & -1.1274 & $\mathrm{O}$ & -4.23666 & -0.49982 & 1.127608 \\
\hline $\mathrm{C}$ & 5.42888 & -0.88059 & -1.84457 & $\mathrm{C}$ & -5.42906 & -0.87992 & 1.844588 \\
\hline $\mathrm{O}$ & -0.85831 & 3.264916 & 1.110141 & $\mathrm{O}$ & 0.85839 & 3.264906 & -1.11002 \\
\hline $\mathrm{C}$ & -1.68146 & 4.359828 & 1.513845 & $\mathrm{C}$ & 1.681281 & 4.36002 & -1.51371 \\
\hline $\mathrm{H}$ & -4.55774 & 0.790904 & -1.36695 & $\mathrm{H}$ & 4.558048 & 0.790785 & 1.366191 \\
\hline $\mathrm{H}$ & -3.83274 & -0.11039 & -2.67812 & $\mathrm{H}$ & 3.833548 & -0.11053 & 2.677586 \\
\hline $\mathrm{H}$ & -1.54251 & 0.413209 & -1.65547 & $\mathrm{H}$ & 1.542922 & 0.413052 & 1.655467 \\
\hline $\mathrm{H}$ & -2.41044 & 1.71852 & -2.44658 & $\mathrm{H}$ & 2.411009 & 1.718315 & 2.446508 \\
\hline $\mathrm{H}$ & -3.13739 & 2.666954 & -0.36315 & $\mathrm{H}$ & 3.137654 & 2.666865 & 0.363029 \\
\hline $\mathrm{H}$ & -2.1034 & -1.90569 & -1.88211 & $\mathrm{H}$ & 2.103467 & -1.9056 & 1.881725 \\
\hline $\mathrm{H}$ & -3.15627 & -4.14619 & -0.90185 & $\mathrm{H}$ & 3.156333 & -4.14619 & 0.90143 \\
\hline $\mathrm{H}$ & -3.08217 & -3.35807 & 0.652057 & $\mathrm{H}$ & 3.081688 & -3.35817 & -0.6525 \\
\hline $\mathrm{H}$ & -1.19275 & -4.90504 & 0.159899 & $\mathrm{H}$ & 1.192325 & -4.90517 & -0.15945 \\
\hline $\mathrm{H}$ & -0.70728 & -3.9239 & -1.20922 & $\mathrm{H}$ & 0.707372 & -3.92366 & 1.209597 \\
\hline $\mathrm{H}$ & 0.320948 & 1.633775 & -1.53047 & $\mathrm{H}$ & -0.32049 & 1.633584 & 1.53069 \\
\hline $\mathrm{H}$ & 1.137434 & 2.649547 & -0.36365 & $\mathrm{H}$ & -1.13715 & 2.649489 & 0.364133 \\
\hline $\mathrm{H}$ & 0.474341 & 1.022412 & 1.467876 & $\mathrm{H}$ & -0.47423 & 1.022359 & -1.46768 \\
\hline $\mathrm{H}$ & -0.29845 & 0.023427 & 0.272265 & $\mathrm{H}$ & 0.298473 & 0.0233 & -0.27207 \\
\hline $\mathrm{H}$ & 1.603354 & -1.28175 & 1.874689 & $\mathrm{H}$ & -1.60358 & -1.28186 & -1.8743 \\
\hline $\mathrm{H}$ & 2.874793 & -1.8009 & 0.786767 & $\mathrm{H}$ & -2.87495 & -1.80075 & -0.78619 \\
\hline $\mathrm{H}$ & 1.892634 & -0.187 & -0.9521 & $\mathrm{H}$ & -1.89276 & -0.18664 & 0.952349 \\
\hline $\mathrm{H}$ & 4.089301 & 2.183136 & 1.696568 & $\mathrm{H}$ & -4.08893 & 2.183649 & -1.69645 \\
\hline $\mathrm{H}$ & 2.270542 & 2.026557 & 2.00652 & $\mathrm{H}$ & -2.27006 & 2.027309 & -2.0059 \\
\hline $\mathrm{H}$ & -5.69927 & -1.1048 & -0.10347 & $\mathrm{H}$ & 5.699359 & -1.10558 & 0.102869 \\
\hline $\mathrm{H}$ & -4.61695 & -2.0231 & 0.946214 & $\mathrm{H}$ & 4.61664 & -2.02322 & -0.94695 \\
\hline $\mathrm{H}$ & -4.58824 & -0.24969 & 0.964555 & $\mathrm{H}$ & 4.588605 & -0.2498 & -0.9649 \\
\hline $\mathrm{H}$ & 0.026265 & -2.40861 & 2.742617 & $\mathrm{H}$ & -0.02709 & -2.40924 & -2.74242 \\
\hline $\mathrm{H}$ & -1.52841 & -1.99573 & 2.001092 & $\mathrm{H}$ & 1.527944 & -1.99656 & -2.00153 \\
\hline $\mathrm{H}$ & -1.21407 & -3.6506 & 2.543772 & $\mathrm{H}$ & 1.212992 & -3.65149 & -2.54363 \\
\hline $\mathrm{H}$ & -1.98904 & 1.654802 & 1.545386 & $\mathrm{H}$ & 1.989044 & 1.654832 & -1.54539 \\
\hline $\mathrm{H}$ & -1.92378 & 4.528459 & -1.18598 & $\mathrm{H}$ & 1.924093 & 4.528364 & 1.186138 \\
\hline $\mathrm{H}$ & -0.15393 & 4.597066 & -1.09866 & $\mathrm{H}$ & 0.154229 & 4.596904 & 1.099026 \\
\hline $\mathrm{H}$ & -0.94566 & 3.554276 & -2.28248 & $\mathrm{H}$ & 0.946128 & 3.554038 & 2.282636 \\
\hline $\mathrm{H}$ & 5.109787 & -1.63615 & -2.5578 & $\mathrm{H}$ & -5.10969 & -1.63394 & 2.559306 \\
\hline $\mathrm{H}$ & 5.850485 & -0.01869 & -2.36246 & $\mathrm{H}$ & -5.85193 & -0.01758 & 2.360696 \\
\hline $\mathrm{H}$ & 6.169738 & -1.29076 & -1.15776 & $\mathrm{H}$ & -6.16896 & -1.29205 & 1.157922 \\
\hline $\mathrm{H}$ & -1.40039 & 5.288463 & 1.009163 & $\mathrm{H}$ & 1.399807 & 5.288637 & -1.00921 \\
\hline $\mathrm{H}$ & -1.51604 & 4.479824 & 2.584851 & $\mathrm{H}$ & 1.516038 & 4.479834 & -2.58476 \\
\hline
\end{tabular}




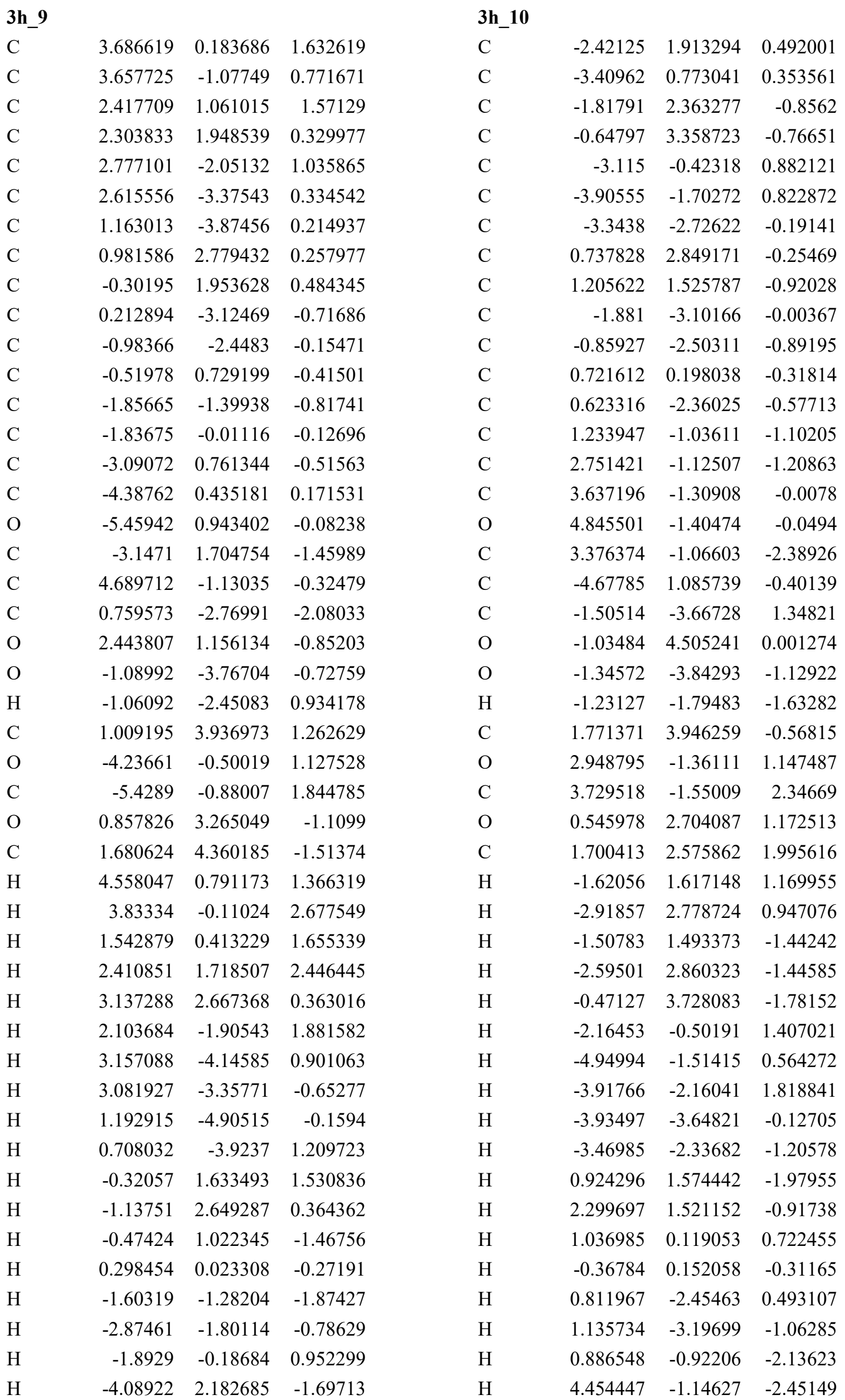




$\begin{array}{lrrrrrrr}\mathrm{H} & -2.27035 & 2.026387 & -2.00665 & \mathrm{H} & 2.82409 & -0.93765 & -3.31274 \\ \mathrm{H} & 5.699568 & -1.10587 & 0.102889 & \mathrm{H} & -4.46141 & 1.356922 & -1.44008 \\ \mathrm{H} & 4.616454 & -2.0225 & -0.94739 & \mathrm{H} & -5.381 & 0.253132 & -0.41753 \\ \mathrm{H} & 4.58925 & -0.24907 & -0.96458 & \mathrm{H} & -5.18305 & 1.949807 & 0.045677 \\ \mathrm{H} & -0.02723 & -2.41024 & -2.7425 & \mathrm{H} & -0.49285 & -4.07049 & 1.336163 \\ \mathrm{H} & 1.52737 & -1.99595 & -2.00155 & \mathrm{H} & -1.56291 & -2.9052 & 2.129897 \\ \mathrm{H} & 1.214007 & -3.6513 & -2.54328 & \mathrm{H} & -2.19075 & -4.4763 & 1.617837 \\ \mathrm{H} & 1.988643 & 1.655228 & -1.54541 & \mathrm{H} & -0.82772 & 4.272049 & 0.91708 \\ \mathrm{H} & 1.923575 & 4.528581 & 1.186212 & \mathrm{H} & 1.455195 & 4.905758 & -0.15697 \\ \mathrm{H} & 0.153684 & 4.596893 & 1.099203 & \mathrm{H} & 2.757458 & 3.695463 & -0.17391 \\ \mathrm{H} & 0.945796 & 3.554073 & 2.282734 & \mathrm{H} & 1.870577 & 4.052633 & -1.65141 \\ \mathrm{H} & -5.10937 & -1.63365 & 2.559907 & \mathrm{H} & 3.009381 & -1.57288 & 3.16037 \\ \mathrm{H} & -5.85189 & -0.01751 & 2.360413 & \mathrm{H} & 4.280259 & -2.48948 & 2.295223 \\ \mathrm{H} & -6.16876 & -1.29272 & 1.158375 & \mathrm{H} & 4.429371 & -0.7244 & 2.476559 \\ \mathrm{H} & 1.39992 & 5.288572 & -1.00841 & \mathrm{H} & 2.365422 & 1.776718 & 1.652181 \\ \mathrm{H} & 1.514319 & 4.480664 & -2.58456 & \mathrm{H} & 1.336566 & 2.321333 & 2.991671 \\ \mathrm{H} & 2.746084 & 4.169403 & -1.34495 & \mathrm{H} & 2.267739 & 3.509673 & 2.057672\end{array}$

\begin{tabular}{|c|c|c|c|c|c|c|c|}
\hline \multicolumn{4}{|c|}{ 3h_11 } & \multicolumn{4}{|c|}{ 3h_12 } \\
\hline $\mathrm{C}$ & 3.593755 & 1.774989 & 0.840694 & $\mathrm{C}$ & -3.65788 & 1.140522 & -1.54284 \\
\hline C & 3.896677 & 0.515484 & 0.031154 & $\mathrm{C}$ & -3.95286 & -0.11915 & -0.73127 \\
\hline C & 2.105976 & 2.007853 & 1.18182 & $\mathrm{C}$ & -2.2005 & 1.646439 & -1.47924 \\
\hline C & 1.261426 & 2.57774 & 0.040565 & $\mathrm{C}$ & -1.8482 & 2.431468 & -0.21578 \\
\hline C & 3.679756 & -0.69391 & 0.56418 & $\mathrm{C}$ & -3.36007 & -1.27709 & -1.04955 \\
\hline C & 3.949029 & -2.04418 & -0.04919 & $\mathrm{C}$ & -3.54243 & -2.62558 & -0.40126 \\
\hline C & 2.920598 & -3.13641 & 0.302214 & $\mathrm{C}$ & -2.27086 & -3.49293 & -0.34013 \\
\hline C & -0.25587 & 2.733298 & 0.383469 & $\mathrm{C}$ & -0.3579 & 2.893861 & -0.13632 \\
\hline C & -0.90276 & 1.471289 & 0.992454 & $\mathrm{C}$ & 0.668883 & 1.77705 & -0.42863 \\
\hline C & 1.514673 & -3.02181 & -0.28336 & $\mathrm{C}$ & -1.13782 & -3.05313 & 0.584984 \\
\hline C & 0.35268 & -2.84739 & 0.624382 & $\mathrm{C}$ & 0.17953 & -2.68891 & 0.005266 \\
\hline C & -0.76457 & 0.15986 & 0.208205 & $\mathrm{C}$ & 0.574515 & 0.502239 & 0.421168 \\
\hline C & -1.04553 & -2.38405 & 0.261913 & $\mathrm{C}$ & 1.31415 & -1.93276 & 0.669518 \\
\hline C & -1.45004 & -1.0359 & 0.906888 & $\mathrm{C}$ & 1.630416 & -0.55099 & 0.043568 \\
\hline C & -2.96156 & -0.8883 & 1.002281 & $\mathrm{C}$ & 3.059326 & -0.15963 & 0.396356 \\
\hline C & -3.74296 & -0.82488 & -0.27445 & $\mathrm{C}$ & 4.106654 & -0.65486 & -0.55455 \\
\hline C & -3.26642 & -0.98782 & -1.37988 & $\mathrm{O}$ & 3.863473 & -1.3299 & -1.53468 \\
\hline C & -3.59734 & -0.83733 & 2.177892 & $\mathrm{C}$ & 3.413632 & 0.517722 & 1.492607 \\
\hline C & 4.472656 & 0.755384 & -1.34029 & $\mathrm{C}$ & -4.95159 & 0.052446 & 0.383631 \\
\hline C & 1.431189 & -2.68098 & -1.75338 & $\mathrm{C}$ & -1.5431 & -2.62033 & 1.975052 \\
\hline C & 1.411907 & 1.772717 & -1.13105 & $\mathrm{O}$ & -2.18945 & 1.658386 & 0.936248 \\
\hline C & 0.703676 & -4.15489 & 0.127078 & $\mathrm{O}$ & -0.04779 & -4.01176 & 0.532215 \\
\hline H & 0.595222 & -2.71975 & 1.681252 & $\mathrm{H}$ & 0.225586 & -2.66784 & -1.08507 \\
\hline C & -0.48144 & 3.915312 & 1.333296 & $\mathrm{C}$ & -0.12864 & 4.0752 & -1.08741 \\
\hline C & -5.04993 & -0.56706 & -0.08402 & $\mathrm{O}$ & 5.35431 & -0.28176 & -0.22202 \\
\hline C & -5.86842 & -0.51251 & -1.26978 & $\mathrm{C}$ & 6.409246 & -0.73432 & -1.09471 \\
\hline C & -0.96076 & 2.922996 & -0.87697 & $\mathrm{O}$ & -0.27156 & 3.335059 & 1.245404 \\
\hline C & -0.85518 & 4.192066 & -1.52287 & $\mathrm{C}$ & 0.924253 & 3.962677 & 1.689873 \\
\hline $\mathrm{H}$ & 3.984726 & 2.652555 & 0.315068 & $\mathrm{H}$ & -4.33281 & 1.944579 & -1.23151 \\
\hline H & 4.142933 & 1.721579 & 1.786885 & $\mathrm{H}$ & -3.89177 & 0.941172 & -2.59415 \\
\hline
\end{tabular}




\begin{tabular}{|c|c|c|c|c|c|c|c|}
\hline $\mathrm{H}$ & 1.679815 & 1.067793 & 1.537149 & $\mathrm{H}$ & -1.53082 & 0.792016 & -1.59422 \\
\hline $\mathrm{H}$ & 2.050588 & 2.715536 & 2.015212 & $\mathrm{H}$ & -2.02627 & 2.302717 & -2.3372 \\
\hline $\mathrm{H}$ & 1.648718 & 3.584514 & -0.18175 & $\mathrm{H}$ & -2.45174 & 3.352656 & -0.20533 \\
\hline $\mathrm{H}$ & 3.290912 & -0.73231 & 1.582677 & $\mathrm{H}$ & -2.67862 & -1.27601 & -1.90139 \\
\hline $\mathrm{H}$ & 4.919411 & -2.41109 & 0.313604 & $\mathrm{H}$ & -4.27767 & -3.20242 & -0.97962 \\
\hline $\mathrm{H}$ & 4.052384 & -1.97131 & -1.13412 & $\mathrm{H}$ & -3.97075 & -2.52808 & 0.598933 \\
\hline $\mathrm{H}$ & 3.314483 & -4.09498 & -0.05732 & $\mathrm{H}$ & -2.56312 & -4.49282 & 0.003351 \\
\hline 11 & 2.83834 & -3.22853 & 1.390234 & $\mathrm{H}$ & -1.86521 & -3.62277 & -1.34892 \\
\hline $\mathrm{H}$ & -0.49639 & 1.333785 & 1.999771 & $\mathrm{H}$ & 0.574662 & 1.509947 & -1.48625 \\
\hline $\mathrm{H}$ & -1.96386 & 1.705732 & 1.12364 & $\mathrm{H}$ & 1.670941 & 2.206526 & -0.33147 \\
\hline $\mathrm{H}$ & -1.18225 & 0.276537 & -0.79311 & $\mathrm{H}$ & 0.648035 & 0.753335 & 1.48364 \\
\hline $\mathrm{H}$ & 0.290326 & -0.08322 & 0.078094 & $\mathrm{H}$ & -0.41129 & 0.054025 & 0.298456 \\
\hline $\mathrm{H}$ & -1.18291 & -2.33664 & -0.81799 & $\mathrm{H}$ & 1.139958 & -1.81754 & 1.742559 \\
\hline $\mathrm{H}$ & -1.72289 & -3.16478 & 0.628313 & $\mathrm{H}$ & 2.195003 & -2.57654 & 0.568107 \\
\hline $\mathrm{H}$ & -1.09546 & -1.05841 & 1.944802 & $\mathrm{H}$ & 1.605678 & -0.68056 & -1.04314 \\
\hline $\mathrm{H}$ & -4.67305 & -0.74232 & 2.240578 & $\mathrm{H}$ & 4.450928 & 0.733062 & 1.709974 \\
\hline $\mathrm{H}$ & -3.04588 & -0.89509 & 3.109904 & $\mathrm{H}$ & 2.676667 & 0.870978 & 2.20375 \\
\hline $\mathrm{H}$ & 5.437748 & 1.270991 & -1.26192 & $\mathrm{H}$ & -5.92812 & 0.344685 & -0.02182 \\
\hline $\mathrm{H}$ & 4.633289 & -0.16072 & -1.90939 & $\mathrm{H}$ & -5.09692 & -0.85094 & 0.976514 \\
\hline $\mathrm{H}$ & 3.803765 & 1.406558 & -1.90965 & $\mathrm{H}$ & -4.62336 & 0.857281 & 1.047282 \\
\hline $\mathrm{H}$ & 0.417678 & -2.80833 & -2.13168 & $\mathrm{H}$ & -0.67582 & -2.50479 & 2.624289 \\
\hline $\mathrm{H}$ & 1.740979 & -1.64801 & -1.9306 & $\mathrm{H}$ & -2.08141 & -1.66965 & 1.942127 \\
\hline $\mathrm{H}$ & 2.092492 & -3.33625 & -2.32872 & $\mathrm{H}$ & -2.20383 & -3.36848 & 2.42377 \\
\hline $\mathrm{H}$ & 0.597431 & 1.909045 & -1.63625 & $\mathrm{H}$ & -1.72902 & 2.093261 & 1.668131 \\
\hline $\mathrm{H}$ & -0.0008 & 4.829031 & 0.978088 & $\mathrm{H}$ & -0.78239 & 4.910345 & -0.82435 \\
\hline $\mathrm{H}$ & -1.55115 & 4.107399 & 1.447689 & $\mathrm{H}$ & 0.906507 & 4.420905 & -1.04988 \\
\hline $\mathrm{H}$ & -0.07488 & 3.689318 & 2.320707 & $\mathrm{H}$ & -0.33606 & 3.787145 & -2.11965 \\
\hline $\mathrm{H}$ & -6.87424 & -0.2994 & -0.91697 & $\mathrm{H}$ & 7.326235 & -0.33021 & -0.67357 \\
\hline $\mathrm{H}$ & -5.52167 & 0.278524 & -1.93559 & $\mathrm{H}$ & 6.445928 & -1.82409 & -1.11447 \\
\hline $\mathrm{H}$ & -5.84227 & -1.46745 & -1.79575 & $\mathrm{H}$ & 6.254443 & -0.35735 & -2.10615 \\
\hline $\mathrm{H}$ & -1.35571 & 4.98159 & -0.95522 & $\mathrm{H}$ & 0.789655 & 4.150464 & 2.755519 \\
\hline 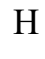 & -1.35667 & 4.081083 & -2.48464 & $\mathrm{H}$ & 1.097645 & 4.919137 & 1.186239 \\
\hline & 0.184657 & 4.485604 & -1.70287 & $\mathrm{H}$ & 1.804685 & 3.326108 & 1.556126 \\
\hline
\end{tabular}

Table S11. SCF energies (Hartree) computed at the PCM/ mPW1PW91/6-31G* level of theory using coordinate files incorporated in Table S10.

3a_1_DP4+

3a_2_DP4+

3a_3_DP4+

3a_4_DP4+

3a_5_DP4+

3a_6_DP4+

3a_7_DP4+

3a_8_DP4+

3a_9_DP4+

3a_10_DP4+

3a_11_DP4+

3a_12_DP4+

3a_13_DP4+
SCF Energy (PCM/mPW1PW91/6-31G*) =-1235.78696109

SCF Energy (PCM/mPW1PW91/6-31G*) $=-1235.78669756$

SCF Energy (PCM/mPW1PW91/6-31G*) $=-1235.78672930$

SCF Energy $\left(\mathrm{PCM} / \mathrm{mPW} 1 \mathrm{PW} 91 / 6-31 \mathrm{G}^{*}\right)=-1235.78762913$

SCF Energy (PCM/mPW1PW91/6-31G*) $=-1235.78709438$

SCF Energy (PCM/mPW1PW91/6-31G*) $=-1235.78732654$

SCF Energy (PCM/mPW1PW91/6-31G*) =-1235.78762715

SCF Energy $\left(\mathrm{PCM} / \mathrm{mPW} 1 \mathrm{PW} 91 / 6-31 \mathrm{G}^{*}\right)=-1235.78665937$

SCF Energy (PCM/mPW1PW91/6-31G*) =-1235.78669793

SCF Energy (PCM/mPW1PW91/6-31G*) =-1235.78764771

SCF Energy (PCM/mPW1PW91/6-31G*) =-1235.78669761

SCF Energy (PCM/mPW1PW91/6-31G*) $=-1235.77983130$

SCF Energy (PCM/mPW1PW91/6-31G*) =-1235.78732562 
3a_14_DP4+ 3a-15 DP4+ 3a_16_DP4+ 3a_17_DP4+ 3a_18_DP4+ 3a_19_DP4+ 3a_20_DP4+ 3b_1_DP4+ 3b_2_DP4+ 3b_3_DP4+ 3b_4_DP4+ 3b 5 DP4+ 3b_6_DP4+ 3b_7_DP4+ 3b_8_DP4+ 3b_9_DP4+ 3b_10_DP4+ 3b_11_DP4+ 3b_12_DP4+ 3b_13_DP4+ 3b_14_DP4+ 3b_15_DP4+ 3b_16_DP4+ 3b_17_DP4+ 3b_18_DP4+ 3c_1_DP4+ 3c_2_DP4+ 3c 3 DP $4+$ 3c_4_DP4+ 3c_5_DP4+ 3c_6_DP4+ 3c_7_DP4+ 3c_8_DP4+ 3c_9_DP4+ 3c_10_DP4+ 3d_1_DP4+ 3d_2_DP4+ 3d_3_DP4+ 3d 4 DP4+ 3d_5_DP4+ 3d_6_DP4+ 3d_7_DP4+ 3d_8_DP4+ 3d_9_DP4+ 3d_10_DP4+ 3d_11_DP4+ 3d_12_DP4+ 3d_13_DP4+ 3d_14_DP4+
SCF Energy (PCM/mPW1PW91/6-31G*) =-1235.78524980 SCF Energy (PCM/mPW1PW91/6-31G*) =-1235.77780977 SCF Energy (PCM/mPW1PW91/6-31G*) =-1235.77839226 SCF Energy (PCM/mPW1PW91/6-31G*) $=-1235.77825980$ SCF Energy (PCM/mPW1PW91/6-31G*) =-1235.78764749 SCF Energy $\left(\mathrm{PCM} / \mathrm{mPW} 1 \mathrm{PW} 91 / 6-31 \mathrm{G}^{*}\right)=-1235.78672929$ SCF Energy $\left(\mathrm{PCM} / \mathrm{mPW} 1 \mathrm{PW} 91 / 6-31 \mathrm{G}^{*}\right)=-1235.77977713$ SCF Energy (PCM/mPW1PW91/6-31G*) $=-1235.78788397$ SCF Energy $\left(\mathrm{PCM} / \mathrm{mPW} 1 \mathrm{PW} 91 / 6-31 \mathrm{G}^{*}\right)=-1235.78417434$ SCF Energy (PCM/mPW1PW91/6-31G*) $=-1235.78362325$ SCF Energy $\left(\mathrm{PCM} / \mathrm{mPW} 1 \mathrm{PW} 91 / 6-31 \mathrm{G}^{*}\right)=-1235.78245959$ SCF Energy $\left(\mathrm{PCM} / \mathrm{mPW} 1 \mathrm{PW} 91 / 6-31 \mathrm{G}^{*}\right)=-1235.78644103$ SCF Energy (PCM/mPW1PW91/6-31G*) =-1235.78526932 SCF Energy (PCM/mPW1PW91/6-31G*) $=-1235.77761455$ SCF Energy $\left(\mathrm{PCM} / \mathrm{mPW} 1 \mathrm{PW} 91 / 6-31 \mathrm{G}^{*}\right)=-1235.77937962$ SCF Energy (PCM/mPW1PW91/6-31G*) $=-1235.78245861$ SCF Energy $\left(\mathrm{PCM} / \mathrm{mPW} 1 \mathrm{PW} 91 / 6-31 \mathrm{G}^{*}\right)=-1235.77736515$ SCF Energy $\left(\mathrm{PCM} / \mathrm{mPW} 1 \mathrm{PW} 91 / 6-31 \mathrm{G}^{*}\right)=-1235.77972886$ SCF Energy (PCM/mPW1PW91/6-31G*) $=-1235.78360895$ SCF Energy $\left(\mathrm{PCM} / \mathrm{mPW} 1 \mathrm{PW} 91 / 6-31 \mathrm{G}^{*}\right)=-1235.78424414$ SCF Energy $\left(\mathrm{PCM} / \mathrm{mPW} 1 \mathrm{PW} 91 / 6-31 \mathrm{G}^{*}\right)=-1235.78592997$ SCF Energy $\left(\mathrm{PCM} / \mathrm{mPW} 1 \mathrm{PW} 91 / 6-31 \mathrm{G}^{*}\right)=-1235.78136345$ SCF Energy (PCM/mPW1PW91/6-31G*) $=-1235.78644108$ SCF Energy (PCM/mPW1PW91/6-31G*) =-1235.78394405 SCF Energy (PCM/mPW1PW91/6-31G*) $=-1235.77825973$ SCF Energy (PCM/mPW1PW91/6-31G*) =-1235.78954012 SCF Energy $\left(\mathrm{PCM} / \mathrm{mPW} 1 \mathrm{PW} 91 / 6-31 \mathrm{G}^{*}\right)=-1235.78903528$ SCF Energy $\left(\mathrm{PCM} / \mathrm{mPW} 1 \mathrm{PW} 91 / 6-31 \mathrm{G}^{*}\right)=-1235.78980631$ SCF Energy (PCM/mPW1PW91/6-31G*) $=-1235.79035888$ SCF Energy $\left(\mathrm{PCM} / \mathrm{mPW} 1 \mathrm{PW} 91 / 6-31 \mathrm{G}^{*}\right)=-1235.78711891$ SCF Energy (PCM/mPW1PW91/6-31G*) =-1235.78095019 SCF Energy (PCM/mPW1PW91/6-31G*) $=-1235.78502444$ SCF Energy (PCM/mPW1PW91/6-31G*) $=-1235.78277613$ SCF Energy (PCM/mPW1PW91/6-31G*) $=-1235.78560078$ SCF Energy (PCM/mPW1PW91/6-31G*) =-1235.79035809 SCF Energy (PCM/mPW1PW91/6-31G*) $=-1235.78872777$ SCF Energy (PCM/mPW1PW91/6-31G*) $=-1235.78785930$ SCF Energy $\left(\mathrm{PCM} / \mathrm{mPW} 1 \mathrm{PW} 91 / 6-31 \mathrm{G}^{*}\right)=-1235.78872822$ SCF Energy (PCM/mPW1PW91/6-31G*) =-1235.78911056 SCF Energy (PCM/mPW1PW91/6-31G*) $=-1235.78480941$ SCF Energy (PCM/mPW1PW91/6-31G*) $=-1235.78413072$ SCF Energy $\left(\mathrm{PCM} / \mathrm{mPW} 1 \mathrm{PW} 91 / 6-31 \mathrm{G}^{*}\right)=-1235.78246653$ SCF Energy (PCM/mPW1PW91/6-31G*) $=-1235.78739851$ SCF Energy (PCM/mPW1PW91/6-31G*) =-1235.78475391 SCF Energy (PCM/mPW1PW91/6-31G*) $=-1235.77934637$ SCF Energy (PCM/mPW1PW91/6-31G*) $=-1235.78156022$ SCF Energy (PCM/mPW1PW91/6-31G*) $=-1235.78440250$ SCF Energy $\left(\mathrm{PCM} / \mathrm{mPW} 1 \mathrm{PW} 91 / 6-31 \mathrm{G}^{*}\right)=-1235.78515247$ SCF Energy (PCM/mPW1PW91/6-31G*) =-1235.78766515 
3d_15_DP4+ 3d_16_DP4+ 3d_17_DP4+ 3e_1_DP4+ 3e_2_DP4+ 3e_3_DP4+ 3e_4_DP4+ 3e_5_DP4+ 3e_6_DP4+ 3e_7_DP4+ 3e_8_DP4+ 3e_9_DP4+ 3e_10_DP4+ 3e_11_DP4+ 3e_12_DP4+ 3e_13_DP4+ 3e_14_DP4+ 3e_15_DP4+ 3e_16_DP4+ 3e_17_DP4+ 3f_1_DP4+ 3f_2_DP4+ 3f_3_DP4+ 3f_4_DP4+ 3f_5_DP4+ 3f 6 DP4+ 3f_7_DP4+ 3f_8_DP4+ 3f_9_DP4+ 3f_10_DP4+ 3f_11_DP4+ $3 f$ - 12 DP4+ 3f_13_DP4+ 3f_14_DP4+ 3f_15_DP4+ 3f_16_DP4+ 3f_17_DP4+ 3f_18_DP4+ 3g_1_DP4+ 3g_2_DP4+ 3g_3_DP4+ 3g_4_DP4+ 3g_5_DP4+ 3g_6_DP4+ 3g_7_DP4+ 3g_8_DP4+ 3g_9_DP4+ 3g_10_DP4+ 3g_11_DP4+
SCF Energy (PCM/mPW1PW91/6-31G*) =-1235.78490733 SCF Energy (PCM/mPW1PW91/6-31G*) $=-1235.78174246$ SCF Energy (PCM/mPW1PW91/6-31G*) =-1235.78226324 SCF Energy (PCM/mPW1PW91/6-31G*) =-1235.78591797 SCF Energy (PCM/mPW1PW91/6-31G*) $=-1235.78454532$ SCF Energy (PCM/mPW1PW91/6-31G*) $=-1235.77949072$ SCF Energy (PCM/mPW1PW91/6-31G*) $=-1235.77949268$ SCF Energy (PCM/mPW1PW91/6-31G*) $=-1235.78659150$ SCF Energy $\left(\mathrm{PCM} / \mathrm{mPW} 1 \mathrm{PW} 91 / 6-31 \mathrm{G}^{*}\right)=-1235.78454491$ SCF Energy (PCM/mPW1PW91/6-31G*) =-1235.78026655 SCF Energy $\left(\mathrm{PCM} / \mathrm{mPW} 1 \mathrm{PW} 91 / 6-31 \mathrm{G}^{*}\right)=-1235.78680699$ SCF Energy (PCM/mPW1PW91/6-31G*) $=-1235.78026610$ SCF Energy $\left(\mathrm{PCM} / \mathrm{mPW} 1 \mathrm{PW} 91 / 6-31 \mathrm{G}^{*}\right)=-1235.77981674$ SCF Energy (PCM/mPW1PW91/6-31G*) $=-1235.78526728$ SCF Energy (PCM/mPW1PW91/6-31G*) =-1235.77949208 SCF Energy (PCM/mPW1PW91/6-31G*) =-1235.77949284 SCF Energy $\left(\mathrm{PCM} / \mathrm{mPW} 1 \mathrm{PW} 91 / 6-31 \mathrm{G}^{*}\right)=-1235.77846889$ SCF Energy (PCM/mPW1PW91/6-31G*) =-1235.78009226 SCF Energy $\left(\mathrm{PCM} / \mathrm{mPW} 1 \mathrm{PW} 91 / 6-31 \mathrm{G}^{*}\right)=-1235.78009129$ SCF Energy (PCM/mPW1PW91/6-31G*) =-1235.77574371 SCF Energy $\left(\mathrm{PCM} / \mathrm{mPW} 1 \mathrm{PW} 91 / 6-31 \mathrm{G}^{*}\right)=-1235.78218040$ SCF Energy $\left(\mathrm{PCM} / \mathrm{mPW} 1 \mathrm{PW} 91 / 6-31 \mathrm{G}^{*}\right)=-1235.78274787$ SCF Energy (PCM/mPW1PW91/6-31G*) $=-1235.78291841$ SCF Energy (PCM/mPW1PW91/6-31G*) =-1235.77546006 SCF Energy (PCM/mPW1PW91/6-31G*) $=-1235.77554960$ SCF Energy $\left(\mathrm{PCM} / \mathrm{mPW} 1 \mathrm{PW} 91 / 6-31 \mathrm{G}^{*}\right)=-1235.77681439$ SCF Energy $\left(\mathrm{PCM} / \mathrm{mPW} 1 \mathrm{PW} 91 / 6-31 \mathrm{G}^{*}\right)=-1235.77991012$ SCF Energy (PCM/mPW1PW91/6-31G*) =-1235.78211665 SCF Energy (PCM/mPW1PW91/6-31G*) $=-1235.79033124$ SCF Energy $\left(\mathrm{PCM} / \mathrm{mPW} 1 \mathrm{PW} 91 / 6-31 \mathrm{G}^{*}\right)=-1235.78276373$ SCF Energy (PCM/mPW1PW91/6-31G*) $=-1235.78365105$ SCF Energy (PCM/mPW1PW91/6-31G*) $=-1235.78305708$ SCF Energy (PCM/mPW1PW91/6-31G*) $=-1235.78308932$ SCF Energy (PCM/mPW1PW91/6-31G*) $=-1235.78116437$ SCF Energy (PCM/mPW1PW91/6-31G*) $=-1235.77553598$ SCF Energy (PCM/mPW1PW91/6-31G*) $=-1235.78292178$ SCF Energy (PCM/mPW1PW91/6-31G*) $=-1235.78158835$ SCF Energy $\left(\mathrm{PCM} / \mathrm{mPW} 1 \mathrm{PW} 91 / 6-31 \mathrm{G}^{*}\right)=-1235.78170139$ SCF Energy (PCM/mPW1PW91/6-31G*) $=-1235.78841622$ SCF Energy (PCM/mPW1PW91/6-31G*) $=-1235.78807355$ SCF Energy (PCM/mPW1PW91/6-31G*) $=-1235.78844143$ SCF Energy $\left(\mathrm{PCM} / \mathrm{mPW} 1 \mathrm{PW} 91 / 6-31 \mathrm{G}^{*}\right)=-1235.78000226$ SCF Energy $\left(\mathrm{PCM} / \mathrm{mPW} 1 \mathrm{PW} 91 / 6-31 \mathrm{G}^{*}\right)=-1235.78170689$ SCF Energy $\left(\mathrm{PCM} / \mathrm{mPW} 1 \mathrm{PW} 91 / 6-31 \mathrm{G}^{*}\right)=-1235.78935138$ SCF Energy (PCM/mPW1PW91/6-31G*) $=-1235.78189250$ SCF Energy $\left(\mathrm{PCM} / \mathrm{mPW} 1 \mathrm{PW} 91 / 6-31 \mathrm{G}^{*}\right)=-1235.78910975$ SCF Energy (PCM/mPW1PW91/6-31G*) $=-1235.78063217$ SCF Energy (PCM/mPW1PW91/6-31G*) =-1235.78281491 SCF Energy (PCM/mPW1PW91/6-31G*) =-1235.78436631 
3g_12_DP4+

3h_1_DP4+

3h_2_DP4+

3h_3_DP4+

3h_4_DP4+

3h_5_DP4+

3h_6_DP4+

3h_7_DP4+

3h_8_DP4+

3h_9_DP4+

3h_10_DP4+

3h_11_DP4+

3h_12_DP4+
SCF Energy (PCM/mPW1PW91/6-31G*) =-1235.78160737

SCF Energy (PCM/mPW1PW91/6-31G*) $=-1235.78474083$

SCF Energy (PCM/mPW1PW91/6-31G*) =-1235.78369445

SCF Energy (PCM/mPW1PW91/6-31G*) $=-1235.78374428$

SCF Energy (PCM/mPW1PW91/6-31G*) $=-1235.78469215$

SCF Energy (PCM/mPW1PW91/6-31G*) =-1235.78041037

SCF Energy $\left(\mathrm{PCM} / \mathrm{mPW} 1 \mathrm{PW} 91 / 6-31 \mathrm{G}^{*}\right)=-1235.78551201$

SCF Energy (PCM/mPW1PW91/6-31G*) =-1235.78447521

SCF Energy (PCM/mPW1PW91/6-31G*) $=-1235.78447382$

SCF Energy (PCM/mPW1PW91/6-31G*) $=-1235.78447314$

SCF Energy (PCM/mPW1PW91/6-31G*) $=-1235.78403608$

SCF Energy $\left(\mathrm{PCM} / \mathrm{mPW} 1 \mathrm{PW} 91 / 6-31 \mathrm{G}^{*}\right)=-1235.78444927$

SCF Energy $\left(\mathrm{PCM} / \mathrm{mPW} 1 \mathrm{PW} 91 / 6-31 \mathrm{G}^{*}\right)=-1235.78530838$

Table S12. Imaginary frequencies and absolute energy values of all conformers of isomers $\mathbf{3 a}-\mathbf{3 h}$ used after optimization at the B3LYP/6-311G(d,p) level of theory as required for DP4+.

3a_1_DP4+ Imaginary Freq $=0$

Zero-point correction $=$

Thermal correction to Energy=

Thermal correction to Enthalpy=

Thermal correction to Gibbs Free Energy=

Sum of electronic and zero-point Energies=

Sum of electronic and thermal Energies=

Sum of electronic and thermal Enthalpies=

Sum of electronic and thermal Free Energies=

3a_2_DP4+ Imaginary Freq $=0$

Zero-point correction $=$

Thermal correction to Energy=

Thermal correction to Enthalpy=

Thermal correction to Gibbs Free Energy=

Sum of electronic and zero-point Energies=

Sum of electronic and thermal Energies=

Sum of electronic and thermal Enthalpies=

Sum of electronic and thermal Free Energies=

3a_3_DP4+ Imaginary Freq $=0$

Zero-point correction $=$

Thermal correction to Energy=

Thermal correction to Enthalpy=

Thermal correction to Gibbs Free Energy=

Sum of electronic and zero-point Energies=

Sum of electronic and thermal Energies=

Sum of electronic and thermal Enthalpies=

Sum of electronic and thermal Free Energies=

3a_4_DP4+ Imaginary Freq $=0$

Zero-point correction=

Thermal correction to Energy=

Thermal correction to Enthalpy=

Thermal correction to Gibbs Free Energy=

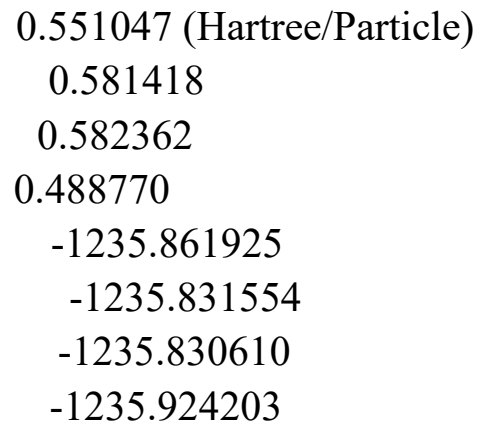

0.550931 (Hartree/Particle)

0.581374

0.582318

0.488408

$-1235.861941$

$-1235.831498$

$-1235.830554$

$-1235.924465$

0.550738 (Hartree/Particle)

0.581243

0.582187

0.488637

$-1235.861927$

$-1235.831422$

$-1235.830478$

$-1235.924028$

0.551054 (Hartree/Particle) 0.581448

0.582393

0.489029 
Sum of electronic and zero-point Energies=

Sum of electronic and thermal Energies=

Sum of electronic and thermal Enthalpies=

Sum of electronic and thermal Free Energies=

3a_5_DP4 + Imaginary Freq $=0$

Zero-point correction=

Thermal correction to Energy=

Thermal correction to Enthalpy=

Thermal correction to Gibbs Free Energy=

Sum of electronic and zero-point Energies=

Sum of electronic and thermal Energies=

Sum of electronic and thermal Enthalpies=

Sum of electronic and thermal Free Energies=

3a_6_DP4+ Imaginary Freq $=0$

Zero-point correction $=$

Thermal correction to Energy=

Thermal correction to Enthalpy=

Thermal correction to Gibbs Free Energy=

Sum of electronic and zero-point Energies=

Sum of electronic and thermal Energies=

Sum of electronic and thermal Enthalpies=

Sum of electronic and thermal Free Energies=

3a_7_DP4+ Imaginary Freq $=0$

Zero-point correction=

Thermal correction to Energy=

Thermal correction to Enthalpy=

Thermal correction to Gibbs Free Energy=

Sum of electronic and zero-point Energies=

Sum of electronic and thermal Energies=

Sum of electronic and thermal Enthalpies=

Sum of electronic and thermal Free Energies=

3a_9_DP4+ Imaginary Freq $=0$

Zero-point correction=

Thermal correction to Energy=

Thermal correction to Enthalpy=

Thermal correction to Gibbs Free Energy=

Sum of electronic and zero-point Energies=

Sum of electronic and thermal Energies=

Sum of electronic and thermal Enthalpies=

Sum of electronic and thermal Free Energies=

3a_10_DP4+ Imaginary Freq $=0$

Zero-point correction $=$

Thermal correction to Energy=

Thermal correction to Enthalpy=

Thermal correction to Gibbs Free Energy=

Sum of electronic and zero-point Energies=

Sum of electronic and thermal Energies=

Sum of electronic and thermal Enthalpies=

Sum of electronic and thermal Free Energies=
$-1235.862668$

$-1235.832273$

$-1235.831329$

$-1235.924692$

0.551010 (Hartree/Particle)

0.580433

0.581377

0.491873

$-1235.862296$

$-1235.832873$

$-1235.831929$

$-1235.921434$

0.550886 (Hartree/Particle)

0.581207

0.582151

0.489174

$-1235.862434$

$-1235.832113$

$-1235.831169$

$-1235.924146$

0.551052 (Hartree/Particle)

0.581447

0.582391

0.489027

$-1235.862669$

$-1235.832274$

$-1235.831330$

$-1235.924694$

0.550933 (Hartree/Particle)

0.581375

0.582319

0.488410

$-1235.861939$

$-1235.831498$

$-1235.830554$

$-1235.924463$

0.550774 (Hartree/Particle)

0.581265

0.582209

0.487717

$-1235.862953$

$-1235.832462$

$-1235.831518$

$-1235.926010$ 
3a_11_DP4+ Imaginary Freq $=0$

Zero-point correction=

Thermal correction to Energy=

Thermal correction to Enthalpy=

Thermal correction to Gibbs Free Energy=

Sum of electronic and zero-point Energies=

Sum of electronic and thermal Energies=

Sum of electronic and thermal Enthalpies=

Sum of electronic and thermal Free Energies=

3a_12_DP4+ Imaginary Freq $=0$

Zero-point correction=

Thermal correction to Energy=

Thermal correction to Enthalpy=

Thermal correction to Gibbs Free Energy=

Sum of electronic and zero-point Energies=

Sum of electronic and thermal Energies=

Sum of electronic and thermal Enthalpies=

Sum of electronic and thermal Free Energies=

3a_13_DP4+ Imaginary Freq $=0$

Zero-point correction=

Thermal correction to Energy=

Thermal correction to Enthalpy=

Thermal correction to Gibbs Free Energy=

Sum of electronic and zero-point Energies=

Sum of electronic and thermal Energies=

Sum of electronic and thermal Enthalpies=

Sum of electronic and thermal Free Energies=

3a_14_DP4+ Imaginary Freq $=0$

Zero-point correction=

Thermal correction to Energy=

Thermal correction to Enthalpy=

Thermal correction to Gibbs Free Energy=

Sum of electronic and zero-point Energies=

Sum of electronic and thermal Energies=

Sum of electronic and thermal Enthalpies=

Sum of electronic and thermal Free Energies=

3a_15_DP4+ Imaginary Freq $=0$

Zero-point correction=

Thermal correction to Energy=

Thermal correction to Enthalpy=

Thermal correction to Gibbs Free Energy=

Sum of electronic and zero-point Energies=

Sum of electronic and thermal Energies=

Sum of electronic and thermal Enthalpies=

Sum of electronic and thermal Free Energies=

3a_16_DP4+ Imaginary Freq $=0$

Zero-point correction=

Thermal correction to Energy=

Thermal correction to Enthalpy=

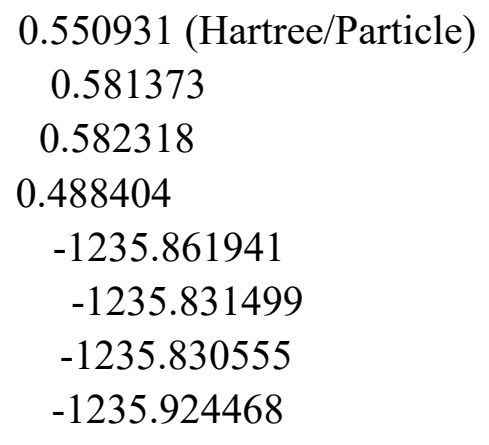

0.550688 (Hartree/Particle)

0.581239

0.582183

0.488274

$-1235.856221$

$-1235.825670$

$-1235.824726$

$-1235.918636$

0.550880 (Hartree/Particle)

0.581204

0.582148

0.489149

$-1235.862439$

$-1235.832115$

$-1235.831171$

$-1235.924170$

0.551582 (Hartree/Particle)

0.581644

0.582588

0.491179

$-1235.859076$

$-1235.829014$

$-1235.828070$

$-1235.919479$

0.550028 (Hartree/Particle)

0.580949

0.581893

0.486604

$-1235.855184$

$-1235.824263$

$-1235.823319$

$-1235.918609$

0.551448 (Hartree/Particle)

0.581395

0.582339 
Thermal correction to Gibbs Free Energy=

Sum of electronic and zero-point Energies=

Sum of electronic and thermal Energies=

Sum of electronic and thermal Enthalpies=

Sum of electronic and thermal Free Energies=

3a_17_DP4+ Imaginary Freq $=0$

Zero-point correction=

Thermal correction to Energy=

Thermal correction to Enthalpy=

Thermal correction to Gibbs Free Energy=

Sum of electronic and zero-point Energies=

Sum of electronic and thermal Energies=

Sum of electronic and thermal Enthalpies=

Sum of electronic and thermal Free Energies=

3a_18_DP4+ Imaginary Freq $=0$

Zero-point correction=

Thermal correction to Energy=

Thermal correction to Enthalpy=

Thermal correction to Gibbs Free Energy=

Sum of electronic and zero-point Energies=

Sum of electronic and thermal Energies=

Sum of electronic and thermal Enthalpies=

Sum of electronic and thermal Free Energies=

3a_19_DP4+ Imaginary Freq $=0$

Zero-point correction=

Thermal correction to Energy=

Thermal correction to Enthalpy=

Thermal correction to Gibbs Free Energy=

Sum of electronic and zero-point Energies=

Sum of electronic and thermal Energies $=$

Sum of electronic and thermal Enthalpies=

Sum of electronic and thermal Free Energies=

3a_20_DP4+ Imaginary Freq $=0$

Zero-point correction=

Thermal correction to Energy=

Thermal correction to Enthalpy=

Thermal correction to Gibbs Free Energy=

Sum of electronic and zero-point Energies=

Sum of electronic and thermal Energies=

Sum of electronic and thermal Enthalpies=

Sum of electronic and thermal Free Energies=

3b_1_DP4+ Imaginary Freq $=0$

Zero-point correction=

Thermal correction to Energy=

Thermal correction to Enthalpy=

Thermal correction to Gibbs Free Energy=

Sum of electronic and zero-point Energies=

Sum of electronic and thermal Energies=

Sum of electronic and thermal Enthalpies=

$$
\begin{aligned}
& 0.491253 \\
& -1235.852881 \\
& -1235.822934 \\
& -1235.821990 \\
& -1235.913076
\end{aligned}
$$

0.551668 (Hartree/Particle)

0.581583

0.582527

0.491167

$-1235.852742$

$-1235.822826$

$-1235.821882$

$-1235.913243$

0.550773 (Hartree/Particle)

0.581265

0.582209

0.487717

$-1235.862954$

$-1235.832462$

$-1235.831518$

$-1235.926010$

0.550741 (Hartree/Particle)

0.581244

0.582188

0.488666

$-1235.861924$

$-1235.831422$

$-1235.830478$

$-1235.924000$

0.550221 (Hartree/Particle)

0.580933

0.581877

0.487814

$-1235.856878$

$-1235.826165$

$-1235.825221$

$-1235.919285$

0.551230 (Hartree/Particle)

0.581346

0.582290

0.490758

$-1235.861091$

$-1235.830975$

$-1235.830031$ 
Sum of electronic and thermal Free Energies=

3b_2_DP4+

3b_3_DP4+

3b_4_DP4+

3b_5_DP4+

3b_6_DP4+

3b_7_DP4+

Imaginary Freq $=0$

Zero-point correction=

Thermal correction to Energy=

Thermal correction to Enthalpy=

Thermal correction to Gibbs Free Energy=

Sum of electronic and zero-point Energies=

Sum of electronic and thermal Energies=

Sum of electronic and thermal Enthalpies=

Sum of electronic and thermal Free Energies=

Imaginary Freq $=0$

Zero-point correction=

Thermal correction to Energy=

Thermal correction to Enthalpy=

Thermal correction to Gibbs Free Energy=

Sum of electronic and zero-point Energies=

Sum of electronic and thermal Energies=

Sum of electronic and thermal Enthalpies=

Sum of electronic and thermal Free Energies=

Imaginary Freq $=0$

Zero-point correction $=$

Thermal correction to Energy=

Thermal correction to Enthalpy=

Thermal correction to Gibbs Free Energy=

Sum of electronic and zero-point Energies=

Sum of electronic and thermal Energies=

Sum of electronic and thermal Enthalpies=

Sum of electronic and thermal Free Energies=

Imaginary Freq $=0$

Zero-point correction=

Thermal correction to Energy=

Thermal correction to Enthalpy=

Thermal correction to Gibbs Free Energy=

Sum of electronic and zero-point Energies=

Sum of electronic and thermal Energies=

Sum of electronic and thermal Enthalpies=

Sum of electronic and thermal Free Energies=

Imaginary Freq $=0$

Zero-point correction=

Thermal correction to Energy=

Thermal correction to Enthalpy=

Thermal correction to Gibbs Free Energy=

Sum of electronic and zero-point Energies=

Sum of electronic and thermal Energies=

Sum of electronic and thermal Enthalpies=

Sum of electronic and thermal Free Energies=

Imaginary Freq $=0$

Zero-point correction=

Thermal correction to Energy=
$-1235.921562$

0.551430 (Hartree/Particle)

0.581424

0.582368

0.491461

$-1235.857591$

$-1235.827597$

$-1235.826653$

$-1235.917561$

0.551506 (Hartree/Particle)

0.581530

0.582474

0.491915

$-1235.857125$

$-1235.827101$

$-1235.826157$

$-1235.916716$

0.550855 (Hartree/Particle)

0.581160

0.582105

0.489653

$-1235.856643$

$-1235.826338$

$-1235.825393$

$-1235.917845$

0.551295 (Hartree/Particle)

0.581407

0.582351

0.490782

$-1235.859840$

$-1235.829728$

$-1235.828784$

$-1235.920353$

0.551474 (Hartree/Particle)

0.581410

0.582354

0.491755

$-1235.858394$

$-1235.828458$

$-1235.827514$

$-1235.918113$

0.550522 (Hartree/Particle)

0.581025 
Thermal correction to Enthalpy=

Thermal correction to Gibbs Free Energy=

Sum of electronic and zero-point Energies=

Sum of electronic and thermal Energies=

Sum of electronic and thermal Enthalpies=

Sum of electronic and thermal Free Energies=

3b_8_DP4+ Imaginary Freq $=0$

Zero-point correction=

Thermal correction to Energy=

Thermal correction to Enthalpy=

Thermal correction to Gibbs Free Energy=

Sum of electronic and zero-point Energies=

Sum of electronic and thermal Energies=

Sum of electronic and thermal Enthalpies=

Sum of electronic and thermal Free Energies=

3b_9_DP4+ Imaginary Freq $=0$

Zero-point correction=

Thermal correction to Energy=

Thermal correction to Enthalpy=

Thermal correction to Gibbs Free Energy=

Sum of electronic and zero-point Energies=

Sum of electronic and thermal Energies=

Sum of electronic and thermal Enthalpies=

Sum of electronic and thermal Free Energies=

3b_10_DP4+ Imaginary Freq $=0$

Zero-point correction=

Thermal correction to Energy=

Thermal correction to Enthalpy=

Thermal correction to Gibbs Free Energy=

Sum of electronic and zero-point Energies=

Sum of electronic and thermal Energies=

Sum of electronic and thermal Enthalpies=

Sum of electronic and thermal Free Energies=

3b_12_DP4+ Imaginary Freq $=0$

Zero-point correction=

Thermal correction to Energy=

Thermal correction to Enthalpy=

Thermal correction to Gibbs Free Energy=

Sum of electronic and zero-point Energies=

Sum of electronic and thermal Energies $=$

Sum of electronic and thermal Enthalpies=

Sum of electronic and thermal Free Energies=

3b_13_DP4+ Imaginary Freq $=0$

Zero-point correction=

Thermal correction to Energy=

Thermal correction to Enthalpy=

Thermal correction to Gibbs Free Energy=

Sum of electronic and zero-point Energies=

Sum of electronic and thermal Energies=

$$
\begin{aligned}
& 0.581969 \\
& 0.489611 \\
& -1235.853002 \\
& -1235.822499 \\
& -1235.821555 \\
& -1235.913913
\end{aligned}
$$

0.550560 (Hartree/Particle)

0.580996

0.581940

0.489635

$-1235.854534$

$-1235.824098$

$-1235.823154$

$-1235.915459$

0.550857 (Hartree/Particle)

0.581162

0.582107

0.489650

$-1235.856641$

$-1235.826336$

$-1235.825391$

$-1235.917848$

0.550612 (Hartree/Particle)

0.581086

0.582030

0.488663

$-1235.852856$

$-1235.822382$

$-1235.821438$

$-1235.914806$

0.551507 (Hartree/Particle)

0.581458

0.582403

0.491634

$-1235.856566$

$-1235.826615$

$-1235.825671$

$-1235.916440$

0.551346 (Hartree/Particle)

0.581417

0.582362

0.490749

$-1235.857724$

$-1235.827653$ 
Sum of electronic and thermal Enthalpies=

Sum of electronic and thermal Free Energies=

3b_15_DP4+ Imaginary Freq $=0$

Zero-point correction $=$

Thermal correction to Energy=

Thermal correction to Enthalpy=

Thermal correction to Gibbs Free Energy=

Sum of electronic and zero-point Energies=

Sum of electronic and thermal Energies=

Sum of electronic and thermal Enthalpies=

Sum of electronic and thermal Free Energies=

3b_16_DP4+ Imaginary Freq $=0$

Zero-point correction=

Thermal correction to Energy=

Thermal correction to Enthalpy=

Thermal correction to Gibbs Free Energy=

Sum of electronic and zero-point Energies=

Sum of electronic and thermal Energies=

Sum of electronic and thermal Enthalpies=

Sum of electronic and thermal Free Energies=

3b_18_DP4+ Imaginary Freq $=0$

Zero-point correction $=$

Thermal correction to Energy=

Thermal correction to Enthalpy=

Thermal correction to Gibbs Free Energy=

Sum of electronic and zero-point Energies=

Sum of electronic and thermal Energies=

Sum of electronic and thermal Enthalpies=

Sum of electronic and thermal Free Energies=

3c_1_DP4+ Imaginary Freq $=0$

Zero-point correction $=$

Thermal correction to Energy=

Thermal correction to Enthalpy=

Thermal correction to Gibbs Free Energy=

Sum of electronic and zero-point Energies=

Sum of electronic and thermal Energies $=$

Sum of electronic and thermal Enthalpies=

Sum of electronic and thermal Free Energies=

3c_3_DP4 $+\quad$ Imaginary Freq $=0$

Zero-point correction $=$

Thermal correction to Energy=

Thermal correction to Enthalpy=

Thermal correction to Gibbs Free Energy=

Sum of electronic and zero-point Energies=

Sum of electronic and thermal Energies=

Sum of electronic and thermal Enthalpies=

Sum of electronic and thermal Free Energies=

3c_4_DP4+
Imaginary Freq $=0$

Zero-point correction=
$-1235.826709$

$-1235.918321$

0.550988 (Hartree/Particle)

0.581211

0.582155

0.490527

$-1235.855907$

$-1235.825684$

$-1235.824740$

$-1235.916368$

0.551295 (Hartree/Particle)

0.581407

0.582351

0.490782

$-1235.859840$

$-1235.829728$

$-1235.828784$

$-1235.920353$

0.550366 (Hartree/Particle)

0.580899

0.581843

0.488555

$-1235.853629$

$-1235.823097$

$-1235.822152$

$-1235.915440$

0.550607 (Hartree/Particle)

0.581015

0.581959

0.489009

$-1235.864281$

$-1235.833872$

$-1235.832928$

$-1235.925879$

0.550749 (Hartree/Particle)

0.581033

0.581977

0.489543

$-1235.864296$

$-1235.834012$

$-1235.833067$

$-1235.925501$

0.550784 (Hartree/Particle) 
Thermal correction to Energy=

Thermal correction to Enthalpy=

Thermal correction to Gibbs Free Energy=

Sum of electronic and zero-point Energies=

Sum of electronic and thermal Energies=

Sum of electronic and thermal Enthalpies=

Sum of electronic and thermal Free Energies=

3c_5_DP4+

3c_6_DP4+

3c_7_DP4+

3c_8_DP4+

3c_9_DP4+
Imaginary Freq $=0$

Zero-point correction=

Thermal correction to Energy=

Thermal correction to Enthalpy=

Thermal correction to Gibbs Free Energy=

Sum of electronic and zero-point Energies=

Sum of electronic and thermal Energies=

Sum of electronic and thermal Enthalpies=

Sum of electronic and thermal Free Energies=

Imaginary Freq $=0$

Zero-point correction=

Thermal correction to Energy=

Thermal correction to Enthalpy=

Thermal correction to Gibbs Free Energy=

Sum of electronic and zero-point Energies=

Sum of electronic and thermal Energies=

Sum of electronic and thermal Enthalpies=

Sum of electronic and thermal Free Energies=

Imaginary Freq $=0$

Zero-point correction=

Thermal correction to Energy=

Thermal correction to Enthalpy=

Thermal correction to Gibbs Free Energy=

Sum of electronic and zero-point Energies=

Sum of electronic and thermal Energies=

Sum of electronic and thermal Enthalpies=

Sum of electronic and thermal Free Energies=

Imaginary Freq $=0$

Zero-point correction=

Thermal correction to Energy=

Thermal correction to Enthalpy=

Thermal correction to Gibbs Free Energy=

Sum of electronic and zero-point Energies=

Sum of electronic and thermal Energies=

Sum of electronic and thermal Enthalpies=

Sum of electronic and thermal Free Energies=

Imaginary Freq $=0$

Zero-point correction $=$

Thermal correction to Energy=

Thermal correction to Enthalpy=

Thermal correction to Gibbs Free Energy=

Sum of electronic and zero-point Energies=
0.581114

0.582058

0.489128

$-1235.864970$

$-1235.834639$

$-1235.833695$

$-1235.926625$

0.551020 (Hartree/Particle)

$$
0.581240
$$

0.582185

0.489703

$-1235.860950$

$-1235.830729$

$-1235.829785$

$-1235.922267$

0.550382 (Hartree/Particle) 0.580887

0.581831

0.488971

$-1235.857427$

$-1235.826921$

$-1235.825977$

$-1235.918837$

0.551044 (Hartree/Particle)

0.581159

0.582103

0.490683

$-1235.859069$

$-1235.828954$

$-1235.828010$

$-1235.919429$

0.550697 (Hartree/Particle)

0.581051

0.581995

0.489775

$-1235.858769$

$-1235.828415$

$-1235.827471$

$-1235.919690$

0.550636 (Hartree/Particle)

0.581116

0.582060

0.488447

$-1235.860649$ 
Sum of electronic and thermal Energies=

Sum of electronic and thermal Enthalpies=

Sum of electronic and thermal Free Energies=

3c_10_DP4+ Imaginary Freq $=0$

Zero-point correction $=$

Thermal correction to Energy=

Thermal correction to Enthalpy=

Thermal correction to Gibbs Free Energy=

Sum of electronic and zero-point Energies=

Sum of electronic and thermal Energies=

Sum of electronic and thermal Enthalpies=

Sum of electronic and thermal Free Energies=

3d_1_DP4+ Imaginary Freq $=0$

Zero-point correction $=$

Thermal correction to Energy=

Thermal correction to Enthalpy=

Thermal correction to Gibbs Free Energy=

Sum of electronic and zero-point Energies=

Sum of electronic and thermal Energies=

Sum of electronic and thermal Enthalpies=

Sum of electronic and thermal Free Energies=

3d_2_DP4+ Imaginary Freq $=0$

Zero-point correction=

Thermal correction to Energy=

Thermal correction to Enthalpy=

Thermal correction to Gibbs Free Energy=

Sum of electronic and zero-point Energies=

Sum of electronic and thermal Energies=

Sum of electronic and thermal Enthalpies=

Sum of electronic and thermal Free Energies=

3d_3_DP4+ Imaginary Freq $=0$

Zero-point correction $=$

Thermal correction to Energy=

Thermal correction to Enthalpy=

Thermal correction to Gibbs Free Energy=

Sum of electronic and zero-point Energies=

Sum of electronic and thermal Energies=

Sum of electronic and thermal Enthalpies=

Sum of electronic and thermal Free Energies=

3d_4_DP4+ Imaginary Freq $=0$

Zero-point correction=

Thermal correction to Energy=

Thermal correction to Enthalpy=

Thermal correction to Gibbs Free Energy=

Sum of electronic and zero-point Energies=

Sum of electronic and thermal Energies=

Sum of electronic and thermal Enthalpies=

Sum of electronic and thermal Free Energies=

3d_5_DP4+

Imaginary Freq $=0$
$-1235.830169$

$-1235.829225$

$-1235.922838$

0.550786 (Hartree/Particle)

0.581116

0.582060

0.489127

$-1235.864968$

$-1235.834638$

$-1235.833694$

$-1235.926627$

0.551442 (Hartree/Particle)

0.581397

0.582341

0.491223

$-1235.862492$

$-1235.832537$

$-1235.831593$

$-1235.922711$

0.551243 (Hartree/Particle)

0.581362

0.582306

0.490454

$-1235.862203$

$-1235.832084$

$-1235.831140$

$-1235.922993$

0.551443 (Hartree/Particle)

0.581398

0.582342

0.491226

$-1235.862491$

$-1235.832536$

$-1235.831592$

$-1235.922708$

0.551398 (Hartree/Particle)

0.581401

0.582346

0.490864

$-1235.863089$

$-1235.833086$

$-1235.832141$

$-1235.923623$ 
Zero-point correction=

Thermal correction to Energy=

Thermal correction to Enthalpy=

Thermal correction to Gibbs Free Energy=

Sum of electronic and zero-point Energies=

Sum of electronic and thermal Energies=

Sum of electronic and thermal Enthalpies=

Sum of electronic and thermal Free Energies=

3d_6_DP4+ Imaginary Freq $=0$

Zero-point correction=

Thermal correction to Energy=

Thermal correction to Enthalpy=

Thermal correction to Gibbs Free Energy=

Sum of electronic and zero-point Energies=

Sum of electronic and thermal Energies=

Sum of electronic and thermal Enthalpies=

Sum of electronic and thermal Free Energies=

3d_7_DP4+ Imaginary Freq $=0$

Zero-point correction $=$

Thermal correction to Energy=

Thermal correction to Enthalpy=

Thermal correction to Gibbs Free Energy=

Sum of electronic and zero-point Energies=

Sum of electronic and thermal Energies=

Sum of electronic and thermal Enthalpies=

Sum of electronic and thermal Free Energies=

3d_8_DP4+ Imaginary Freq $=0$

Zero-point correction=

Thermal correction to Energy=

Thermal correction to Enthalpy=

Thermal correction to Gibbs Free Energy=

Sum of electronic and zero-point Energies=

Sum of electronic and thermal Energies=

Sum of electronic and thermal Enthalpies=

Sum of electronic and thermal Free Energies=

3d_9_DP4+ Imaginary Freq $=0$

Zero-point correction $=$

Thermal correction to Energy=

Thermal correction to Enthalpy=

Thermal correction to Gibbs Free Energy=

Sum of electronic and zero-point Energies=

Sum of electronic and thermal Energies=

Sum of electronic and thermal Enthalpies=

Sum of electronic and thermal Free Energies=

3d_10_DP4+ Imaginary Freq $=0$

Zero-point correction=

Thermal correction to Energy=

Thermal correction to Enthalpy=

Thermal correction to Gibbs Free Energy=
0.551367 (Hartree/Particle)

0.581384

0.582328

0.490748

$-1235.858940$

$-1235.828923$

$-1235.827979$

$-1235.919558$

0.551180 (Hartree/Particle)

0.581302

0.582246

0.491035

$-1235.857711$

$-1235.827588$

$-1235.826644$

$-1235.917856$

0.550274 (Hartree/Particle)

0.580782

0.581726

0.488796

$-1235.857586$

$-1235.827078$

$-1235.826134$

$-1235.919064$

0.551387 (Hartree/Particle)

0.581436

0.582380

0.490805

$-1235.861584$

$-1235.831535$

$-1235.830591$

$-1235.922165$

0.550861 (Hartree/Particle)

0.581221

0.582165

0.488952

$-1235.859546$

$-1235.829186$

$-1235.828242$

$-1235.921456$

0.550803 (Hartree/Particle)

0.581153

0.582097

0.490123 
Sum of electronic and zero-point Energies=

Sum of electronic and thermal Energies=

Sum of electronic and thermal Enthalpies=

Sum of electronic and thermal Free Energies=

3d_11_DP4+ Imaginary Freq $=0$

Zero-point correction=

Thermal correction to Energy=

Thermal correction to Enthalpy=

Thermal correction to Gibbs Free Energy=

Sum of electronic and zero-point Energies=

Sum of electronic and thermal Energies=

Sum of electronic and thermal Enthalpies=

Sum of electronic and thermal Free Energies=

3d_12_DP4+ Imaginary Freq $=0$

Zero-point correction=

Thermal correction to Energy=

Thermal correction to Enthalpy=

Thermal correction to Gibbs Free Energy=

Sum of electronic and zero-point Energies=

Sum of electronic and thermal Energies=

Sum of electronic and thermal Enthalpies=

Sum of electronic and thermal Free Energies=

3d_13_DP4+ Imaginary Freq $=0$

Zero-point correction=

Thermal correction to Energy=

Thermal correction to Enthalpy=

Thermal correction to Gibbs Free Energy=

Sum of electronic and zero-point Energies=

Sum of electronic and thermal Energies=

Sum of electronic and thermal Enthalpies=

Sum of electronic and thermal Free Energies=

3d_14_DP4+ Imaginary Freq $=0$

Zero-point correction=

Thermal correction to Energy=

Thermal correction to Enthalpy=

Thermal correction to Gibbs Free Energy=

Sum of electronic and zero-point Energies=

Sum of electronic and thermal Energies=

Sum of electronic and thermal Enthalpies=

Sum of electronic and thermal Free Energies=

3d_16_DP4+ Imaginary Freq $=0$

Zero-point correction=

Thermal correction to Energy=

Thermal correction to Enthalpy=

Thermal correction to Gibbs Free Energy=

Sum of electronic and zero-point Energies=

Sum of electronic and thermal Energies=

Sum of electronic and thermal Enthalpies=

Sum of electronic and thermal Free Energies=
$-1235.855335$

$-1235.824986$

$-1235.824041$

$-1235.916016$

0.551126 (Hartree/Particle)

0.581185

0.582130

0.491216

$-1235.856712$

$-1235.826653$

$-1235.825708$

$-1235.916622$

0.551176 (Hartree/Particle)

0.581344

0.582289

0.490059

$-1235.858506$

$-1235.828338$

$-1235.827394$

$-1235.919623$

0.551379 (Hartree/Particle)

0.581455

0.582400

0.491095

$-1235.858476$

$-1235.828399$

$-1235.827455$

$-1235.918759$

0.551042 (Hartree/Particle)

0.581117

0.582061

0.490186

$-1235.861859$

$-1235.831784$

$-1235.830839$

$-1235.922715$

0.550140 (Hartree/Particle) 0.580689

0.581633

0.488714

$-1235.857248$

$-1235.826700$

$-1235.825756$

$-1235.918675$ 
3d_17_DP4+ Imaginary Freq $=0$

Zero-point correction=

Thermal correction to Energy=

Thermal correction to Enthalpy=

Thermal correction to Gibbs Free Energy=

Sum of electronic and zero-point Energies=

Sum of electronic and thermal Energies=

Sum of electronic and thermal Enthalpies=

Sum of electronic and thermal Free Energies=

3e_1_DP4+ Imaginary Freq $=0$

Zero-point correction=

Thermal correction to Energy=

Thermal correction to Enthalpy=

Thermal correction to Gibbs Free Energy=

Sum of electronic and zero-point Energies=

Sum of electronic and thermal Energies=

Sum of electronic and thermal Enthalpies=

Sum of electronic and thermal Free Energies=

3e_2_DP4 $+\quad$ Imaginary Freq $=0$

Zero-point correction=

Thermal correction to Energy=

Thermal correction to Enthalpy=

Thermal correction to Gibbs Free Energy=

Sum of electronic and zero-point Energies=

Sum of electronic and thermal Energies=

Sum of electronic and thermal Enthalpies=

Sum of electronic and thermal Free Energies=

3e_3_DP4+ Imaginary Freq $=0$

Zero-point correction=

Thermal correction to Energy=

Thermal correction to Enthalpy=

Thermal correction to Gibbs Free Energy=

Sum of electronic and zero-point Energies=

Sum of electronic and thermal Energies=

Sum of electronic and thermal Enthalpies=

Sum of electronic and thermal Free Energies=

3e_4_DP4+ Imaginary Freq $=0$

Zero-point correction=

Thermal correction to Energy=

Thermal correction to Enthalpy=

Thermal correction to Gibbs Free Energy=

Sum of electronic and zero-point Energies=

Sum of electronic and thermal Energies=

Sum of electronic and thermal Enthalpies=

Sum of electronic and thermal Free Energies=

3e_5_DP4+ Imaginary Freq $=0$

Zero-point correction=

Thermal correction to Energy=

Thermal correction to Enthalpy=

0.550614 (Hartree/Particle)

0.581115

0.582059

0.487949

$-1235.857542$

$-1235.827041$

$-1235.826097$

$-1235.920207$

0.551218 (Hartree/Particle)

0.581557

0.582502

0.489559

$-1235.860448$

$-1235.830109$

$-1235.829165$

$-1235.922108$

0.551145 (Hartree/Particle)

0.581486

0.582430

0.489523

$-1235.859440$

$-1235.829098$

$-1235.828154$

$-1235.921062$

0.550534 (Hartree/Particle)

0.581143

0.582087

0.488183

$-1235.856384$

$-1235.825774$

$-1235.824830$

$-1235.918734$

0.550538 (Hartree/Particle)

0.581145

0.582089

0.488197

$-1235.856380$

$-1235.825773$

$-1235.824828$

$-1235.918720$

0.551085 (Hartree/Particle)

0.581439

0.582383 
Thermal correction to Gibbs Free Energy=

Sum of electronic and zero-point Energies=

Sum of electronic and thermal Energies=

Sum of electronic and thermal Enthalpies=

Sum of electronic and thermal Free Energies=

3e_6_DP4+ Imaginary Freq $=0$

Zero-point correction $=$

Thermal correction to Energy=

Thermal correction to Enthalpy=

Thermal correction to Gibbs Free Energy=

Sum of electronic and zero-point Energies=

Sum of electronic and thermal Energies=

Sum of electronic and thermal Enthalpies=

Sum of electronic and thermal Free Energies=

3e_7_DP4+

Imaginary Freq $=0$

Zero-point correction=

Thermal correction to Energy=

Thermal correction to Enthalpy=

Thermal correction to Gibbs Free Energy=

Sum of electronic and zero-point Energies=

Sum of electronic and thermal Energies=

Sum of electronic and thermal Enthalpies=

Sum of electronic and thermal Free Energies=

3e_8_DP4 $+\quad$ Imaginary Freq $=0$

Zero-point correction=

Thermal correction to Energy=

Thermal correction to Enthalpy=

Thermal correction to Gibbs Free Energy=

Sum of electronic and zero-point Energies=

Sum of electronic and thermal Energies=

Sum of electronic and thermal Enthalpies=

Sum of electronic and thermal Free Energies=

3e_9_DP4+ Imaginary Freq $=0$

Zero-point correction $=$

Thermal correction to Energy=

Thermal correction to Enthalpy=

Thermal correction to Gibbs Free Energy=

Sum of electronic and zero-point Energies=

Sum of electronic and thermal Energies=

Sum of electronic and thermal Enthalpies=

Sum of electronic and thermal Free Energies=

3e_10_DP4+ Imaginary Freq $=0$

Zero-point correction=

Thermal correction to Energy=

Thermal correction to Enthalpy=

Thermal correction to Gibbs Free Energy=

Sum of electronic and zero-point Energies=

Sum of electronic and thermal Energies=

Sum of electronic and thermal Enthalpies=

$$
\begin{aligned}
& 0.489125 \\
& -1235.861317 \\
& -1235.830963 \\
& -1235.830019 \\
& -1235.923276
\end{aligned}
$$

0.551143 (Hartree/Particle)

0.581486

0.582430

0.489518

$-1235.859441$

$-1235.829098$

$-1235.828154$

$-1235.921066$

0.550390 (Hartree/Particle)

0.581063

0.582007

0.487591

$-1235.857246$

$-1235.826573$

$-1235.825629$

$-1235.920044$

0.551285 (Hartree/Particle)

0.581581

0.582525

0.489710

$-1235.861198$

$-1235.830903$

$-1235.829959$

$-1235.922773$

0.550388 (Hartree/Particle)

0.581062

0.582006

0.487571

$-1235.857247$

$-1235.826574$

$-1235.825629$

$-1235.920064$

0.550463 (Hartree/Particle)

0.581006

0.581950

0.488385

$-1235.857142$

$-1235.826599$

$-1235.825655$ 
Sum of electronic and thermal Free Energies=

3e_11_DP4+

Imaginary Freq $=0$

Zero-point correction=

Thermal correction to Energy=

Thermal correction to Enthalpy=

Thermal correction to Gibbs Free Energy=

Sum of electronic and zero-point Energies=

Sum of electronic and thermal Energies=

Sum of electronic and thermal Enthalpies=

Sum of electronic and thermal Free Energies=

3e_12_DP4+ Imaginary Freq $=0$

Zero-point correction=

Thermal correction to Energy=

Thermal correction to Enthalpy=

Thermal correction to Gibbs Free Energy=

Sum of electronic and zero-point Energies=

Sum of electronic and thermal Energies=

Sum of electronic and thermal Enthalpies=

Sum of electronic and thermal Free Energies=

3e_13_DP4+ Imaginary Freq $=0$

Zero-point correction=

Thermal correction to Energy=

Thermal correction to Enthalpy=

Thermal correction to Gibbs Free Energy=

Sum of electronic and zero-point Energies=

Sum of electronic and thermal Energies $=$

Sum of electronic and thermal Enthalpies=

Sum of electronic and thermal Free Energies=

3e_14_DP4+ Imaginary Freq $=0$

Zero-point correction=

Thermal correction to Energy=

Thermal correction to Enthalpy=

Thermal correction to Gibbs Free Energy=

Sum of electronic and zero-point Energies=

Sum of electronic and thermal Energies=

Sum of electronic and thermal Enthalpies=

Sum of electronic and thermal Free Energies=

3e_15_DP4+ Imaginary Freq $=0$

Zero-point correction $=$

Thermal correction to Energy=

Thermal correction to Enthalpy=

Thermal correction to Gibbs Free Energy=

Sum of electronic and zero-point Energies=

Sum of electronic and thermal Energies=

Sum of electronic and thermal Enthalpies=

Sum of electronic and thermal Free Energies=

3e_16_DP4+ Imaginary Freq $=0$

Zero-point correction $=$

Thermal correction to Energy=
$-1235.919219$

0.550749 (Hartree/Particle)

0.581278

0.582222

0.488224

$-1235.860510$

$-1235.829981$

$-1235.829037$

$-1235.923035$

0.550538 (Hartree/Particle)

0.581145

0.582089

0.488203

$-1235.856380$

$-1235.825773$

$-1235.824829$

$-1235.918715$

0.550538 (Hartree/Particle)

0.581145

0.582089

0.488199

$-1235.856380$

$-1235.825772$

$-1235.824828$

$-1235.918718$

0.550576 (Hartree/Particle)

0.581096

0.582040

0.488843

$-1235.855397$

$-1235.824877$

$-1235.823933$

$-1235.917130$

0.551013 (Hartree/Particle)

0.581320

0.582264

0.489685

$-1235.856714$

$-1235.826408$

$-1235.825463$

$-1235.918043$

0.551013 (Hartree/Particle)

0.581319 
Thermal correction to Enthalpy=

Thermal correction to Gibbs Free Energy=

Sum of electronic and zero-point Energies=

Sum of electronic and thermal Energies=

Sum of electronic and thermal Enthalpies=

Sum of electronic and thermal Free Energies=

3e_17_DP4+

Imaginary Freq $=0$

Zero-point correction=

Thermal correction to Energy=

Thermal correction to Enthalpy=

Thermal correction to Gibbs Free Energy=

Sum of electronic and zero-point Energies=

Sum of electronic and thermal Energies=

Sum of electronic and thermal Enthalpies=

Sum of electronic and thermal Free Energies=

3f_1_DP4+ Imaginary Freq $=0$

Zero-point correction=

Thermal correction to Energy=

Thermal correction to Enthalpy=

Thermal correction to Gibbs Free Energy=

Sum of electronic and zero-point Energies=

Sum of electronic and thermal Energies=

Sum of electronic and thermal Enthalpies=

Sum of electronic and thermal Free Energies=

3f_2_DP4+ Imaginary Freq $=0$

Zero-point correction=

Thermal correction to Energy=

Thermal correction to Enthalpy=

Thermal correction to Gibbs Free Energy=

Sum of electronic and zero-point Energies=

Sum of electronic and thermal Energies=

Sum of electronic and thermal Enthalpies=

Sum of electronic and thermal Free Energies=

3f_3_DP4+ Imaginary Freq $=0$

Zero-point correction=

Thermal correction to Energy=

Thermal correction to Enthalpy=

Thermal correction to Gibbs Free Energy=

Sum of electronic and zero-point Energies=

Sum of electronic and thermal Energies $=$

Sum of electronic and thermal Enthalpies=

Sum of electronic and thermal Free Energies=

3f_4_DP4+ Imaginary Freq $=0$

Zero-point correction=

Thermal correction to Energy=

Thermal correction to Enthalpy=

Thermal correction to Gibbs Free Energy=

Sum of electronic and zero-point Energies=

Sum of electronic and thermal Energies=

$$
\begin{aligned}
& 0.582263 \\
& 0.489688 \\
& -1235.856715 \\
& -1235.826408 \\
& -1235.825464 \\
& -1235.918040
\end{aligned}
$$

0.550326 (Hartree/Particle)

0.580885

0.581829

0.488503

$-1235.852317$

$-1235.821758$

$-1235.820814$

$-1235.914140$

0.551153 (Hartree/Particle)

0.581394

0.582338

0.490364

$-1235.855727$

$-1235.825486$

$-1235.824542$

$-1235.916516$

0.550812 (Hartree/Particle)

0.581221

0.582165

0.489268

$-1235.857718$

$-1235.827309$

$-1235.826365$

$-1235.919263$

0.551094 (Hartree/Particle)

0.581465

0.582409

0.488757

$-1235.858238$

$-1235.827866$

$-1235.826922$

$-1235.920575$

0.550393 (Hartree/Particle)

0.581005

0.581949

0.488876

$-1235.850971$

$-1235.820359$ 
Sum of electronic and thermal Enthalpies=

Sum of electronic and thermal Free Energies=

3f_5_DP4+ Imaginary Freq $=0$

Zero-point correction=

Thermal correction to Energy=

Thermal correction to Enthalpy=

Thermal correction to Gibbs Free Energy=

Sum of electronic and zero-point Energies=

Sum of electronic and thermal Energies=

Sum of electronic and thermal Enthalpies=

Sum of electronic and thermal Free Energies=

3f_6_DP4+ Imaginary Freq $=0$

Zero-point correction=

Thermal correction to Energy=

Thermal correction to Enthalpy=

Thermal correction to Gibbs Free Energy=

Sum of electronic and zero-point Energies=

Sum of electronic and thermal Energies=

Sum of electronic and thermal Enthalpies=

Sum of electronic and thermal Free Energies=

3f_7_DP4+ Imaginary Freq $=0$

Zero-point correction $=$

Thermal correction to Energy=

Thermal correction to Enthalpy=

Thermal correction to Gibbs Free Energy=

Sum of electronic and zero-point Energies=

Sum of electronic and thermal Energies=

Sum of electronic and thermal Enthalpies=

Sum of electronic and thermal Free Energies=

3f_8_DP4+ Imaginary Freq $=0$

Zero-point correction=

Thermal correction to Energy=

Thermal correction to Enthalpy=

Thermal correction to Gibbs Free Energy=

Sum of electronic and zero-point Energies=

Sum of electronic and thermal Energies $=$

Sum of electronic and thermal Enthalpies=

Sum of electronic and thermal Free Energies=

3f_9_DP4+ Imaginary Freq $=0$

Zero-point correction=

Thermal correction to Energy=

Thermal correction to Enthalpy=

Thermal correction to Gibbs Free Energy=

Sum of electronic and zero-point Energies=

Sum of electronic and thermal Energies=

Sum of electronic and thermal Enthalpies=

Sum of electronic and thermal Free Energies=

3f_10_DP4+ Imaginary Freq $=0$

Zero-point correction $=$
$-1235.819415$

$-1235.912488$

0.549869 (Hartree/Particle)

0.580845

0.581789

0.486922

$-1235.853169$

$-1235.822193$

$-1235.821249$

$-1235.916116$

0.550463 (Hartree/Particle)

0.581052

0.581996

0.488716

$-1235.853733$

$-1235.823145$

$-1235.822200$

$-1235.915480$

0.550956 (Hartree/Particle)

0.581337

0.582281

0.489428

$-1235.853858$

$-1235.823477$

$-1235.822533$

$-1235.915386$

0.551247 (Hartree/Particle)

0.581473

0.582417

0.490508

$-1235.856045$

$-1235.825818$

$-1235.824874$

$-1235.916783$

0.550984 (Hartree/Particle)

0.581202

0.582146

0.489491

$-1235.865508$

$-1235.835290$

$-1235.834346$

$-1235.927002$

0.550799 (Hartree/Particle) 
Thermal correction to Energy=

Thermal correction to Enthalpy=

Thermal correction to Gibbs Free Energy=

Sum of electronic and zero-point Energies=

Sum of electronic and thermal Energies=

Sum of electronic and thermal Enthalpies=

Sum of electronic and thermal Free Energies=

3f_11_DP4+ Imaginary Freq $=0$

Zero-point correction $=$

Thermal correction to Energy=

Thermal correction to Enthalpy=

Thermal correction to Gibbs Free Energy=

Sum of electronic and zero-point Energies=

Sum of electronic and thermal Energies=

Sum of electronic and thermal Enthalpies=

Sum of electronic and thermal Free Energies=

3f_12_DP4+ Imaginary Freq $=0$

Zero-point correction=

Thermal correction to Energy=

Thermal correction to Enthalpy=

Thermal correction to Gibbs Free Energy=

Sum of electronic and zero-point Energies=

Sum of electronic and thermal Energies=

Sum of electronic and thermal Enthalpies=

Sum of electronic and thermal Free Energies=

3f_13_DP4+ Imaginary Freq $=0$

Zero-point correction=

Thermal correction to Energy=

Thermal correction to Enthalpy=

Thermal correction to Gibbs Free Energy=

Sum of electronic and zero-point Energies=

Sum of electronic and thermal Energies=

Sum of electronic and thermal Enthalpies=

Sum of electronic and thermal Free Energies=

3f_14_DP4+ Imaginary Freq $=0$

Zero-point correction=

Thermal correction to Energy=

Thermal correction to Enthalpy=

Thermal correction to Gibbs Free Energy=

Sum of electronic and zero-point Energies=

Sum of electronic and thermal Energies=

Sum of electronic and thermal Enthalpies=

Sum of electronic and thermal Free Energies=

3f_15_DP4+ Imaginary Freq $=0$

Zero-point correction=

Thermal correction to Energy=

Thermal correction to Enthalpy=

Thermal correction to Gibbs Free Energy=

Sum of electronic and zero-point Energies=
0.581228

0.582172

0.489151

$-1235.857408$

$-1235.826979$

$-1235.826035$

$-1235.919057$

0.550841 (Hartree/Particle)

0.581234

0.582178

0.489348

$-1235.858475$

$-1235.828082$

$-1235.827137$

$-1235.919968$

0.550953 (Hartree/Particle)

0.581306

0.582250

0.489022

$-1235.858205$

$-1235.827853$

$-1235.826908$

$-1235.920137$

0.551338 (Hartree/Particle)

0.581527

0.582471

0.490456

$-1235.857808$

$-1235.827620$

$-1235.826675$

$-1235.918690$

0.551108 (Hartree/Particle)

0.581389

0.582333

0.489826

$-1235.855211$

$-1235.824930$

$-1235.823986$

$-1235.916494$

0.550785 (Hartree/Particle)

0.581282

0.582226

0.489770

$-1235.851407$ 
Sum of electronic and thermal Energies=

Sum of electronic and thermal Enthalpies=

Sum of electronic and thermal Free Energies=

3f_16_DP4+ Imaginary Freq $=0$

Zero-point correction=

Thermal correction to Energy=

Thermal correction to Enthalpy=

Thermal correction to Gibbs Free Energy=

Sum of electronic and zero-point Energies=

Sum of electronic and thermal Energies=

Sum of electronic and thermal Enthalpies=

Sum of electronic and thermal Free Energies=

3f_17_DP4+ Imaginary Freq $=0$

Zero-point correction=

Thermal correction to Energy=

Thermal correction to Enthalpy=

Thermal correction to Gibbs Free Energy=

Sum of electronic and zero-point Energies=

Sum of electronic and thermal Energies=

Sum of electronic and thermal Enthalpies=

Sum of electronic and thermal Free Energies=

3f_18_DP4+ Imaginary Freq $=0$

Zero-point correction=

Thermal correction to Energy=

Thermal correction to Enthalpy=

Thermal correction to Gibbs Free Energy=

Sum of electronic and zero-point Energies=

Sum of electronic and thermal Energies=

Sum of electronic and thermal Enthalpies=

Sum of electronic and thermal Free Energies=

3g_1_DP4+ Imaginary Freq $=0$

Zero-point correction=

Thermal correction to Energy=

Thermal correction to Enthalpy=

Thermal correction to Gibbs Free Energy=

Sum of electronic and zero-point Energies=

Sum of electronic and thermal Energies=

Sum of electronic and thermal Enthalpies=

Sum of electronic and thermal Free Energies=

3g_3_DP4+ Imaginary Freq $=0$

Zero-point correction $=$

Thermal correction to Energy=

Thermal correction to Enthalpy=

Thermal correction to Gibbs Free Energy=

Sum of electronic and zero-point Energies=

Sum of electronic and thermal Energies=

Sum of electronic and thermal Enthalpies=

Sum of electronic and thermal Free Energies=

3g_4_DP4+ Imaginary Freq $=0$
$-1235.820910$

$-1235.819966$

$-1235.912421$

0.551067 (Hartree/Particle)

0.581474

0.582419

0.488928

$-1235.858257$

$-1235.827849$

$-1235.826905$

$-1235.920396$

0.551120 (Hartree/Particle)

0.581445

0.582389

0.489010

$-1235.856917$

$-1235.826592$

$-1235.825648$

$-1235.919027$

0.551237 (Hartree/Particle)

0.581451

0.582395

0.490327

$-1235.855620$

$-1235.825406$

$-1235.824462$

$-1235.916530$

0.550784 (Hartree/Particle)

0.581149

0.582093

0.489368

$-1235.862861$

$-1235.832496$

$-1235.831552$

$-1235.924277$

0.550651 (Hartree/Particle)

0.581075

0.582019

0.488955

$-1235.862929$

$-1235.832505$

$-1235.831561$

$-1235.924624$ 
Zero-point correction=

Thermal correction to Energy=

Thermal correction to Enthalpy=

Thermal correction to Gibbs Free Energy=

Sum of electronic and zero-point Energies=

Sum of electronic and thermal Energies=

Sum of electronic and thermal Enthalpies=

Sum of electronic and thermal Free Energies=

3g_5_DP4+ Imaginary Freq $=0$

Zero-point correction $=$

Thermal correction to Energy=

Thermal correction to Enthalpy=

Thermal correction to Gibbs Free Energy=

Sum of electronic and zero-point Energies=

Sum of electronic and thermal Energies=

Sum of electronic and thermal Enthalpies=

Sum of electronic and thermal Free Energies=

3g_6_DP4+ Imaginary Freq $=0$

Zero-point correction $=$

Thermal correction to Energy=

Thermal correction to Enthalpy=

Thermal correction to Gibbs Free Energy=

Sum of electronic and zero-point Energies=

Sum of electronic and thermal Energies=

Sum of electronic and thermal Enthalpies=

Sum of electronic and thermal Free Energies=

3g_7_DP4+ Imaginary Freq $=0$

Zero-point correction=

Thermal correction to Energy=

Thermal correction to Enthalpy=

Thermal correction to Gibbs Free Energy=

Sum of electronic and zero-point Energies=

Sum of electronic and thermal Energies=

Sum of electronic and thermal Enthalpies=

Sum of electronic and thermal Free Energies=

3g_8_DP4+ Imaginary Freq $=0$

Zero-point correction $=$

Thermal correction to Energy=

Thermal correction to Enthalpy=

Thermal correction to Gibbs Free Energy=

Sum of electronic and zero-point Energies=

Sum of electronic and thermal Energies=

Sum of electronic and thermal Enthalpies=

Sum of electronic and thermal Free Energies=

3g_9_DP4+ Imaginary Freq $=0$

Zero-point correction=

Thermal correction to Energy=

Thermal correction to Enthalpy=

Thermal correction to Gibbs Free Energy=
0.549447 (Hartree/Particle)

0.579707

0.580651

0.487729

$-1235.857973$

$-1235.827713$

$-1235.826769$

$-1235.919691$

0.550441 (Hartree/Particle)

0.581022

0.581967

0.488670

$-1235.857975$

$-1235.827394$

$-1235.826450$

$-1235.919746$

0.550928 (Hartree/Particle)

0.581250

0.582194

0.489747

$-1235.863586$

$-1235.833263$

$-1235.832319$

$-1235.924766$

0.550346 (Hartree/Particle)

0.580914

0.581858

0.488440

$-1235.858095$

$-1235.827527$

$-1235.826582$

$-1235.920001$

0.550828 (Hartree/Particle)

0.581169

0.582114

0.489194

$-1235.863426$

$-1235.833084$

$-1235.832140$

$-1235.925060$

0.550015 (Hartree/Particle)

0.580799

0.581744

0.487122 
Sum of electronic and zero-point Energies=

Sum of electronic and thermal Energies=

Sum of electronic and thermal Enthalpies=

Sum of electronic and thermal Free Energies=

3g_10_DP4+ Imaginary Freq $=0$

Zero-point correction=

Thermal correction to Energy=

Thermal correction to Enthalpy=

Thermal correction to Gibbs Free Energy=

Sum of electronic and zero-point Energies=

Sum of electronic and thermal Energies=

Sum of electronic and thermal Enthalpies=

Sum of electronic and thermal Free Energies=

3g_11_DP4+ Imaginary Freq $=0$

Zero-point correction=

Thermal correction to Energy=

Thermal correction to Enthalpy=

Thermal correction to Gibbs Free Energy=

Sum of electronic and zero-point Energies=

Sum of electronic and thermal Energies=

Sum of electronic and thermal Enthalpies=

Sum of electronic and thermal Free Energies=

3g_12_DP4+ Imaginary Freq $=0$

Zero-point correction=

Thermal correction to Energy=

Thermal correction to Enthalpy=

Thermal correction to Gibbs Free Energy=

Sum of electronic and zero-point Energies=

Sum of electronic and thermal Energies=

Sum of electronic and thermal Enthalpies=

Sum of electronic and thermal Free Energies=

3h_1_DP4+ Imaginary Freq $=0$

Zero-point correction=

Thermal correction to Energy=

Thermal correction to Enthalpy=

Thermal correction to Gibbs Free Energy=

Sum of electronic and zero-point Energies=

Sum of electronic and thermal Energies=

Sum of electronic and thermal Enthalpies=

Sum of electronic and thermal Free Energies=

3h_2_DP4+ Imaginary Freq $=0$

Zero-point correction $=$

Thermal correction to Energy=

Thermal correction to Enthalpy=

Thermal correction to Gibbs Free Energy=

Sum of electronic and zero-point Energies=

Sum of electronic and thermal Energies=

Sum of electronic and thermal Enthalpies=

Sum of electronic and thermal Free Energies=
$-1235.857750$

$-1235.826966$

$-1235.826021$

$-1235.920643$

0.550378 (Hartree/Particle)

0.580970

0.581914

0.488469

$-1235.858901$

$-1235.828309$

$-1235.827365$

$-1235.920810$

0.550832 (Hartree/Particle)

0.581154

0.582098

0.489528

$-1235.858296$

$-1235.827974$

$-1235.827030$

$-1235.919600$

0.549967 (Hartree/Particle)

0.580792

0.581736

0.487122

$-1235.858624$

$-1235.827800$

$-1235.826856$

$-1235.921470$

0.550862 (Hartree/Particle)

0.581017

0.581961

0.490463

$-1235.859554$

$-1235.829399$

$-1235.828455$

$-1235.919953$

0.550780 (Hartree/Particle)

0.580903

0.581847

0.490176

$-1235.858743$

$-1235.828620$

$-1235.827676$

$-1235.919347$ 
3h_4_DP4+ Imaginary Freq $=0$

Zero-point correction=

Thermal correction to Energy=

Thermal correction to Enthalpy=

Thermal correction to Gibbs Free Energy=

Sum of electronic and zero-point Energies=

Sum of electronic and thermal Energies=

Sum of electronic and thermal Enthalpies=

Sum of electronic and thermal Free Energies=

3h_5_DP4+ Imaginary Freq $=0$

Zero-point correction=

Thermal correction to Energy=

Thermal correction to Enthalpy=

Thermal correction to Gibbs Free Energy=

Sum of electronic and zero-point Energies=

Sum of electronic and thermal Energies=

Sum of electronic and thermal Enthalpies=

Sum of electronic and thermal Free Energies=

3h_6_DP4+ Imaginary Freq $=0$

Zero-point correction=

Thermal correction to Energy=

Thermal correction to Enthalpy=

Thermal correction to Gibbs Free Energy=

Sum of electronic and zero-point Energies=

Sum of electronic and thermal Energies=

Sum of electronic and thermal Enthalpies=

Sum of electronic and thermal Free Energies=

3h_7_DP4+ Imaginary Freq $=0$

Zero-point correction=

Thermal correction to Energy=

Thermal correction to Enthalpy=

Thermal correction to Gibbs Free Energy=

Sum of electronic and zero-point Energies=

Sum of electronic and thermal Energies=

Sum of electronic and thermal Enthalpies=

Sum of electronic and thermal Free Energies=

3h_8_DP4+ Imaginary Freq $=0$

Zero-point correction=

Thermal correction to Energy=

Thermal correction to Enthalpy=

Thermal correction to Gibbs Free Energy=

Sum of electronic and zero-point Energies=

Sum of electronic and thermal Energies=

Sum of electronic and thermal Enthalpies=

Sum of electronic and thermal Free Energies=

3h_9_DP4+ Imaginary Freq $=0$

Zero-point correction=

Thermal correction to Energy=

Thermal correction to Enthalpy=

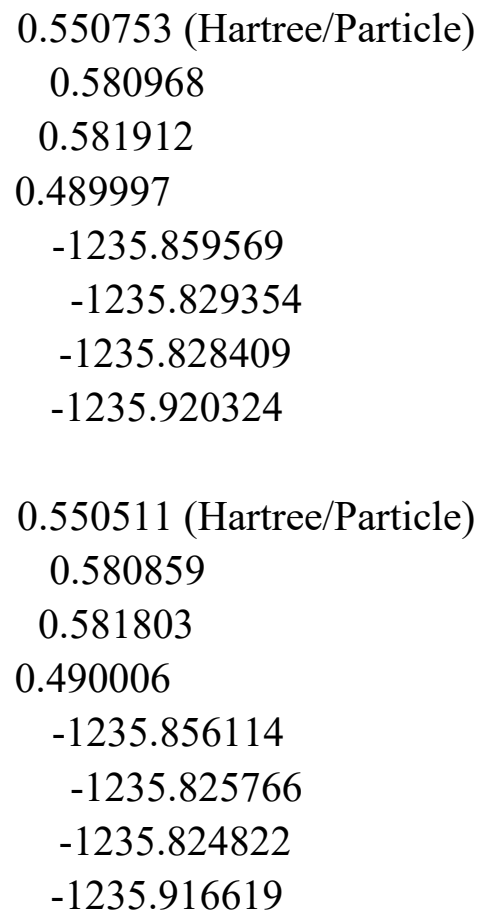

0.550788 (Hartree/Particle) 0.580900

0.581844

0.490380

$-1235.859177$

$-1235.829065$

$-1235.828121$

$-1235.919585$

0.550786 (Hartree/Particle) 0.580899

0.581843

0.490373

$-1235.859179$

$-1235.829066$

$-1235.828122$

$-1235.919592$

0.550784 (Hartree/Particle)

0.580897

0.581842 
Thermal correction to Gibbs Free Energy=

Sum of electronic and zero-point Energies=

Sum of electronic and thermal Energies $=$

Sum of electronic and thermal Enthalpies=

Sum of electronic and thermal Free Energies=

3h_10_DP4+ Imaginary Freq $=0$

Zero-point correction=

Thermal correction to Energy=

Thermal correction to Enthalpy=

Thermal correction to Gibbs Free Energy=

Sum of electronic and zero-point Energies=

Sum of electronic and thermal Energies=

Sum of electronic and thermal Enthalpies=

Sum of electronic and thermal Free Energies=

3h_11_DP4+ Imaginary Freq $=0$

Zero-point correction=

Thermal correction to Energy=

Thermal correction to Enthalpy=

Thermal correction to Gibbs Free Energy=

Sum of electronic and zero-point Energies=

Sum of electronic and thermal Energies=

Sum of electronic and thermal Enthalpies=

Sum of electronic and thermal Free Energies=

3h_12_DP4+ Imaginary Freq $=0$

Zero-point correction=

Thermal correction to Energy=

Thermal correction to Enthalpy=

Thermal correction to Gibbs Free Energy=

Sum of electronic and zero-point Energies=

Sum of electronic and thermal Energies=

Sum of electronic and thermal Enthalpies=

Sum of electronic and thermal Free Energies=

$$
\begin{aligned}
& 0.490368 \\
& -1235.859181 \\
& -1235.829067 \\
& -1235.828123 \\
& -1235.919597
\end{aligned}
$$

0.551189 (Hartree/Particle)

0.581347

0.582291

0.490479

$-1235.858176$

$-1235.828018$

$-1235.827074$

$-1235.918887$

0.550821 (Hartree/Particle)

0.580942

0.581887

0.490263

$-1235.859476$

$-1235.829354$

$-1235.828410$

$-1235.920033$

0.550555 (Hartree/Particle)

0.580820

0.581764

0.489116

$-1235.860426$

$-1235.830160$

$-1235.829216$

$-1235.921865$

Table S13. Experiment and calculated ${ }^{13} \mathrm{C}$ NMR chemical shifts of stereoisomers $\mathbf{3 a}-\mathbf{3 h}$.

\begin{tabular}{llllllllll}
\hline & & \multicolumn{8}{c}{$\delta_{\text {calc. }}$} \\
\cline { 2 - 10 } No. & $\delta_{\text {exp. }}$ & $\mathbf{3 a}$ & $\mathbf{3 b}$ & $\mathbf{3 c}$ & $\mathbf{3 d}$ & $\mathbf{3 e}$ & $\mathbf{3 f}$ & $\mathbf{3 g}$ & $\mathbf{3 h}$ \\
\hline 1 & 38.6 & 42.8 & 39.9 & 43.0 & 37.4 & 39.6 & 41.2 & 42.3 & 41.7 \\
2 & 26.1 & 23.4 & 25.0 & 23.4 & 25.1 & 24.9 & 24.0 & 24.5 & 23.8 \\
3 & 31.8 & 30.6 & 29.4 & 30.3 & 27.1 & 31.3 & 31.2 & 31.9 & 31.6 \\
4 & 78.6 & 81.2 & 81.7 & 80.3 & 81.4 & 81.3 & 79.7 & 79.5 & 77.8 \\
5 & 74.8 & 72.5 & 71.7 & 71.1 & 72.1 & 74.9 & 76.3 & 72.4 & 76.9 \\
6 & 29.4 & 27.0 & 28.5 & 31.7 & 28.5 & 25.6 & 29.4 & 30.8 & 31.8 \\
7 & 34.2 & 33.4 & 31.8 & 32.2 & 35.2 & 32.6 & 32.7 & 31.1 & 32.7
\end{tabular}




\begin{tabular}{llllllllll}
8 & 136.6 & 136.1 & 135.9 & 136.5 & 134.6 & 136.2 & 137.1 & 137.1 & 139.7 \\
9 & 122.9 & 121.2 & 122.5 & 125.3 & 126.0 & 119.3 & 120.8 & 124.3 & 121.7 \\
10 & 23.3 & 25.4 & 25.0 & 24.7 & 25.7 & 25.5 & 23.7 & 23.9 & 23.3 \\
11 & 37.8 & 38.9 & 38.0 & 38.2 & 39.2 & 38.8 & 38.9 & 37.8 & 37.0 \\
12 & 60.1 & 60.6 & 61.4 & 61.5 & 62.0 & 60.6 & 60.1 & 61.3 & 61.1 \\
13 & 61.2 & 63.1 & 62.8 & 65.3 & 64.7 & 63.5 & 61.8 & 64.9 & 65.5 \\
14 & 34.5 & 35.9 & 37.0 & 34.5 & 35.6 & 35.0 & 35.5 & 34.6 & 34.0 \\
15 & 143.3 & 141.8 & 141.3 & 140.6 & 141.5 & 141.4 & 141.5 & 140.7 & 140.8 \\
16 & 167.5 & 164.0 & 164.0 & 161.9 & 163.4 & 164.0 & 163.6 & 162.8 & 162.2 \\
17 & 124.5 & 130.5 & 130.6 & 130.5 & 128.1 & 131.9 & 130.7 & 130.2 & 129.3 \\
18 & 19.1 & 16.5 & 17.4 & 16.1 & 17.7 & 19.5 & 21.2 & 18.4 & 20.0 \\
19 & 18.1 & 18.2 & 19.4 & 18.7 & 16.6 & 18.2 & 16.5 & 18.7 & 18.1 \\
20 & 17.2 & 16.2 & 15.6 & 15.4 & 17.0 & 15.5 & 14.2 & 14.7 & 13.7 \\
21 & 52.1 & 52.5 & 52.5 & 51.4 & 52.6 & 52.4 & 51.5 & 51.3 & 50.6 \\
22 & 49.3 & 49.2 & 49.7 & 48.3 & 49.8 & 49.0 & 49.3 & 47.9 & 47.4 \\
\hline
\end{tabular}

Table S14. Mean absolute error (MAE) values, maximum deviation (MD) values and correlation coefficient $\left(R^{2}\right)$ analysis of four stereoisomers $\mathbf{3 a}-\mathbf{3 h}$.

\begin{tabular}{llll}
\hline Stereoisomers & MAE $(\mathrm{ppm})$ & $\mathrm{MD}(\mathrm{ppm})$ & $R^{2}$ \\
\hline 3a & 1.8 & 5.9 & 0.9974 \\
3b & 1.8 & 5.9 & 0.9976 \\
3c & 2.2 & 6.0 & 0.9962 \\
3d & 2.0 & 4.5 & 0.9974 \\
3e & 1.7 & 7.3 & 0.9972 \\
3f & 1.6 & 6.2 & 0.9978 \\
3g & 1.8 & 5.7 & 0.9972 \\
3h & 2.0 & 5.3 & 0.9970 \\
\hline
\end{tabular}




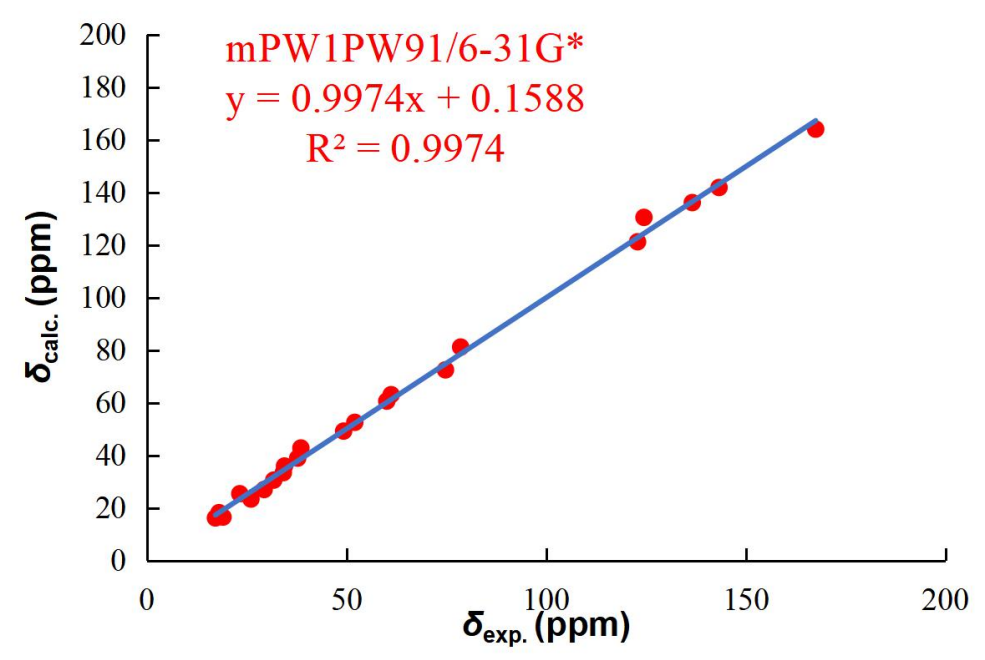

Figure S48. Regression analysis of experimental versus calculated ${ }^{13} \mathrm{C}$ NMR chemical shifts at mPW1PW91/6-31G* level using GIAO method of 3a; linear fitting is shown as a line (blue).

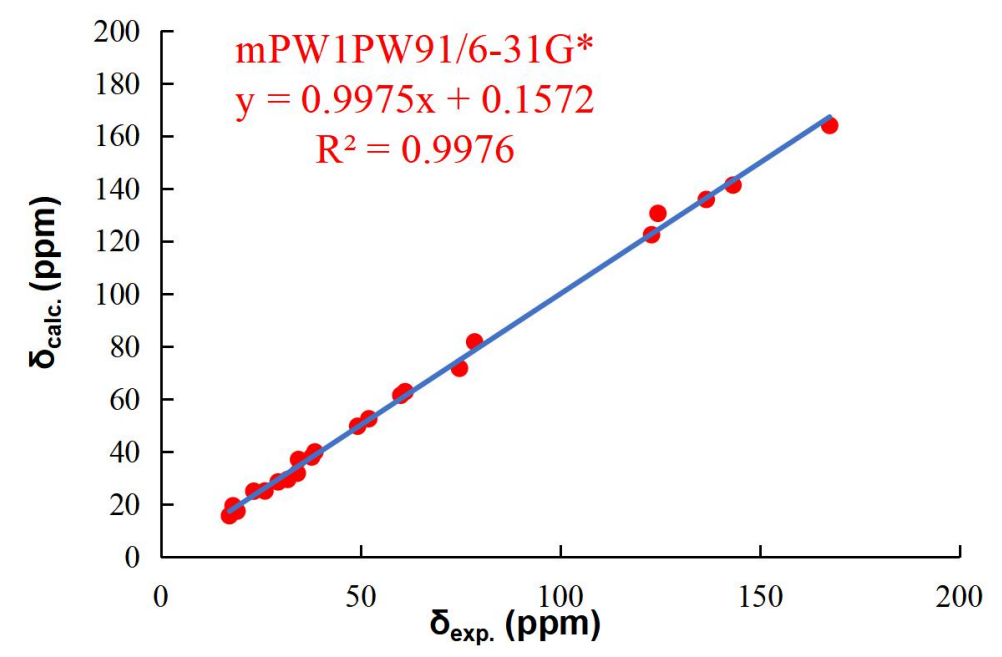

Figure S49. Regression analysis of experimental versus calculated ${ }^{13} \mathrm{C}$ NMR chemical shifts at mPW1PW91/6-31G* level using GIAO method of $\mathbf{3 b}$; linear fitting is shown as a line (blue).

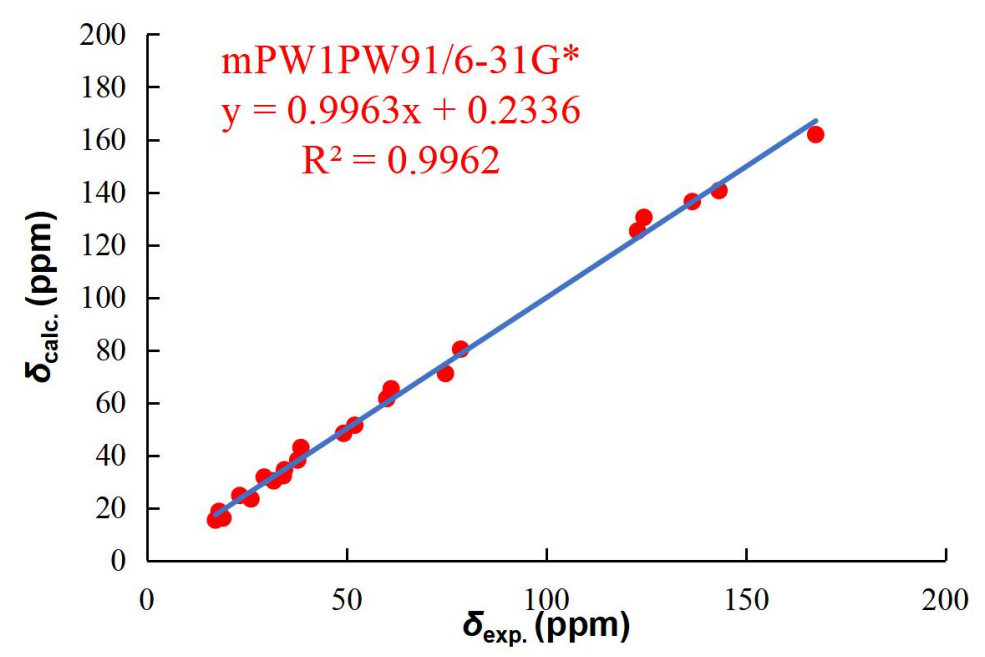

Figure S50. Regression analysis of experimental versus calculated ${ }^{13} \mathrm{C}$ NMR chemical shifts at mPW1PW91/6-31G* level using GIAO method of 3c; linear fitting is shown as a line (blue). 


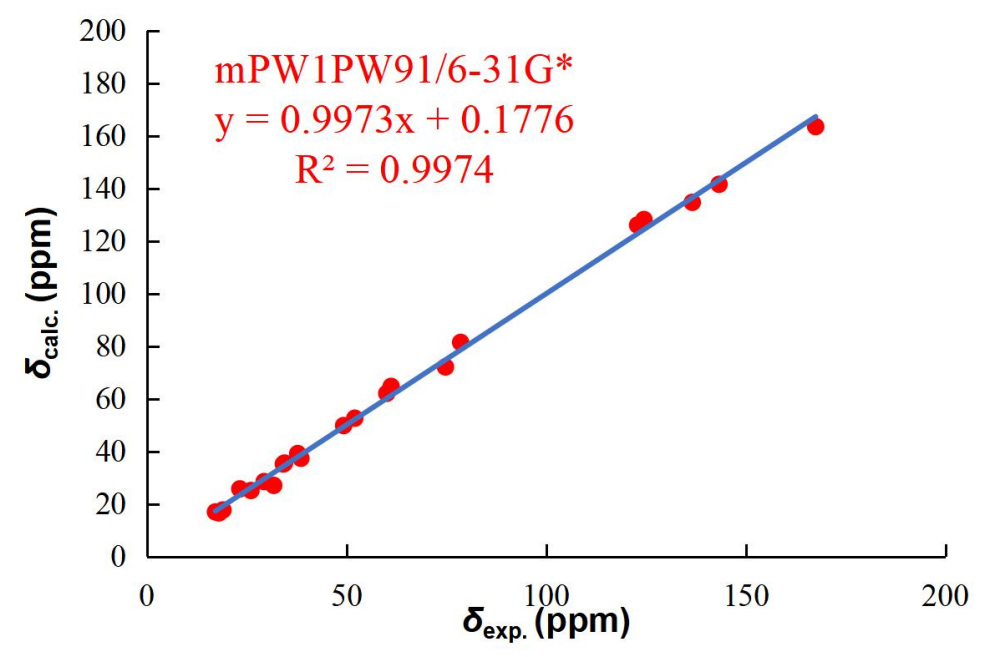

Figure S51. Regression analysis of experimental versus calculated ${ }^{13} \mathrm{C}$ NMR chemical shifts at mPW1PW91/6-31G* level using GIAO method of 3d; linear fitting is shown as a line (blue).

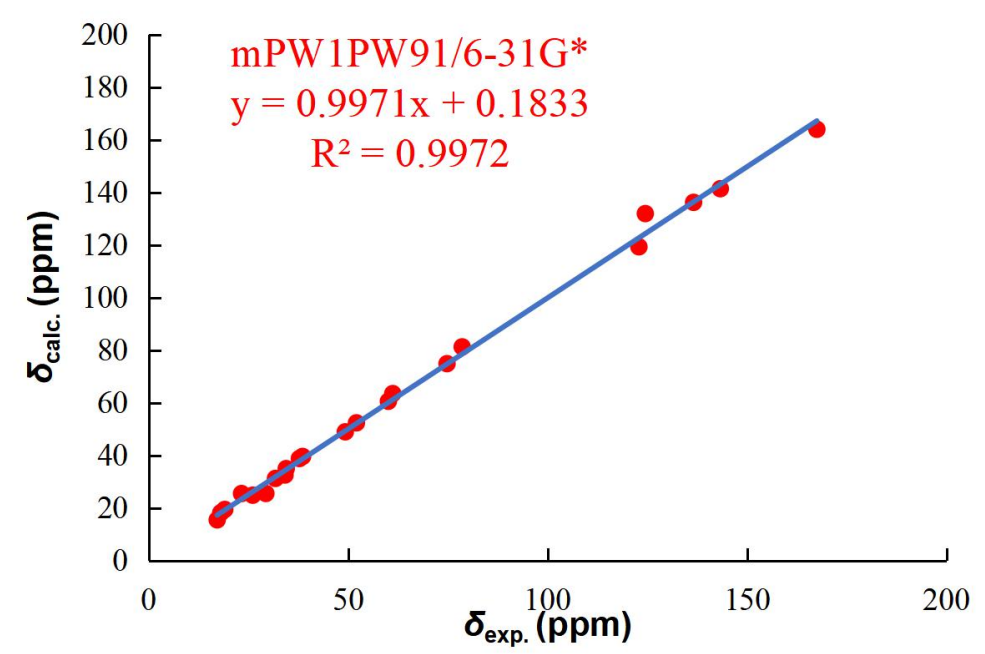

Figure S52. Regression analysis of experimental versus calculated ${ }^{13} \mathrm{C}$ NMR chemical shifts at mPW1PW91/6-31G* level using GIAO method of 3e; linear fitting is shown as a line (blue).

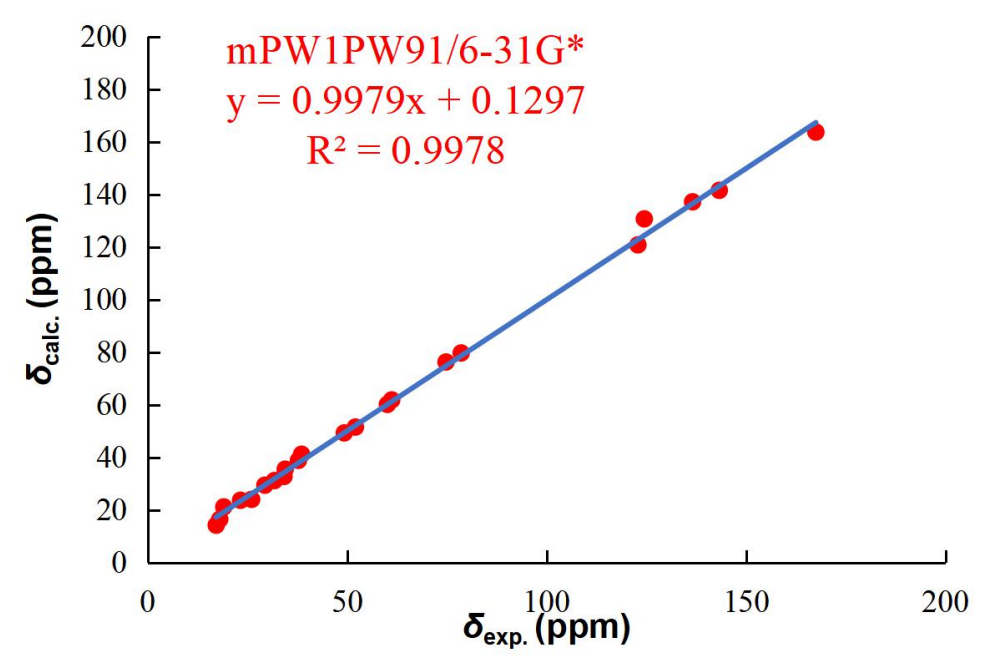

Figure S53. Regression analysis of experimental versus calculated ${ }^{13} \mathrm{C}$ NMR chemical shifts at mPW1PW91/6-31G* level using GIAO method of 3f; linear fitting is shown as a line (blue). 


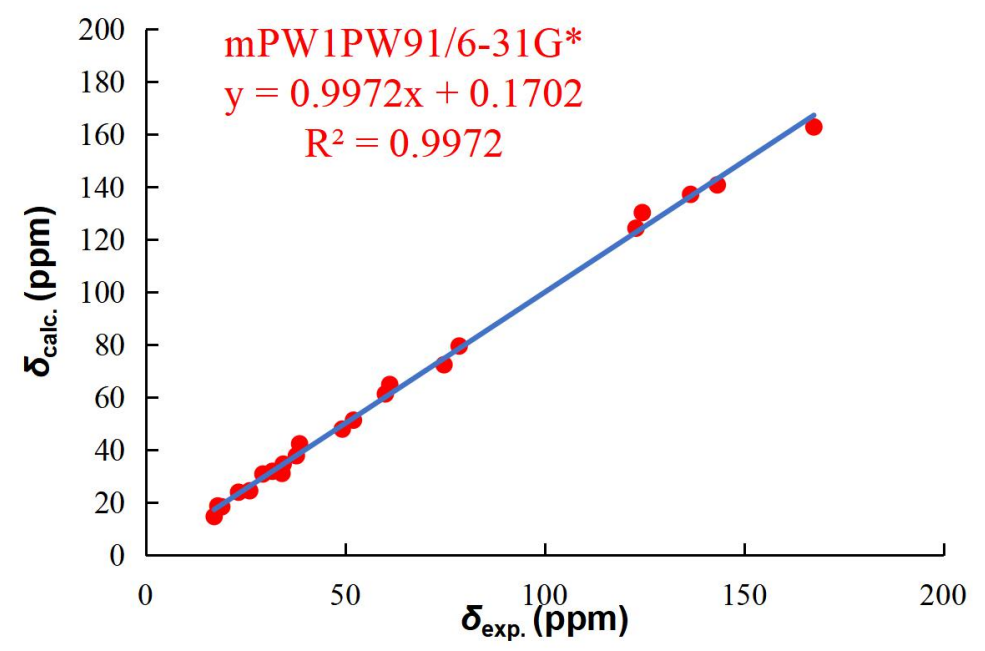

Figure S54. Regression analysis of experimental versus calculated ${ }^{13} \mathrm{C}$ NMR chemical shifts at mPW1PW91/6-31G* level using GIAO method of 3g; linear fitting is shown as a line (blue).

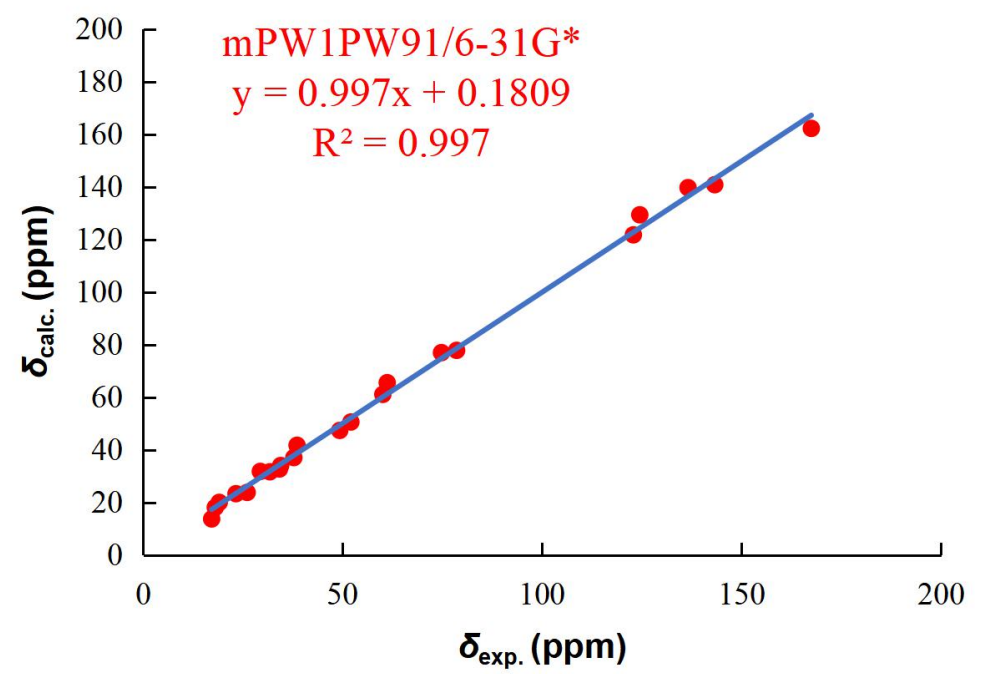

Figure S55. Regression analysis of experimental versus calculated ${ }^{13} \mathrm{C}$ NMR chemical shifts at mPW1PW91/6-31G* level using GIAO method of $\mathbf{3 h}$; linear fitting is shown as a line (blue). 


\begin{tabular}{|c|c|c|c|c|c|c|c|c|c|c|c|c|c|c|c|}
\hline \multirow{2}{*}{\multicolumn{2}{|c|}{$\begin{array}{l}\text { Functional } \\
\text { nPu1Pt91 }\end{array}$}} & \multirow{2}{*}{\multicolumn{3}{|c|}{$\begin{array}{l}\text { Solvent? } \\
\text { PCI }\end{array}$}} & \multirow{2}{*}{\multicolumn{3}{|c|}{$\begin{array}{l}\text { Basis Set } \\
6-31 G(d)\end{array}$}} & \multicolumn{4}{|c|}{ Type of Data } & & & & \\
\hline & & & & & & & & \multicolumn{4}{|c|}{ Shielding Tensors } & & & & \\
\hline & & \multirow{2}{*}{$\frac{\text { DP4+ }}{\text { xperinenta }}$} & \multicolumn{2}{|c|}{$\Delta 0.00 \mathrm{x}$} & $3.04 \mathrm{~s}$ & \multicolumn{2}{|c|}{$0.00 \mathrm{~s}$} & \multicolumn{2}{|c|}{$0.00 \mathrm{~s}$} & \multicolumn{2}{|c|}{$96.88 \mathrm{x}$} & \multicolumn{2}{|c|}{$0.00 \mathrm{x}$} & $0.08 \%$ & $0.00 \mathrm{~s}$ \\
\hline Huclei & sp2? & & \multicolumn{2}{|c|}{ Isoner 1} & \begin{tabular}{|l|} 
Isoner 2 \\
\end{tabular} & \multicolumn{2}{|c|}{ Isonex 3} & \multirow{2}{*}{\multicolumn{2}{|c|}{\begin{tabular}{|l|} 
Isonex 4 \\
156709587
\end{tabular}}} & \multicolumn{2}{|c|}{ Isoner 5} & \multicolumn{2}{|c|}{ Isoner 6} & Isonex 7 & Isonex 8 \\
\hline $\mathrm{C}$ & & 38.6 & & 3.605716 & 154.145184 & 150 & 545506 & & & 154.5 & 57542 & 156.88 & 3177 & 150.906 & $\begin{array}{ll}31 & 150.8558\end{array}$ \\
\hline $\mathrm{C}$ & & 26.1 & & 2.549929 & 168.553718 & 169. & 496998 & 168.6 & 92655 & 168.73 & 30868 & 173.85 & 2795 & 168.0622 & 168.066 \\
\hline $\mathrm{C}$ & & 31.8 & & 5.521993 & 164.291896 & & 2.84306 & 166.7 & 95562 & 162.60 & 01839 & 166.79 & 0175 & 160.9491 & 160.5388 \\
\hline $\mathrm{C}$ & & 78.6 & & 6.173687 & 113.582694 & 114. & 348405 & 114.0 & 65628 & 114.54 & 47973 & $118.85 \mathrm{C}$ & 3997 & 115.1595 & 116.1942 \\
\hline $\mathrm{C}$ & & 74.8 & & 24.64239 & 123.321912 & 123. & 322201 & 123.1 & 35912 & 120.68 & 89829 & $122.24 \mathrm{C}$ & 801 & 122.011 & 117.0208 \\
\hline $\mathrm{C}$ & & 29.4 & & 9.013322 & 165.197821 & 161. & 441649 & 165.3 & 98312 & 168.09 & 95502 & $168.51 \mathrm{c}$ & 4993 & 161.9391 & 160.3958 \\
\hline $\mathrm{C}$ & & 34.2 & & 2.751641 & 161.991263 & 160 & 954327 & 158.9 & 18363 & 161.35 & 54137 & $165.24 \mathrm{C}$ & 196 & $161.721 C$ & 159.5047 \\
\hline $\mathrm{C}$ & $\mathrm{x}$ & 136.6 & & 6100993 & 61.0693583 & 59.9 & 893204 & 62.42 & 66637 & 61.772 & 29624 & 62.0733 & 3072 & 59.7233 & 56.77982 \\
\hline $\mathrm{C}$ & $\mathrm{x}$ & 122.9 & & 1311264 & 74.0726318 & 70.8 & 165782 & 70.78 & 82353 & 78.008 & 84353 & 78.23 & 7969 & $72.0966 \mathrm{~S}$ & 74.02618 \\
\hline C & & 23.3 & & 0.557145 & 168.611656 & 168. & 221339 & 168.0 & 87978 & 168.12 & 23169 & $174.18 \mathrm{~s}$ & 961 & 168.5980 & 168.5349 \\
\hline $\mathrm{C}$ & & 37.8 & & 7.359727 & 156.024197 & 155 & 174331 & 155.0 & 55699 & 155.34 & 42778 & 159.19 & 7808 & 155.256 & 155.3523 \\
\hline $\mathrm{C}$ & & 60.1 & & 6.220304 & 133.259806 & 132. & 558264 & 132 & 85286 & 134.43 & 30778 & $138.17 \mathrm{~s}$ & 176 & $132.656 \mathrm{C}$ & 132.2797 \\
\hline C & & 61.2 & & 3.837926 & 131.919575 & & 3.91734 & 130.2 & 93676 & 131.61 & 16791 & 136.48 & 1547 & 129.2162 & 128.0039 \\
\hline C & & 34.5 & & 0.286718 & 156.92696 & & 3.75435 & 158.5 & 56289 & 159.02 & 21576 & $162.50 \mathrm{C}$ & 5606 & 158.329 & 158.2516 \\
\hline C & $\mathrm{x}$ & 143.3 & & .0025473 & 55.7819286 & 55.9 & 450078 & 55.75 & 85895 & 56.788 & 88428 & $57.754 \mathrm{C}$ & 0443 & 56.2644 & 55.7146 \\
\hline $\mathrm{C}$ & $\mathrm{x}$ & 167.5 & & 35.36754 & 33.7851487 & 35.3 & 146453 & 34.49 & 93533 & 35.092 & 27199 & $35.875 \mathrm{~S}$ & 917 & 35.0748 & 35.15919 \\
\hline C & $\mathrm{x}$ & 124.5 & & .0830583 & 66.1935857 & 65.7 & 958887 & 68.75 & 67212 & 65.877 & 75205 & $68.480^{3}$ & 3355 & 66.3771 & 66.70139 \\
\hline C & & 19.1 & & 9.229509 & 175.971702 & 176. & 530668 & 175.8 & 59234 & 173.93 & 36924 & 176.67 & 591 & 173.9201 & 171.7039 \\
\hline C & & 18.1 & & 7.587547 & 173.998479 & 174. & 074069 & 176.9 & 45706 & 175.13 & 30497 & $181.31 \mathrm{C}$ & 253 & 173.6057 & 173.5576 \\
\hline C & & 17.2 & & 9.543467 & 177.662286 & 177. & 282419 & 176.5 & 85181 & 177.73 & 31655 & 183.55 & 453 & 177.4803 & 177.7547 \\
\hline C & & 52.1 & & 4.098483 & 141.919114 & 142. & 357399 & 142.0 & 24597 & 142.30 & 07157 & 146.69 & 863 & 142.268 & 142.3654 \\
\hline C & & 49.3 & & 7.343041 & 144.665285 & & .34805 & 144.7 & 43629 & 145.58 & 88066 & 148.878 & 125 & 145.5247 & 145.4328 \\
\hline Fur & nal & & olv & ent? & & Basi & Set & & & Type 0 & of $\mathrm{Da}$ & ata & & & \\
\hline & W91 & & PC & & & $6-31$ & $G(d)$ & & & ielding & g Ter & nsors & & & \\
\hline & & Isomer & & Isomer 2 & IsomeI & 3 & Isome & er 4 & Isol & mer 5 & Iso & omer 6 & & omer 7 & Isomer 8 \\
\hline sDP4+ & I data) & - & & - & - & & - & - & & - & & - & & - & - \\
\hline sDP4+ & data) & 페 $0.33 \%$ & & ⿹ㅔ $0.91 \%$ & जी 0.00 & & जी 0.0 & $2 \%$ & 에 14. & . $96 \%$ & 네 83 & $3.34 \%$ & & $0.43 \%$ & $0.01 \%$ \\
\hline sDP4+ & 1 data) & 페 $0.33 \%$ & & 펭 $0.91 \%$ & 페 0.00 & & ज्ञn 0.0 & $2 \%$ & 메 14. & $.96 \%$ & -183 & $3.34 \%$ & & $0.43 \%$ & जी $0.01 \%$ \\
\hline uDP4+ & I data) & - & & - & - & & - & - & & - & & - & & - & - \\
\hline uDP4+ & data) & 페 $0.00 \%$ & & 페 $32.43 \%$ & 페 0.01 & & 페 2.4 & $15 \%$ & ज्ञl 63. & $18 \%$ & & $.00 \%$ & & 1. $75 \%$ & 게 $0.18 \%$ \\
\hline uDP4+ & 1 data) & 正 $0.00 \%$ & & 페 $32.43 \%$ & 페 0.01 & & 페 2.4 & $15 \%$ & 네 63. & $18 \%$ & जnll 0. & $.00 \%$ & & 1. $75 \%$ & 페 $0.18 \%$ \\
\hline DP4+ & data) & - & & - & - & & - & - & & - & & - & & - & - \\
\hline DP4+ & data) & 메 $0.00 \%$ & & ⿹ㅔ $3.04 \%$ & 페 0.00 & & 0.0 & $0 \%$ & -196. & $.88 \%$ & 正 0 . & $.00 \%$ & & $0.08 \%$ & $0.00 \%$ \\
\hline DP4+ & 1 data) & 페 $0.00 \%$ & & ज्ञll $3.04 \%$ & 페 0.00 & & 데 0.0 & $0 \%$ & 96. & $.88 \%$ & 正 0. & $.00 \%$ & & $0.08 \%$ & $0.00 \%$ \\
\hline
\end{tabular}

Figure S56. DP4+ evaluation of theoretical and experimental data of $\mathbf{3}$.

\subsection{ECD calculation for compound 1}

Conformational searches were carried out using the torsional sampling (MCMM) method and OPLS_2005 force field in the Macromodel 9.9.223 software applying an energy window of 21 $\mathrm{kJ} / \mathrm{mol}$. Conformers above $1 \%$ population were re-optimized with Gaussian 09 at the B3LYP/6-311G(d,p) level with IEFPCM solvent model for acetonitrile. For the resulting geometries, ECD spectra were obtained by TDDFT calculations performed with the same functional, basis set and solvent model as the energy optimization. Finally, the Boltzmann-averaged ECD spectra of 1 was obtained with SpecDis1.62 software.

The initial torsional sampling (MCMM) and OPLS_2005 force field conformational searches of $(1 R, 14 S, 15 S)-1$ afforded 87 conformers within the $21 \mathrm{~kJ} / \mathrm{mol}$ energy window, respectively. The Boltzmann populations of the conformers were obtained based on the potential energy provided by the OPLS_2005 force field, leading to 16 conformers for $\mathbf{1}$ above $1 \%$ population for further re-optimization. The resulting geometries were re-optimized at the B3LYP/6-311G(d,p) level with IEFPCM solvent model for $\mathrm{CH}_{3} \mathrm{CN}$, and frequency analysis was performed as well to confirm that the re-optimized geometries were at the energy minima. Finally, the Boltzmann-averaged ECD 
spectra of $(1 R, 14 S, 15 S)-1$ and its enantiomer were matched to the experimental curves of sinusiaetone A (1)

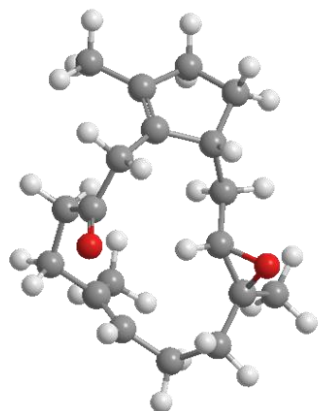

conf. $128.384 \%$

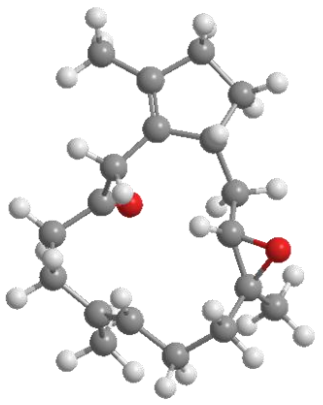

conf. $55.383 \%$

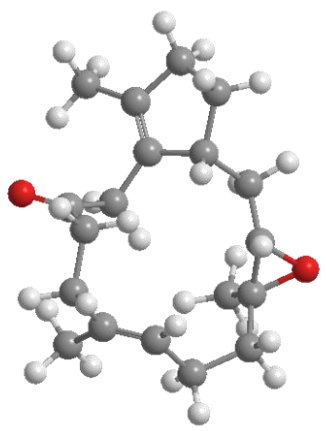

conf. $93.208 \%$

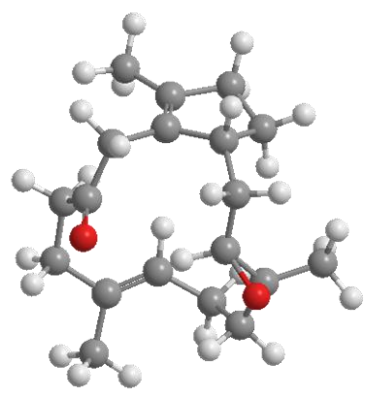

conf. $131.936 \%$

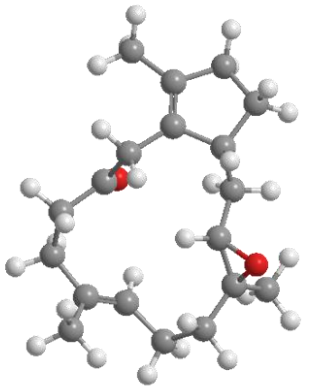

conf. $212.285 \%$

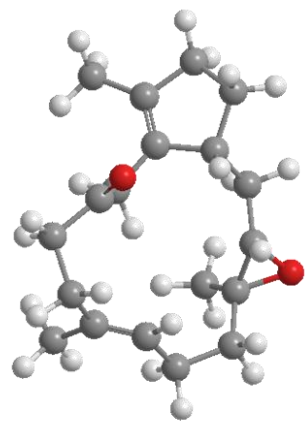

conf. $6 \quad 4.307 \%$

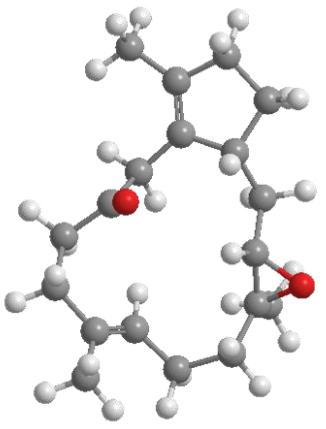

conf. $102.939 \%$

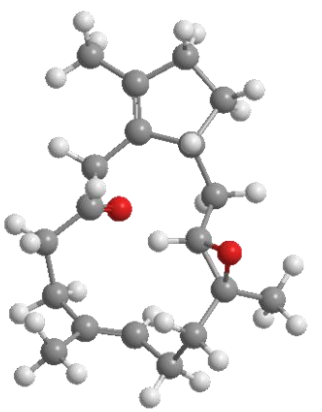

conf. $141.746 \%$

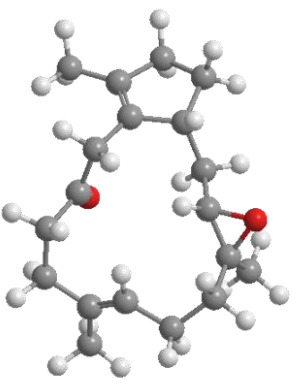

conf. $38.468 \%$

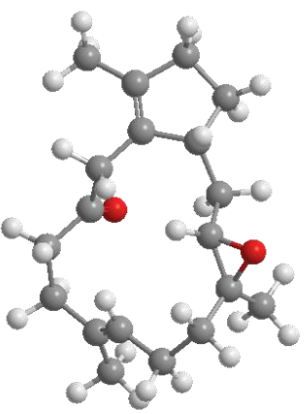

conf. $74.199 \%$

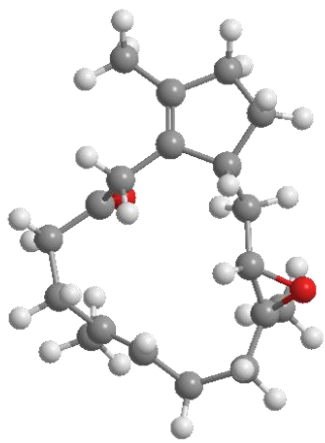

conf. $112.762 \%$

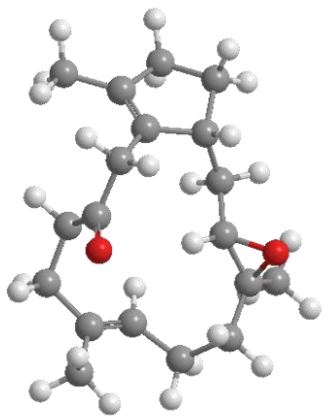

conf. $15 \quad 1.571 \%$

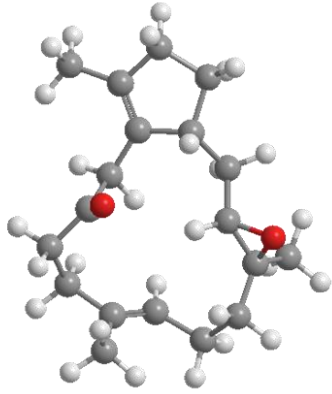

conf. $45.438 \%$

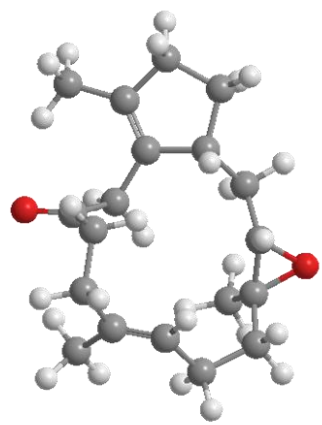

conf. $83.235 \%$

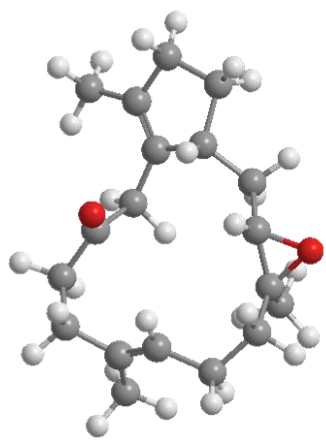

conf. $122.483 \%$

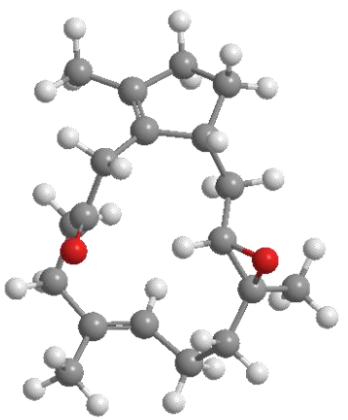

conf. $16 \quad 1.222 \%$

Figure S57. Re-optimized conformers above 1\% population (OPLS_2005) of $(1 R, 14 S, 15 S)-1$ calculated at the B3LYP/6-311G(d,p) level with IEFPCM solvent model for acetonitrile.

Table S15. Cartesian coordinates for the re-optimized conformers of 1 at the B3LYP/6-311G(d,p) level with IEFPCM solvent model for acetonitrile. 


\begin{tabular}{|c|c|c|c|c|c|c|c|c|c|}
\hline \multicolumn{2}{|c|}{$\begin{array}{c}\text { Sinusiaetone A (1) } \\
\text { Conf. } 1\end{array}$} & \multicolumn{3}{|c|}{$\begin{array}{c}\text { Standard Orientation } \\
\text { (Ångstroms) }\end{array}$} & \multicolumn{2}{|c|}{$\begin{array}{c}\text { Sinusiaetone A (1) } \\
\text { Conf. } 2\end{array}$} & \multicolumn{3}{|c|}{$\begin{array}{c}\text { Standard Orientation } \\
\text { (Ångstroms) }\end{array}$} \\
\hline I & atom & $\mathrm{X}$ & $\mathrm{Y}$ & Z & I & atom & $\mathrm{X}$ & Y & Z \\
\hline 1 & $\mathrm{C}$ & 0.787306 & 2.980546 & 0.459776 & 1 & $\mathrm{C}$ & -1.88128 & -2.85446 & 0.498344 \\
\hline 2 & $\mathrm{C}$ & 1.81368 & 1.936853 & 0.870145 & 2 & $\mathrm{C}$ & -2.80965 & -1.70341 & 0.151073 \\
\hline 3 & $\mathrm{C}$ & -0.59121 & 2.375765 & 0.132812 & 3 & $\mathrm{C}$ & -0.60048 & -2.88168 & -0.35839 \\
\hline 4 & $\mathrm{C}$ & 2.664739 & 1.440332 & -0.03682 & 4 & $\mathrm{C}$ & -2.78087 & -0.56301 & 0.85436 \\
\hline 5 & $\mathrm{C}$ & 3.696692 & 0.355393 & 0.115636 & 5 & $\mathrm{C}$ & -3.60143 & 0.681008 & 0.609735 \\
\hline 6 & $\mathrm{C}$ & 3.370398 & -0.89846 & -0.73318 & 6 & $\mathrm{C}$ & -2.97357 & 1.672378 & -0.39846 \\
\hline 7 & $\mathrm{C}$ & 2.219423 & -1.73942 & -0.20039 & 7 & $\mathrm{C}$ & -1.62392 & 2.257275 & -0.00787 \\
\hline 8 & $\mathrm{C}$ & 0.838544 & -1.35442 & -0.54816 & 8 & $\mathrm{C}$ & -0.39951 & 1.559077 & -0.44181 \\
\hline 9 & $\mathrm{C}$ & -0.37399 & -1.66914 & 0.297768 & 9 & $\mathrm{C}$ & 0.958565 & 1.664399 & 0.217402 \\
\hline 10 & $\mathrm{C}$ & -0.67299 & 1.719912 & -1.23654 & 10 & $\mathrm{C}$ & 0.450632 & -1.87285 & 0.092975 \\
\hline 11 & $\mathrm{C}$ & -1.88606 & 0.832714 & -1.54698 & 11 & $\mathrm{C}$ & 1.431881 & -1.38983 & -0.97888 \\
\hline 12 & $\mathrm{C}$ & -2.34289 & -0.18435 & -0.53039 & 12 & $\mathrm{C}$ & 2.407699 & -0.33598 & -0.53533 \\
\hline 13 & $\mathrm{C}$ & -1.73758 & -1.59052 & -0.44317 & 13 & $\mathrm{C}$ & 2.119535 & 1.156359 & -0.68662 \\
\hline 14 & $\mathrm{C}$ & -2.84333 & -2.3655 & 0.321751 & 14 & $\mathrm{C}$ & 3.49323 & 1.799291 & -0.34583 \\
\hline 15 & $\mathrm{C}$ & -3.58989 & -1.29557 & 1.141615 & 15 & $\mathrm{C}$ & 4.278151 & 0.728769 & 0.441011 \\
\hline 16 & $\mathrm{C}$ & -3.34537 & -0.02727 & 0.350964 & 16 & $\mathrm{C}$ & 3.574547 & -0.56227 & 0.085184 \\
\hline 17 & $\mathrm{C}$ & -4.17456 & 1.191391 & 0.616088 & 17 & $\mathrm{C}$ & 4.169629 & -1.87932 & 0.479656 \\
\hline 18 & $\mathrm{O}$ & 0.143669 & 1.946332 & -2.10829 & 18 & $\mathrm{O}$ & 0.530469 & -1.51774 & 1.250819 \\
\hline 19 & $\mathrm{C}$ & 1.75614 & 1.521654 & 2.319662 & 19 & $\mathrm{C}$ & -3.71574 & -1.94825 & -1.0309 \\
\hline 20 & $\mathrm{C}$ & 2.540991 & -2.55875 & 1.031689 & 20 & $\mathrm{C}$ & -1.62971 & 3.194225 & 1.179605 \\
\hline 21 & $\mathrm{O}$ & 1.484016 & -2.46039 & -1.22783 & 21 & $\mathrm{O}$ & -0.86933 & 2.726905 & -1.1597 \\
\hline 22 & $\mathrm{H}$ & -1.5954 & -2.01555 & -1.44399 & 22 & $\mathrm{H}$ & 1.848029 & 1.393564 & -1.72323 \\
\hline 23 & $\mathrm{H}$ & 0.747981 & -0.48256 & -1.1902 & 23 & $\mathrm{H}$ & -0.57083 & 0.639742 & -0.99836 \\
\hline 24 & $\mathrm{H}$ & 1.138979 & 3.528885 & -0.41615 & 24 & $\mathrm{H}$ & -2.41108 & -3.80254 & 0.359992 \\
\hline 25 & $\mathrm{H}$ & 0.660366 & 3.707245 & 1.269034 & 25 & $\mathrm{H}$ & -1.58431 & -2.79762 & 1.547207 \\
\hline 26 & $\mathrm{H}$ & -1.35967 & 3.161304 & 0.131325 & 26 & $\mathrm{H}$ & -0.11871 & -3.86557 & -0.27784 \\
\hline 27 & $\mathrm{H}$ & -0.91669 & 1.661622 & 0.893708 & 27 & $\mathrm{H}$ & -0.81921 & -2.74398 & -1.42063 \\
\hline 28 & $\mathrm{H}$ & 2.583779 & 1.834277 & -1.0482 & 28 & $\mathrm{H}$ & -2.07324 & -0.50709 & 1.678126 \\
\hline 29 & $\mathrm{H}$ & 3.840635 & 0.073542 & 1.160884 & 29 & $\mathrm{H}$ & -3.76741 & 1.191208 & 1.56394 \\
\hline 30 & $\mathrm{H}$ & 4.663644 & 0.740385 & -0.23009 & 30 & $\mathrm{H}$ & -4.59464 & 0.418952 & 0.234812 \\
\hline 31 & $\mathrm{H}$ & 4.26299 & -1.53152 & -0.79621 & 31 & $\mathrm{H}$ & -3.67072 & 2.505001 & -0.55379 \\
\hline 32 & $\mathrm{H}$ & 3.126527 & -0.59178 & -1.75486 & 32 & $\mathrm{H}$ & -2.8543 & 1.173654 & -1.36446 \\
\hline 33 & $\mathrm{H}$ & -0.25827 & -2.68421 & 0.682506 & 33 & $\mathrm{H}$ & 1.135281 & 2.714572 & 0.458712 \\
\hline 34 & $\mathrm{H}$ & -0.38978 & -1.00113 & 1.167279 & 34 & $\mathrm{H}$ & 0.955242 & 1.104535 & 1.157402 \\
\hline 35 & $\mathrm{H}$ & -1.66582 & 0.346559 & -2.50117 & 35 & $\mathrm{H}$ & 0.845957 & -1.05109 & -1.84069 \\
\hline 36 & $\mathrm{H}$ & -2.70268 & 1.539957 & -1.74125 & 36 & $\mathrm{H}$ & 1.952094 & -2.28895 & -1.33375 \\
\hline 37 & $\mathrm{H}$ & -3.53489 & -2.80832 & -0.40046 & 37 & $\mathrm{H}$ & 4.02265 & 2.026016 & -1.27499 \\
\hline 38 & $\mathrm{H}$ & -2.4428 & -3.17744 & 0.932855 & 38 & $\mathrm{H}$ & 3.387562 & 2.737534 & 0.203046 \\
\hline 39 & $\mathrm{H}$ & -3.19157 & -1.19879 & 2.160839 & 39 & $\mathrm{H}$ & 4.237051 & 0.895744 & 1.526035 \\
\hline 40 & $\mathrm{H}$ & -4.65772 & -1.51351 & 1.247611 & 40 & $\mathrm{H}$ & 5.340902 & 0.70809 & 0.175458 \\
\hline 41 & $\mathrm{H}$ & -4.01957 & 1.547255 & 1.641674 & 41 & $\mathrm{H}$ & 4.232011 & -1.96096 & 1.571425 \\
\hline 42 & $\mathrm{H}$ & -3.94831 & 2.019202 & -0.05768 & 42 & $\mathrm{H}$ & 3.592406 & -2.73186 & 0.117417 \\
\hline 43 & $\mathrm{H}$ & -5.24153 & 0.959038 & 0.526023 & 43 & $\mathrm{H}$ & 5.193598 & -1.97472 & 0.101071 \\
\hline
\end{tabular}




\begin{tabular}{|l|l|c|c|c|c|c|c|c|c|}
\hline 44 & $\mathrm{H}$ & 1.995359 & 2.372607 & 2.968362 & 44 & $\mathrm{H}$ & -4.33468 & -1.08713 & -1.28202 \\
\hline 45 & $\mathrm{H}$ & 0.746955 & 1.197293 & 2.597102 & 45 & $\mathrm{H}$ & -3.13478 & -2.20954 & -1.92255 \\
\hline 46 & $\mathrm{H}$ & 2.443534 & 0.711727 & 2.562274 & 46 & $\mathrm{H}$ & -4.37837 & -2.79918 & -0.83407 \\
\hline 47 & $\mathrm{H}$ & 3.367953 & -3.24052 & 0.810496 & 47 & $\mathrm{H}$ & -2.33024 & 4.016092 & 1.001837 \\
\hline 48 & $\mathrm{H}$ & 1.69155 & -3.15343 & 1.363941 & 48 & $\mathrm{H}$ & -0.64439 & 3.619916 & 1.364683 \\
\hline 50 & $\mathrm{H}$ & 2.85255 & -1.91669 & 1.85966 & 50 & $\mathrm{H}$ & -1.95127 & 2.67187 & 2.084725 \\
\hline
\end{tabular}

B3LYP/6-311G(d,p) Energy $/$ Hartree $=-891.35454825$ a.u. Population $=8.468 \%$

\begin{tabular}{|c|c|c|c|c|c|c|c|c|c|}
\hline \multicolumn{2}{|c|}{$\begin{array}{c}\text { Sinusiaetone A (1) } \\
\text { Conf. } 3\end{array}$} & \multicolumn{3}{|c|}{$\begin{array}{c}\text { Standard Orientation } \\
\text { (Ångstroms) }\end{array}$} & \multicolumn{2}{|c|}{$\begin{array}{c}\text { Sinusiaetone A (1) } \\
\text { Conf. } 4\end{array}$} & \multicolumn{3}{|c|}{$\begin{array}{c}\text { Standard Orientation } \\
\text { (Ångstroms) }\end{array}$} \\
\hline I & atom & $\mathrm{X}$ & $\mathrm{Y}$ & Z & I & atom & $\mathrm{X}$ & $\mathrm{Y}$ & $\mathrm{Z}$ \\
\hline 1 & $\mathrm{C}$ & -1.85305 & -2.94781 & -0.11073 & 1 & $\mathrm{C}$ & 1.447984 & 2.847106 & 0.814701 \\
\hline 2 & $\mathrm{C}$ & -2.74345 & -1.7573 & 0.214802 & 2 & $\mathrm{C}$ & 2.483299 & 1.854594 & 0.324268 \\
\hline 3 & $\mathrm{C}$ & -0.62657 & -2.67808 & -0.98363 & 3 & $\mathrm{C}$ & 0.294968 & 3.122416 & -0.18431 \\
\hline 4 & $\mathrm{C}$ & -2.77497 & -0.65084 & -0.53676 & 4 & $\mathrm{C}$ & 2.665477 & 0.6922 & 0.966824 \\
\hline 5 & $\mathrm{C}$ & -3.61228 & 0.589604 & -0.34227 & 5 & $\mathrm{C}$ & 3.645373 & -0.40215 & 0.616715 \\
\hline 6 & $\mathrm{C}$ & -2.89239 & 1.879064 & -0.78572 & 6 & $\mathrm{C}$ & 3.123366 & -1.42173 & -0.42116 \\
\hline 7 & $\mathrm{C}$ & -1.64603 & 2.254055 & 0.003275 & 7 & $\mathrm{C}$ & 1.86603 & -2.1829 & -0.03026 \\
\hline 8 & $\mathrm{C}$ & -0.32724 & 1.804401 & -0.48076 & 8 & $\mathrm{C}$ & 0.554687 & -1.67101 & -0.47213 \\
\hline 9 & $\mathrm{C}$ & 0.917034 & 1.642878 & 0.3731 & 9 & $\mathrm{C}$ & -0.77348 & -1.97094 & 0.204643 \\
\hline 10 & $\mathrm{C}$ & 0.435473 & -1.83167 & -0.29032 & 10 & $\mathrm{C}$ & -0.53028 & 1.900044 & -0.55763 \\
\hline 11 & $\mathrm{C}$ & 1.485329 & -1.18153 & -1.19903 & 11 & $\mathrm{C}$ & -1.20128 & 1.147633 & 0.590449 \\
\hline 12 & $\mathrm{C}$ & 2.439084 & -0.2398 & -0.51673 & 12 & $\mathrm{C}$ & -2.27375 & 0.168576 & 0.20276 \\
\hline 13 & $\mathrm{C}$ & 2.182999 & 1.263709 & -0.45124 & 13 & $\mathrm{C}$ & -1.97042 & -1.20485 & -0.39914 \\
\hline 14 & $\mathrm{C}$ & 3.512541 & 1.810694 & 0.142891 & 14 & $\mathrm{C}$ & -3.31704 & -1.94435 & -0.22323 \\
\hline 15 & $\mathrm{C}$ & 4.22753 & 0.600448 & 0.780925 & 15 & $\mathrm{C}$ & -4.37872 & -0.83112 & -0.17628 \\
\hline 16 & $\mathrm{C}$ & 3.544533 & -0.59418 & 0.153581 & 16 & $\mathrm{C}$ & -3.59647 & 0.371549 & 0.312429 \\
\hline 17 & $\mathrm{C}$ & 4.087928 & -1.97301 & 0.371888 & 17 & $\mathrm{C}$ & -4.32177 & 1.594638 & 0.784625 \\
\hline 18 & $\mathrm{O}$ & 0.472021 & -1.72948 & 0.918163 & 18 & $\mathrm{O}$ & -0.64332 & 1.548153 & -1.71622 \\
\hline 19 & $\mathrm{C}$ & -3.59798 & -1.97203 & 1.439581 & 19 & $\mathrm{C}$ & 3.265121 & 2.273544 & -0.89676 \\
\hline 20 & $\mathrm{C}$ & -1.86524 & 2.616855 & 1.455276 & 20 & $\mathrm{C}$ & 1.980695 & -3.09137 & 1.173928 \\
\hline 21 & $\mathrm{O}$ & -0.77751 & 3.158499 & -0.73214 & 21 & $\mathrm{O}$ & 1.192753 & -2.76766 & -1.17482 \\
\hline 22 & $\mathrm{H}$ & 2.042197 & 1.669684 & -1.46125 & 22 & $\mathrm{H}$ & -1.77211 & -1.06995 & -1.47167 \\
\hline 23 & $\mathrm{H}$ & -0.34282 & 1.196736 & -1.38448 & 23 & $\mathrm{H}$ & 0.574566 & -0.74894 & -1.04898 \\
\hline 24 & $\mathrm{H}$ & -2.46364 & -3.72742 & -0.58368 & 24 & $\mathrm{H}$ & 1.93589 & 3.809833 & 1.008386 \\
\hline 25 & $\mathrm{H}$ & -1.50664 & -3.37767 & 0.834044 & 25 & $\mathrm{H}$ & 1.036922 & 2.510895 & 1.769698 \\
\hline 26 & $\mathrm{H}$ & -0.8864 & -2.23529 & -1.94851 & 26 & $\mathrm{H}$ & 0.675774 & 3.554022 & -1.11153 \\
\hline 27 & $\mathrm{H}$ & -0.1405 & -3.63166 & -1.22943 & 27 & $\mathrm{H}$ & -0.38209 & 3.856242 & 0.268218 \\
\hline 28 & $\mathrm{H}$ & -2.14124 & -0.61534 & -1.42007 & 28 & $\mathrm{H}$ & 2.03784 & 0.498673 & 1.834847 \\
\hline 29 & $\mathrm{H}$ & -3.9466 & 0.684303 & 0.693324 & 29 & $\mathrm{H}$ & 3.928645 & -0.93018 & 1.532512 \\
\hline 30 & $\mathrm{H}$ & -4.52711 & 0.510772 & -0.94484 & 30 & $\mathrm{H}$ & 4.57007 & 0.025514 & 0.21891 \\
\hline 31 & $\mathrm{H}$ & -3.59565 & 2.717918 & -0.72272 & 31 & $\mathrm{H}$ & 3.914549 & -2.15512 & -0.62083 \\
\hline 32 & $\mathrm{H}$ & -2.60796 & 1.788918 & -1.83899 & 32 & $\mathrm{H}$ & 2.923213 & -0.9061 & -1.36437 \\
\hline 33 & $\mathrm{H}$ & 1.091857 & 2.595501 & 0.878095 & 33 & $\mathrm{H}$ & -0.95546 & -3.04424 & 0.101819 \\
\hline 34 & $\mathrm{H}$ & 0.746168 & 0.890412 & 1.148789 & 34 & $\mathrm{H}$ & -0.71431 & -1.77177 & 1.281393 \\
\hline 35 & $\mathrm{H}$ & 0.961364 & -0.68848 & -2.02453 & 35 & $\mathrm{H}$ & -1.57976 & 1.886725 & 1.302061 \\
\hline
\end{tabular}




\begin{tabular}{|l|l|l|l|l|l|l|l|l|c|}
\hline 36 & $\mathrm{H}$ & 2.023527 & -2.01464 & -1.66952 & 36 & $\mathrm{H}$ & -0.39048 & 0.635285 & 1.125021 \\
\hline 37 & $\mathrm{H}$ & 4.128721 & 2.212189 & -0.66561 & 37 & $\mathrm{H}$ & -3.49219 & -2.68402 & -1.00815 \\
\hline 38 & $\mathrm{H}$ & 3.343926 & 2.623712 & 0.852117 & 38 & $\mathrm{H}$ & -3.3074 & -2.47526 & 0.735066 \\
\hline 39 & $\mathrm{H}$ & 4.107306 & 0.573237 & 1.87246 & 39 & $\mathrm{H}$ & -5.22554 & -1.07308 & 0.474836 \\
\hline 40 & $\mathrm{H}$ & 5.307037 & 0.605451 & 0.593245 & 40 & $\mathrm{H}$ & -4.79976 & -0.62862 & -1.1713 \\
\hline 41 & $\mathrm{H}$ & 4.047702 & -2.23813 & 1.435139 & 41 & $\mathrm{H}$ & -4.9901 & 1.35309 & 1.619093 \\
\hline 42 & $\mathrm{H}$ & 3.538855 & -2.73786 & -0.18009 & 42 & $\mathrm{H}$ & -3.65124 & 2.393068 & 1.106146 \\
\hline 43 & $\mathrm{H}$ & 5.141701 & -2.02864 & 0.076159 & 43 & $\mathrm{H}$ & -4.95655 & 1.995695 & -0.01455 \\
\hline 44 & $\mathrm{H}$ & -4.30333 & -1.16191 & 1.621789 & 44 & $\mathrm{H}$ & 2.62703 & 2.291253 & -1.78674 \\
\hline 45 & $\mathrm{H}$ & -4.17299 & -2.90039 & 1.342783 & 45 & $\mathrm{H}$ & 3.659242 & 3.288245 & -0.77268 \\
\hline 46 & $\mathrm{H}$ & -2.96971 & -2.08826 & 2.330262 & 46 & $\mathrm{H}$ & 4.100831 & 1.606536 & -1.10799 \\
\hline 47 & $\mathrm{H}$ & -2.59823 & 3.425826 & 1.532429 & 47 & $\mathrm{H}$ & 2.80829 & -3.79416 & 1.036926 \\
\hline 48 & $\mathrm{H}$ & -0.94175 & 2.945268 & 1.931083 & 48 & $\mathrm{H}$ & 1.066892 & -3.66463 & 1.328167 \\
\hline 50 & $\mathrm{H}$ & -2.25149 & 1.760784 & 2.014942 & 50 & $\mathrm{H}$ & 2.179002 & -2.51424 & 2.081343 \\
\hline
\end{tabular}

B3LYP/6-311G(d,p) Energy / Hartree =-891.35976601 a.u. Population $=5.383 \%$

\begin{tabular}{|c|c|c|c|c|c|c|c|c|c|}
\hline \multicolumn{2}{|c|}{$\begin{array}{c}\text { Sinusiaetone A (1) } \\
\text { Conf. } 5\end{array}$} & \multicolumn{3}{|c|}{$\begin{array}{c}\text { Standard Orientation } \\
\text { (Ångstroms) }\end{array}$} & \multicolumn{2}{|c|}{$\begin{array}{c}\text { Sinusiaetone A (1) } \\
\text { Conf. } 6\end{array}$} & \multicolumn{3}{|c|}{$\begin{array}{c}\text { Standard Orientation } \\
\text { (Ångstroms) }\end{array}$} \\
\hline I & atom & $X$ & $\mathrm{Y}$ & Z & I & atom & $X$ & $\mathrm{Y}$ & $\mathrm{Z}$ \\
\hline 1 & $\mathrm{C}$ & 1.344503 & 2.935799 & -0.74591 & 1 & $\mathrm{C}$ & -2.14042 & -2.70895 & -0.79401 \\
\hline 2 & $\mathrm{C}$ & 2.33628 & 1.950385 & -0.15729 & 2 & $\mathrm{C}$ & -2.72886 & -1.56539 & 0.007637 \\
\hline 3 & $\mathrm{C}$ & 0.061558 & 3.150245 & 0.099045 & 3 & $\mathrm{C}$ & -0.74059 & -3.19225 & -0.32381 \\
\hline 4 & $\mathrm{C}$ & 2.734767 & 0.897461 & -0.88406 & 4 & $\mathrm{C}$ & -2.86731 & -0.35384 & -0.54779 \\
\hline 5 & $\mathrm{C}$ & 3.721019 & -0.18315 & -0.52885 & 5 & $\mathrm{C}$ & -3.46533 & 0.888437 & 0.056721 \\
\hline 6 & $\mathrm{C}$ & 3.202198 & -1.60371 & -0.83934 & 6 & $\mathrm{C}$ & -2.72742 & 2.185338 & -0.3221 \\
\hline 7 & $\mathrm{C}$ & 2.035096 & -2.07499 & 0.014726 & 7 & $\mathrm{C}$ & -1.31933 & 2.379467 & 0.228644 \\
\hline 8 & $\mathrm{C}$ & 0.657814 & -1.81259 & -0.44109 & 8 & $\mathrm{C}$ & -0.15636 & 2.247894 & -0.68437 \\
\hline 9 & $\mathrm{C}$ & -0.55496 & -1.72492 & 0.46848 & 9 & $\mathrm{C}$ & 1.303689 & 2.064973 & -0.31869 \\
\hline 10 & $\mathrm{C}$ & -0.6919 & 1.867435 & 0.414271 & 10 & $\mathrm{C}$ & 0.354217 & -2.18654 & -0.64886 \\
\hline 11 & $\mathrm{C}$ & -1.33658 & 1.135454 & -0.76662 & 11 & $\mathrm{C}$ & 0.724639 & -1.18521 & 0.443186 \\
\hline 12 & $\mathrm{C}$ & -2.29729 & 0.034601 & -0.40333 & 12 & $\mathrm{C}$ & 1.961575 & -0.38415 & 0.167685 \\
\hline 13 & $\mathrm{C}$ & -1.88358 & -1.43307 & -0.26555 & 13 & $\mathrm{C}$ & 1.980295 & 0.76531 & -0.8378 \\
\hline 14 & $\mathrm{C}$ & -3.11039 & -2.05139 & 0.444503 & 14 & $\mathrm{C}$ & 3.502492 & 0.953217 & -1.07766 \\
\hline 15 & $\mathrm{C}$ & -4.29137 & -1.13599 & 0.077185 & 15 & $\mathrm{C}$ & 4.182511 & 0.417645 & 0.197316 \\
\hline 16 & $\mathrm{C}$ & -3.6189 & 0.187109 & -0.22673 & 16 & $\mathrm{C}$ & 3.159158 & -0.55344 & 0.750174 \\
\hline 17 & $\mathrm{C}$ & -4.43549 & 1.438092 & -0.33816 & 17 & $\mathrm{C}$ & 3.550963 & -1.51991 & 1.826374 \\
\hline 18 & $\mathrm{O}$ & -0.76989 & 1.455323 & 1.555448 & 18 & $\mathrm{O}$ & 0.877939 & -2.17864 & -1.74627 \\
\hline 19 & $\mathrm{C}$ & 2.824227 & 2.268108 & 1.235048 & 19 & $\mathrm{C}$ & -3.16524 & -1.91762 & 1.408824 \\
\hline 20 & $\mathrm{C}$ & 2.339572 & -2.31082 & 1.477866 & 20 & $\mathrm{C}$ & -1.17168 & 2.274575 & 1.72974 \\
\hline 21 & $\mathrm{O}$ & 1.254063 & -3.12164 & -0.62193 & 21 & $\mathrm{O}$ & -0.69721 & 3.548355 & -0.36339 \\
\hline 22 & $\mathrm{H}$ & -1.80609 & -1.86062 & -1.27783 & 22 & $\mathrm{H}$ & 1.48554 & 0.473215 & -1.76929 \\
\hline 23 & $\mathrm{H}$ & 0.566489 & -1.27503 & -1.38374 & 23 & $\mathrm{H}$ & -0.38957 & 1.934738 & -1.70372 \\
\hline 24 & $\mathrm{H}$ & 1.071111 & 2.620584 & -1.75601 & 24 & $\mathrm{H}$ & -2.07214 & -2.43225 & -1.84945 \\
\hline 25 & $\mathrm{H}$ & 1.82281 & 3.918195 & -0.84373 & 25 & $\mathrm{H}$ & -2.81381 & -3.57169 & -0.73342 \\
\hline 26 & $\mathrm{H}$ & 0.303696 & 3.625059 & 1.051146 & 26 & $\mathrm{H}$ & -0.49653 & -4.11227 & -0.86018 \\
\hline 27 & $\mathrm{H}$ & -0.60248 & 3.823938 & -0.45235 & 27 & $\mathrm{H}$ & -0.75702 & -3.41545 & 0.745158 \\
\hline
\end{tabular}




\begin{tabular}{|c|c|c|c|c|c|c|c|c|c|}
\hline 28 & $\mathrm{H}$ & 2.314669 & 0.799767 & -1.8853 & 28 & $\mathrm{H}$ & -2.54467 & -0.24008 & -1.5827 \\
\hline 29 & $\mathrm{H}$ & 4.032424 & -0.1169 & 0.515483 & 29 & $\mathrm{H}$ & -3.54565 & 0.80722 & 1.142638 \\
\hline 30 & $\mathrm{H}$ & 4.63132 & -0.03694 & -1.12507 & 30 & $\mathrm{H}$ & -4.49543 & 0.994467 & -0.3095 \\
\hline 31 & $\mathrm{H}$ & 4.025541 & -2.31705 & -0.71625 & 31 & $\mathrm{H}$ & -3.32052 & 3.03603 & 0.034678 \\
\hline 32 & $\mathrm{H}$ & 2.895486 & -1.65586 & -1.88873 & 32 & $\mathrm{H}$ & -2.68332 & 2.275445 & -1.41215 \\
\hline 33 & $\mathrm{H}$ & -0.64426 & -2.68723 & 0.97964 & 33 & $\mathrm{H}$ & 1.814676 & 2.915548 & -0.78514 \\
\hline 34 & $\mathrm{H}$ & -0.39995 & -0.96676 & 1.241472 & 34 & $\mathrm{H}$ & 1.456712 & 2.172727 & 0.757723 \\
\hline 35 & $\mathrm{H}$ & -0.51346 & 0.753015 & -1.3823 & 35 & $\mathrm{H}$ & 0.796867 & -1.73152 & 1.387528 \\
\hline 36 & $\mathrm{H}$ & -1.82089 & 1.893149 & -1.39124 & 36 & $\mathrm{H}$ & -0.15854 & -0.54044 & 0.553811 \\
\hline 37 & $\mathrm{H}$ & -3.26573 & -3.09843 & 0.173201 & 37 & $\mathrm{H}$ & 3.806134 & 0.336704 & -1.92837 \\
\hline 38 & $\mathrm{H}$ & -2.94616 & -2.01136 & 1.526676 & 38 & $\mathrm{H}$ & 3.768785 & 1.986854 & -1.30983 \\
\hline 39 & $\mathrm{H}$ & -5.03529 & -1.05613 & 0.877003 & 39 & $\mathrm{H}$ & 4.391111 & 1.212896 & 0.926359 \\
\hline 40 & $\mathrm{H}$ & -4.82788 & -1.49874 & -0.81114 & 40 & $\mathrm{H}$ & 5.141916 & -0.06795 & -0.01083 \\
\hline 41 & $\mathrm{H}$ & -4.91244 & 1.675352 & 0.619983 & 41 & $\mathrm{H}$ & 3.967716 & -0.98758 & 2.689539 \\
\hline 42 & $\mathrm{H}$ & -3.84711 & 2.305876 & -0.64097 & 42 & $\mathrm{H}$ & 2.718115 & -2.13072 & 2.178138 \\
\hline 43 & $\mathrm{H}$ & -5.24638 & 1.307314 & -1.06435 & 43 & $\mathrm{H}$ & 4.336767 & -2.19696 & 1.471436 \\
\hline 44 & $\mathrm{H}$ & 3.623338 & 1.605121 & 1.564269 & 44 & $\mathrm{H}$ & -3.54879 & -1.06213 & 1.963109 \\
\hline 45 & $\mathrm{H}$ & 3.200062 & 3.296643 & 1.281755 & 45 & $\mathrm{H}$ & -3.95543 & -2.67689 & 1.376299 \\
\hline 46 & $\mathrm{H}$ & 2.008533 & 2.197609 & 1.961615 & 46 & $\mathrm{H}$ & -2.34368 & -2.35044 & 1.988858 \\
\hline 47 & $\mathrm{H}$ & 3.161286 & -3.02671 & 1.577409 & 47 & $\mathrm{H}$ & -1.91192 & 2.912782 & 2.222093 \\
\hline 48 & $\mathrm{H}$ & 1.474275 & -2.70593 & 2.008931 & 48 & $\mathrm{H}$ & -0.18191 & 2.593372 & 2.054521 \\
\hline 50 & $\mathrm{H}$ & 2.644153 & -1.38167 & 1.966926 & 50 & $\mathrm{H}$ & -1.33317 & 1.249258 & 2.072622 \\
\hline & & & & & & & & & \\
\hline
\end{tabular}

B3LYP/6-311G(d,p) Energy / Hartree =-891.35644974 a.u. B3LYP/6-311G(d,p) Energy / Hartree =-891.36095057 a.u. Population $=4.199 \%$ Population $=3.235 \%$

\begin{tabular}{|c|c|c|c|c|c|c|c|c|c|}
\hline \multicolumn{2}{|c|}{$\begin{array}{c}\text { Sinusiaetone A (1) } \\
\text { Conf. } 7\end{array}$} & \multicolumn{3}{|c|}{$\begin{array}{c}\text { Standard Orientation } \\
\text { (Ångstroms) }\end{array}$} & \multicolumn{2}{|c|}{$\begin{array}{c}\text { Sinusiaetone A (1) } \\
\text { Conf. } 8\end{array}$} & \multicolumn{3}{|c|}{$\begin{array}{c}\text { Standard Orientation } \\
\text { (Ångstroms) }\end{array}$} \\
\hline I & atom & $\mathrm{X}$ & $\mathrm{Y}$ & $\mathrm{Z}$ & I & atom & $\mathrm{X}$ & $\mathrm{Y}$ & $\mathrm{Z}$ \\
\hline 1 & $\mathrm{C}$ & 1.804272 & 2.946758 & 0.10812 & 1 & $\mathrm{C}$ & 0.959785 & 2.830916 & -1.25689 \\
\hline 2 & $\mathrm{C}$ & 2.753386 & 1.763001 & 0.225339 & 2 & $\mathrm{C}$ & 2.00942 & 2.135563 & -0.40067 \\
\hline 3 & $\mathrm{C}$ & 0.539386 & 2.745105 & -0.72831 & 3 & $\mathrm{C}$ & -0.42669 & 2.132897 & -1.24036 \\
\hline 4 & $\mathrm{C}$ & 2.748868 & 0.740471 & -0.63852 & 4 & $\mathrm{C}$ & 2.631865 & 1.046999 & -0.8695 \\
\hline 5 & $\mathrm{C}$ & 3.63218 & -0.48242 & -0.64605 & 5 & $\mathrm{C}$ & 3.663416 & 0.186405 & -0.19304 \\
\hline 6 & $\mathrm{C}$ & 2.881838 & -1.77118 & -1.04106 & 6 & $\mathrm{C}$ & 3.392083 & -1.3232 & -0.34432 \\
\hline 7 & $\mathrm{C}$ & 1.74138 & -2.17304 & -0.11812 & 7 & $\mathrm{C}$ & 2.155161 & -1.87456 & 0.354323 \\
\hline 8 & $\mathrm{C}$ & 0.361098 & -1.7973 & -0.47748 & 8 & $\mathrm{C}$ & 0.942148 & -2.17719 & -0.44628 \\
\hline 9 & $\mathrm{C}$ & -0.77789 & -1.63339 & 0.511521 & 9 & $\mathrm{C}$ & -0.47806 & -2.37887 & 0.050878 \\
\hline 10 & $\mathrm{C}$ & -0.46815 & 1.798819 & -0.08323 & 10 & $\mathrm{C}$ & -1.00664 & 2.059222 & 0.163328 \\
\hline 11 & $\mathrm{C}$ & -1.45294 & 1.117075 & -1.03407 & 11 & $\mathrm{C}$ & -1.00857 & 0.704703 & 0.863523 \\
\hline 12 & $\mathrm{C}$ & -2.44634 & 0.182945 & -0.40078 & 12 & $\mathrm{C}$ & -1.997 & -0.25212 & 0.222741 \\
\hline 13 & $\mathrm{C}$ & -2.14404 & -1.2938 & -0.12673 & 13 & $\mathrm{C}$ & -1.56748 & -1.47755 & -0.58252 \\
\hline 14 & $\mathrm{C}$ & -3.32853 & -1.70967 & 0.778159 & 14 & $\mathrm{C}$ & -2.90776 & -2.23885 & -0.76395 \\
\hline 15 & $\mathrm{C}$ & -4.45627 & -0.71248 & 0.460579 & 15 & $\mathrm{C}$ & -4.01577 & -1.18943 & -0.56601 \\
\hline 16 & $\mathrm{C}$ & -3.71369 & 0.490164 & -0.08368 & 16 & $\mathrm{C}$ & -3.33258 & -0.10741 & 0.241145 \\
\hline 17 & $\mathrm{C}$ & -4.42779 & 1.796767 & -0.24947 & 17 & $\mathrm{C}$ & -4.13974 & 0.964437 & 0.907658 \\
\hline 18 & $\mathrm{O}$ & -0.50809 & 1.642968 & 1.119958 & 18 & $\mathrm{O}$ & -1.44249 & 3.058463 & 0.705929 \\
\hline 19 & $\mathrm{C}$ & 3.704593 & 1.884286 & 1.390757 & 19 & $\mathrm{C}$ & 2.26091 & 2.757525 & 0.951144 \\
\hline
\end{tabular}




\begin{tabular}{|c|c|c|c|c|c|c|c|c|c|}
\hline 20 & $\mathrm{C}$ & 2.120428 & -2.46457 & 1.317309 & 20 & $\mathrm{C}$ & 2.095264 & -1.67427 & 1.851705 \\
\hline 21 & $\mathrm{O}$ & 0.843008 & -3.14172 & -0.72293 & 21 & $\mathrm{O}$ & 1.865302 & -3.22628 & -0.08307 \\
\hline 22 & $\mathrm{H}$ & -2.2089 & -1.83919 & -1.08159 & 22 & $\mathrm{H}$ & -1.20293 & -1.15103 & -1.56808 \\
\hline 23 & $\mathrm{H}$ & 0.254228 & -1.23491 & -1.40323 & 23 & $\mathrm{H}$ & 0.997837 & -1.91785 & -1.50468 \\
\hline 24 & $\mathrm{H}$ & 2.360817 & 3.807072 & -0.2847 & 24 & $\mathrm{H}$ & 1.291646 & 2.872064 & -2.29765 \\
\hline 25 & $\mathrm{H}$ & 1.495167 & 3.23261 & 1.118462 & 25 & $\mathrm{H}$ & 0.8288 & 3.863758 & -0.92199 \\
\hline 26 & $\mathrm{H}$ & 0.020439 & 3.707346 & -0.8314 & 26 & $\mathrm{H}$ & -0.33469 & 1.135277 & -1.67285 \\
\hline 27 & $\mathrm{H}$ & 0.757324 & 2.425372 & -1.75005 & 27 & $\mathrm{H}$ & -1.11877 & 2.713889 & -1.85688 \\
\hline 28 & $\mathrm{H}$ & 2.041587 & 0.773196 & -1.46387 & 28 & $\mathrm{H}$ & 2.377047 & 0.726532 & -1.88055 \\
\hline 29 & $\mathrm{H}$ & 4.125169 & -0.62157 & 0.318231 & 29 & $\mathrm{H}$ & 3.769863 & 0.444351 & 0.862605 \\
\hline 30 & $\mathrm{H}$ & 4.4388 & -0.34411 & -1.37827 & 30 & $\mathrm{H}$ & 4.643392 & 0.379766 & -0.64914 \\
\hline 31 & $\mathrm{H}$ & 3.598098 & -2.60062 & -1.07688 & 31 & $\mathrm{H}$ & 4.253584 & -1.8744 & 0.051367 \\
\hline 32 & $\mathrm{H}$ & 2.477446 & -1.66254 & -2.05246 & 32 & $\mathrm{H}$ & 3.323297 & -1.5762 & -1.4071 \\
\hline 33 & $\mathrm{H}$ & -0.87728 & -2.57776 & 1.053439 & 33 & $\mathrm{H}$ & -0.72026 & -3.41606 & -0.20902 \\
\hline 34 & $\mathrm{H}$ & -0.5284 & -0.86513 & 1.249119 & 34 & $\mathrm{H}$ & -0.53327 & -2.32578 & 1.141449 \\
\hline 35 & $\mathrm{H}$ & -0.86316 & 0.607296 & -1.80484 & 35 & $\mathrm{H}$ & -1.24371 & 0.873239 & 1.916907 \\
\hline 36 & $\mathrm{H}$ & -1.95956 & 1.926768 & -1.57319 & 36 & $\mathrm{H}$ & 0.001062 & 0.289884 & 0.802696 \\
\hline 37 & $\mathrm{H}$ & -3.61461 & -2.75432 & 0.635073 & 37 & $\mathrm{H}$ & -2.9632 & -2.75683 & -1.72404 \\
\hline 38 & $\mathrm{H}$ & -3.02714 & -1.59161 & 1.824427 & 38 & $\mathrm{H}$ & -2.98829 & -2.99707 & 0.021517 \\
\hline 39 & $\mathrm{H}$ & -5.0687 & -0.4681 & 1.335082 & 39 & $\mathrm{H}$ & -4.90062 & -1.59381 & -0.06312 \\
\hline 40 & $\mathrm{H}$ & -5.14613 & -1.10265 & -0.30115 & 40 & $\mathrm{H}$ & -4.36492 & -0.776 & -1.5224 \\
\hline 41 & $\mathrm{H}$ & -4.76572 & 2.176263 & 0.721795 & 41 & $\mathrm{H}$ & -4.87144 & 0.519544 & 1.591843 \\
\hline 42 & $\mathrm{H}$ & -3.80874 & 2.568232 & -0.71028 & 42 & $\mathrm{H}$ & -3.52561 & 1.6712 & 1.465359 \\
\hline 43 & $\mathrm{H}$ & -5.32727 & 1.670689 & -0.86347 & 43 & $\mathrm{H}$ & -4.71492 & 1.533015 & 0.166815 \\
\hline 44 & $\mathrm{H}$ & 4.448646 & 1.089499 & 1.426101 & 44 & $\mathrm{H}$ & 2.932946 & 2.170693 & 1.576765 \\
\hline 45 & $\mathrm{H}$ & 4.23874 & 2.840481 & 1.345777 & 45 & $\mathrm{H}$ & 2.703631 & 3.753036 & 0.82727 \\
\hline 46 & $\mathrm{H}$ & 3.153586 & 1.881157 & 2.338267 & 46 & $\mathrm{H}$ & 1.325838 & 2.906274 & 1.500481 \\
\hline 47 & $\mathrm{H}$ & 2.915703 & -3.21565 & 1.350214 & 47 & $\mathrm{H}$ & 3.019125 & -2.0403 & 2.310089 \\
\hline 48 & $\mathrm{H}$ & 1.270286 & -2.84076 & 1.885221 & 48 & $\mathrm{H}$ & 1.261347 & -2.21888 & 2.293529 \\
\hline 50 & $\mathrm{H}$ & 2.489447 & -1.56239 & 1.812268 & 50 & $\mathrm{H}$ & 1.992115 & -0.61714 & 2.108497 \\
\hline
\end{tabular}

B3LYP/6-311G(d,p) Energy / Hartree =-891.36146774 a.u. B3LYP/6-311G(d,p) Energy $/$ Hartree =-891.35870641 a.u. Population $=3.208 \%$

\begin{tabular}{|c|c|c|c|c|c|c|c|c|c|}
\hline \multicolumn{2}{|c|}{$\begin{array}{c}\text { Sinusiaetone A (1) } \\
\text { Conf. 9 }\end{array}$} & \multicolumn{3}{c|}{$\begin{array}{c}\text { Standard Orientation } \\
\text { (̊ngstroms) }\end{array}$} & $\begin{array}{c}\text { Sinusiaetone A (1) } \\
\text { Conf. 10 }\end{array}$ & \multicolumn{3}{c|}{$\begin{array}{c}\text { Standard Orientation } \\
\text { (Ångstroms) }\end{array}$} \\
\hline I & atom & $\mathrm{X}$ & $\mathrm{Y}$ & $\mathrm{Z}$ & $\mathrm{I}$ & atom & $\mathrm{X}$ & $\mathrm{Y}$ & $\mathrm{Z}$ \\
\hline 1 & $\mathrm{C}$ & 1.280433 & 2.821785 & -1.2278 & 1 & $\mathrm{C}$ & -2.03147 & -2.91449 & -0.10757 \\
\hline 2 & $\mathrm{C}$ & 2.163601 & 2.01425 & -0.28684 & 2 & $\mathrm{C}$ & -2.95417 & -1.70863 & -0.12987 \\
\hline 3 & $\mathrm{C}$ & -0.17353 & 2.287433 & -1.37652 & 3 & $\mathrm{C}$ & -0.71202 & -2.76504 & 0.650086 \\
\hline 4 & $\mathrm{C}$ & 2.737818 & 0.881911 & -0.71301 & 4 & $\mathrm{C}$ & -2.68585 & -0.57996 & 0.539052 \\
\hline 5 & $\mathrm{C}$ & 3.619225 & -0.06948 & 0.050871 & 5 & $\mathrm{C}$ & -3.48633 & 0.694557 & 0.575079 \\
\hline 6 & $\mathrm{C}$ & 3.258944 & -1.55387 & -0.15909 & 6 & $\mathrm{C}$ & -2.86986 & 1.834429 & -0.26704 \\
\hline 7 & $\mathrm{C}$ & 1.917769 & -2.0243 & 0.391231 & 7 & $\mathrm{C}$ & -1.50687 & 2.351618 & 0.173137 \\
\hline 8 & $\mathrm{C}$ & 0.786776 & -2.23026 & -0.54653 & 8 & $\mathrm{C}$ & -0.28282 & 1.895546 & -0.52585 \\
\hline 9 & $\mathrm{C}$ & -0.69383 & -2.31244 & -0.22835 & 9 & $\mathrm{C}$ & 1.136738 & 1.960232 & 0.029301 \\
\hline 10 & $\mathrm{C}$ & -0.902 & 2.212655 & -0.04589 & 10 & $\mathrm{C}$ & 0.301384 & -1.8535 & -0.03351 \\
\hline 11 & $\mathrm{C}$ & -0.89069 & 0.87813 & 0.696955 & 11 & $\mathrm{C}$ & 1.251436 & -1.10664 & 0.897933 \\
\hline
\end{tabular}




\begin{tabular}{|c|c|c|c|c|c|c|c|c|c|}
\hline 12 & $\mathrm{C}$ & -1.92395 & -0.0749 & 0.130378 & 12 & $\mathrm{C}$ & 2.364177 & -0.35126 & 0.234814 \\
\hline 13 & $\mathrm{C}$ & -1.56272 & -1.18214 & -0.85577 & 13 & $\mathrm{C}$ & 2.12781 & 0.931278 & -0.56451 \\
\hline 14 & $\mathrm{C}$ & -2.96116 & -1.66329 & -1.33603 & 14 & $\mathrm{C}$ & 3.561829 & 1.497267 & -0.70301 \\
\hline 15 & $\mathrm{C}$ & -3.95639 & -1.21687 & -0.24558 & 15 & $\mathrm{C}$ & 4.489264 & 0.276115 & -0.58003 \\
\hline 16 & $\mathrm{C}$ & -3.22383 & -0.09995 & 0.465553 & 16 & $\mathrm{C}$ & 3.657814 & -0.71023 & 0.214685 \\
\hline 17 & $\mathrm{C}$ & -3.9407 & 0.778671 & 1.443992 & 17 & $\mathrm{C}$ & 4.304582 & -1.9252 & 0.806698 \\
\hline 18 & $\mathrm{O}$ & -1.46632 & 3.187372 & 0.416081 & 18 & $\mathrm{O}$ & 0.359313 & -1.75723 & -1.24277 \\
\hline 19 & $\mathrm{C}$ & 2.321567 & 2.59122 & 1.098796 & 19 & $\mathrm{C}$ & -4.18022 & -1.92306 & -0.98348 \\
\hline 20 & $\mathrm{C}$ & 1.707345 & -1.83795 & 1.876774 & 20 & $\mathrm{C}$ & -1.43121 & 2.924581 & 1.571299 \\
\hline 21 & $\mathrm{O}$ & 1.58225 & -3.34815 & -0.10034 & 21 & $\mathrm{O}$ & -0.88938 & 3.168828 & -0.8512 \\
\hline 22 & $\mathrm{H}$ & -1.00616 & -0.7827 & -1.71147 & 22 & $\mathrm{H}$ & 1.758081 & 0.644885 & -1.55848 \\
\hline 23 & $\mathrm{H}$ & 0.985688 & -1.96372 & -1.586 & 23 & $\mathrm{H}$ & -0.43092 & 1.151976 & -1.30815 \\
\hline 24 & $\mathrm{H}$ & 1.718029 & 2.837506 & -2.22936 & 24 & $\mathrm{H}$ & -1.80325 & -3.18826 & -1.1439 \\
\hline 25 & $\mathrm{H}$ & 1.229842 & 3.860001 & -0.88686 & 25 & $\mathrm{H}$ & -2.58369 & -3.76758 & 0.305818 \\
\hline 26 & $\mathrm{H}$ & -0.14089 & 1.302611 & -1.84653 & 26 & $\mathrm{H}$ & -0.22379 & -3.74638 & 0.713153 \\
\hline 27 & $\mathrm{H}$ & -0.72865 & 2.967636 & -2.02738 & 27 & $\mathrm{H}$ & -0.86891 & -2.44729 & 1.684111 \\
\hline 28 & $\mathrm{H}$ & 2.567379 & 0.598149 & -1.75231 & 28 & $\mathrm{H}$ & -1.77089 & -0.54153 & 1.122927 \\
\hline 29 & $\mathrm{H}$ & 3.630393 & 0.164912 & 1.117192 & 29 & $\mathrm{H}$ & -3.58313 & 1.028578 & 1.614572 \\
\hline 30 & $\mathrm{H}$ & 4.654635 & 0.055116 & -0.29296 & 30 & $\mathrm{H}$ & -4.50428 & 0.530523 & 0.216126 \\
\hline 31 & $\mathrm{H}$ & 4.03269 & -2.16894 & 0.315853 & 31 & $\mathrm{H}$ & -3.55714 & 2.689627 & -0.25162 \\
\hline 32 & $\mathrm{H}$ & 3.289087 & -1.78872 & -1.22777 & 32 & $\mathrm{H}$ & -2.79135 & 1.510453 & -1.3088 \\
\hline 33 & $\mathrm{H}$ & -1.02489 & -3.2693 & -0.64773 & 33 & $\mathrm{H}$ & 1.506401 & 2.962692 & -0.20696 \\
\hline 34 & $\mathrm{H}$ & -0.8639 & -2.37831 & 0.848759 & 34 & $\mathrm{H}$ & 1.140427 & 1.891081 & 1.122042 \\
\hline 35 & $\mathrm{H}$ & -1.08681 & 1.085304 & 1.750446 & 35 & $\mathrm{H}$ & 1.634587 & -1.83444 & 1.620783 \\
\hline 36 & $\mathrm{H}$ & 0.103773 & 0.436064 & 0.608339 & 36 & $\mathrm{H}$ & 0.623208 & -0.43227 & 1.496809 \\
\hline 37 & $\mathrm{H}$ & -3.20764 & -1.15946 & -2.27435 & 37 & $\mathrm{H}$ & 3.699017 & 2.060608 & -1.62912 \\
\hline 38 & $\mathrm{H}$ & -2.98898 & -2.73781 & -1.52813 & 38 & $\mathrm{H}$ & 3.754173 & 2.181088 & 0.130693 \\
\hline 39 & $\mathrm{H}$ & -4.19413 & -2.02382 & 0.46096 & 39 & $\mathrm{H}$ & 5.442914 & 0.508864 & -0.09425 \\
\hline 40 & $\mathrm{H}$ & -4.9124 & -0.88393 & -0.66358 & 40 & $\mathrm{H}$ & 4.736446 & -0.14805 & -1.56348 \\
\hline 41 & $\mathrm{H}$ & -4.38232 & 0.18027 & 2.249872 & 41 & $\mathrm{H}$ & 5.10557 & -1.64031 & 1.498554 \\
\hline 42 & $\mathrm{H}$ & -3.28886 & 1.532617 & 1.885709 & 42 & $\mathrm{H}$ & 3.603907 & -2.56537 & 1.344951 \\
\hline 43 & $\mathrm{H}$ & -4.77072 & 1.301611 & 0.955035 & 43 & $\mathrm{H}$ & 4.773688 & -2.5305 & 0.02192 \\
\hline 44 & $\mathrm{H}$ & 2.866341 & 1.937725 & 1.779415 & 44 & $\mathrm{H}$ & -4.8638 & -1.07501 & -0.97957 \\
\hline 45 & $\mathrm{H}$ & 2.861135 & 3.544263 & 1.04685 & 45 & $\mathrm{H}$ & -3.89203 & -2.11712 & -2.02292 \\
\hline 46 & $\mathrm{H}$ & 1.349398 & 2.816659 & 1.549614 & 46 & $\mathrm{H}$ & -4.73394 & -2.80633 & -0.64487 \\
\hline 47 & $\mathrm{H}$ & 2.537009 & -2.29393 & 2.425844 & 47 & $\mathrm{H}$ & -2.23594 & 3.65016 & 1.72382 \\
\hline 48 & $\mathrm{H}$ & 0.782942 & -2.30789 & 2.21081 & 48 & $\mathrm{H}$ & -0.48057 & 3.430573 & 1.738309 \\
\hline 50 & $\mathrm{H}$ & 1.672467 & -0.7793 & 2.145008 & 50 & $\mathrm{H}$ & -1.53959 & 2.140791 & 2.326046 \\
\hline
\end{tabular}

B3LYP/6-311G(d,p) Energy / Hartree =-891.35729008 a.u. B3LYP/6-311G(d,p) Energy $/$ Hartree =-891.36007325 a.u. Population $=2.762 \%$ Population $=2.483 \%$

\begin{tabular}{|c|c|c|c|c|c|c|c|c|c|}
\hline $\begin{array}{c}\text { Sinusiaetone A (1) } \\
\text { Conf. 11 }\end{array}$ & \multicolumn{3}{c|}{$\begin{array}{c}\text { Standard Orientation } \\
\text { (̊ngstroms) }\end{array}$} & $\begin{array}{c}\text { Sinusiaetone A (1) } \\
\text { Conf. 12 }\end{array}$ & \multicolumn{3}{c|}{$\begin{array}{c}\text { Standard Orientation } \\
\text { (Angstroms) }\end{array}$} \\
\hline I & atom & $\mathrm{X}$ & $\mathrm{Y}$ & $\mathrm{Z}$ & $\mathrm{I}$ & atom & $\mathrm{X}$ & $\mathrm{Y}$ & $\mathrm{Z}$ \\
\hline 1 & $\mathrm{C}$ & -1.47991 & -2.83157 & -0.9427 & 1 & $\mathrm{C}$ & 1.908805 & 2.892932 & -0.64193 \\
\hline 2 & $\mathrm{C}$ & -2.38908 & -1.88599 & -0.18129 & 2 & $\mathrm{C}$ & 2.754387 & 1.75665 & -0.09851 \\
\hline 3 & $\mathrm{C}$ & -0.15039 & -3.1936 & -0.22651 & 3 & $\mathrm{C}$ & 0.531237 & 2.990962 & 0.046227 \\
\hline
\end{tabular}




\begin{tabular}{|c|c|c|c|c|c|c|c|c|c|}
\hline 4 & $\mathrm{C}$ & -2.80433 & -0.75481 & -0.76652 & 4 & $\mathrm{C}$ & 2.8587 & 0.607291 & -0.77928 \\
\hline 5 & $\mathrm{C}$ & -3.72181 & 0.311004 & -0.22728 & 5 & $\mathrm{C}$ & 3.638407 & -0.62613 & -0.41386 \\
\hline 6 & $\mathrm{C}$ & -3.22349 & 1.741032 & -0.52497 & 6 & $\mathrm{C}$ & 2.876744 & -1.94375 & -0.64939 \\
\hline 7 & $\mathrm{C}$ & -1.93479 & 2.140127 & 0.177904 & 7 & $\mathrm{C}$ & 1.642536 & -2.19993 & 0.208841 \\
\hline 8 & $\mathrm{C}$ & -0.64434 & 1.851016 & -0.47251 & 8 & $\mathrm{C}$ & 0.296707 & -2.19629 & -0.41854 \\
\hline 9 & $\mathrm{C}$ & 0.674128 & 1.666918 & 0.258826 & 9 & $\mathrm{C}$ & -1.04915 & -2.07079 & 0.279788 \\
\hline 10 & $\mathrm{C}$ & 0.675839 & -1.98137 & 0.176518 & 10 & $\mathrm{C}$ & -0.41686 & 1.879231 & $\begin{array}{l}-0.39091 \\
\end{array}$ \\
\hline 11 & $\mathrm{C}$ & 1.489567 & -1.30511 & -0.93082 & 11 & $\mathrm{C}$ & -1.13646 & 1.12318 & 0.720959 \\
\hline 12 & $\mathrm{C}$ & 2.338823 & -0.13456 & -0.51437 & 12 & $\mathrm{C}$ & -2.27477 & 0.254969 & 0.273292 \\
\hline 13 & $\mathrm{C}$ & 1.871434 & 1.30889 & -0.67726 & 13 & $\mathrm{C}$ & -2.06871 & -1.10966 & -0.3814 \\
\hline 14 & $\mathrm{C}$ & 3.17093 & 2.112737 & -0.39456 & 14 & $\mathrm{C}$ & -3.505 & -1.69266 & -0.38102 \\
\hline 15 & $\mathrm{C}$ & 4.08401 & 1.162468 & 0.408006 & 15 & $\mathrm{C}$ & -4.44071 & -0.47356 & -0.32223 \\
\hline 16 & $\mathrm{C}$ & 3.53635 & -0.21066 & 0.083577 & 16 & $\mathrm{C}$ & -3.5723 & 0.599159 & 0.300845 \\
\hline 17 & $\mathrm{C}$ & 4.291477 & -1.44162 & 0.481387 & 17 & $\mathrm{C}$ & -4.19079 & 1.875659 & 0.783865 \\
\hline 18 & $\mathrm{O}$ & 0.686979 & -1.59094 & 1.327619 & 18 & $\mathrm{O}$ & -0.58154 & 1.624875 & -1.56731 \\
\hline 19 & $\mathrm{C}$ & -2.7893 & -2.3334 & 1.203158 & 19 & $\mathrm{C}$ & 3.434531 & 2.042746 & 1.218403 \\
\hline 20 & $\mathrm{C}$ & -2.02751 & 2.318436 & 1.677367 & 20 & $\mathrm{C}$ & 1.817292 & -2.01628 & 1.699262 \\
\hline 21 & $\mathrm{O}$ & -1.20577 & 3.186594 & -0.51896 & 21 & $\mathrm{O}$ & 0.995104 & -3.43754 & -0.17638 \\
\hline 22 & $\mathrm{H}$ & 1.5488 & 1.489454 & -1.70997 & 22 & $\mathrm{H}$ & -1.74869 & -0.93782 & -1.41833 \\
\hline 23 & $\mathrm{H}$ & -0.70215 & 1.346185 & -1.43619 & 23 & $\mathrm{H}$ & 0.268807 & -1.93251 & -1.47652 \\
\hline 24 & $\mathrm{H}$ & -1.2628 & -2.41315 & -1.92876 & 24 & $\mathrm{H}$ & 1.744563 & 2.76618 & $\begin{array}{l}-1.71404 \\
\end{array}$ \\
\hline 25 & $\mathrm{H}$ & -2.00515 & -3.77936 & -1.11506 & 25 & $\mathrm{H}$ & 2.434951 & 3.843296 & -0.50231 \\
\hline 26 & $\mathrm{H}$ & 0.434642 & -3.8286 & -0.89837 & 26 & $\mathrm{H}$ & 0.63052 & 3.003741 & 1.133894 \\
\hline 27 & $\mathrm{H}$ & -0.35488 & -3.76215 & 0.682349 & 27 & $\mathrm{H}$ & 0.045029 & 3.932544 & -0.23678 \\
\hline 28 & $\mathrm{H}$ & -2.4557 & -0.56771 & -1.78209 & 28 & $\mathrm{H}$ & 2.335828 & 0.54509 & -1.73308 \\
\hline 29 & $\mathrm{H}$ & -3.89312 & 0.187525 & 0.844104 & 29 & $\mathrm{H}$ & 3.993434 & -0.58226 & 0.61753 \\
\hline 30 & $\mathrm{H}$ & -4.7057 & 0.207461 & -0.70398 & 30 & $\mathrm{H}$ & 4.541528 & -0.66958 & -1.03743 \\
\hline 31 & $\mathrm{H}$ & -4.00513 & 2.456786 & -0.24475 & 31 & $\mathrm{H}$ & 3.564894 & -2.7761 & -0.45809 \\
\hline 32 & $\mathrm{H}$ & -3.06733 & 1.852232 & -1.6026 & 32 & $\mathrm{H}$ & 2.586848 & -2.01811 & -1.70237 \\
\hline 33 & $\mathrm{H}$ & 0.89055 & 2.598341 & 0.787239 & 33 & $\mathrm{H}$ & -1.47768 & -3.07864 & 0.253516 \\
\hline 34 & $\mathrm{H}$ & 0.564237 & 0.889105 & 1.019113 & 34 & $\mathrm{H}$ & -0.93103 & -1.82962 & 1.340051 \\
\hline 35 & $\mathrm{H}$ & 0.787535 & -1.02139 & -1.72407 & 35 & $\mathrm{H}$ & -1.45829 & 1.856595 & 1.466771 \\
\hline 36 & $\mathrm{H}$ & 2.105727 & -2.09456 & -1.37799 & 36 & $\mathrm{H}$ & -0.35512 & 0.537832 & 1.227079 \\
\hline 37 & $\mathrm{H}$ & 3.654333 & 2.356579 & -1.34452 & 37 & $\mathrm{H}$ & -3.6893 & -2.34595 & -1.23713 \\
\hline 38 & $\mathrm{H}$ & 2.975163 & 3.055681 & 0.120369 & 38 & $\mathrm{H}$ & -3.63954 & -2.29221 & 0.52541 \\
\hline 39 & $\mathrm{H}$ & 4.02792 & 1.345498 & 1.489868 & 39 & $\mathrm{H}$ & -5.35535 & -0.6625 & 0.250085 \\
\hline 40 & $\mathrm{H}$ & 5.139859 & 1.265115 & 0.134306 & 40 & $\mathrm{H}$ & -4.76147 & -0.15704 & -1.32479 \\
\hline 41 & $\mathrm{H}$ & 4.39132 & -1.49653 & 1.57204 & 41 & $\mathrm{H}$ & -4.96013 & 1.673251 & 1.53775 \\
\hline 42 & $\mathrm{H}$ & 3.807721 & -2.36101 & 0.146929 & 42 & $\mathrm{H}$ & -3.46489 & 2.565912 & 1.216245 \\
\hline 43 & $\mathrm{H}$ & 5.309345 & -1.42418 & 0.075658 & 43 & $\mathrm{H}$ & -4.69348 & 2.395171 & -0.04079 \\
\hline 44 & $\mathrm{H}$ & -3.54462 & -1.68958 & 1.652475 & 44 & $\mathrm{H}$ & 3.93754 & 1.174268 & 1.642611 \\
\hline 45 & $\mathrm{H}$ & -3.19221 & -3.35233 & 1.17196 & 45 & $\mathrm{H}$ & 4.184055 & 2.832774 & 1.090437 \\
\hline 46 & $\mathrm{H}$ & -1.92305 & -2.35581 & 1.871461 & 46 & $\mathrm{H}$ & 2.720092 & 2.412046 & 1.961441 \\
\hline 47 & $\mathrm{H}$ & -2.79476 & 3.060273 & 1.919457 & 47 & $\mathrm{H}$ & 2.701493 & -2.56217 & 2.042621 \\
\hline 48 & $\mathrm{H}$ & -1.08097 & 2.652424 & 2.10115 & 48 & $\mathrm{H}$ & 0.954519 & -2.39367 & 2.247643 \\
\hline 50 & $\mathrm{H}$ & -2.30488 & 1.378892 & 2.162856 & 50 & $\mathrm{H}$ & 1.954765 & -0.96166 & 1.951603 \\
\hline
\end{tabular}


B3LYP/6-311G(d,p) Energy / Hartree =-891.36049857 a.u. Population $=1.936 \%$
B3LYP/6-311G(d,p) Energy / Hartree =-891.35852576 a.u. Population $=1.746 \%$

\begin{tabular}{|c|c|c|c|c|c|c|c|c|c|}
\hline \multicolumn{2}{|c|}{$\begin{array}{c}\text { Sinusiaetone A (1) } \\
\text { Conf. } 13\end{array}$} & \multicolumn{3}{|c|}{$\begin{array}{c}\text { Standard Orientation } \\
\text { (Ångstroms) }\end{array}$} & \multicolumn{2}{|c|}{$\begin{array}{c}\text { Sinusiaetone A (1) } \\
\text { Conf. } 14\end{array}$} & \multicolumn{3}{|c|}{$\begin{array}{c}\text { Standard Orientation } \\
\text { (Ångstroms) }\end{array}$} \\
\hline I & atom & $\mathrm{X}$ & Y & Z & I & atom & $X$ & Y & Z \\
\hline 1 & $\mathrm{C}$ & 1.307742 & -3.00862 & -0.06158 & 1 & $\mathrm{C}$ & 1.82271 & 2.835949 & 0.68974 \\
\hline 2 & $\mathrm{C}$ & 2.117066 & -1.86237 & -0.64379 & 2 & $\mathrm{C}$ & 2.760082 & 1.778861 & 0.134021 \\
\hline 3 & $\mathrm{C}$ & -0.18056 & -2.76602 & 0.184893 & 3 & $\mathrm{C}$ & 0.477035 & 2.919764 & -0.05965 \\
\hline 4 & $\mathrm{C}$ & 1.565779 & -0.72073 & -1.07604 & 4 & $\mathrm{C}$ & 2.881496 & 0.587572 & 0.735678 \\
\hline 5 & $\mathrm{C}$ & 2.290027 & 0.503659 & -1.5855 & 5 & $\mathrm{C}$ & 3.741272 & -0.57635 & 0.303362 \\
\hline 6 & $\mathrm{C}$ & 2.628745 & 1.521681 & -0.46774 & 6 & $\mathrm{C}$ & 3.040584 & -1.56954 & -0.6549 \\
\hline 7 & $\mathrm{C}$ & 1.438974 & 2.265021 & 0.132359 & 7 & $\mathrm{C}$ & 1.761554 & -2.2001 & -0.12586 \\
\hline 8 & $\mathrm{C}$ & 0.557795 & 1.535249 & 1.070441 & 8 & $\mathrm{C}$ & 0.479602 & -1.53859 & -0.43187 \\
\hline 9 & $\mathrm{C}$ & -0.87499 & 1.861095 & 1.439467 & 9 & $\mathrm{C}$ & -0.78617 & -1.66046 & 0.385345 \\
\hline 10 & $\mathrm{C}$ & -0.49331 & -1.86501 & 1.371809 & 10 & $\mathrm{C}$ & -0.50246 & 1.818057 & 0.333315 \\
\hline 11 & $\mathrm{C}$ & -1.89558 & -1.24889 & 1.43568 & 11 & $\mathrm{C}$ & -1.42762 & 1.317497 & -0.77368 \\
\hline 12 & $\mathrm{C}$ & -2.18223 & -0.22237 & 0.361748 & 12 & $\mathrm{C}$ & -2.42204 & 0.255492 & -0.3938 \\
\hline 13 & $\mathrm{C}$ & -2.03113 & 1.288793 & 0.589681 & 13 & $\mathrm{C}$ & -2.07668 & -1.23712 & -0.35474 \\
\hline 14 & $\mathrm{C}$ & -2.1313 & 1.845343 & -0.8467 & 14 & $\mathrm{C}$ & -3.33426 & -1.85229 & 0.307458 \\
\hline 15 & $\mathrm{C}$ & -2.9933 & 0.818387 & -1.59848 & 15 & $\mathrm{C}$ & -4.46617 & -0.83698 & 0.074872 \\
\hline 16 & $\mathrm{C}$ & -2.76275 & -0.46396 & -0.82725 & 16 & $\mathrm{C}$ & -3.72807 & 0.463437 & -0.16188 \\
\hline 17 & $\mathrm{C}$ & -3.26715 & -1.76056 & -1.3828 & 17 & $\mathrm{C}$ & -4.48636 & 1.755821 & -0.17243 \\
\hline 18 & $\mathrm{O}$ & 0.311196 & -1.67883 & 2.263639 & 18 & $\mathrm{O}$ & -0.56106 & 1.40511 & 1.473742 \\
\hline 19 & $\mathrm{C}$ & 3.600635 & -2.14089 & -0.65786 & 19 & $\mathrm{C}$ & 3.506752 & 2.176514 & -1.11592 \\
\hline 20 & $\mathrm{C}$ & 0.995812 & 3.491202 & -0.63421 & 20 & $\mathrm{C}$ & 1.923291 & -3.12555 & 1.059594 \\
\hline 21 & $\mathrm{O}$ & 1.550435 & 2.469861 & 1.56483 & 21 & $\mathrm{O}$ & 0.901414 & -2.70271 & -1.18536 \\
\hline 22 & $\mathrm{H}$ & -2.93792 & 1.596937 & 1.134952 & 22 & $\mathrm{H}$ & -1.9957 & -1.59773 & -1.39224 \\
\hline 23 & $\mathrm{H}$ & 0.844333 & 0.506479 & 1.263683 & 23 & $\mathrm{H}$ & 0.568835 & -0.62373 & -1.01295 \\
\hline 24 & $\mathrm{H}$ & 1.771727 & -3.29845 & 0.88795 & 24 & $\mathrm{H}$ & 2.309501 & 3.81547 & 0.63528 \\
\hline 25 & $\mathrm{H}$ & 1.41843 & -3.88067 & -0.71855 & 25 & $\mathrm{H}$ & 1.609561 & 2.637026 & 1.74184 \\
\hline 26 & $\mathrm{H}$ & -0.66746 & -3.7236 & 0.415868 & 26 & $\mathrm{H}$ & -0.02632 & 3.862818 & 0.191589 \\
\hline 27 & $\mathrm{H}$ & -0.68635 & -2.39518 & -0.70727 & 27 & $\mathrm{H}$ & 0.616195 & 2.93228 & -1.14354 \\
\hline 28 & $\mathrm{H}$ & 0.486561 & -0.61729 & -1.01467 & 28 & $\mathrm{H}$ & 2.282707 & 0.421065 & 1.628522 \\
\hline 29 & $\mathrm{H}$ & 1.681495 & 0.993644 & -2.35339 & 29 & $\mathrm{H}$ & 4.080152 & -1.11463 & 1.194529 \\
\hline 30 & $\mathrm{H}$ & 3.226053 & 0.22533 & -2.07604 & 30 & $\mathrm{H}$ & 4.647723 & -0.2232 & -0.19548 \\
\hline 31 & $\mathrm{H}$ & 3.333517 & 2.263038 & -0.86203 & 31 & $\mathrm{H}$ & 3.74353 & -2.37391 & -0.90449 \\
\hline 32 & $\mathrm{H}$ & 3.139135 & 0.996866 & 0.344082 & 32 & $\mathrm{H}$ & 2.798932 & -1.05711 & -1.5904 \\
\hline 33 & $\mathrm{H}$ & -1.00723 & 1.532676 & 2.477503 & 33 & $\mathrm{H}$ & -0.89701 & -2.70568 & 0.684632 \\
\hline 34 & $\mathrm{H}$ & -0.99098 & 2.948485 & 1.46034 & 34 & $\mathrm{H}$ & -0.69074 & -1.0663 & 1.298856 \\
\hline 35 & $\mathrm{H}$ & -2.00446 & -0.80934 & 2.429612 & 35 & $\mathrm{H}$ & -0.78731 & 0.98126 & -1.5991 \\
\hline 36 & $\mathrm{H}$ & -2.61185 & -2.07418 & 1.355917 & 36 & $\mathrm{H}$ & -1.93033 & 2.207472 & -1.17068 \\
\hline 37 & $\mathrm{H}$ & -2.53723 & 2.859141 & -0.87557 & 37 & $\mathrm{H}$ & -3.55808 & -2.8507 & -0.07528 \\
\hline 38 & $\mathrm{H}$ & -1.13573 & 1.871885 & -1.29501 & 38 & $\mathrm{H}$ & -3.1507 & -1.94795 & 1.382781 \\
\hline 39 & $\mathrm{H}$ & -2.73075 & 0.728032 & -2.65772 & 39 & $\mathrm{H}$ & -5.16404 & -0.7775 & 0.916992 \\
\hline 40 & $\mathrm{H}$ & -4.06054 & 1.081294 & -1.56395 & 40 & $\mathrm{H}$ & -5.06939 & -1.08806 & -0.80895 \\
\hline 41 & $\mathrm{H}$ & -2.70879 & -2.04994 & -2.28098 & 41 & $\mathrm{H}$ & -4.9421 & 1.941726 & 0.806996 \\
\hline 42 & $\mathrm{H}$ & -3.2076 & -2.58639 & -0.67221 & 42 & $\mathrm{H}$ & -3.86439 & 2.61792 & -0.41801 \\
\hline
\end{tabular}




\begin{tabular}{|l|c|c|c|c|c|c|c|c|c|}
\hline 43 & $\mathrm{H}$ & -4.3135 & -1.65441 & -1.69103 & 43 & $\mathrm{H}$ & -5.30984 & 1.714131 & -0.895 \\
\hline 44 & $\mathrm{H}$ & 3.98423 & -2.22356 & 0.365735 & 44 & $\mathrm{H}$ & 4.143981 & 1.381853 & -1.50301 \\
\hline 45 & $\mathrm{H}$ & 3.806938 & -3.10072 & -1.145 & 45 & $\mathrm{H}$ & 2.813265 & 2.462186 & -1.91447 \\
\hline 46 & $\mathrm{H}$ & 4.178167 & -1.37122 & -1.16879 & 46 & $\mathrm{H}$ & 4.1346 & 3.054219 & -0.92282 \\
\hline 47 & $\mathrm{H}$ & 1.7999 & 4.233584 & -0.61763 & 47 & $\mathrm{H}$ & 2.63326 & -3.9217 & 0.814911 \\
\hline 48 & $\mathrm{H}$ & 0.102605 & 3.946644 & -0.20793 & 48 & $\mathrm{H}$ & 0.978909 & -3.58723 & 1.344701 \\
\hline 50 & $\mathrm{H}$ & 0.792626 & 3.246636 & -1.68044 & 50 & $\mathrm{H}$ & 2.314678 & -2.58513 & 1.925784 \\
\hline
\end{tabular}

B3LYP/6-311G(d,p) Energy / Hartree =-891.35897701 a.u. Population $=1.571 \%$

\begin{tabular}{|c|c|c|c|c|c|c|c|c|c|}
\hline \multicolumn{2}{|c|}{$\begin{array}{c}\text { Sinusiaetone A (1) } \\
\text { Conf. } 15\end{array}$} & \multicolumn{3}{|c|}{$\begin{array}{c}\text { Standard Orientation } \\
\text { (Ångstroms) }\end{array}$} & \multicolumn{2}{|c|}{$\begin{array}{c}\text { Sinusiaetone A (1) } \\
\text { Conf. } 16\end{array}$} & \multicolumn{3}{|c|}{$\begin{array}{c}\text { Standard Orientation } \\
\text { (Ångstroms) }\end{array}$} \\
\hline I & atom & $\mathrm{X}$ & $\mathrm{Y}$ & Z & $\mathrm{I}$ & atom & $X$ & $\mathrm{Y}$ & $\mathrm{Z}$ \\
\hline 1 & $\mathrm{C}$ & 1.292095 & -2.71109 & -0.91753 & 1 & $\mathrm{C}$ & 1.292821 & 2.71127 & 0.916932 \\
\hline 2 & $\mathrm{C}$ & 2.463672 & -1.77974 & -0.61065 & 2 & $\mathrm{C}$ & 2.464249 & 1.779608 & 0.610506 \\
\hline 3 & $\mathrm{C}$ & -0.09333 & -2.14549 & -0.57756 & 3 & $\mathrm{C}$ & -0.09275 & 2.145803 & 0.577377 \\
\hline 4 & $\mathrm{C}$ & 2.464373 & -0.51373 & -1.04491 & 4 & $\mathrm{C}$ & 2.464404 & 0.513534 & 1.044587 \\
\hline 5 & $\mathrm{C}$ & 3.470571 & 0.569622 & -0.74888 & 5 & $\mathrm{C}$ & 3.470386 & -0.57009 & 0.748888 \\
\hline 6 & $\mathrm{C}$ & 3.013535 & 1.515655 & 0.390496 & 6 & $\mathrm{C}$ & 3.013277 & -1.51625 & -0.39038 \\
\hline 7 & $\mathrm{C}$ & 1.69549 & 2.229606 & 0.12977 & 7 & $\mathrm{C}$ & 1.695082 & -2.22991 & -0.1296 \\
\hline 8 & $\mathrm{C}$ & 0.442741 & 1.549862 & 0.511273 & 8 & $\mathrm{C}$ & 0.442516 & -1.54987 & -0.51119 \\
\hline 9 & $\mathrm{C}$ & -0.89195 & 1.783964 & -0.15873 & 9 & $\mathrm{C}$ & -0.89225 & -1.78355 & 0.158809 \\
\hline 10 & $\mathrm{C}$ & -0.29992 & -1.85221 & 0.896402 & 10 & $\mathrm{C}$ & -0.29961 & 1.852031 & -0.89647 \\
\hline 11 & $\mathrm{C}$ & -1.64585 & -1.26708 & 1.348521 & 11 & $\mathrm{C}$ & -1.64601 & 1.26779 & -1.3482 \\
\hline 12 & $\mathrm{C}$ & -2.37204 & -0.25872 & 0.492285 & 12 & $\mathrm{C}$ & -2.37215 & 0.25922 & -0.49215 \\
\hline 13 & $\mathrm{C}$ & -2.13237 & 1.2526 & 0.607425 & 13 & $\mathrm{C}$ & -2.1325 & -1.2521 & -0.60759 \\
\hline 14 & $\mathrm{C}$ & -3.44012 & 1.828197 & 0.001508 & 14 & $\mathrm{C}$ & -3.44041 & -1.82773 & -0.00213 \\
\hline 15 & $\mathrm{C}$ & -3.94675 & 0.737567 & -0.96207 & 15 & $\mathrm{C}$ & -3.94697 & -0.73742 & 0.961853 \\
\hline 16 & $\mathrm{C}$ & -3.35752 & -0.53058 & -0.38036 & 16 & $\mathrm{C}$ & -3.35763 & 0.53088 & 0.380557 \\
\hline 17 & $\mathrm{C}$ & -3.88824 & -1.86558 & -0.80352 & 17 & $\mathrm{C}$ & -3.88837 & 1.865749 & 0.804101 \\
\hline 18 & $\mathrm{O}$ & 0.537821 & -2.13078 & 1.732502 & 18 & $\mathrm{O}$ & 0.538283 & 2.129723 & -1.73272 \\
\hline 19 & $\mathrm{C}$ & 3.566588 & -2.40446 & 0.203873 & 19 & $\mathrm{C}$ & 3.567599 & 2.40408 & -0.20361 \\
\hline 20 & $\mathrm{C}$ & 1.733219 & 3.291256 & -0.94829 & 20 & $\mathrm{C}$ & 1.732578 & -3.29146 & 0.948552 \\
\hline 21 & $\mathrm{O}$ & 0.980607 & 2.611987 & 1.336687 & 21 & $\mathrm{O}$ & 0.980102 & -2.61223 & -1.33648 \\
\hline 22 & $\mathrm{H}$ & -2.02873 & 1.55027 & 1.657786 & 22 & $\mathrm{H}$ & -2.02859 & -1.54957 & -1.65798 \\
\hline 23 & $\mathrm{H}$ & 0.571244 & 0.574877 & 0.974492 & 23 & $\mathrm{H}$ & 0.571305 & -0.57496 & -0.97449 \\
\hline 24 & $\mathrm{H}$ & 1.429619 & -3.64418 & -0.36557 & 24 & $\mathrm{H}$ & 1.430476 & 3.64403 & 0.364437 \\
\hline 25 & $\mathrm{H}$ & 1.300336 & -2.98141 & -1.97944 & 25 & $\mathrm{H}$ & 1.301215 & 2.982216 & 1.978692 \\
\hline 26 & $\mathrm{H}$ & -0.87741 & -2.85252 & -0.87852 & 26 & $\mathrm{H}$ & -0.87661 & 2.85315 & 0.878171 \\
\hline 27 & $\mathrm{H}$ & -0.31459 & -1.23606 & -1.14367 & 27 & $\mathrm{H}$ & -0.31415 & 1.236647 & 1.143869 \\
\hline 28 & $\mathrm{H}$ & 1.610693 & -0.18664 & -1.63571 & 28 & $\mathrm{H}$ & 1.61035 & 0.186603 & 1.634932 \\
\hline 29 & $\mathrm{H}$ & 3.649743 & 1.157713 & -1.65577 & 29 & $\mathrm{H}$ & 3.649323 & -1.15807 & 1.655901 \\
\hline 30 & $\mathrm{H}$ & 4.437302 & 0.147419 & -0.46615 & 30 & $\mathrm{H}$ & 4.437243 & -0.14817 & 0.466165 \\
\hline 31 & $\mathrm{H}$ & 3.791626 & 2.268232 & 0.566606 & 31 & $\mathrm{H}$ & 3.791281 & -2.26897 & -0.56629 \\
\hline 32 & $\mathrm{H}$ & 2.909637 & 0.937966 & 1.313248 & 32 & $\mathrm{H}$ & 2.909547 & -0.9387 & -1.31324 \\
\hline 33 & $\mathrm{H}$ & -1.01417 & 2.861155 & -0.29056 & 33 & $\mathrm{H}$ & -1.01467 & -2.86068 & 0.290922 \\
\hline 34 & $\mathrm{H}$ & -0.87446 & 1.343526 & -1.16326 & 34 & $\mathrm{H}$ & -0.87473 & -1.34285 & 1.163216 \\
\hline
\end{tabular}

B3LYP/6-311G(d,p) Energy / Hartree =-891.35897985 a.u.

Population $=1.222 \%$
Sinusiaetone A (1) Conf. 16 dard Orientation

atom 


\begin{tabular}{|l|l|l|l|l|l|l|l|l|c|}
\hline 35 & $\mathrm{H}$ & -1.47387 & -0.86466 & 2.350562 & 35 & $\mathrm{H}$ & -1.47479 & 0.86584 & -2.35057 \\
\hline 36 & $\mathrm{H}$ & -2.28511 & -2.14957 & 1.482242 & 36 & $\mathrm{H}$ & -2.28502 & 2.150601 & -1.48101 \\
\hline 37 & $\mathrm{H}$ & -4.17425 & 1.968699 & 0.799741 & 37 & $\mathrm{H}$ & -4.17447 & -1.96768 & -0.80054 \\
\hline 38 & $\mathrm{H}$ & -3.28969 & 2.796474 & -0.4814 & 38 & $\mathrm{H}$ & -3.29026 & -2.79627 & 0.480336 \\
\hline 39 & $\mathrm{H}$ & -3.59474 & 0.894134 & -1.9908 & 39 & $\mathrm{H}$ & -3.5949 & -0.89435 & 1.990506 \\
\hline 40 & $\mathrm{H}$ & -5.03963 & 0.694524 & -1.01411 & 40 & $\mathrm{H}$ & -5.03984 & -0.69432 & 1.013937 \\
\hline 41 & $\mathrm{H}$ & -3.69336 & -2.03864 & -1.8687 & 41 & $\mathrm{H}$ & -3.69457 & 2.037923 & 1.86962 \\
\hline 42 & $\mathrm{H}$ & -3.44974 & -2.69668 & -0.24861 & 42 & $\mathrm{H}$ & -3.44903 & 2.697127 & 0.250286 \\
\hline 43 & $\mathrm{H}$ & -4.97569 & -1.9063 & -0.67632 & 43 & $\mathrm{H}$ & -4.97568 & 1.906901 & 0.675787 \\
\hline 44 & $\mathrm{H}$ & 3.168746 & -2.72836 & 1.170611 & 44 & $\mathrm{H}$ & 3.170325 & 2.727454 & -1.17077 \\
\hline 45 & $\mathrm{H}$ & 3.950577 & -3.30098 & -0.29729 & 45 & $\mathrm{H}$ & 3.951124 & 3.300917 & 0.29733 \\
\hline 46 & $\mathrm{H}$ & 4.406304 & -1.73329 & 0.383494 & 46 & $\mathrm{H}$ & 4.407532 & 1.732959 & -0.38237 \\
\hline 47 & $\mathrm{H}$ & 2.484221 & 4.045865 & -0.69504 & 47 & $\mathrm{H}$ & 2.483594 & -4.04613 & 0.695519 \\
\hline 48 & $\mathrm{H}$ & 0.773142 & 3.792409 & -1.06223 & 48 & $\mathrm{H}$ & 0.772464 & -3.79258 & 1.062365 \\
\hline 50 & $\mathrm{H}$ & 2.009591 & 2.858382 & -1.9135 & 50 & $\mathrm{H}$ & 2.00879 & -2.85853 & 1.913782 \\
\hline
\end{tabular}

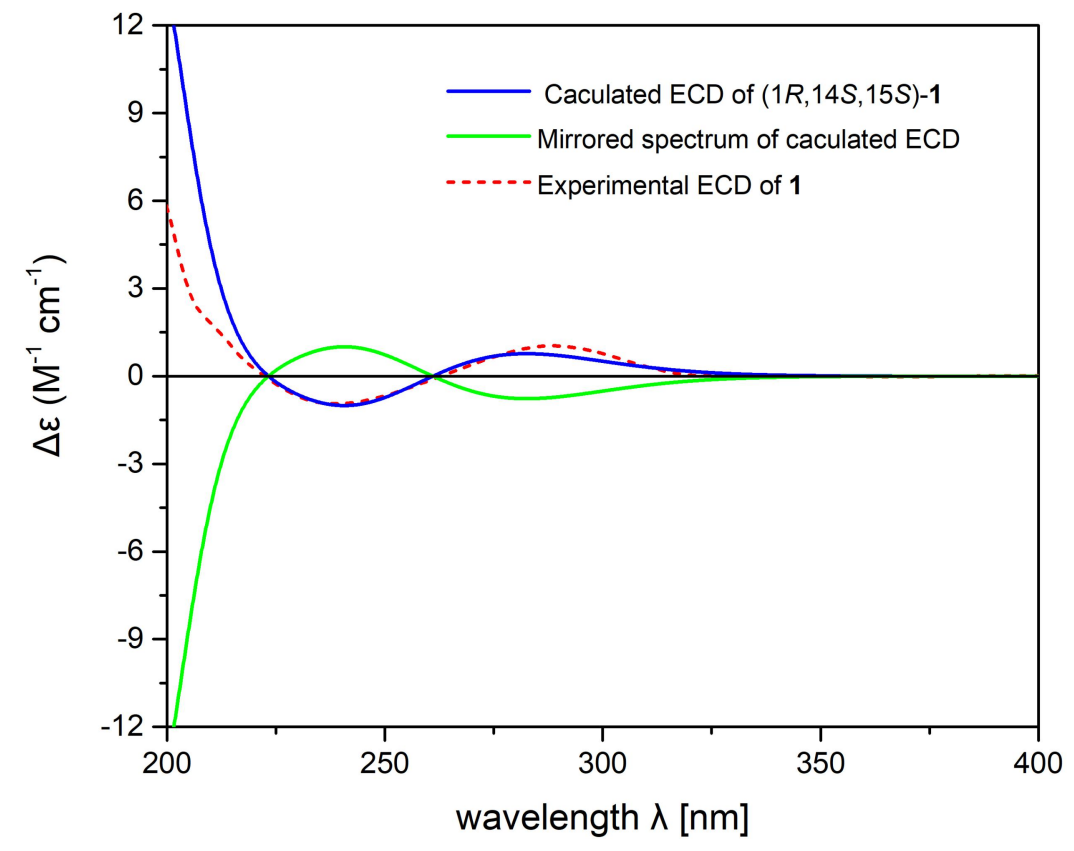

Figure S58. Experimental ECD spectrum of Sinuflexitone A (1) (dash red, $1 R, 14 S, 15 S$ ), calculated ECD spectrum of 1 (solid blue, $1 R, 14 S, 15 S$ ) and the enantiomer (solid green, $1 S, 14 R, 15 R$ ). 